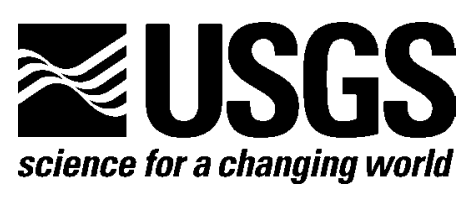

\title{
Magnetotelluric Data, San Luis Valley, Colorado
}

By Brian D. Rodriguez and Jackie M. Williams

Open-File Report 2007-1405

U.S. Department of the Interior U.S. Geological Survey 


\title{
U.S. Department of the Interior DIRK KEMPTHORNE, Secretary
}

\author{
U.S. Geological Survey \\ Mark D. Myers, Director
}

U.S. Geological Survey, Reston, Virginia 2008

For product and ordering information:

World Wide Web: http://www.usgs.gov/pubprod

Telephone: 1-888-ASK-USGS

For more information on the USGS - the Federal source for science about the Earth, its natural and living resources, natural hazards, and the environment:

World Wide Web: http://www.usgs.gov

Telephone: 1-888-ASK-USGS

Suggested citation:

Rodriguez, B.D. and Williams, J.M., 2008, Magnetotelluric data, San Luis Valley, Colorado: U.S. Geological Survey Open-File Report 2007-1405, 227 p.

Any use of trade, product, or firm names is for descriptive purposes only and does not imply endorsement by the U.S. Government.

Although this report is in the public domain, permission must be secured from the individual copyright owners to reproduce any copyrighted material contained within this report. 


\section{Contents}

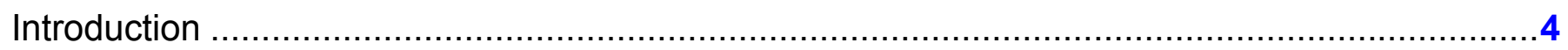

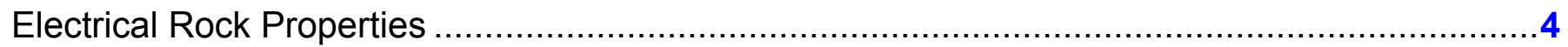

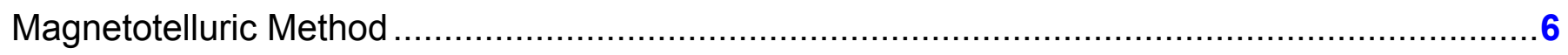

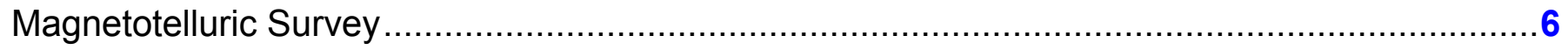

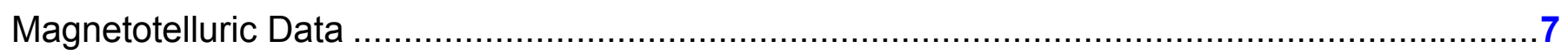

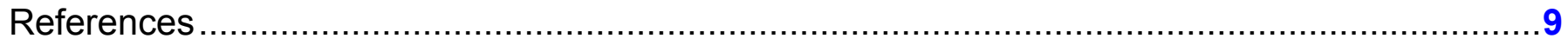

\section{Figure}

1. Index image showing magnetotelluric stations in the San Luis Valley Area .......................... 5

\section{Table}

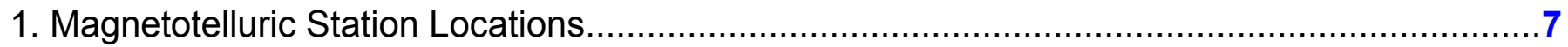

\section{Appendix}

Magnetotelluric Data Plots 


\section{Introduction}

The San Luis Valley region population is growing. Water shortfalls could have serious consequences. Future growth and land management in the region depend on accurate assessment and protection of the region's ground-water resources. An important issue in managing the ground-water resources is a better understanding of the hydrogeology of the Santa Fe Group and the nature of the sedimentary deposits that fill the Rio Grande rift, which contain the principal ground-water aquifers. The shallow unconfined aquifer and the deeper confined Santa Fe Group aquifer in the San Luis Basin are the main sources of municipal water for the region.

The U.S. Geological Survey (USGS) is conducting a series of multidisciplinary studies of the San Luis Basin located in southern Colorado. Detailed geologic mapping, high-resolution airborne magnetic surveys, gravity surveys, an electromagnetic survey (called magnetotellurics, or MT), and hydrologic and lithologic data are being used to better understand the aquifers. The MT survey primary goal is to map changes in electrical resistivity with depth that are related to differences in rock types. These various rock types help control the properties of aquifers. This report does not include any data interpretation. Its purpose is to release the MT data acquired at 24 stations shown in figure 1. Two of the stations were collected near Santa Fe, New Mexico, near deep wildcat wells. Well logs from those wells will help tie future interpretations of this data with geologic units from the Santa Fe Group sediments to Precambrian basement.

\section{Electrical Rock Properties}

Electromagnetic geophysical methods detect variations in the electrical properties of rocks. Electrical resistivity, or its inverse, electrical conductivity, is of particular interest. Electrical resistivity can be correlated with geologic units on the surface and at depth using lithologic logs to provide a three-dimensional (3-D) picture of subsurface geology. In the upper crust the resistivity of geologic units is largely dependent upon their fluid content, pore-volume porosity, interconnected fracture porosity, and conductive mineral content (Keller, 1989). Although there is not a one-to-one relationship between lithology and resistivity, there are general correlations that can be made using typical resistivity values even though values vary from one location to another (Palacky, 1987). Fluids within the pore spaces and fracture openings, especially if saline, can reduce resistivities in what would otherwise be a resistive rock matrix. Resistivities can also be lowered by electrically conductive clay minerals, graphitic carbon, and metallic mineralization. For example, it is common for altered volcanic rocks to contain replacement minerals that have resistivities ten times lower than those of the surrounding rocks (Nelson and Anderson, 1992). Fine-grained sediments, such as clay-rich alluvium, marine shales, and other mudstones, are normally conductive from a few ohm-meters (ohm-m) to a few tens of ohm-m (Palacky, 1987). Metamorphic rocks and unaltered, unfractured igneous rocks are normally moderately to highly resistive (hundreds to thousands of ohm-m). Carbonate rocks can also have high resistivities depending on their fluid content, porosity, and impurities (Keller, 1987). Fault zones may be moderately conductive (tens of ohm-m) when comprised of rocks fractured enough to have hosted fluid transport and consequent mineralogical alteration (Eberhart-Phillips and others, 1995). Higher subsurface temperatures cause higher ionic mobility that reduces rock resistivities (Keller, 1987). Tables of electrical resistivities for a variety of rocks, minerals, and geological environments may be found in Keller (1987) and Palacky (1987). 


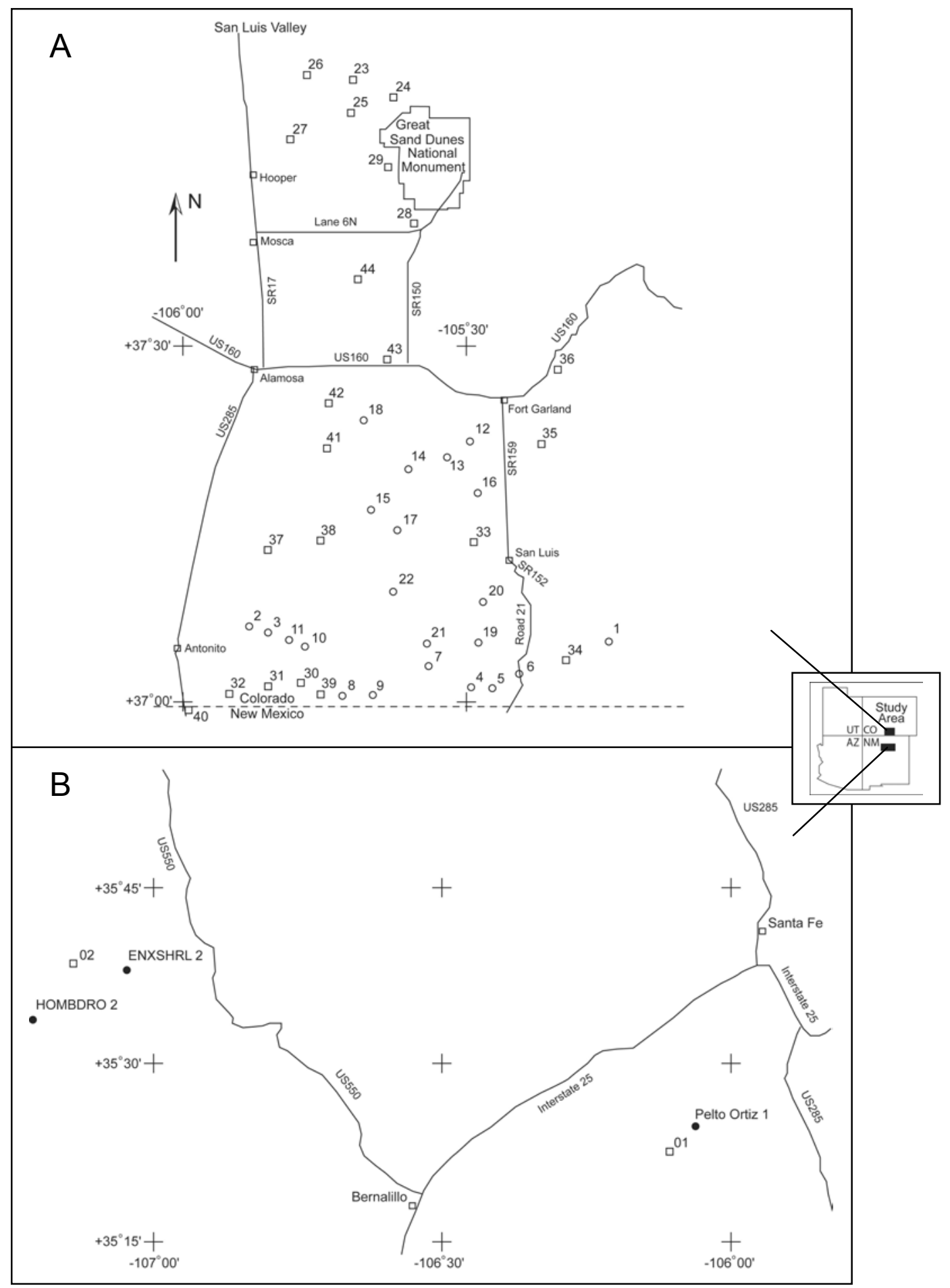

Figure 1. A. Magnetotelluric (MT) stations in the San Luis Valley Area. Open squares (23-44) are MT stations collected in July 2007. Open circles (1-22) are MT stations from Williams and Rodriguez (2007). B. MT stations (01-02) collected near Santa Fe, New Mexico. Solid black circles are deep wildcat wells. 


\section{Magnetotelluric Method}

The MT method is a passive-surface electromagnetic geophysical technique that measures variations in the Earth's natural electromagnetic fields to investigate the electrical resistivity structure of the subsurface from depths of tens of meters to tens of kilometers (Vozoff, 1991). Worldwide lightning activity at frequencies of 10,000 to $1 \mathrm{Hertz}(\mathrm{Hz})$ and geomagnetic micro-pulsations at frequencies of 1 to $0.001 \mathrm{~Hz}$ provide the majority of natural signal used by the MT method. A small amount of electromagnetic energy reflects and propagates vertically into the earth due to the resistivity contrast at the air-earth interface (Vozoff, 1972).

The natural fields are recorded in the $x y z$ direction for the magnetic field and the $x y$ direction for the electric field at the Earth's surface. The resulting time-series signals are used to derive tensor apparent-resistivities and phases by first converting them to complex cross-spectra using Fourier-transform techniques. Least squares, cross-spectral analysis (Bendat and Piersol, 1971) is used to solve for a tensor transfer function. Prior to conversion to apparent resistivity and phase, the tensor is normally rotated into principal directions that usually correspond to the direction of maximum and minimum apparent resistivity. For a two-dimensional (2-D) Earth, in which Earth's resistivity structure varies with depth and in one lateral direction, the MT fields can be decoupled into transverse-electric (TE) and transverse-magnetic (TM) modes; 2-D resistivity modeling is generally computed to fit both modes. When the geologic structures are assumed to be primarily 2-D, the MT data for the TE mode represents electric fields that are oriented parallel to geologic strike, and the data for the TM mode represents electric fields oriented perpendicular to strike.

The MT method is well suited for studying complicated geological environments because the electric and magnetic fields are sensitive to vertical and horizontal variations in resistivity. The method is capable of establishing whether the electromagnetic fields are responding to subsurface rock bodies of effectively 1-, 2-, or 3-dimensions. An introduction to the MT method and references for a more advanced understanding are contained in Dobrin and Savit (1988) and Vozoff (1991).

\section{Magnetotelluric Survey}

In July of 2006 we collected 22 MT stations in the San Luis Valley of south central Colorado (numbered 23-44, fig. 1). Two additional stations (01 and 02, fig. 1) were collected west of Santa Fe, New Mexico, near deep wildcat wells. Well logs from those wells will help tie future interpretations of this data with geologic units from the Santa Fe Group sediments to Precambrian basement. The station locations were chosen to constrain the geologic and hydrostratigraphic interpretation, for proximity to roads, and to avoid, where possible, electrical noise from power lines and vehicles. The MT data were collected with Electromagnetic Instruments, Inc., (EMI) MT-1 system (EMI, 1996). Horizontal electric fields were measured using three copper/copper sulfate porous-pot electrodes placed in an L-shaped array with dipole lengths of 30 meters $(\mathrm{m})$. The orthogonal magnetic fields in the direction of the electric-field measurement array were sensed using EMI's high-magnetic-permeability, mu-metal-cored induction coils. The MT data were recorded as non-remote referenced single stations.

Table 1 lists the 24 MT station locations as recorded using a GPS during field acquisition. Coordinates are referenced to the 1866 Clarke spheroid and North American 1927 Western United States datum. Longitude and latitude format is degrees, minutes, seconds. Station elevation is given in meters. Universal Transverse Mercator (UTM) Zone 13 Northing and 
Easting units are in meters. The accuracy of the $x, y$ component is about $\pm 5 \mathrm{~m}$. The accuracy of the $z$ component is about $\pm 10 \mathrm{~m}$. The $\mathrm{X}$ direction is given in degrees and defines the direction of the measured magnetic (Hx) and electric (Ex) fields.

Table 1. Magnetotelluric Station Locations

\begin{tabular}{|c|c|c|c|c|c|c|}
\hline Station & $\begin{array}{l}\text { Latitude } \\
\left(d^{\circ} \mathrm{m}^{\prime} \mathrm{s}^{\prime \prime}\right)\end{array}$ & $\begin{array}{l}\text { Longitude } \\
\left(d^{\circ} \mathrm{m}^{\prime} \mathrm{s}^{\prime \prime}\right)\end{array}$ & $\begin{array}{l}\text { Elevation } \\
(\mathrm{m})\end{array}$ & $\begin{array}{l}\text { Northing } \\
(\mathrm{m})\end{array}$ & $\begin{array}{l}\text { Easting } \\
(\mathrm{m})\end{array}$ & $\begin{array}{l}\text { X-Dir } \\
\text { (deg) }\end{array}$ \\
\hline 01 & $35^{\circ} 22^{\prime} 37.3^{\prime \prime}$ & $106^{\circ} 06^{\prime} 16.3^{\prime \prime}$ & 1905 & 3915216 & 399671 & 143 \\
\hline 02 & $35^{\circ} 38^{\prime} 29.1^{\prime \prime}$ & $107^{\circ} 08^{\prime} 05.3^{\prime \prime}$ & 1866 & 3946078 & 306708 & 64 \\
\hline 23 & $37^{\circ} 53^{\prime} 02.7^{\prime \prime}$ & $105^{\circ} 41^{\prime} 55.1^{\prime \prime}$ & 2336 & 4192978 & 438564 & 90 \\
\hline 24 & $37^{\circ} 51^{\prime} 29.2^{\prime \prime}$ & $105^{\circ} 37^{\prime} 37.3^{\prime \prime}$ & 2402 & 4190052 & 444841 & 165 \\
\hline 25 & $37^{\circ} 50^{\prime} 12.9^{\prime \prime}$ & $105^{\circ} 42^{\prime} 13.3^{\prime \prime}$ & 2331 & 4187750 & 438080 & 166 \\
\hline 26 & $37^{\circ} 53^{\prime} 25.7^{\prime \prime}$ & $105^{\circ} 46^{\prime} 52.1^{\prime \prime}$ & 2304 & 4193745 & 431314 & 149 \\
\hline 27 & $37^{\circ} 47^{\prime} 58.3^{\prime \prime}$ & $105^{\circ} 48^{\prime} 34.1^{\prime \prime}$ & 2297 & 4183676 & 428735 & 91 \\
\hline 28 & $37^{\circ} 40^{\prime} 52.7^{\prime \prime}$ & $105^{\circ} 35^{\prime} 27.1^{\prime \prime}$ & 2360 & 4170417 & 447898 & 181 \\
\hline 29 & $37^{\circ} 45^{\prime} 37.0^{\prime \prime}$ & $105^{\circ} 38^{\prime} 12.8^{\prime \prime}$ & 2331 & 4179204 & 443899 & 198 \\
\hline 30 & $37^{\circ} 02^{\prime} 04.4^{\prime \prime}$ & $105^{\circ} 47^{\prime} 30.4^{\prime \prime}$ & 2323 & 4098796 & 429580 & 249 \\
\hline 31 & $37^{\circ} 01^{\prime} 43.8^{\prime \prime}$ & $105^{\circ} 51^{\prime} 01.3^{\prime \prime}$ & 2326 & 4098205 & 424364 & 188 \\
\hline 32 & $37^{\circ} 01^{\prime} 07.8^{\prime \prime}$ & $105^{\circ} 55^{\prime} 03.3^{\prime \prime}$ & 2369 & 4097151 & 418374 & 127 \\
\hline 33 & $37^{\circ} 13^{\prime} 56.7^{\prime \prime}$ & $105^{\circ} 29^{\prime} 03.2^{\prime \prime}$ & 2403 & 4120563 & 457047 & 6 \\
\hline 34 & $37^{\circ} 04^{\prime} 00.7^{\prime \prime}$ & $105^{\circ} 19^{\prime} 19.1^{\prime \prime}$ & 2873 & 4102135 & 471376 & 265 \\
\hline 35 & $37^{\circ} 22^{\prime} 14.7^{\prime \prime}$ & $105^{\circ} 21^{\prime} 52.5^{\prime \prime}$ & 2516 & 4135861 & 467719 & 291 \\
\hline 36 & $37^{\circ} 28^{\prime} 33.3^{\prime \prime}$ & $105^{\circ} 20^{\prime} 11.2^{\prime \prime}$ & 2475 & 4147518 & 470252 & 206 \\
\hline 37 & $37^{\circ} 13^{\prime} 15.9^{\prime \prime}$ & $105^{\circ} 50^{\prime} 57.8^{\prime \prime}$ & 2369 & 4119532 & 424641 & 215 \\
\hline 38 & $37^{\circ} 14^{\prime} 06.8^{\prime \prime}$ & $105^{\circ} 45^{\prime} 23.3^{\prime \prime}$ & 2298 & 4121031 & 432896 & 247 \\
\hline 39 & $37^{\circ} 01^{\prime} 07.6 "$ & $105^{\circ} 45^{\prime} 23.3^{\prime \prime}$ & 2297 & 4097019 & 432705 & 101 \\
\hline 40 & $36^{\circ} 59^{\prime} 46.9^{\prime \prime}$ & $105^{\circ} 59^{\prime} 25.2^{\prime \prime}$ & 2448 & 4094723 & 411876 & 296 \\
\hline 41 & $37^{\circ} 21^{\prime} 53.5^{\prime \prime}$ & $105^{\circ} 44^{\prime} 39.5^{\prime \prime}$ & 2300 & 4135404 & 434089 & 126 \\
\hline 42 & $37^{\circ} 25^{\prime} 43.9^{\prime \prime}$ & $105^{\circ} 44^{\prime} 31.5^{\prime \prime}$ & 2301 & 4142505 & 434342 & 244 \\
\hline 43 & $37^{\circ} 29^{\prime} 21.7^{\prime \prime}$ & $105^{\circ} 38^{\prime} 19.4^{\prime \prime}$ & 2318 & 4149150 & 443533 & 273 \\
\hline 44 & $37^{\circ} 36^{\prime} 07.2^{\prime \prime}$ & $105^{\circ} 41^{\prime} 28.6^{\prime \prime}$ & 2294 & 4161677 & 438980 & 162 \\
\hline
\end{tabular}

\section{Magnetotelluric Data}

The recorded time-series data were transformed to the frequency domain and processed to determine the impedance tensor, which is used to derive apparent resistivities and phases at each site. Rotation of the impedance tensor allows for decoupling into the TE and TM modes. The data provided here have not been rotated from the original acquisition orientation (X-dir) listed in Table 1 above. During the analysis and interpretation process, each station will be rotated to a fixed angle determined by the given nominal profile orientation. Cross-power files were sorted to select optimal signal-to-noise time-series data sets (see Appendix 1).

The effects of near-surface resistivity anomalies can cause what are known as "static shifts" in the data (Sternberg and others, 1988). Cultural features also can affect the measured magnetotelluric responses. These features include fences, pipelines, communication lines, railways, and other manmade conductors. 
The figures in appendix 1 represent the field-processed MT data for each station, after the time-series data were converted to the frequency domain and the tensor-transfer function was developed.

For each station, nine separate plots are given:

1. Apparent Resistivity ( $\mathrm{x}$ and o symbols are $x y$ and $y x$ components)

2. Impedance Phase ( $\mathrm{x}$ and o symbols are $x y$ and $y x$ components)

3. Rotation Angle

4. Impedance Skew

5. Multiple Coherency ( $\mathrm{x}$ and o symbols are $x y$ and $y x$ components)

6. Impedance Polar Plots

7. Tipper Magnitude

8. Tipper Strike

9. HzHx (x symbol) and HzHy (o symbol) Coherency

Error bars (],[) on the Apparent Resistivity, Impedance Phase, Skew, Tipper Magnitude, and Tipper Strike plots represent probable errors within one standard deviation of the sample variance (Gamble and others, 1979).

Apparent resistivity is the approximate ratio of the electric-field strength to the magneticfield strength at a given frequency. The impedance phase is proportional to the slope of the apparent resistivity curve on a log-log plot, but from baselines at \pm 45 degrees (Vozoff, 1991). A measure of the dimensionality for MT data is provided by the impedance skew of the impedance tensor (Vozoff, 1972). If the effective measured resistivity response to the geology beneath a MT station truly is one or two dimensional, then the skew will be zero. Instrumental and environmental sources of electrical noise can cause non-zero skew values. Skew values typically are small (about 0.1) for relatively low-noise recordings. Higher skews (above 0.2) are an indication of either the resistivity response to 3-D geology or higher levels of noise. Manmade electrical noise, such as power lines, power generators, and moving vehicles and trains, can have a negative effect on MT data quality. All of these local disturbances can produce incoherent noise that mainly affects frequencies above $1 \mathrm{~Hz}$. Other manmade electrical noise, such as direct-current electric trains and active cathodic protection of pipelines, produces coherent electromagnetic signals that mainly affect frequencies below $1 \mathrm{~Hz}$.

In the survey area, noise from small power lines and small moving vehicles was negligible at distances greater than $0.4 \mathrm{~km}$ from the noise source. Power-line signal levels were measured at each site and were typically less than 20 percent of the maximum recordable signals. Noise from larger power lines, power generators, pipelines, and trains was negligible at distances greater than $5 \mathrm{~km}$. Local lightning, wind, and rainstorms may also degrade data quality. Burying the magnetic induction coils and the electric dipole wires minimized wind noise.

Predicted values of the electric field can be computed from the measured values of the magnetic field (Vozoff, 1991). The coherence of the predicted electric field with the measured electric field is a measure of the signal-to-noise ratio provided in the multiple coherency plots. Values are normalized between 0 and 1; values at 0.5 signify signal levels equal to noise levels. For this data set, coherencies were generally at an acceptable level, except at times in the frequency ranges of about 0.1 to $5 \mathrm{~Hz}$ (traditionally referred to as the "dead band").

The field-processed MT data include some scatter and poor signal-to-noise ratios. Spectral results were inspected visually for noisy data, and the best signal-to-noise field data were combined into the final plots. 
The magnetotelluric impedance polar plots provide a measure of MT data dimensionality (Reddy and others, 1977). For 1-D resistivity structures, the principal impedance polar diagram (dashed line) is a circle. For 2-D or 3-D resistivity structures, the principal impedance polar diagram (dashed line) elongates either parallel or perpendicular to strike direction. Over resistors, the principal impedance polar diagram elongates perpendicular to strike direction, and over conductors, it elongates parallel to strike direction. For 2-D resistivity structures, the additional impedance polar diagram (solid line) attains the shape of a symmetric clover leaf. For 3-D resistivity structures, the additional impedance polar diagram (solid line) elongates in one direction, and its amplitude is comparable to that of the principal impedance polar diagram (dashed line).

The magnetotelluric "tipper" is calculated from the vertical component of the magnetic field. The tipper magnitude is a measure of the "tipping" of the magnetic field out of the horizontal plane (Vozoff, 1991). It will equal zero for the 1-D case. Typically, tipper value increases from 0.1 to 0.5 and seldom approaches 1 , as it responds primarily to vertical and subvertical structures. The tipper strike is used to help resolve the 90-degree ambiguity in the impedance rotation angle. The HzHx and HzHy coherency is a measure of the signal-to-noise ratio of the vertical magnetic field with respect to each of the orthogonal, horizontal magneticfield directions. Values are normalized between 0 and 1; values at 0.5 signify signal levels equal

to noise levels. These three-component magnetic-field coherencies provide a check on the signalto-noise ratio of the measured values in the tipper magnitude and tipper strike plots.

\section{References}

Bendat, J.S., and Piersol, A.G., 1971, Random data analysis and measurement procedures: New York, Wiley Interscience, 407 p.

Dobrin, M.D., and Savit, C.H., 1988, Introduction to geophysical prospecting (4th ed.): New York, McGraw-Hill, 867 p.

Eberhart-Phillips, Donna, Stanley, W.D., Rodriguez, B.D., and Lutter, W.J., 1995, Surface seismic and electrical methods to detect fluids related to faulting: Journal of Geophysical Research, v. 100, no. B7, p. 12,919-12,936.

EMI, Inc., 1996, MT-1 magnetotelluric system operation manual, version 3.2: Richmond, Calif., ElectroMagnetic Instruments, Inc., 220 p.

Gamble, T.D., Goubau, W.M., and Clarke, J., 1979, Error analysis for remote reference magnetotellurics: Geophysics, v. 44, no. 5, p. 959-968.

Keller, G.V., 1987, Rock and mineral properties, in Nabighian, M.N., ed., Electromagnetic methods in applied geophysics theory: Tulsa, Okla., Society of Exploration Geophysicists, v. 1, p. 13-51.

Keller, G.V., 1989, Electrical properties, in Carmichael, R.S., ed., Practical handbook of physical properties of rocks and minerals: Boca Raton, Fla., CRC Press, p. 359-427. 
Nelson, P.H., and Anderson, L.A., 1992, Physical properties of ash flow tuff from Yucca Mountain, Nevada: Journal of Geophysical Research, v. 97, no. B5, p. 6,823-6,841.

Palacky, G.J., 1987, Resistivity characteristics of geologic targets, in Nabighian, M.N., ed., Electromagnetic methods in applied geophysics theory: Tulsa, Okla., Society of Exploration Geophysicists, v. 1, p. 53-129.

Reddy, I.K., Rankin, David, and Phillips, R.J., 1977, Three dimensional modelling in magnetotelluric and magnetic variational sounding: Geophysics Journal of the Royal Astronomical Society, v. 51, p. 313-325.

Sternberg, B.K., Washburne, J.C., and Pellerin, Louise, 1988, Correction for the static shift in magnetotellurics using transient electromagnetic soundings: Geophysics, v. 53, p. 1,4591,468 .

Vozoff, Keeva, 1972, The magnetotelluric method in the exploration of sedimentary basins: Geophysics, v. 37, p. 980-1041.

Vozoff, Keeva, 1991, The magnetotelluric method, in Nabighian, M.N., Electromagnetic methods in applied geophysics: Tulsa, Okla., Society of Exploration Geophysicists, v. 2, pt. B, p. 641-711.

Williams, J.M., and Rodriguez, B.D., 2007, Magnetotelluric data, southern San Luis Valley, Colorado: U.S. Geological Survey Open-File Report 2007-1291, 208 p. 


\section{Appendix 1 Magnetotelluric Data Plots}

There are nine separate plots for each station:

1. Apparent Resistivity for the $\mathrm{x}-\mathrm{y}$ direction ( $\mathrm{x}$ symbol) and $\mathrm{y}-\mathrm{x}$ direction (o symbol) modes

2. Impedance Phase for the $\mathrm{x}-\mathrm{y}$ direction ( $\mathrm{x}$ symbol) and $\mathrm{y}-\mathrm{x}$ direction (o symbol) modes

3. Rotation Angle for the impedance tensor (corresponds to the $\mathrm{x}-\mathrm{y}$ direction)

4. Impedance Skew for the impedance tensor

5. Multiple Coherency for the $\mathrm{x}-\mathrm{y}$ direction ( $\mathrm{x}$ symbol) and $\mathrm{y}-\mathrm{x}$ direction (o symbol) modes of the electric field

6. Impedance Polar Plots (at 12 selected frequencies)

7. Tipper Magnitude for the vertical magnetic field

8. Tipper Strike for the vertical magnetic field

9. HzHx (x symbol) and HzHy (o symbol) Coherency

plots.

Refer to the "Magnetotelluric Data" section in this report for an explanation of these 


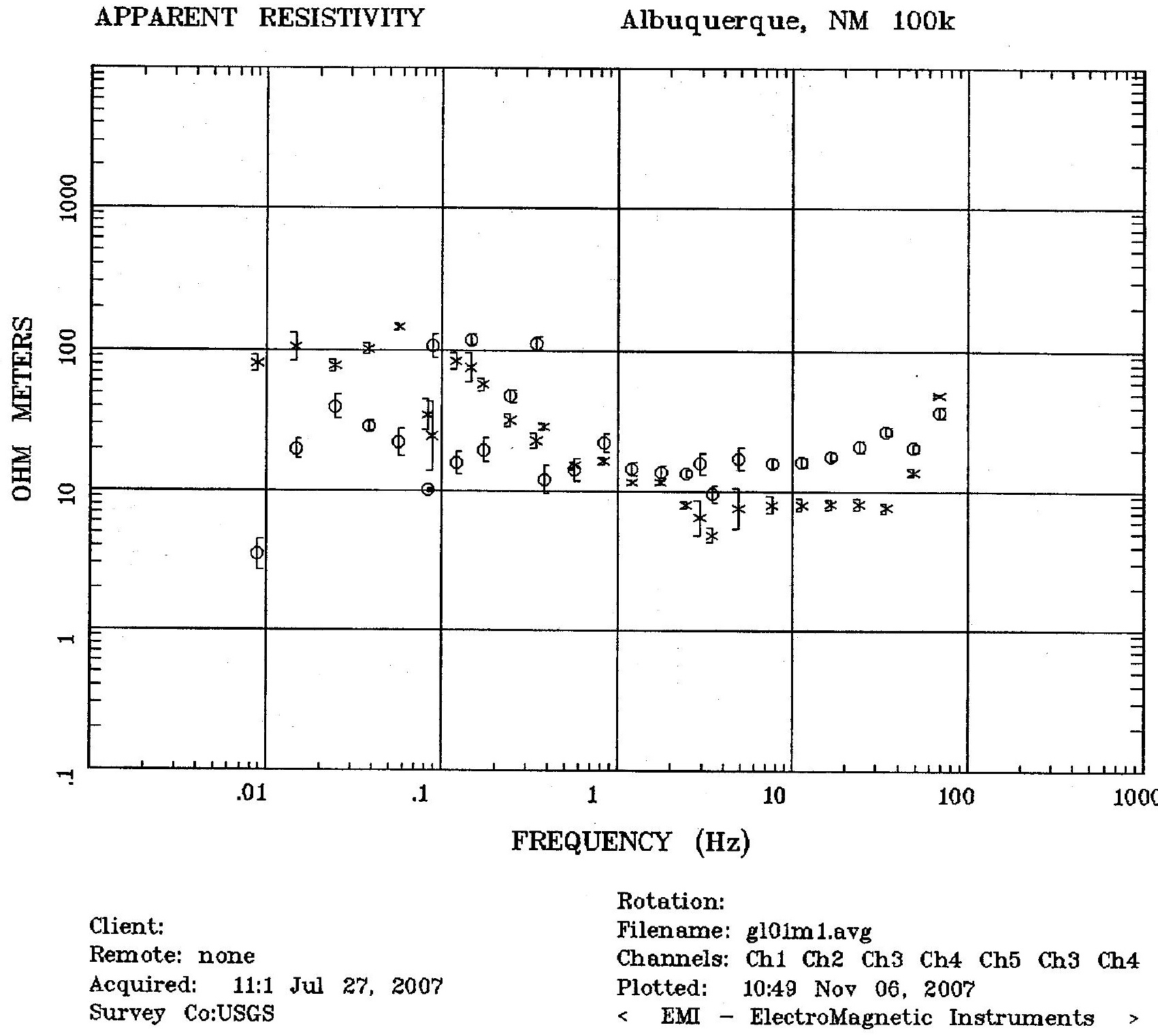


Albuquerque, NM 100k

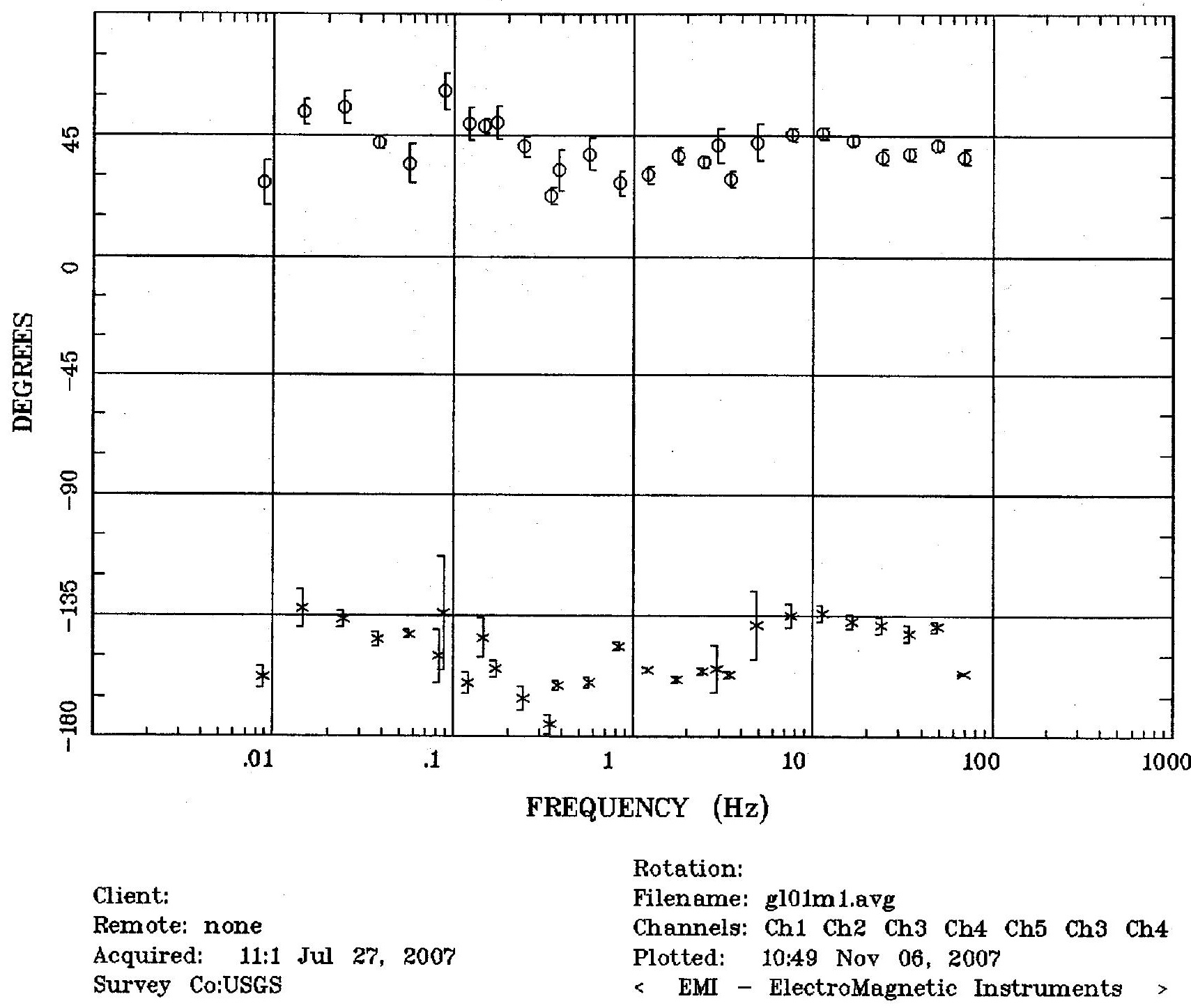


ROTATION ANGLE

Albuquerque, NM 100k

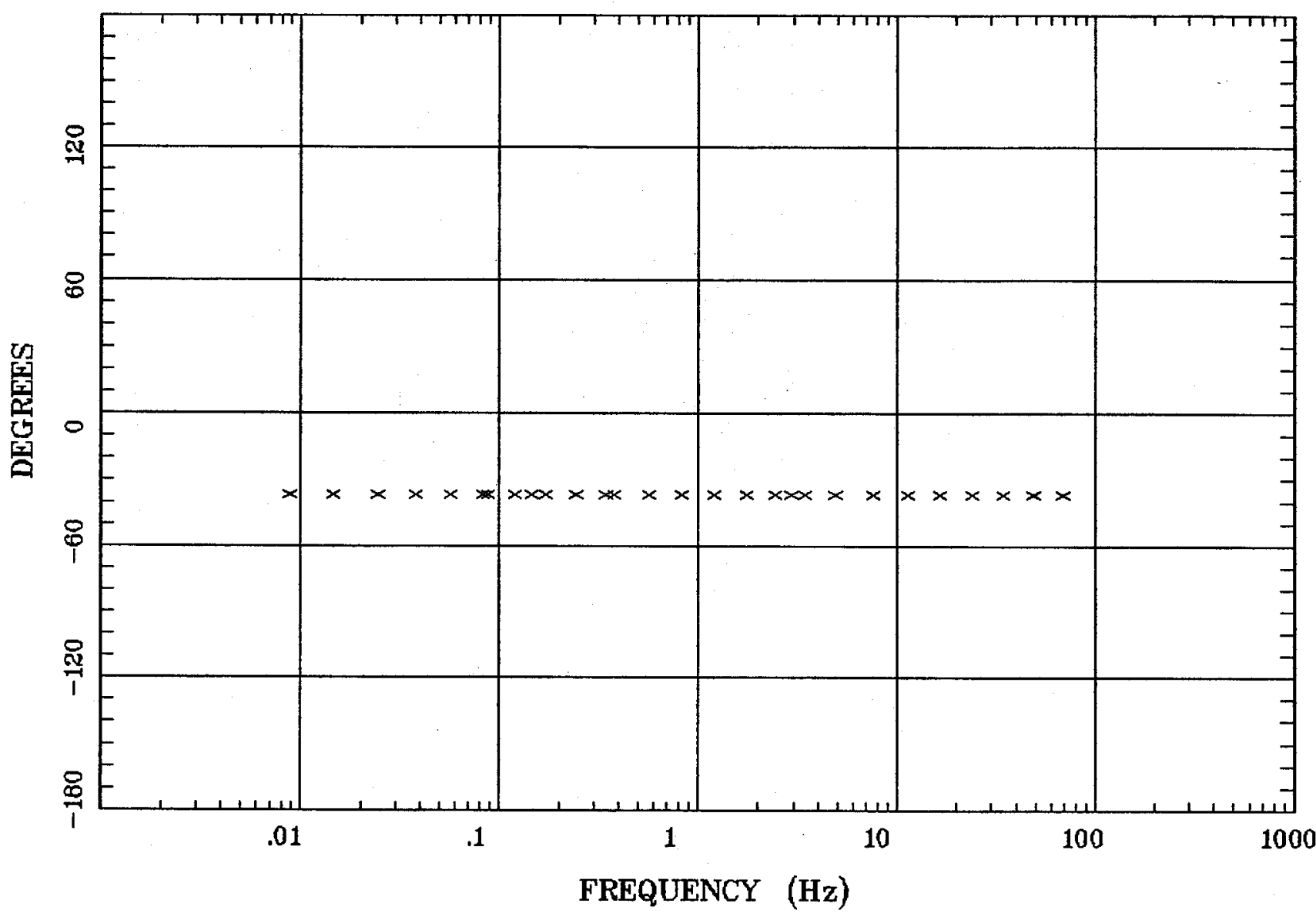

Client:

Remate: none

Acquired: 11:1 Jul 27, 2007

Survey Co:USGS
Rotation:

Filename: gl01m1.avg

Channels: Ch1 Ch2 Ch3 Ch4 Ch5 Ch3 Ch4

Plotted: $10: 49$ Nov 06, 2007

< EMI - ElectroMagnetic Instruments 
Albuquerque, NM 100k

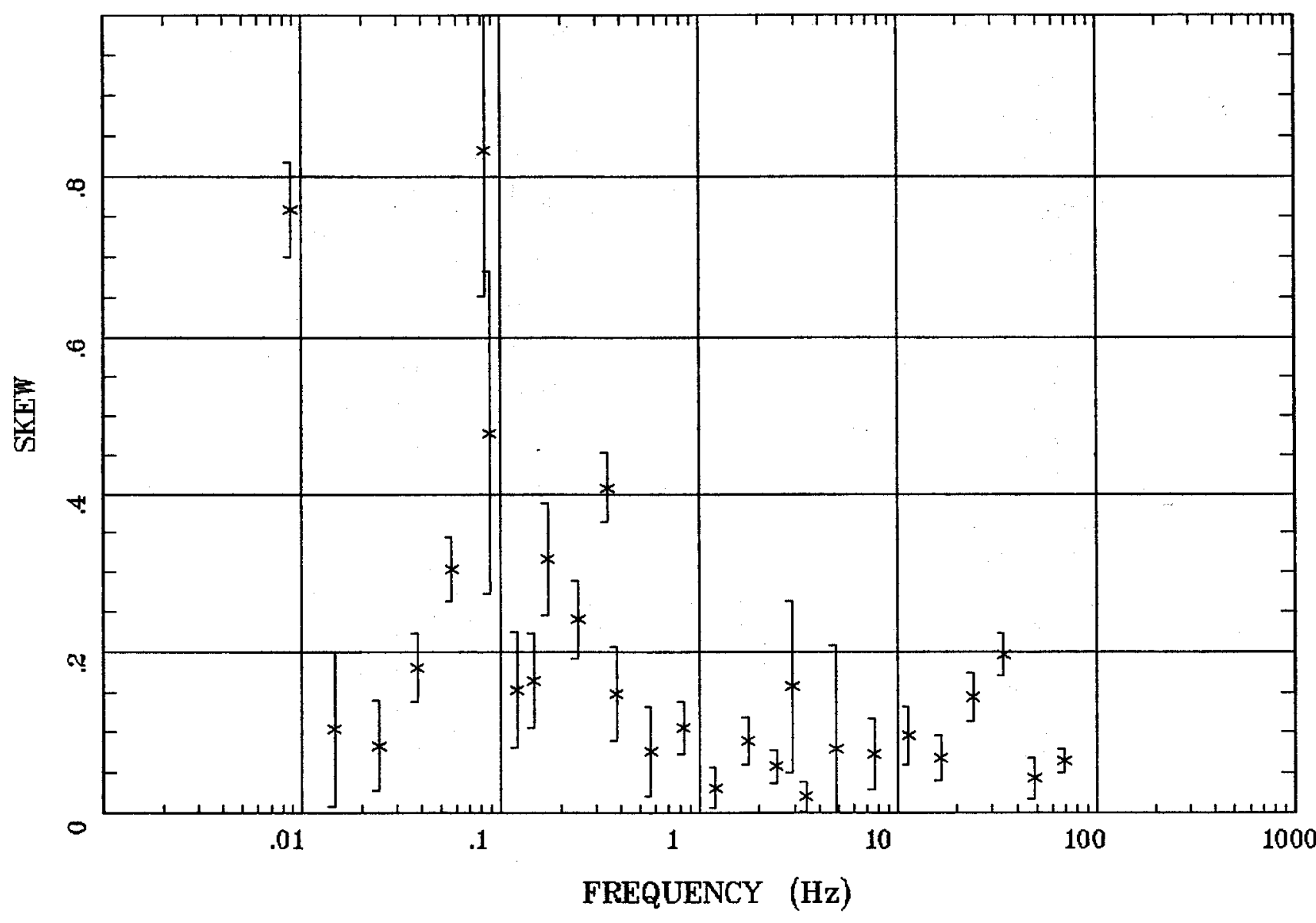

Client:

Remote: none Acquired: 11:1 Jul 27, 2007 Survey Ca:USGS
Rotation:

Filename: gl01m1.avg

Channels: Ch1 Ch2 Ch3 Ch4 Ch5 Ch3 Ch4

Plotted: 10:49 Nov 06, 2007

< EMI - ElectroMagnetic Instruments 


\section{Station 01}

E MULT Coh.

Albuquerque, NM $100 \mathrm{k}$

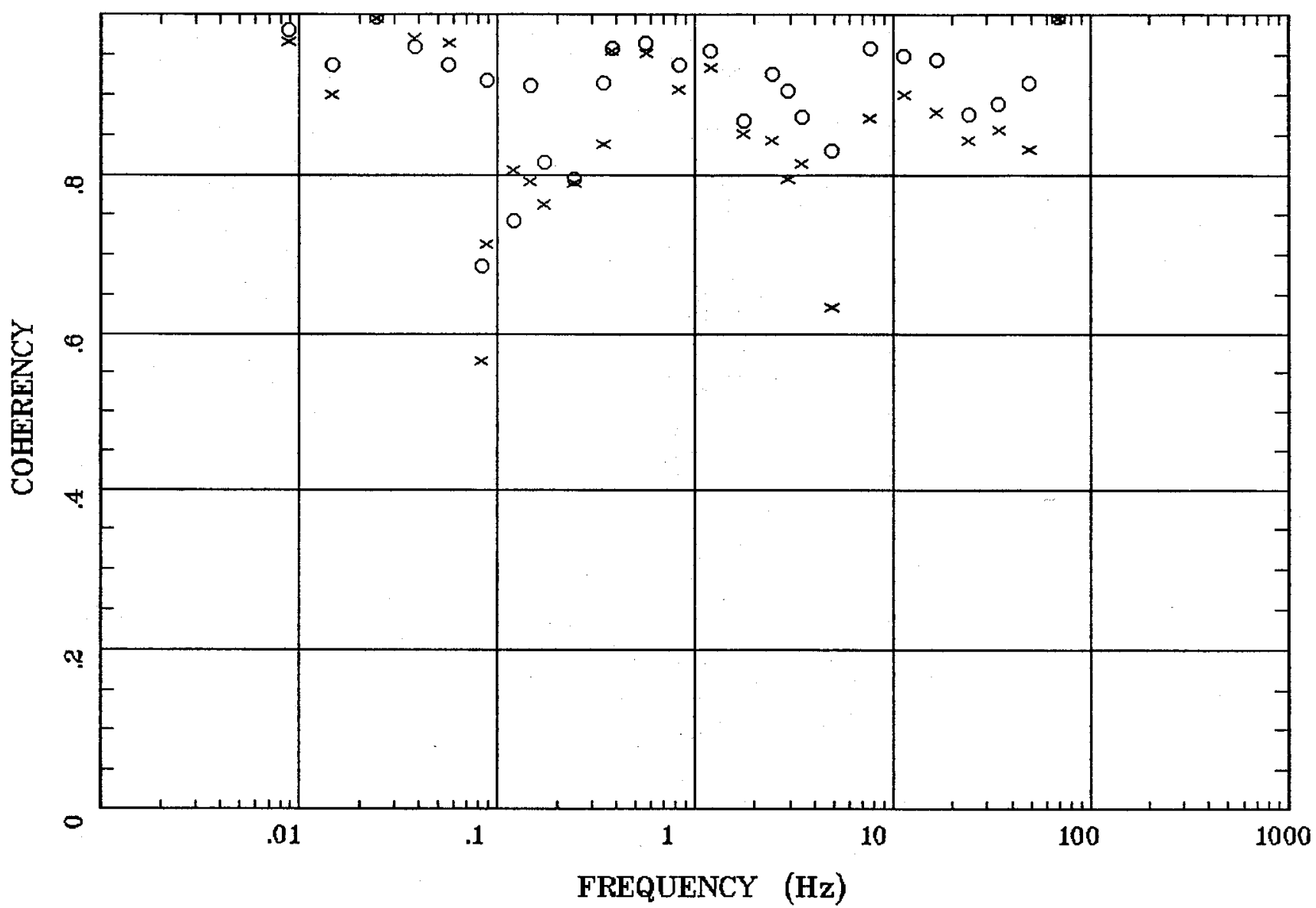

Client:

Remote: none

Acquired: 11:1 Jul 27, 2007

Surrey Co:USGS

Rotation:

Filename: glo1m1.avg

Channels: Ch1 Ch2 Ch3 Ch4 Ch5 Ch3 Ch4

Plotted: 10:49 Nov 06, 2007

< EMI - ElectroMagnetic Instruments > 


\section{Station 01}

POLAR PLOTS

Albuquerque, NM 100k

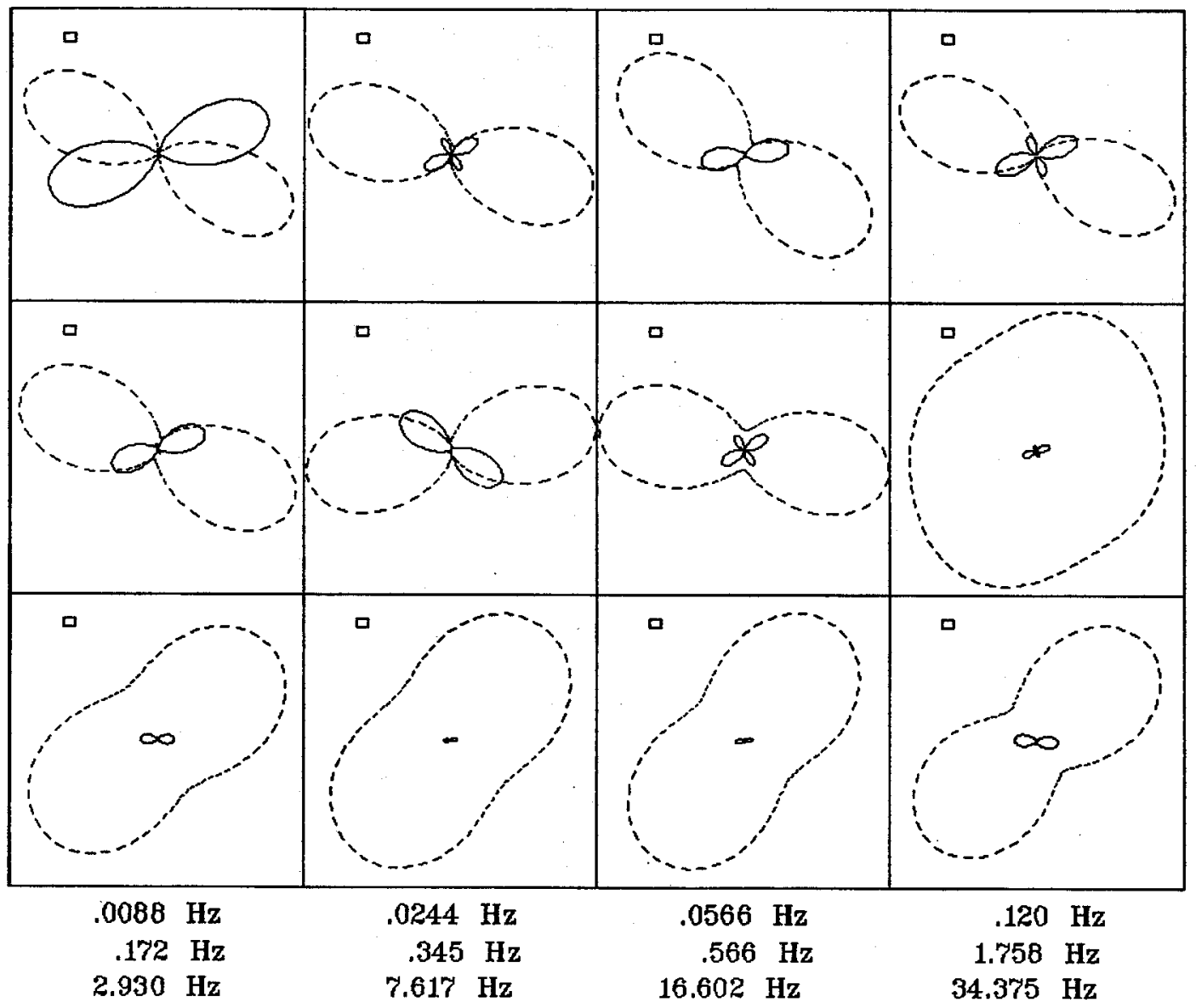

Client:

Remote: none

Acquired: 11:1 Jul 27, 2007 Survey Co:USGS
Rotation:

Filename: glotm1.avg

Channels: Ch1 Ch2 Ch3 ch4 Ch5 Ch3 Ch4 Plotted: 10:49 Nov 06, 2007

< EMI - ElectroMagnetic Instruments > 
Albuquerque, NM $100 \mathrm{k}$

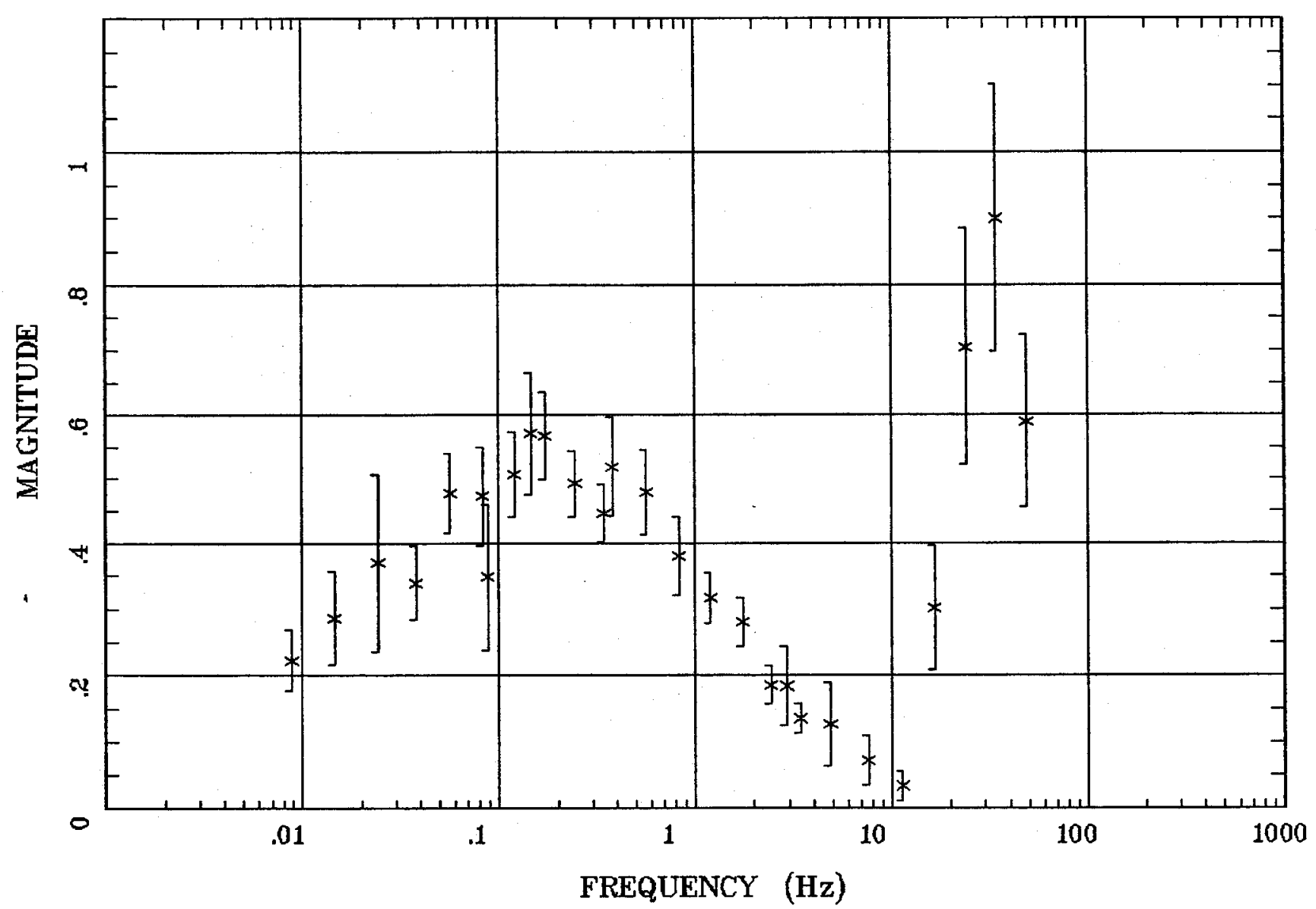

Client:

Remote: none

Acquired: 11:1 Jul 27, 2007 Survey Co:USGS
Rotation:

Filename: glo1m1.avg

Channels: Ch1 Ch2 Ch3 Ch4 Ch5 Ch3 Ch4

Plotted: $10: 49$ Nov 06, 2007

< EMI - ElectroMagnetic Instruments > 
Albuquerque, NM 100k

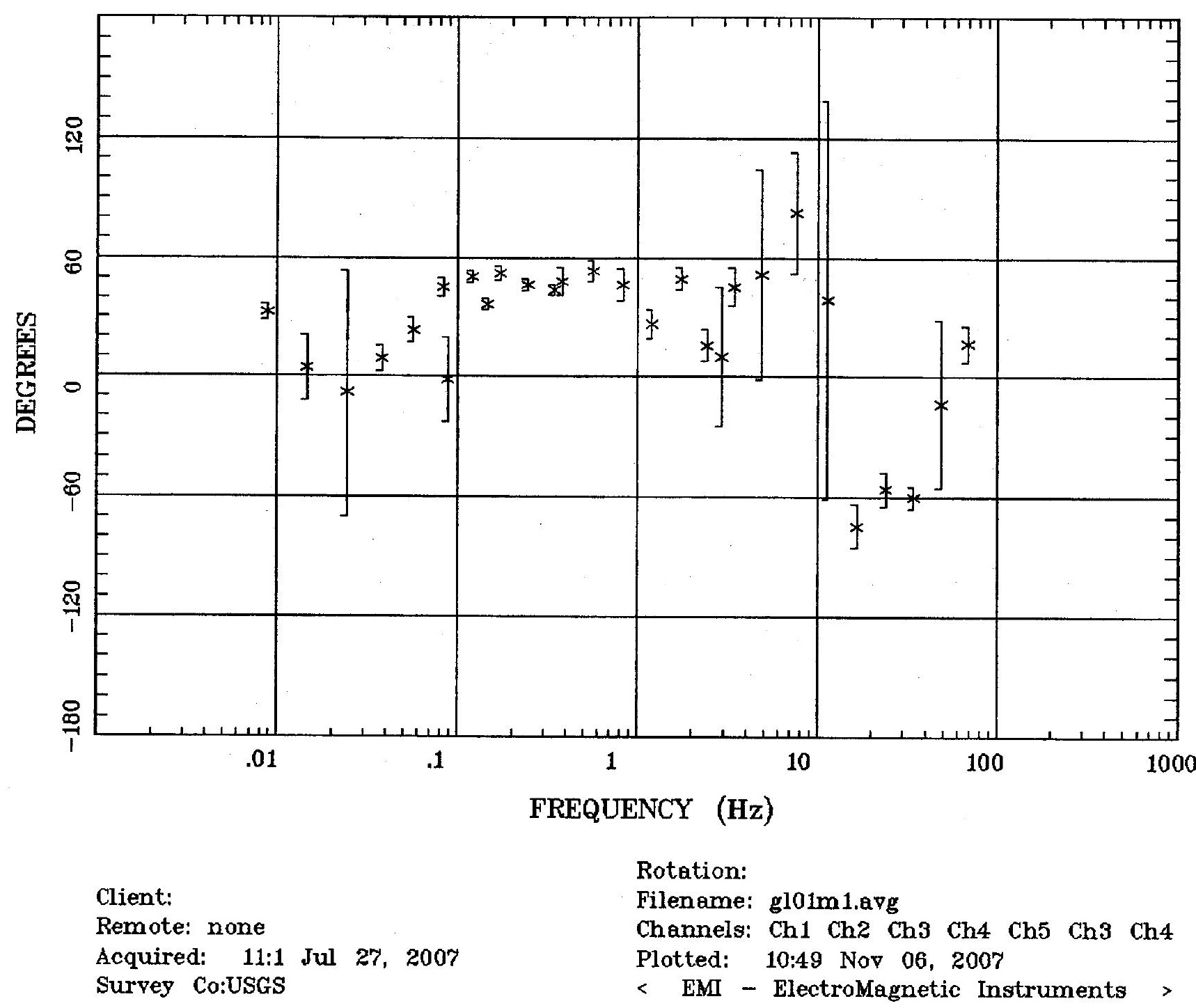


HzHx.x Coh HzHy.o

Albuquerque, NM 100k

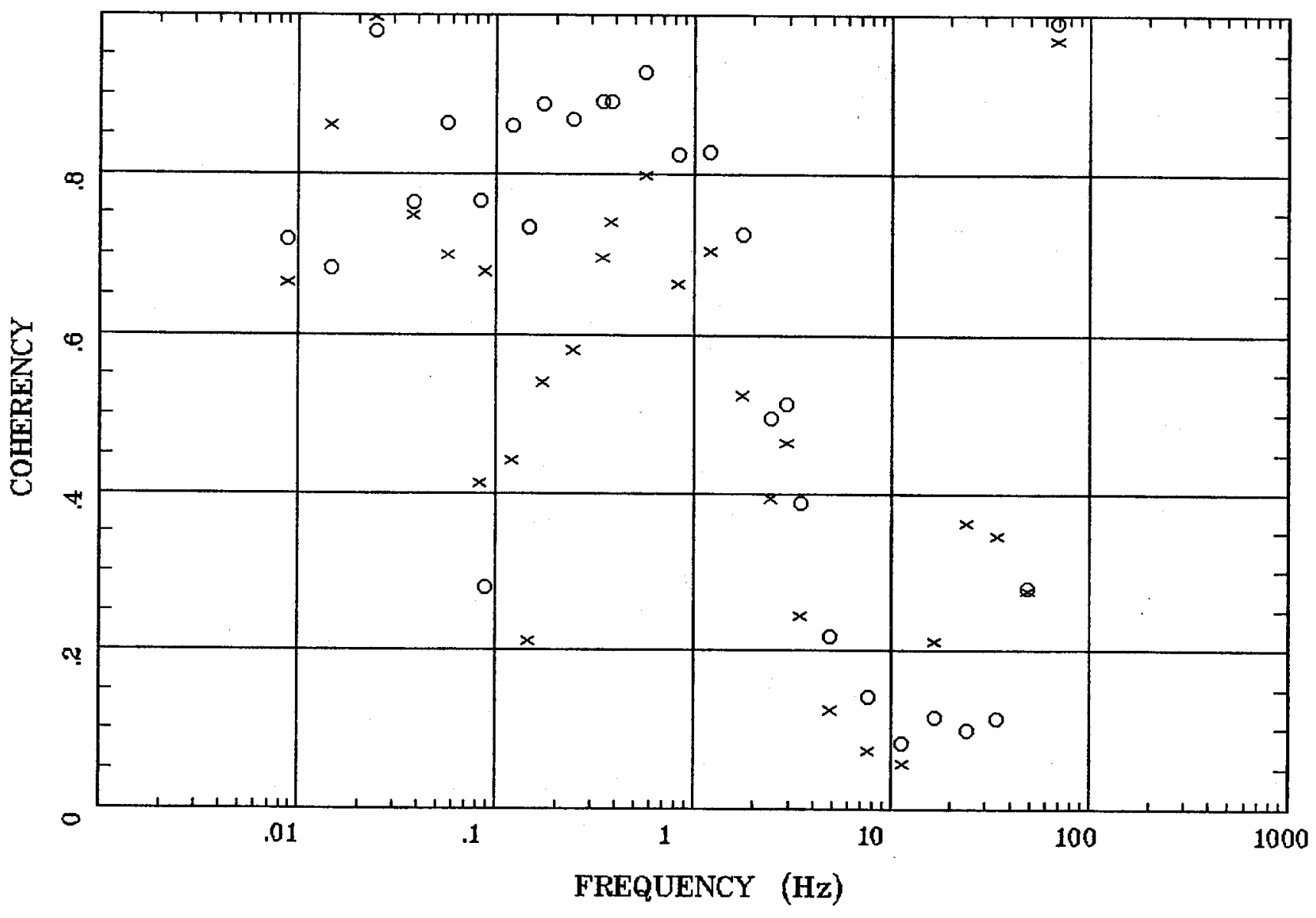

Client:

Remote: none Acquired: 11:1 Jul 27, 2007 Survey Co:USGS
Rotation:

Filename: glo1m1.evg

Channels: Ch1 Ch2 Ch3 Ch4 Ch5 Ch3 Ch4

Plotted: 10:49 Nov 06, 2007

< EMI - ElectroMagnetic Instruments 


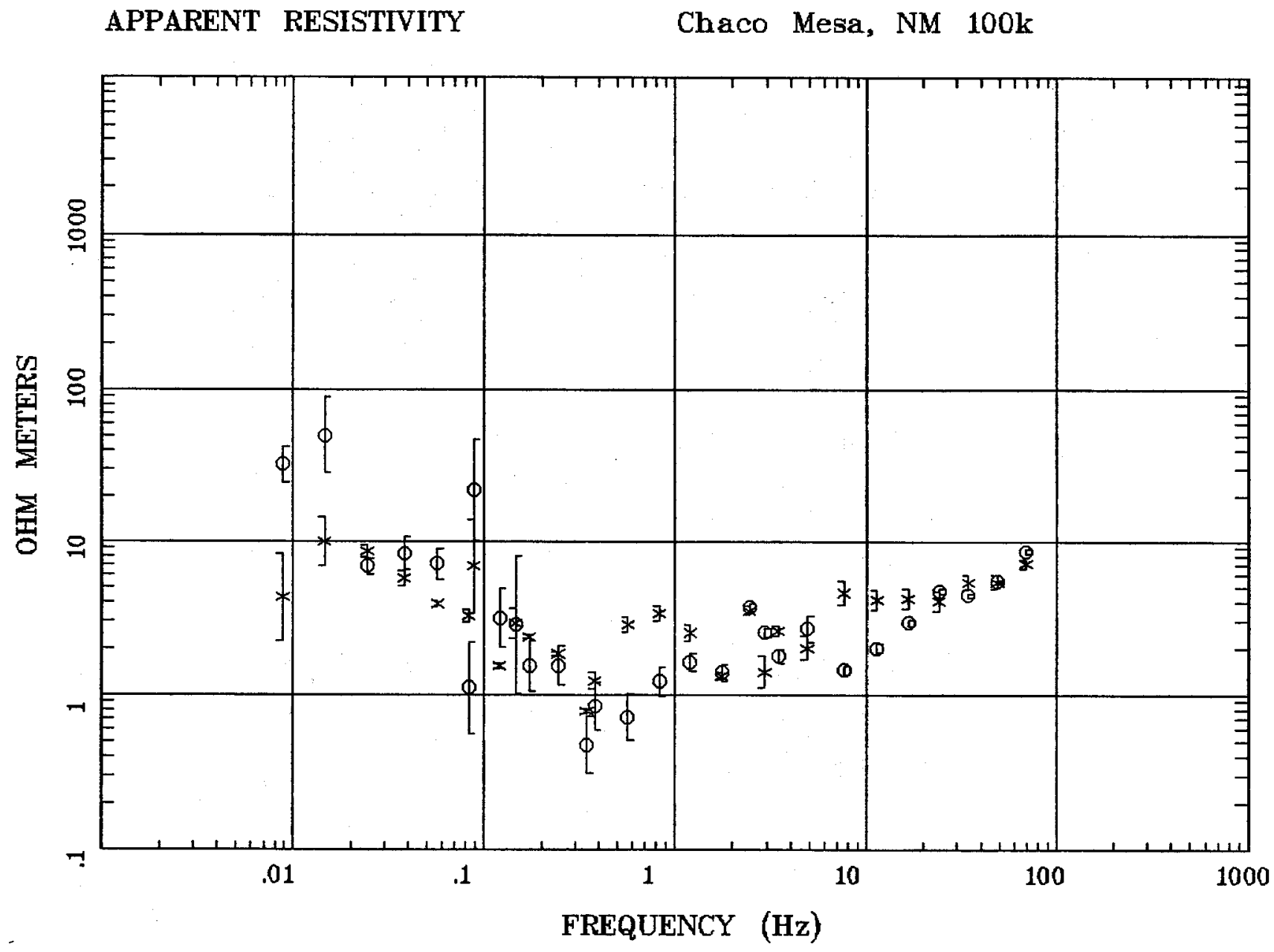

Client:

Remate: none

Acquired: 10:4 Jul 28, 2007

Survey Co:USGS
Rotation:

Filename: glozm1.avg

Channels: Ch1 Ch2 Ch3 Ch4 Ch5 Ch3 Ch4 Plotted: 10:50 Nop 06, 2007

< EMI - ElectroMagnetic Instruments > 
IMPEDANCE PHASE

Chaco Mesa, NM 100k

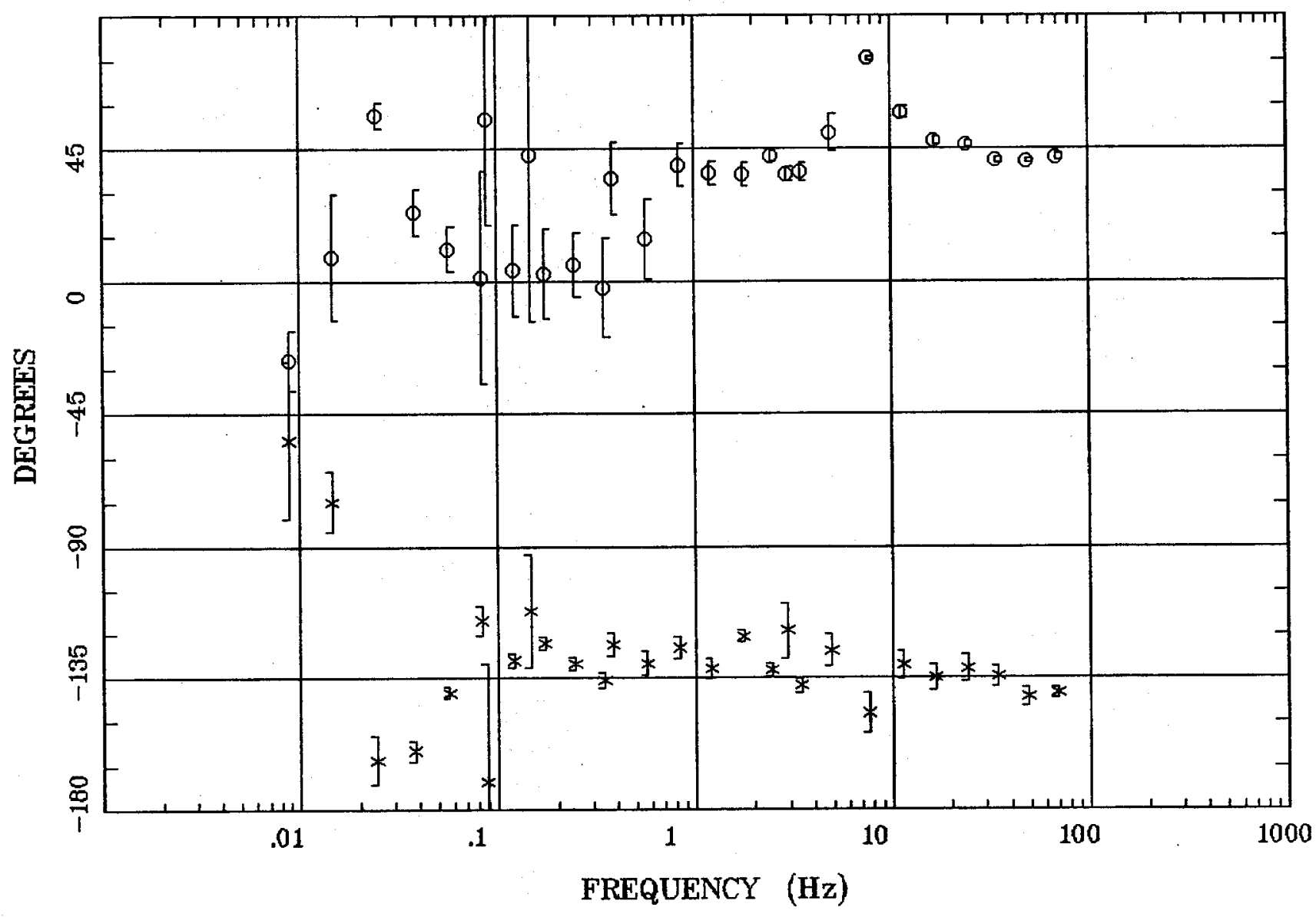

Client:

Remote: none

Acquired: 10:4 Jul 28, 2007

Survey Co:USGS
Rotation:

Filename: glozm 1.avg

Channels: Ch1 Ch2 Ch3 Ch4 Ch5 Ch3 Ch4

Plotted: 10:50 Nov 06, 2007

< EMI - ElectroMagnetic Instruments > 
ROTATION ANGLE

Chaco Mesa, NM $100 \mathrm{k}$

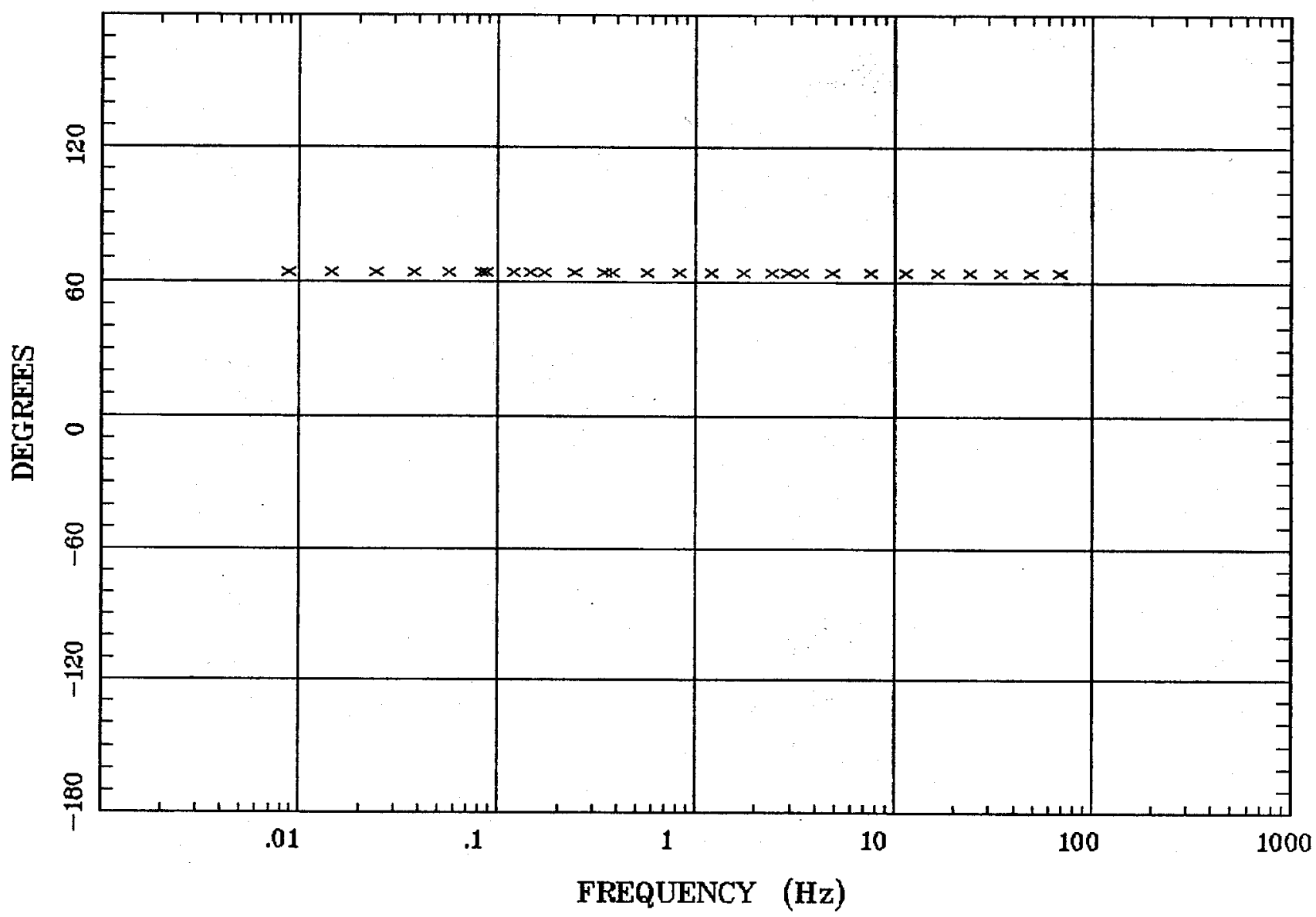

client:

Remote: none

Acquired: 10:4 Jul 28, 2007

Survey Co:USGS
Rotation:

Filename: gl02m1.avg

Channels: Ch1 Ch2 Ch3 Ch4 Ch5 Ch3 Ch4

Plotted: $10: 50$ Nov 06, 2007

< EMI - ElectroMagnetic Instruments > 
Station 02

IMPEDANCE SKEW

Chaco Mesa, NM 100k

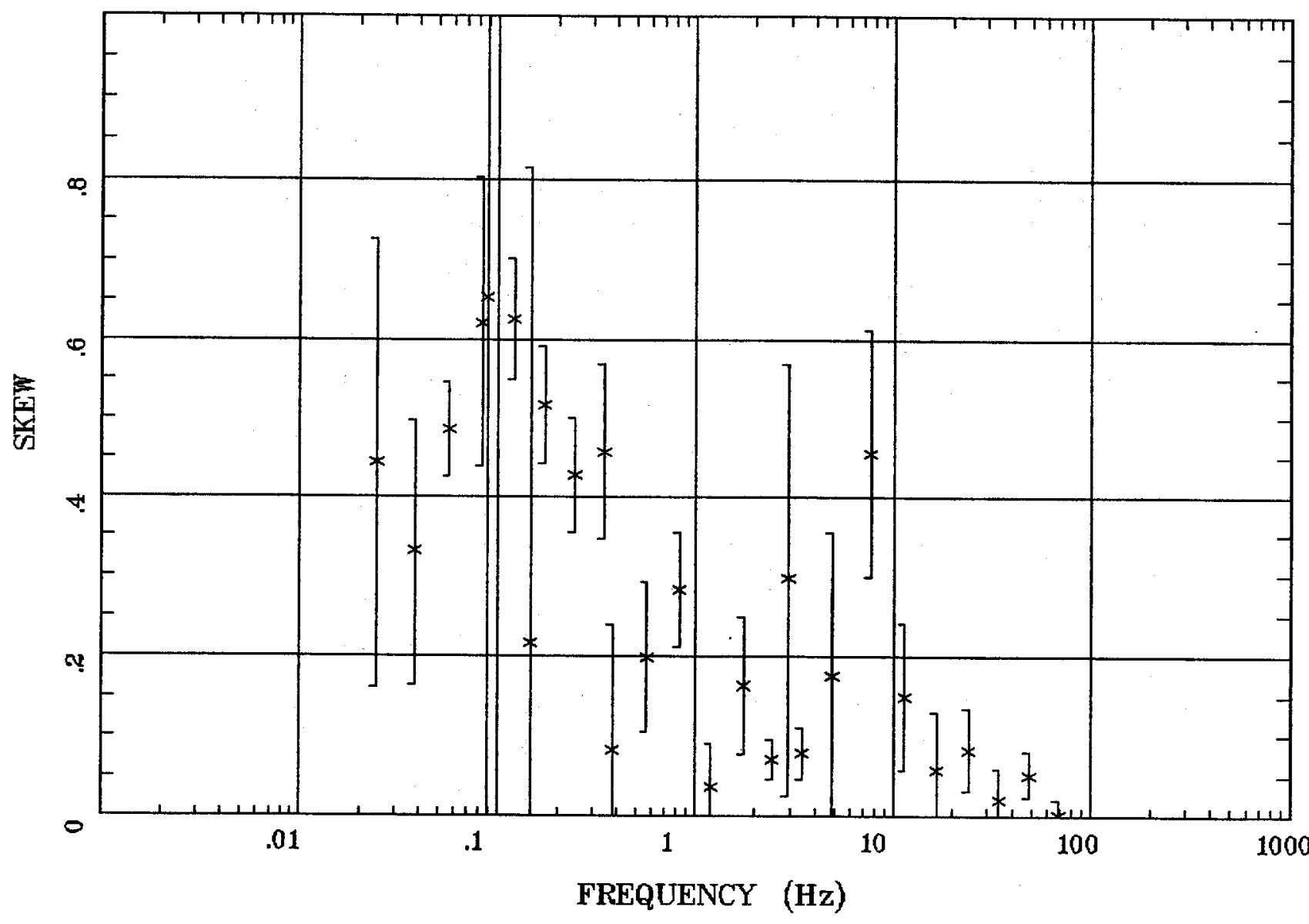

Client:

Remote: none

Acquired: 10:4 Jul 28, 2007 Survey Co:USGS

Rotation:

Filename: gl02m1.avg

Channels: Ch1 Ch2 Ch3 Ch4 Ch5 Ch3 Ch4

Plotted: 10:50 Nor 06, 2007

< EMI - ElectroMagnetic Instruments > 
E MULT Coh.

Chaco Mesa, NM 100k

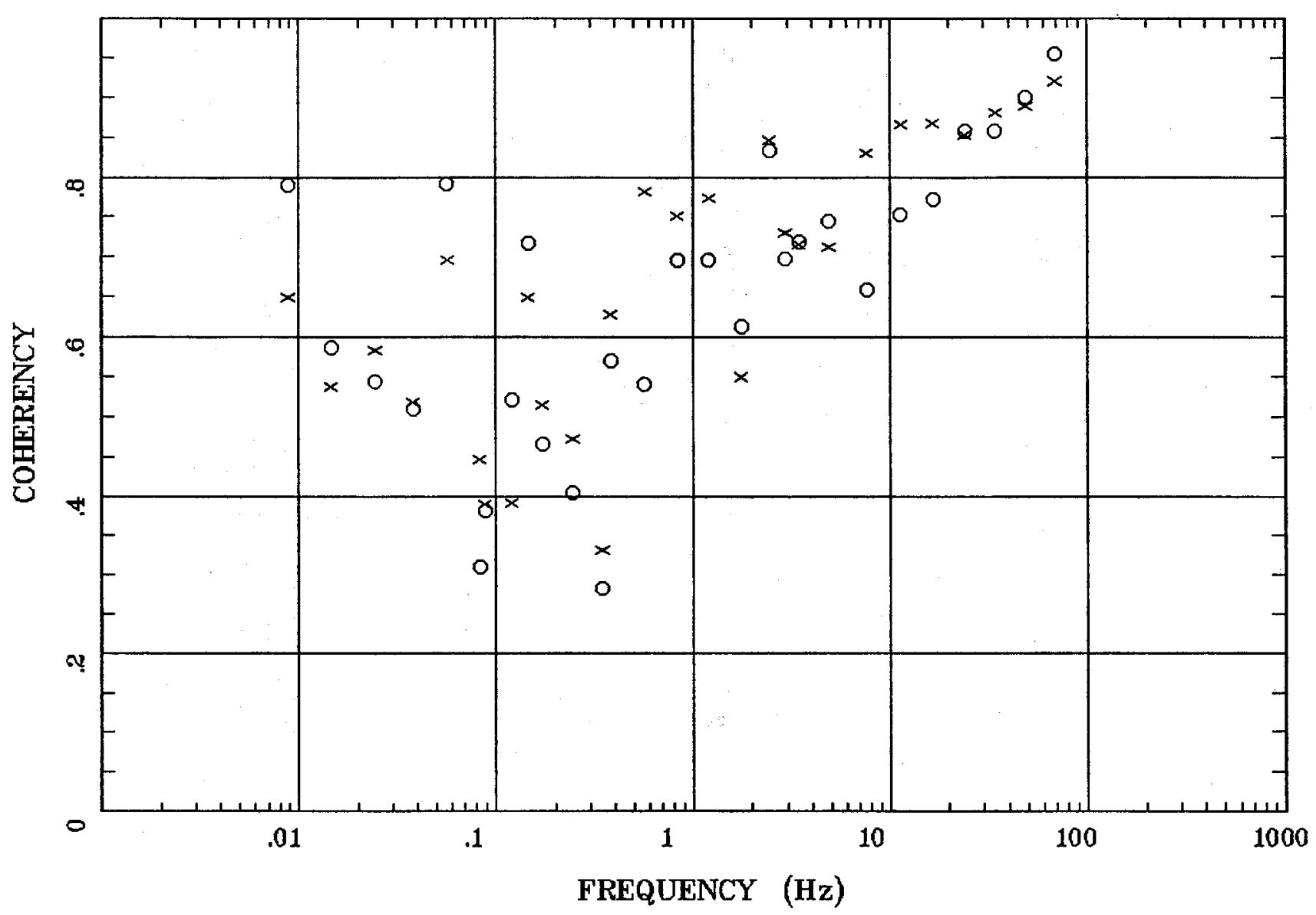

Client:

Remote: none

Acquired: 10:4 Jul 28, 2007

Survey Co:USGS
Rotation:

Filename: gl02m1.avg

Channels: Ch1 Ch2 Ch3 Ch4 Ch5 Ch3 Ch4

Plotted: $\quad$ 10:50 Nov 06, 2007

$<$ EMI - ElectroMagnetic Instruments 
POLAR PLOTS

Chaco Mesa, NM 100k

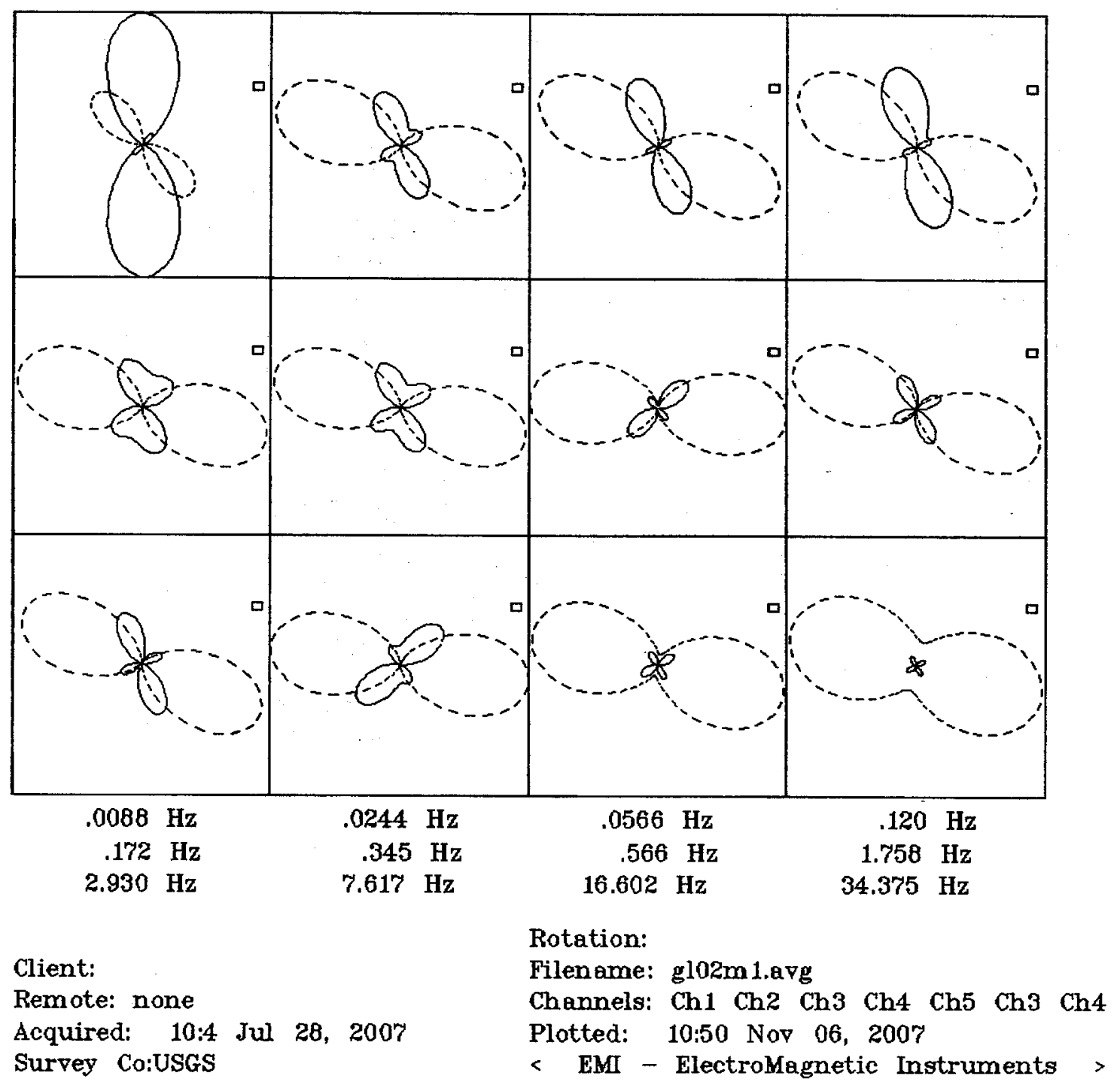


TIPPER MAGNITUDE

Chaco Mesa, NM 100k

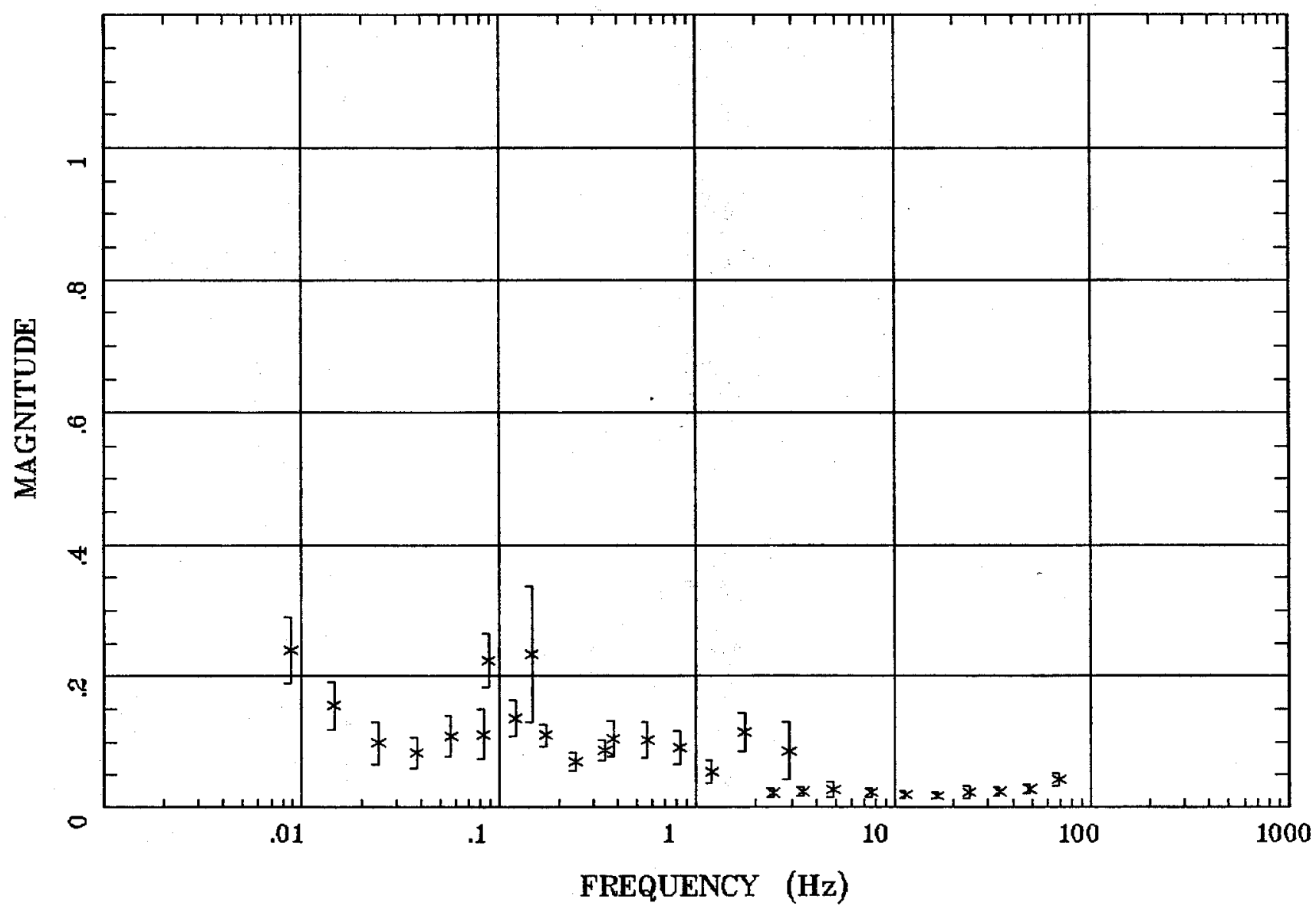

Client:

Remote: none Acquired: 10:4 Jul 28, 2007 Survey Co:USGS
Rotation:

Filenome: g102m1.avg

Channels: Ch1 Ch2 Ch3 Ch4 Ch5 Ch3 Ch4 Plotted: 10:50 Nov 06, 2007

$<$ EMI - ElectroMagnetic Instruments 
TIPPER STRIKE

Chaco Mesa, NM 100k

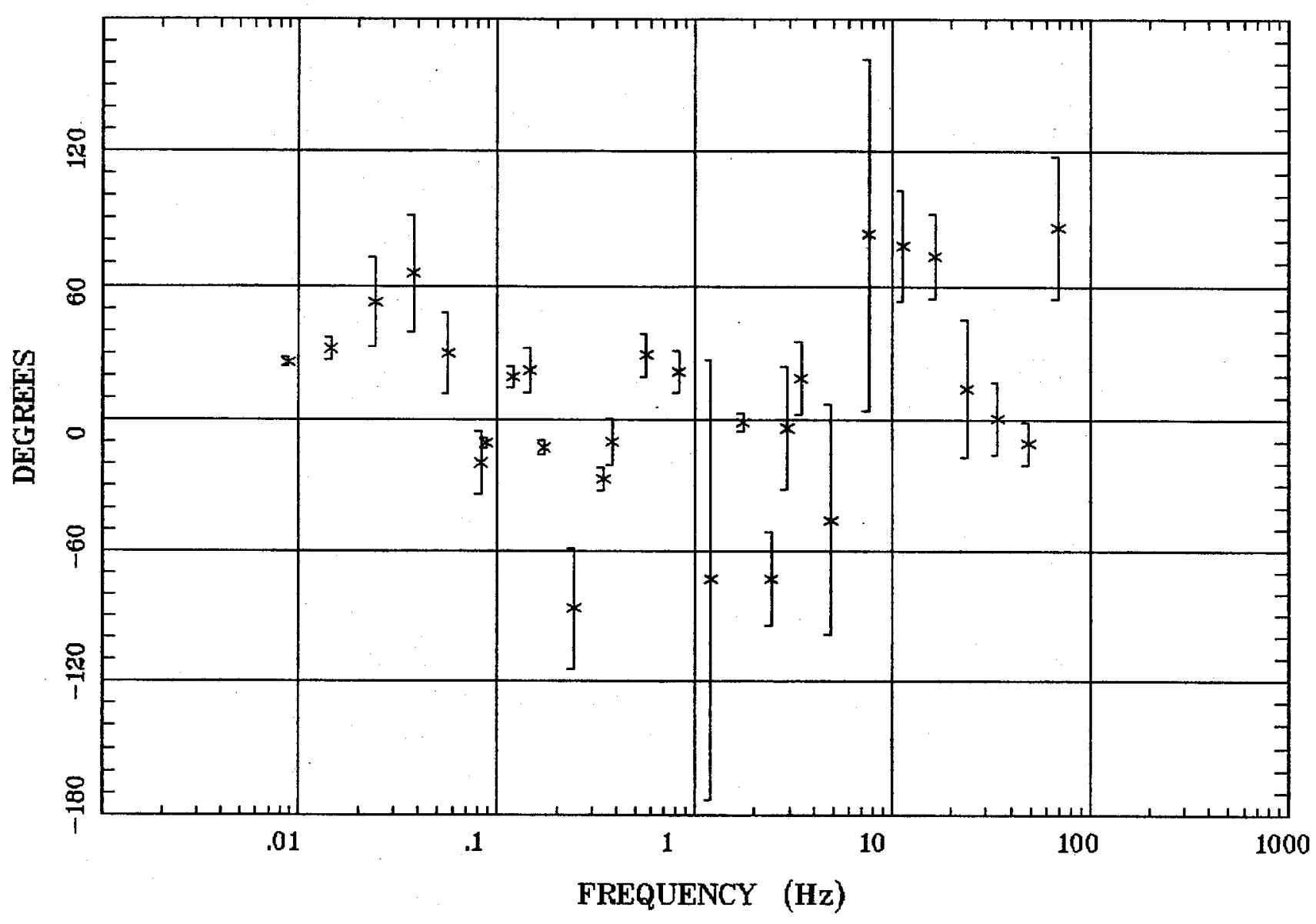

Client:

Remote: none

Acquired: 10:4 Jul 28, 2007

Survey Co:USGS
Rotation:

Filename: g102m1.avg

Channels: Ch1 Ch2 Ch3 Ch4 Ch5 Ch3 Ch4

Plotted: 10:50 Nor 06, 2007

< EMI - ElectroMagnetic Instruments > 
HzHx.x Coh HzHy.o

Chaco Mesa, NM 100k

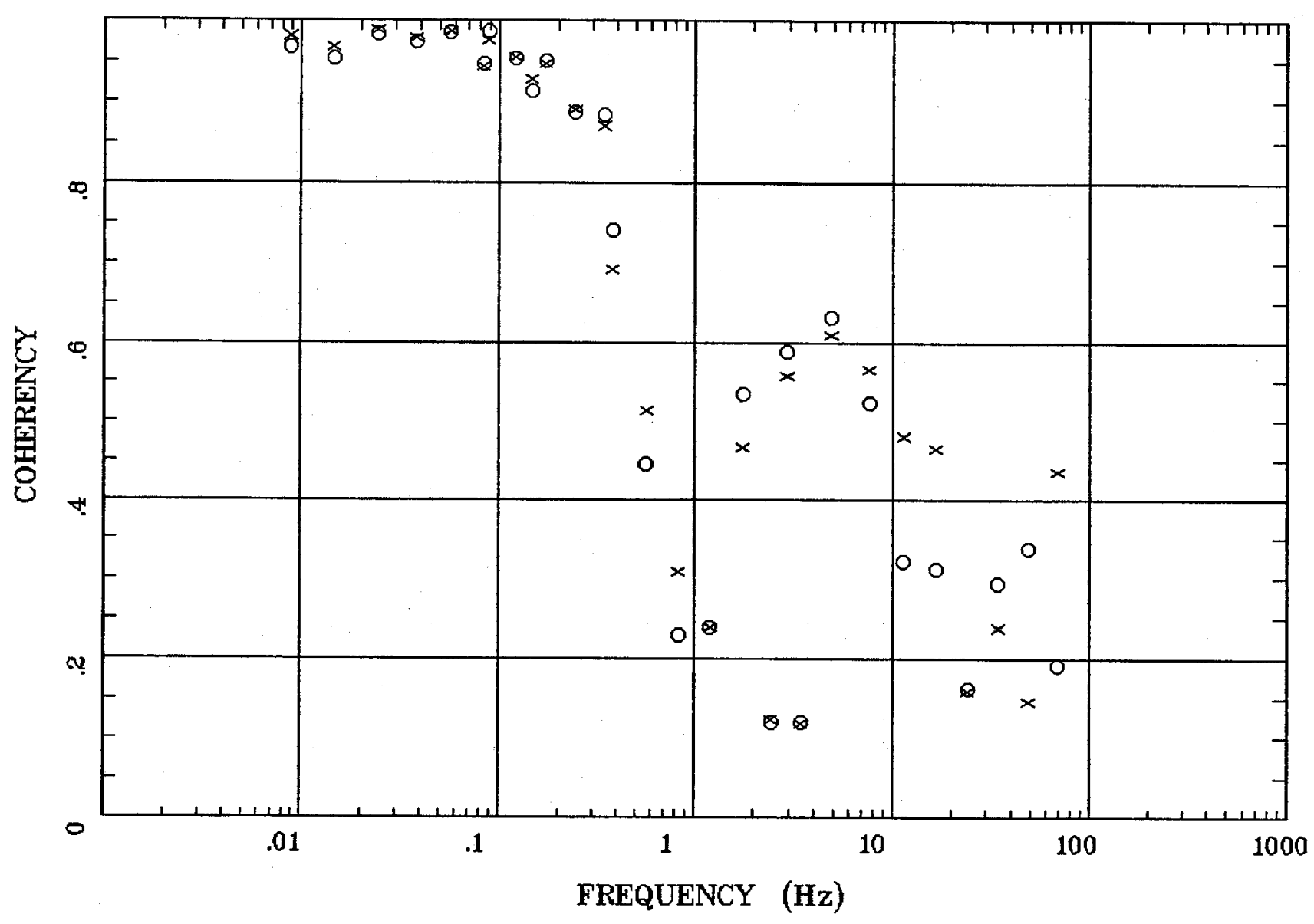

Client:

Remote: none

Acquired: 10:4 Jul 28, 2007

Survey Co:USGS
Rotation:

Filename: glo2m1.avg

Channels: Ch1 Ch2 Ch3 Ch4 Ch5 Ch3 Ch4

Plotted: 10:50 Nov 06, 2007

< EMI - ElectroMagnetic Instruments > 
APPARENT RESISTIVITY

Blanca Peak, 100k

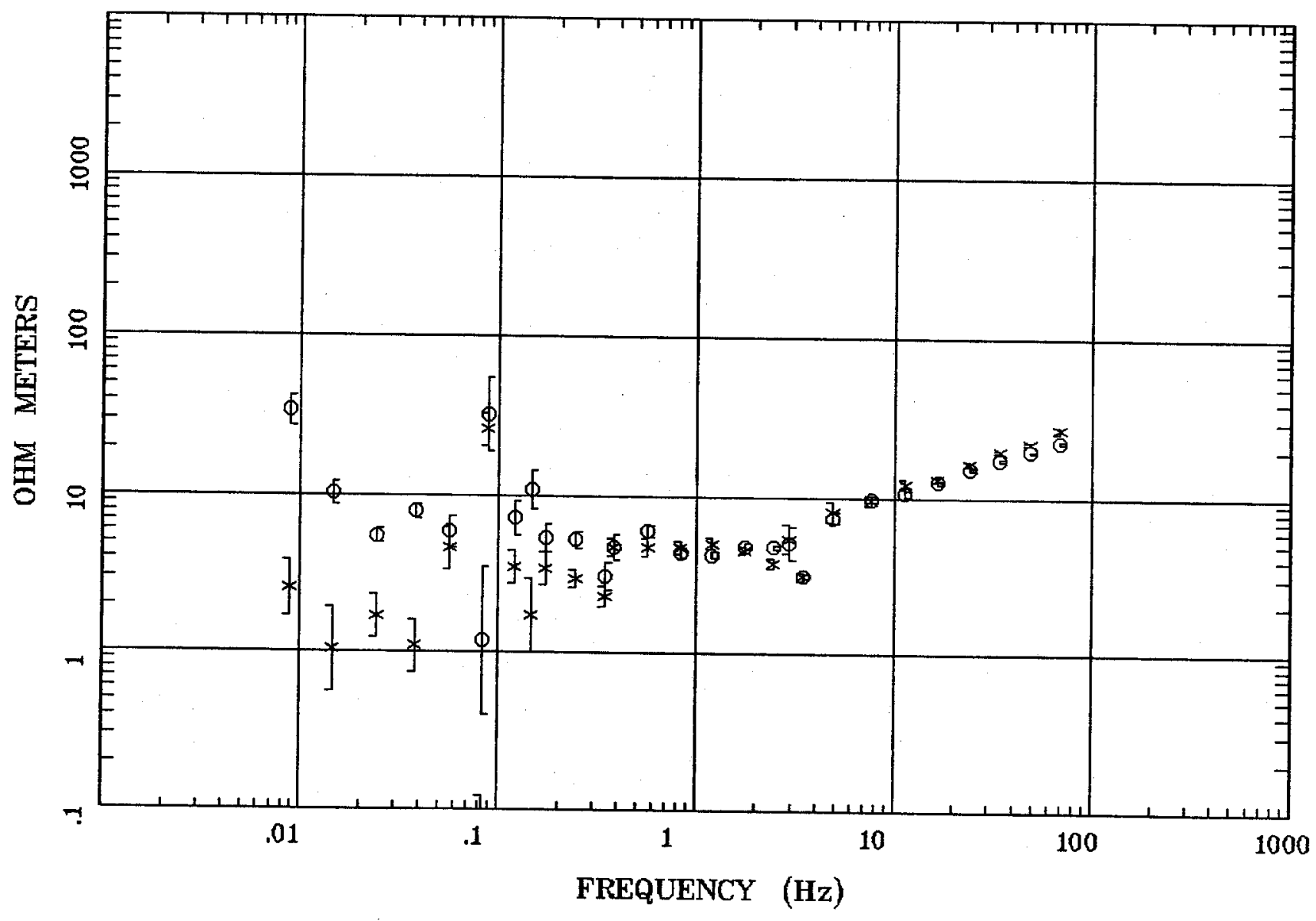

Client:

Remote: none

Acquired: $17: 4 \cdot \operatorname{Jan} 14,1996$

Survey Co:USGS
Rotation:

Filename: slizm.avg

Channels: Ch1 Ch2 Ch3 ch4 Ch5 Ch3 Ch4 Plotted: 11:06 Nov 06, 2007

$<$ EMI - ElectroMagnetic Instruments 
IMPEDANCE PHASE

Blanca Peak, 100k

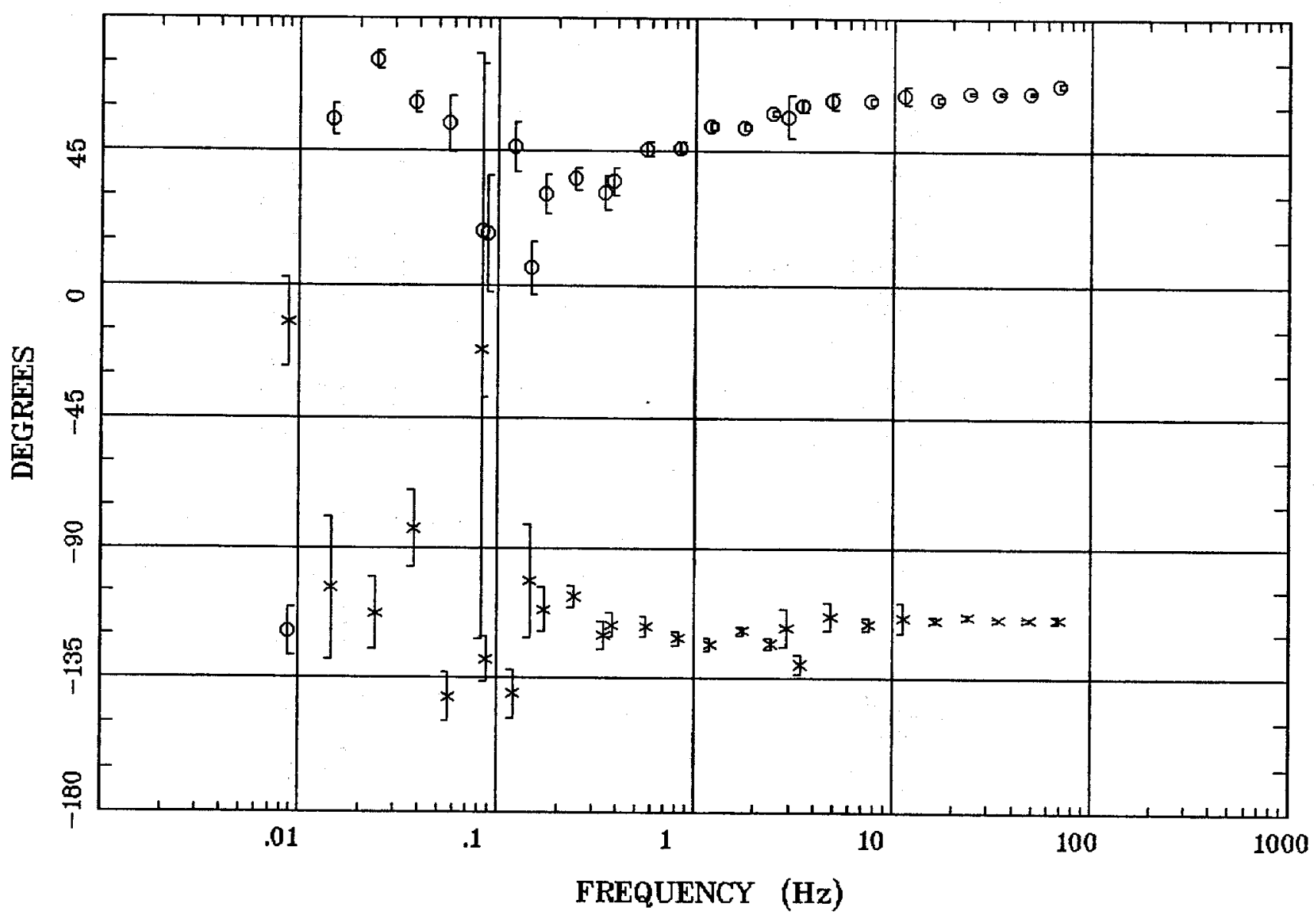

Client:

Remote: none

Acquired: $17: 4$ Jan 14, 1996 Survey Co:USGS
Rotation:

Filename: sl23m.avg

Channels: Ch1 Ch2 Ch3 Ch4 Ch5 Ch3 Ch4

Plotted: 11:06 Nov 06, 2007

< EMI - ElectroMagnetic Instruments > 
Blanca Peak, 100k

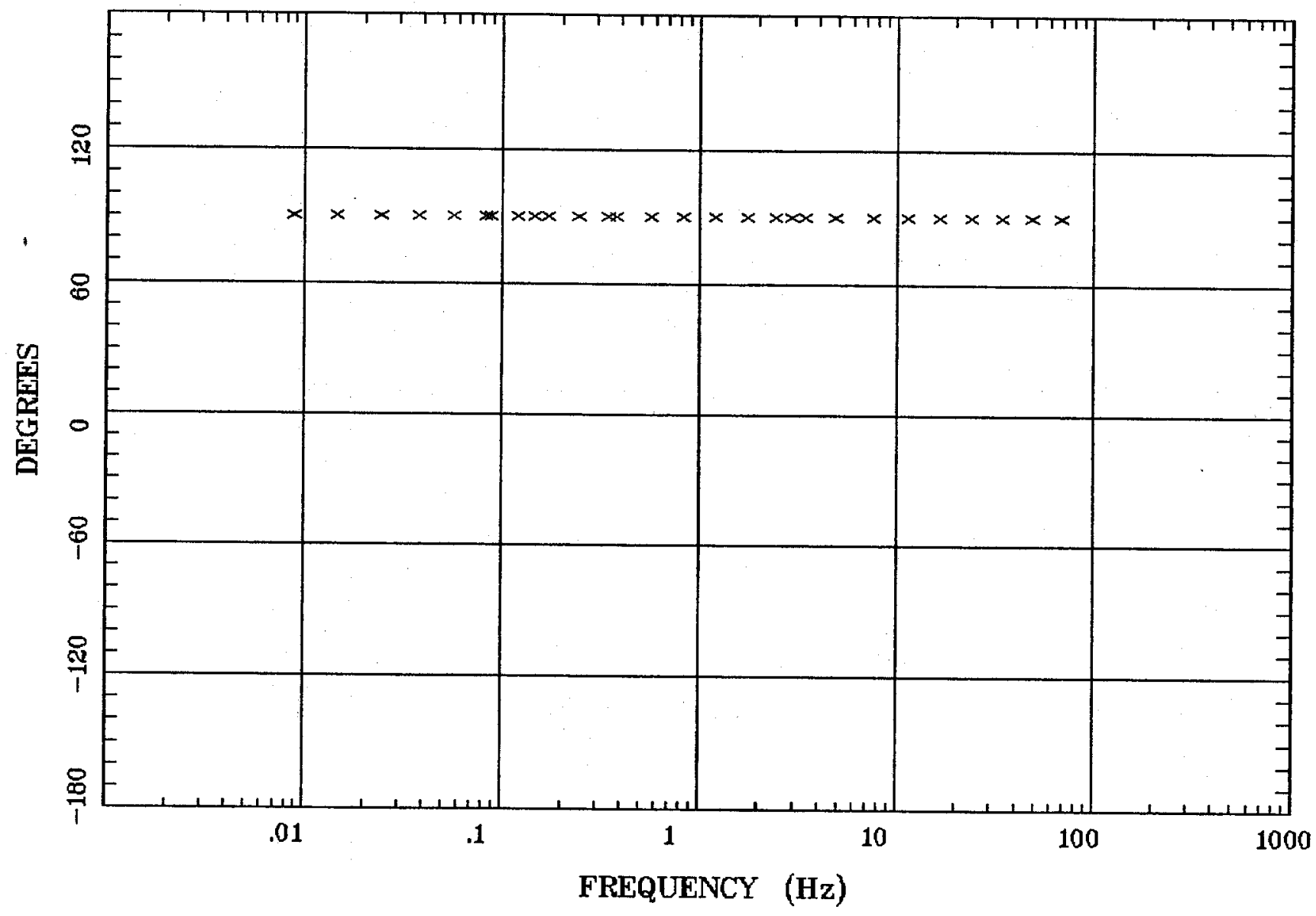

Client:

Remote: none

Acquired: $17: 4$ Jan 14, 1996 Survey Co:USGS
Rotation:

Filename: sl23m.avg

Channels: Ch1 Ch2 Ch3 Ch4 Ch5 Ch3 Ch4 Plotted: 11:06 Nov 06, 2007

< EMI - ElectroMagnetic Instruments > 
Blanca Peak, 100k

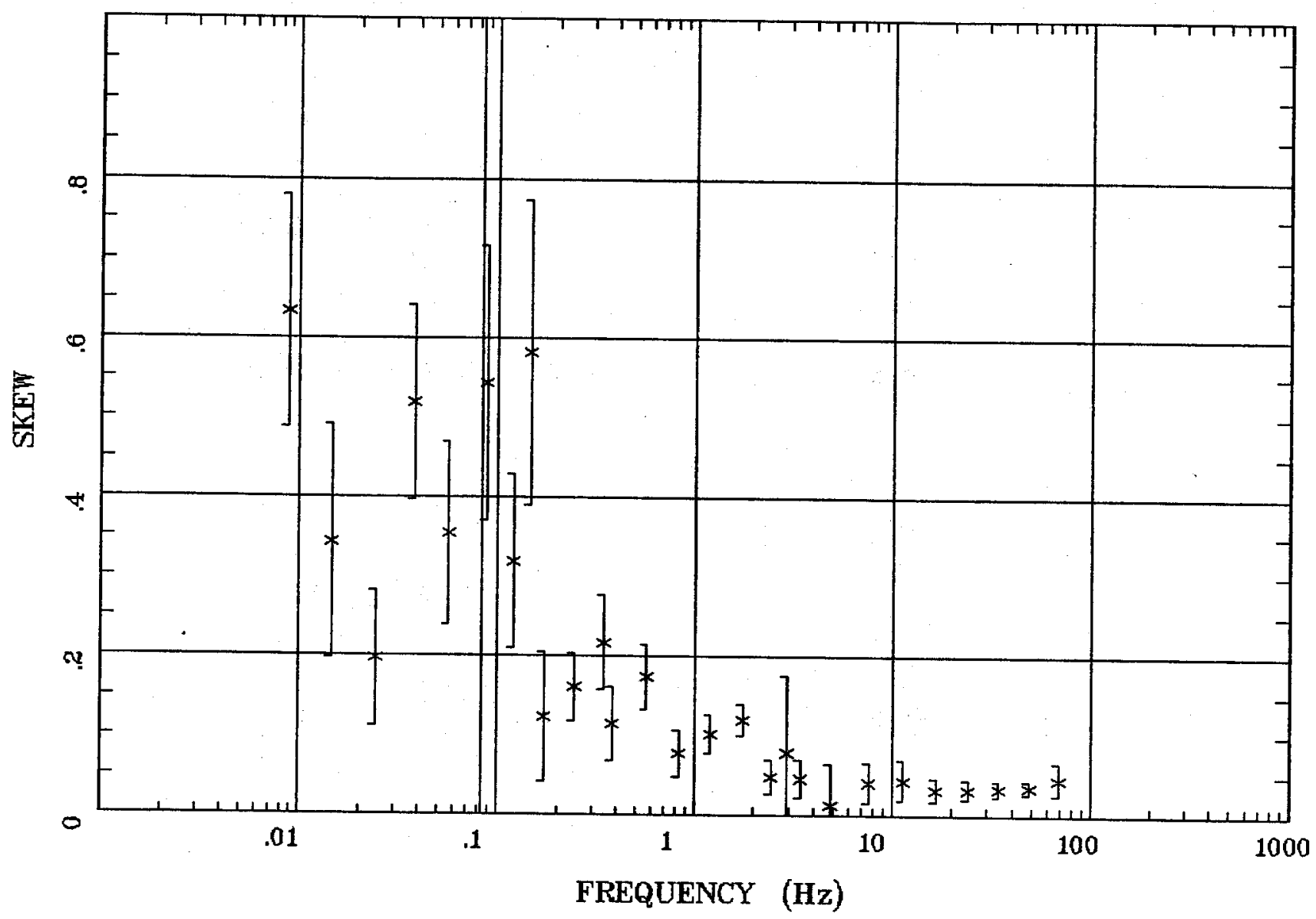

Client:

Remote: none

Acquired: $17: 4 \operatorname{Jan} 14,1996$

Survey Co:USGS
Rotation:

Filename: sl23m.avg

Channels: Ch1 Ch2 Ch3 Ch4 Ch5 Ch3 Ch4 Plotted: 11:06 Nov 06, 2007

< EMI - ElectroMagnetic Instruments > 
Blanca Peak, 100k

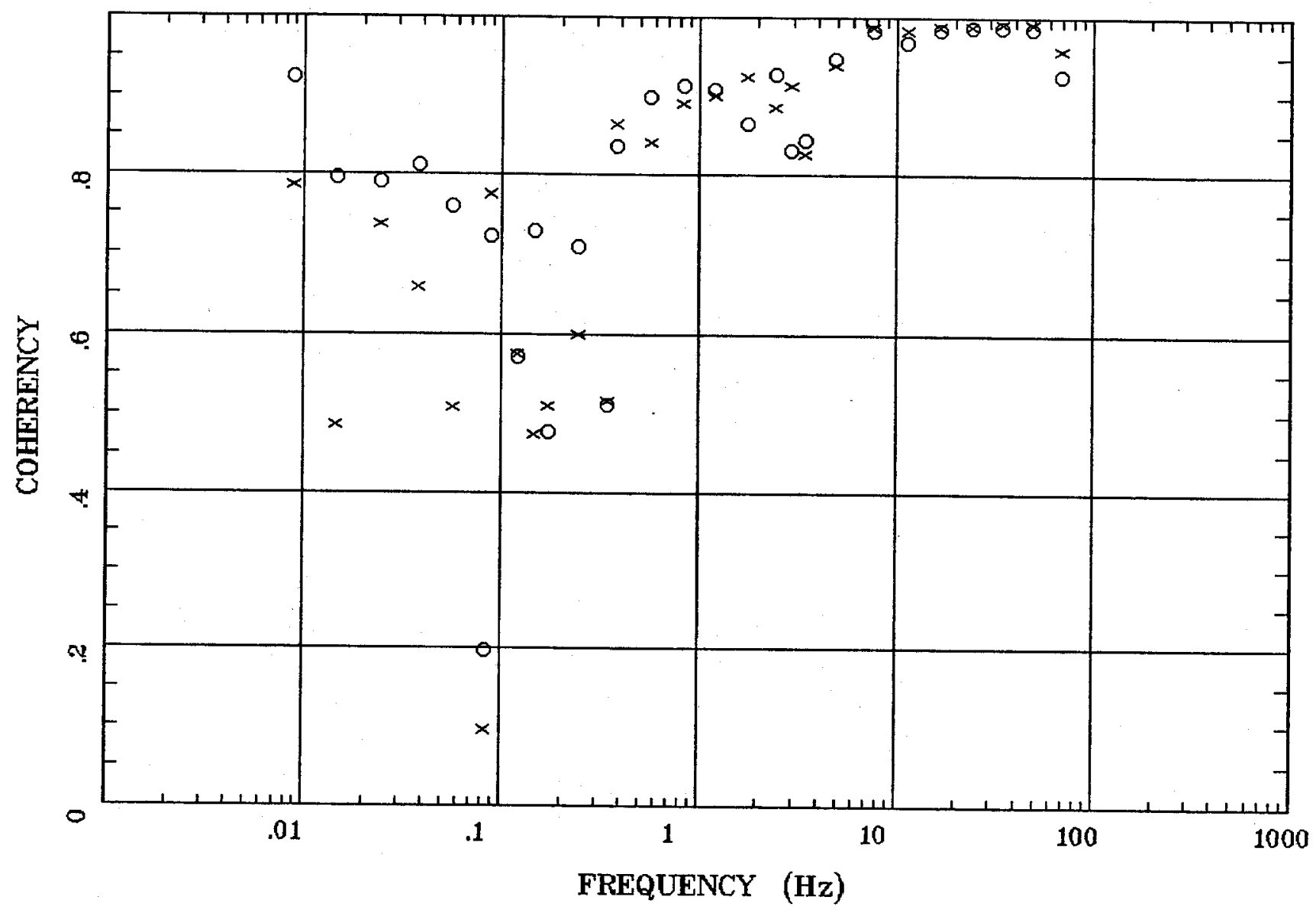

Client:

Remote: none

Acquired: $17: 4 \operatorname{Jan} 14,1996$

Survey Co:USGS
Rotation:

Filename: sl23m.avg

Channels: Ch1 Ch2 Ch3 Ch4 Ch5 Ch3 Ch4 Plotted: 11:06 Nov 06, 2007

< EMI - ElectroMagnetic Instruments > 
Station 23

\section{POLAR PLOTS}

Blanca Peak, 100k

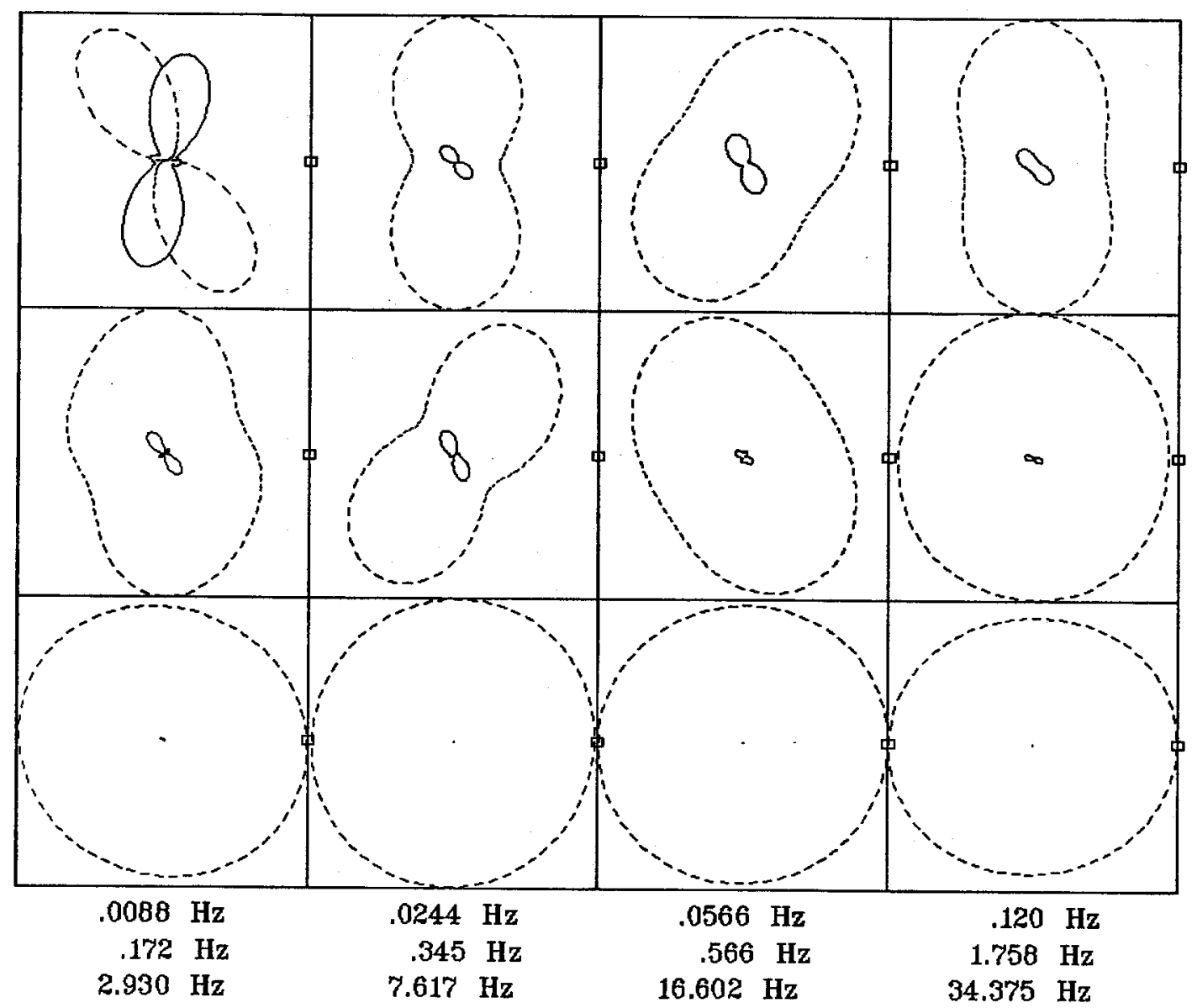

Client:

Remote: none

Acquired: 17:4 Jan 14, 1996 Survey Co:USGS
Rotation:

Filename: sl23m.avg

Channels: Ch1 Ch2 Ch3 Ch4 Ch5 Ch3 Ch4 Plotted: 11:06 Nov 06, 2007

< EMI - ElectroMagnetic Instruments > 
Station 23

TIPPER MAGNITUDE

Blanca Peak, 100k

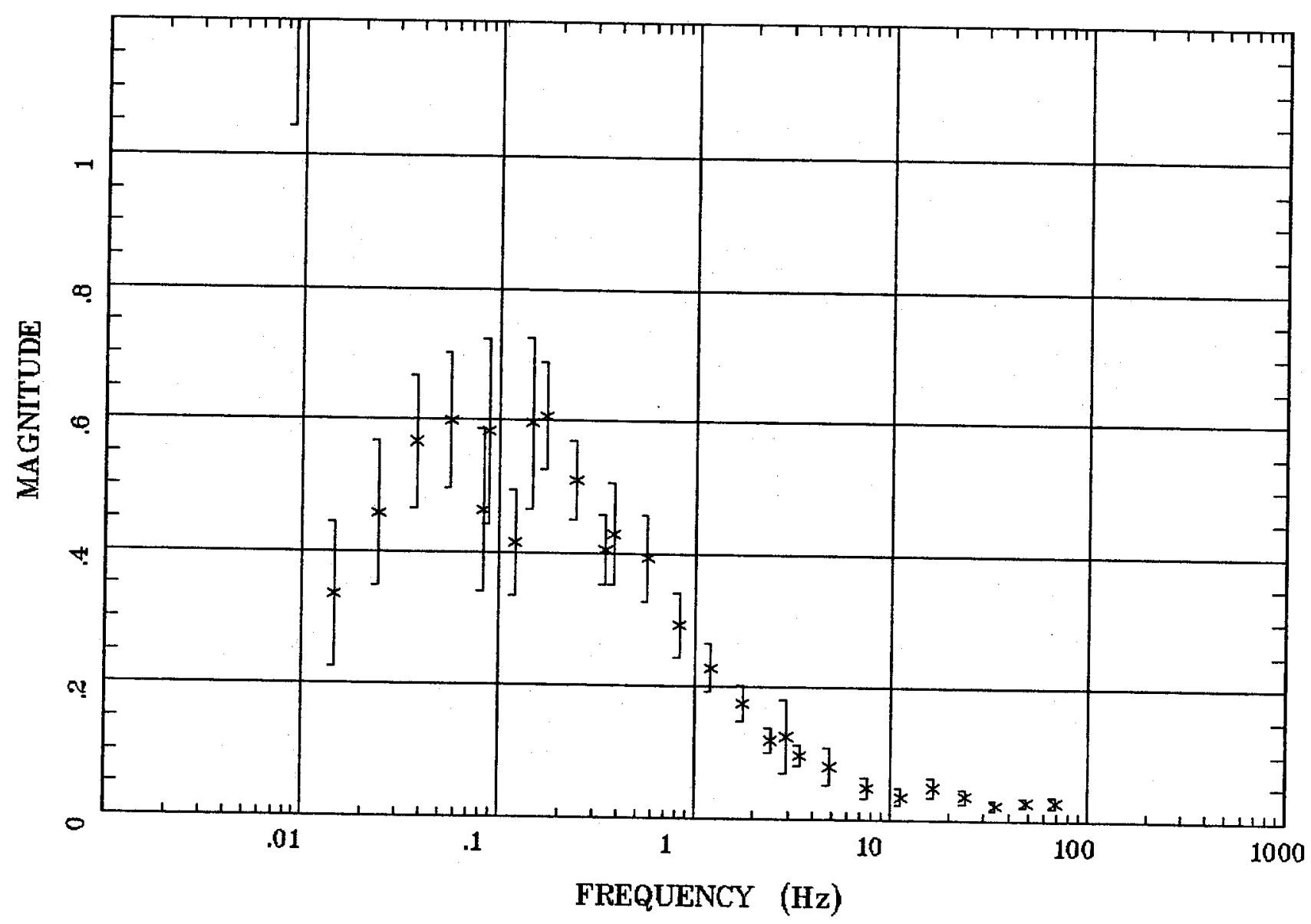

Client:

Remote: none

Acquired: $17: 4$ Jan 14, 1996

Survey Co:USGS
Rotation:

Filename: sl23m.avg

Channels: Ch1 Ch2 Ch3 Ch4 Ch5 Ch3 Ch4 Plotted: 11:06 Nov 06, 2007

< EMI - ElectroMagnetic Instruments 


\section{Station 23}

TIPPER STRIKE

Blanca Peak, 100k

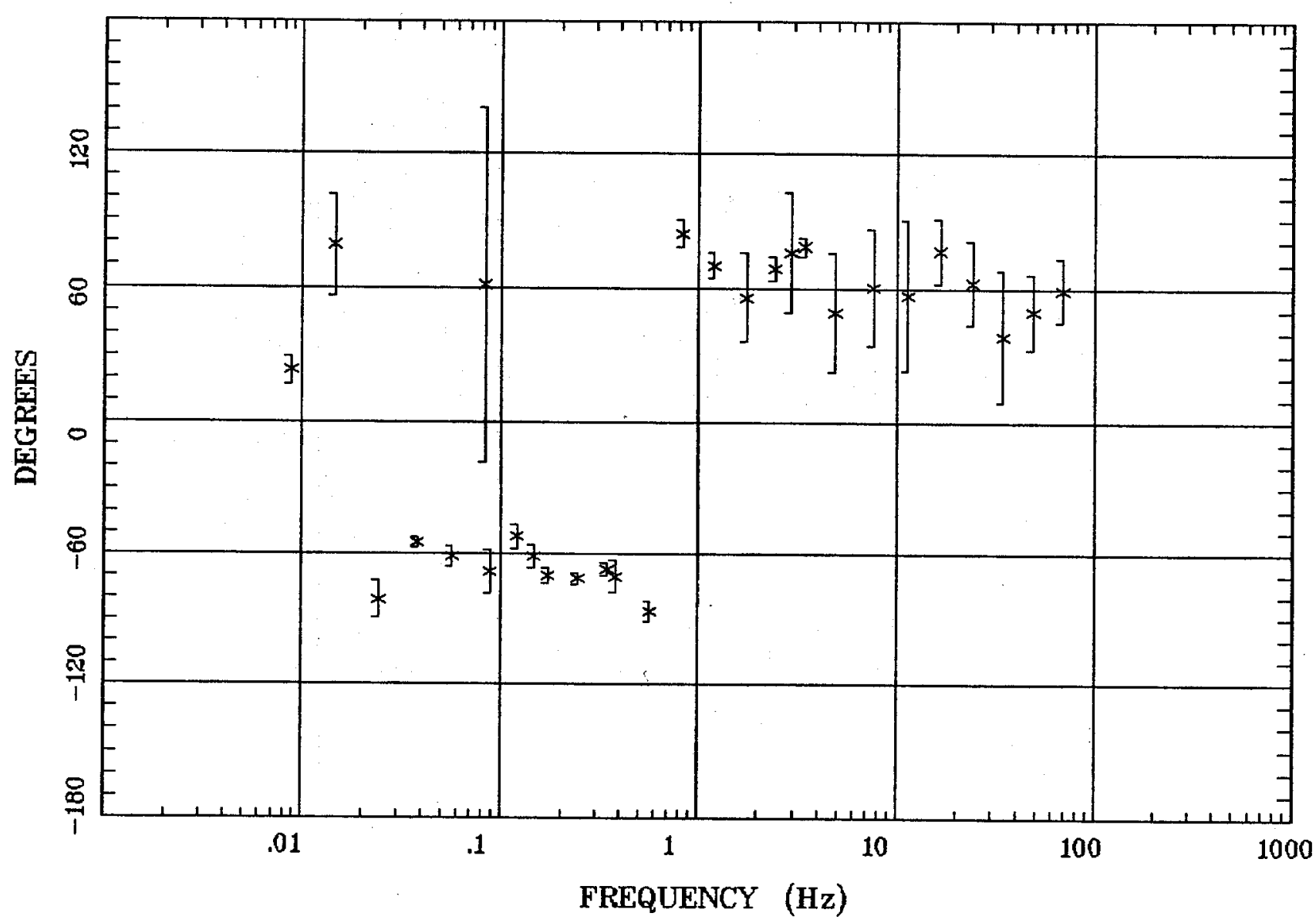

Client:

Remote: none

Acquired: $17: 4$ Jan 14, 1996

Survey Co:USGS
Rotation:

Filename: sl23m.avg

Channels: Ch1 Ch2 Ch3 ch4 Ch5 Ch3 Ch4

Plotted: 11:06 Nov 06, 2007

< EMI - ElectroMagnetic Instruments 
HzHx.x Coh HzHy.o

Blanca Peak, 100k

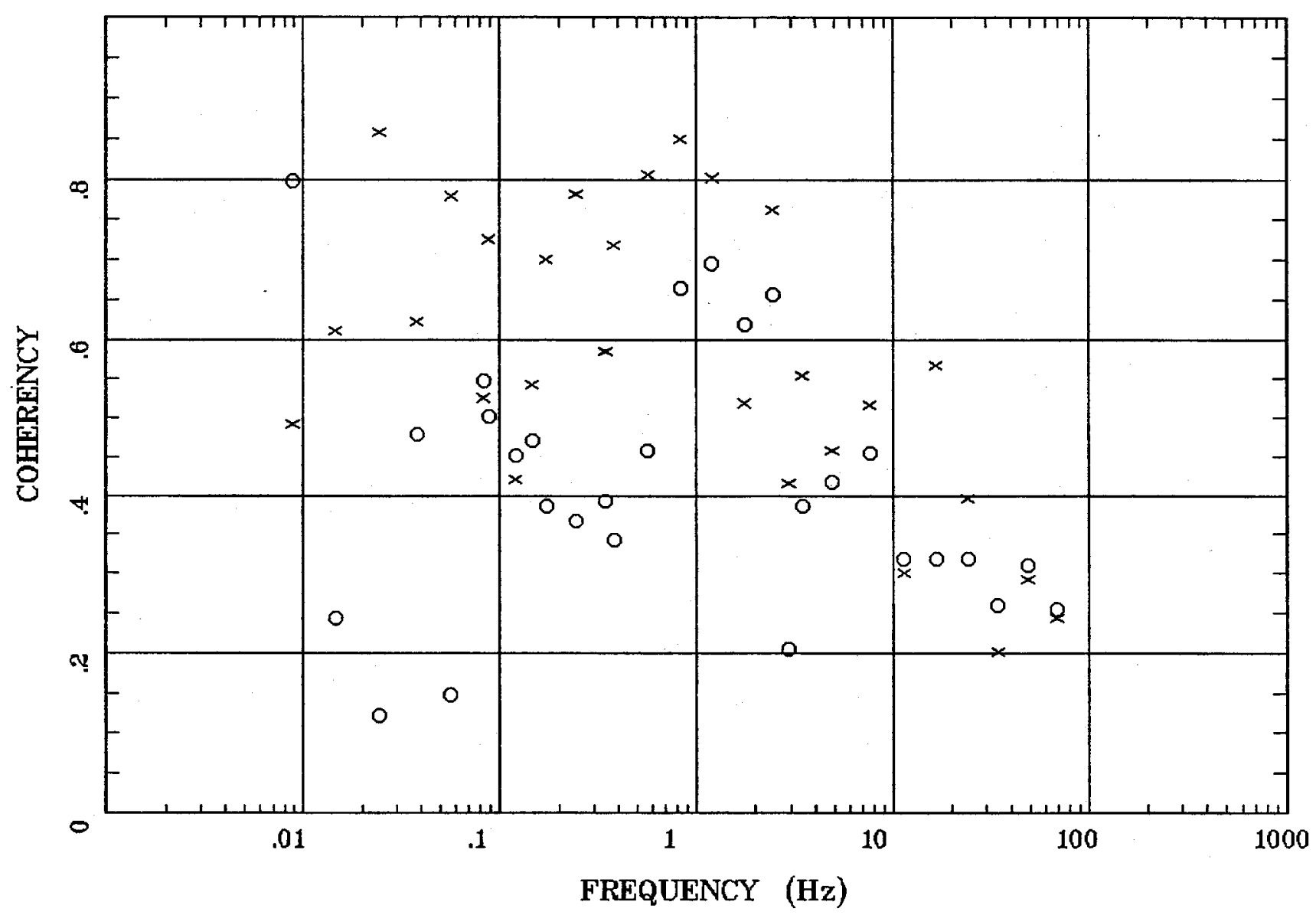

Client:

Remote: none

Acquired: $17: 4$ Jan 14, 1996

Survey Co:USGS
Rotation:

Filename: sl23m.avg

Channels: Ch1 Ch2 Ch3 Ch4 Ch5 Ch3 Ch4

Plotted: 11:06 Nov 06, 2007

< EMI - ElectroMagnetic Instruments > 
Station 24

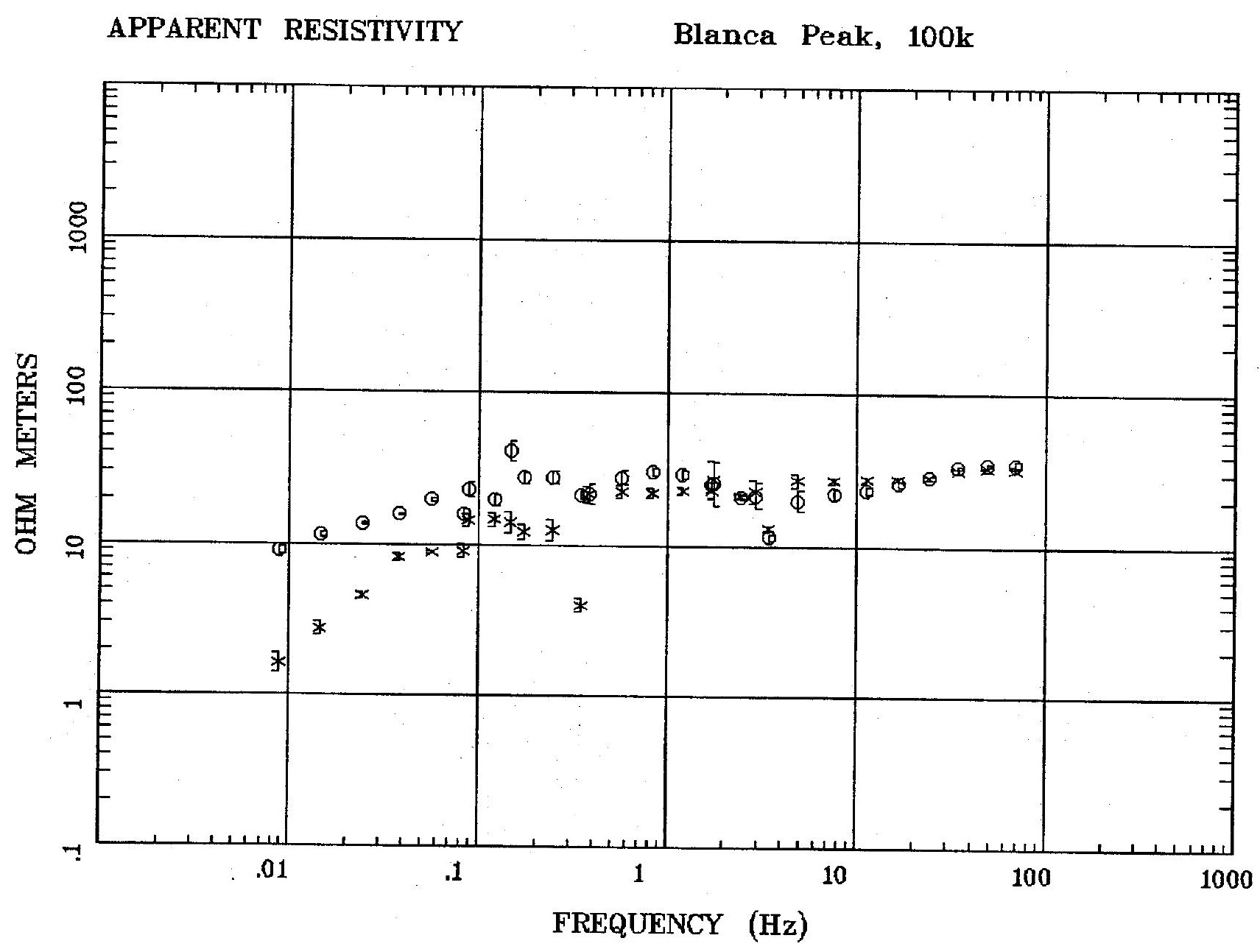

Client:

Remote: none

Acquired: 10:4 Jul 11, 2007

Survey Co:USGS
Rotation:

Filename: sl24mall.avg

Channels: Ch1 Ch2 Ch3 Ch4 Ch5 Ch3 Ch4 Plotted: 11:07 Nov 06, 2007

< EMI - ElectroMagnetic Instruments > 
Blanca Peak, 100k

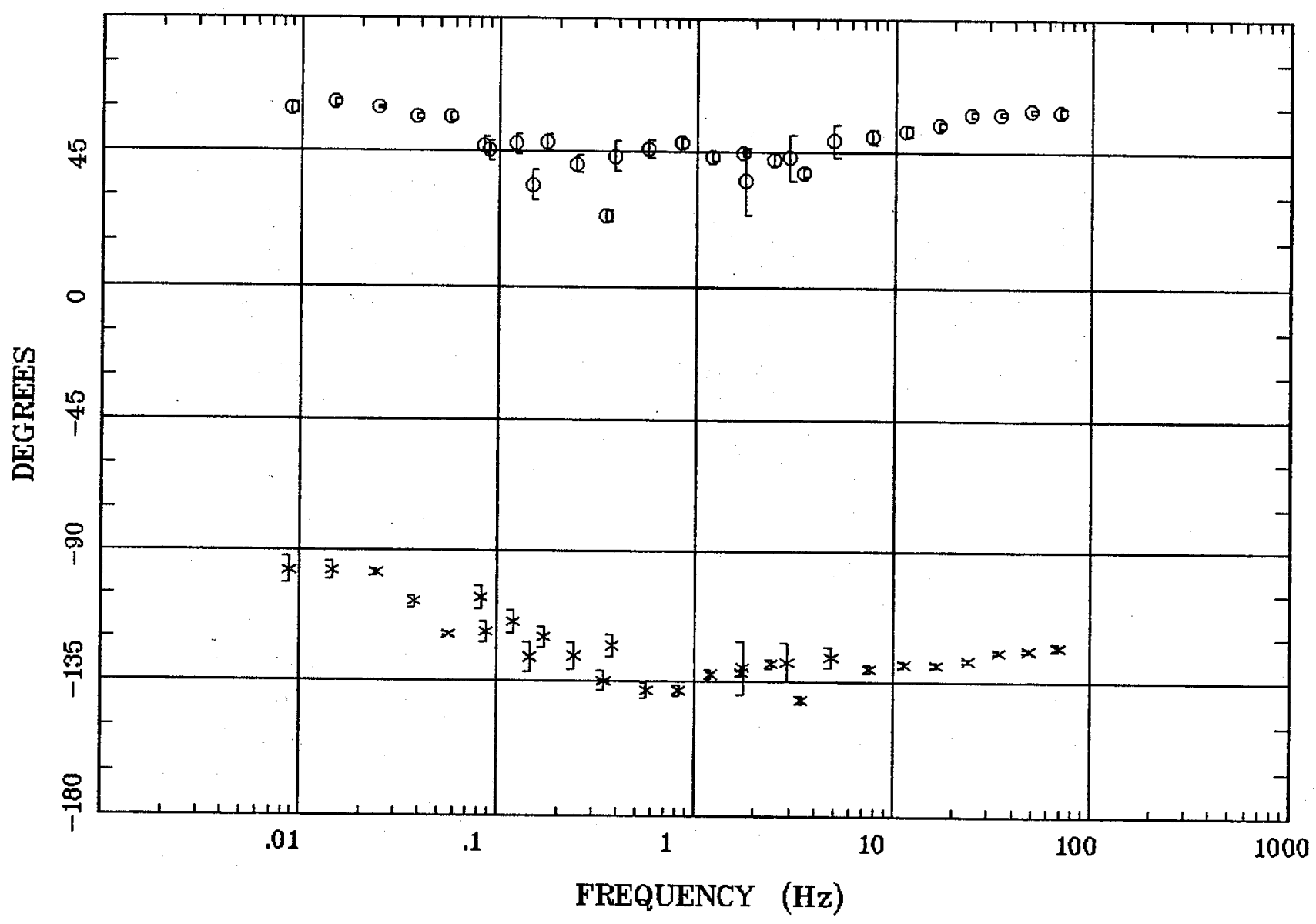

Client:

Remote: none

Acquired: 10:4 Jul 11, 2007 Survey Ca:USGS
Rotation:

Filename: sl24mall.avg

Channels: Ch1 Ch2 Ch3 Ch4 Ch5 Ch3 Ch4 Plotted: 11:07 Nov 06, 2007

< EMI - ElectroMagnetic Instruments > 


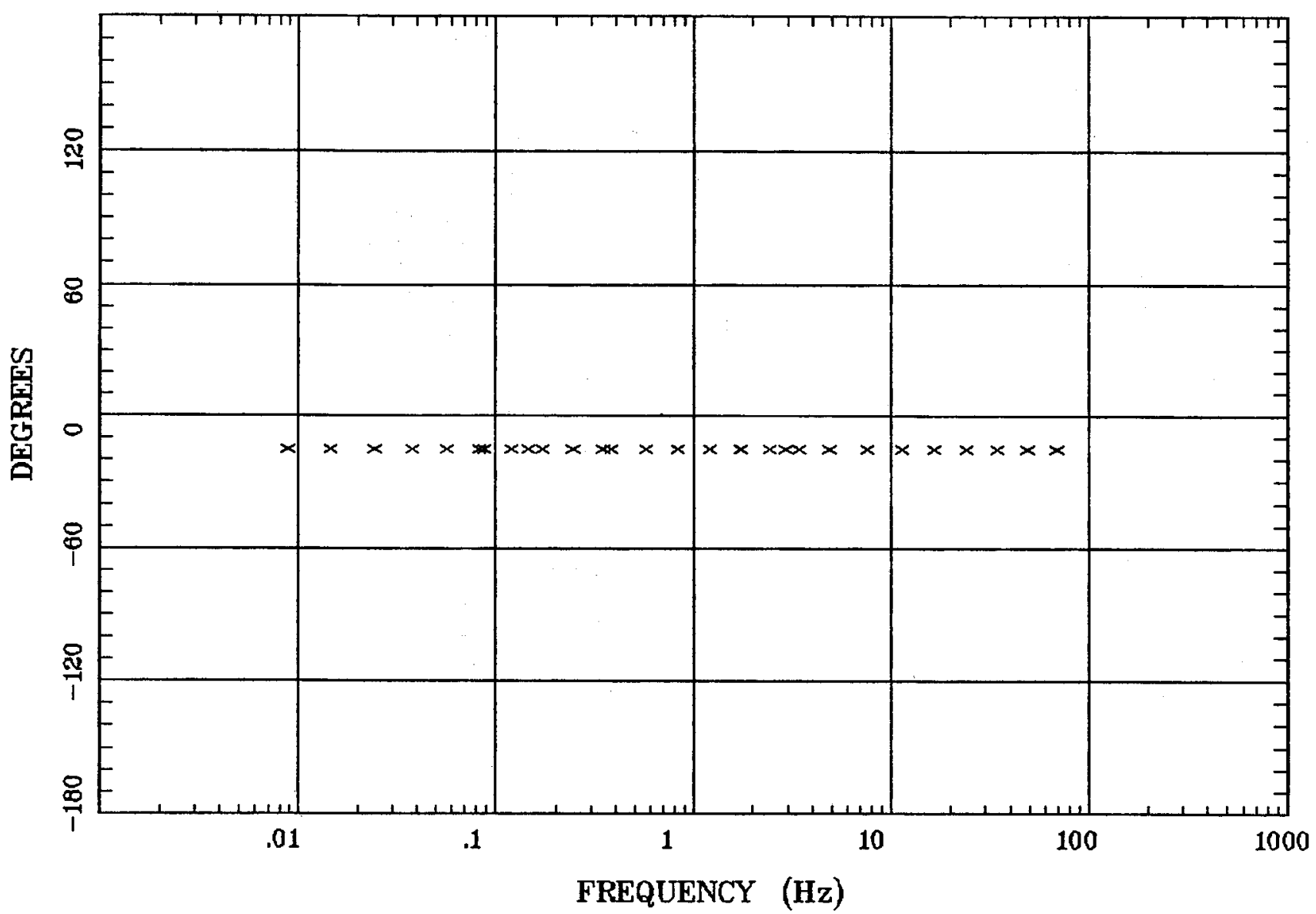

Client: Remote: none Acquired: 10:4 Jul 11, 2007 Survey Co:USGS
Rotation:

Filename: sl24mall.avg Channels: Ch1 Ch2 Ch3 Ch4 Ch5 Ch3 Ch4 Plotted: 11:07 Nov 06, 2007

< EMI - ElectroMagnetic Instruments 
Blanca Peak, 100k

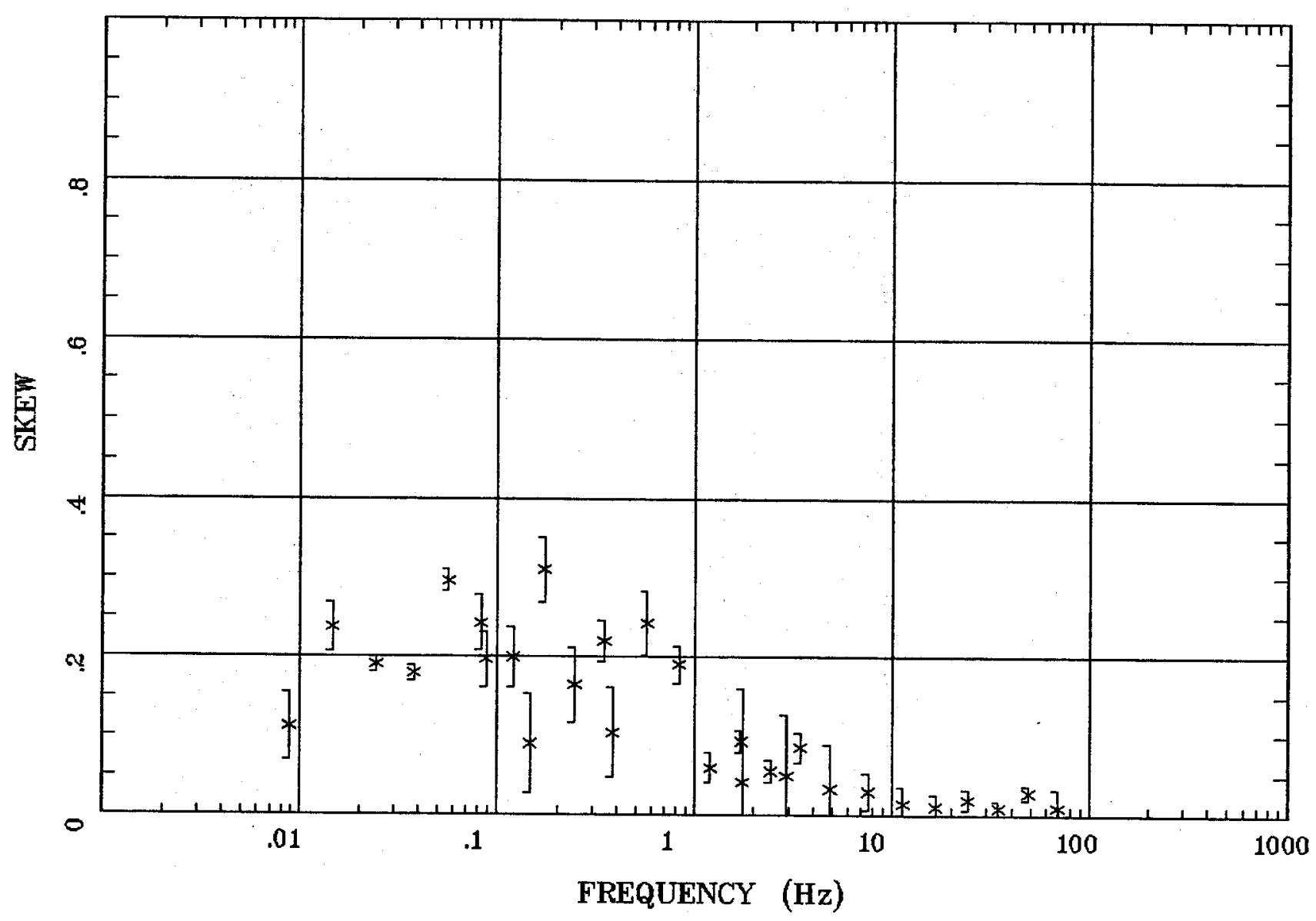

Client:

Remote: none

Acquired: 10:4 Jul 11, 2007 Survey Co:USGS
Rotation:

Filename: sl24mall.avg

Channels: Ch1 Ch2 Ch3 Ch4 Ch5 Ch3 Ch4 Plotted: 11:07 Nov 06, 2007

$<$ EMI - ElectroMagnetic Instruments > 
Station 24

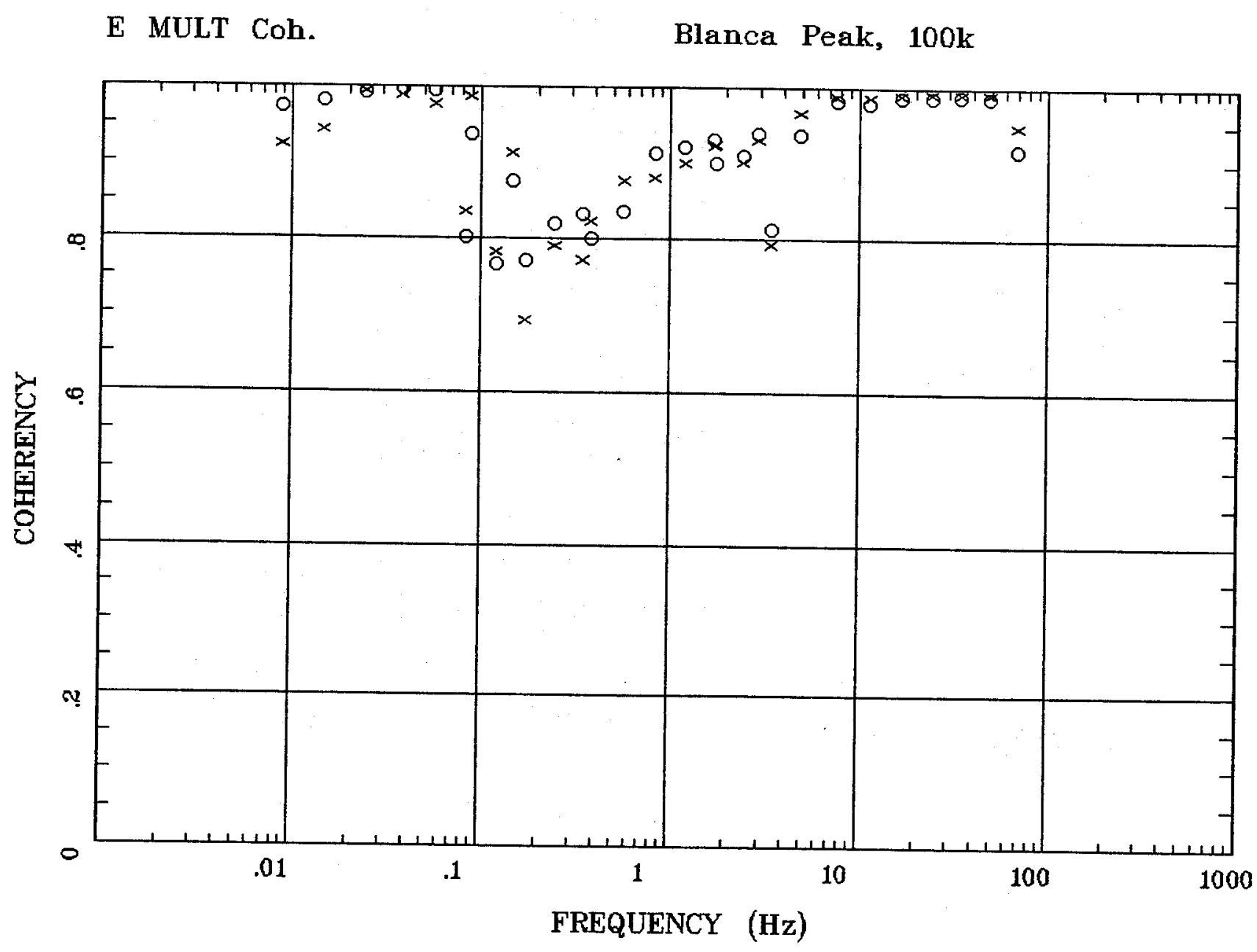

Client:

Remote: none

Acquired: 10:4 Jul 11, 2007 Survey Co:USGS
Rotation:

Filename: sl24mall.avg

Channels: Ch1 Ch2 Ch3 Ch4 Ch5 Ch3 Ch4 Platted: 11:07 Nov 06, 2007

< EMI - ElectroMagnetic Instruments > 


\section{POLAR PLOTS}

Blanca Peak, 100k

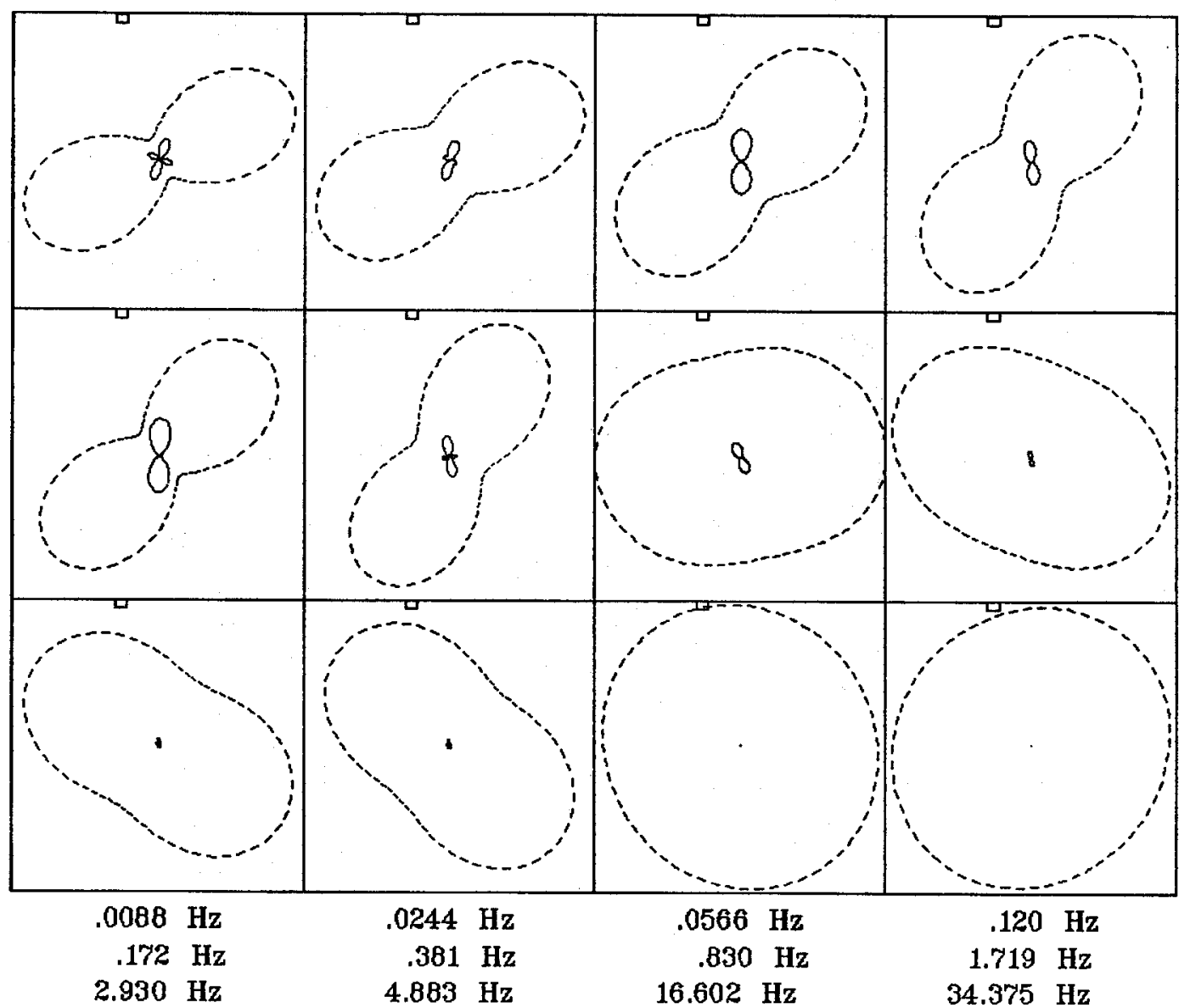

Client:

Remote: none

Acquired: 10:4 Jul 11, 2007 Survey Co:UsGS

\section{Rotation:}

Filename: sl24mall.avg

Channels: Ch1 Ch2 Ch3 Ch4 Ch5 Ch3 Ch4 Plotted: 11:07 Nov 06, 2007

< EMI - ElectroMagnetic Instruments > 


\section{Station 24}

TIPPER MAGNITUDE

Blanca Peak, 100k

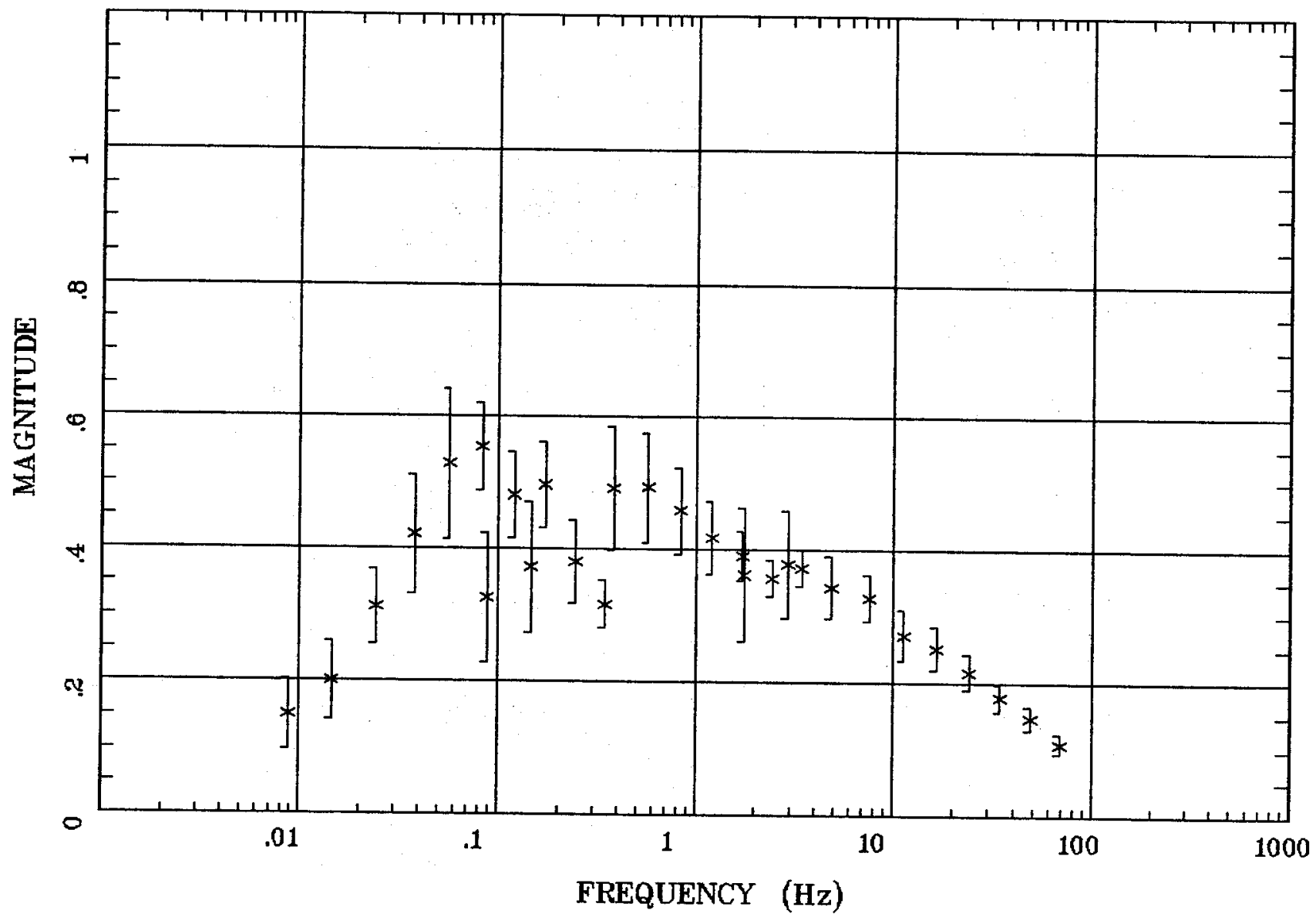

Client:

Remote: none

Acquired: 10:4 Jul 11, 2007

Survey Co:USGS
Rotation:

Filename: sl24mall.avg

Channels: Ch1 Ch2 Ch3 ch4 ch5 Ch3 Ch4 Plotted: 11:07 Nov 06, 2007

< EMI - ElectroMagnetic Instruments > 


\section{Station 24}

TIPPER STRIKE

Blanca Peak, 100k

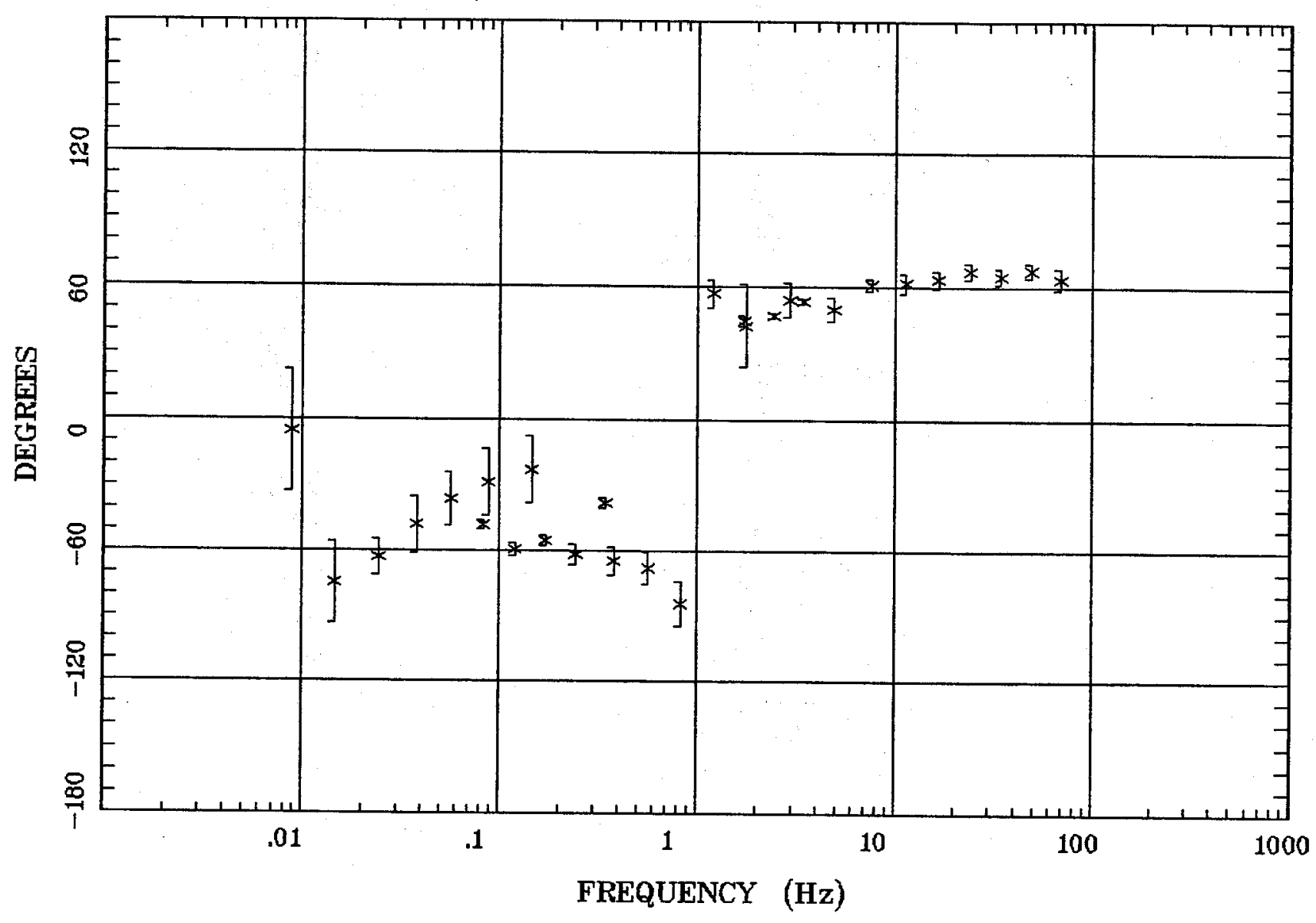

Client:

Remote: none

Acquired: 10:4 Jul 11, 2007 Survey Co:USGS
Rotation:

Filename: sl24mall.avg

Channels: Ch1 Ch2 Ch3 Ch4 Ch5 Ch3 Ch4 Plotted: 11:07 Nov 06, 2007

$<$ EMI - ElectroMagnetic Instruments > 
HzHx.x Coh HzHy.o

Blanca Peak, 100k

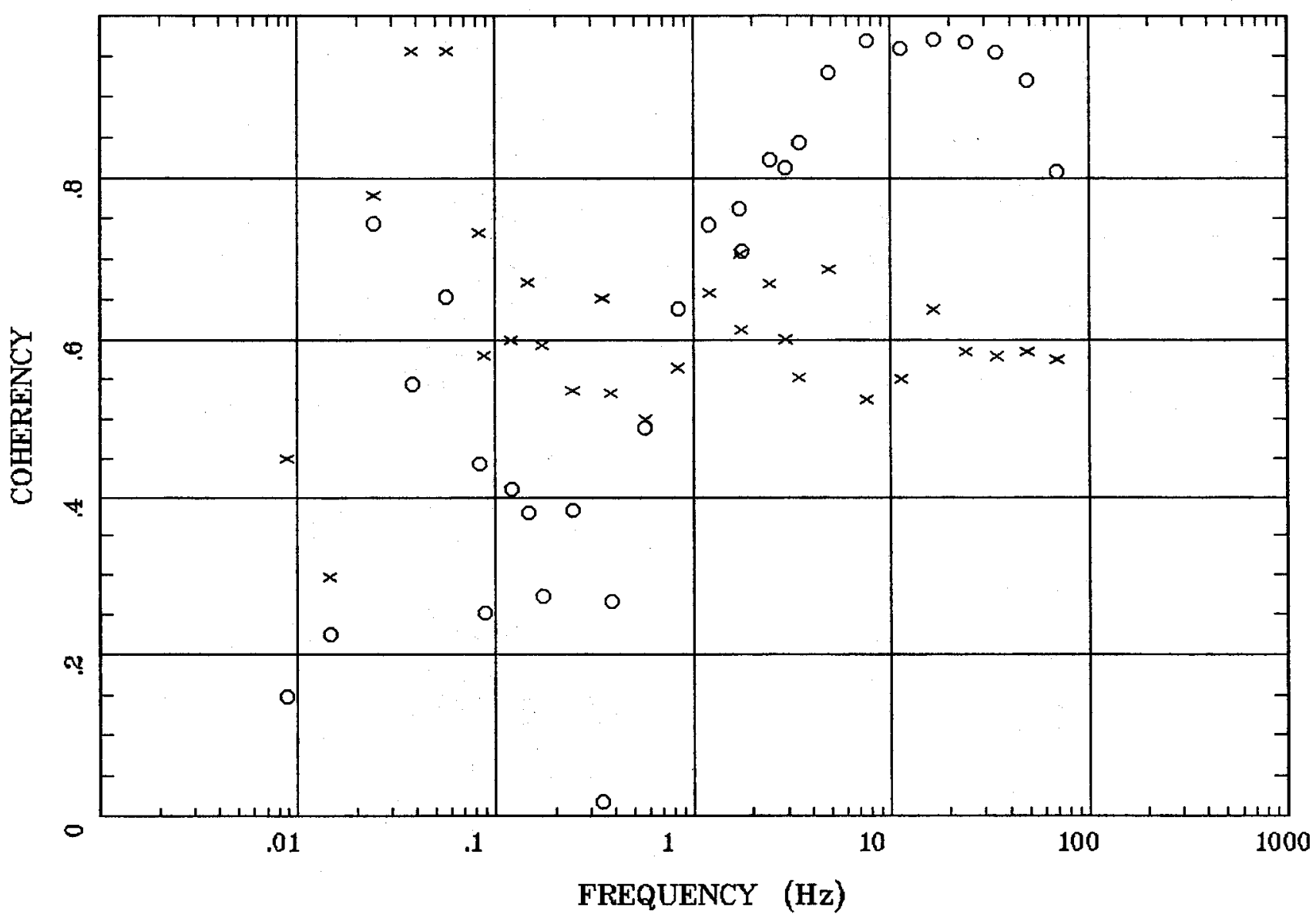

Client:

Remote: none

Acquired: 10:4 Jul 11, 2007

Survey Co:USGS
Rotation:

Filename: sl24mall.avg

Channels: Ch1 Ch2 Ch3 Ch4 Ch5 Ch3 Ch4

Plotted: 11:07 Nov 06, 2007

$<$ EMI - ElectroMagnetic Instruments 


\section{Station 25}

Blanca Peak, 100k

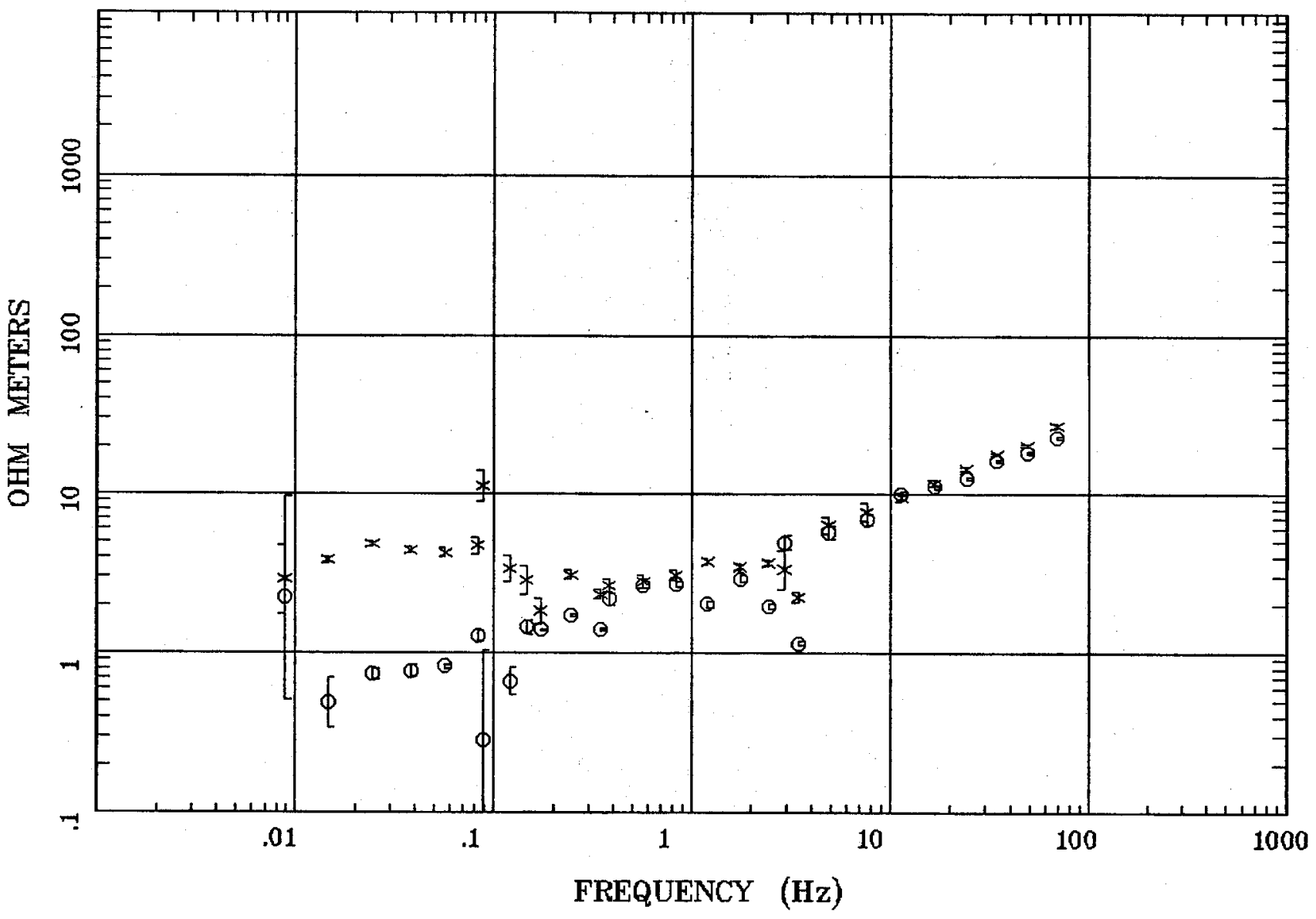

Client:

Remote: none

Acquired: 15:3 Jul 11, 2007

Survey Co:USGS
Rotation:

Filename: sl25m1.avg

Channels: Ch1 Ch2 Ch3 Ch4 Ch5 Ch3 Ch4

Plotted: 11:07 Nov 06, 2007

< EMI - ElectroMagnetic Instruments 
Blanca Peak, 100k

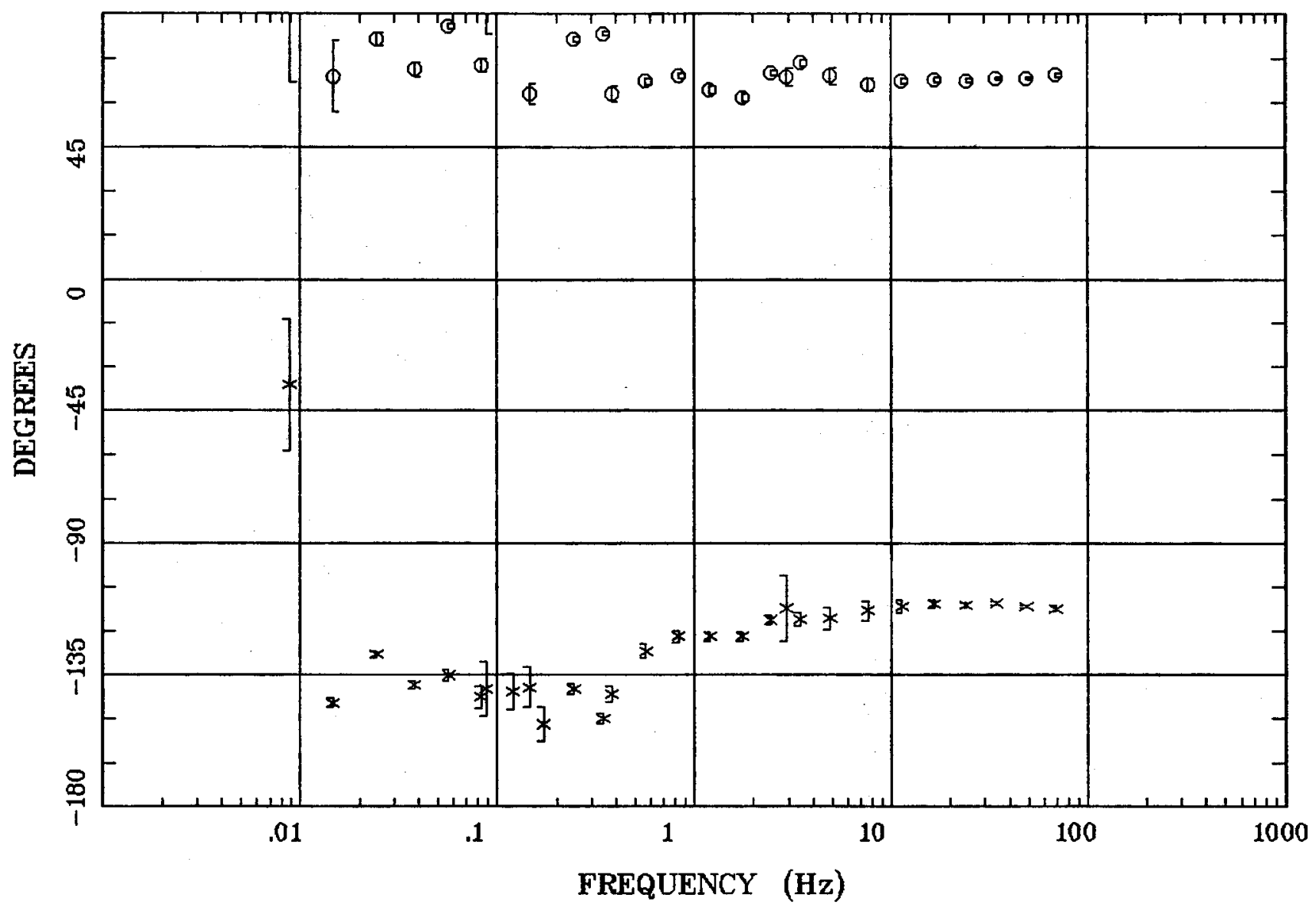

Client:

Remote: none

Acquired: 15:3 Jul 11, 2007

Survey Co:USGS
Rotation:

Filename: sl25m1.erg

Channels: Ch1 Ch2 Ch3 Ch4 Ch5 Ch3 Ch4

Plotted: 11:07 Nov 06, 2007

$<$ EMI - ElectroMagnetic Instruments 
Station 25

ROTATION ANGLE

Blanca Peak, 100k

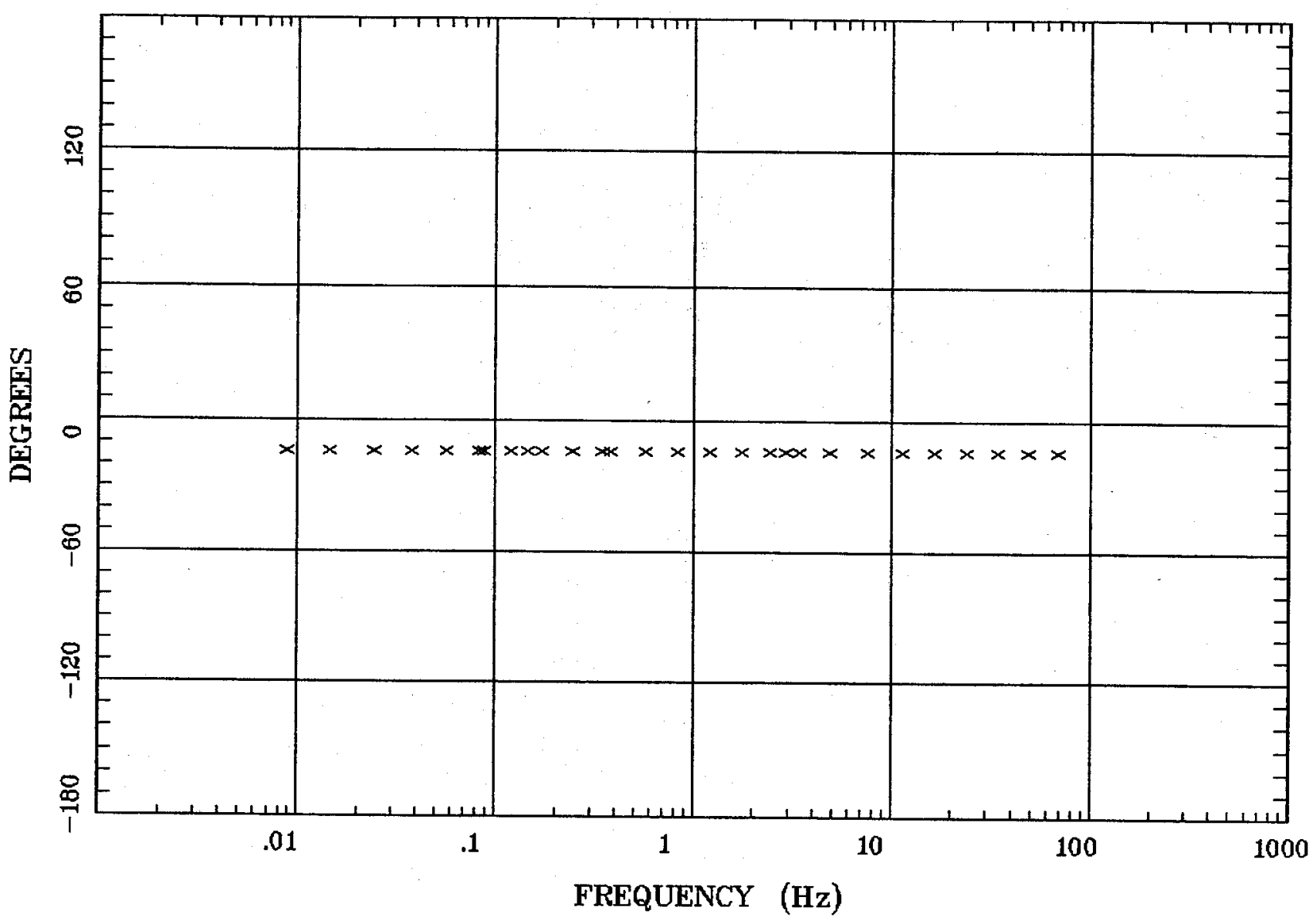

Client:

Remote: none

Acquired: 15:3 Jul 11, 2007

Survey Co:USGS
Rotation:

Filename: sl25m1.avg

Channels: Ch1 Ch2 Ch3 Ch4 Ch5 Ch3 Ch4 Plotted: 11:07 Nov 06, 2007

< EMI - ElectroMagnetic Instruments > 
IMPEDANCE SKEW

Blanca Peak, 100k

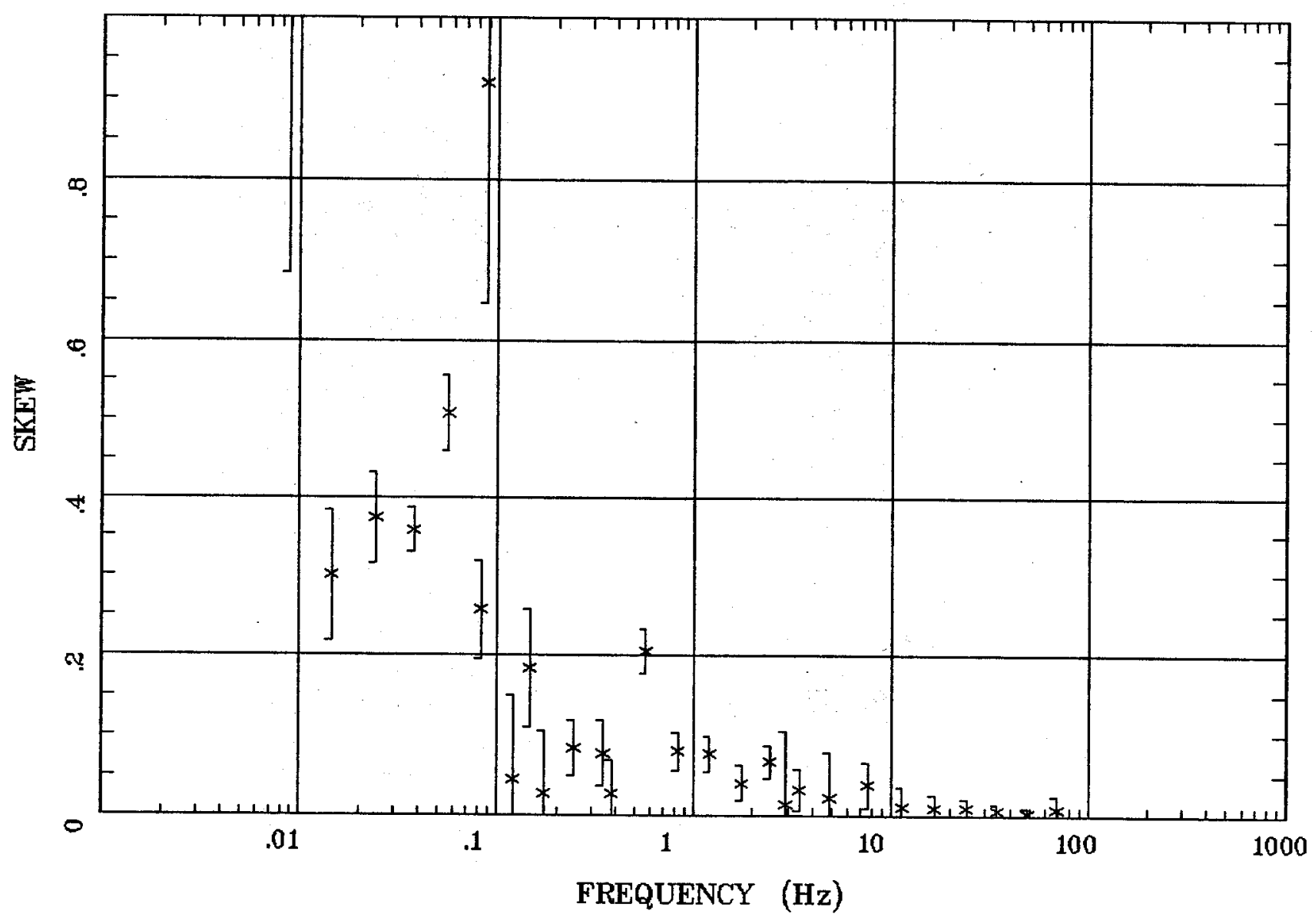

Client:

Remote: none

Acquired: 15:3 Jul 11, 2007

Survey Co:USGS
Rotation:

Filename: sl25m1.avg

Channels: Ch1 Ch2 Ch3 Ch4 Ch5 Ch3 Ch4

Plotted: 11:07 Nov 06, 2007

< EMI - ElectroMagnetic Instruments 
Station 25

E MULT Coh.

Blanca Peak, 100k

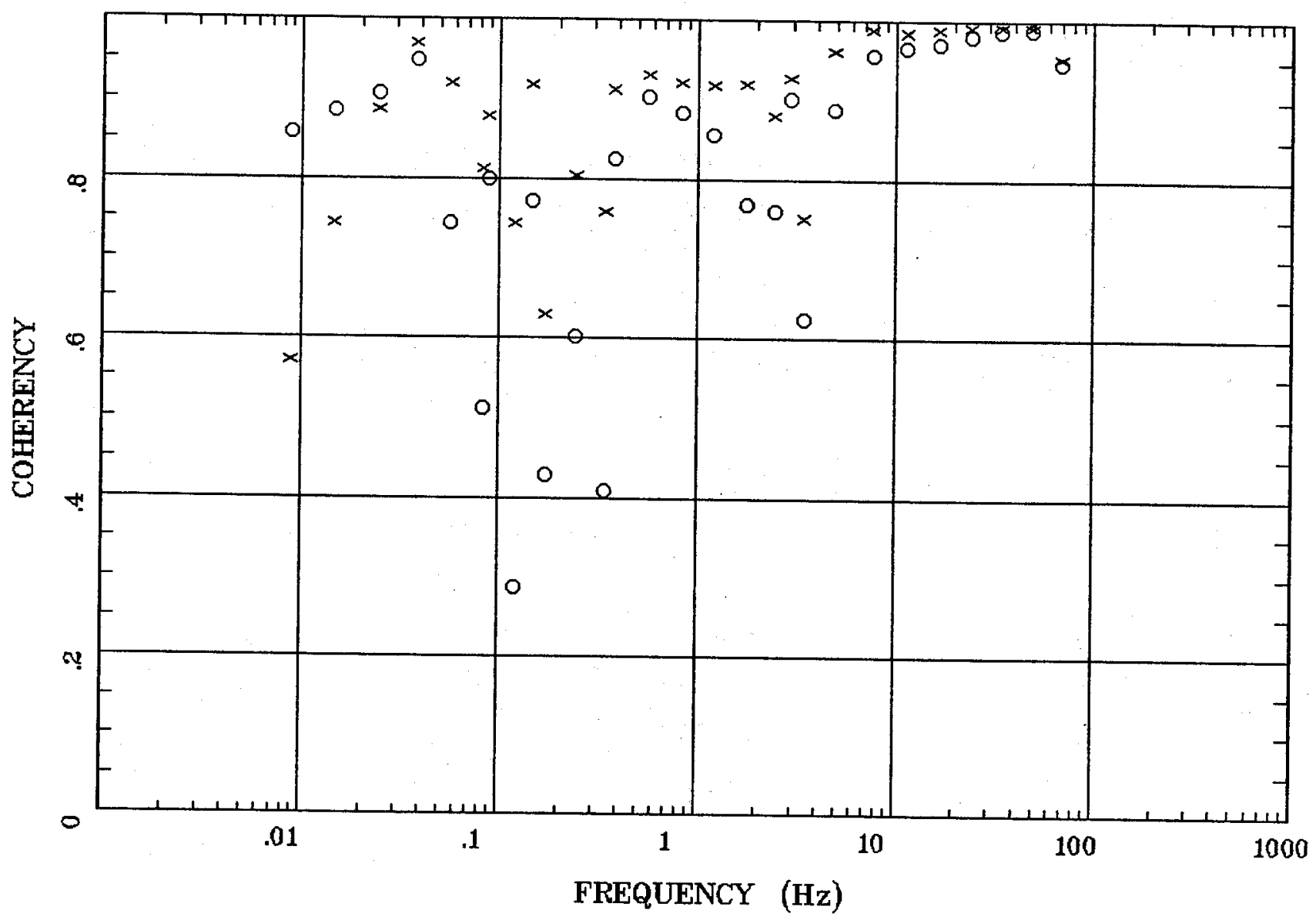

Client:

Remate: none

Acquired: 15:3 Jul 11, 2007

Survey Co:USGS
Rotation:

Filename: sl25m1.avg

Channels: Ch1 Ch2 Ch3 Ch4 Ch5 Ch3 Ch4

Platted: 11:07 Nov 06, 2007

$<$ EMI - ElectroMagnetic Instruments 
POLAR PLOTS

Blanca Peak, 100k

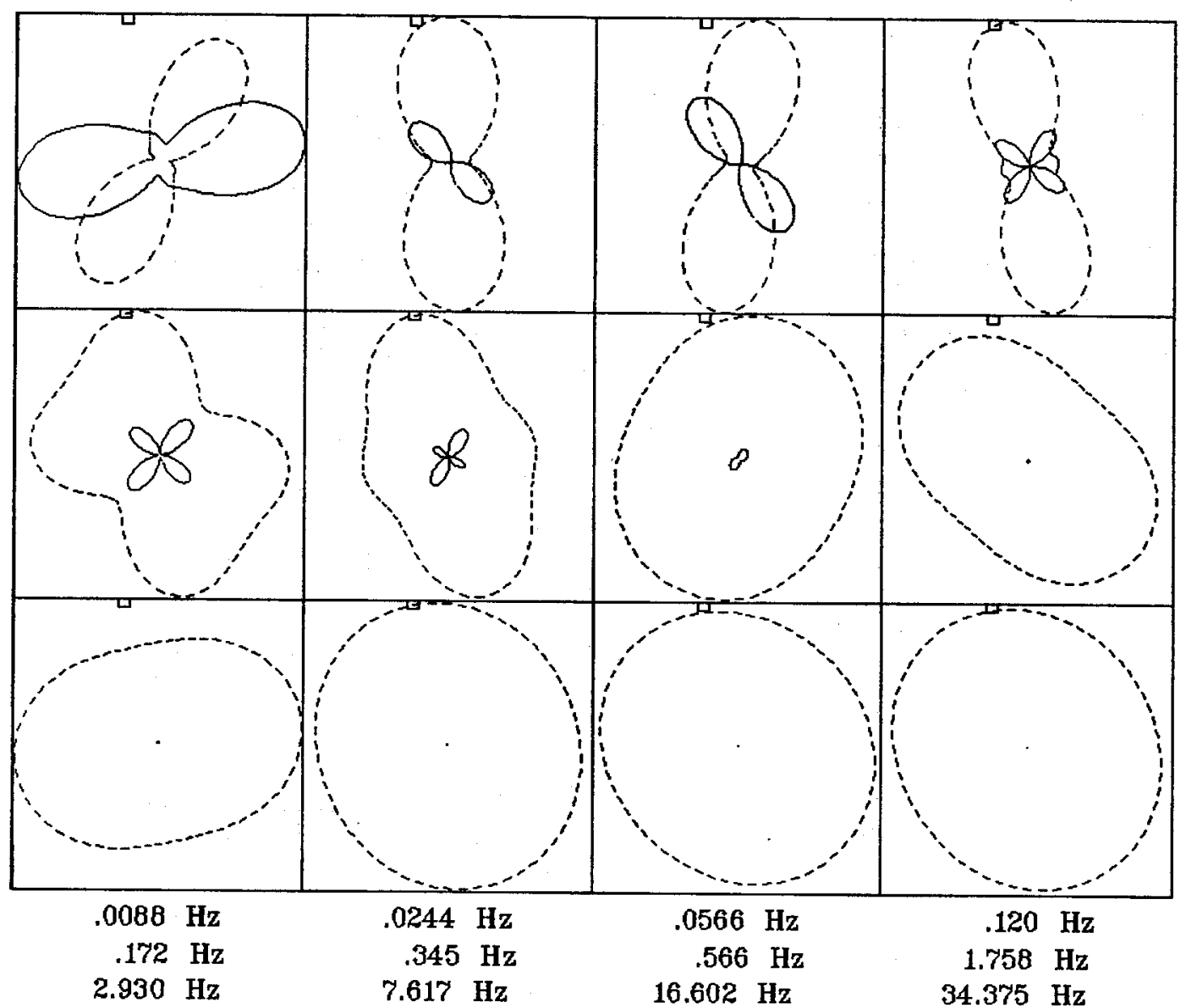

Client:

Remote: none

Acquired: 15:3 Jul 11, 2007

Survey Co:USGS

\section{Rotation:}

Filename: sl25m1.avg

Channels: Ch1 Ch2 Ch3 Ch4 Ch5 Ch3 Ch4 Plotted: 11:07 Nov 06, 2007

< EMI - ElectroMagnetic Instruments > 
Station 25

TIPPER MAGNITUDE

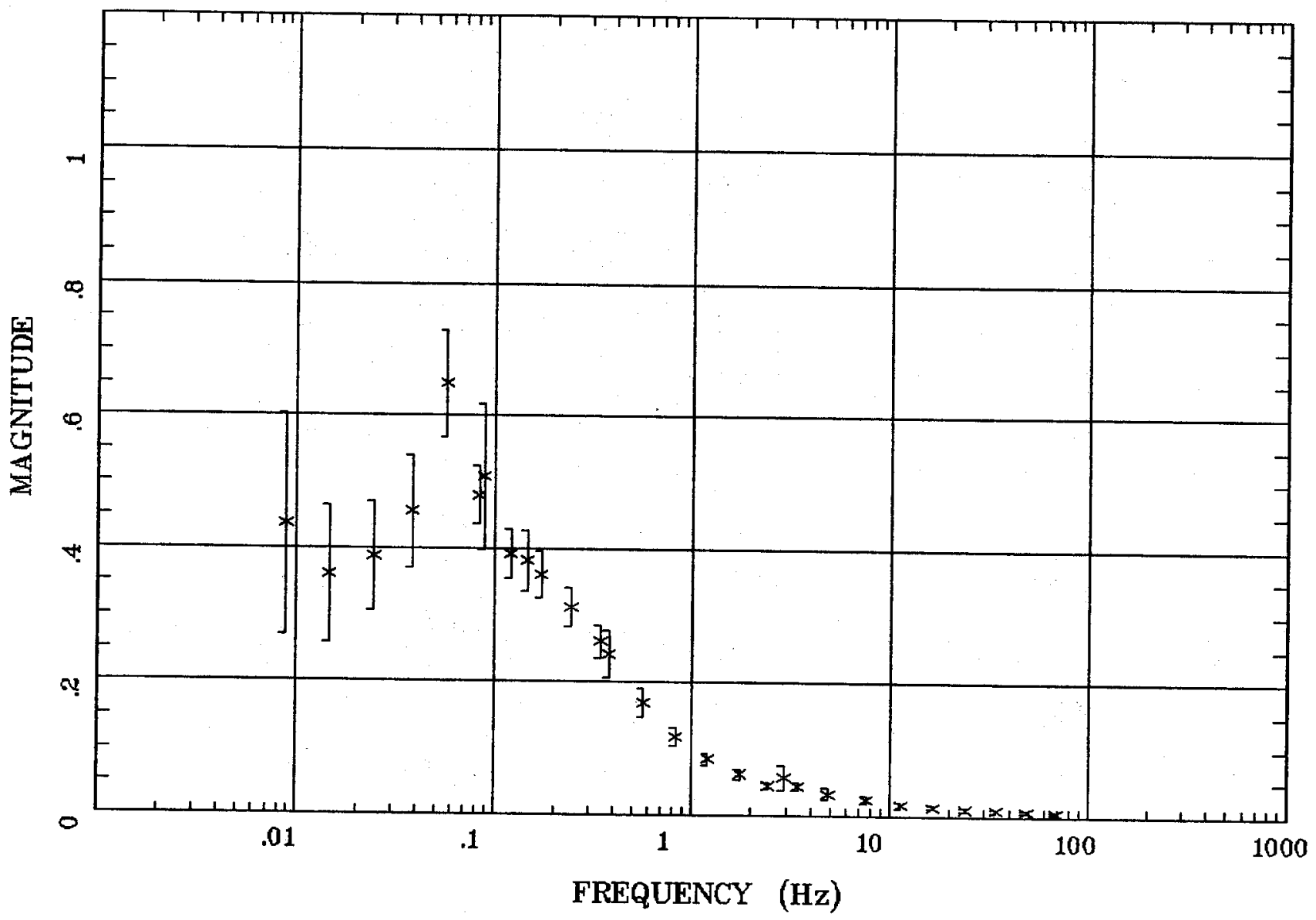

Client:

Remote: none Acquired: 15:3 Jul 11, 2007 Survey Co:USGS
Blanca Peak, 100k

Rotation:

Filename: sl25m1.avg

Channels: Ch1 Ch2 Ch3 Ch4 Ch5 Ch3 Ch4 Plotted: 11:07 Nov 06, 2007

< EMI - ElectroMagnetic Instruments > 


\section{Station 25}

TIPPER STRIKE

Blanca Peak, 100k

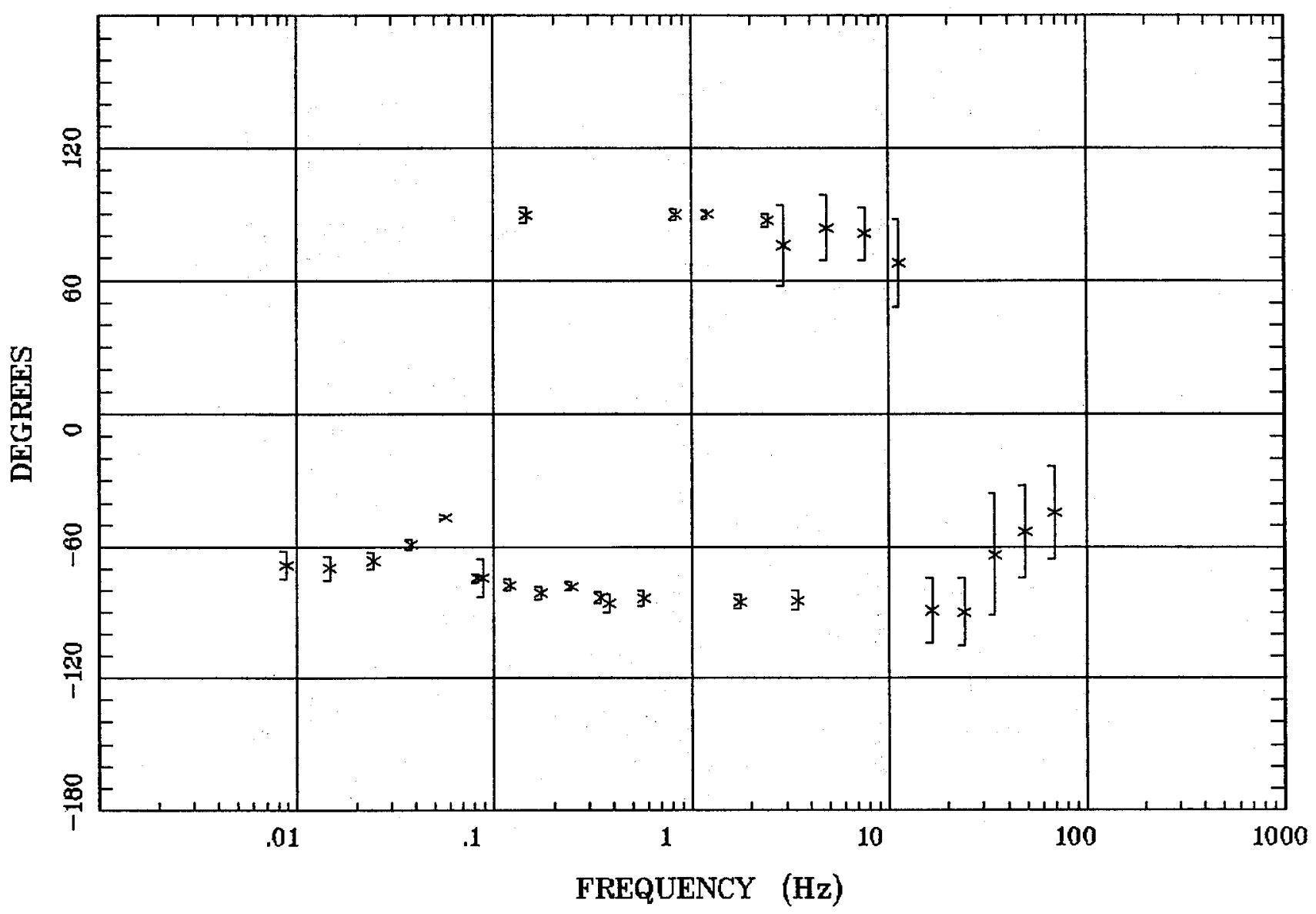

Client:

Remote: none

Acquired: 15:3 Jul 11, 2007

Survey Co:USGS
Rotation:

Filename: sl25m1.avg

Channels: Ch1 Ch2 Ch3 Ch4 Ch5 Ch3 Ch4 Plotted: 11:07 Nov 06, 2007

< EMI - ElectroMagnetic Instruments > 
HzHx.x Coh HzHy.o

Blanca Peak, 100k

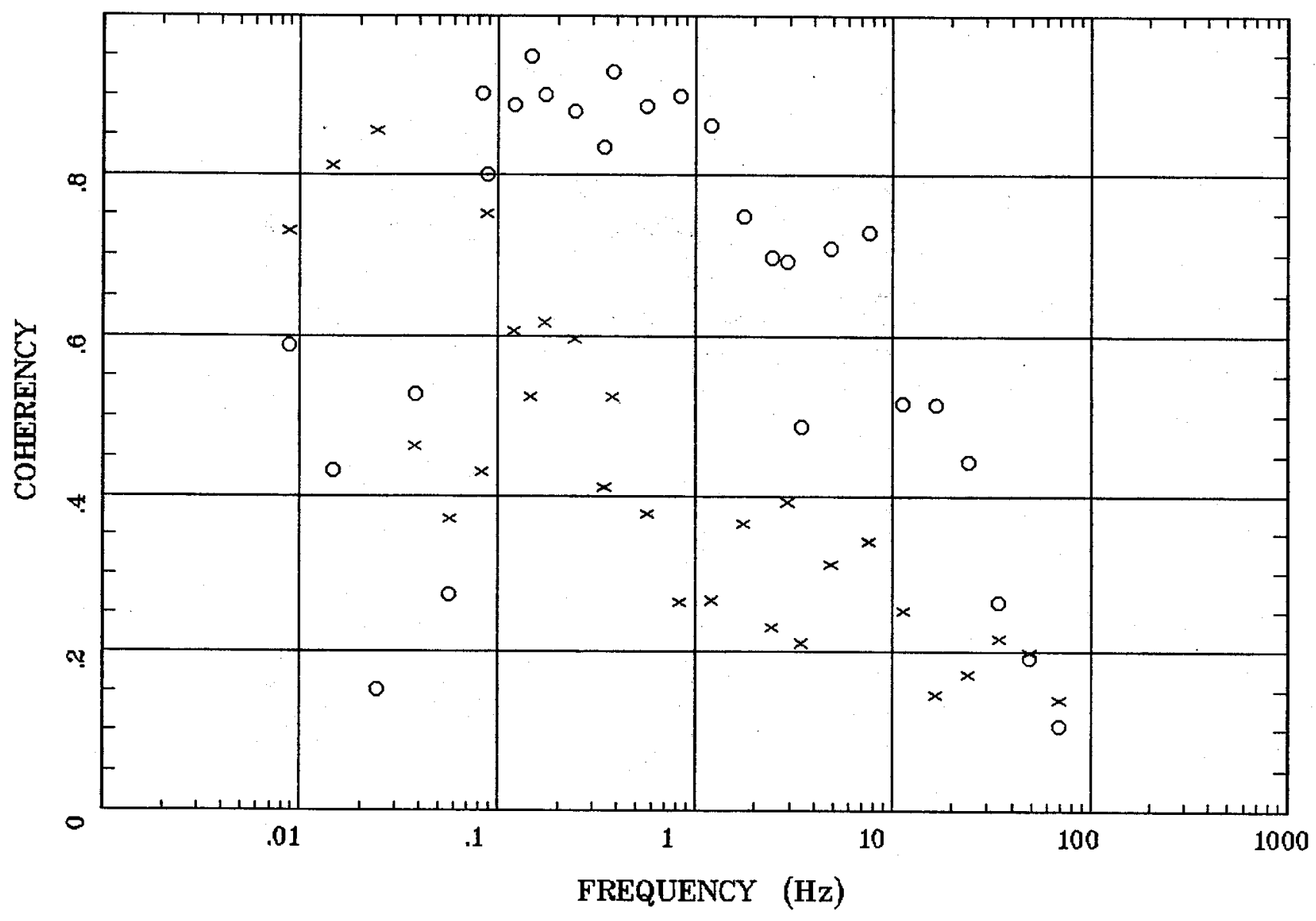

Client:

Remote: none

Acquired: 15:3 Jul 11, 2007

Survey Co:USGS
Rotation:

Filename: sl75m1.avg

Channels: Ch1 Ch2 Ch3 Ch4 Ch5 Ch3 Ch4

Plotted: 11:07 Nov 06, 2007

$<$ EMI - ElectroMagnetic Instruments 
Blanca Peak, 100k

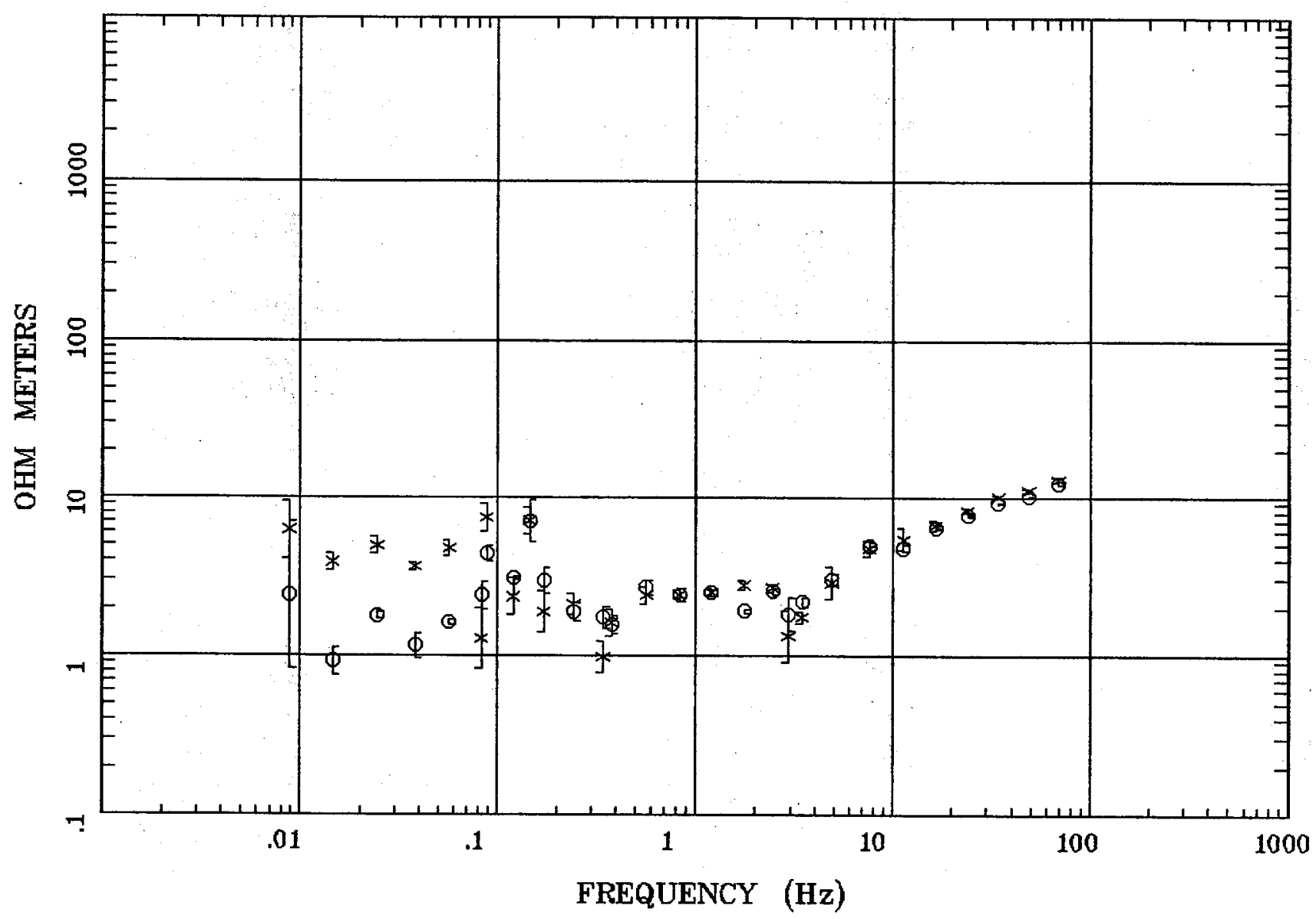

Client:

Remote: none

Acquired: 12:2 Jul 12, 2007 Survey Co:USGS
Rotation:

Filename: sl26m2,avg

Channels: Ch1 Ch2 Ch3 Ch4 Ch5 Ch3 Ch4 Plotted: 11:08 Nov 06, 2007

< EMI - ElectroMagnetic Instruments 
Blanca Peak, 100k

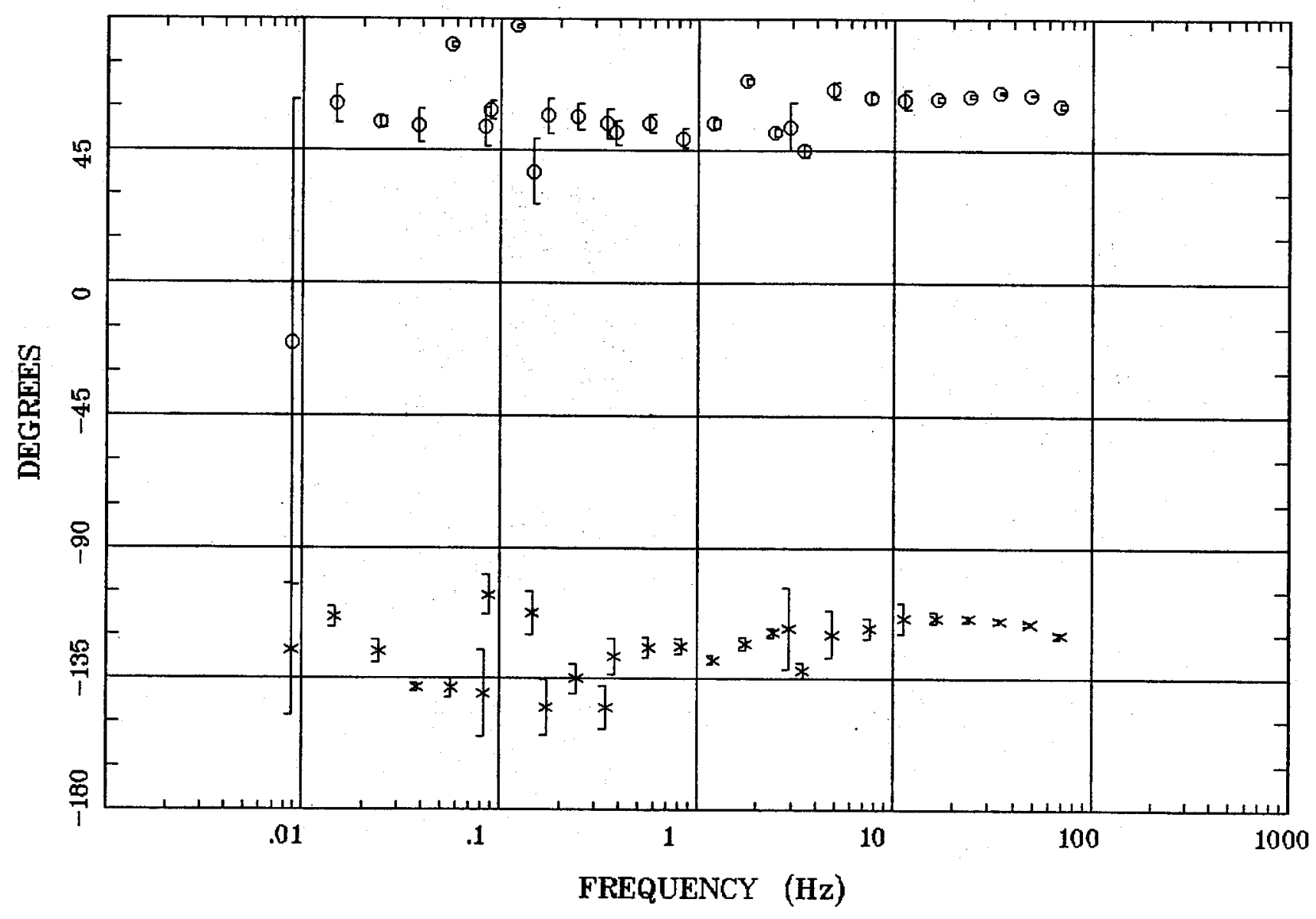

Client:

Remote: none

Acquired: 12:2 Jul 12, 2007

Survey Co:USGS
Rotation:

Filename: sl26m2.avg

Channels: Ch1 Ch2 Ch3 Ch4 Ch5 Ch3 Ch4 Plotted: 11:08 Nov 06, 2007

< EMI - ElectroMagnetic Instruments > 


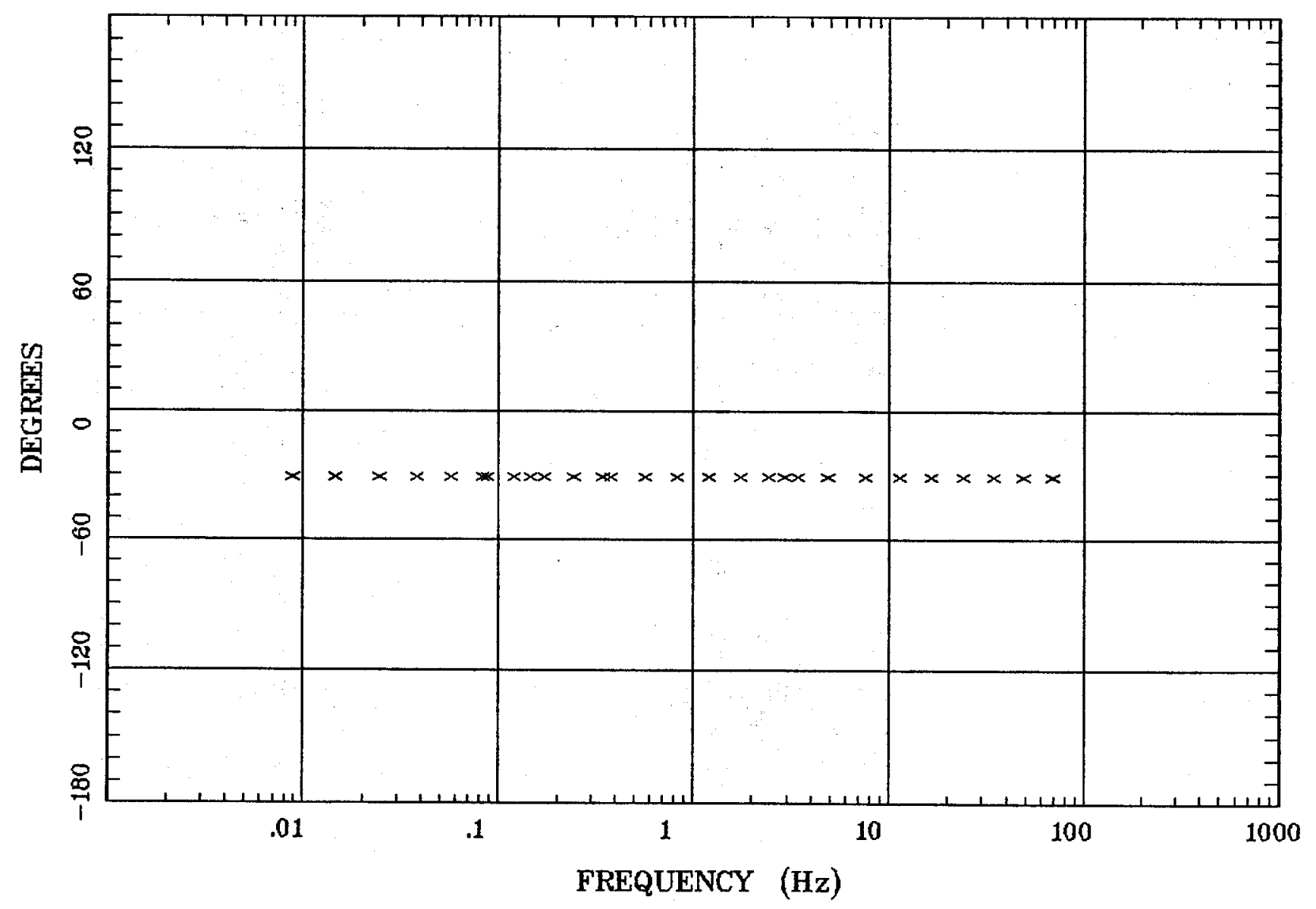

Client:

Remote: none

Acquired: 12:2 Jul 12, 2007

Survey Co:USGS
Rotation:

Filename: sl26m2.avg

Channels: Ch1 Ch2 Ch3 Ch4 Ch5 Ch3 Ch4

Plotted: 11:08 Nov 06, 2007

< EMI - ElectroMagnetic Instruments 
Station 26

IMPEDANCE SKEW

Blanca Peak, 100k

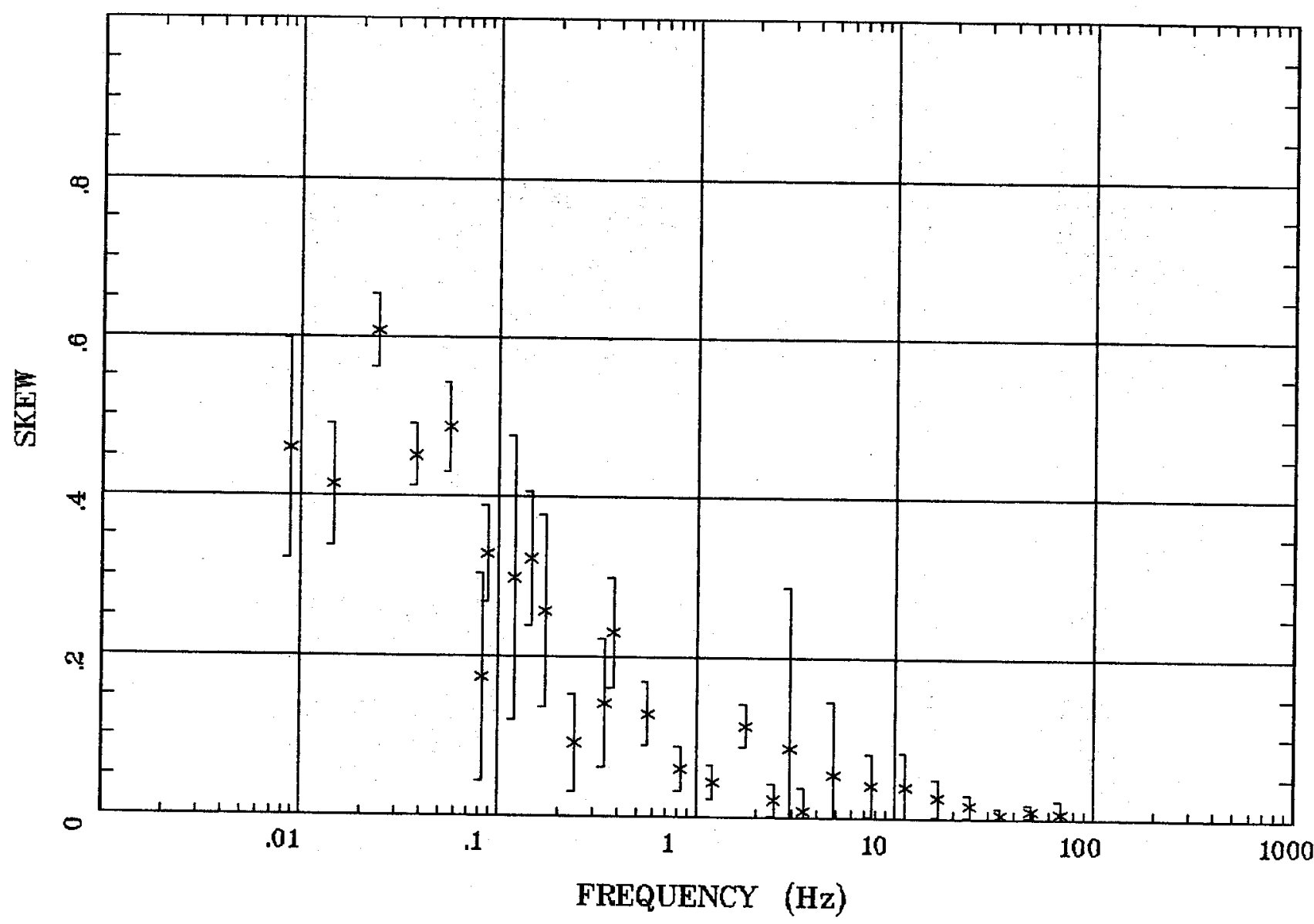

Client:

Rotation:

Remote: none

Acquired: 12:2 Jul 12, 2007

Surrey Co:USGS

Filename: sl26m2.avg

Channels: Ch1 Ch2 Ch3 Ch4 Ch5 Ch3 Ch4

Plotted: 11:08 Nov 06, 2007

< EMI - ElectroMagnetic Instruments 


\section{E MULT Coh.}

Blanca Peak, 100k

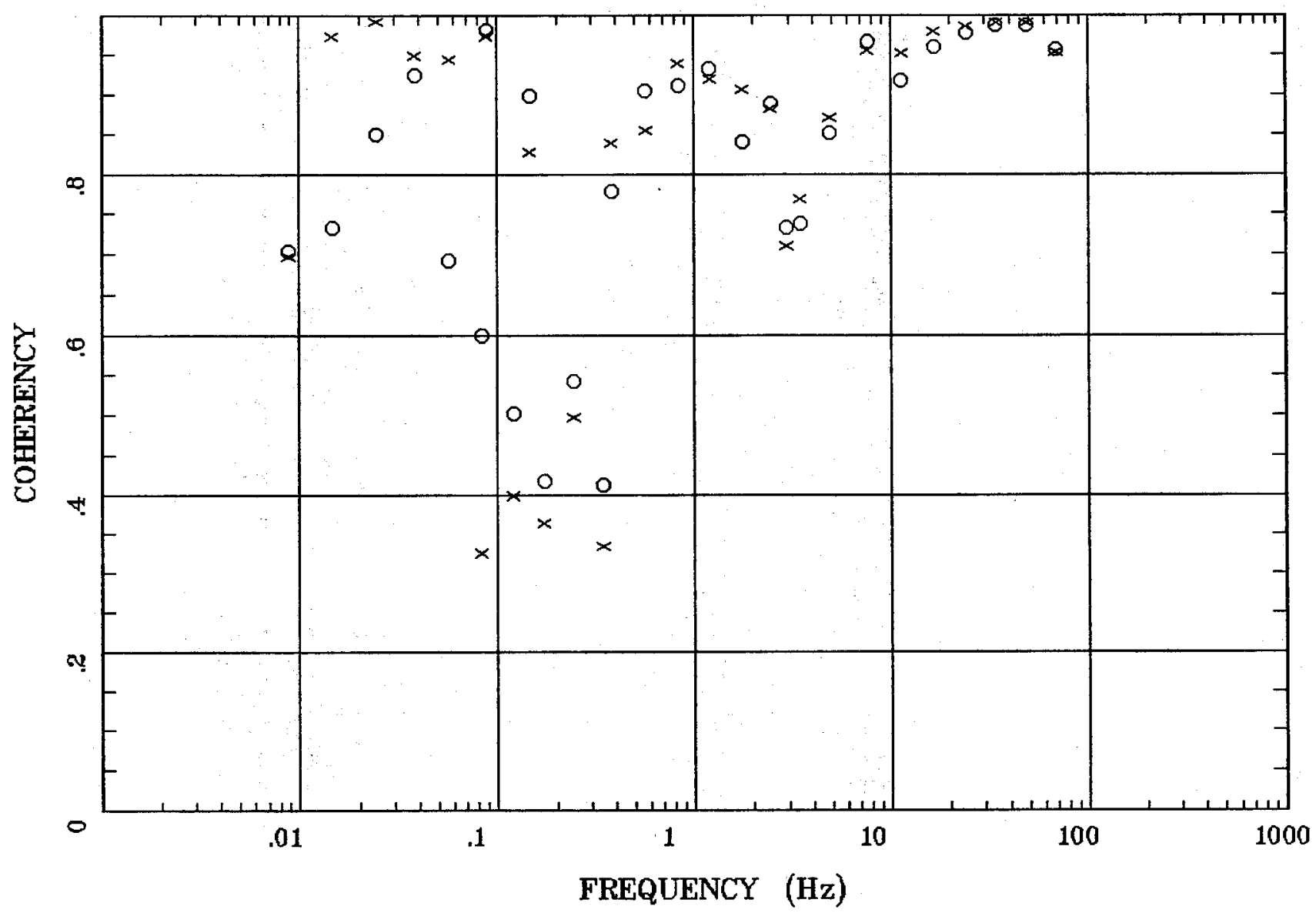

Client:

Remote: none

Acquired: 12:2 Jul 12, 2007 Survey Co:USGS
Rotation:

Filename: sl26m2.avg

Channels: Ch1 Ch2 Ch3 Ch4 Ch5 Ch3 Ch4 Plotted: 11:08 Nor 06, 2007

< EMI - ElectroMagnetic Instruments 


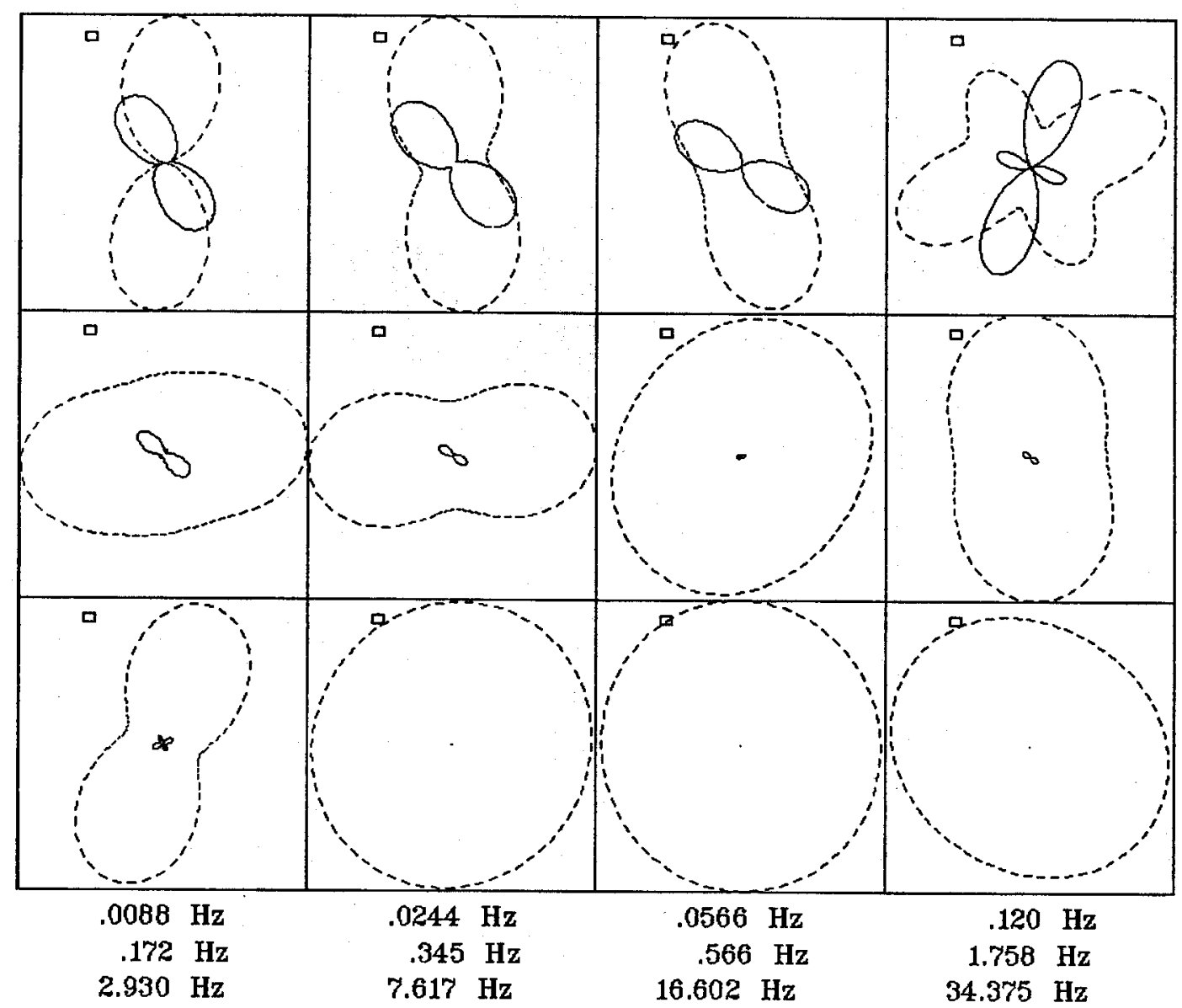

Client:

Remote: none

Acquired: 12:2 Jul 12, 2007

Survey Co:USGS
Rotation:

Filename: sl26mz.avg

Channels: Ch1 Ch2 Ch3 Ch4 Ch5 Ch3 Ch4

Plotted: 11:08 Nov 06, 2007

< EMI - ElectroMagnetic Instruments 


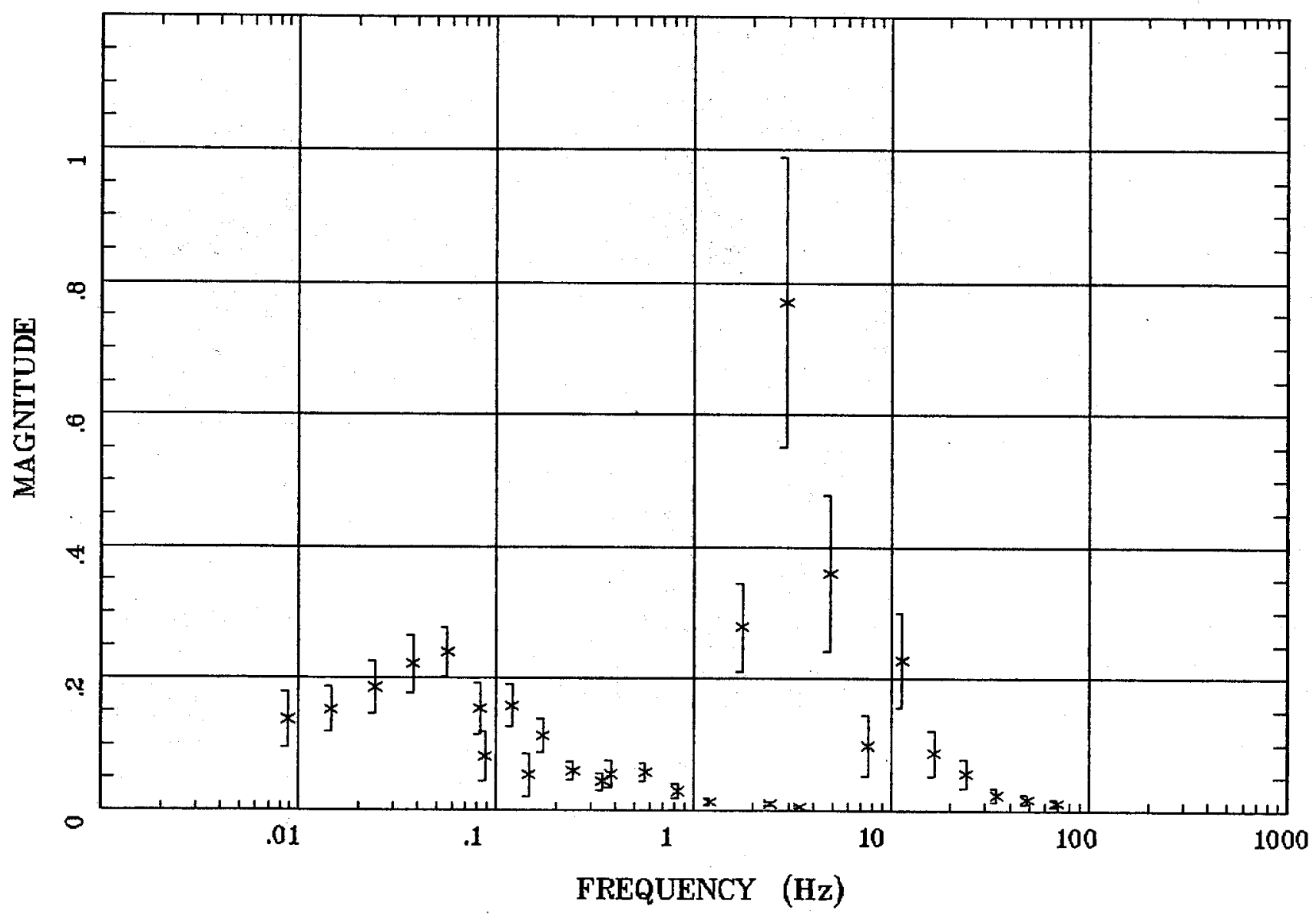

Client:

Remote: none

Acquired: 12:2 Jul 12, 2007

Survey Co:USGS
Rotation:

Filename: sl26m2.avg

Channels: Ch1 Ch2 Ch3 Ch4 Ch5 Ch3 Ch4

Plotted: 11:08 Nor 06, 2007

< EMI - ElectroMagnetic Instruments > 


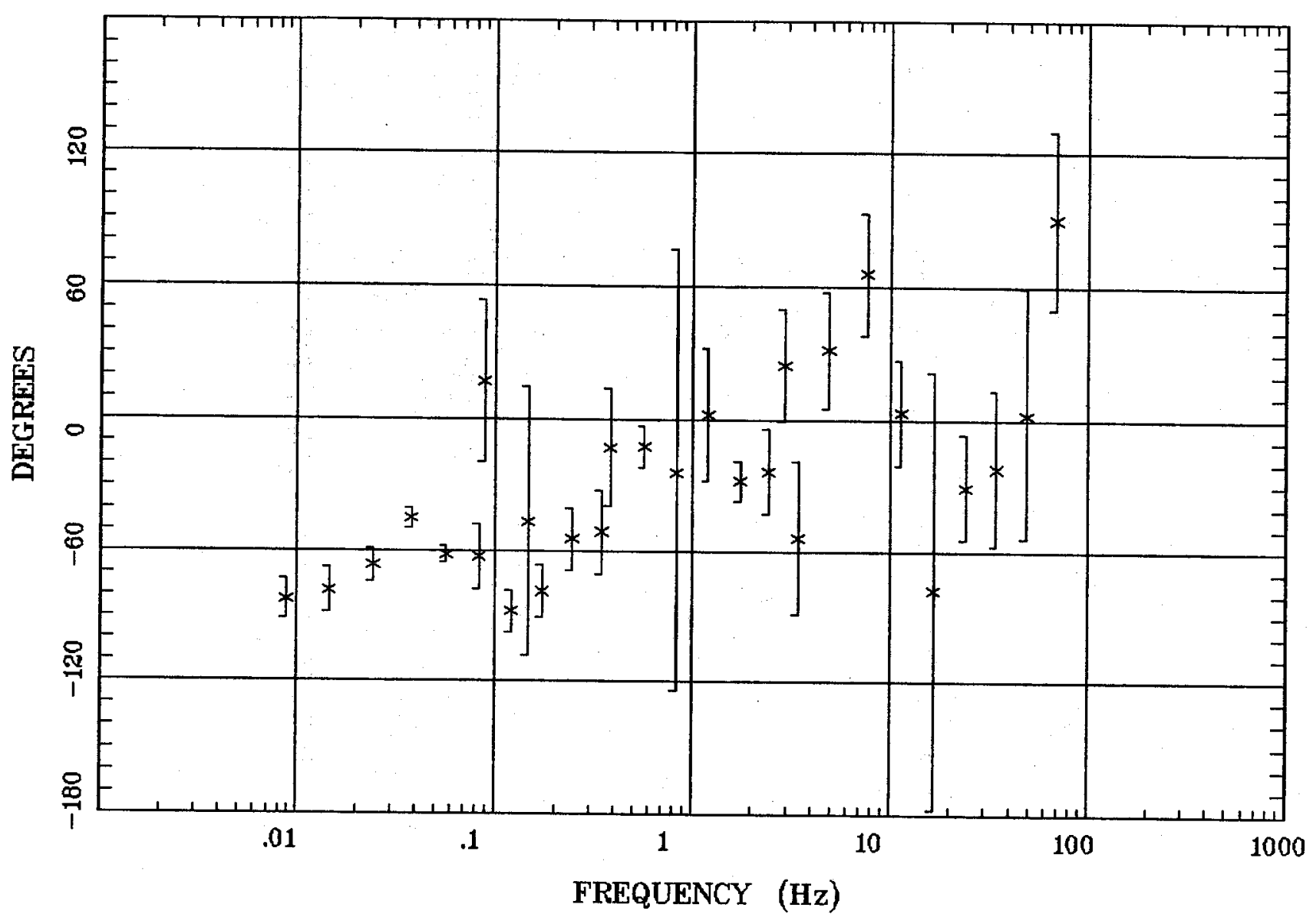

Client:

Remote: none

Acquired: 12:2 Jul 12, 2007 Survey Co:USGS
Rotation:

Filename: sl26m2.avg

Channels: Ch1 Ch2 Ch3 Ch4 Ch5 Ch3 Ch4 Plotted: 11:08 Nov 06, 2007

< EMI - ElectroMagnetic Instruments > 
HzHx.x Coh HzHy.o

Blanca Peak, 100k

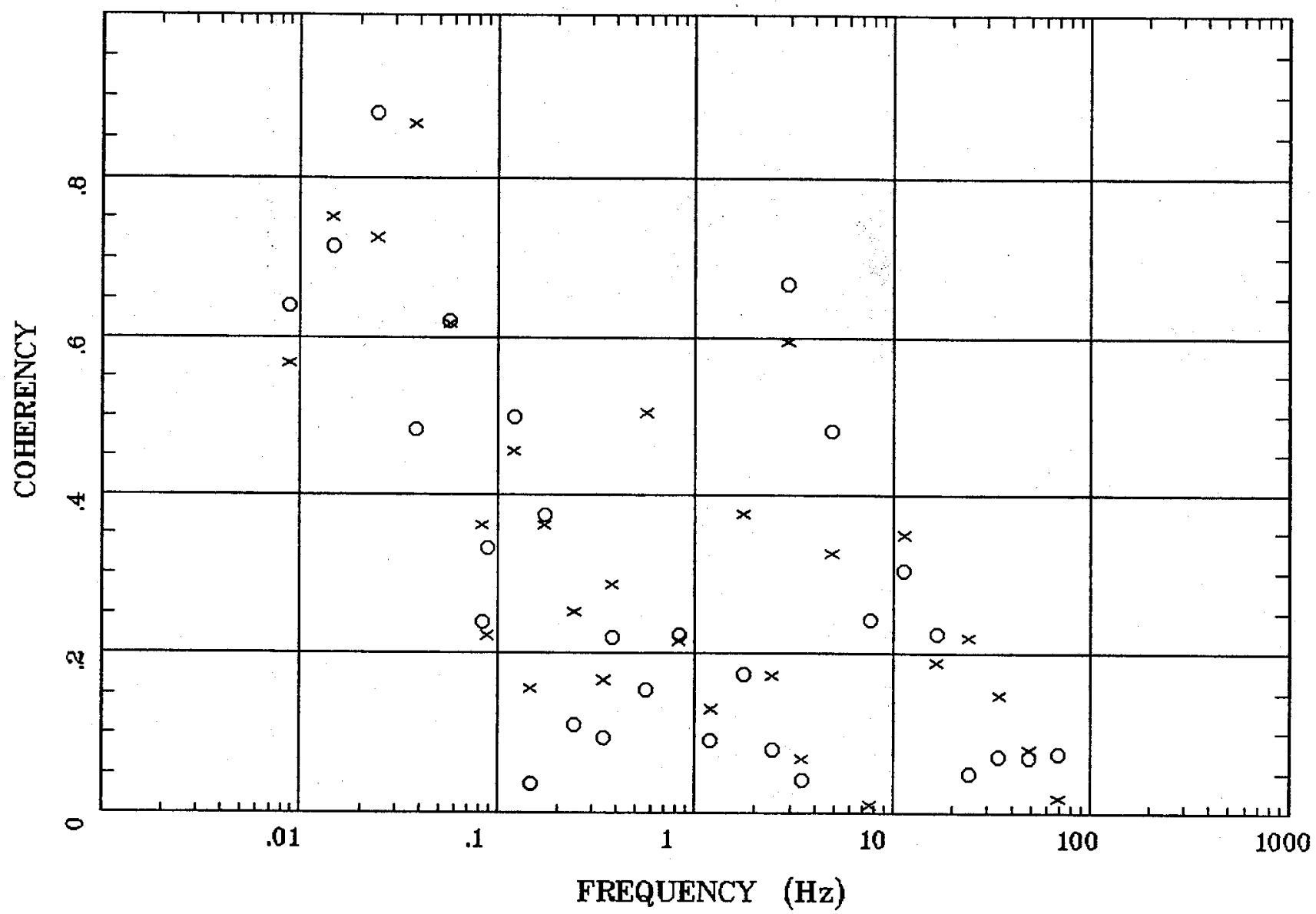

Client:

Remote: none

Acquired: 12:2 Jul 12, 2007

Survey Co:USGS
Rotation:

Filename: sl26m2.avg

Channels: Ch1 Ch2 Ch3 Ch4 Ch5 Ch3 Ch4

Plotted: 11:08 Nov 06, 2007

$<$ EMI - ElectroMagnetic Instruments 


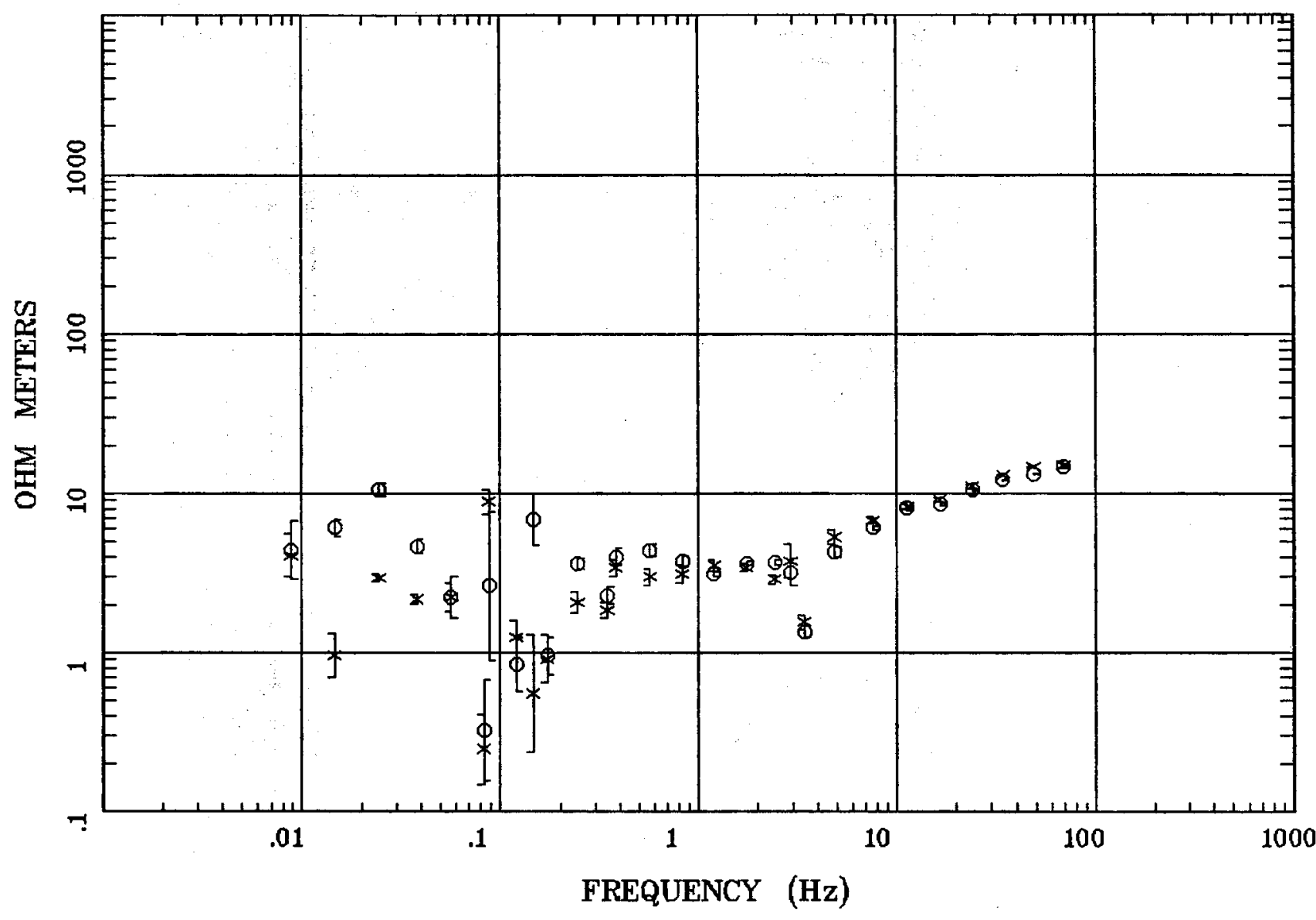

Client:

Remote: none

Acquired: 10:5 Jul 13, 2007

Survey Co:USGS
Rotation:

Filename: slarm2.avg

Channels: Ch1 Ch2 Ch3 Ch4 Ch5 Ch3 Ch4 Plotted: 11:08 Nor 06, 2007

< EMI - ElectroMagnetic Instruments > 
Blanca Peak, 100k

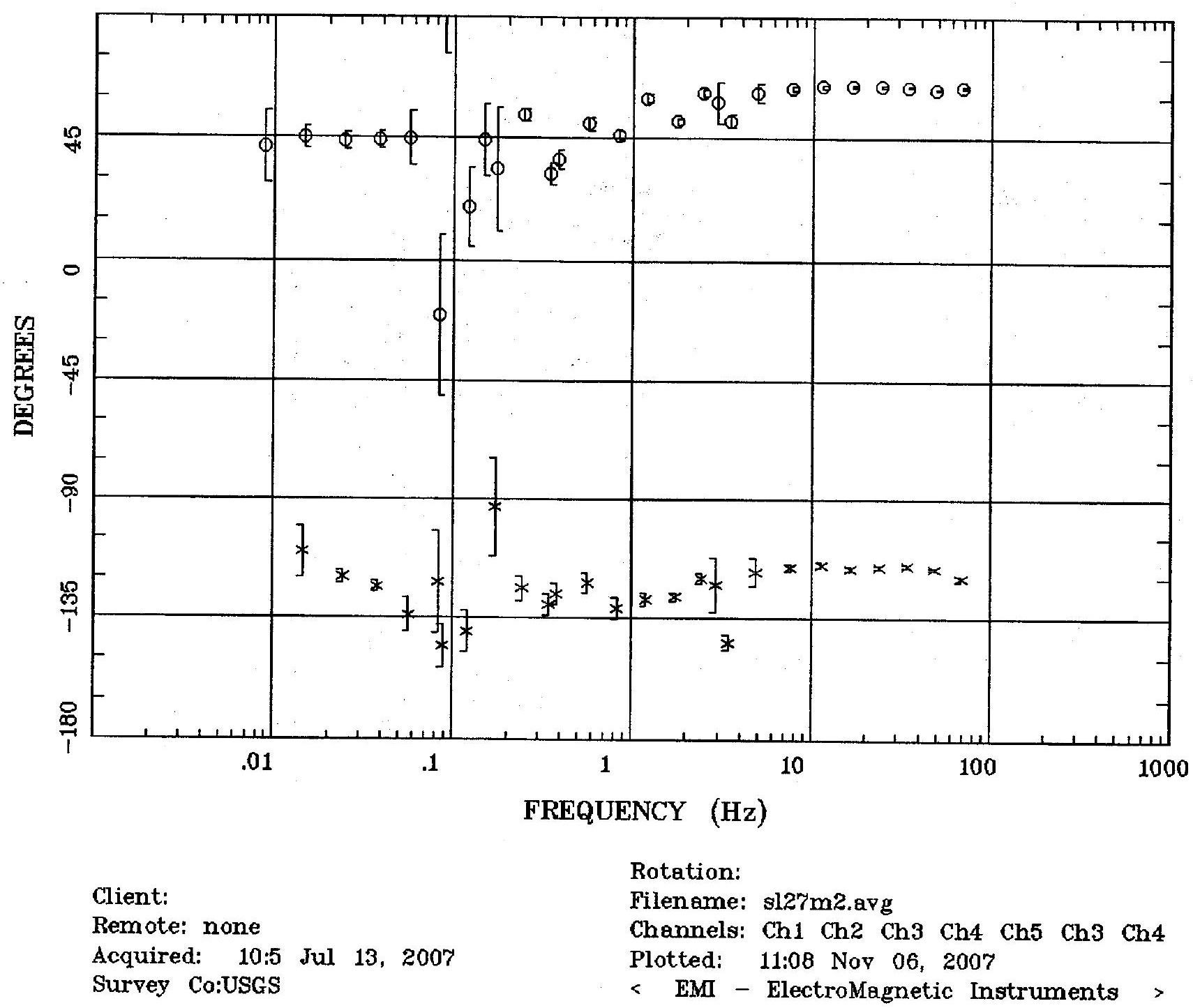


Blanca Peak, 100k

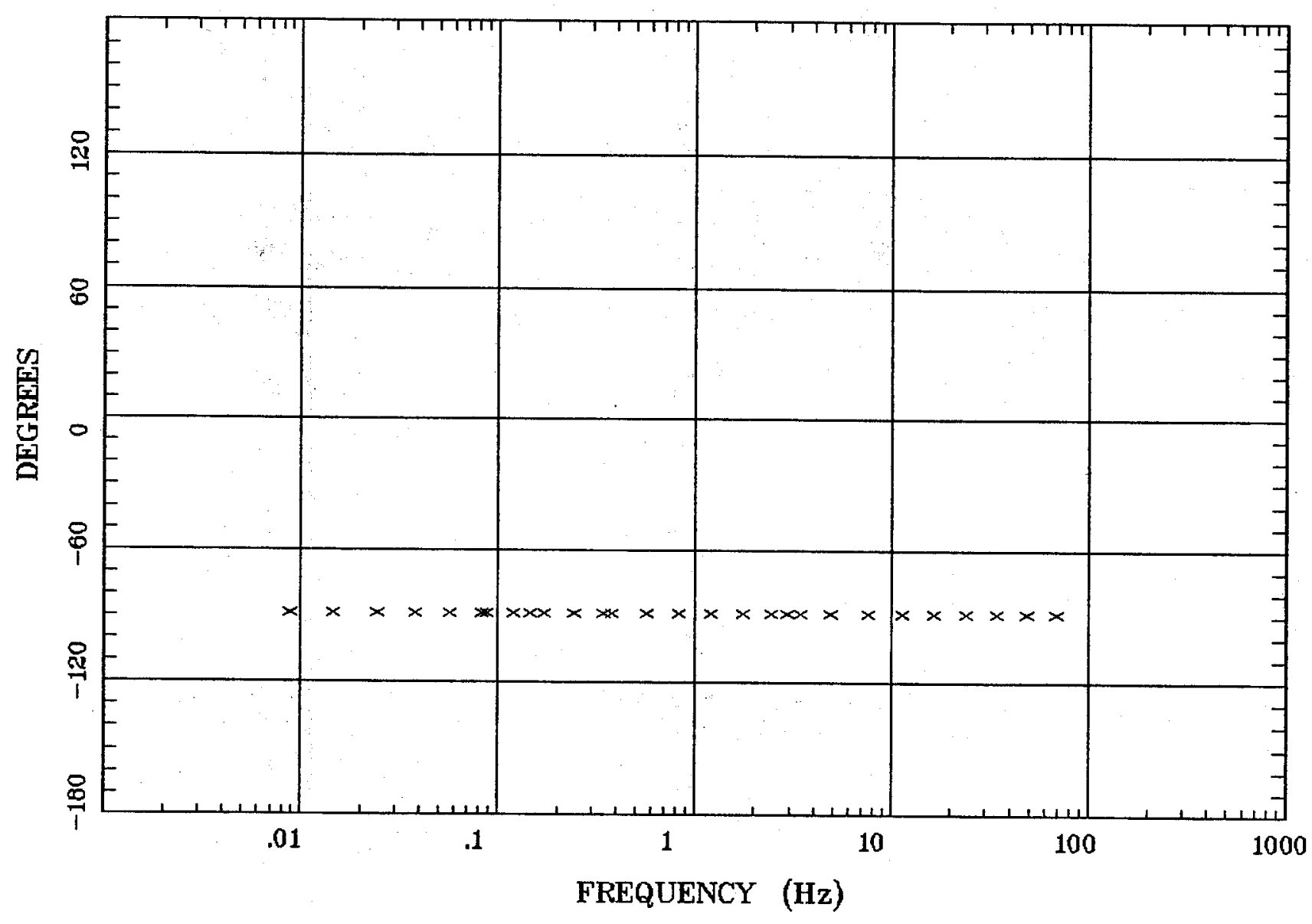

Client:

Remote: none

Acquired: 10:5 Jul 13, 2007

Survey Co:USGS
Rotation:

Filename: sl27m2.avg

Channels: Ch1 Ch2 Ch3 Ch4 Ch5 Ch3 Ch4 Plotted: 11:08 Nov 06, 2007

$<$ EMI - ElectroMagnetic Instruments 
Station 27

IMPEDANCE SKEW

Blanca Peak, 100k

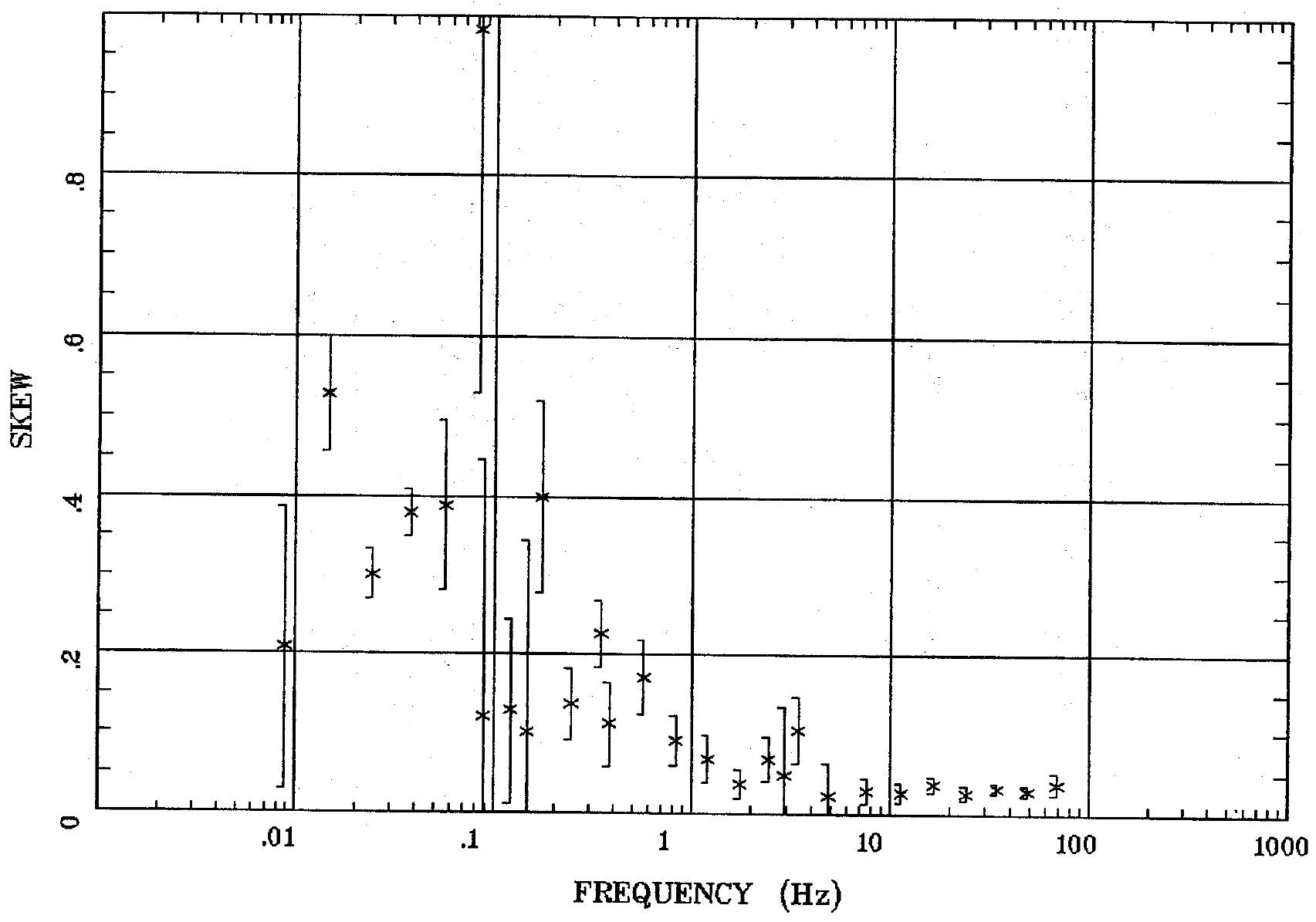

Client:

Remote: none

Acquired: 10:5 Jul 13, 2007 Survey Ca:USGS
Rotation:

Filename: sl27m2.avg

Channels: Ch1 Ch2 Ch3 Ch4 Ch5 Ch3 Ch4 Plotted: 11:08 Nov 06, 2007

< EMI - ElectroMagnetic Instruments > 
E MULT Coh.

Blanca Peak, 100k

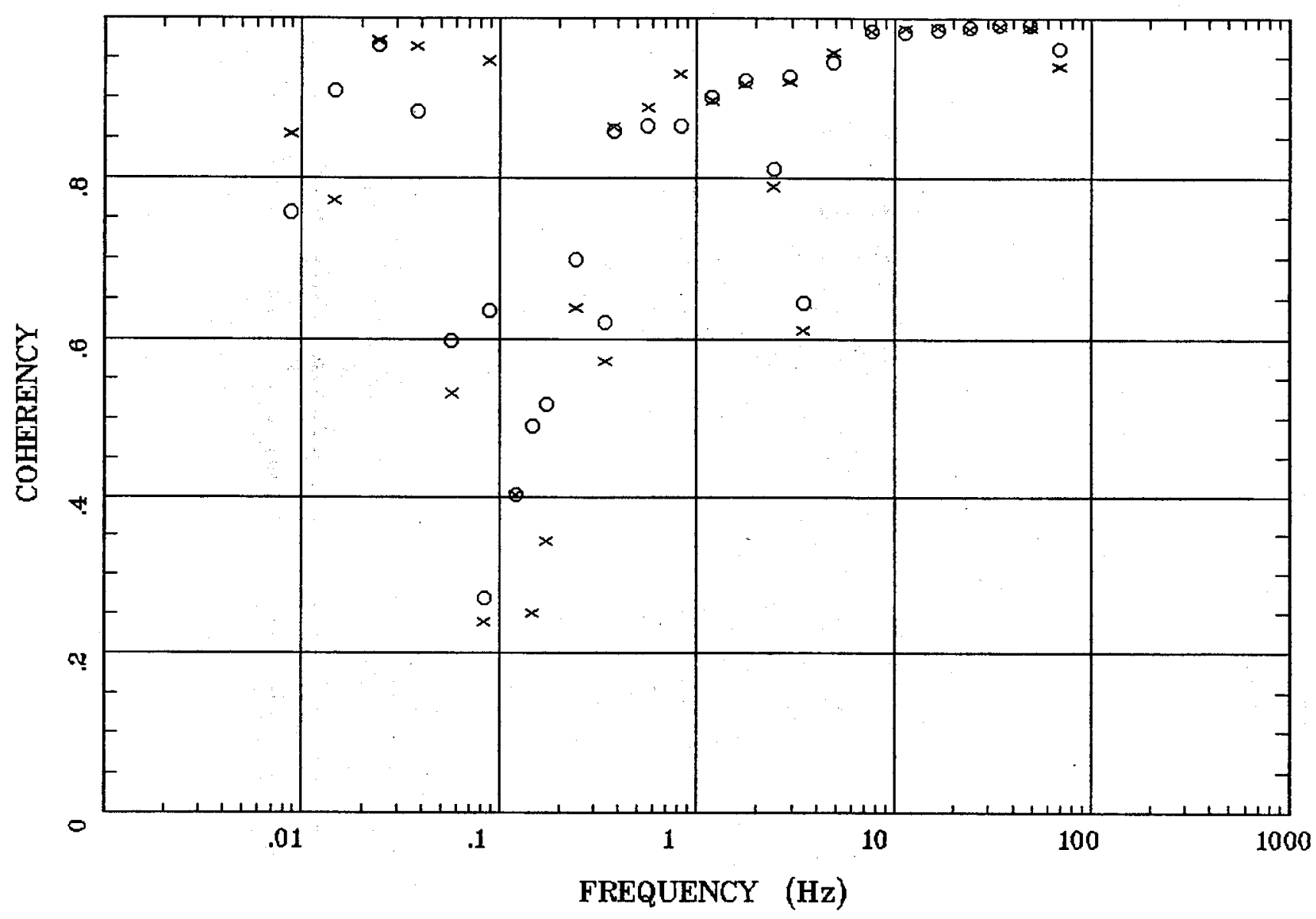

Client:

Remote: none

Acquired: $10: 5$ Jul 13, 2007 Survey Co:USGS
Rotation:

Filename: sl27m2.avg

Channels: Ch1 Ch2 Ch3 Ch4 Ch5 Ch3 Ch4

Plotted: 11:08 Nov 06, 2007

< EMI - ElectroMagnetic Instruments > 
Blanca Peak, 100k

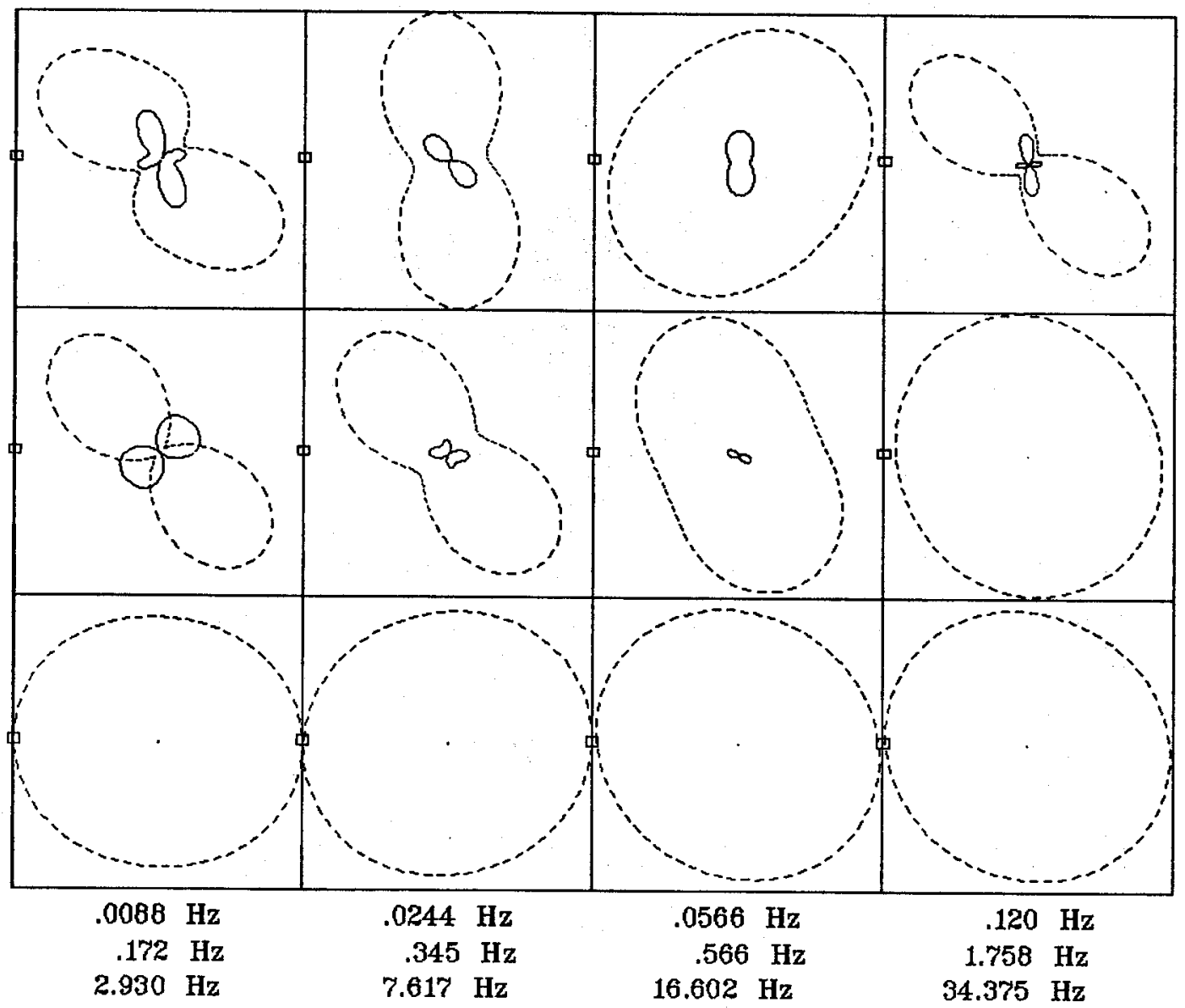

Client:

Remote: none

Acquired: 10:5 Jul 13, 2007

Survey Co:USGS
Rotation:

Filename: sl27m2.avg

Channels: Ch1 Ch2 Ch3 Ch4 Ch5 Ch3 Ch4 Plotted: 11:08 Nov 06, 200r

< EMI - ElectroMagnetic Instruments > 
Station 27

TIPPER MAGNITUDE

Blanca Peak, 100k

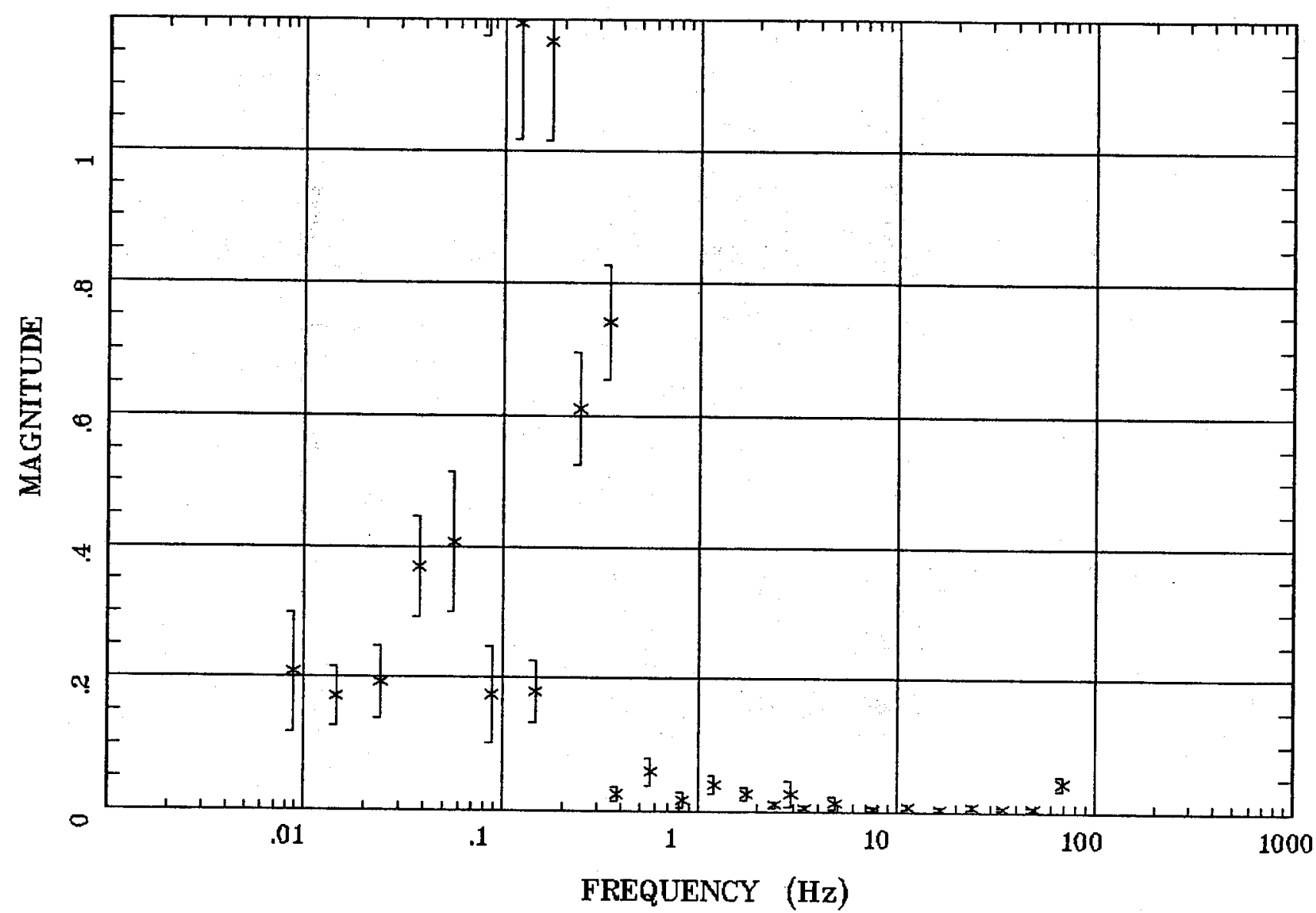

Client:

Remote: none

Acquired: 10:5 Jul 13, 2007

Survey Co:USGS

Rotation:

Filename: slarmz.avg

Channels: Ch1 Ch2 Ch3 Ch4 Ch5 Ch3 Ch4

Platted: 11:08 Nor 06, 2007

< EMI - ElectroMagnetic Instruments > 


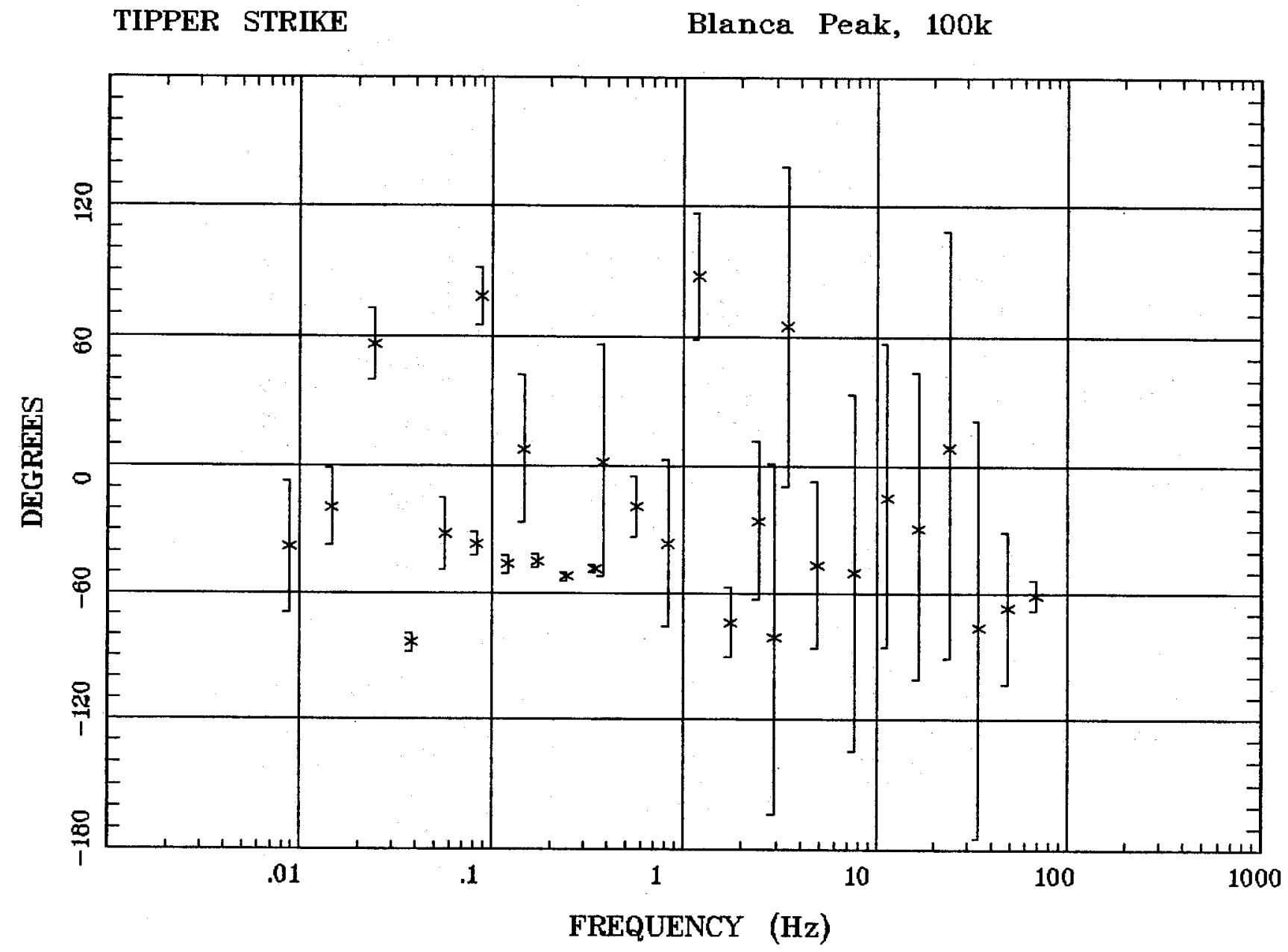

Client:

Remote: none

Acquired: 10:5 Jul 13, 2007 Survey Co:USGS
Rotation:

Filename: sl27m2.avg

Channels: Ch1 Ch2 Ch3 Ch4 Ch5 Ch3 Ch4 Plotted: 11:08 Nov 06, 2007

< EMI - ElectroMagnetic Instruments > 


\section{HzHx.x Coh HzHy.o Blanca Peak, 100k}

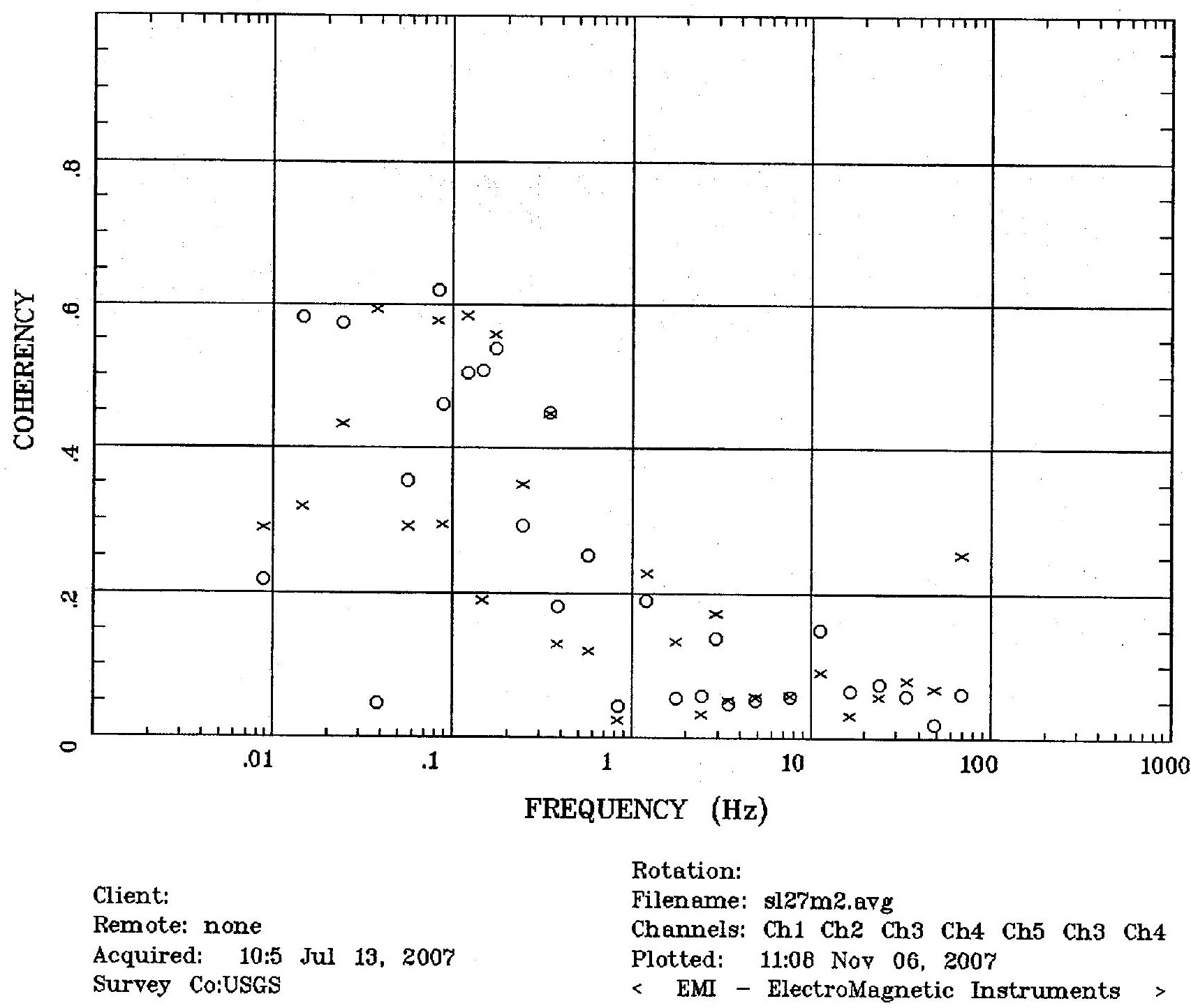


APPARENT RESISTIVITY

Blanca Peak, 100k

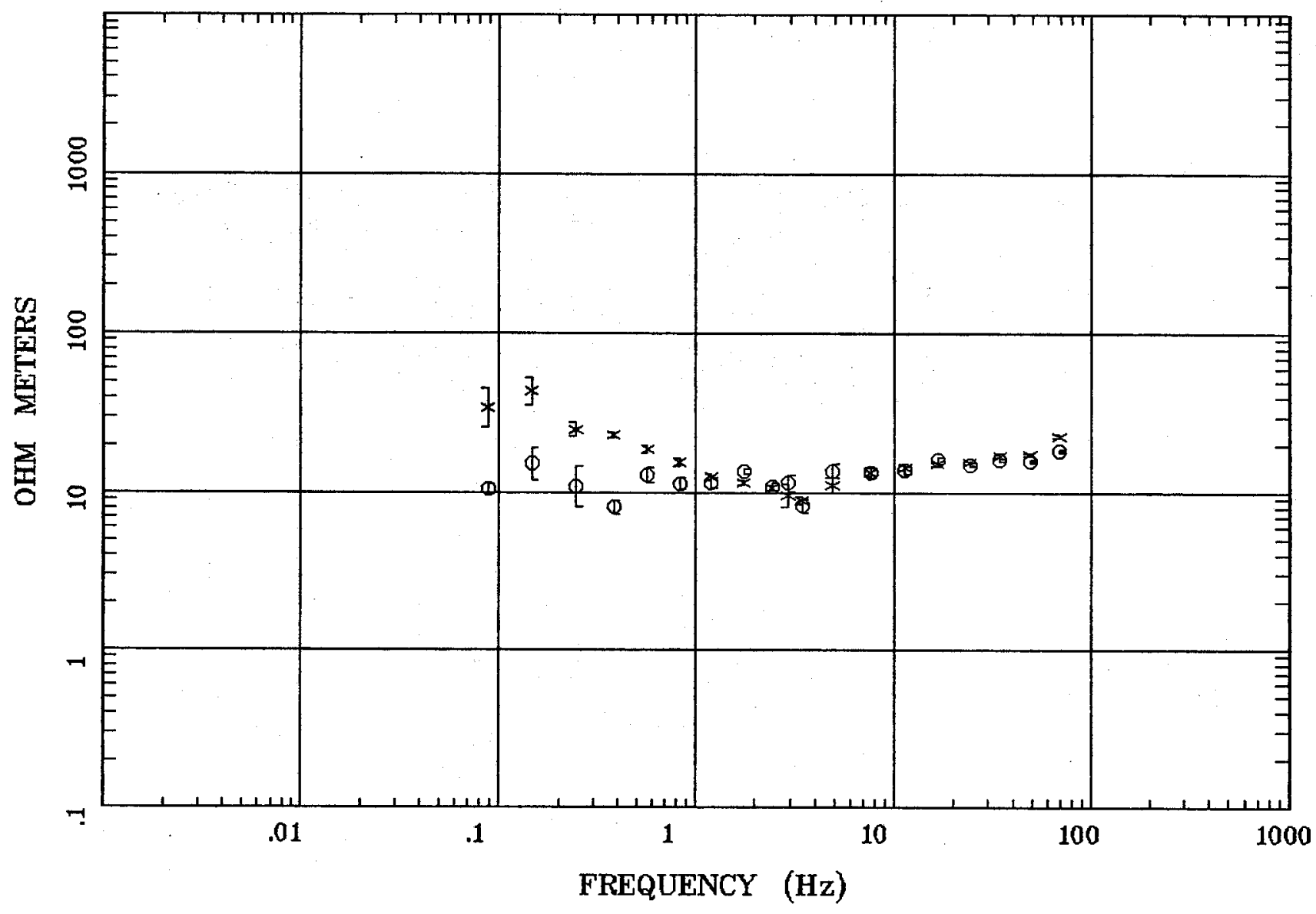

Client:

Remote: none

Acquired: 15:2 Jul 13, 2007

Survey Co:USGS
Rotation:

Filename: sl28m.avg

Channels: Ch1 Ch2 Ch3 Ch4 Ch5 Ch3 Ch4 Plotted: 11:09 Nop 06, 2007

< EMI - ElectroMagnetic Instruments 
Blanca Peak, 100k

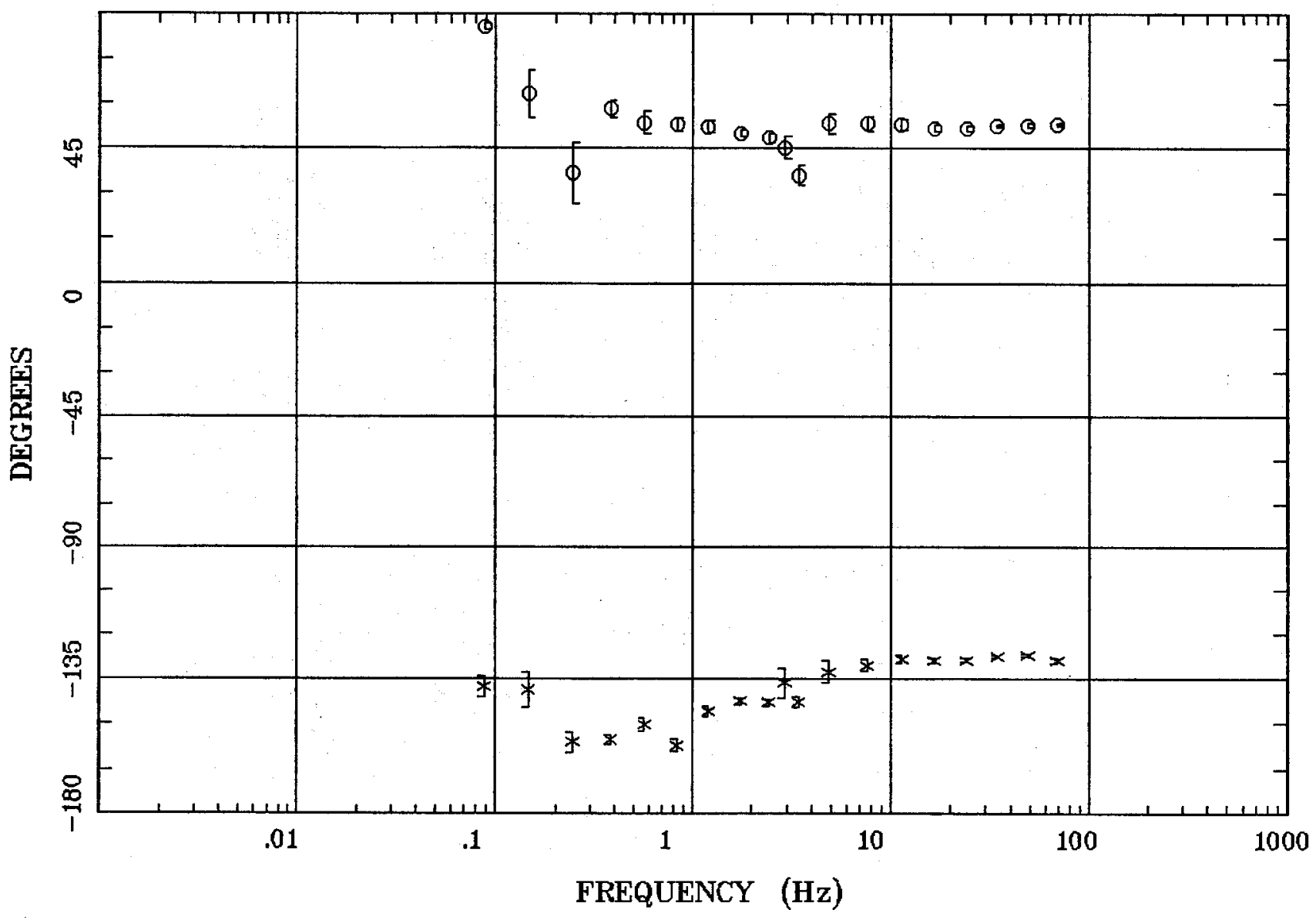

Client:

Remote: none

Acquired: 15:2 Jul 13, 2007 Survey Co:USGS
Rotation:

Filename: sl28m.avg

Channels: Ch1 Ch2 Ch3 Ch4 Ch5 Ch3 Ch4

Plotted: 11:09 Nov 06, 2007

< EMI - ElectroMagnetic Instruments 
Blanca Peak, 100k

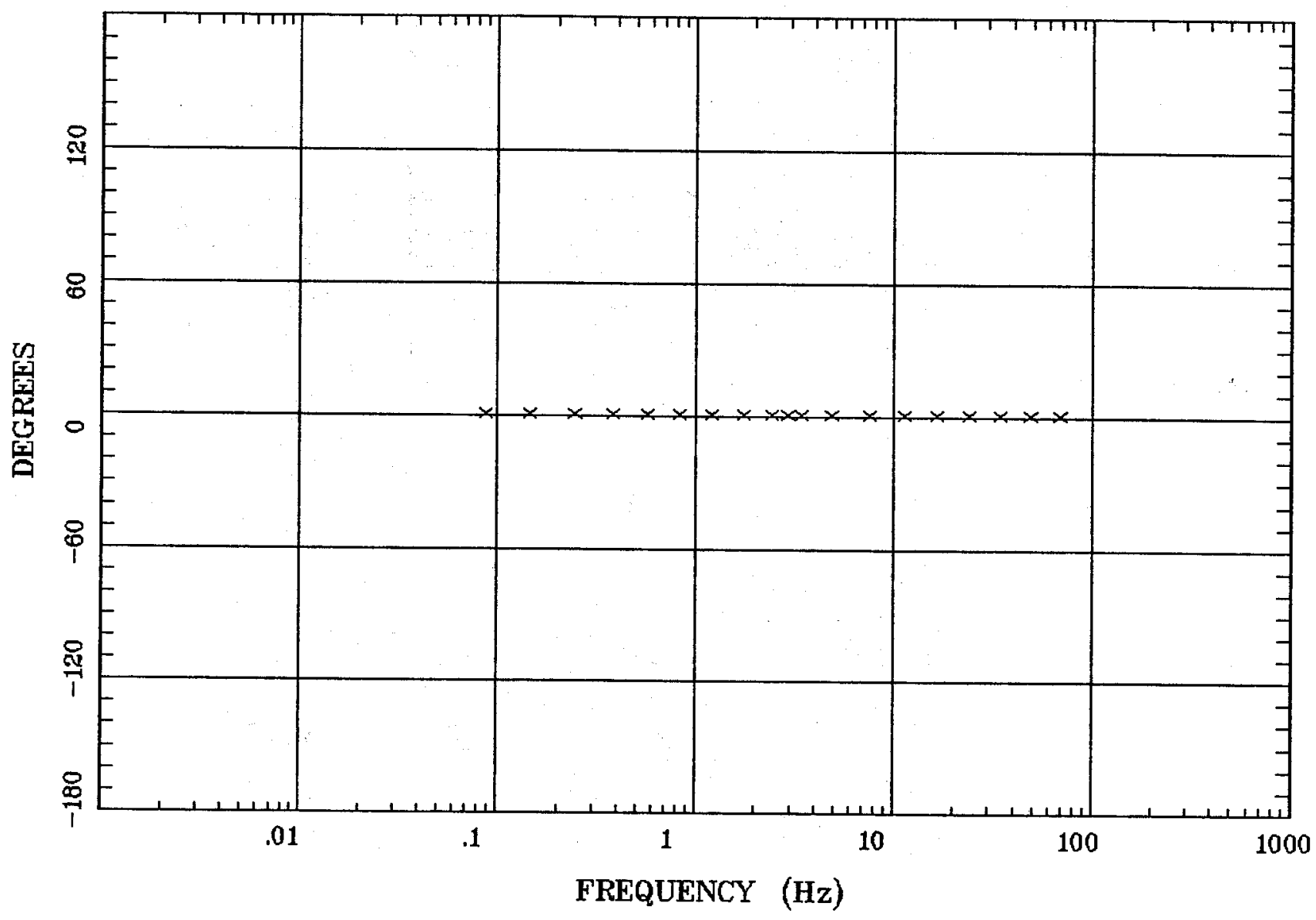

Client:

Remote: none Acquired: 15:2 Jul 13, 2007 Survey Co:USGS
Rotation:

Filename: sl28m.avg

Channels: Ch1 Ch2 Ch3 Ch4 Ch5 Ch3 Ch4 Plotted: 11:09 Nov 06, 2007

$<$ EMI - ElectroMagnetic Instruments > 


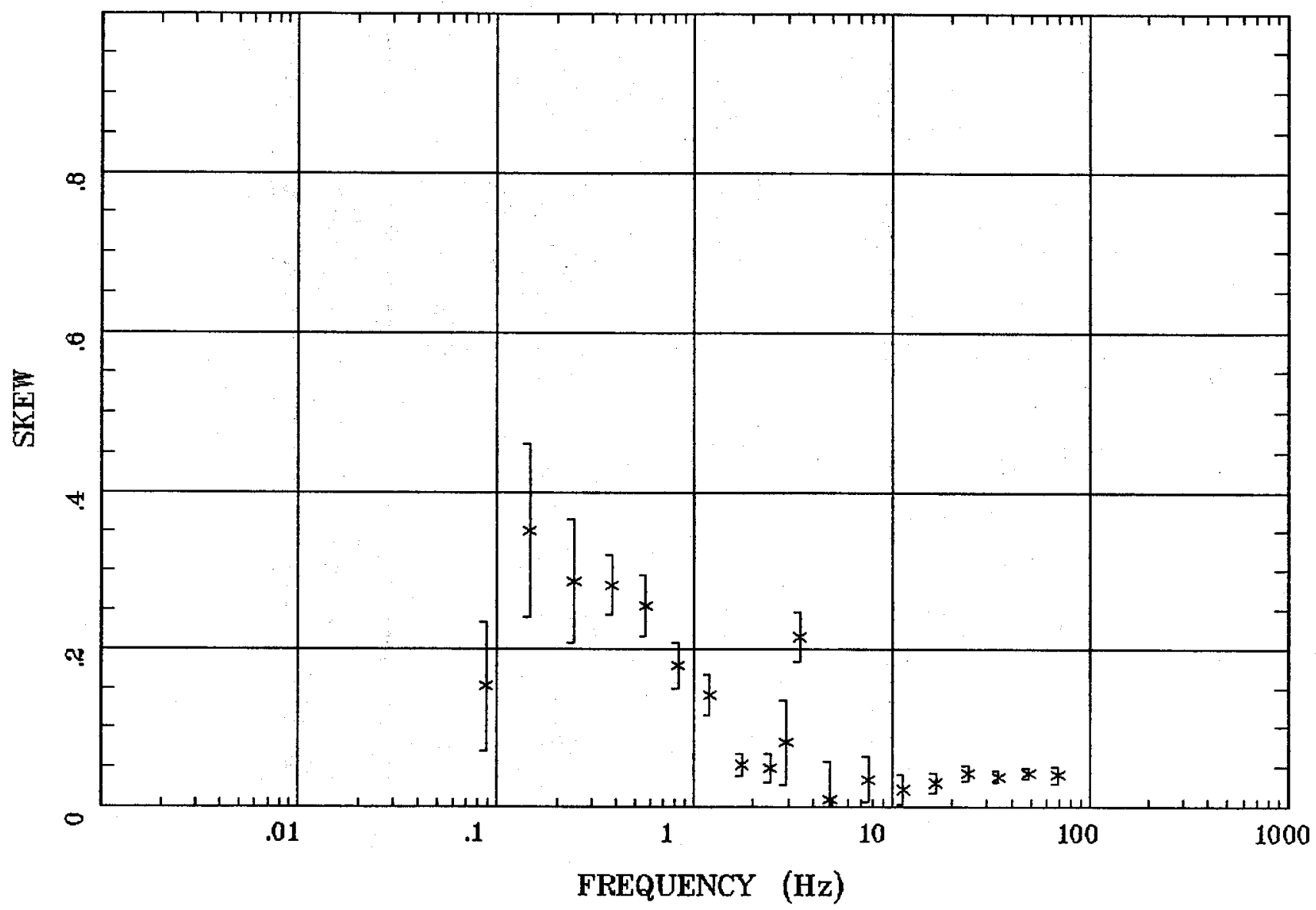

Client:

Remote: none Acquired: 15:2 Jul 13, 2007 Survey Co:USGS
Rotation:

Filename: sl28m.avg

Channels: Ch1 Ch2 Ch3 Ch4 Ch5 Ch3 Ch4

Plotted: 11:09 Nov 06, 2007

< EMI - ElectroMagnetic Instruments 
E MULT Coh.

Blanca Peak, 100k

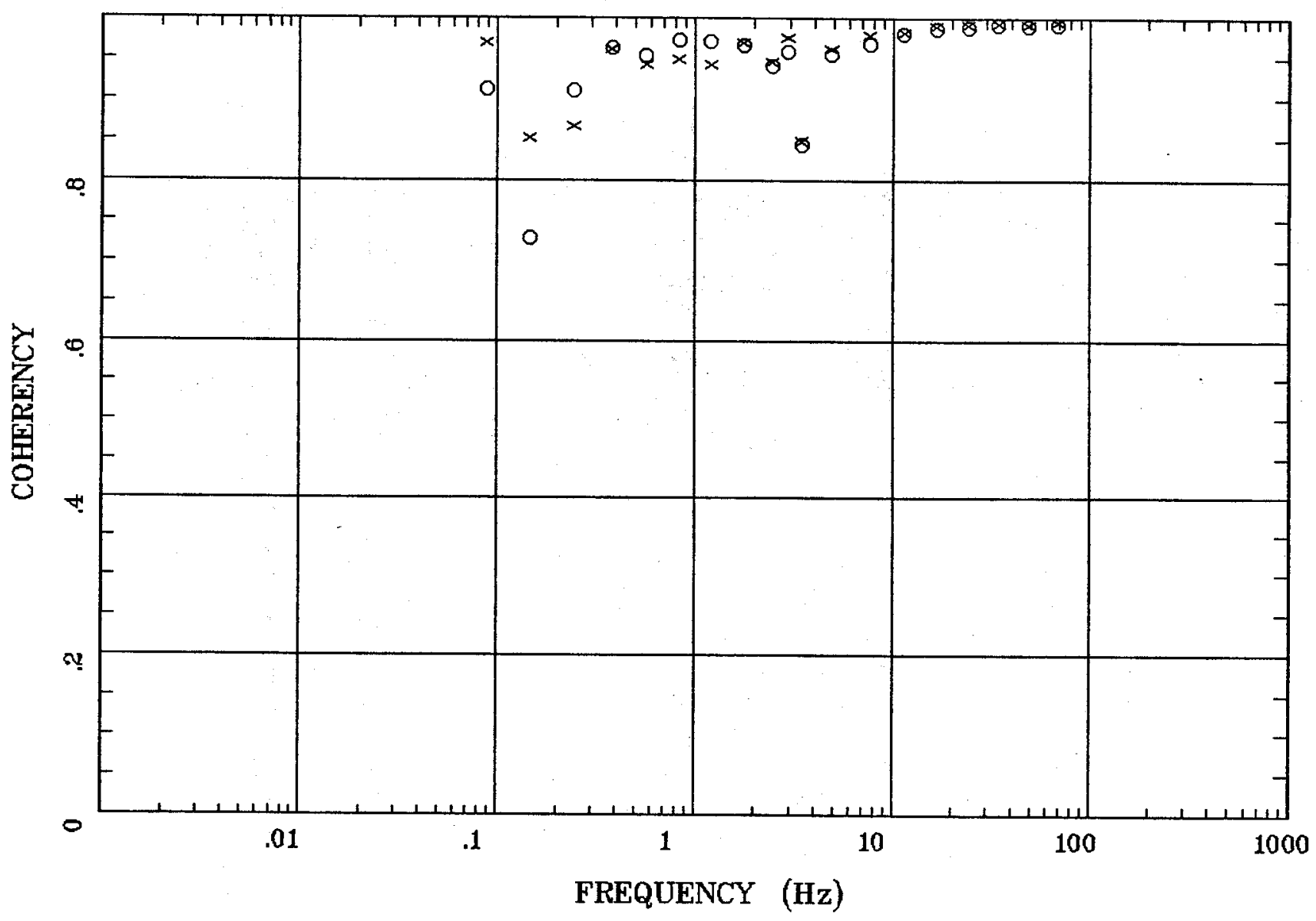

Client:

Remote: none

Acquired: 15:2 Jul 13, 2007 Survey Co:USGS
Rotation:

Filename: sl28m.avg

Channels: Ch1 Ch2 Ch3 Ch4 Ch5 Ch3 Ch4

Plotted: 11:09 Nop 06, 2007

$<$ EMI - ElectroMagnetic Instruments > 
Blanca Peak, 100k

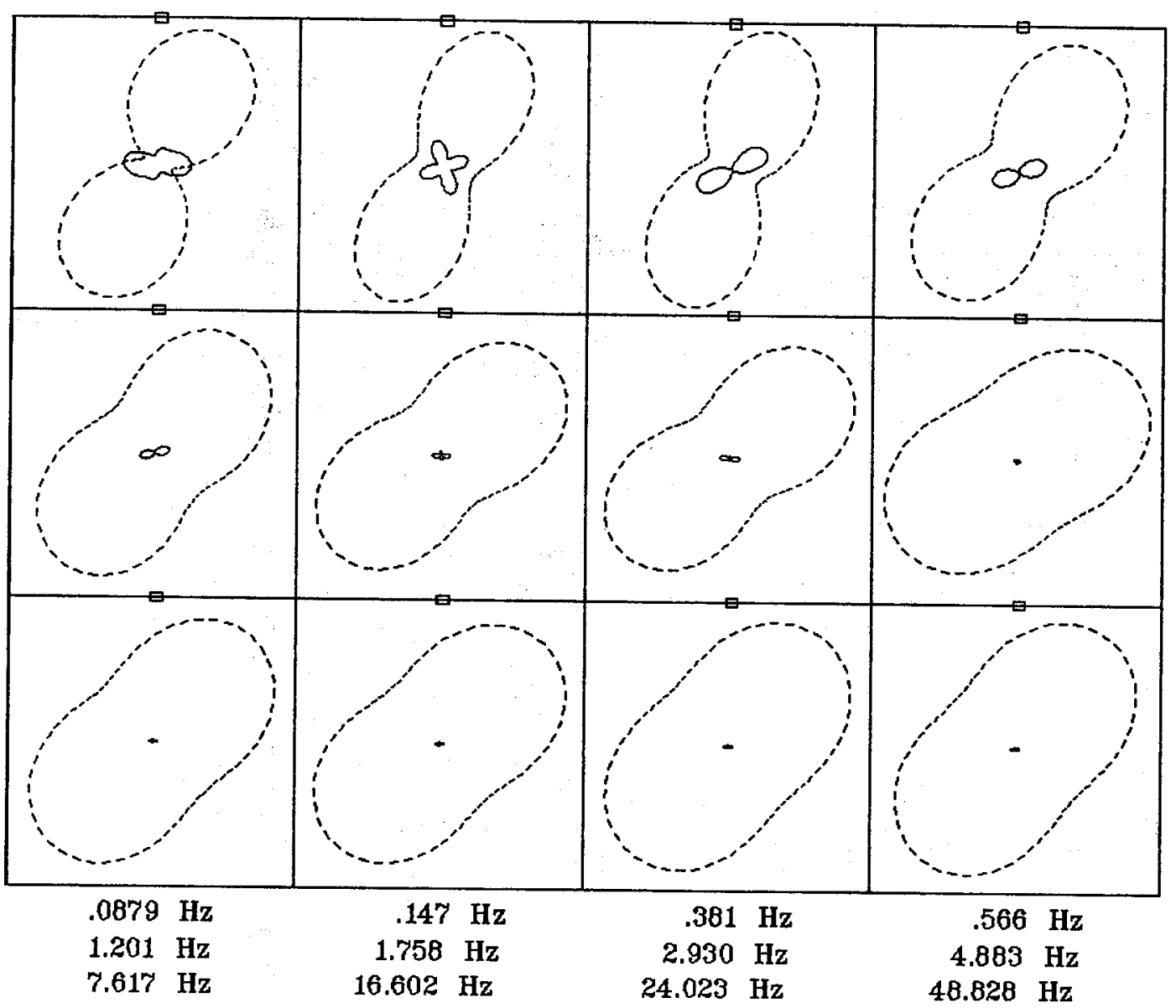

Client:

Remote: none

Acquired: 15:2 Jul 13, 2007 Survey Co:USGS
Rotation:

Filename: sl28m.avg

Channels: Ch1 Ch2 Ch3 Ch4 Ch5 Ch3 Ch4 Plotted: 11:09 Nov 06, 2007

< EMI - ElectroMagnetic Instruments > 
Blanca Peak, 100k

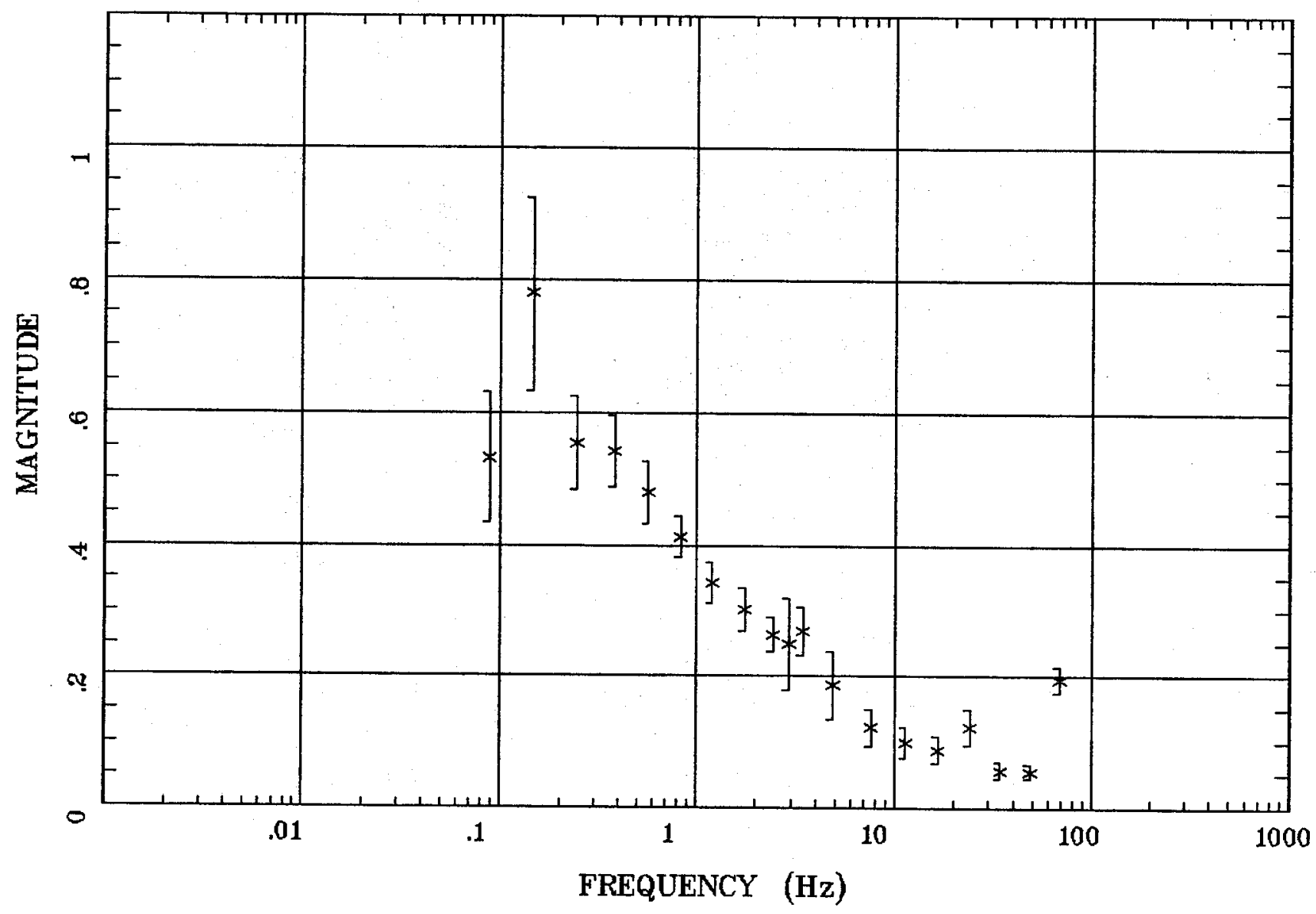

Client:

Remote: none

Acquired: 15:2 Jul 13, 2007

Survey Co:USGS
Rotation:

Filename: sl28m.avg

Channels: Ch1 Ch2 Ch3 Ch4 Ch5 Ch3 Ch4

Plotted: 11:09 Nov 06, 2007

$<$ EMI - ElectroMagnetic Instruments 


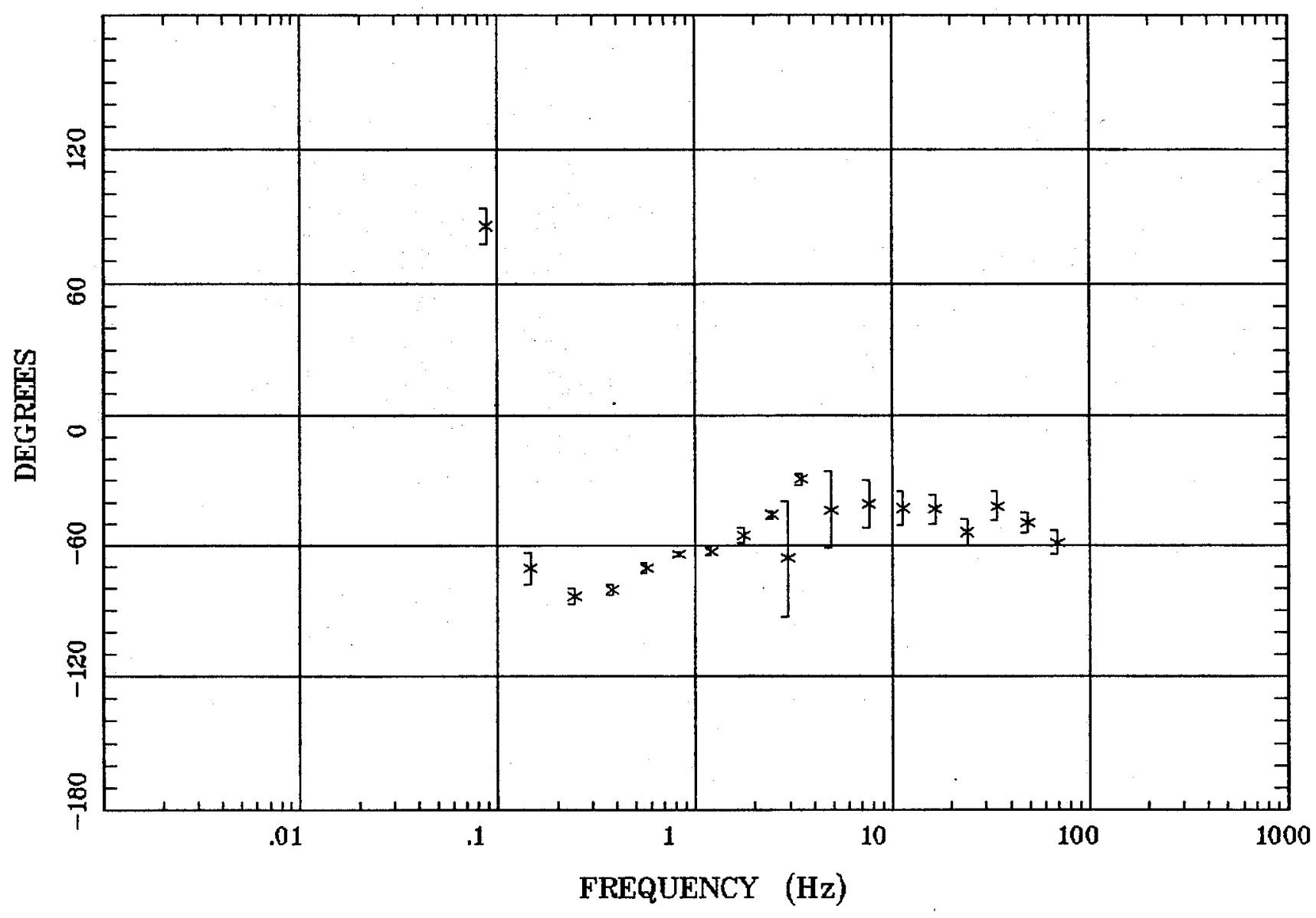

Client:

Remote: none

Acquired: 15:2 Jul 13, 2007 Survey Co:USGS
Rotation:

Filename: sl2bm.arg

Channels: Ch1 Ch2 Ch3 Ch4 Ch5 Ch3 Ch4 Plotted: 11:09 Nov 06, 2007

$<$ EMI - ElectroMagnetic Instruments 
HzHx.x Coh HzHy.o Blanca Peak, 100k

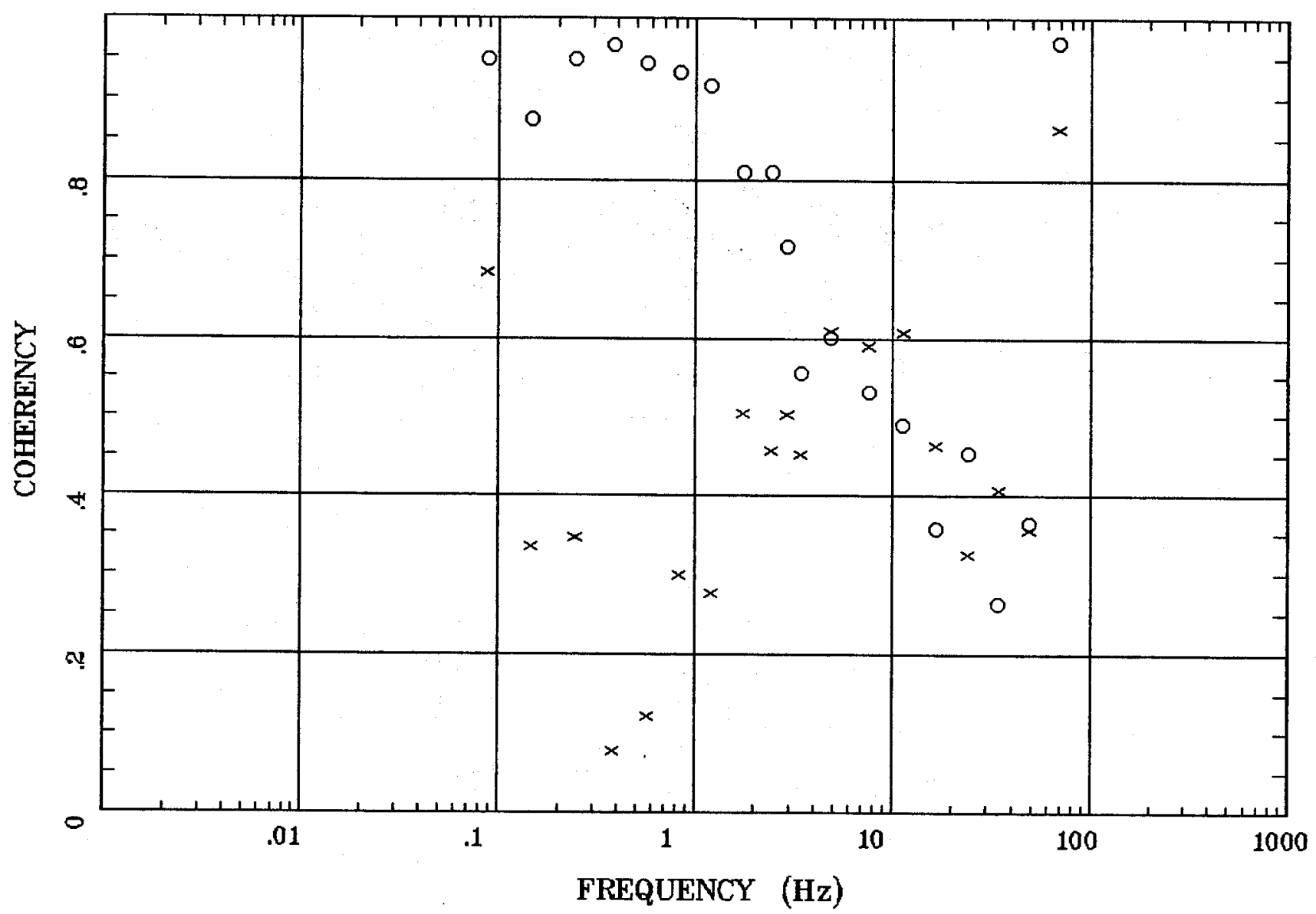

Client:

Remote: none

Acquired: 15:2 Jul 13, 2007

Survey Co:USGS
Rotation:

Filename: sl28m.avg

Channels: Ch1 Ch2 Ch3 Ch4 Ch5 Ch3 Ch4

Plotted: 11:09 Nov 06, 2007

$<$ EMI - ElectroMagnetic Instruments 


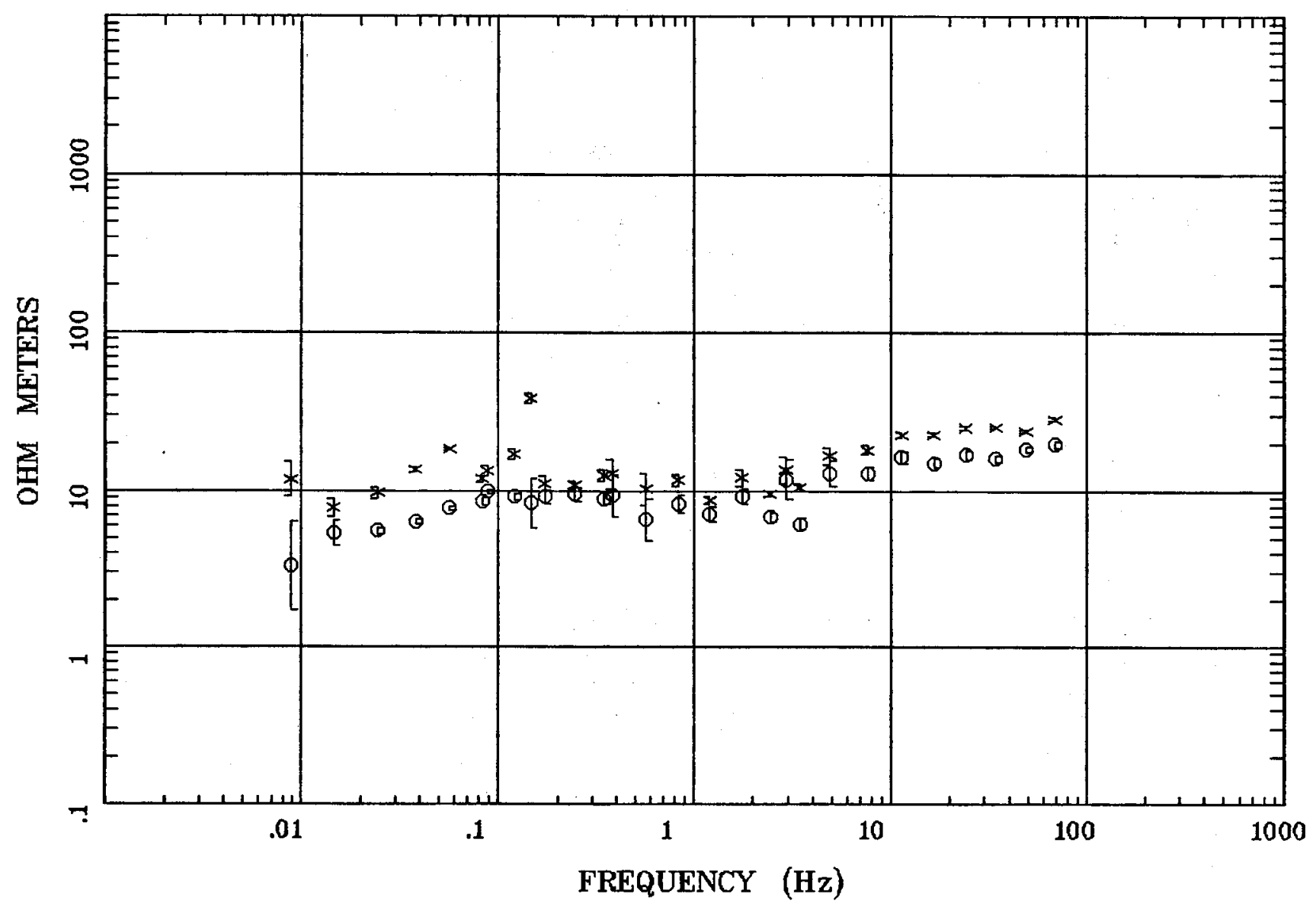

Client:

Remote: none

Acquired: 10:5 Jul 14, 2007 Survey Co:USGS
Rotation:

Filename: sl29m.avg

Channels: Ch1 Ch2 Ch3 Ch4 Ch5 Ch3 Ch4 Plotted: 11:09 Nov 06, 2007

< EMI - ElectroMagnetic Instruments > 
IMPEDANCE PHASE

Blanca Peak, 100k

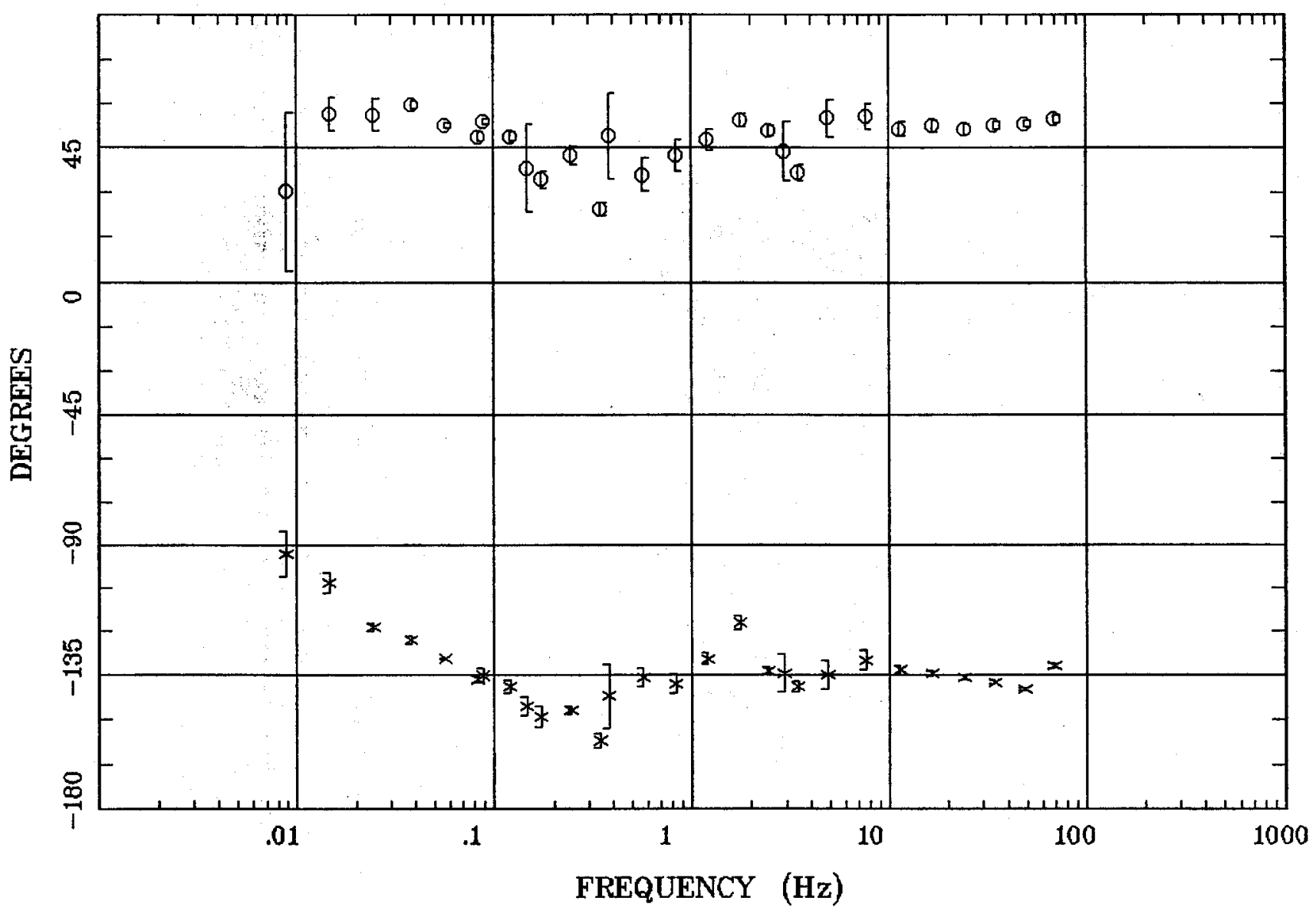

Client:

Remote: none

Acquired: 10:5 Jul 14, 2007

Survey Co:USGS
Rotation:

Filename: sl29m.avg

Channels: Ch1 Ch2 Ch3 Ch4 Ch5 Ch3 Ch4

Plotted: 11:09 Nov 06, 2007

$<$ EMI - ElectroMagnetic Instruments 
Blanca Peak, 100k

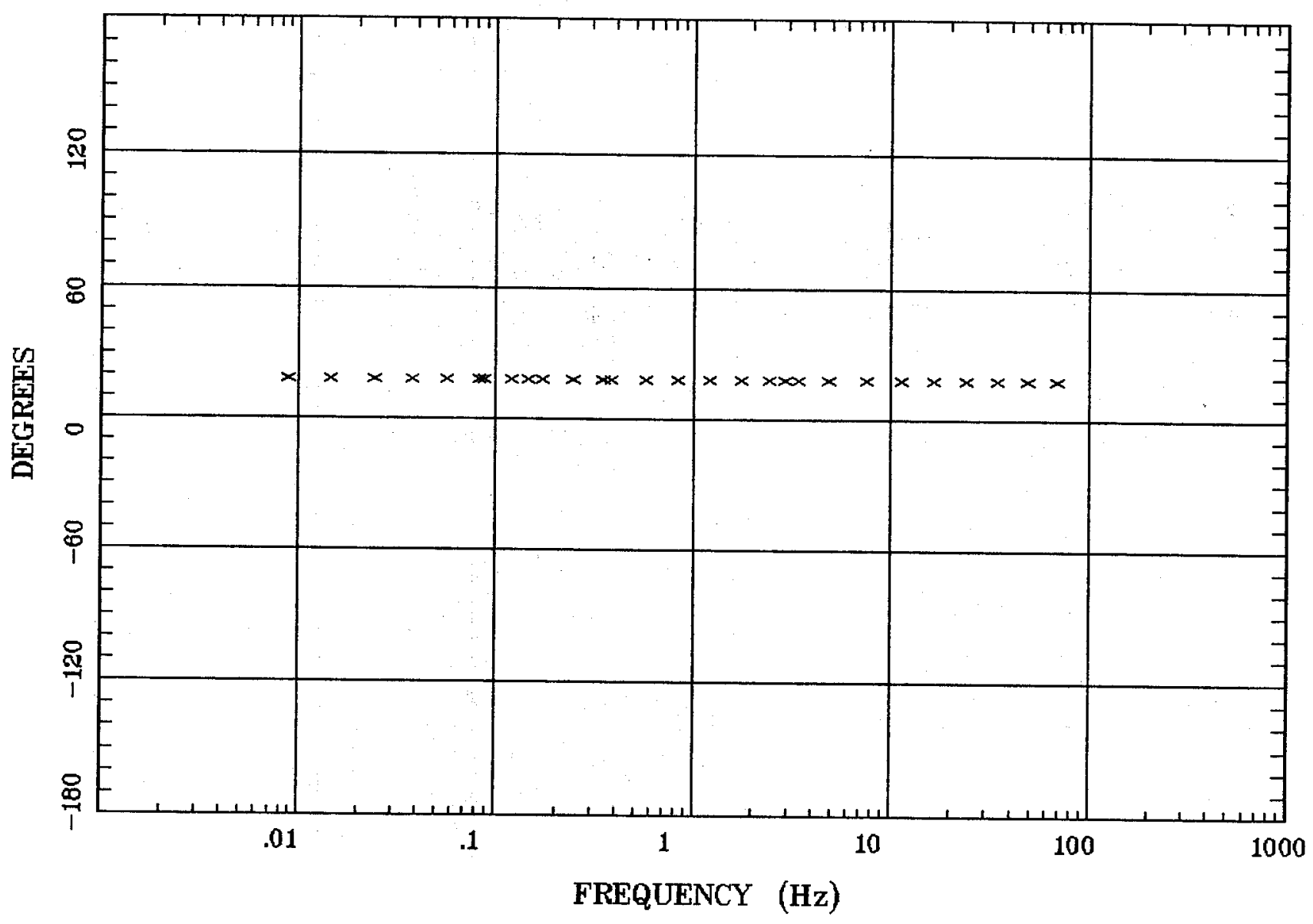

Client:

Remote: none

Acquired: 10:5 Jul 14, 2007

Survey Co:USGS
Rotation:

Filename: sl29m.evg

Channels: Ch1 Ch2 Ch3 Ch4 Ch5 Ch3 Ch4 Plotted: 11:09 Nov 06, 2007

< EMI - ElectroMagnetic Instruments 
Blanca Peak, 100k

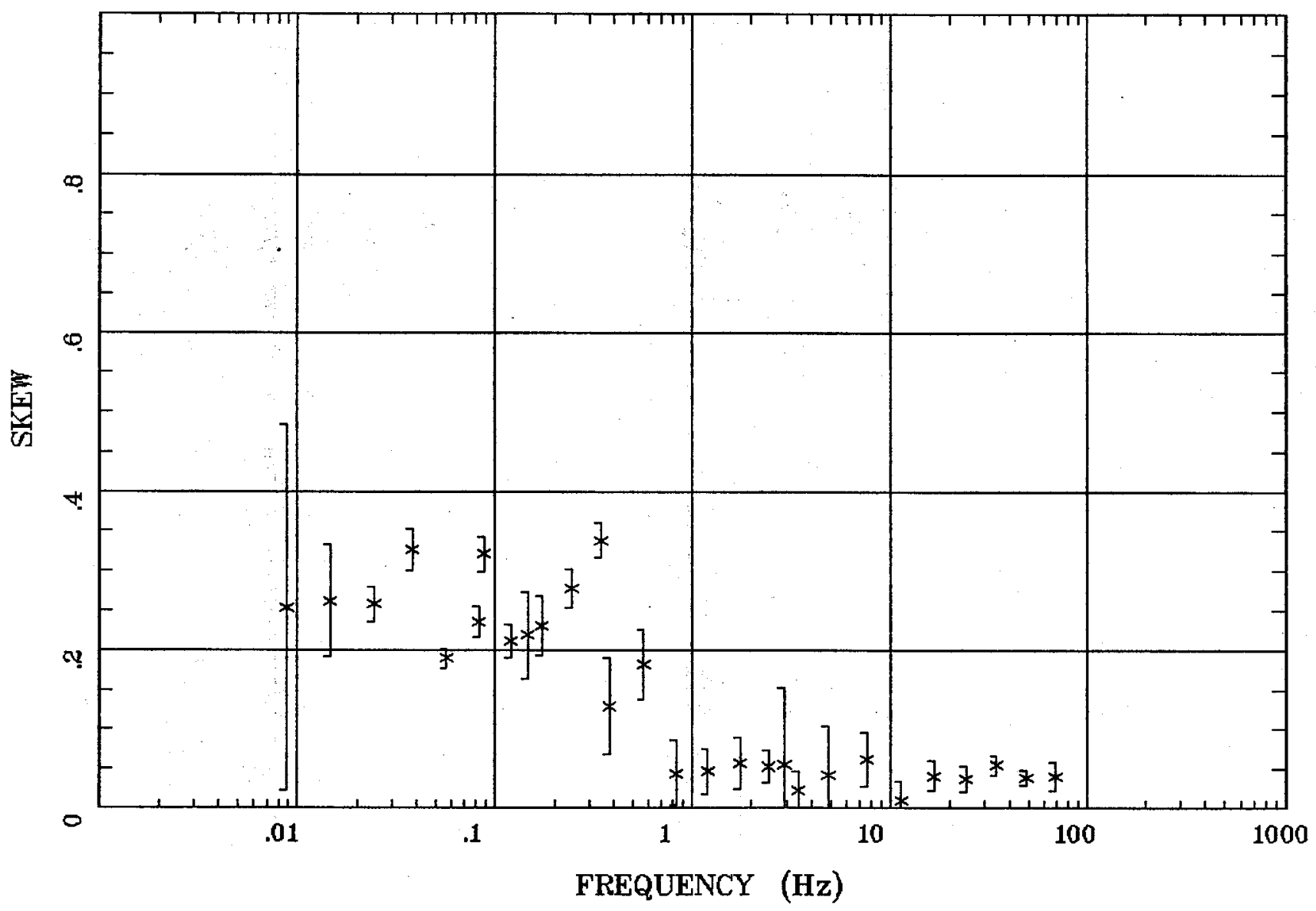

Client:

Remote: none

Acquired: $10: 5 \mathrm{Jul} \mathrm{14,2007}$

Survey Co:USGS
Rotation:

Filename: sl29m.avg

Channels: Ch1 Ch2 Ch3 Ch4 Ch5 Ch3 Ch4

Plotted: 11:09 Nov 06, 2007

< EMI - ElectroMagnetic Instruments 
E MULT Coh.

Blanca Peak, 100k

Station 29

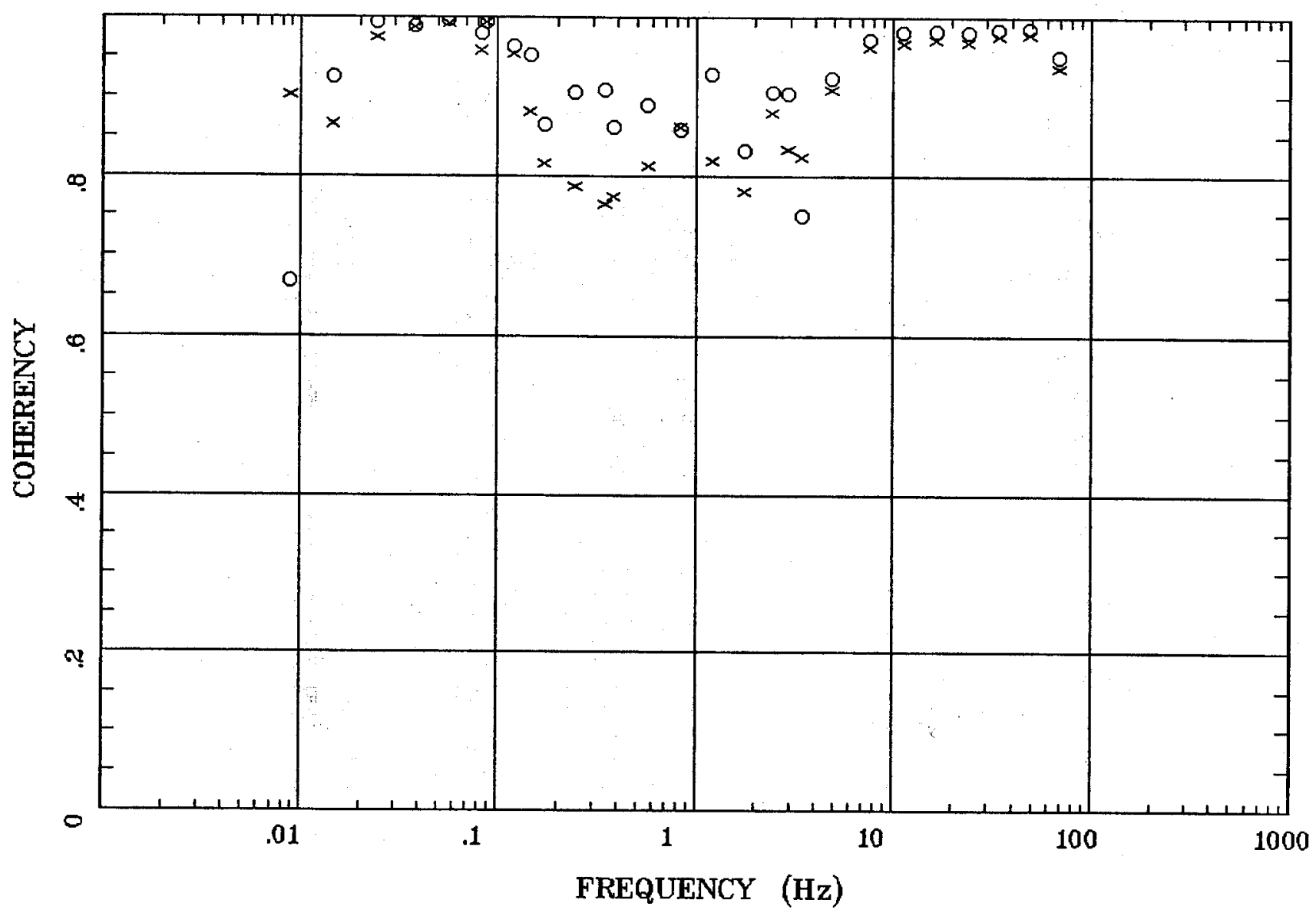

Client:

Remate: none

Acquired: 10:5 Jul 14, 2007

Survey Co:USGS
Rotation:

Filename: sl29m.avg

Channels: Ch1 Ch2 Ch3 Ch4 Ch5 Ch3 Ch4 Plotted: 11:09 Nor 06, 2007

< EMI - ElectroMagnetic Instruments 
Blanca Peak, 100k

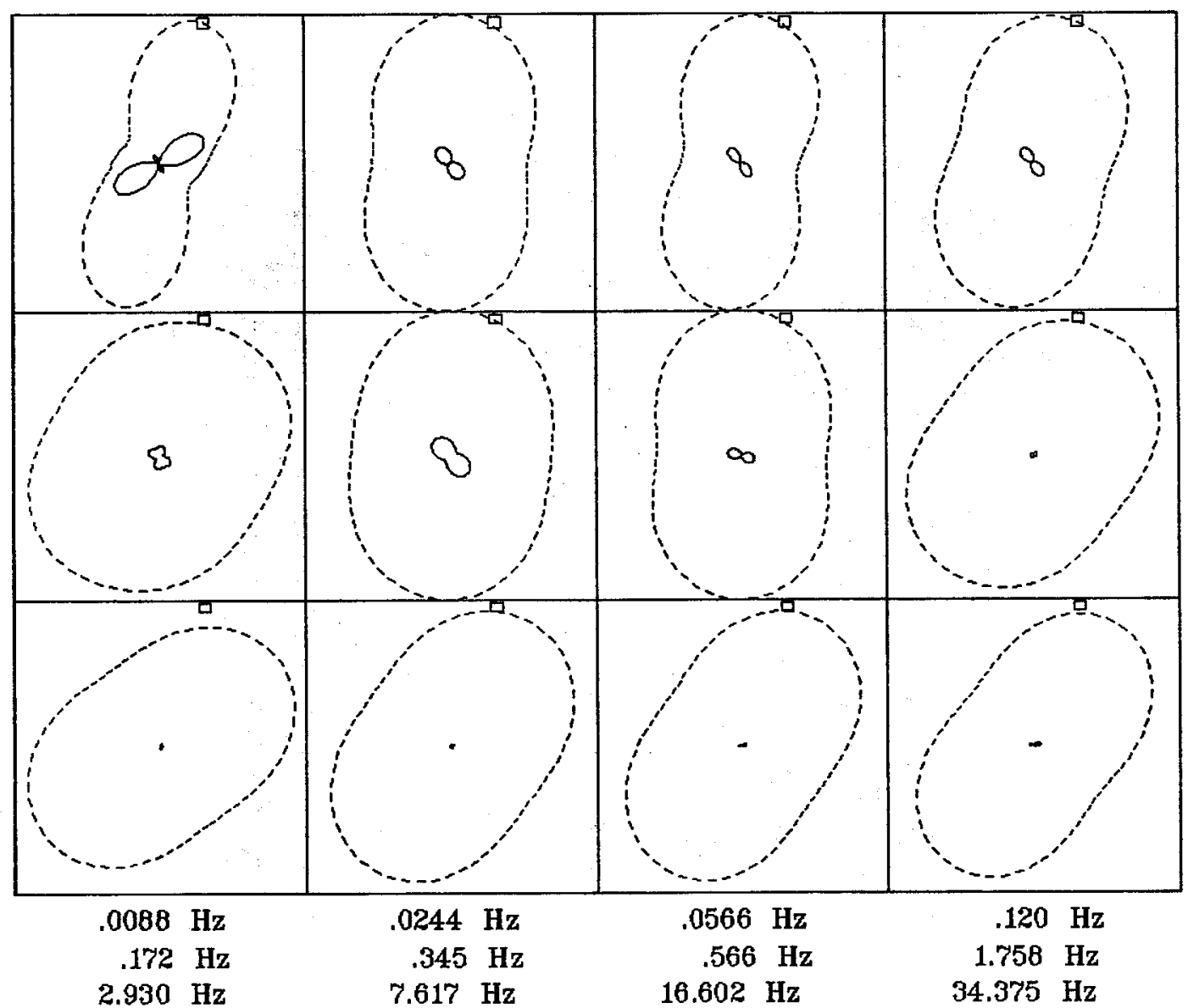

Client:

Remote: none

Acquired: 10:5 Jul 14, 2007 Survey Co:USGS
Rotation:

Filename: slagm.avg

Channels: Ch1 Ch2 Ch3 Ch4 Ch5 Ch3 Ch4 Plotted: 11:09 Nov 06, 2007

< EMI - ElectroMagnetic Instruments > 


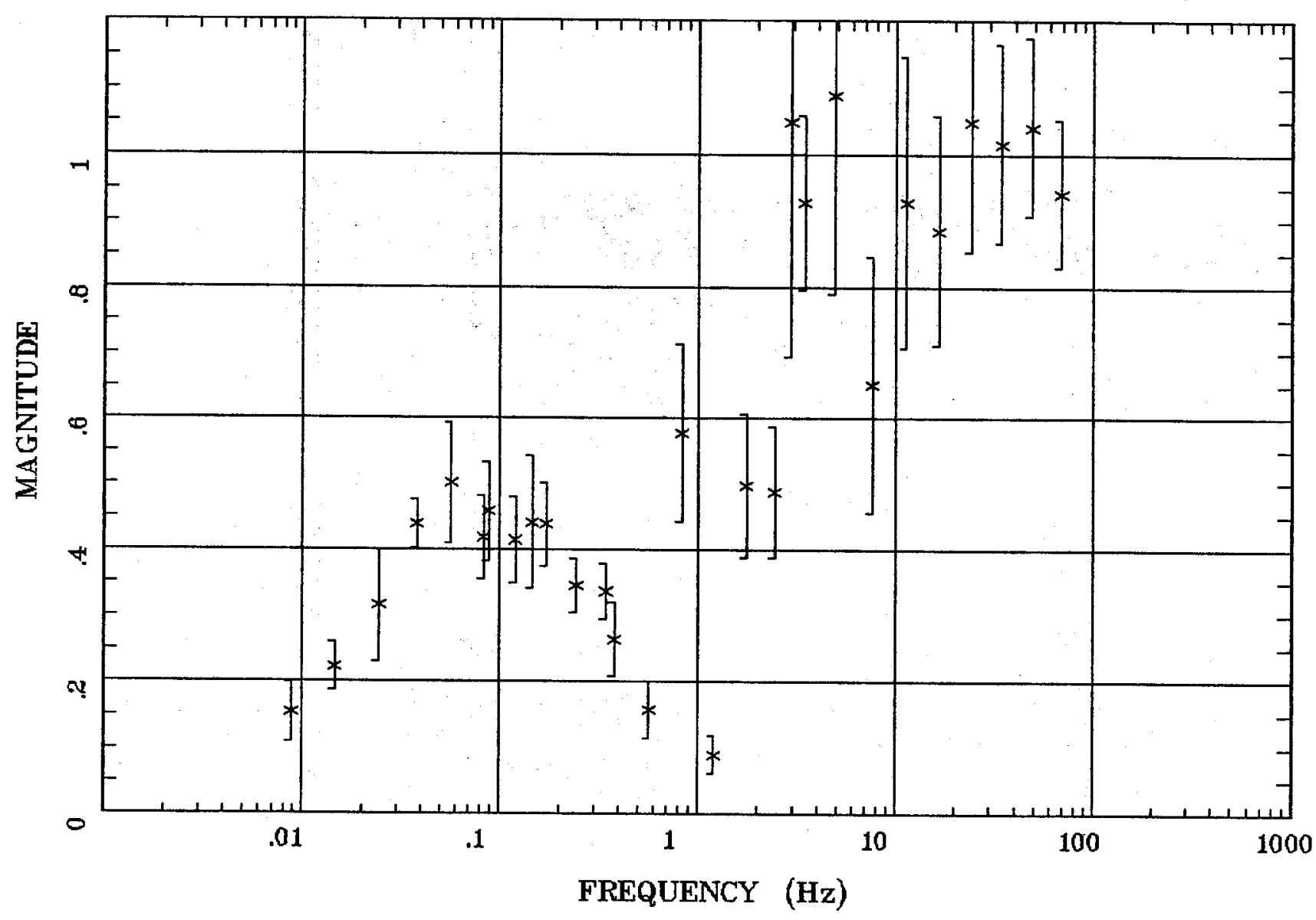

Client:

Remote: none

Acquired: 10:5 Jul 14, 2007

Survey Co:USGS
Rotation:

Filename: sl29m.avg

Channels: Ch1 Ch2 Ch3 Ch4 Ch5 Ch3 Ch4 Plotted: 11:09 Nov 06, 2007

$<$ EMI - ElectroMagnetic Instruments 
TIPPER STRIKE

Blanca Peak, 100k

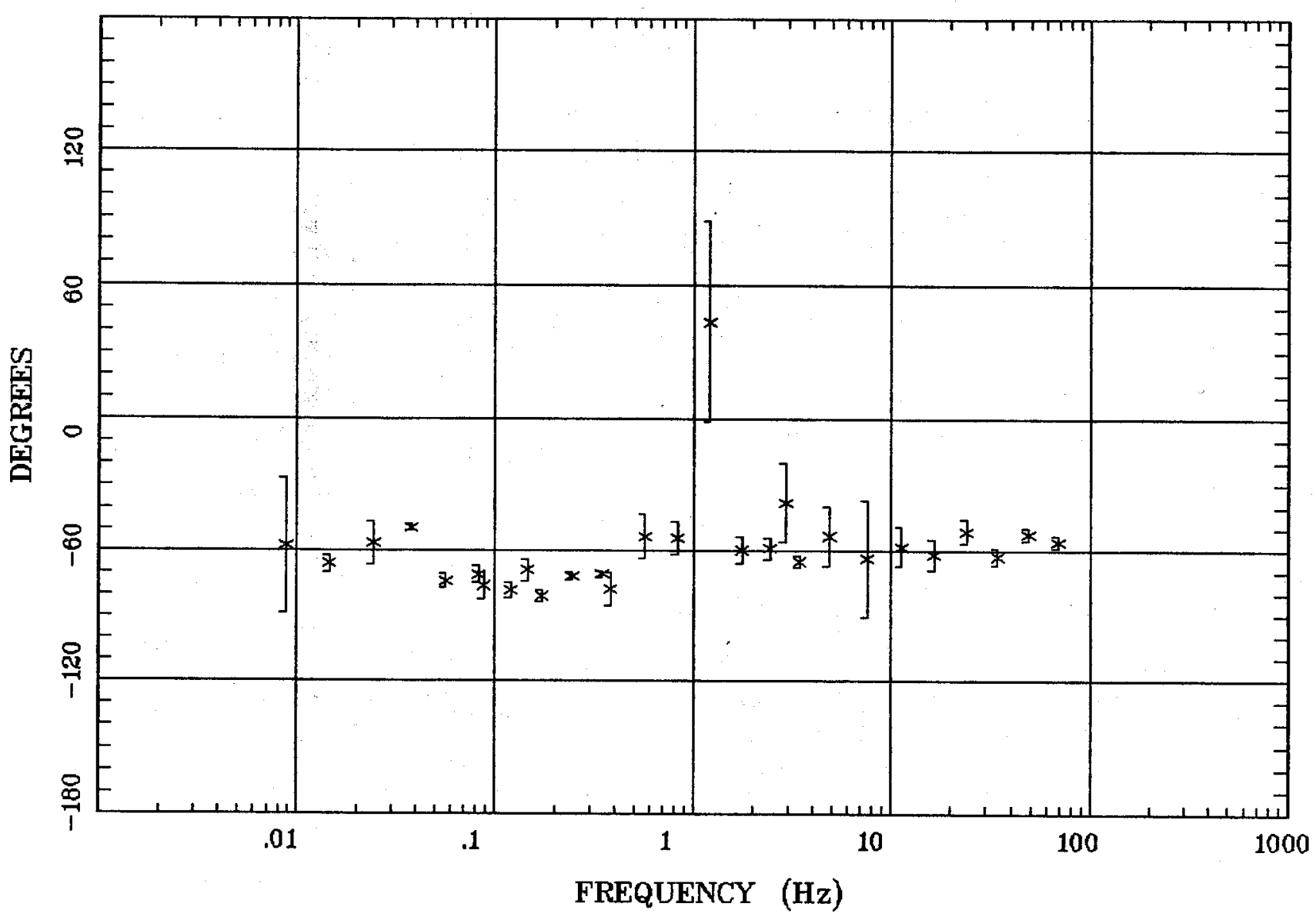

Client:

Remote: none

Acquired: 10:5 Jul 14, 2007

Survey Co:USGS
Rotation:

Filename: sl29m.avg

Channels: Ch1 Ch2 Ch3 Ch4 Ch5 Ch3 Ch4

Plotted: 11:09 Nop 06, 2007

< EMI - ElectroMagnetic Instruments > 


\section{Station 29}

HzHx.x Coh HzHy.o

Blanca Peak, 100k

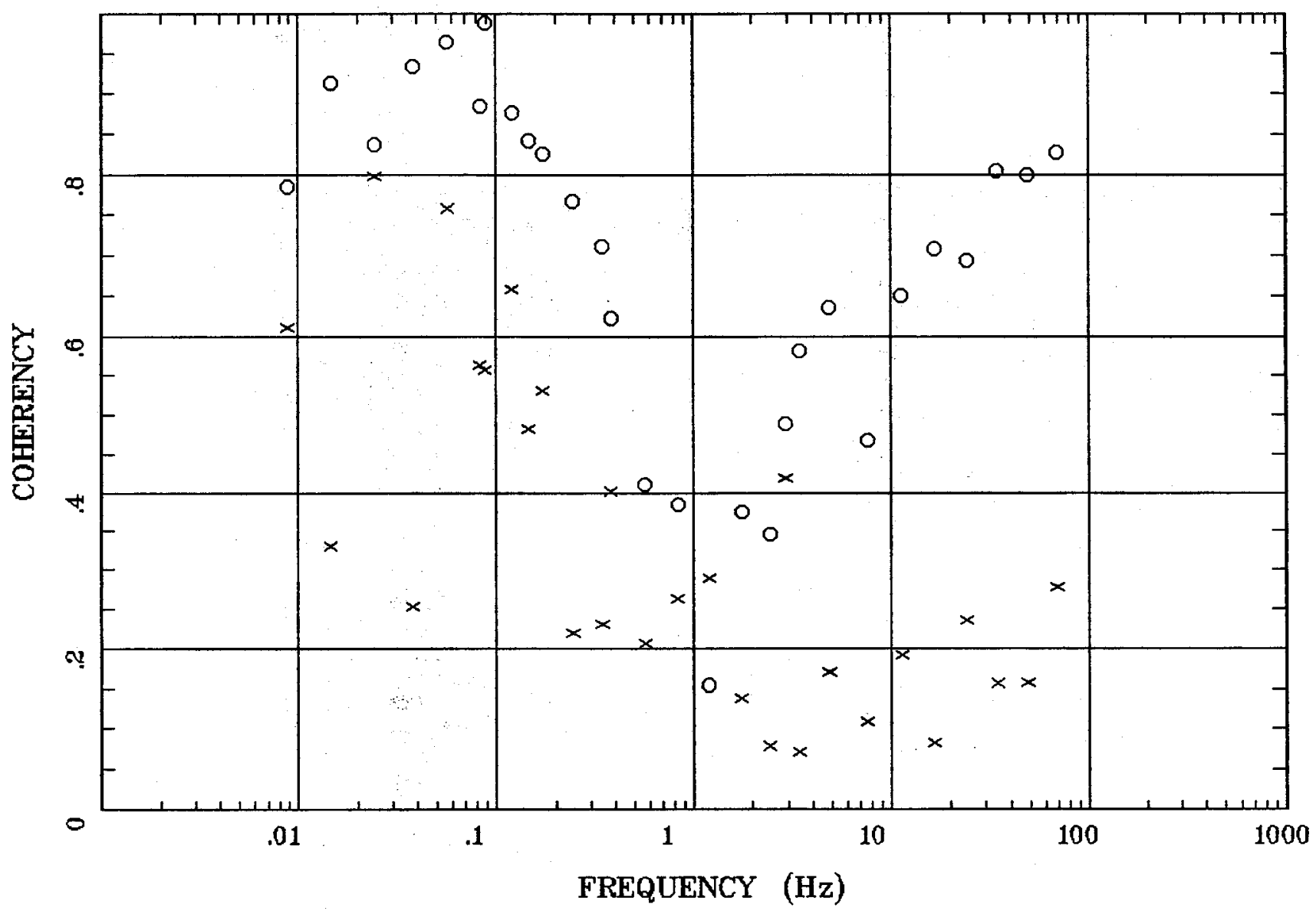

Client:

Remote: none

Acquired: 10:5 Jul 14, 2007 Survey Co:USGS
Rotation:

Filename: sl29m.avg

Channels: Ch1 Ch2 Ch3 Ch4 Ch5 Ch3 Ch4 Plotted: 11:09 Nov 06, 2007

< EMI - ElectroMagnetic Instruments 


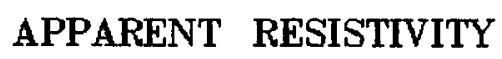

Alamosa, Co 100k

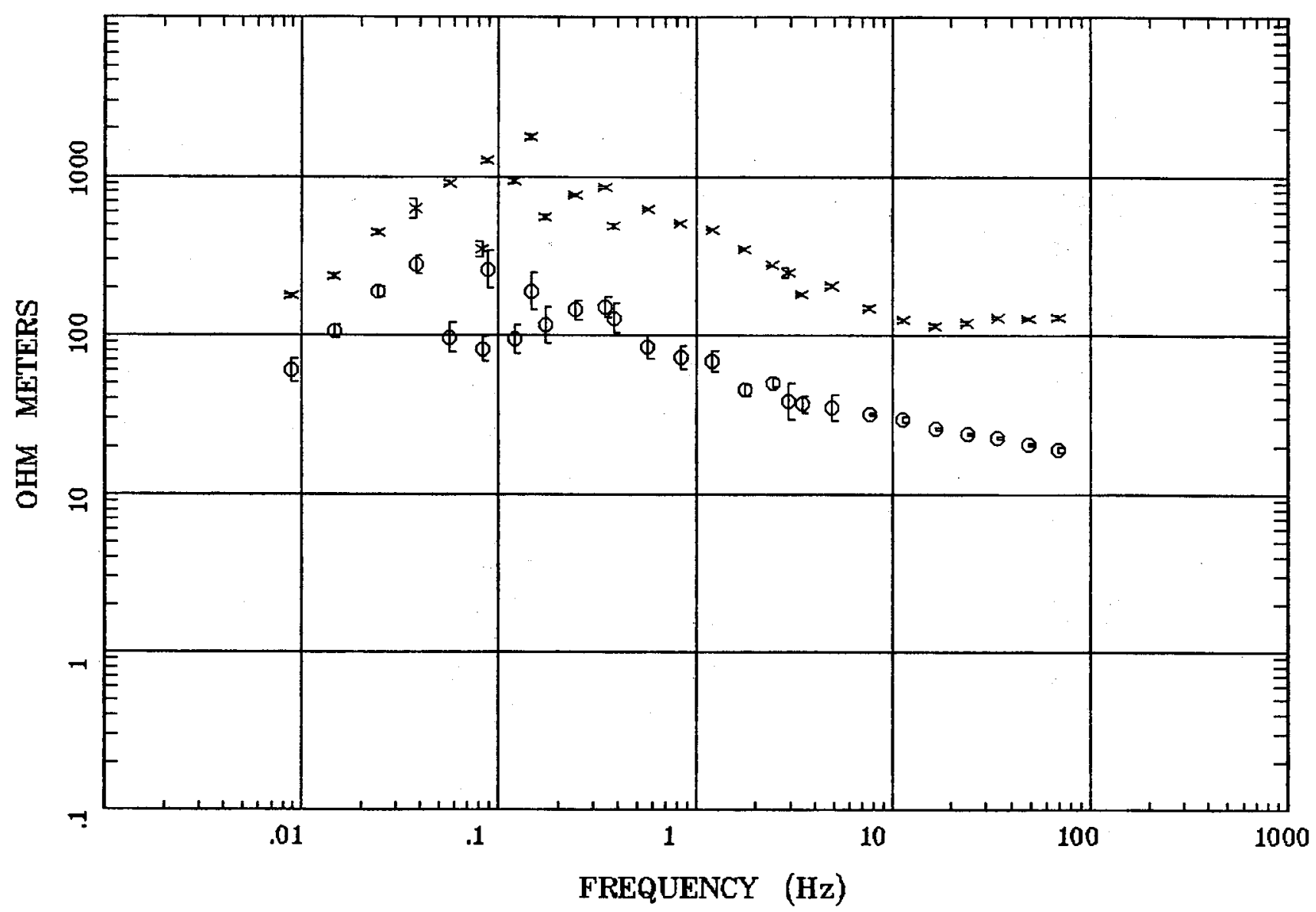

Client:

Remote: none

Acquired: 09:0 Jul 15, 2007

Survey Co:USGS
Rotation:

Filename: sl30m2.avg

Channels: Ch1 Ch2 Ch3 Ch4 Ch5 Ch3 Ch4 Plotted: 11:11 Nor 06, 2007

< EMI - ElectroMagnetic Instruments 
Station 30

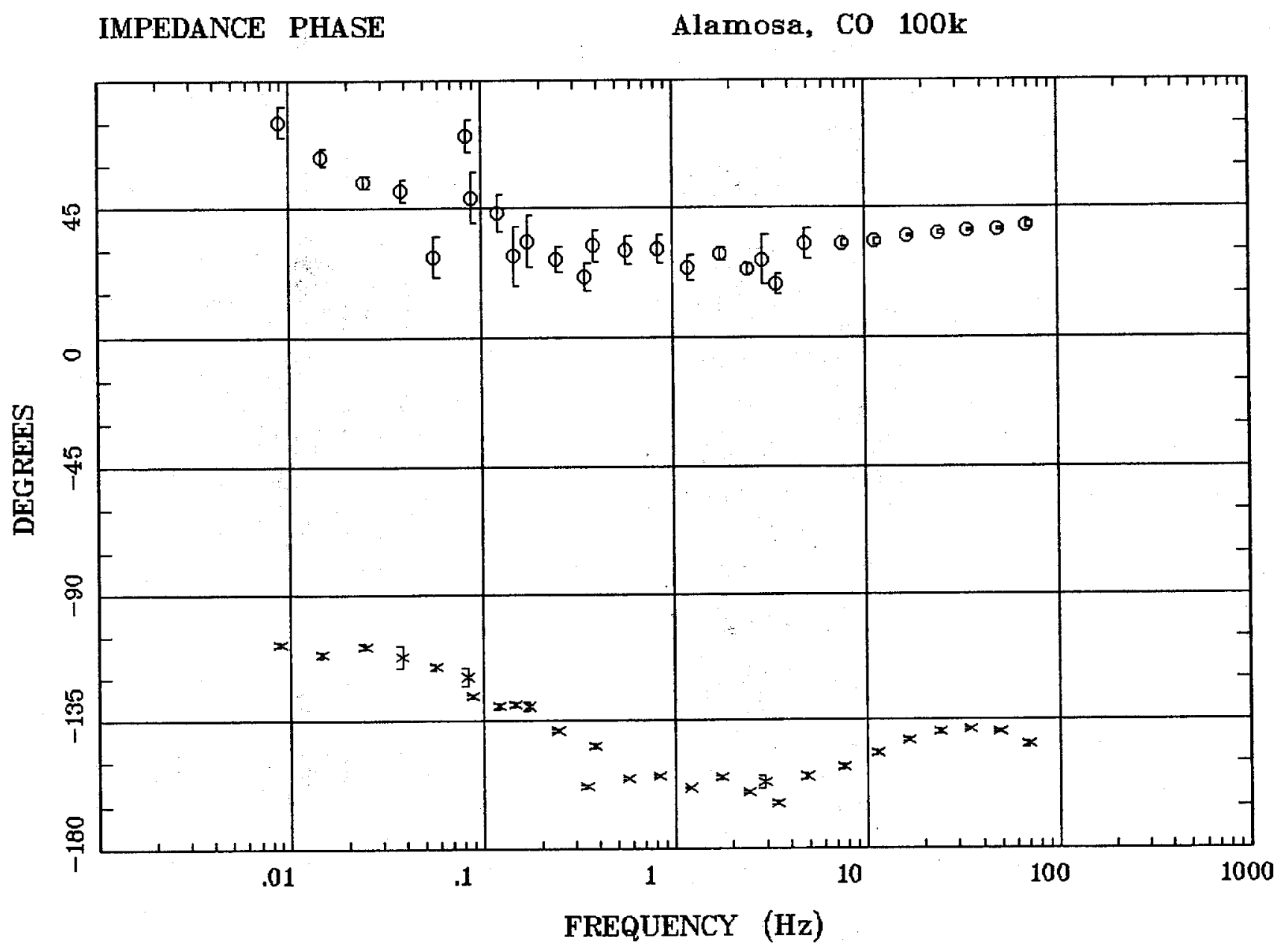

Client:

Remote: none

Acquired: 09:0 Jul 15, 2007 Survey Co:USGS
Rotation:

Filename: sl30m2.avg

Channels: Ch1 Ch2 Ch3 Ch4 Ch5 Ch3 Ch4 Plotted: 11:11 Nov 06, 2007

< EMI - ElectroMagnetic Instruments > 


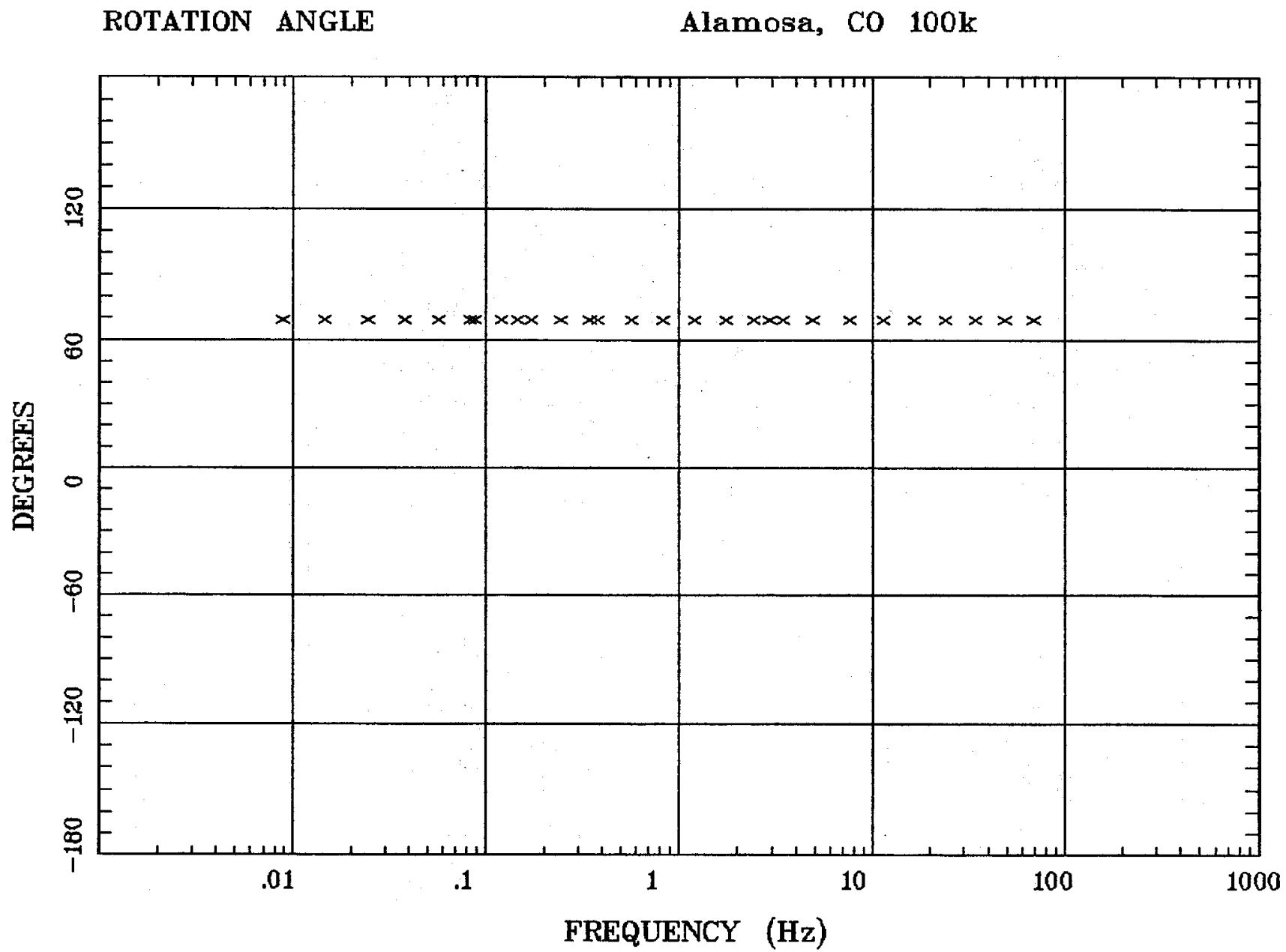

Client:

Remote: none

Acquired: 09:0 Jul 15, 2007

Survey Co:USGS
Rotation:

Filename: sl30mz.avg

Channels: Ch1 Ch2 Ch3 Ch4 Ch5 Ch3 Ch4

Plotted: 11:11 Nov 06, 2007

< EMI - ElectroMagnetic Instruments 
Alamosa, CO 100k

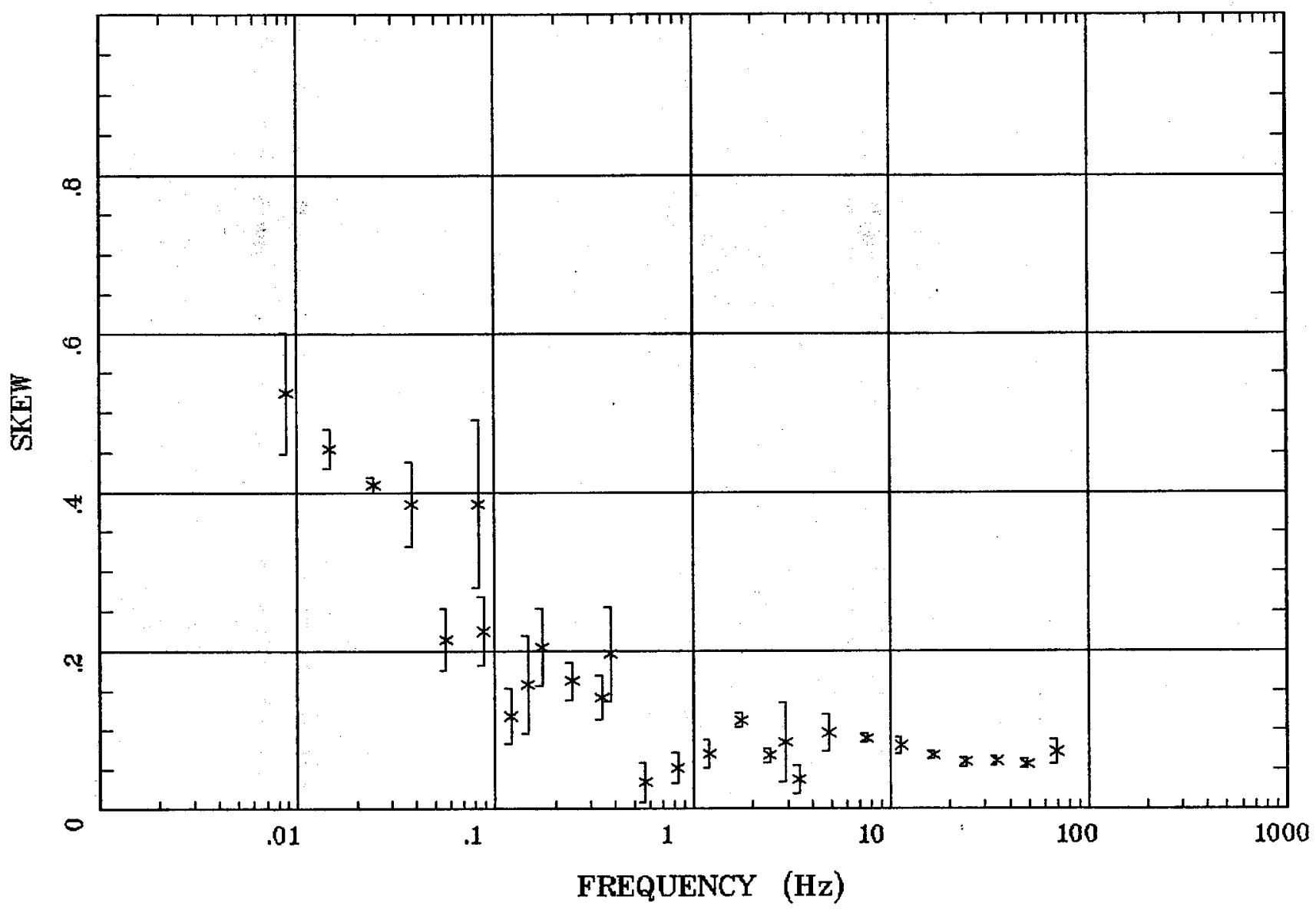

Client:

Remote: none

Acquired: 09:0 Jul 15, 2007 Survey Co:USGS
Rotation:

Filename: sl30m2.avg

Channels: Ch1 Ch2 Ch3 Ch4 Ch5 Ch3 Ch4 Plotted: 11:11 Nov 06, 2007

< EMI - ElectroMagnetic Instruments 
E MULT Coh.

Alamosa, CO 100k

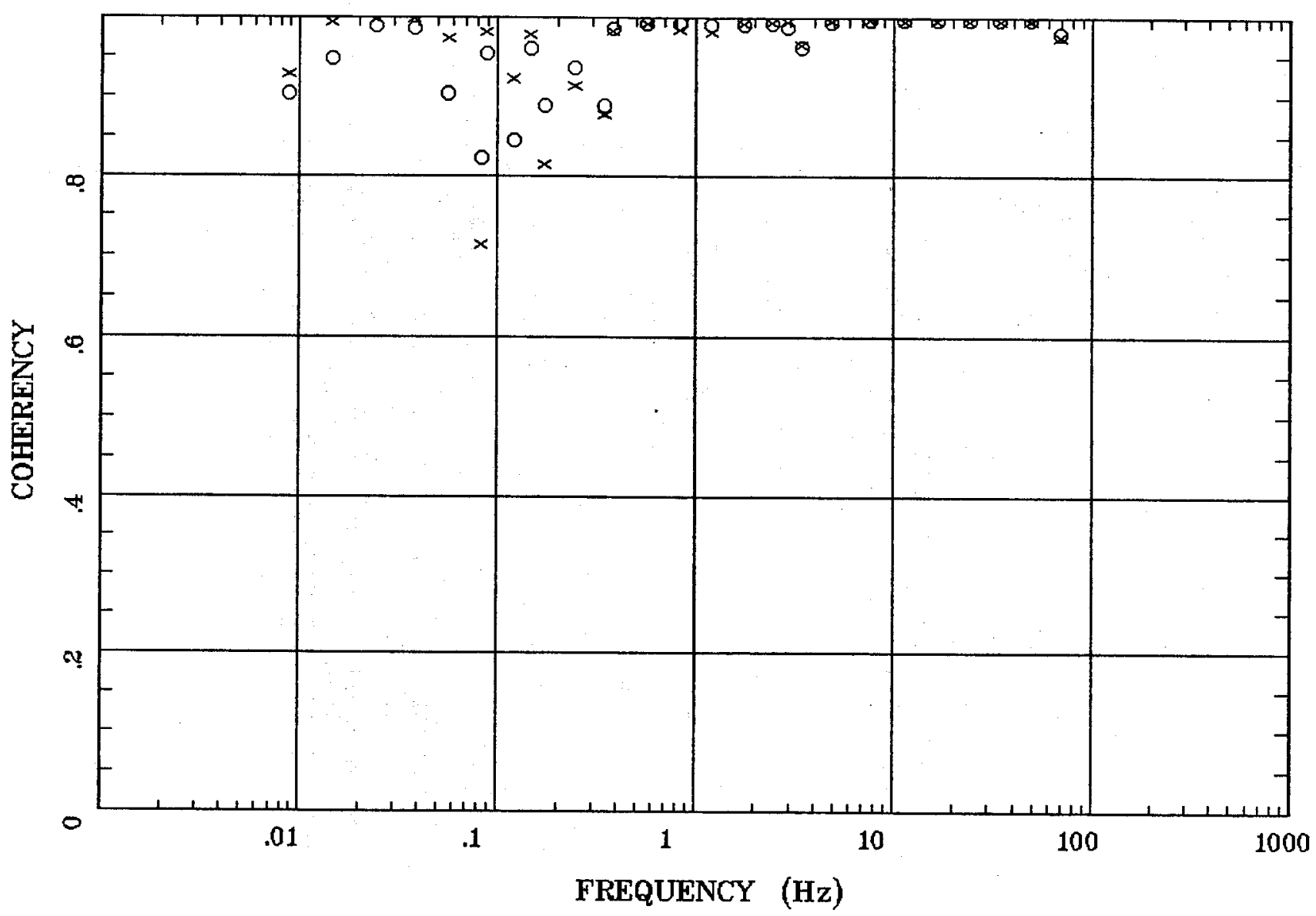

Client:

Remote: none

Acquired: 09:0 Jul 15, 2007

Survey Co:USGS
Rotation:

Filename: sl30m2.avg

Channels: Ch1 Ch2 Ch3 Ch4 Ch5 Ch3 Ch4 Plotted: 11:11 Nov 06, 2007

< EMI - ElectroMagnetic Instruments > 


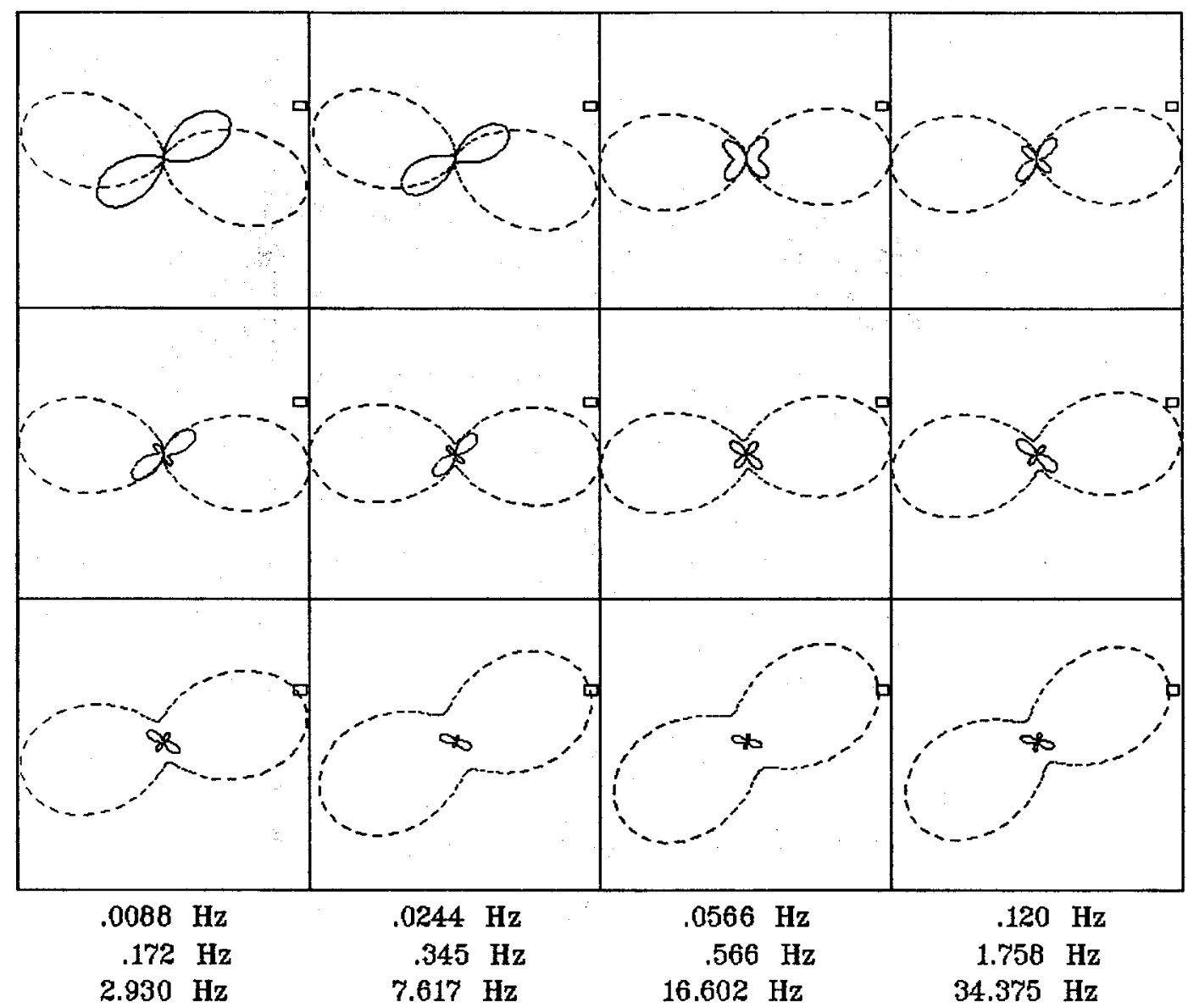

Client:

Remote: none

Acquired: 09:0 Jul 15, 2007 Survey Co:USGS

\section{Rotation:}

Filename: sl30m2.avg

Channels: Ch1 Ch2 Ch3 Ch4 Ch5 Ch3 Ch4 Plotted: 11:11 Nov 06, 2007

$<$ EMI - ElectroMagnetic Instruments > 


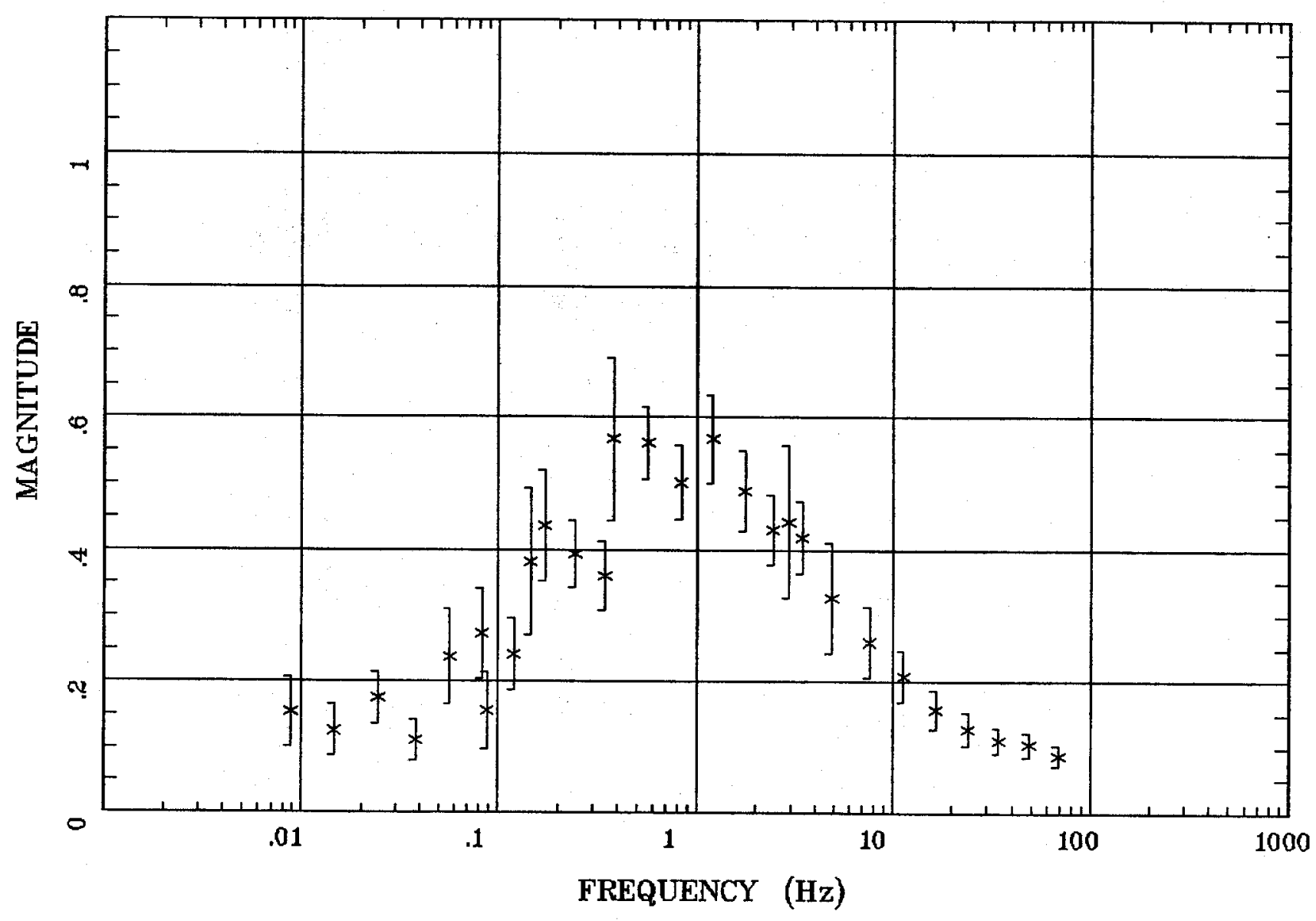

Client:

Remote: none

Acquired: 09:0 Jul 15, 2007

Survey Co:USGS
Rotation:

Filename: sl30m2.avg

Channels: Ch1 Ch2 Ch3 Ch4 Ch5 Ch3 Ch4 Plotted: 11:11 Nov 06, 2007

<EMI - ElectroMagnetic Instruments > 
Alamosa, Co 100k

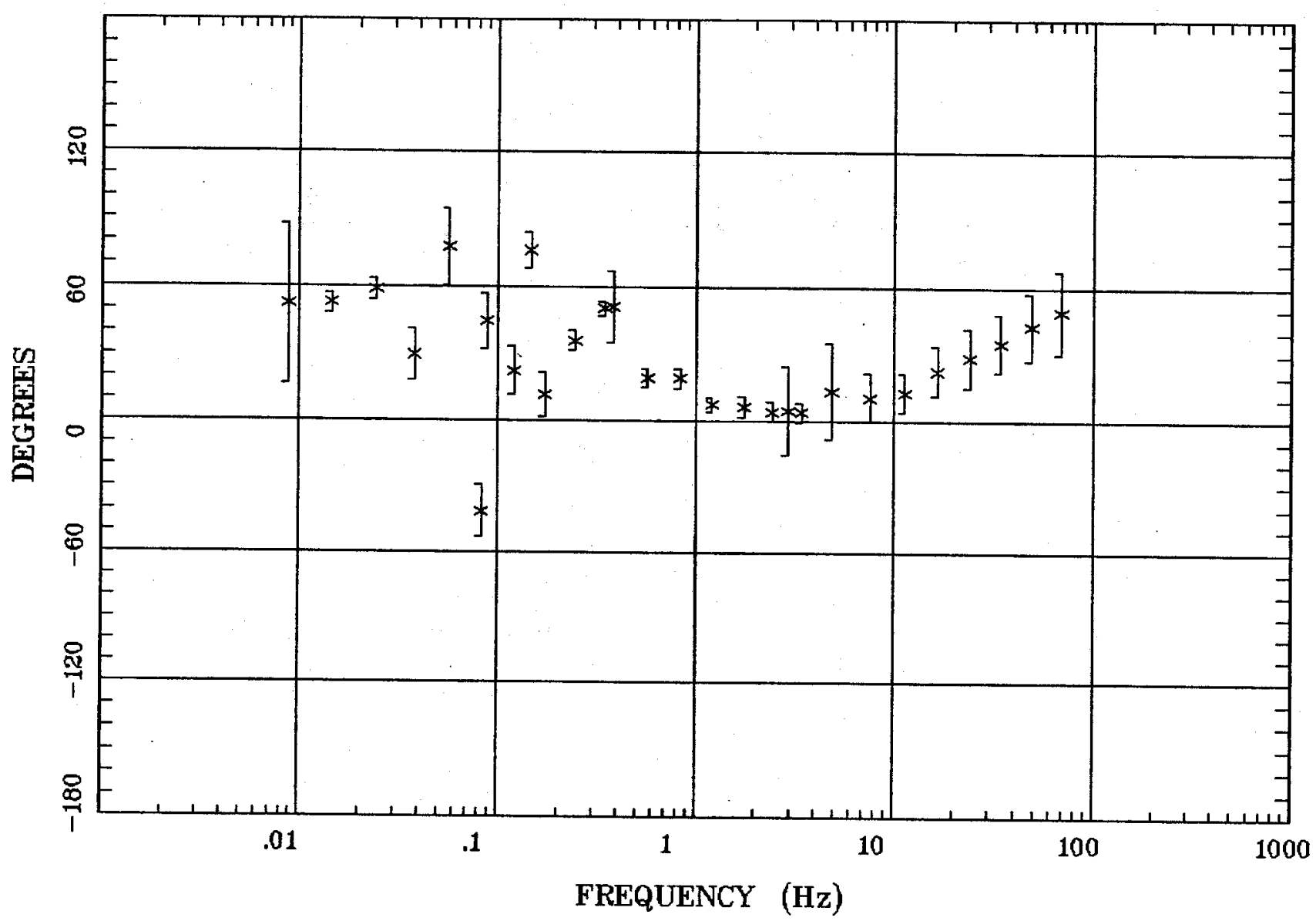

Client:

Remote: none

Acquired: 09:0 Jul 15, 2007 Survey Co:USGS
Rotation:

Filename: sl30m2.avg

Channels: Ch1 Ch2 Ch3 Ch4 Ch5 Ch3 Ch4

Plotted: 11:11 Nor 06, 2007

< EMI - ElectroMagnetic Instruments 
HzHx.x Coh HzHy.o

Alamosa, CO $100 \mathrm{k}$

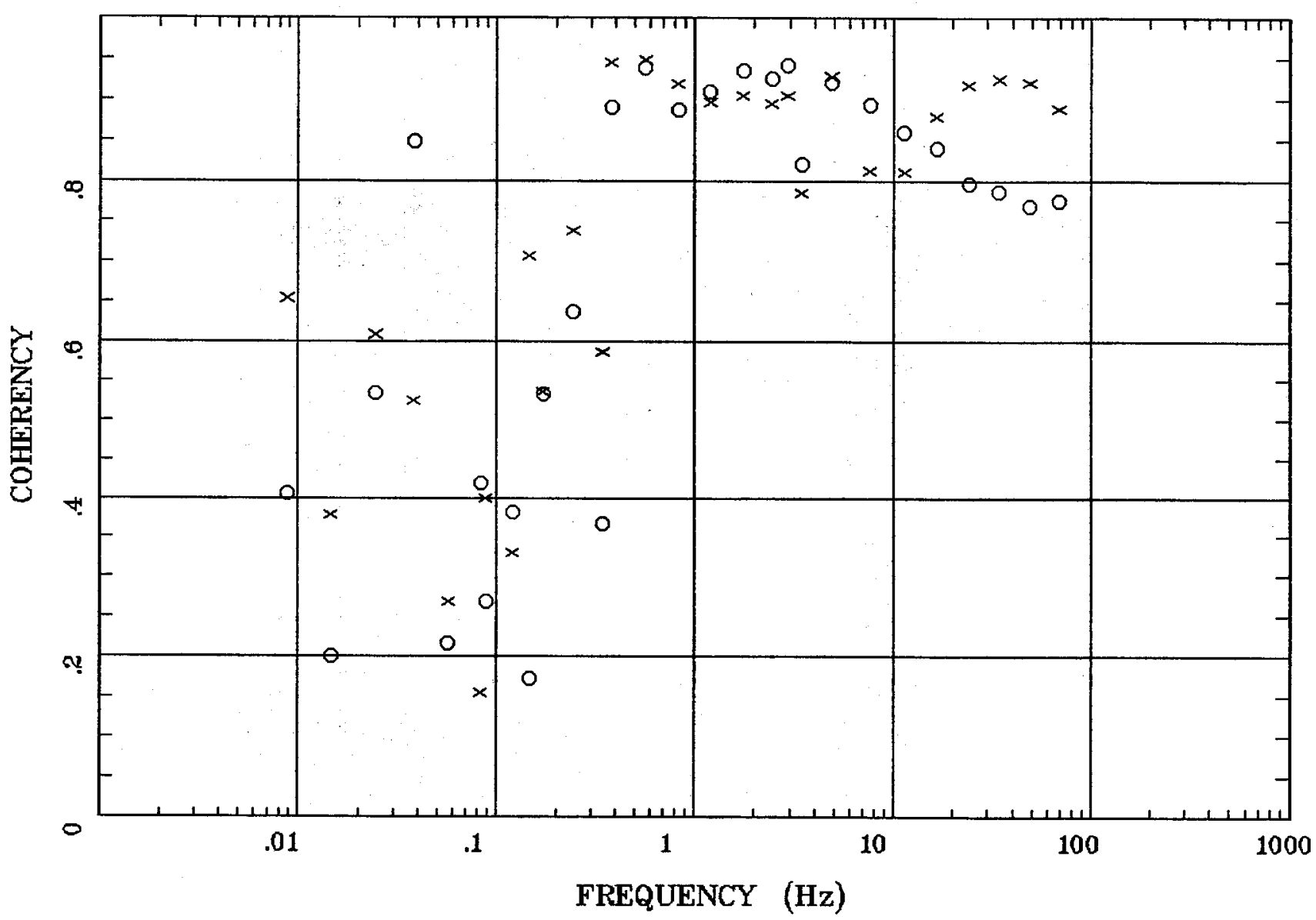

Client:

Remote: none

Acquired: 09:0 Jul 15, 2007 Survey Co:USGS
Rotation:

Filename: sl30m2.avg

Channels: Ch1 Ch2 Ch3 Ch4 Ch5 Ch3 Ch4

Plotted: 11:11 Nov 06, 2007

< EMI - ElectroMagnetic Instruments 
Station 31

APPARENT RESISTIVITY

Alamosa, CO $100 \mathrm{k}$

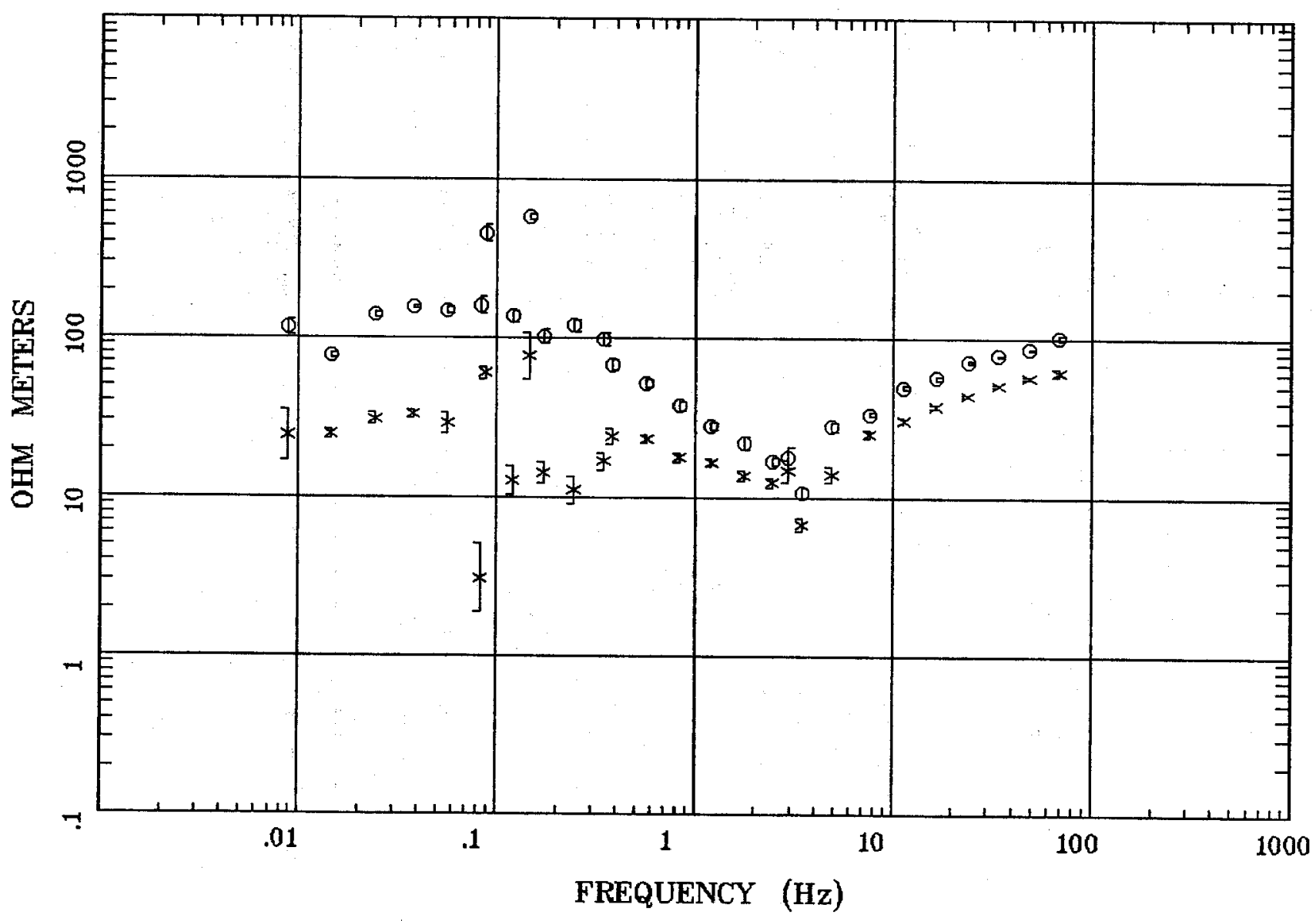

Client:

Remote: none

Acquired: 14:0 Jul 15, 2007

Survey Co:USGS

\section{Rotation:}

Filename: sl31m1.avg

Channels: Ch1 Ch2 Ch3 Ch4 Ch5 Ch3 Ch4 Plotted: 11:13 Nov 06, 2007

< EMI - ElectroMagnetic Instruments > 


\section{IMPEDANCE PHASE}

Alamosa, CO 100k

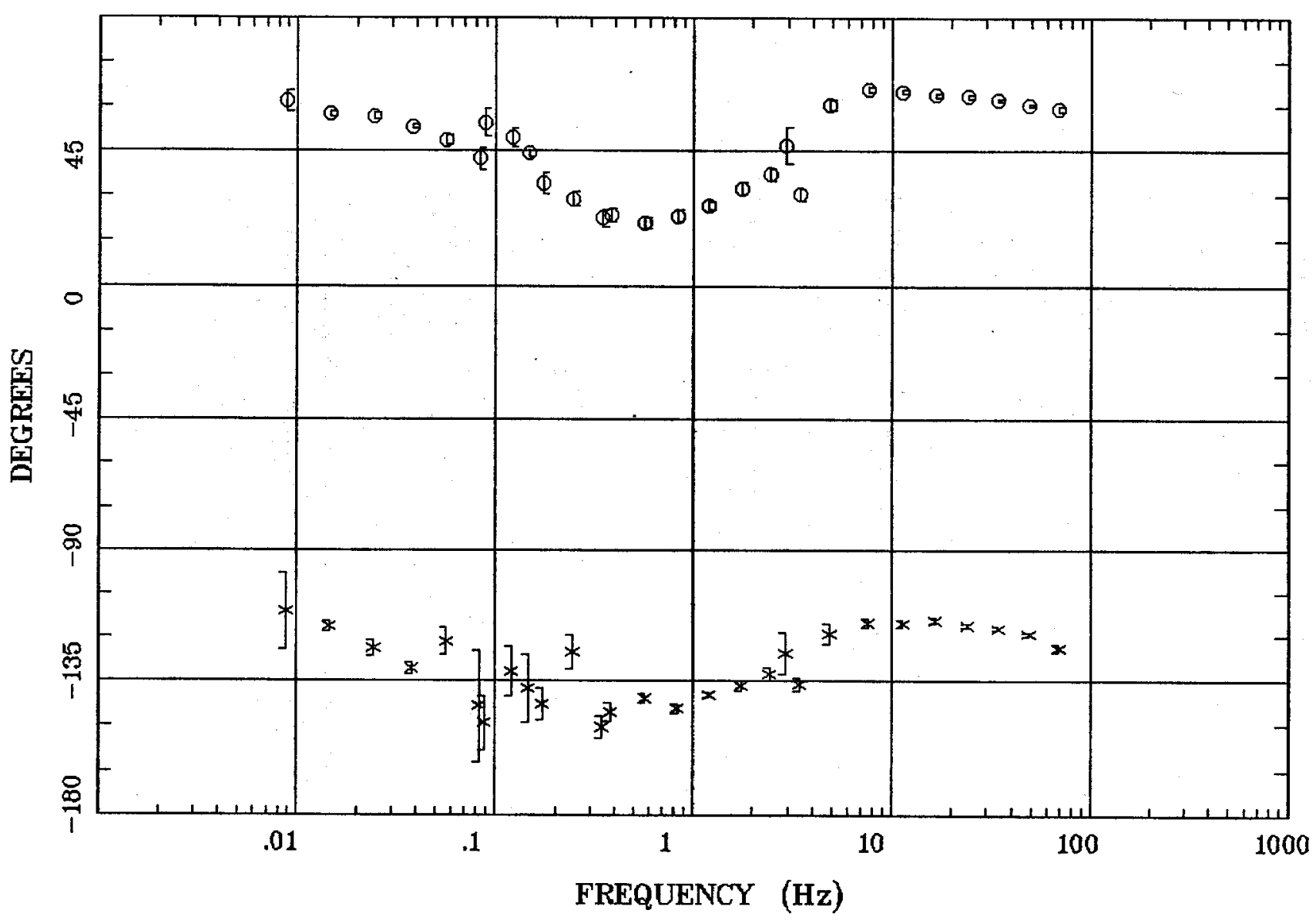

Client:

Remote: none

Acquired: 14:0 Jul 15, 2007 Survey Co:USGS
Rotation:

Filename: sl31m1.avg

Channels: Ch1 Ch2 Ch3 Ch4 Ch5 Ch3 Ch4

Plotted: 11:13 Nov 06, 2007

$<$ EMI - ElectroMagnetic Instruments > 
Station 31

ROTATION ANGLE

Alamosa, CO 100k

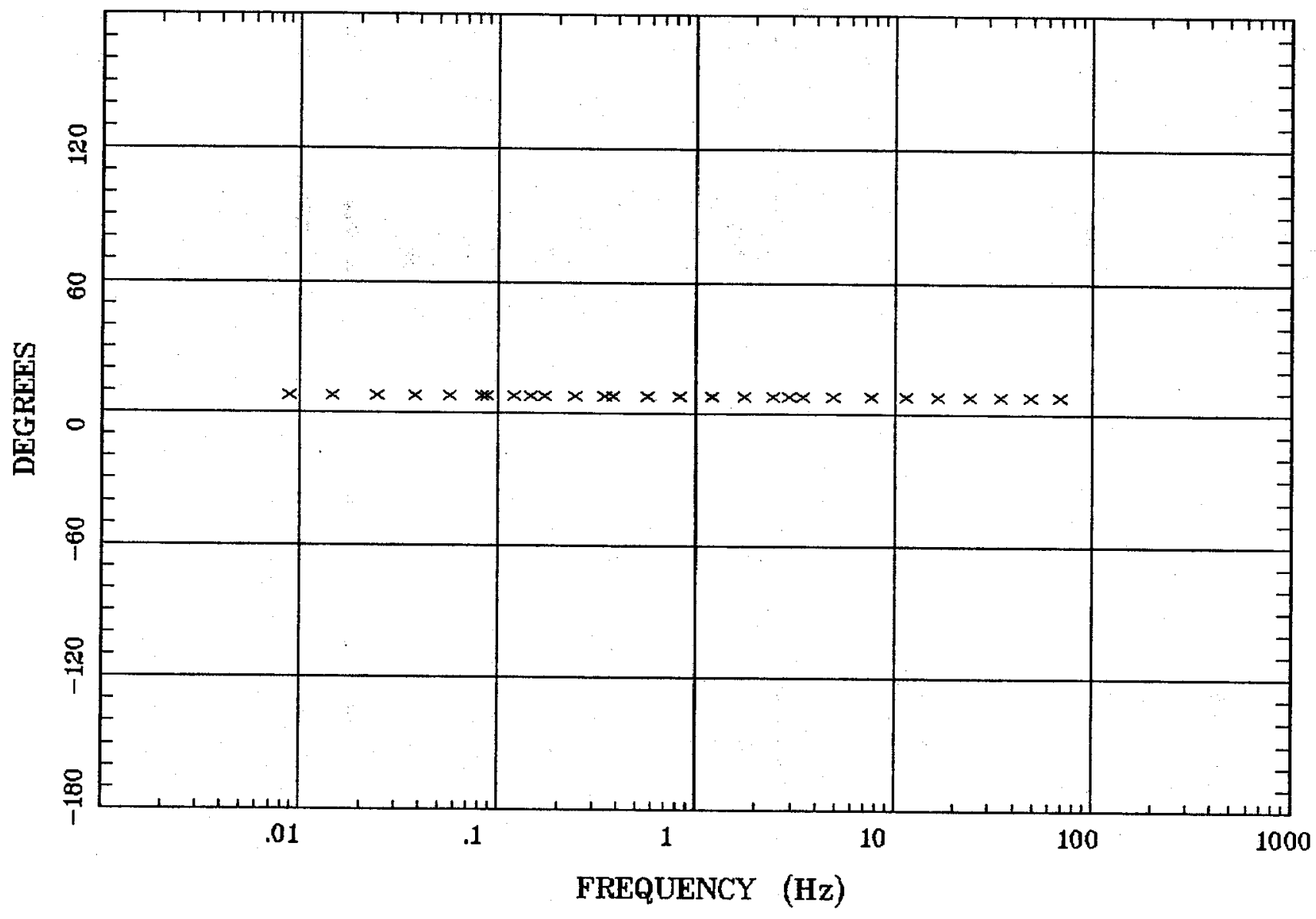

Client:

Remote: none

Acquired: 14:0 Jul 15, 2007

Survey Co:USGS
Rotation:

Filename: sl31m1.avg

Channels: Ch1 Ch2 Ch3 Ch4 Ch5 Ch3 Ch4 Plotted: 11:13 Nov 06, 2007

< EMI - ElectroMagnetic Instruments > 
Alamosa, CO 100k

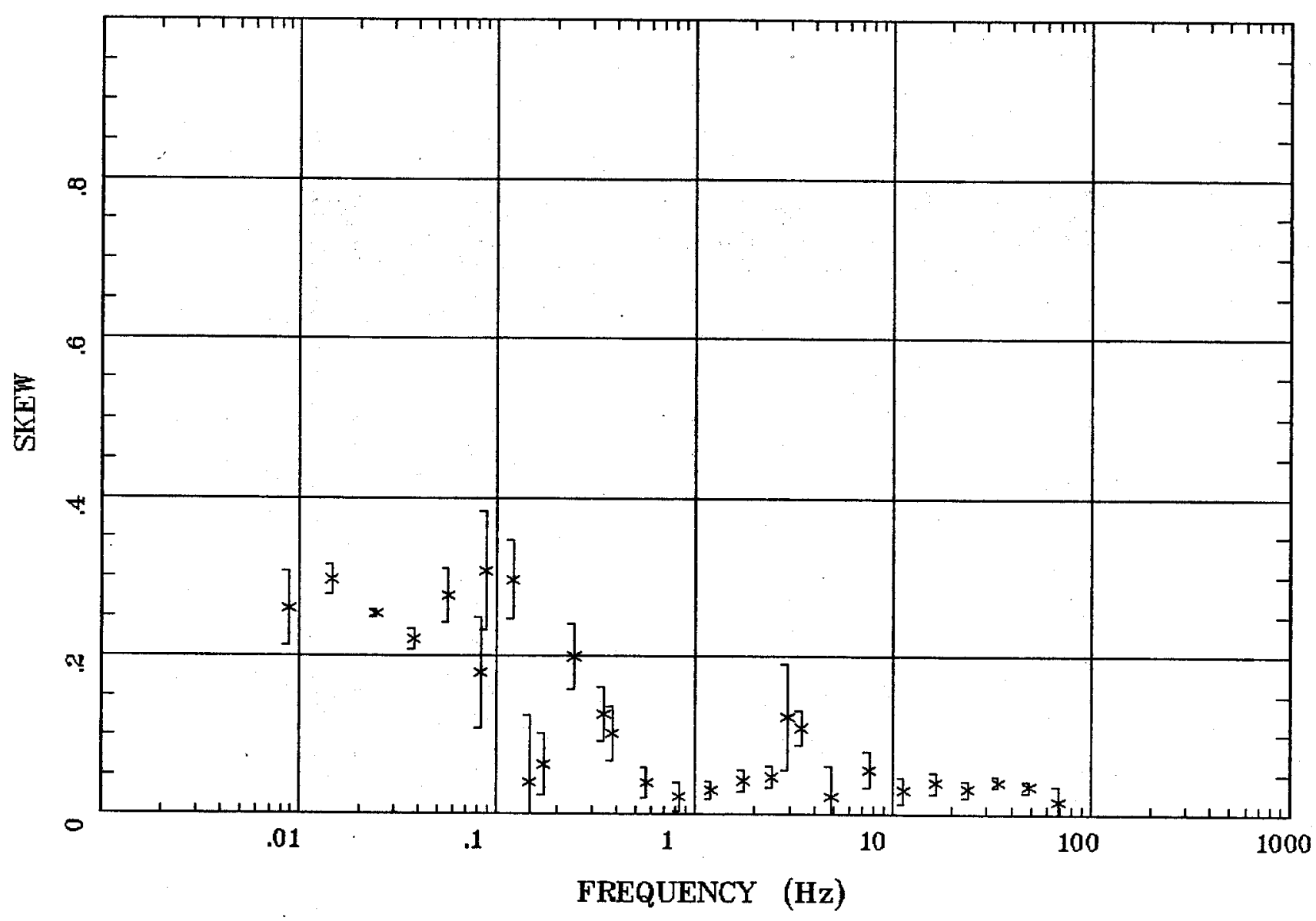

Client:

Remote: none

Acquired: 14:0 Jul 15, 2007 Survey Co:USGS
Rotation:

Filename: sl31m1.avg

Channels: Ch1 Ch2 Ch3 ch4 Ch5 Ch3 Ch4 Plotted: 11:13 Nov 06, 2007

< EMI - ElectroMagnetic Instruments > 
E MULT Coh.

Alamosa, CO 100k

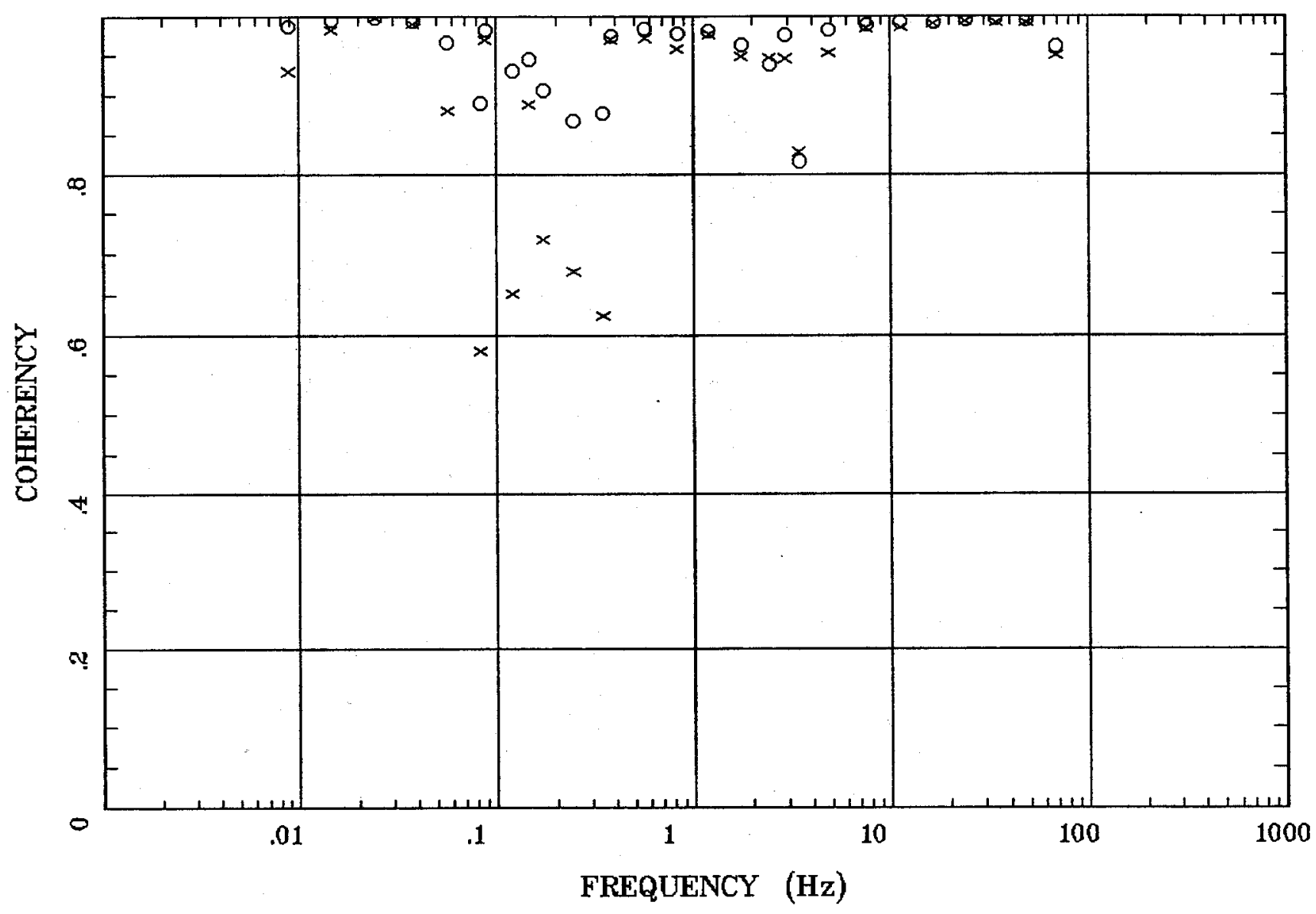

Client:

Remote: none

Acquired: 14:0 Jul 15, 2007

Survey Co:USGS
Rotation:

Filename: sl31m1.avg

Channels: Ch1 Ch2 Ch3 Ch4 Ch5 Ch3 Ch4

Plotted: 11:13 Nov 06, 2007

$<$ EMI - ElectroMagnetic Instruments 
Alamosa, Co 100k

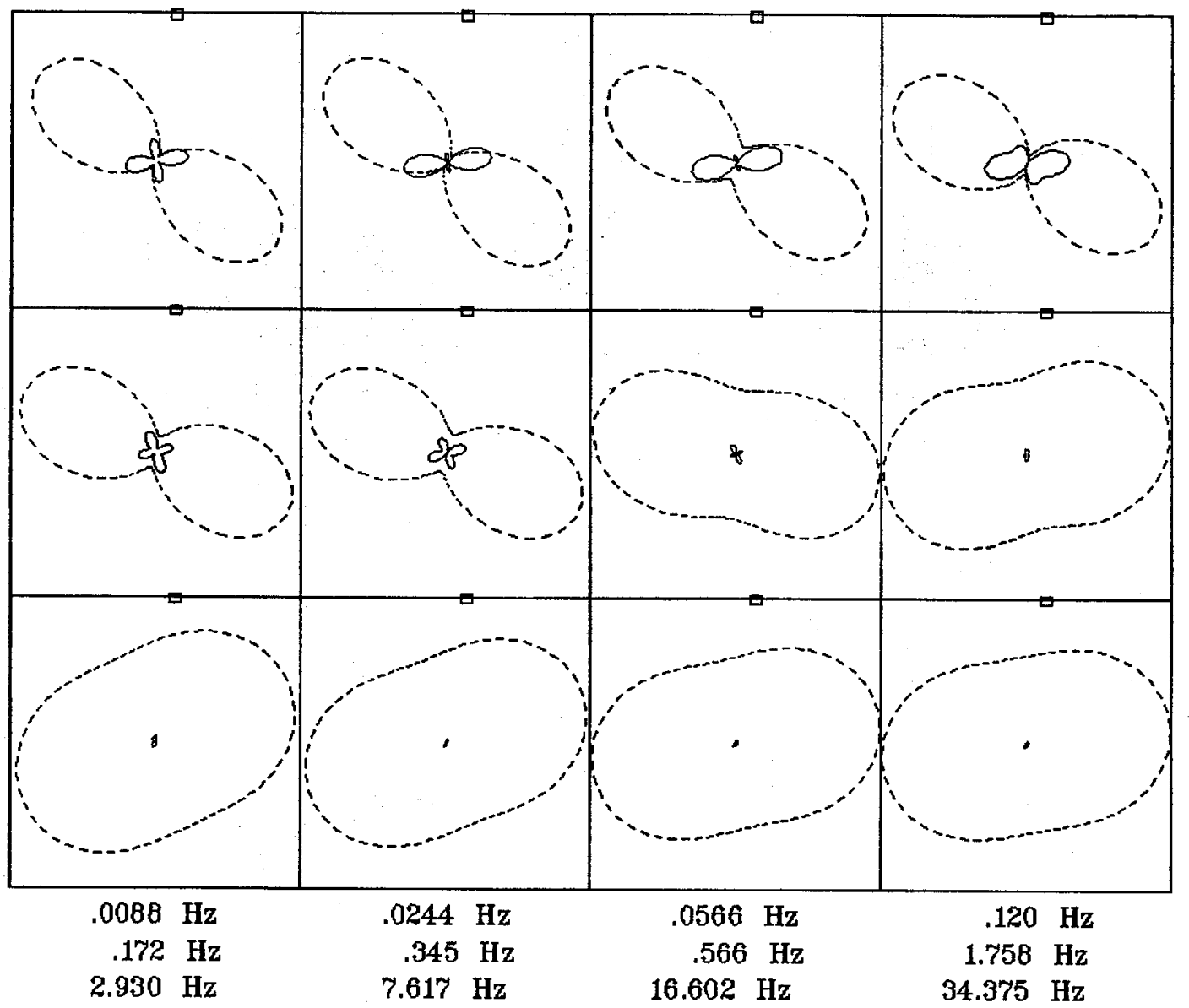

Client:

Remote: none

Acquired: 14:0 Jul 15, 2007 Survey Co:USGS
Rotation:

Filename: sl31m1.avg

Channels: Ch1 Ch2 Ch3 Ch4 Ch5 Ch3 Ch4 Plotted: 11:13 Nov 06, 2007

< EMI - ElectroMagnetic Instruments > 
Alamosa, CO 100k

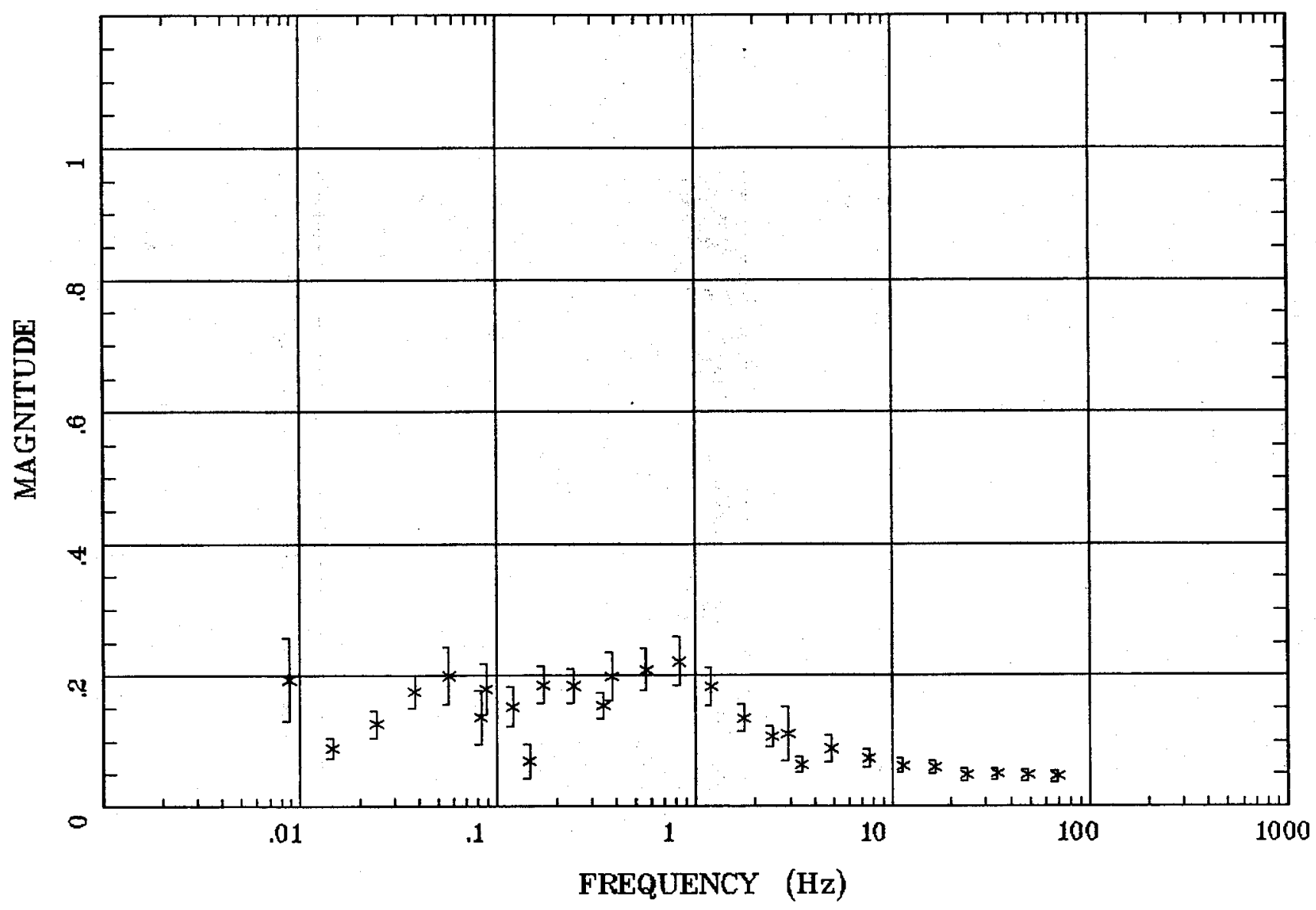

Client:

Remote: none

Acquired: 14:0 Jul 15, 2007

Survey Co:USGS
Rotation:

Filename: sl31m1.avg

Channels: Ch1 Ch2 Ch3 Ch4 Ch5 Ch3 Ch4

Plotted: 11:13 Nor 06, 2007

< EMI - ElectroMagnetic Instruments > 
Station 31

Alamosa, CO 100k

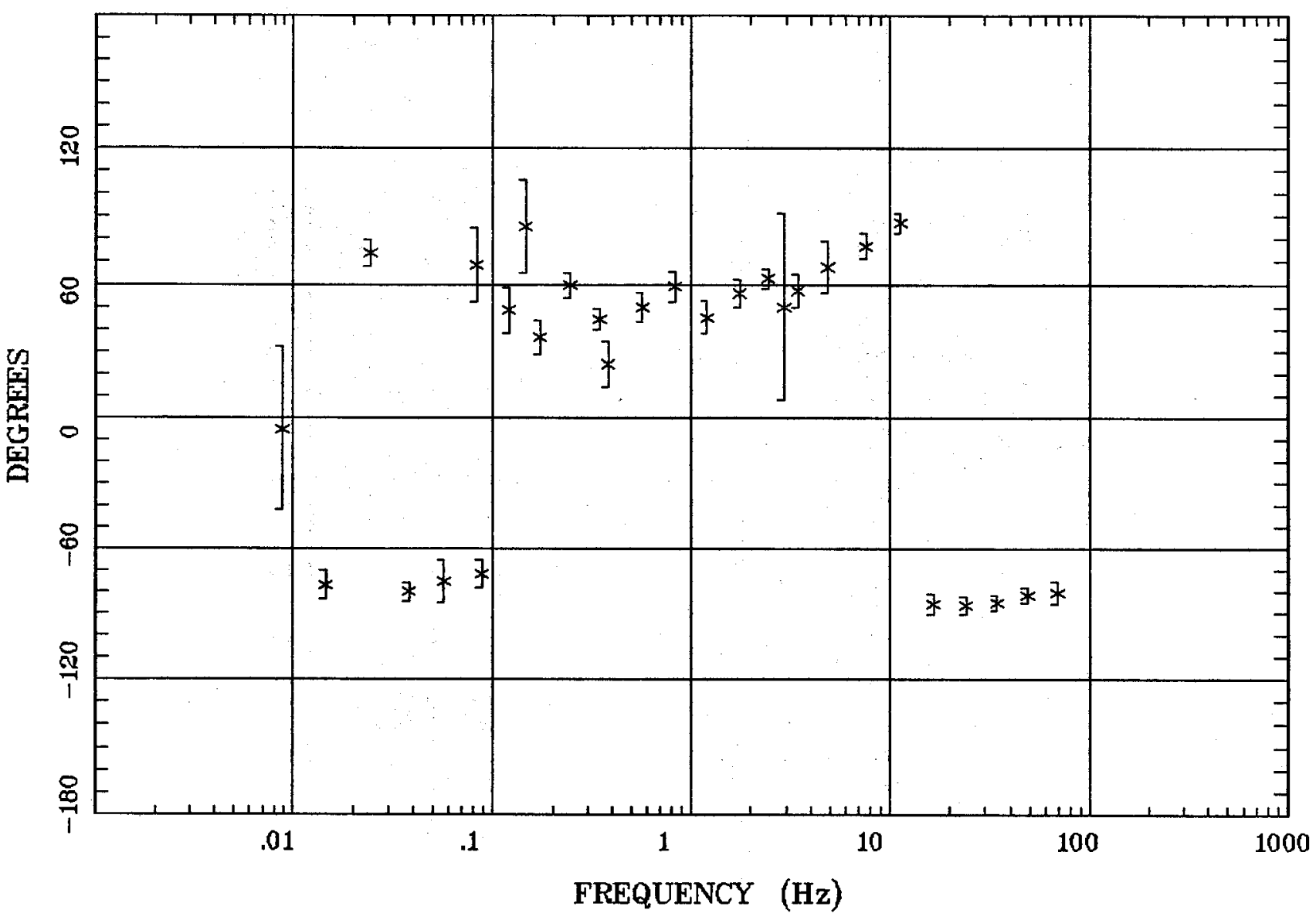

Client:

Remote: none

Acquired: 14:0 Jul 15, 2007

Survey Co:USGS
Rotation:

Filename: sl31m1.avg

Channels: Ch1 Ch2 Ch3 Ch4 Ch5 Ch3 Ch4 Plotted: 11:13 Nov 06, 2007

< EMI - ElectroMagnetic Instruments > 
HzHx.x Coh HzHy.o Alamosa, CO 100k

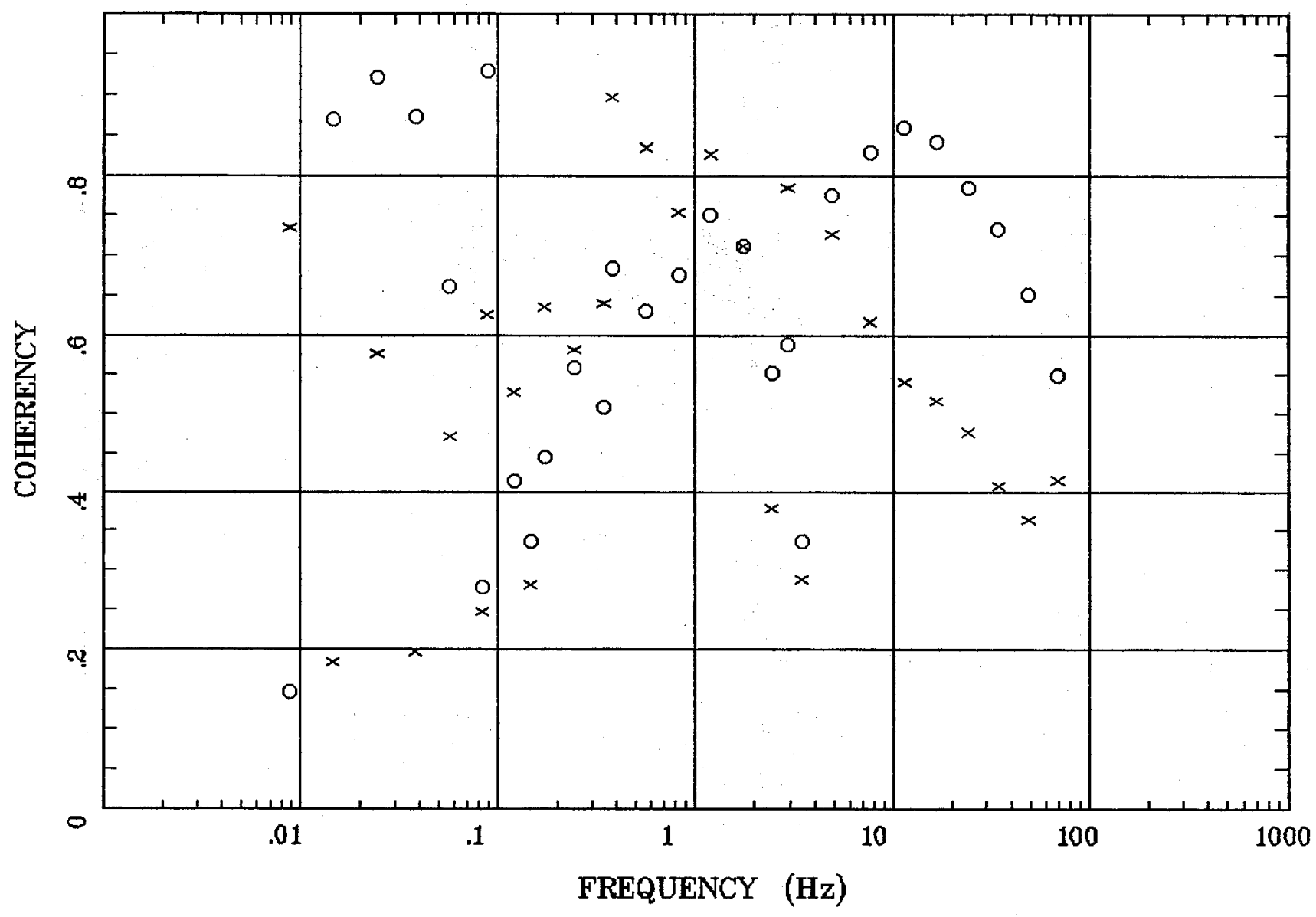

Client:

Remote: none

Acquired: 14:0 Jul 15, 2007

Survey Co:USGS
Rotation:

Filename: sl31m1.avg

Channels: Ch1 Ch2 Ch3 Ch4 Ch5 Ch3 Ch4 Plotted: 11:13 Nov 06, 2007

< EMI - ElectroMagnetic Instruments 
Alamosa, CO 100k

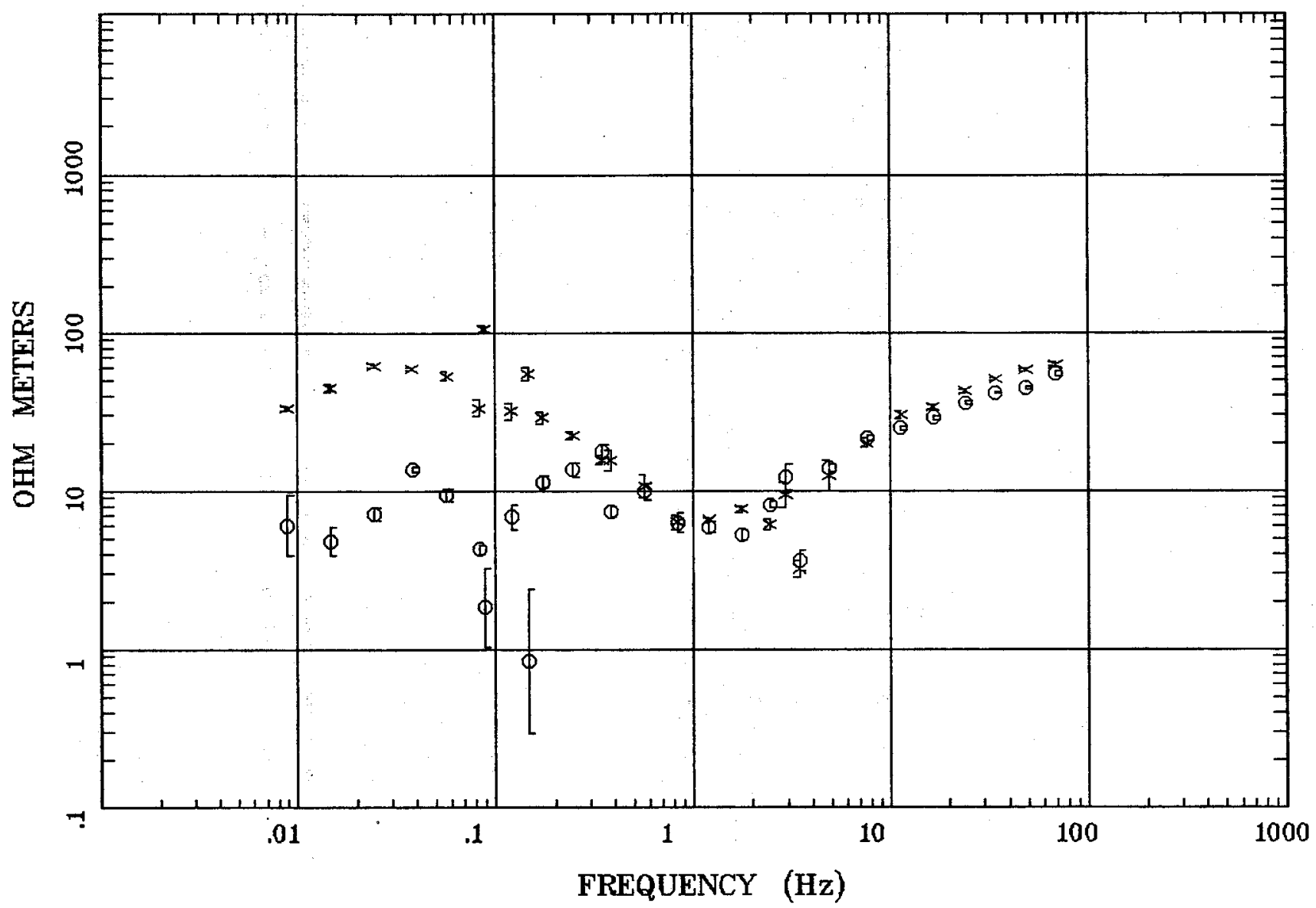

Client:

Remote: none

Acquired: 09:0 Jul 16, 2007

Survey Co:USGS

\section{Rotation:}

Filename: sl32m1.avg

Channels: Ch1 Ch2 Ch3 Ch4 Ch5 Ch3 Ch4

Plotted: 11:11 Nov 06, 2007

< EMI - ElectroMagnetic Instruments 


\section{IMPEDANCE PHASE}

Station 32

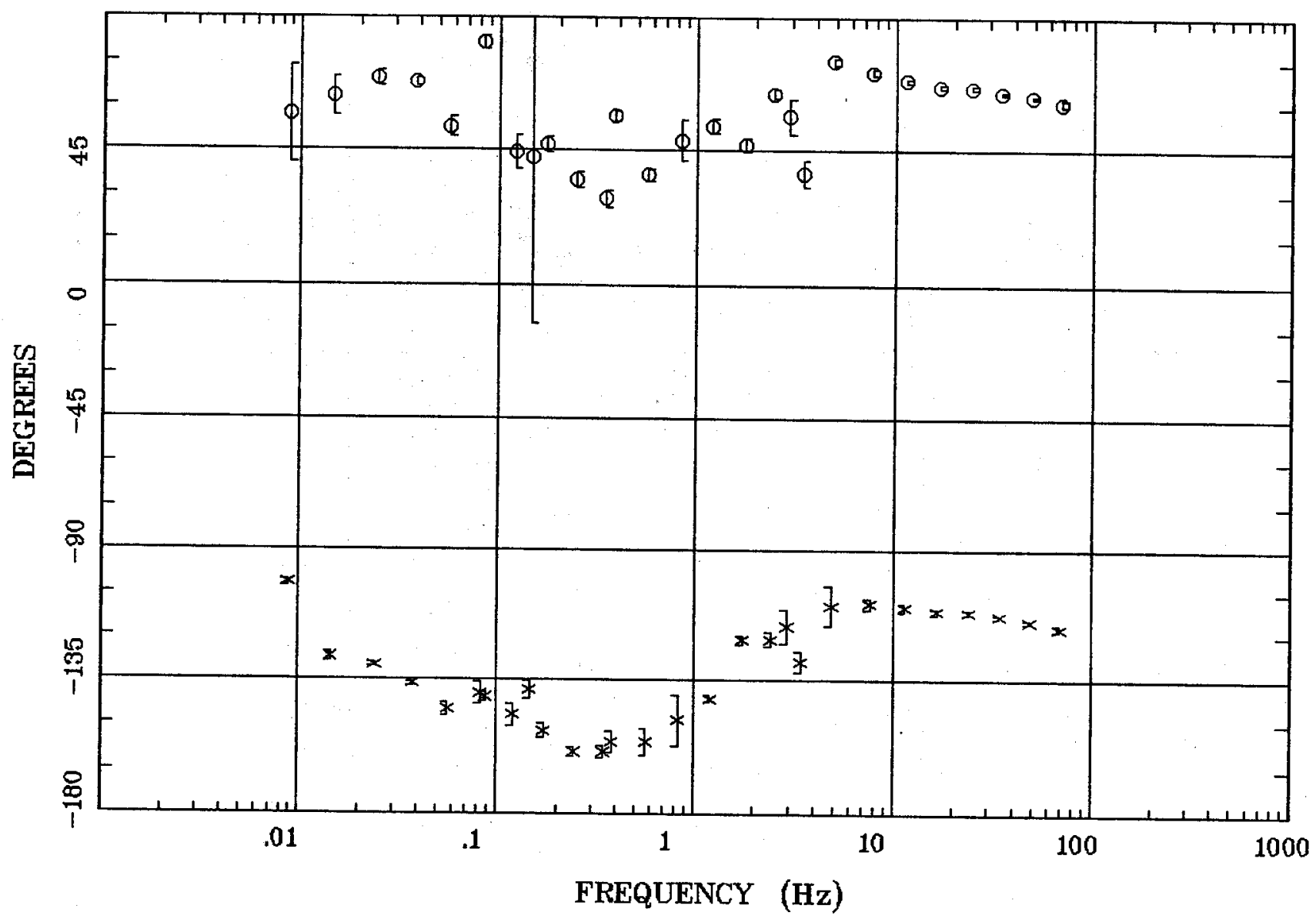

Client:

Remote: none

Acquired: 09:0 Jul 16, 2007

Survey Co:USGS
Rotation:

Filename: sl32m1.avg

Channels: Ch1 Ch2 Ch3 Ch4 Ch5 Ch3 Ch4

Plotted: 11:11 Nor 06, 2007

$<$ EMI - ElectroMagnetic Instruments 


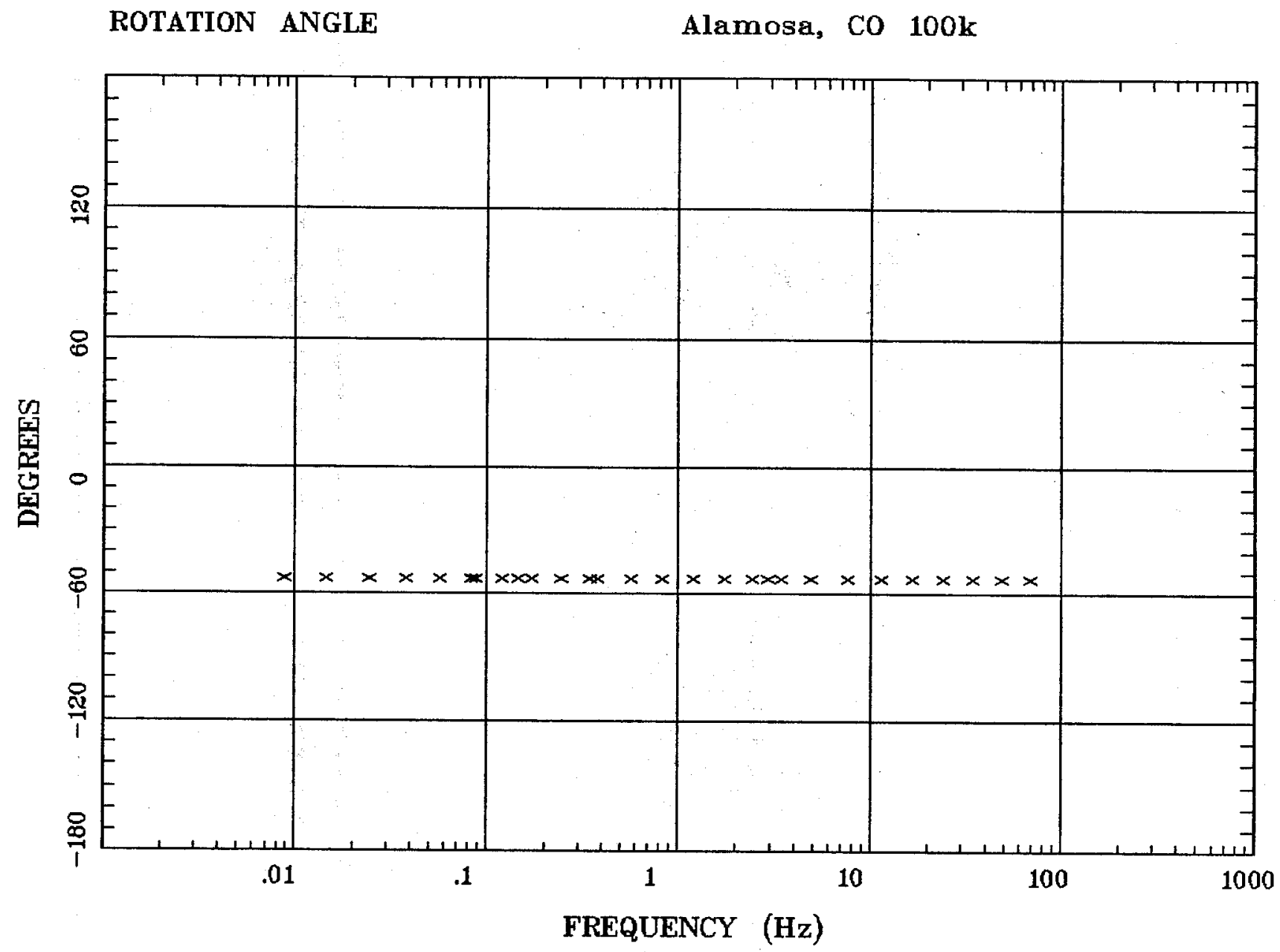

Client:

Remote: none Acquired: 09:0 Jul 16, 2007 Survey Co:USGS
Rotation:

Filename: sl32m1.avg

Channels: Ch1 Ch2 Ch3 Ch4 Ch5 Ch3 Ch4

Plotted: 11:11 Nov 06, 200"7

< EMI - ElectroMagnetic Instruments 
Alamosa, CO 100k

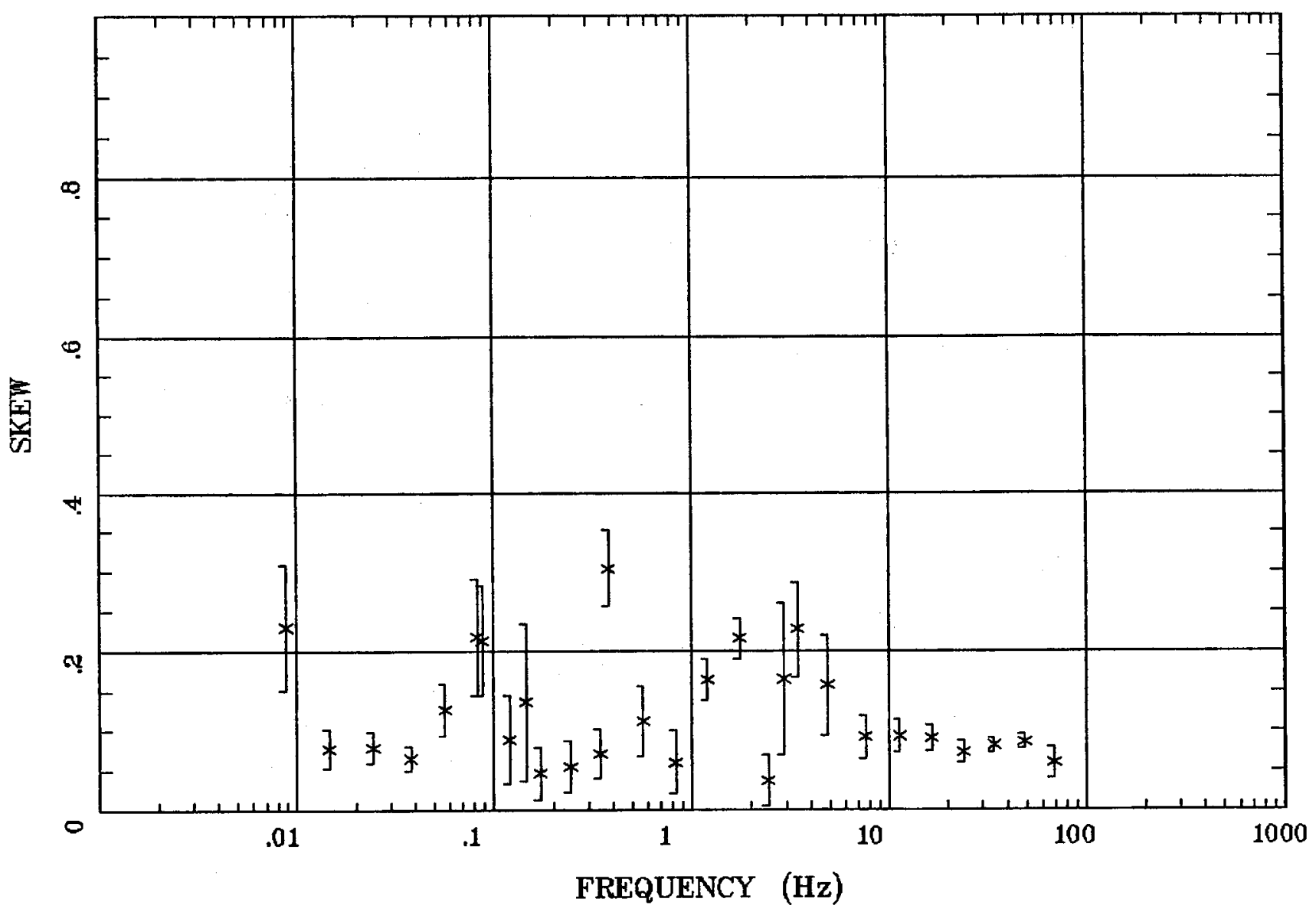

Client:

Remote: none

Acquired: 09:0 Jul 16, 2007

Survey Co:USGS
Rotation:

Filename: sl32m1.avg

Channels: Ch1 Ch2 Ch3 Ch4 Ch5 Ch3 Ch4 Platted: 11:11 Nov 06, 2007

< EMI - ElectroMagnetic Instruments > 
E MULT Coh.

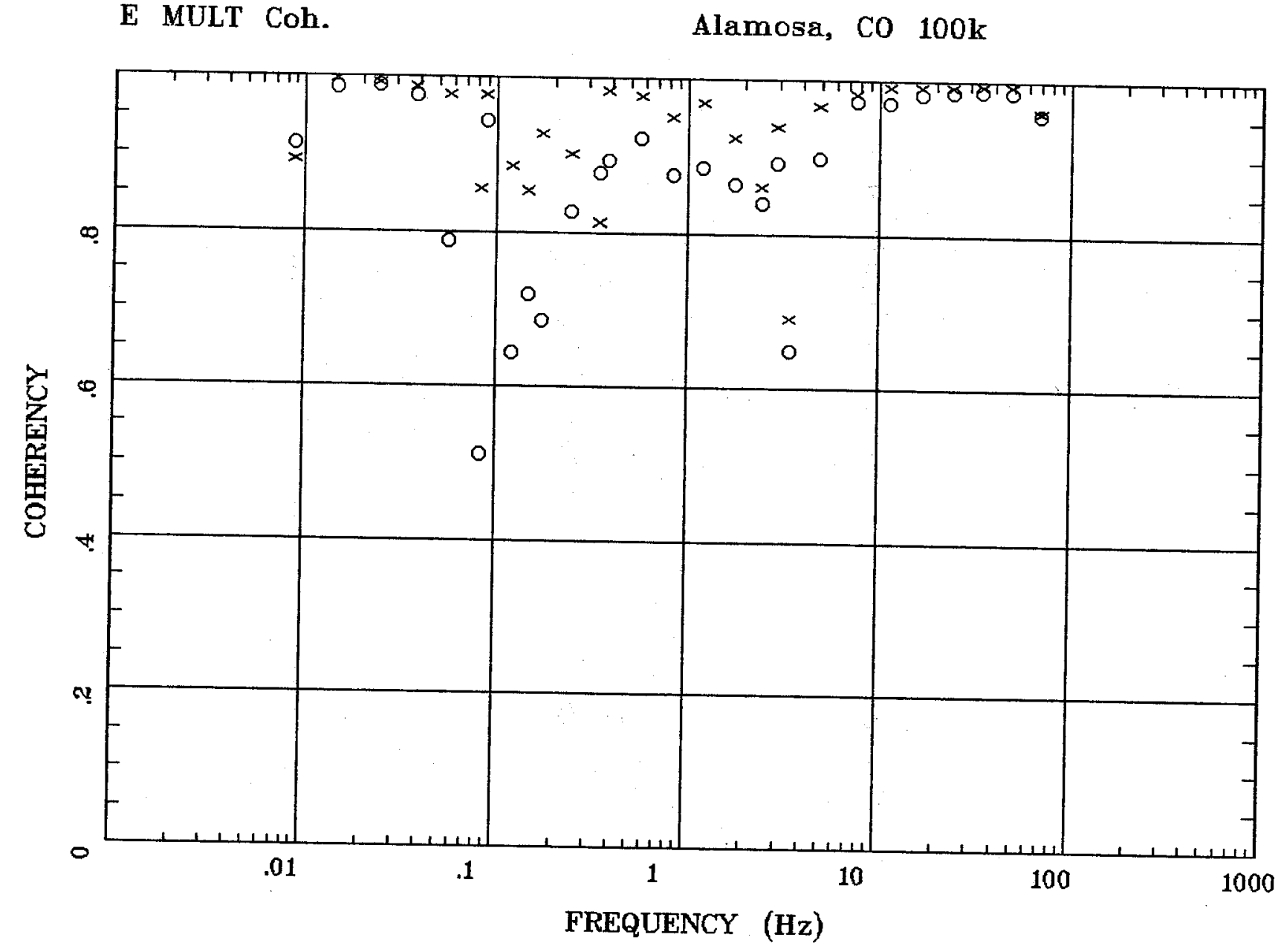

Client:

Remote: none

Acquired: 09:0 Jul 16, 2007

Survey Co:USGS
Rotation:

Filename: sl32m1.avg

Channels: Ch1 Ch2 Ch3 Ch4 Ch5 Ch3 Ch4 Plotted: 11:11 Nov 06, 2007

< EMI - ElectroMagnetic Instruments > 
Alamosa, CO $100 \mathrm{k}$

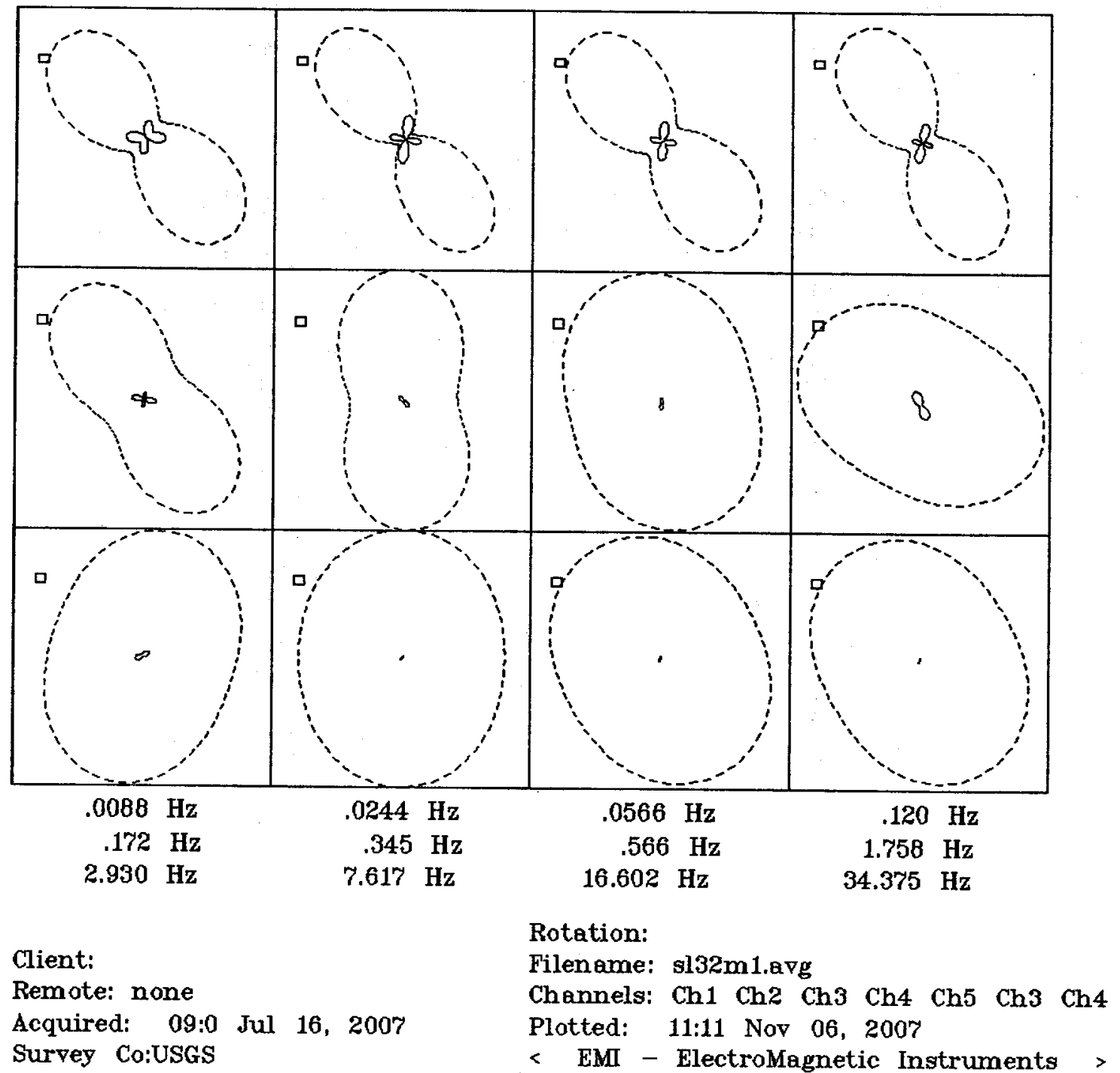


Alamosa, Co 100k

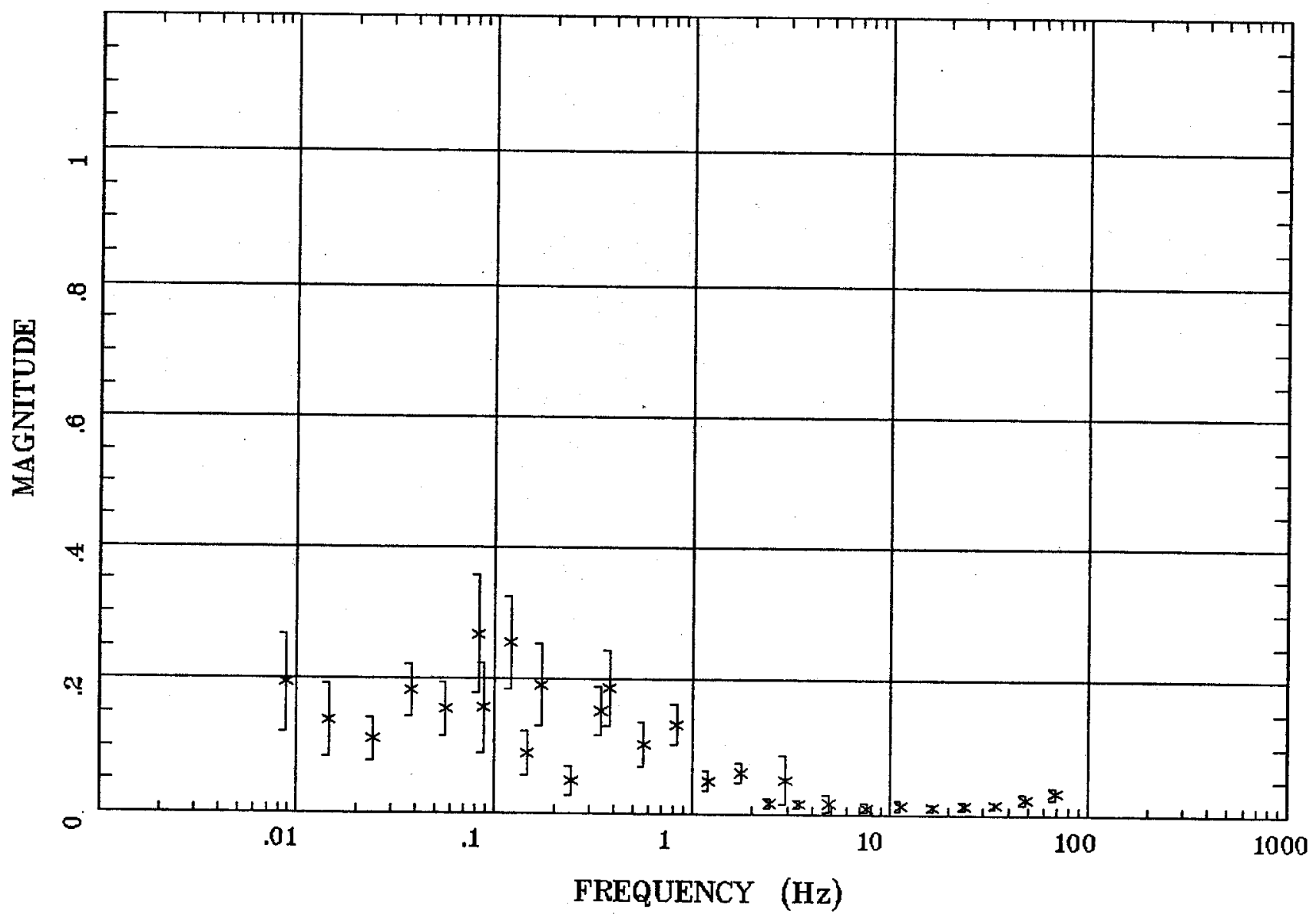

Client:

Remote: none

Acquired: 09:0 Jul 16, 2007

Survey Co:USGS
Rotation:

Filename: sl32m1.avg

Channels: Ch1 Ch2 Ch3 Ch4 Ch5 Ch3 Ch4

Plotted: 11:11 Nov 06, 2007

$<$ EMI - ElectroMagnetic Instruments > 
Alamosa, CO 100k

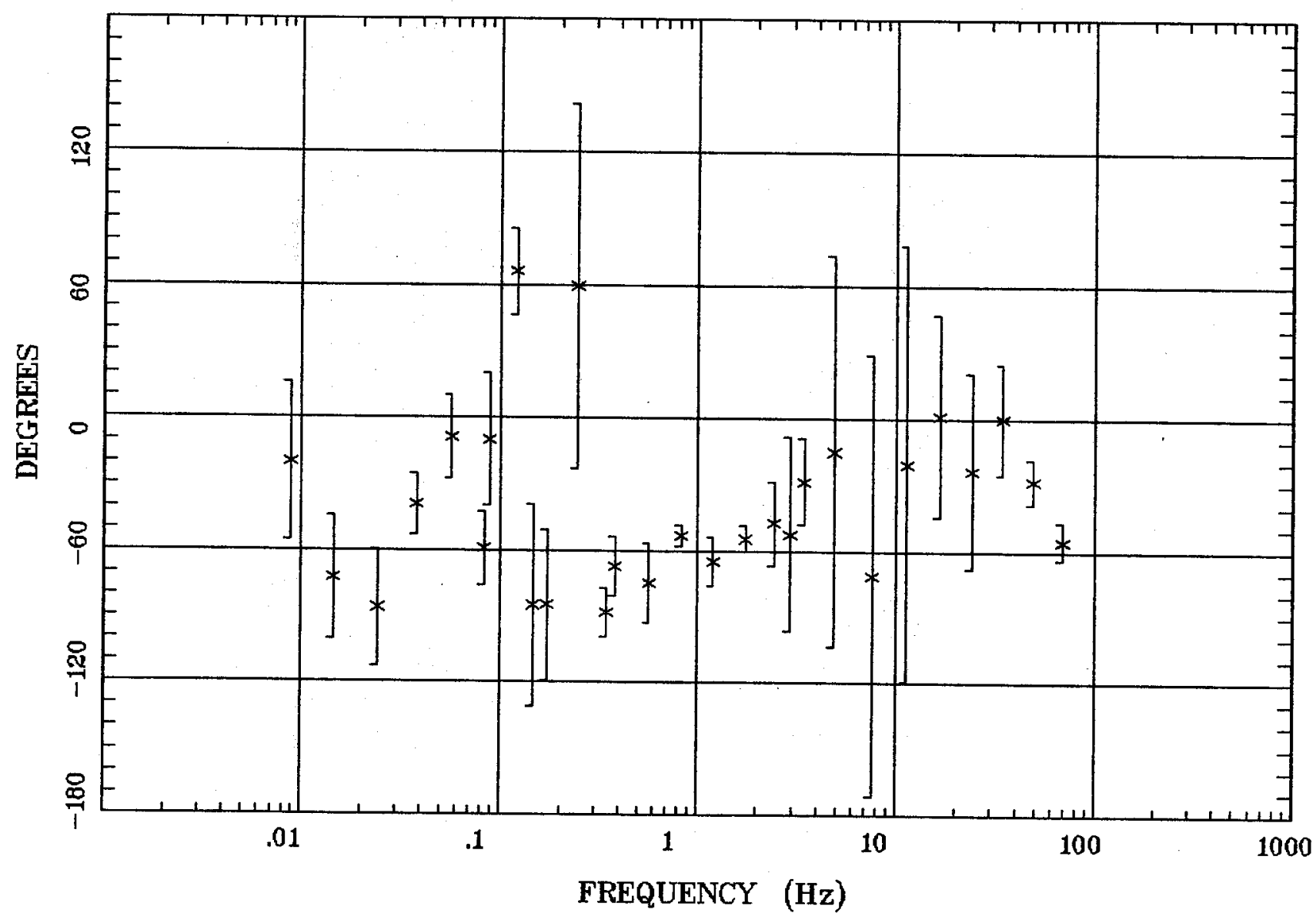

Client:

Remote: none

Acquired: 09:0 Jul 16, 2007

Survey Co:USGS
Ratation:

Filename: sl32m1.avg

Channels: Ch1 Ch2 Ch3 Ch4 Ch5 Ch3 Ch4

Plotted: 11:11 Nop 06, 2007

$<$ EMI - ElectroMagnetic Instruments 


\section{HzHx.x Coh HzHy.o}

Alamosa, CO 100k

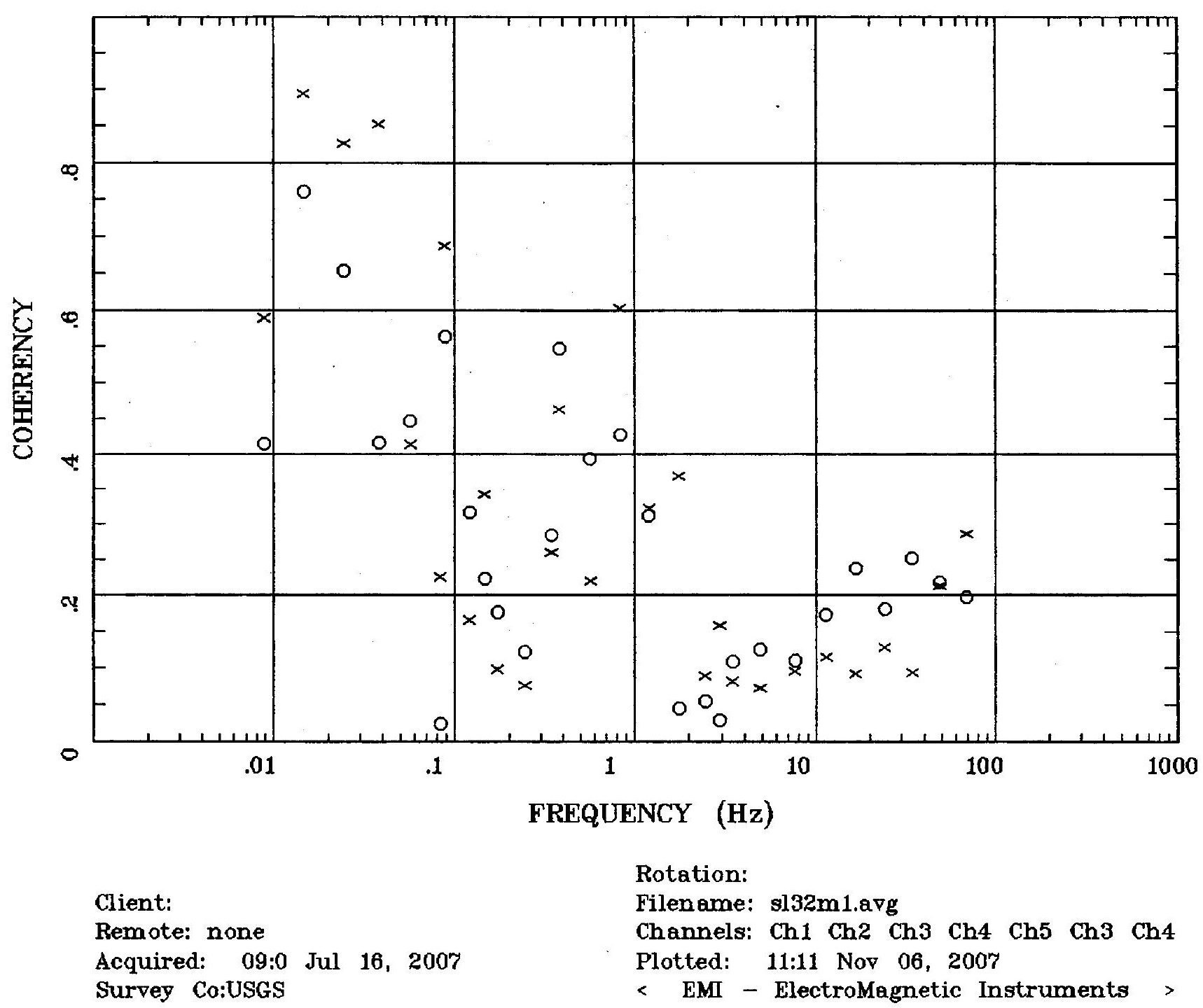


Alamosa, CO 100k

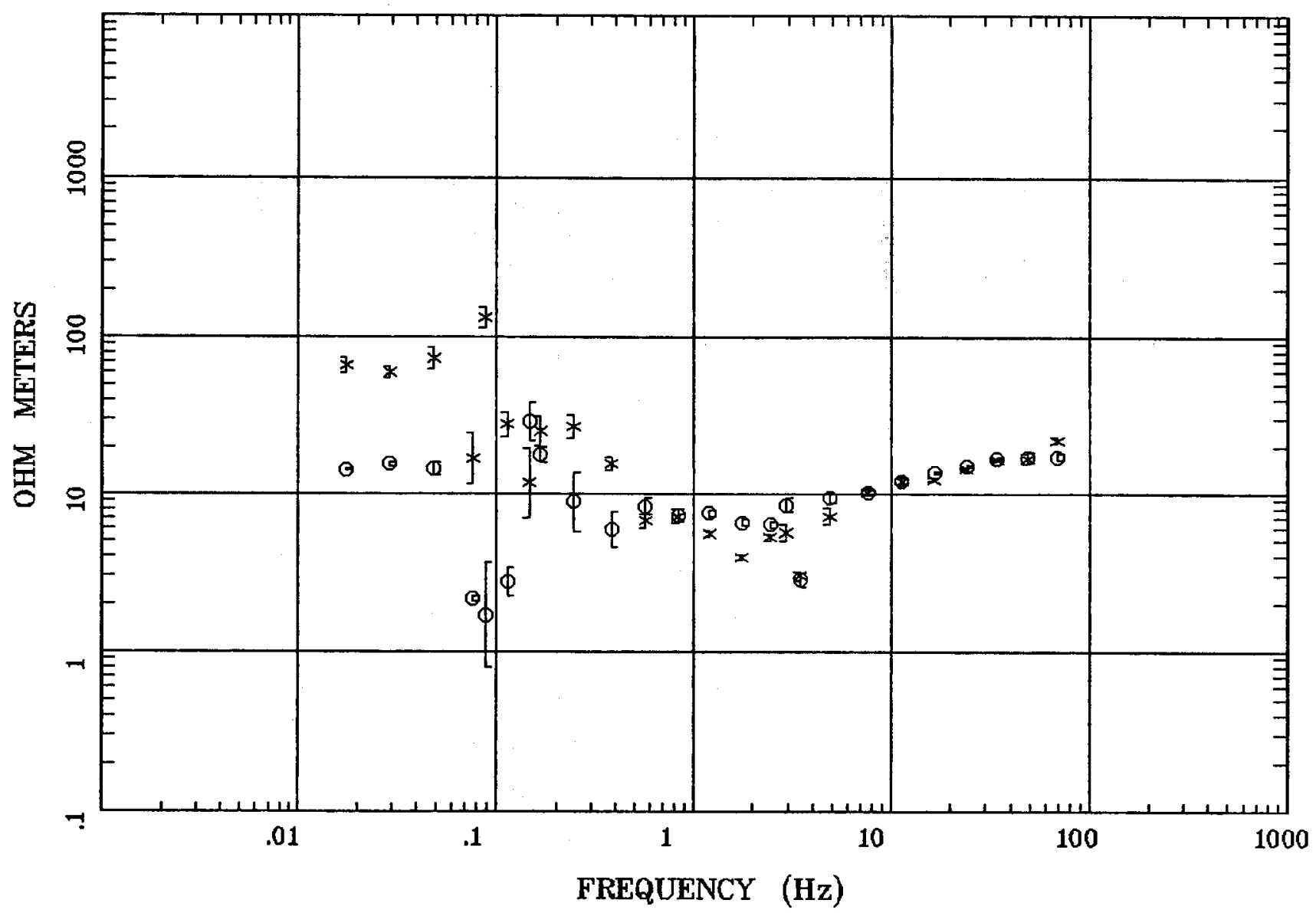

Client:

Remote: none Acquired: 14:0 Jul 17, 2007 Survey Co:USGS
Rotation:

Filename: sl33m1.avg

Channels: Ch1 Ch2 Ch3 Ch4 Ch5 Ch3 Ch4 Plotted: 11:18 Nov 06, 2007

< EMI - ElectroMagnetic Instruments > 
IMPEDANCE PHASE

Alamosa, CO 100k

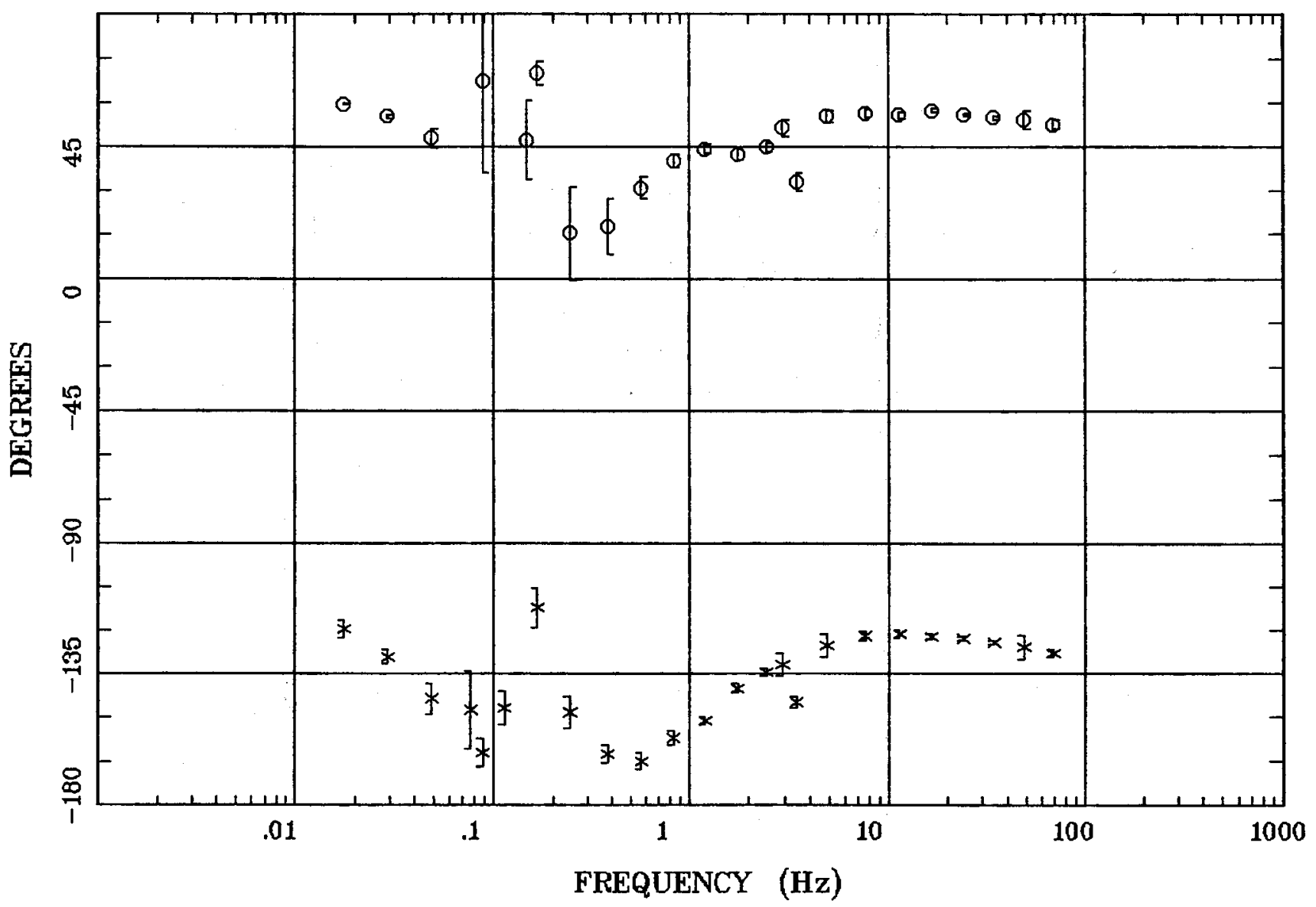

Client:

Remote: none

Acquired: 14:0 Jul 17, 2007

Survey Co:USGS
Rotation:

Filename: sl33m1.avg

Channels: Ch1 Ch2 Ch3 Ch4 Ch5 Ch3 Ch4

Plotted: 11:18 Nov 06, 2007

< EMI - ElectroMagnetic Instruments 
ROTATION ANGLE

Alamosa, CO 100k

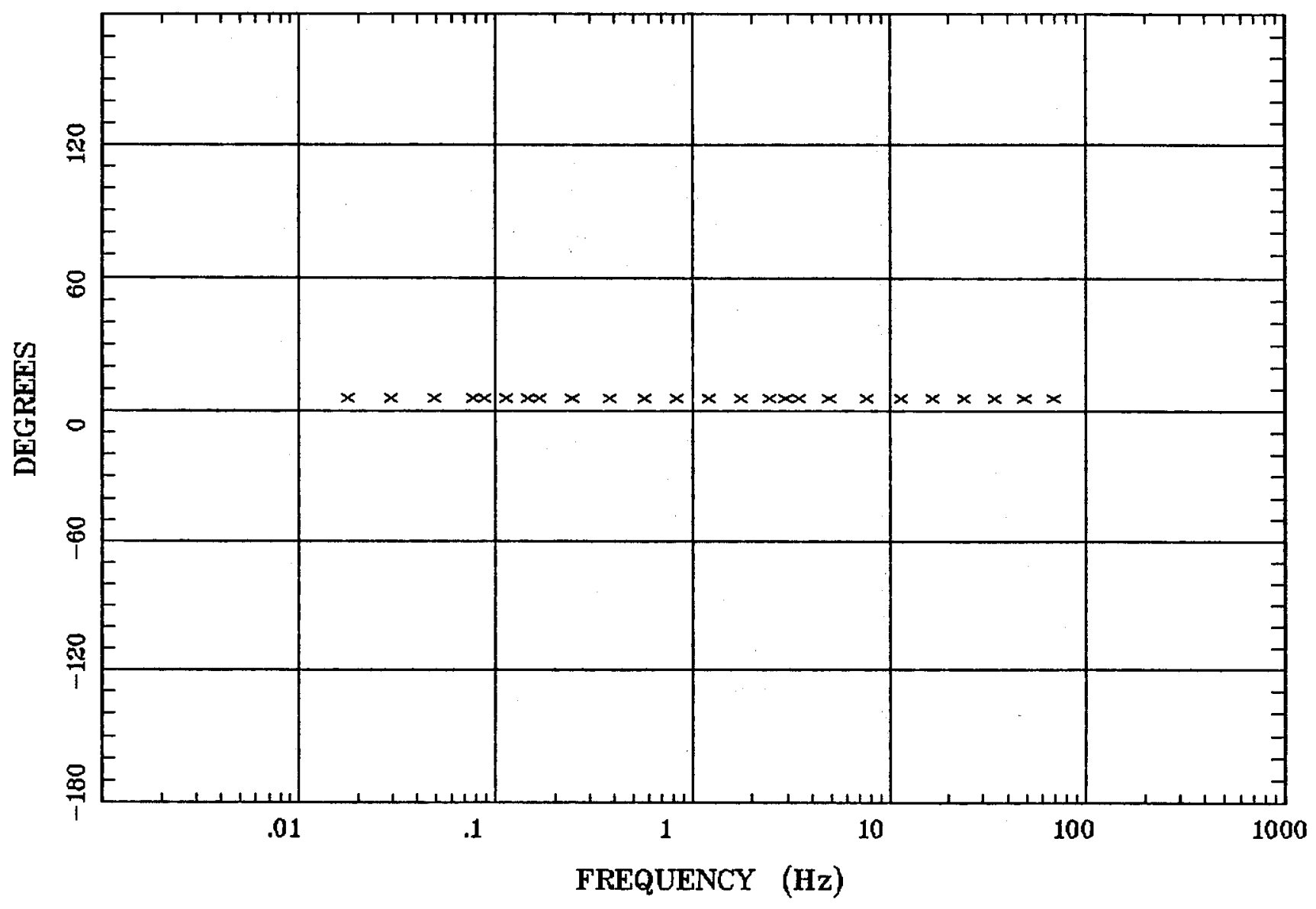

Client:

Remote: none

Acquired: 14:0 Jul 17, 2007 Survey Co:USGS
Rotation:

Filename: sl33m1.avg

Channels: Ch1 Ch2 Ch3 Ch4 Ch5 Ch3 Ch4

Plotted: 11:18 Nov 06, 2007

$<$ EMI - ElectroMagnetic Instruments 
Alamosa, CO 100k

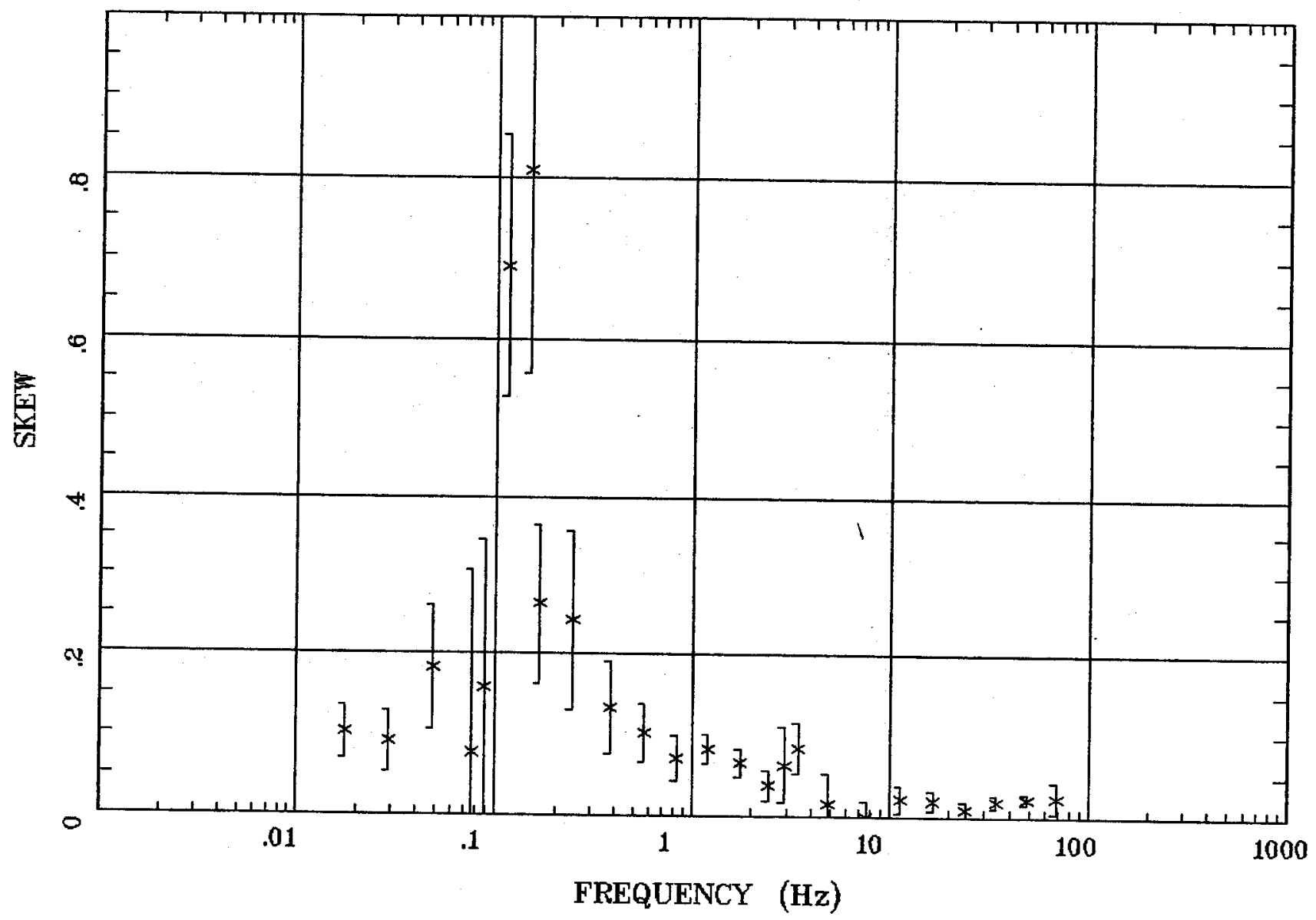

Client:

Remote: none Acquired: 14:0 Jul 17, 2007 Survey Co:USGS
Rotation:

Filename: sl33m1.avg

Channels: Ch1 Ch2 Ch3 Ch4 Ch5 Ch3 Ch4

Plotted: 11:18 Nov 06, 2007

< EMI - ElectroMagnetic Instruments 
E MULT Coh.

Alamosa, CO 100k

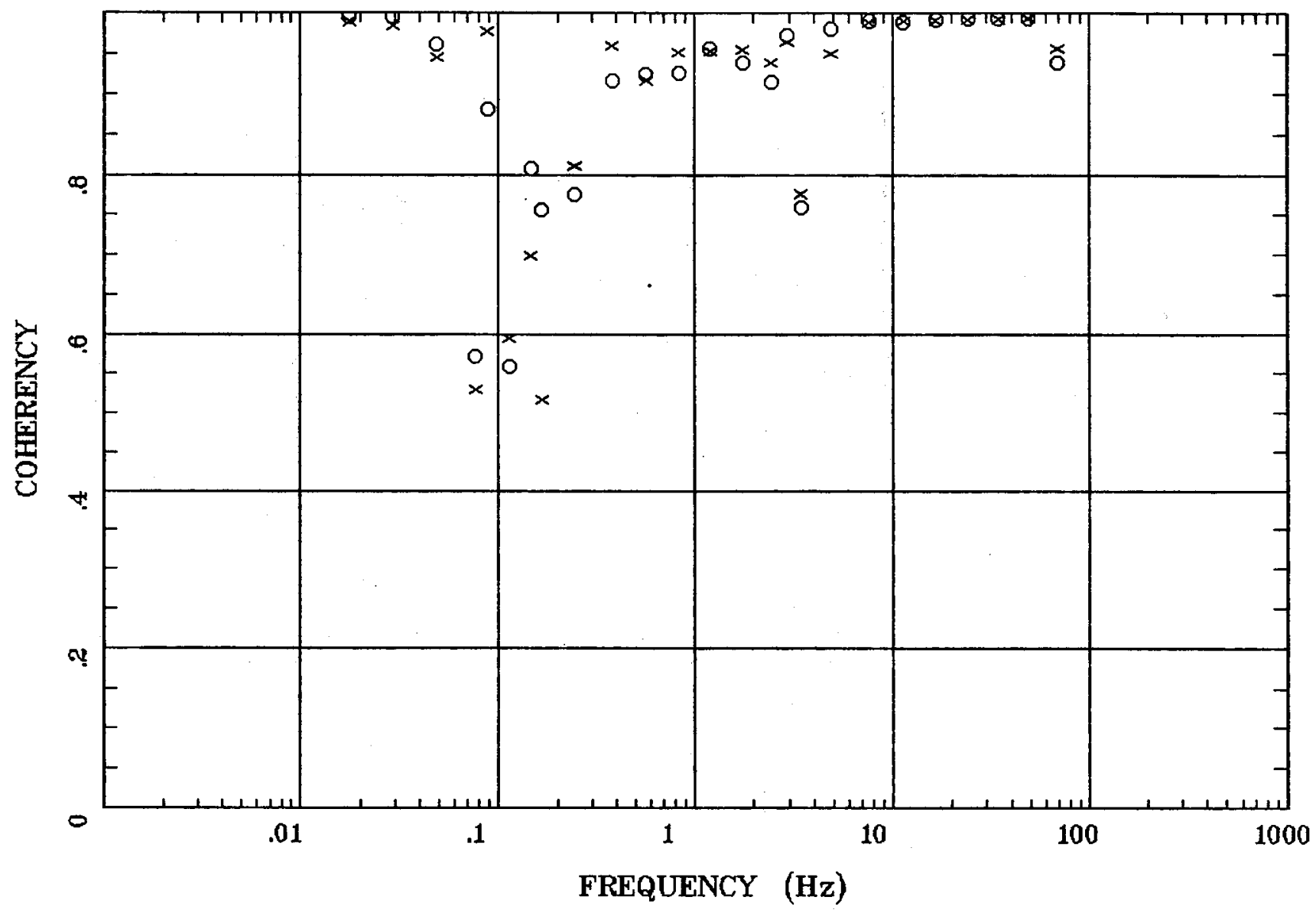

Client:

Remote: none

Acquired: 14:0 Jul 17, 2007 Survey Co:USGS
Rotation:

Filename: sl33m1.avg

Channels: Ch1 Ch2 Ch3 Ch4 Ch5 Ch3 Ch4 Plotted: 11:18 Nor 06, 2007

< EMI - ElectroMagnetic Instruments > 
Station 33

POLAR PLOTS

Alamosa, Co 100k

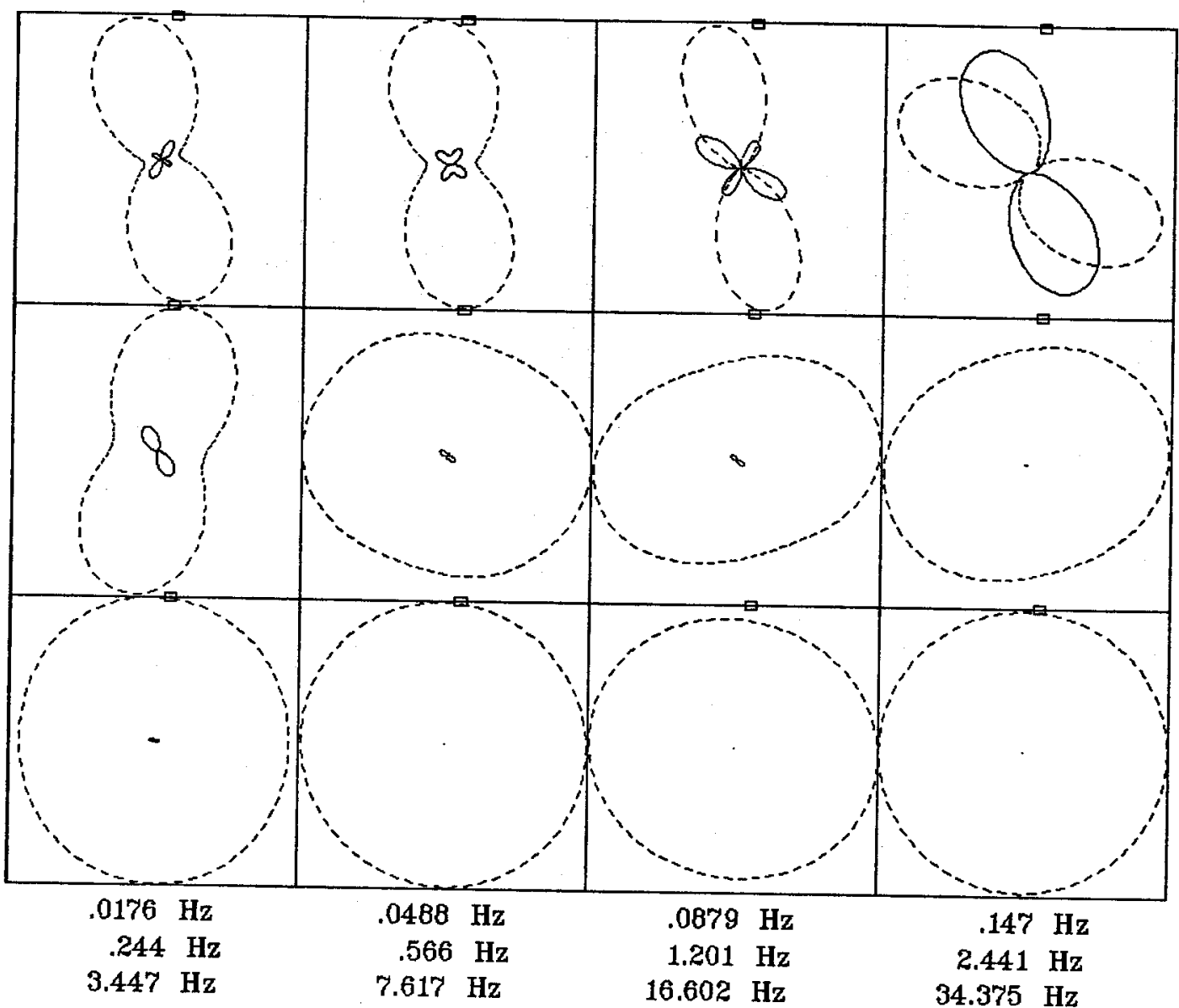

Client:

Rotation:

Remote: none

Acquired: 14:0 Jul 17, 2007

Survey Co:USGS
Filename: sl33m1.avg

Channels: Ch1 Ch2 Ch3 Ch4 Ch5 Ch3 Ch4 Plotted: 11:19 Nov 06, 2007

< EMI - ElectroMagnetic Instruments > 
TIPPER MAGNITUDE

Alamosa, CO 100k

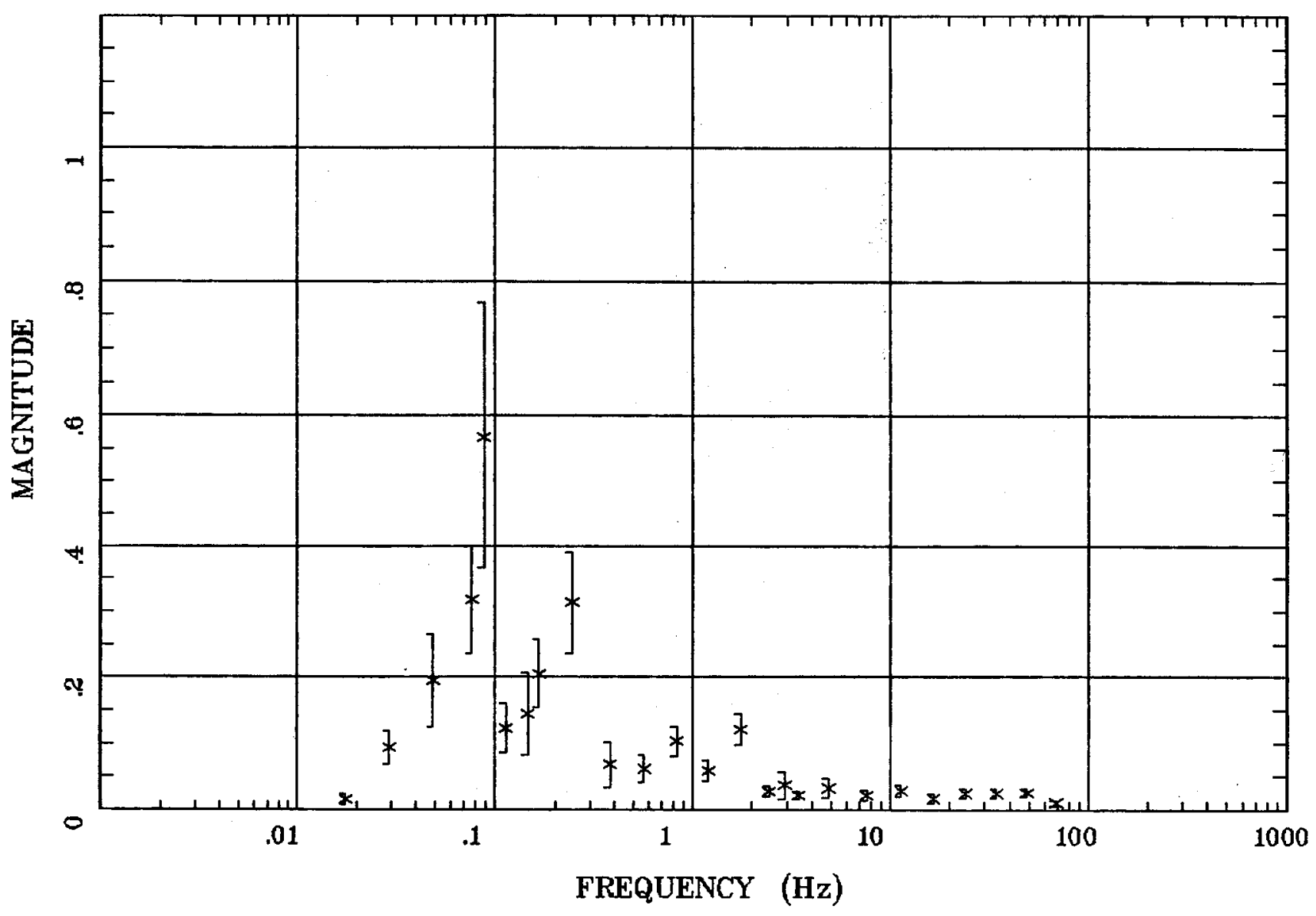

Client:

Remote: none

Acquired: 14:0 Jul 17, 2007 Survey Co:USGS
Rotation:

Filename: sl33m1.avg

Channels: Ch1 Ch2 Ch3 Ch4 Ch5 Ch3 Ch4

Plotted: 11:19 Nov 06, 2007

$<$ EMI - ElectroMagnetic Instruments 
Alamosa, CO 100k

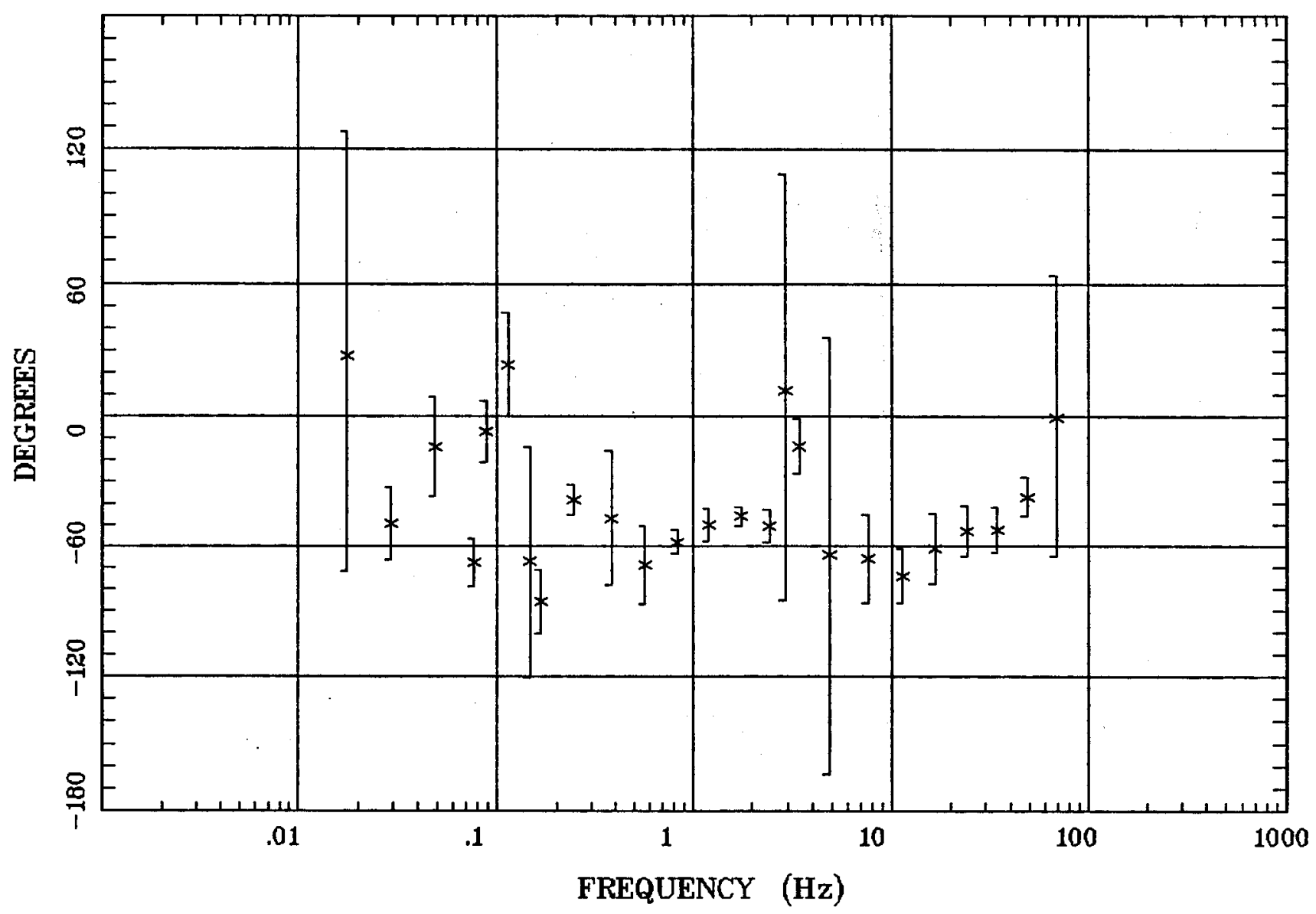

Client:

Remote: none

Acquired: 14:0 Jul 17, 2007 Survey Co:USGS
Rotation:

Filename: sl33m1.avg

Channels: Ch1 Ch2 Ch3 Ch4 Ch5 Ch3 Ch4

Plotted: 11:19 Nov 06, 2007

< EMI - ElectroMagnetic Instruments > 
Station 33

HzHx.x Coh HzHy.o

Alamosa, Co 100k

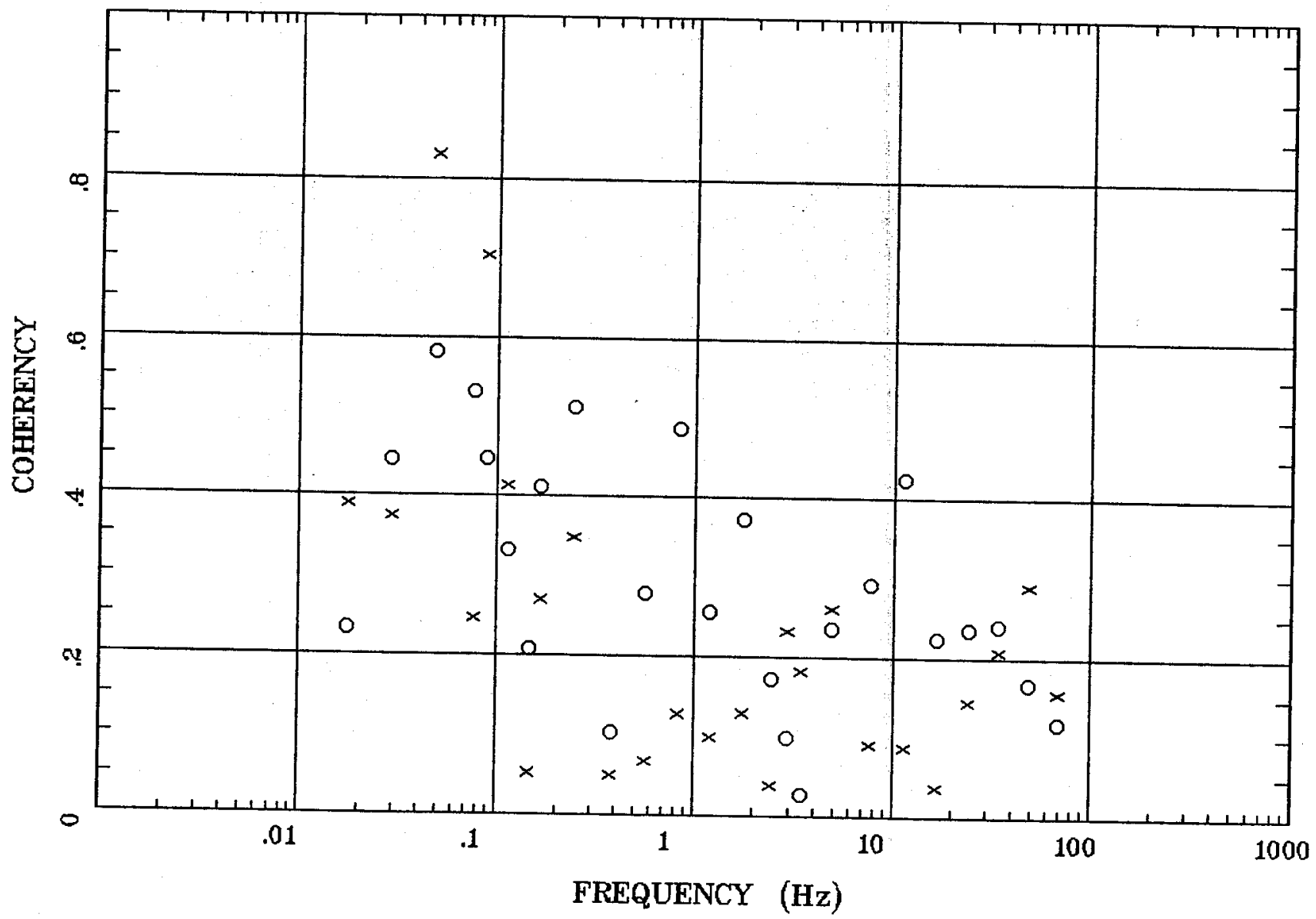

Client:

Remote: none

Acquired: 14:0 Jul 17, 2007

Survey Co:USGS
Rotation:

Filename: sl33m1.avg

Channels: Ch1 Ch2 Ch3 Ch4 Ch5 Ch3 Ch4 Plotted: 11:19 Nov 06, 2007

< EMI - ElectroMagnetic Instruments 
APPARENT RESISTIVITY Alamosa, CO 100k

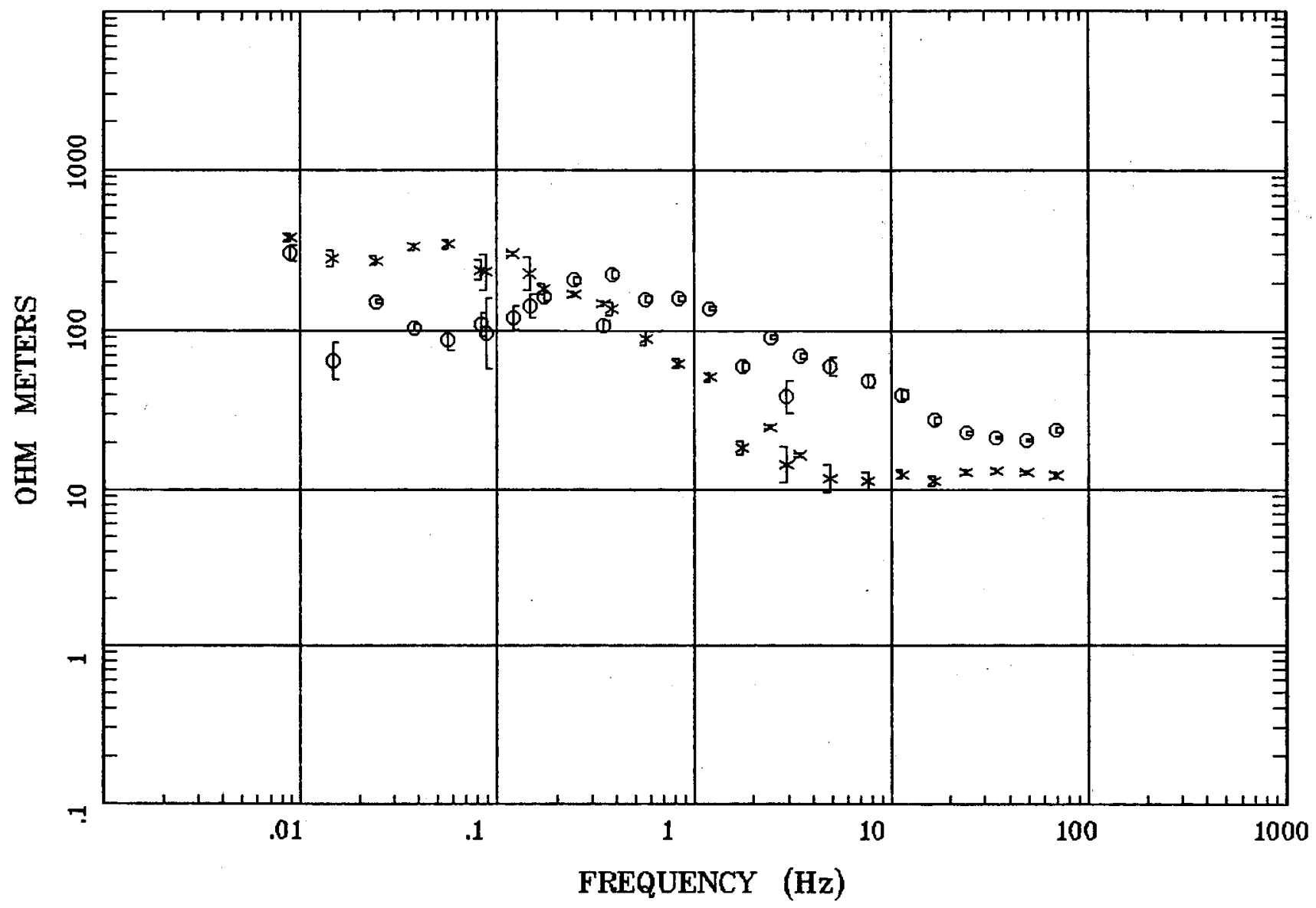

Client:

Remote: none

Acquired: 10:4 Jul 18, 2007

Survey Co:USGS
Ratation:

Filename: sl34m1.avg

Channels: Ch1 Ch2 Ch3 Ch4 Ch5 Ch3 Ch4

Plotted: 11:12 Nov 06, 2007

$<$ EMI - ElectroMagnetic Instruments > 


\section{Station 34}

IMPEDANCE PHASE

Alamosa, CO 100k

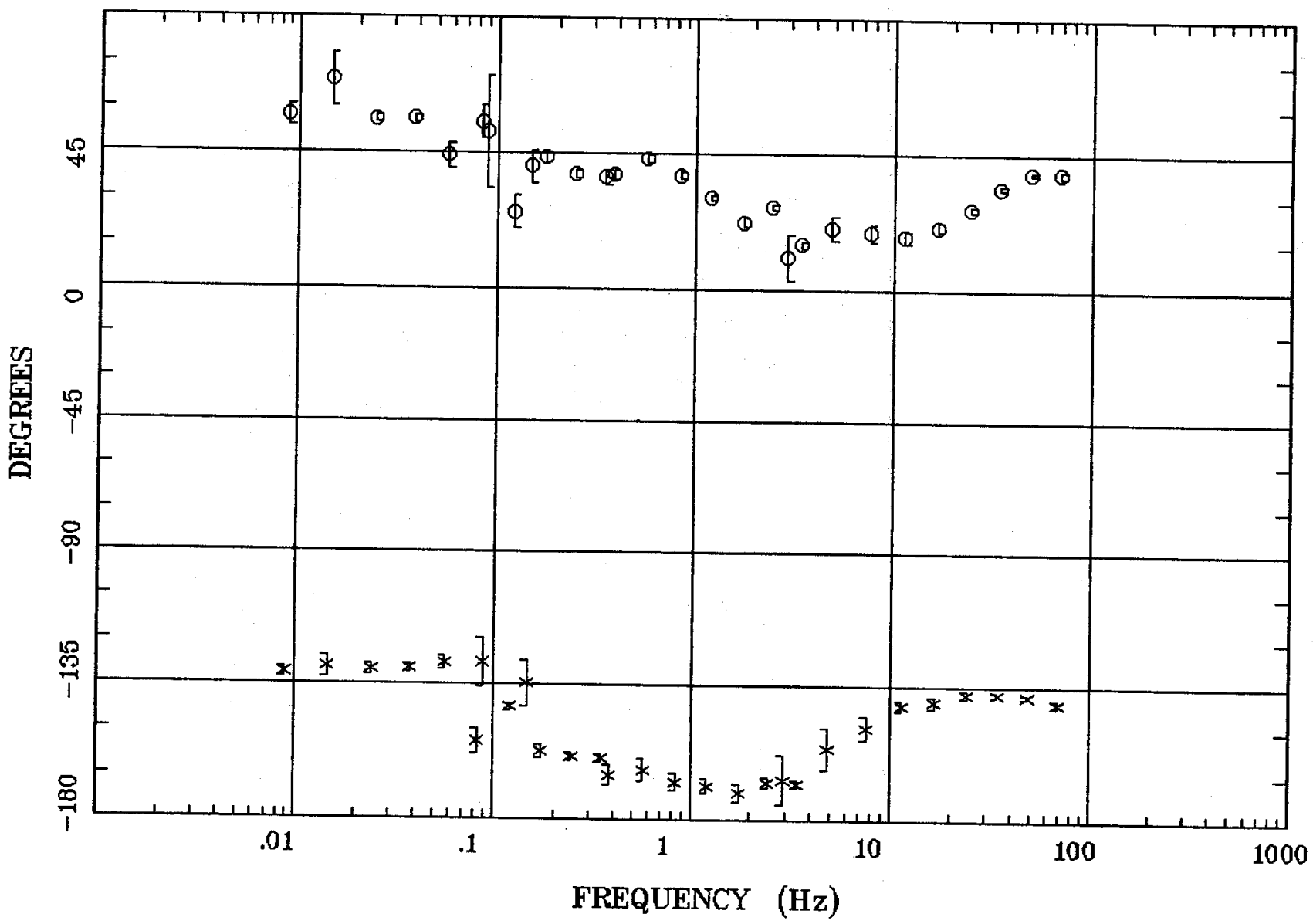

Client:

Remote: none

Acquired: 10:4 Jul 18, 2007 Survey Co:USGS
Rotation:

Filename: sl34m1.avg

Channels: Ch1 Ch2 Ch3 Ch4 Ch5 Ch3 Ch4

Plotted: 11:12 Nov 06, 2007

< EMI - ElectroMagnetic Instruments > 
ROTATION ANGLE Alamosa, CO 100k

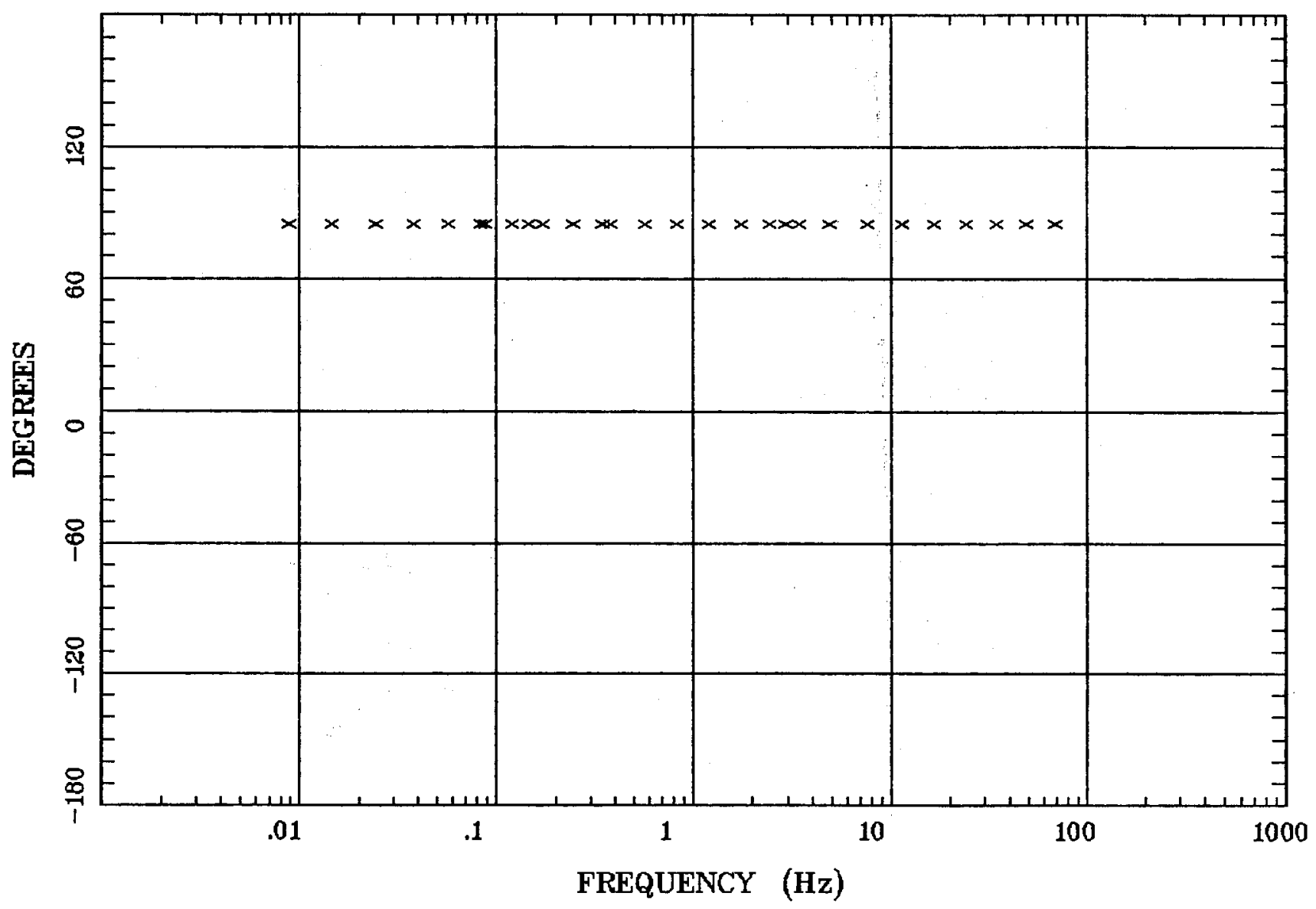

Client:

Remote: none

Acquired: 10:4 Jul 18, 2007 Survey Co:USGS
Rotation:

Filename: sl34m1.avg

Channels: Ch1 Ch2 Ch3 Ch4 Ch5 Ch3 Ch4 Plotted: 11:12 Nov 06, 2007

$<$ EMI - ElectroMagnetic Instruments 
Alamosa, Co 100k

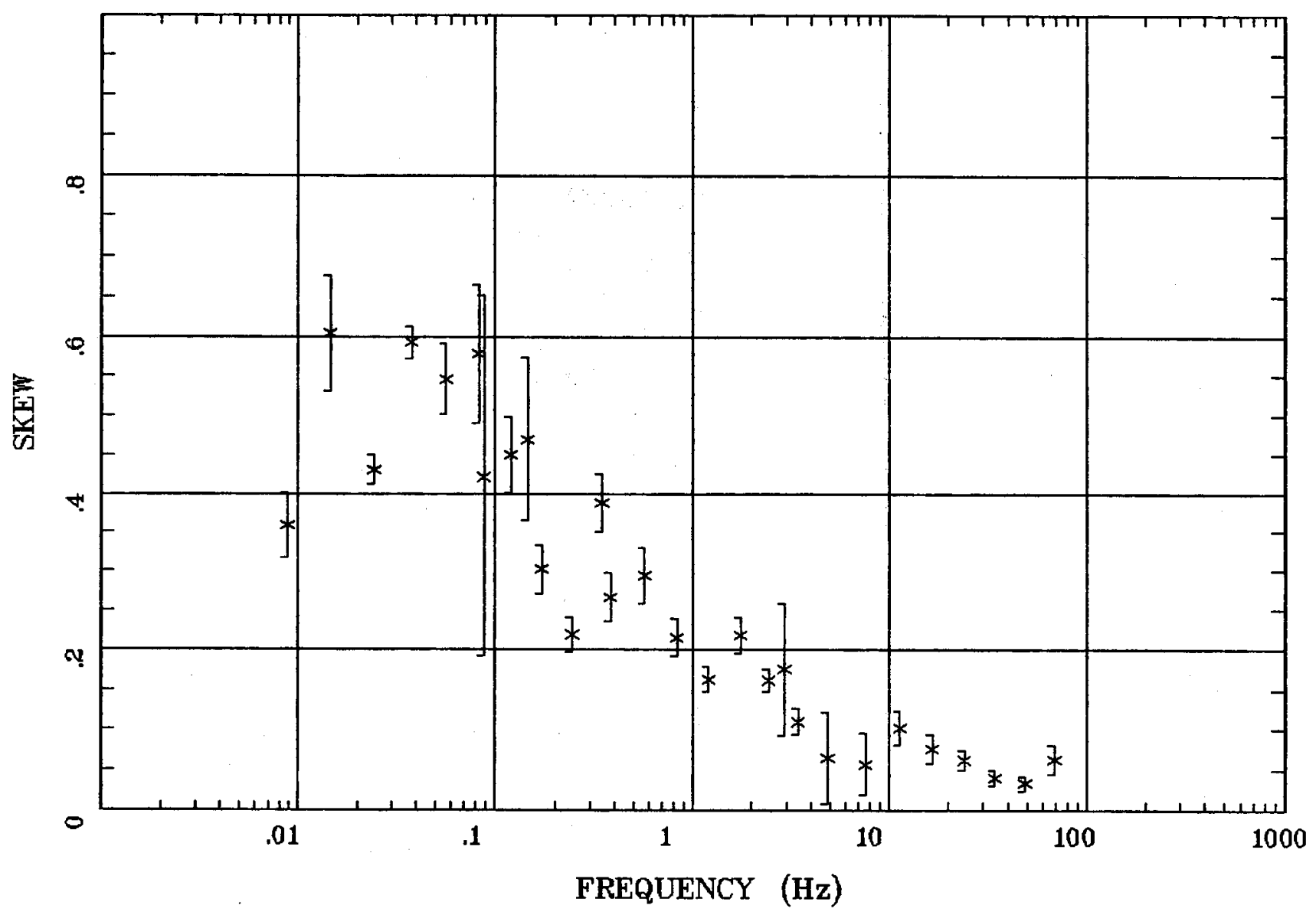

Client:

Remote: none

Acquired: 10:4 Jul 18, 2007

Survey Co:USGS
Rotation:

Filename: sl34m1.avg

Channels: Ch1 Ch2 Ch3 Ch4 Ch5 Ch3 Ch4 Plotted: 11:12 Nov 06, 2007

$<$ EMI - ElectroMagnetic Instruments 
Station 34

E MULT Coh.

Alamosa, CO 100k

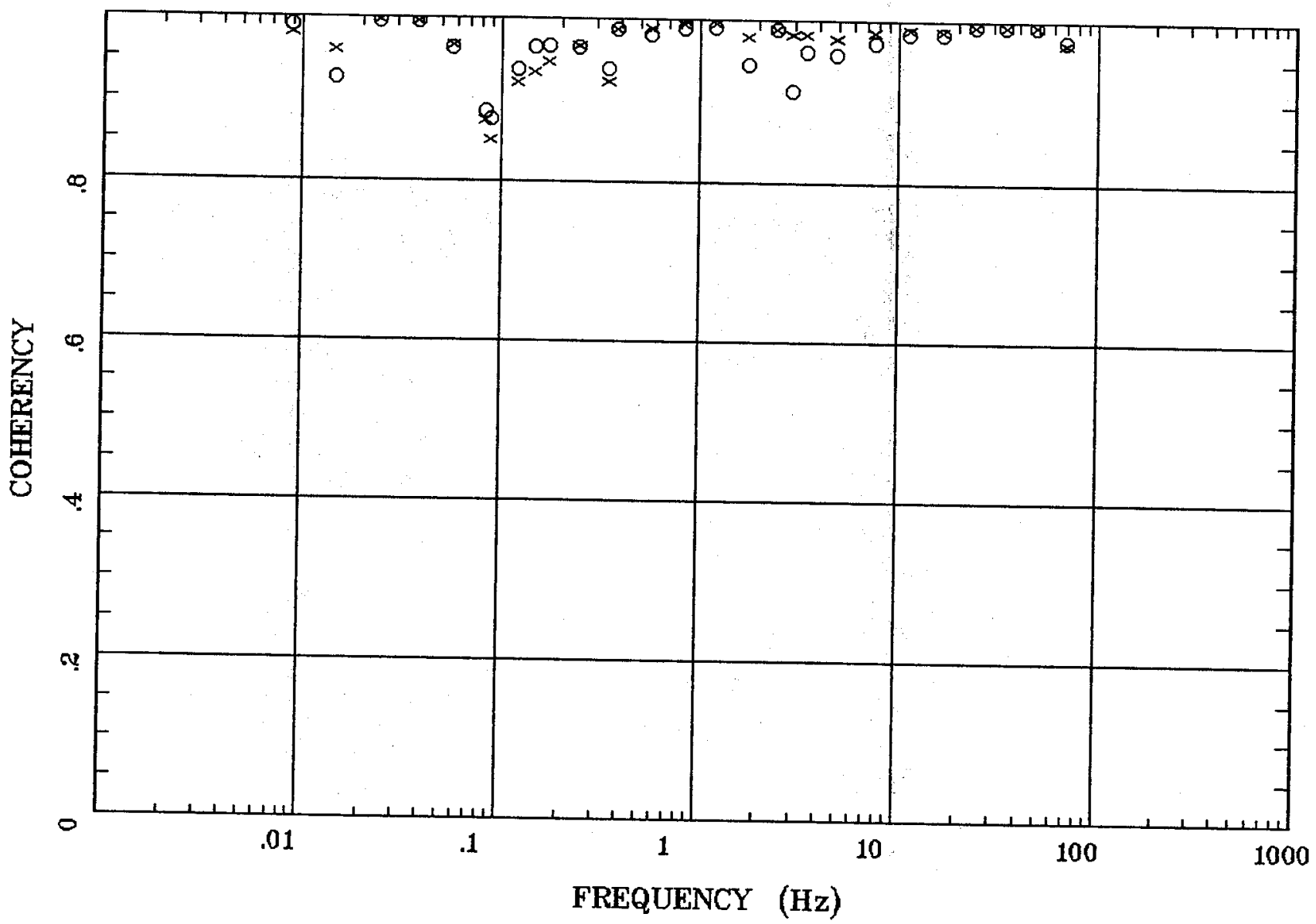

Client:

Remote: none

Acquired: 10:4 Jul 18, 2007

Survey Co:USGS
Rotation:

Filename: sl34m1.avg

Channels: Ch1 Ch2 Ch3 Ch4 Ch5 Ch3 Ch4 Plotted: 11:12 Nov 06, 2007

< EMI - ElectroMagnetic Instruments > 
Alamosa, Co 100k

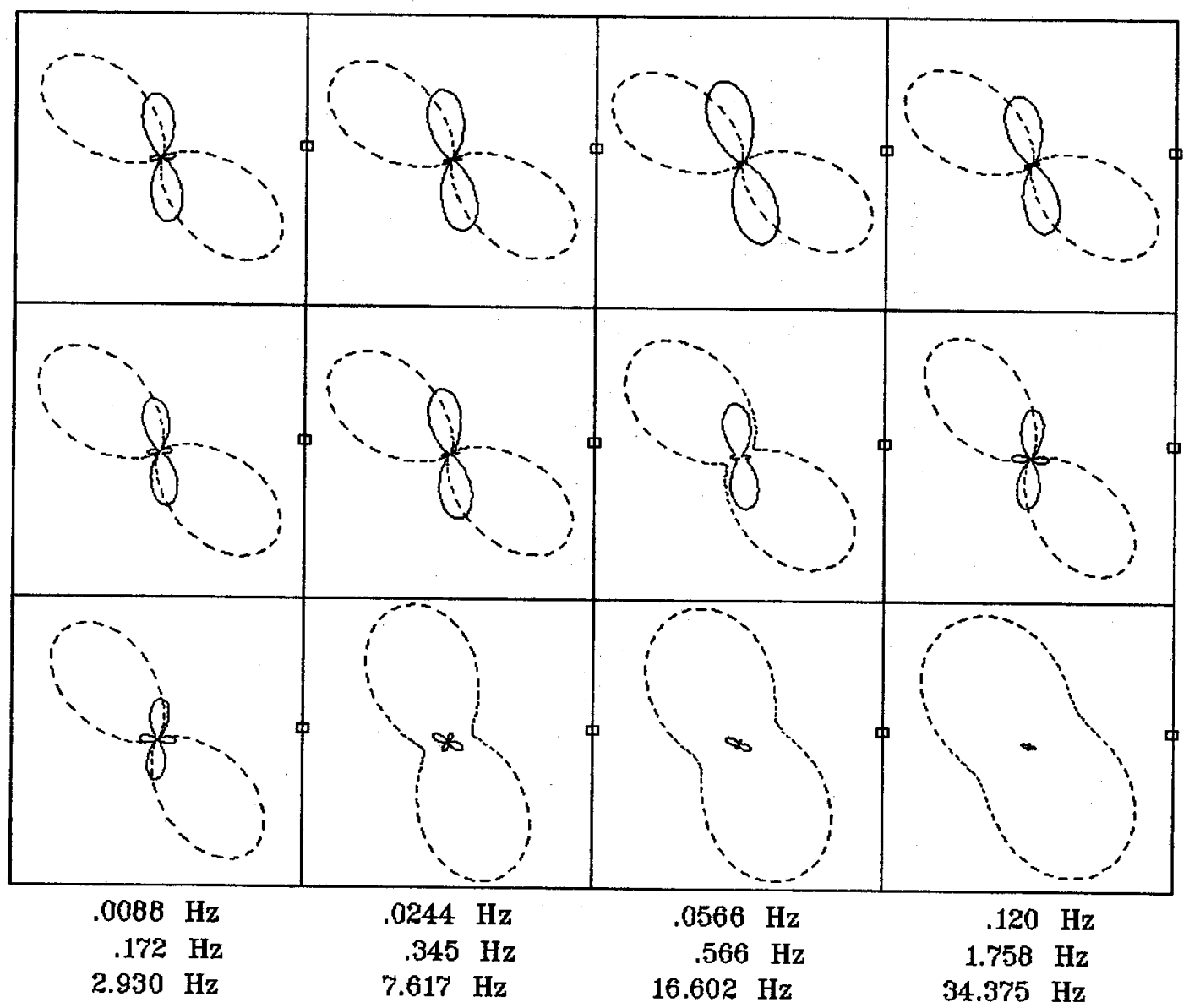

Client:

Remote: none

Acquired: 10:4 Jul 18, 2007 Survey Co:USGS
Rotation:

Filename: sl34m1.avg

Channels: Ch1 Ch2 Ch3 Ch4 Ch5 Ch3 Ch4 Plotted: 11:12 Nov 06, 2007

< EMI - ElectroMagnetic Instruments > 
Alamosa, Co 100k

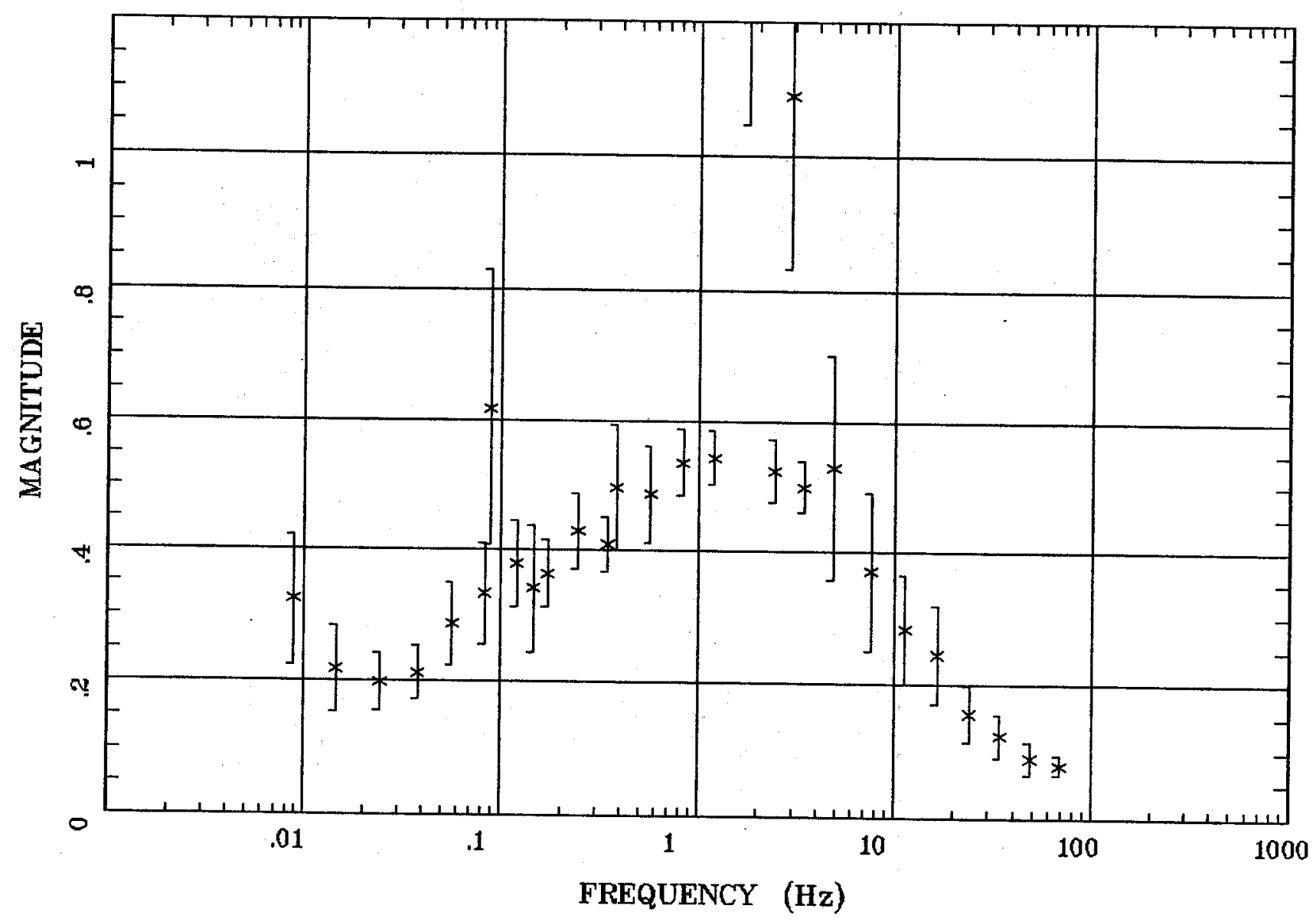

Client:

Remote: none

Acquired: 10:4 Jul 18, 2007

Survey Co:USGS
Rotation:

Filename: sl34m1.avg

Channels: Ch1 Ch2 Ch3 Ch4 Ch5 Ch3 Ch4 Plotted: 11:12 Nov 06, 2007

< EMI - ElectroMagnetic Instruments > 


\section{Station 34}

Alamosa, CO $100 \mathrm{k}$

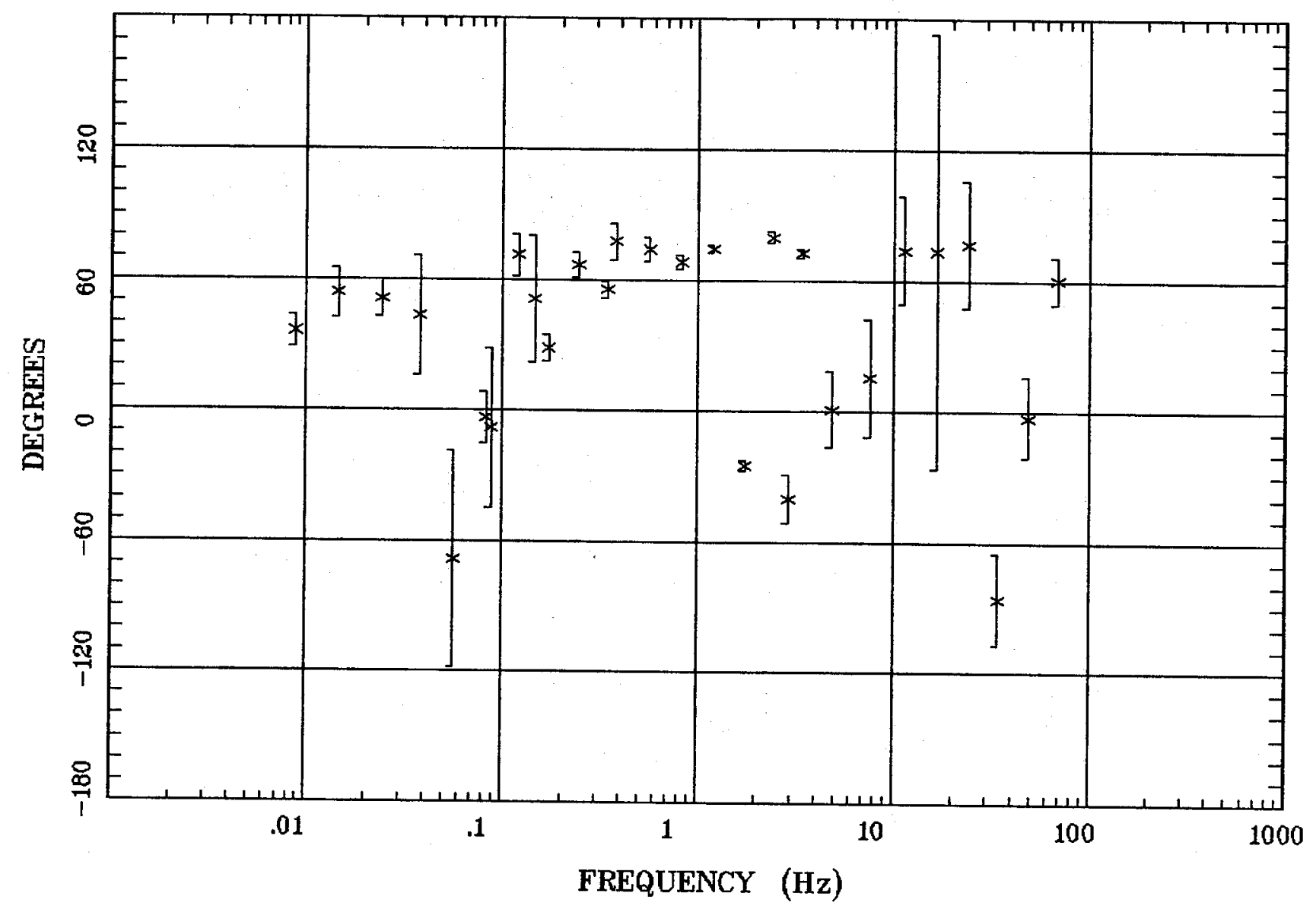

Client:

Remote: none

Acquired: 10:4 Jul 18, 2007

Survey Co:USGS

\section{Rotation:}

Filename: sl34m1.avg

Channels: Ch1 Ch2 Ch3 Ch4 Ch5 Ch3 Ch4 Plotted: 11:12 Nov 06, 2007

< EMI - ElectroMagnetic Instruments > 


\section{Station 34}

HzHx.x Coh HzHy.o

Alamosa, CO 100k

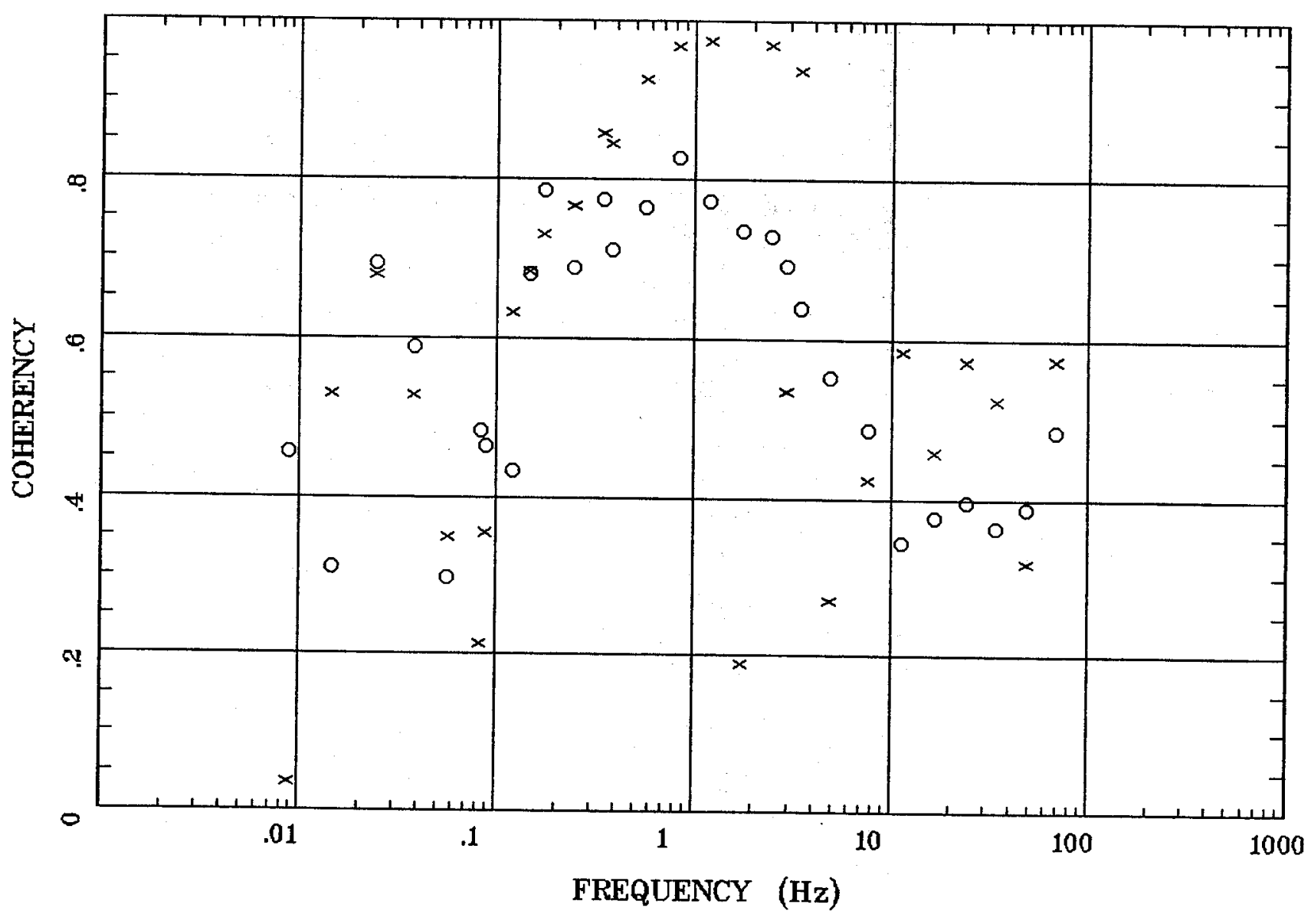

Client:

Remote: none

Acquired: 10:4 Jul 18, 2007

Survey Co:USGS
Rotation:

Filename: sl34m1.avg

Channels: Ch1 Ch2 Ch3 Ch4 Ch5 Ch3 Ch4

Plotted: 11:12 Nov 06, 2007

< EMI - ElectroMagnetic Instruments > 


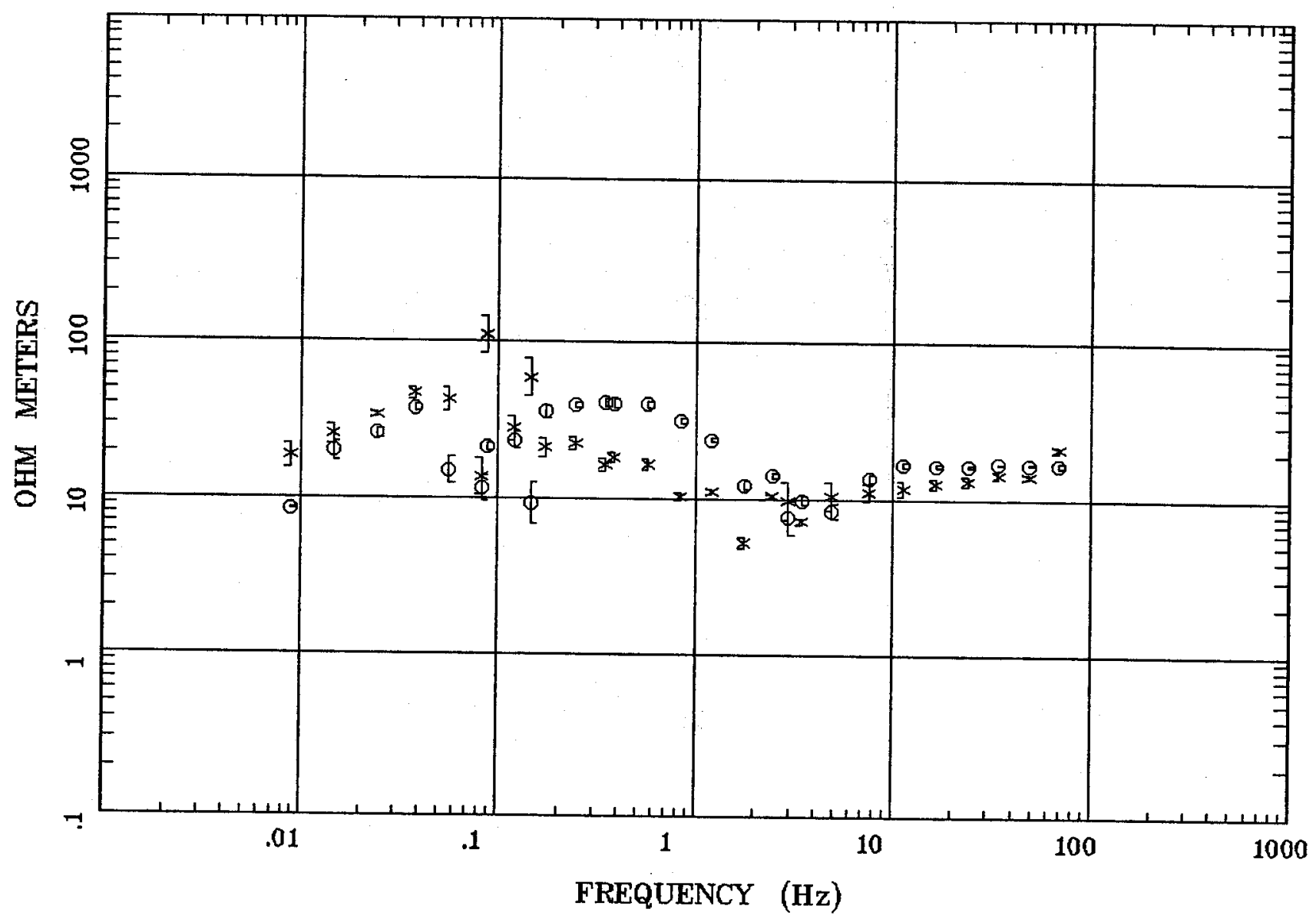

Client:

Remote: none Acquired: 09:1 Jul 19, 2007 Survey Co:USGS
Rotation:

Filename: sl35m.avg

Channels: Ch1 Ch2 Ch3 Ch4 Ch5 Ch3 Ch4

Plotted: 11:14 Nop 06, 2007

< EMI - ElectroMagnetic Instruments > 
Alamosa, CO 100k

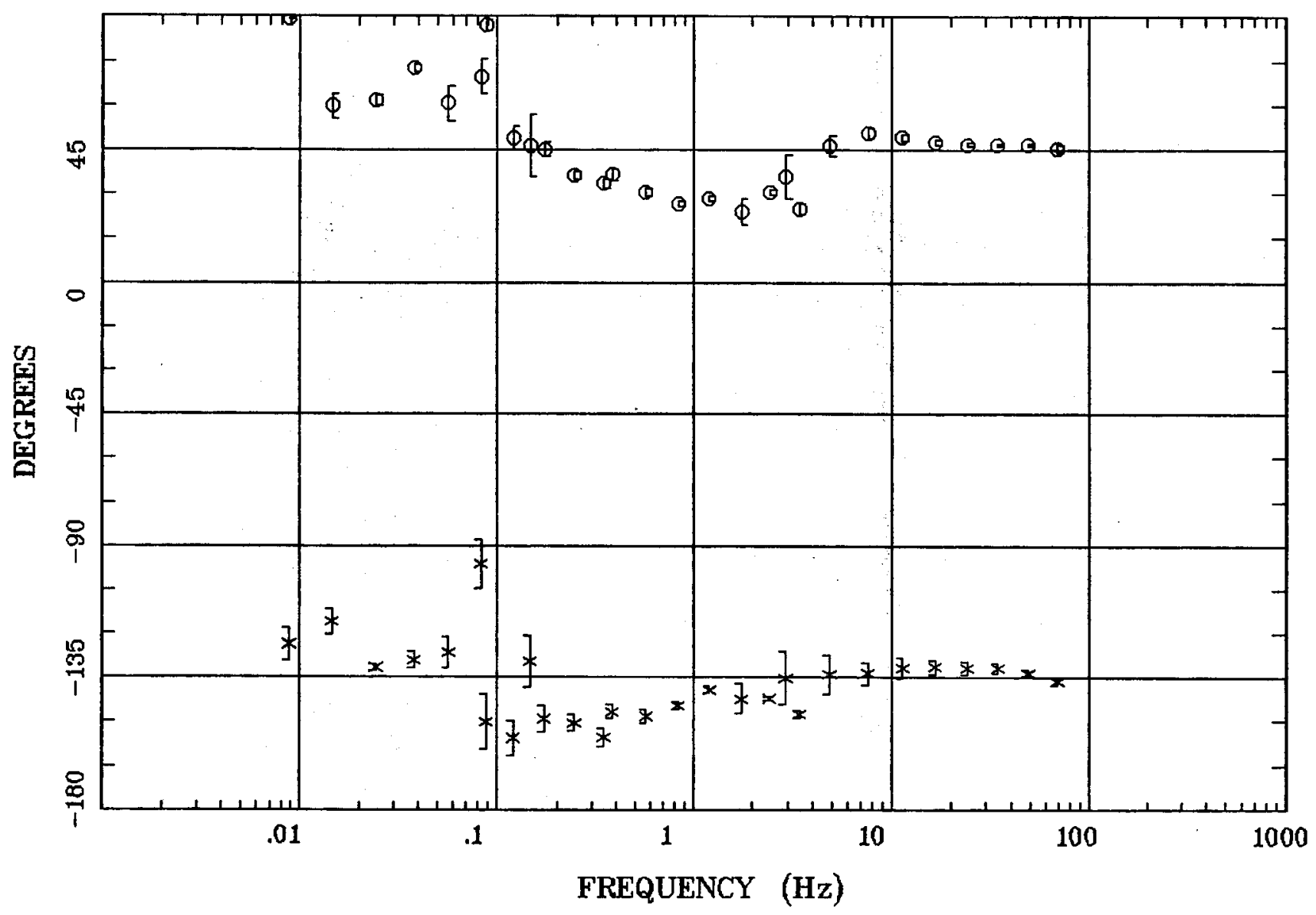

Client:

Remote: none

Acquired: 09:1 Jul 19, 2007

Survey Co:USGS
Rotation:

Filename: sl35m.avg

Channels: Ch1 Ch2 Ch3 Ch4 Ch5 Ch3 Ch4

Plotted: 11:14 Nor 06, 2007

< EMI - ElectroMagnetic Instruments > 
Alamosa, CO 100k

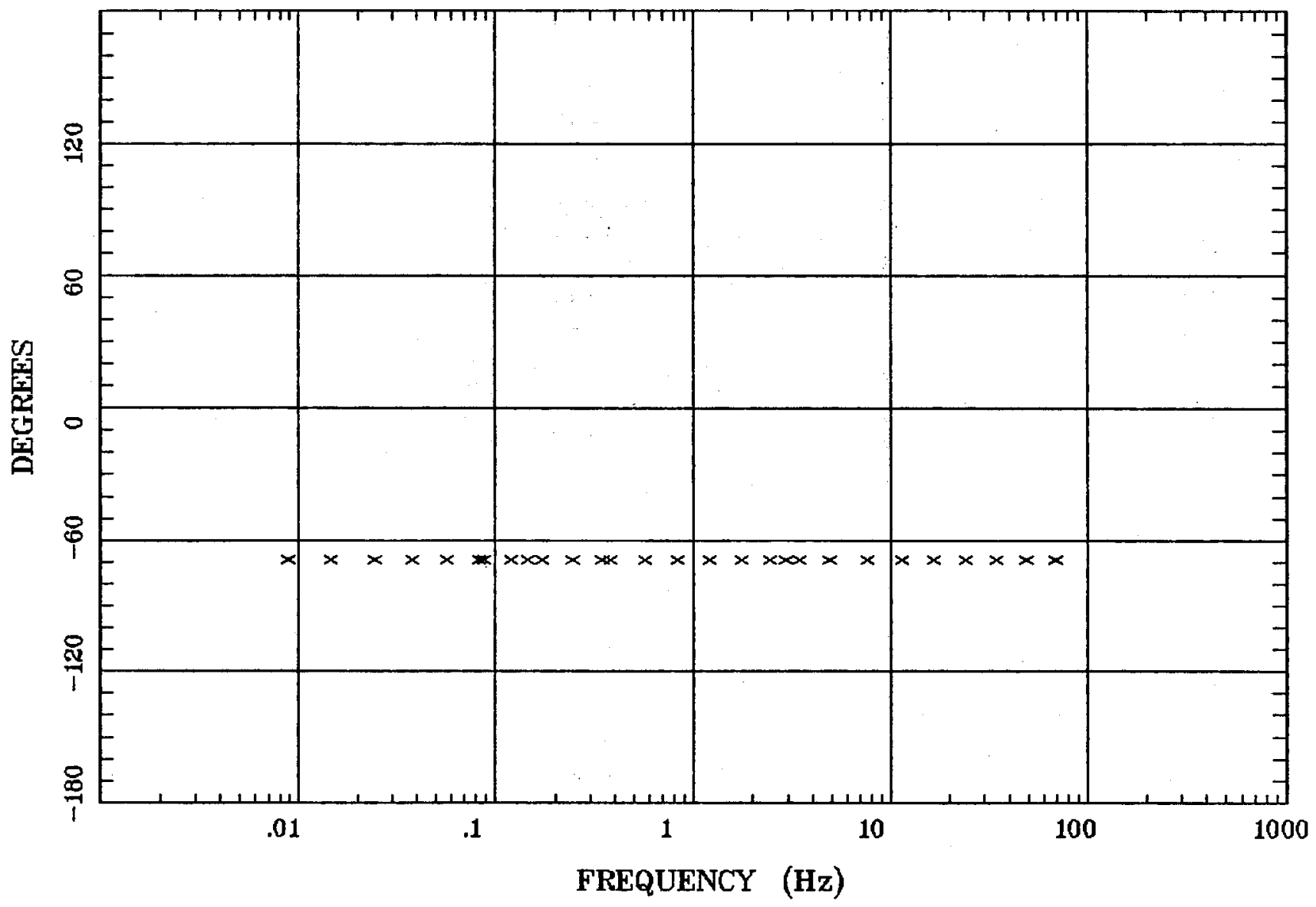

Client:

Remote: none

Acquired: 09:1 Jul 19, 2007

Survey Co:USGS
Rotation:

Filename: sl35m.avg

Channels: Ch1 Ch2 Ch3 Ch4 Ch5 Ch3 Ch4

Plotted: 11:14 Nop 06, 2007

< EMI - ElectroMagnetic Instruments 


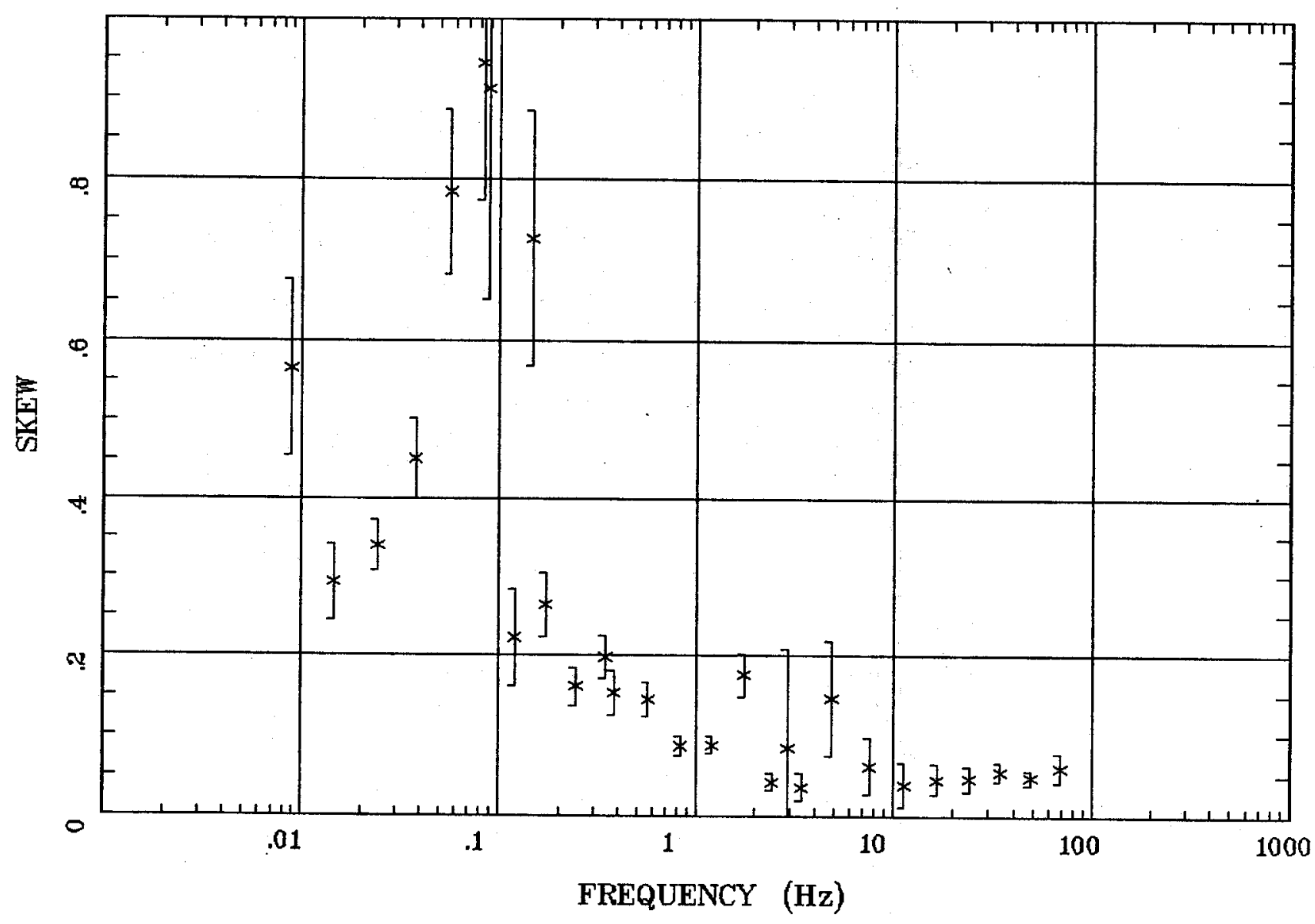

Client:

Remote: none

Acquired: 09:1 Jul 19, 2007

Survey Co:USGS
Rotation:

Filename: sl35m.avg

Channels: Ch1 Ch2 Ch3 Ch4 Ch5 Ch3 Ch4 Plotted: 11:14 Nov 06, 2007

< EMI - ElectroMagnetic Instruments > 
E MULT Coh.

Alamosa, CO 100k

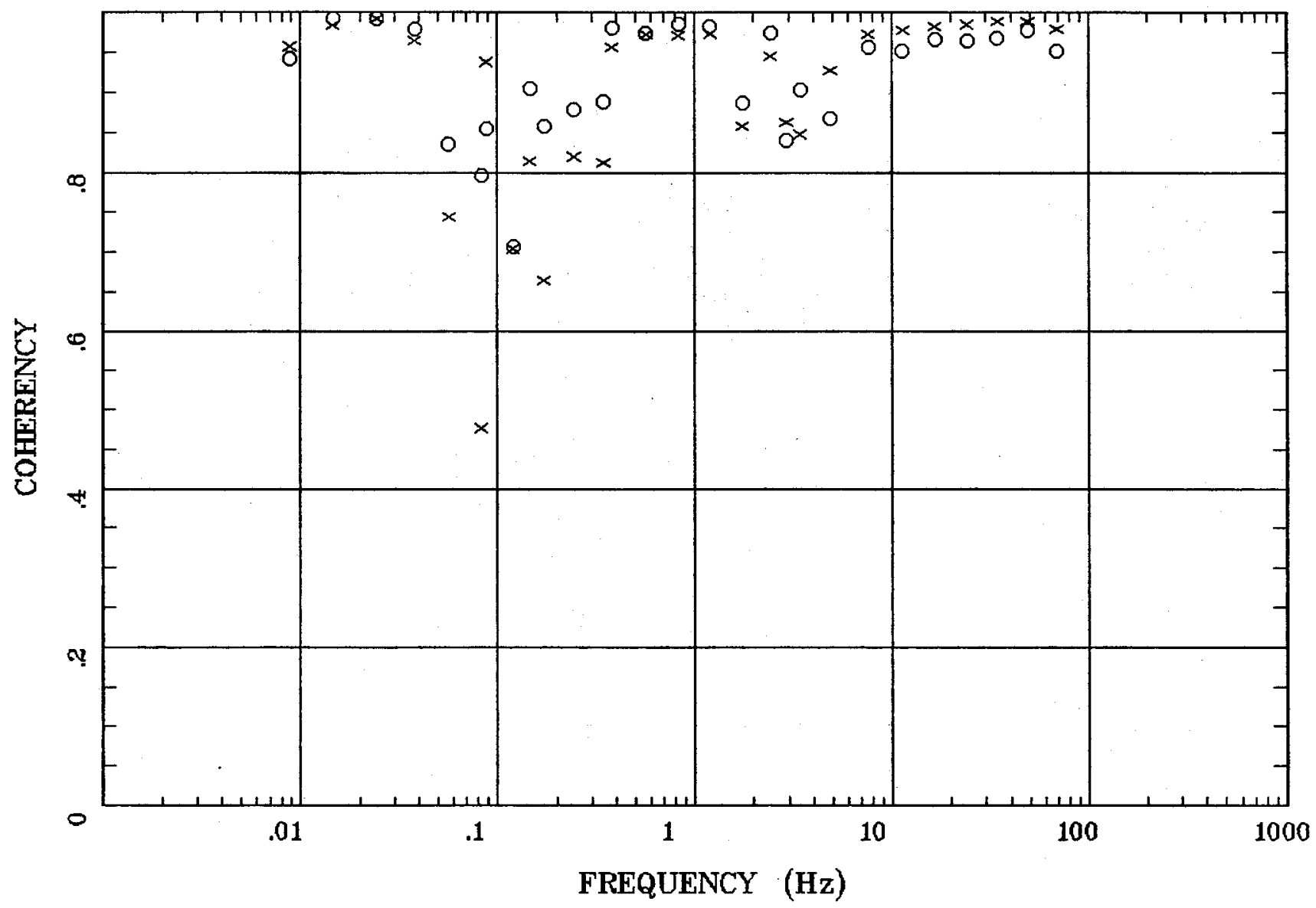

Client:

Remote: none

Acquired: 09:1 Jul 19, 2007

Survey Co:USGS
Rotation:

Filename: sl35m.avg

Channels: Ch1 Ch2 Ch3 Ch4 Ch5 Ch3 Ch4

Plotted: 11:14 Nov 06, 2007

< EMI - ElectroMagnetic Instruments > 


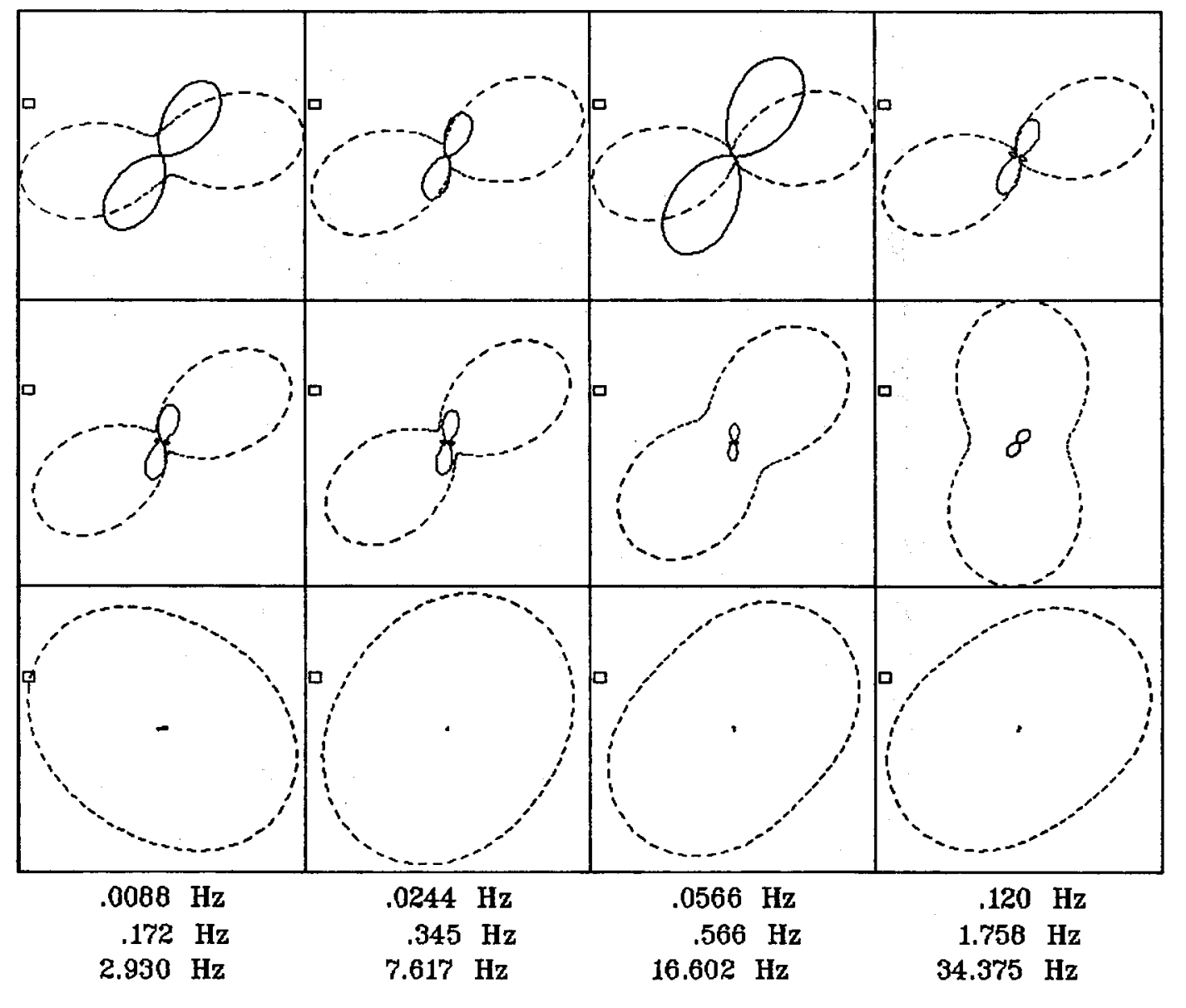

Rotation:

Client:

Remote: none

Acquired: 09:1 Jul 19, 2007 Survey Co:USGS
Filename: sl35m.avg

Channels: Ch1 Ch2 Ch3 Ch4 Ch5 Ch3 Ch4

Plotted: 11:14 Nov 06, 2007

$<$ EMI - ElectroMagnetic Instruments > 
Alamosa, CO 100k

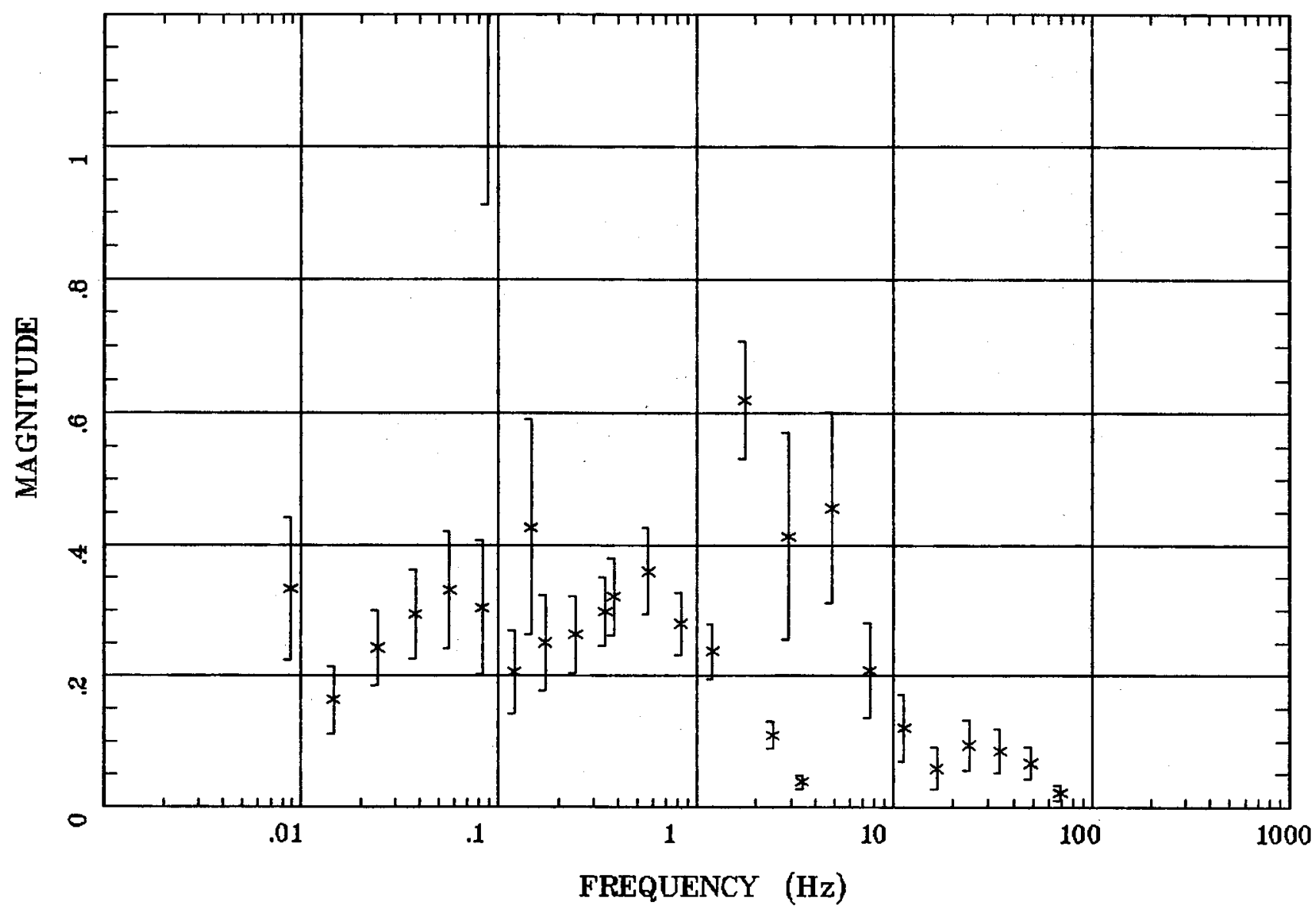

Client:

Remote: none

Acquired: 09:1 Jul 19, 2007

Survey Co:USGS
Rotation:

Filename: sl35m.avg

Channels: Ch1 Ch2 Ch3 Ch4 Ch5 Ch3 Ch4

Plotted: 11:14 Nov 06, 2007

< EMI - ElectroMagnetic Instruments 
Alamosa, CO 100k

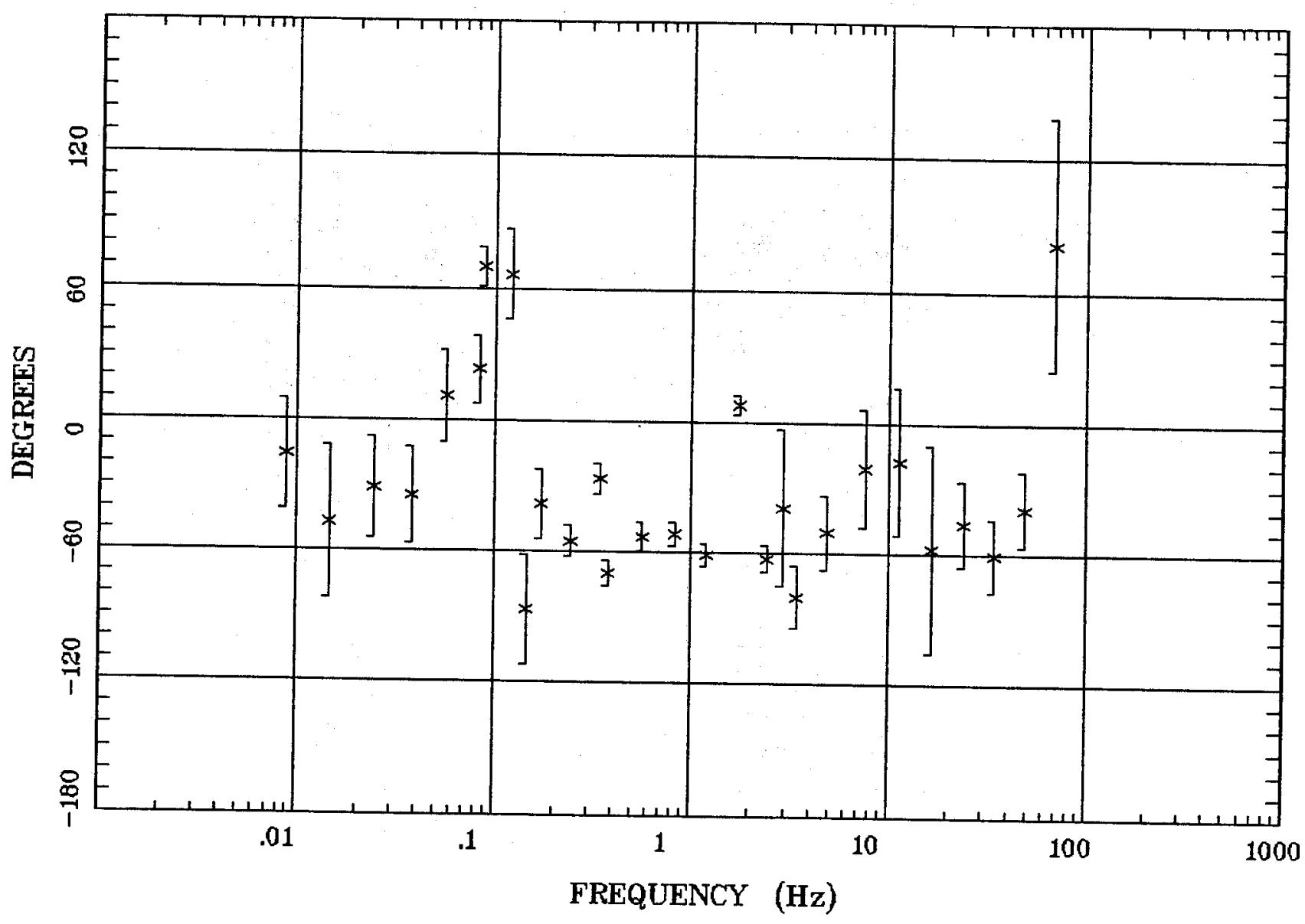

Client:

Remote: none

Acquired: 09:1 Jul 19, 2007

Survey Co:USGS
Rotation:

Filename: sl35m.avg

Channels: Ch1 Ch2 Ch3 Ch4 Ch5 Ch3 Ch4 Plotted: 11:14 Nov 06, 2007

< EMI - ElectroMagnetic Instruments > 


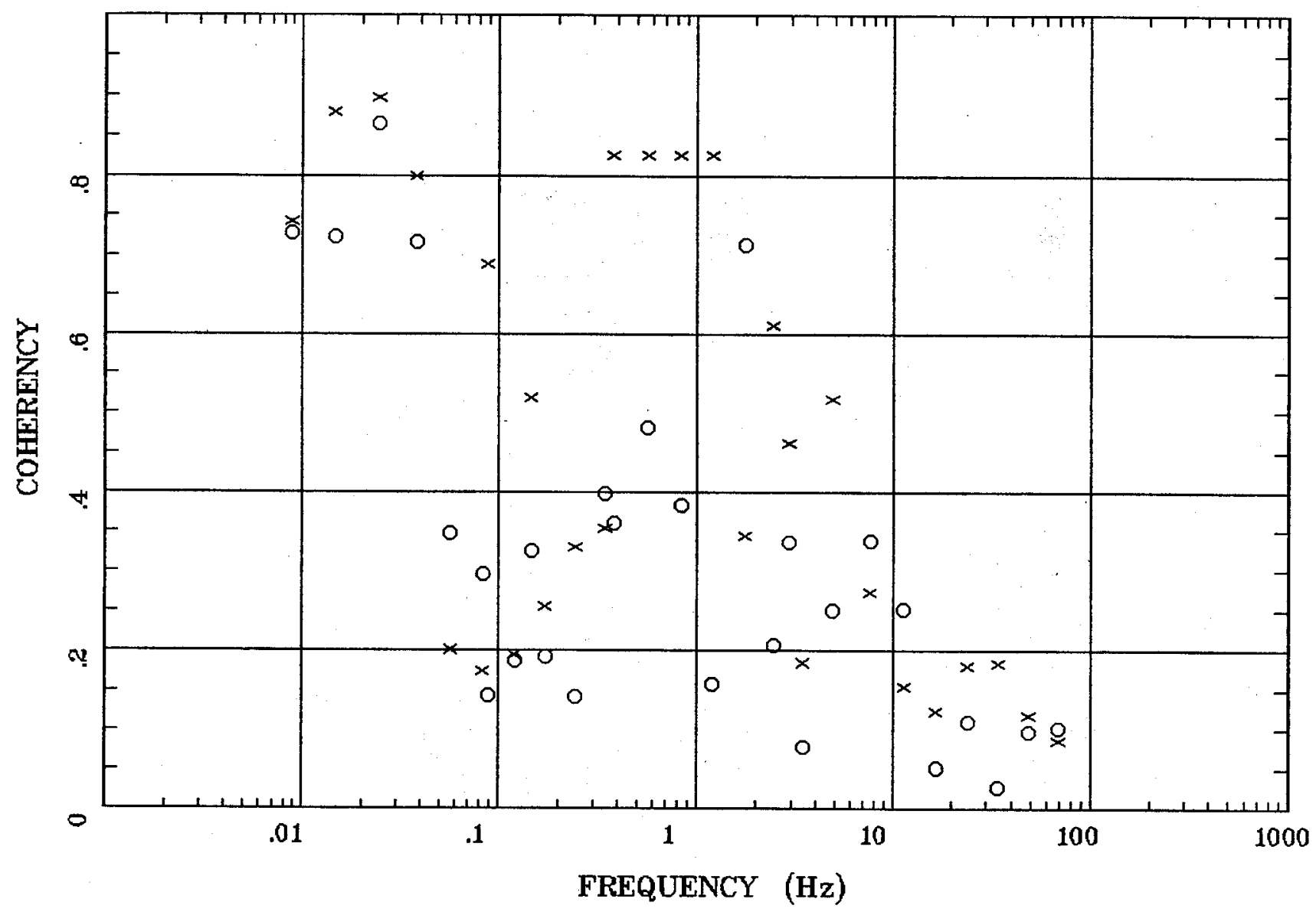

Client:

Remote: none

Acquired: 09:1 Jul 19, 2007 Survey Co:USGS
Rotation:

Filename: sl35m.avg

Channels: Ch1 Ch2 Ch3 Ch4 Ch5 Ch3 Ch4

Plotted: 11:14 Nov 06, 2007

$<$ EMI - ElectroMagnetic Instruments 


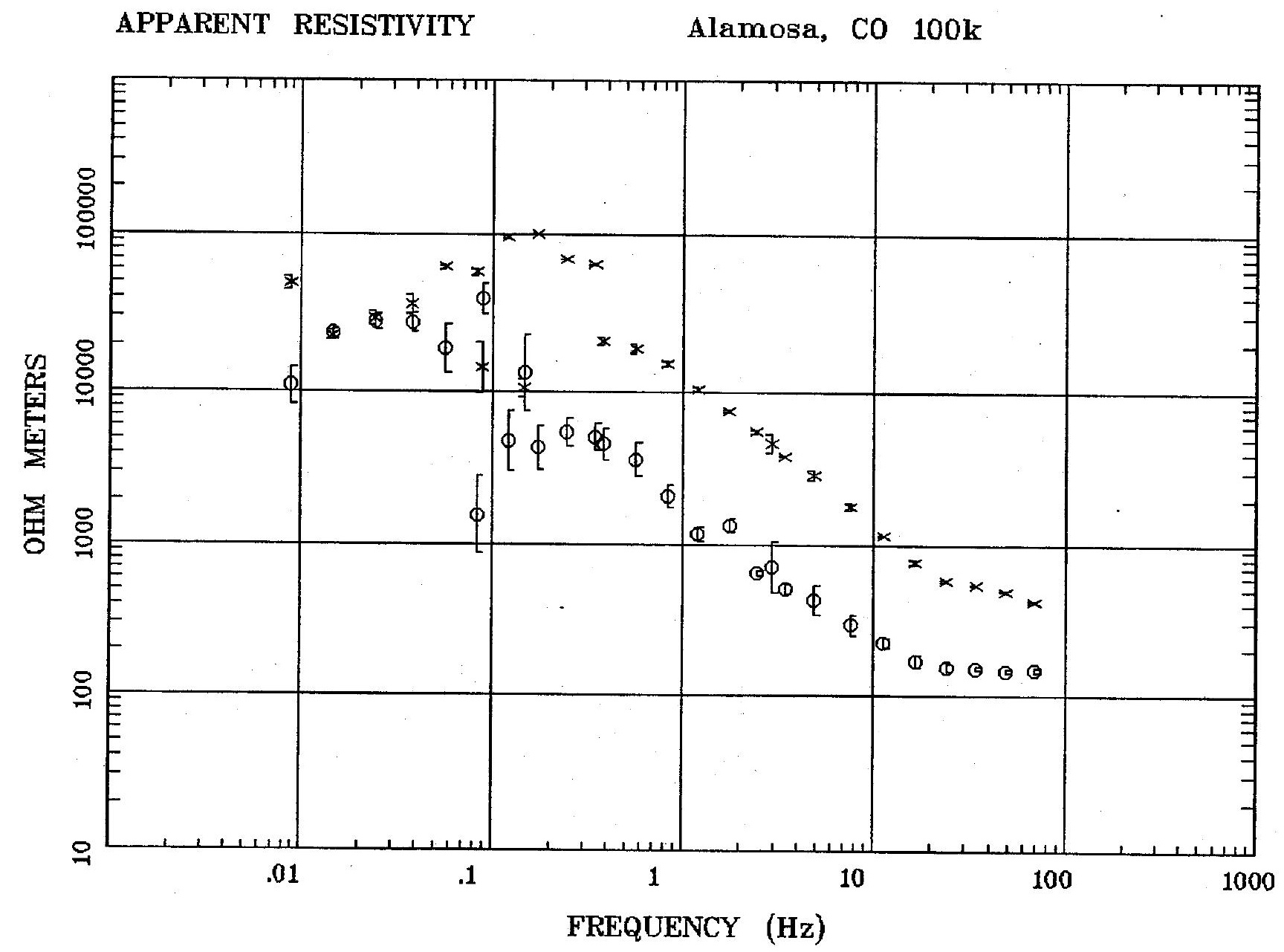

Client:

Remote: none

Acquired: 12:0 Jul 19, 2007

Survey Co:USGS
Rotation:

Filename: sl36m1.avg

Channels: Ch1 Ch2 Ch3 Ch4 Ch5 Ch3 Ch4 Plotted: 11:17 Nov 06, 2007

< EMI - ElectroMagnetic Instruments > 
Alamosa, CO 100k

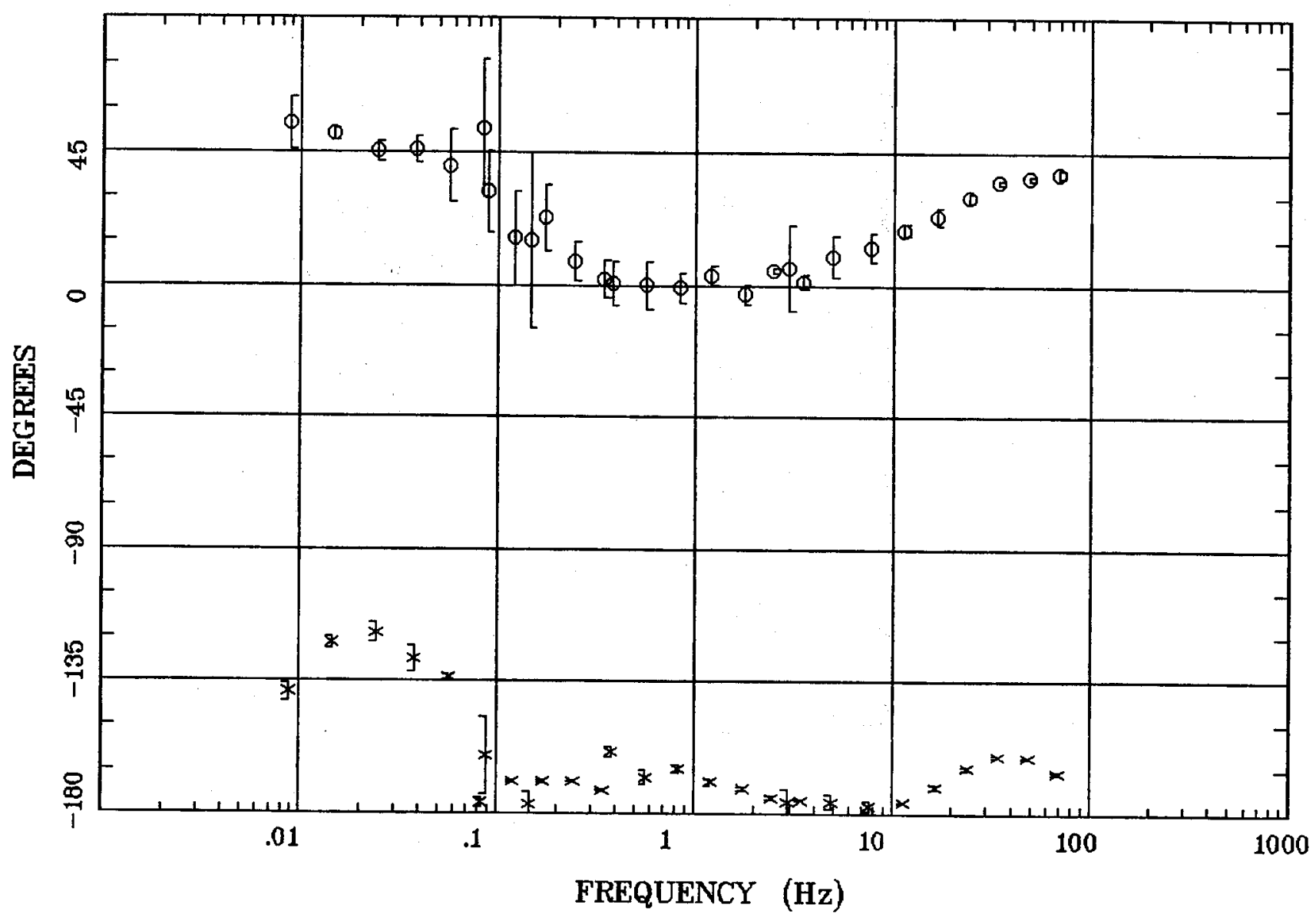

Client:

Remote: none

Acquired: 12:0 Jul 19, 2007

Survey Co:USGS
Rotation:

Filen ame: sl36m1.avg

Channels: Ch1 Ch2 Ch3 Ch4 Ch5 Ch3 Ch4 Plotted: 11:17 Nov 06, 2007

< EMI - ElectroMagnetic Instruments > 
Station 36

ROTATION ANGLE

Alamosa, CO 100k

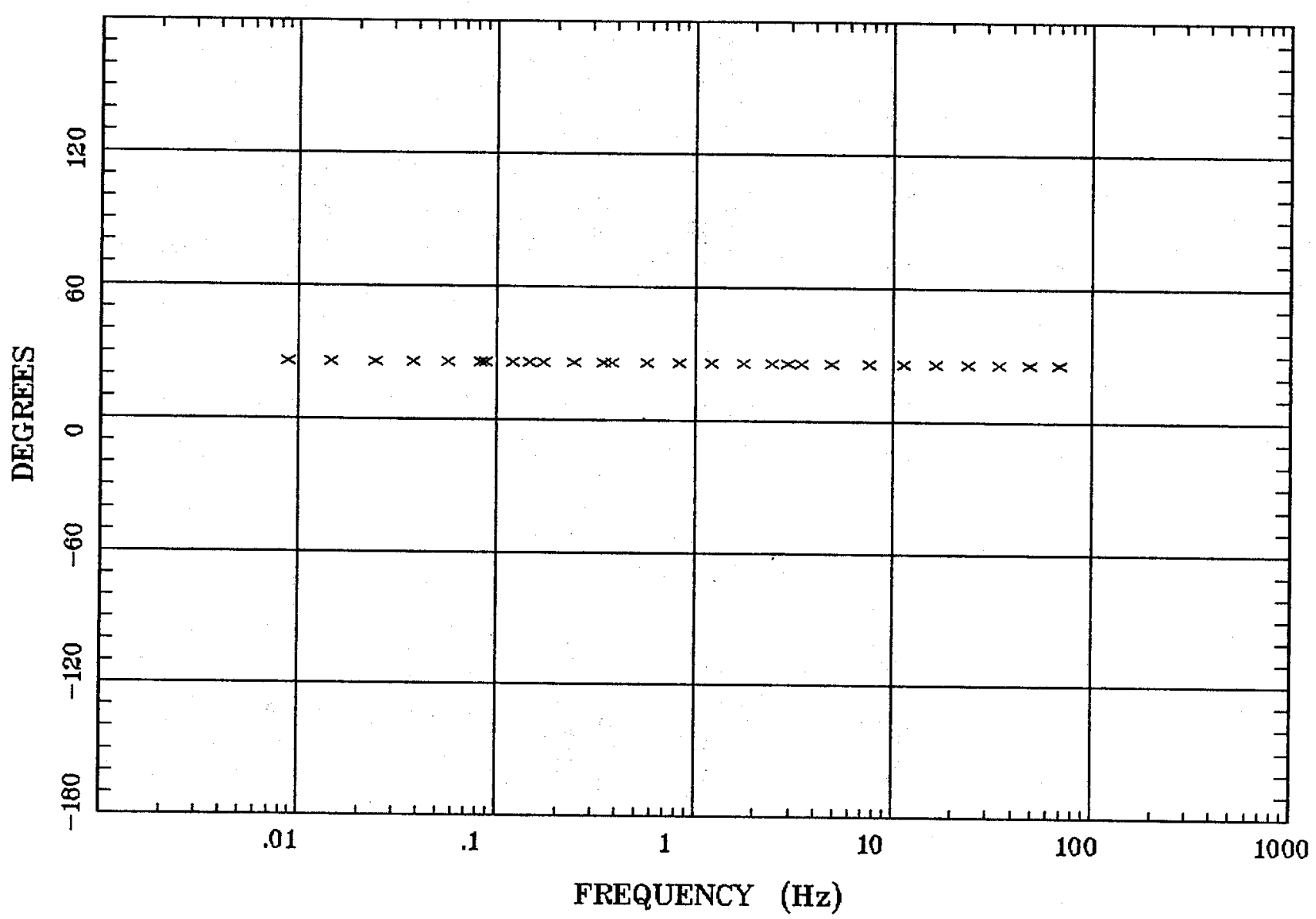

Client:

Remote: none

Acquired: 12:0 Jul 19, 2007

Survey Co:USGS
Rotation:

Filename: sl36m1.avg

Channels: Ch1 Ch2 Ch3 Ch4 Ch5 Ch3 Ch4

Plotted: 11:18 Nov 06, 2007

< EMI - ElectroMagnetic Instruments 
Alamosa, CO 100k

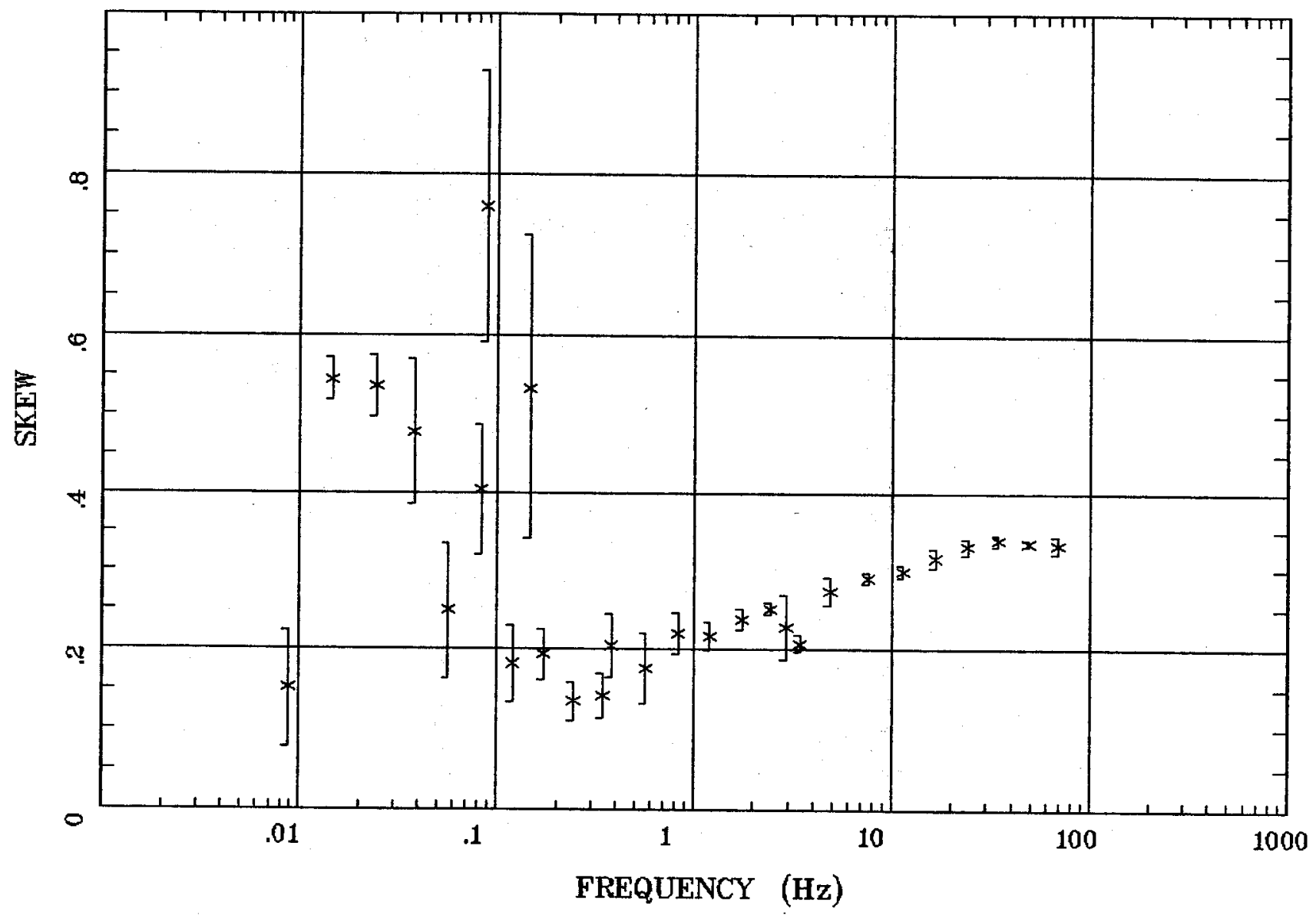

Client:

Remote: none

Acquired: 12:0 Jul 19, 2007

Survey Co:USGS
Rotation:

Filename: sl36m1.avg

Channels: Ch1 Ch2 Ch3 Ch4 Ch5 Ch3 Ch4

Plotted: 11:18 Nov 06, 2007

$<$ EMI - ElectroMagnetic Instruments 


\section{E MULT Coh.}

Alamosa, CO 100k

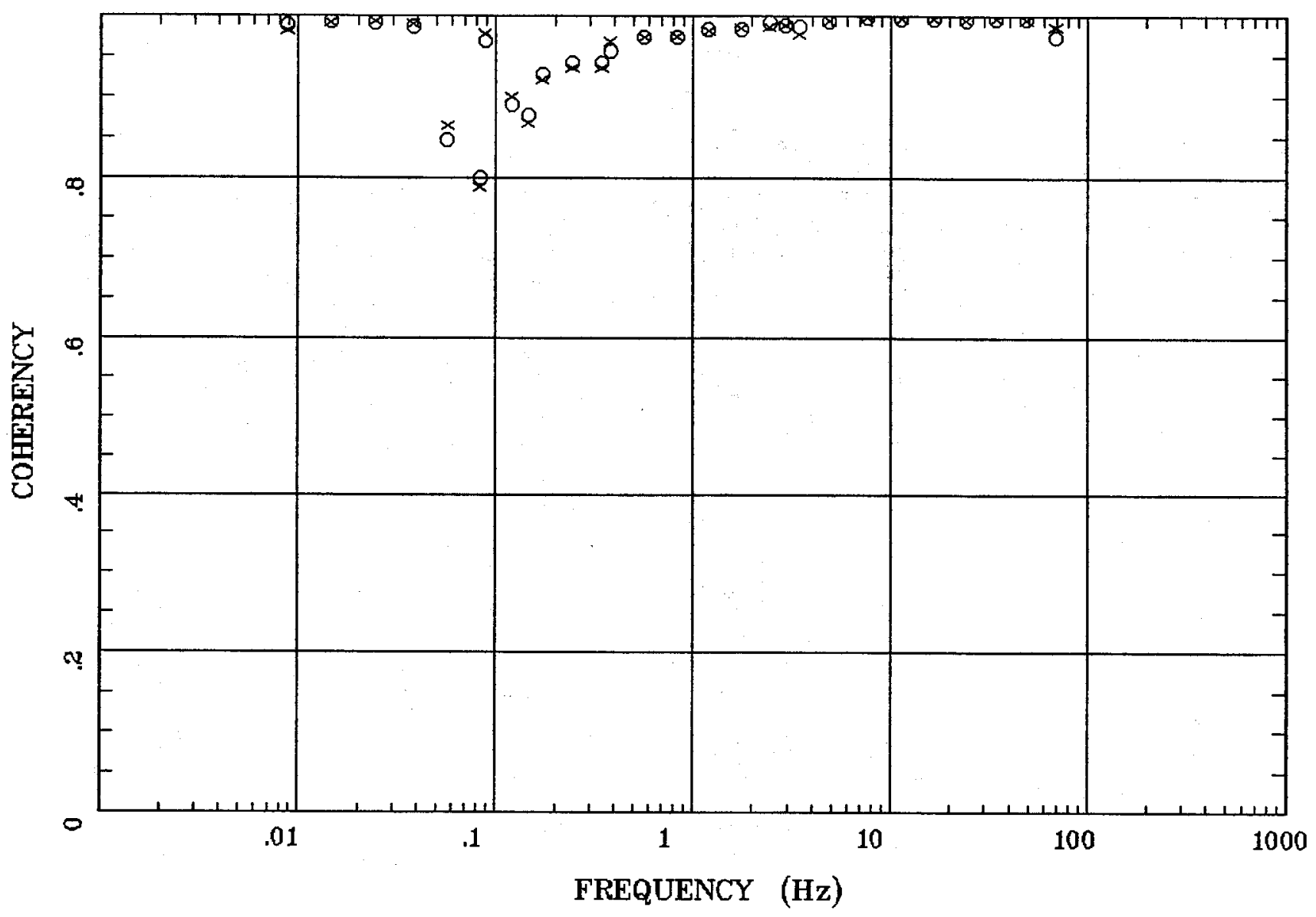

Client:

Remote: none Acquired: 12:0 Jul 19, 2007 Survey Co:USGS
Rotation:

Filename: sl36m1.avg

Channels: Ch1 Ch2 Ch3 Ch4 Ch5 Ch3 Ch4

Platted: 11:18 Nov 06, 2007

< EMI - ElectroMagnetic Instruments 
POLAR PLOTS

Alamosa, CO 100k

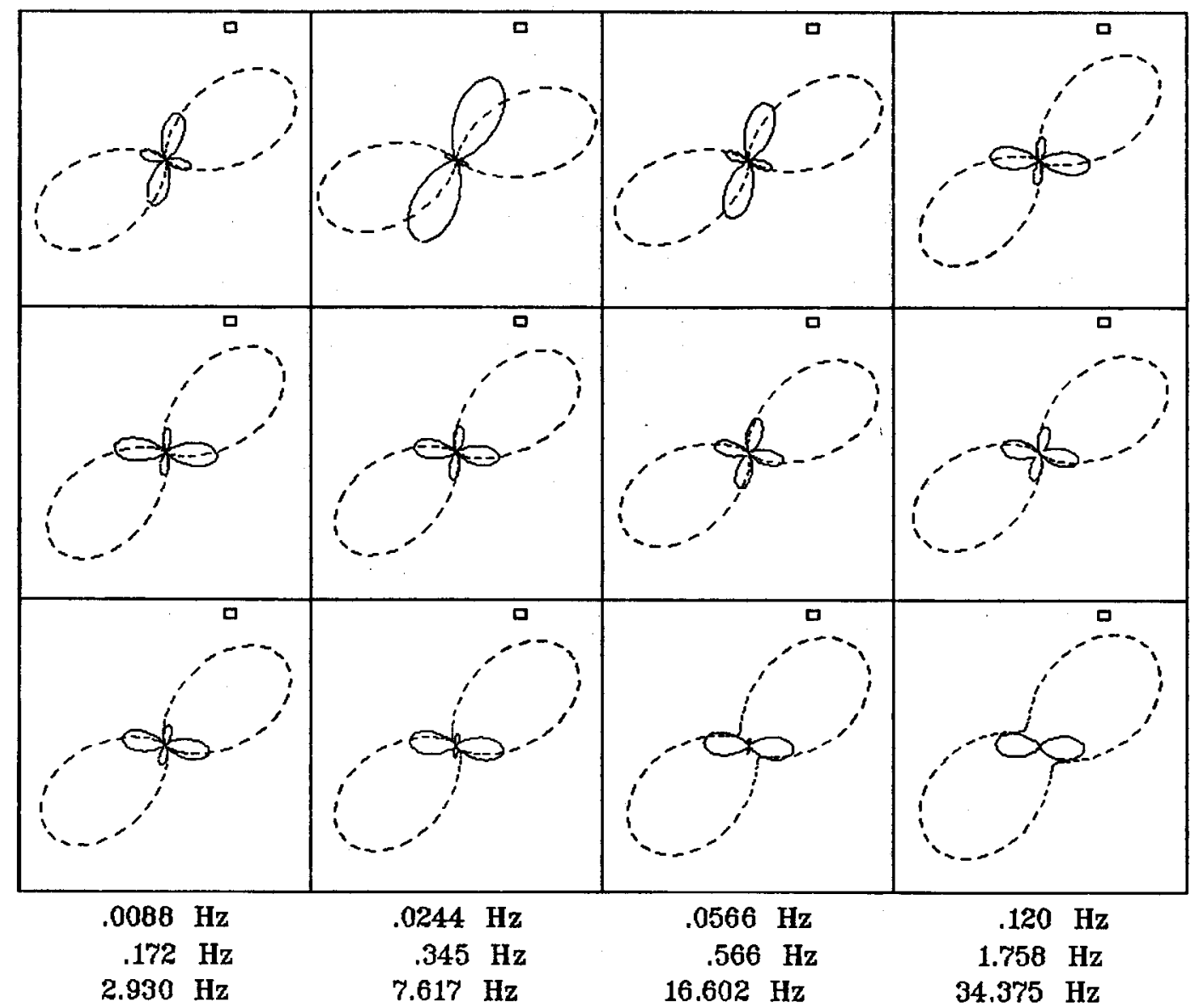

Client:

Remote: none

Acquired: 12:0 Jul 19, 2007 Survey Co:USGS
Rotation:

Filename: sl36m1.avg

Channels: Ch1 Ch2 Ch3 Ch4 Ch5 Ch3 Ch4

Plotted: 11:18 Nov 06, 2007

< EMI - ElectroMagnetic Instruments > 
Station 36

TIPPER MAGNITUDE

Alamosa, Co $100 \mathbf{k}$

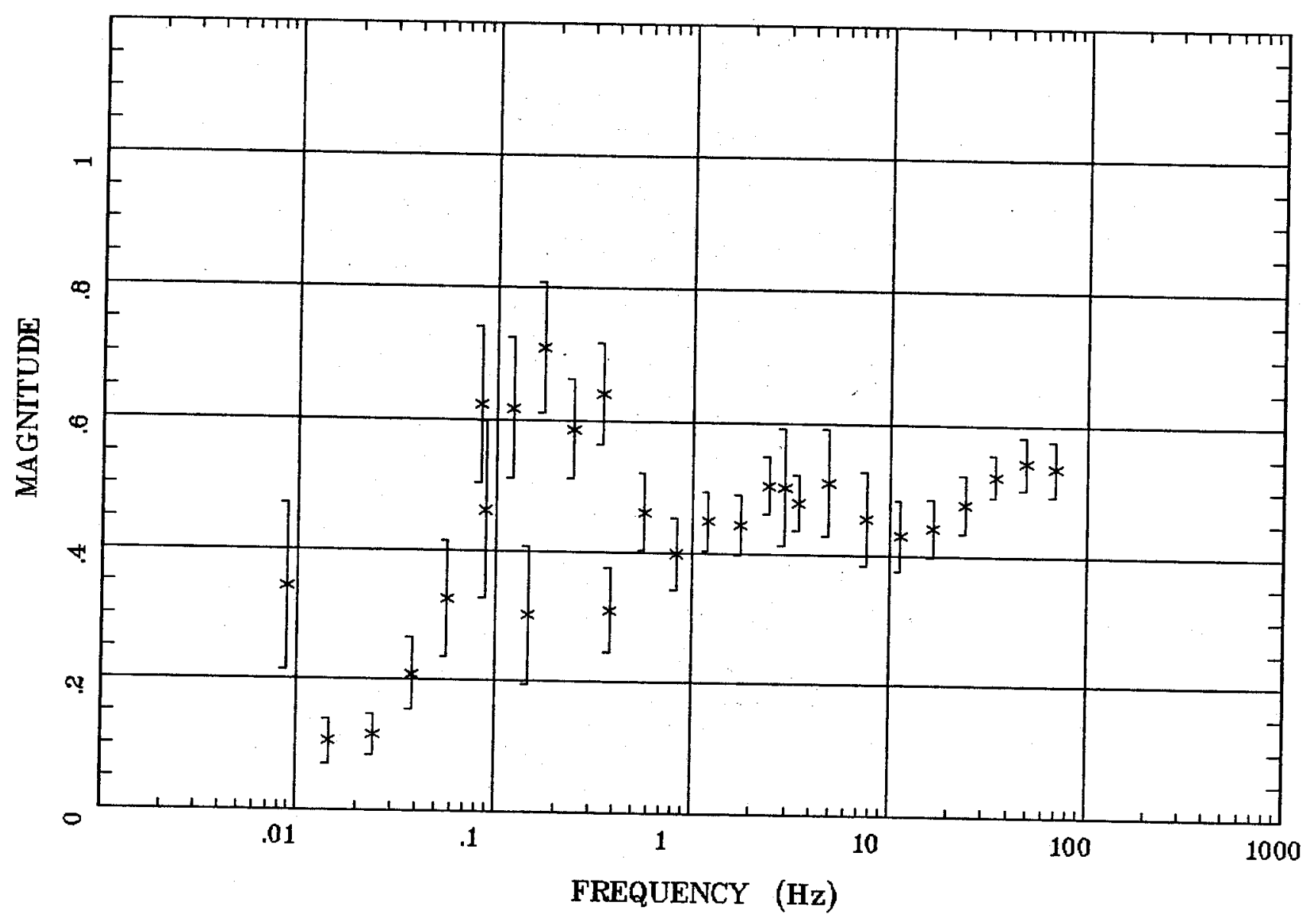

Client:

Remote: none

Acquired: 12:0 Jul 19, 2007

Survey Co:USGS
Rotation:

Filename: sl36m1.avg

Channels: Ch1 Ch2 Ch3 Ch4 Ch5 Ch3 Ch4 Plotted: 11:18 Nov 06, 2007

< EMI - ElectroMagnetic Instruments > 
Alamosa, CO 100k

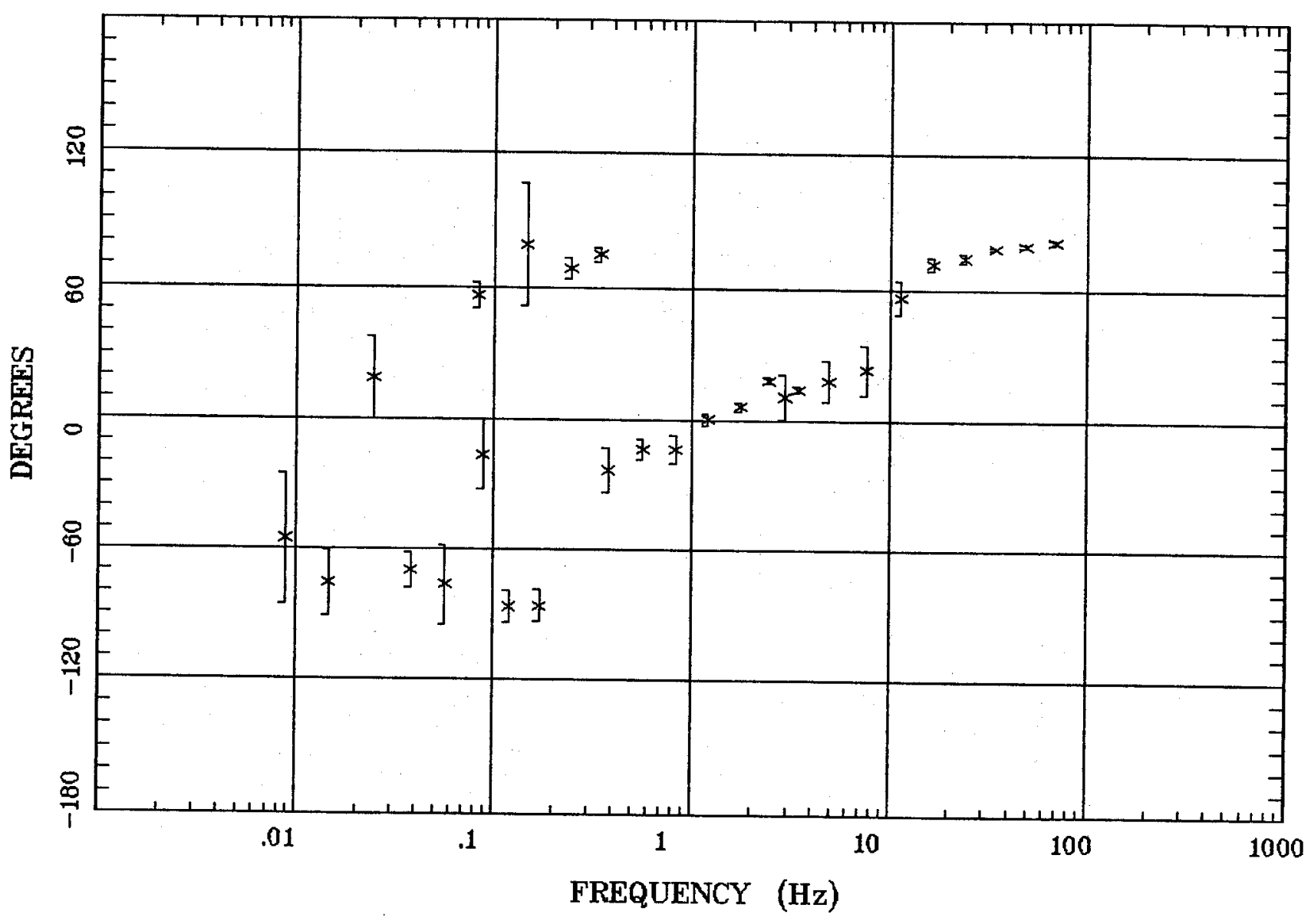

Client:

Remote: none

Acquired: 12:0 Jul 19, 2007

Survey Co:USGS
Rotation:

Filename: sl36m1.avg

Channels: Ch1 Ch2 Ch3 Ch4 Ch5 Ch3 Ch4 Plotted: 11:18 Nov 06, 2007

< EMI - ElectroMagnetic Instruments > 
HzHx.x Coh HzHy.o

Alamosa, CO 100k

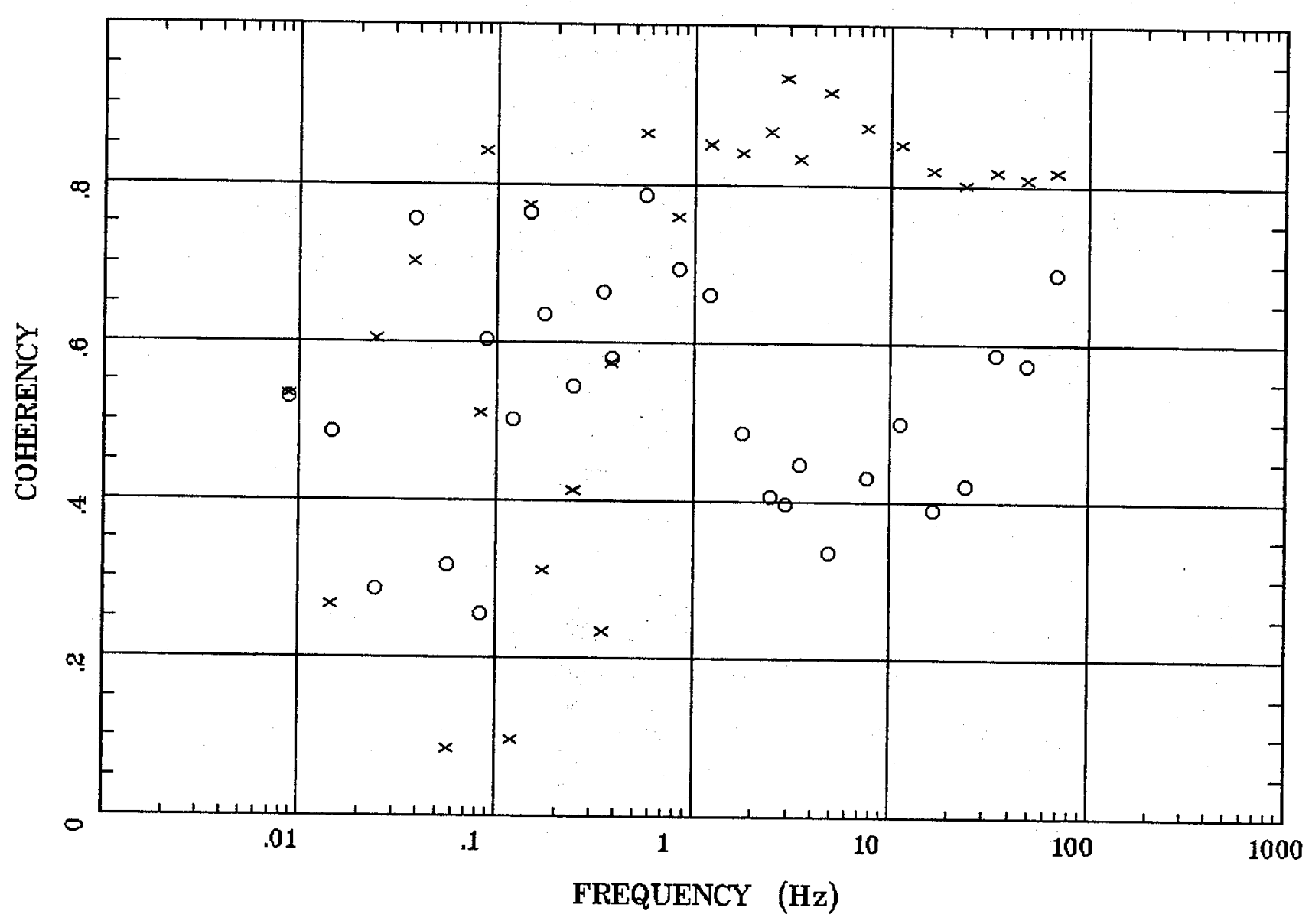

Client:

Remote: none

Acquired: 12:0 Jul 19, 2007

Survey Co:USGS
Rotation:

Filename: sl36m1.avg

Channels: Ch1 Ch2 Ch3 Ch4 Ch5 Ch3 Ch4 Plotted: 11:18 Nov 06, 2007

< EMI - ElectroMagnetic Instruments > 
Alamosa, CO 100k

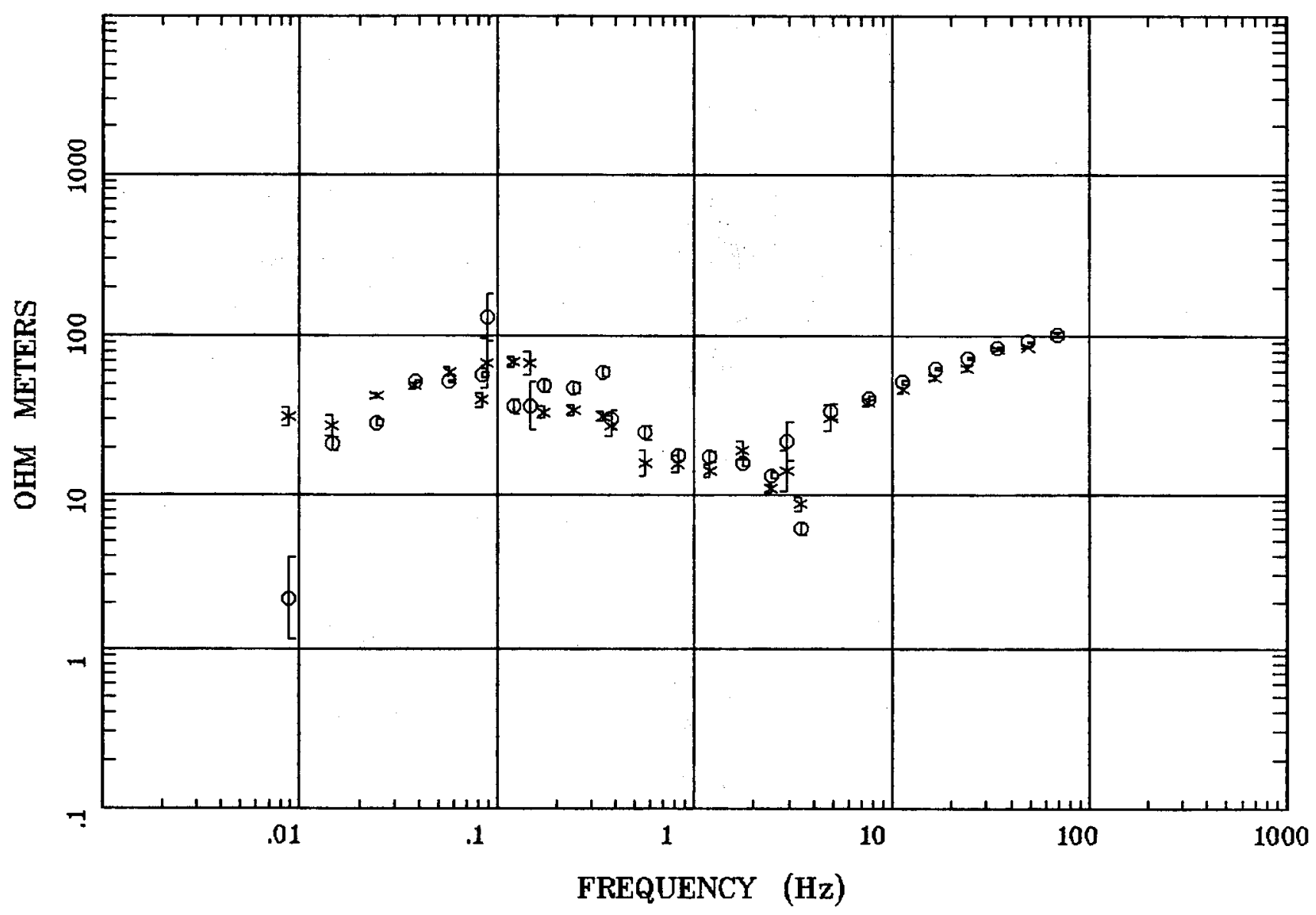

Client:

Remote: none

Acquired: 09:0 Jul 20, 2007 Survey Co:USGS
Rotation:

Filename: sl37m1.avg

Channels: Ch1 Ch2 Ch3 Ch4 Ch5 Ch3 Ch4 Plotted: 11:13 Nov 06, 2007

< EMI - ElectroMagnetic Instruments 
IMPEDANCE PHASE

Alamosa, CO 100k

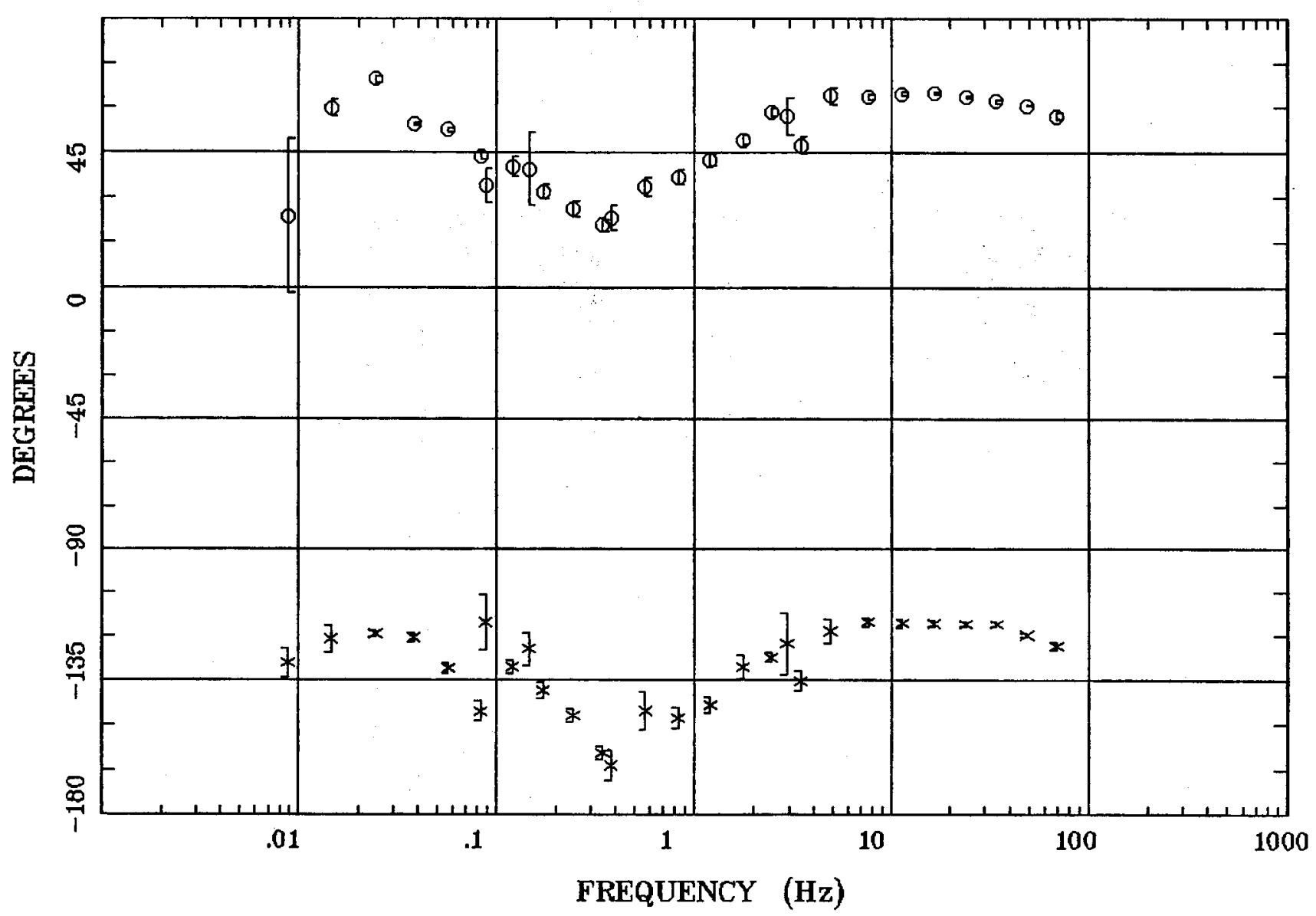

Client:

Remote: none

Acquired: 09:0 Jul 20, 2007 Survey Co:USGS
Rotation:

Filename: sl37m1.avg

Channels: Ch1 Ch2 Ch3 Ch4 Ch5 Ch3 Cb4 Plotted: 11:13 Nov 06, 2007

$<$ EMI - ElectroMagnetic Instruments > 


\section{ROTATION ANGLE}

Station 37

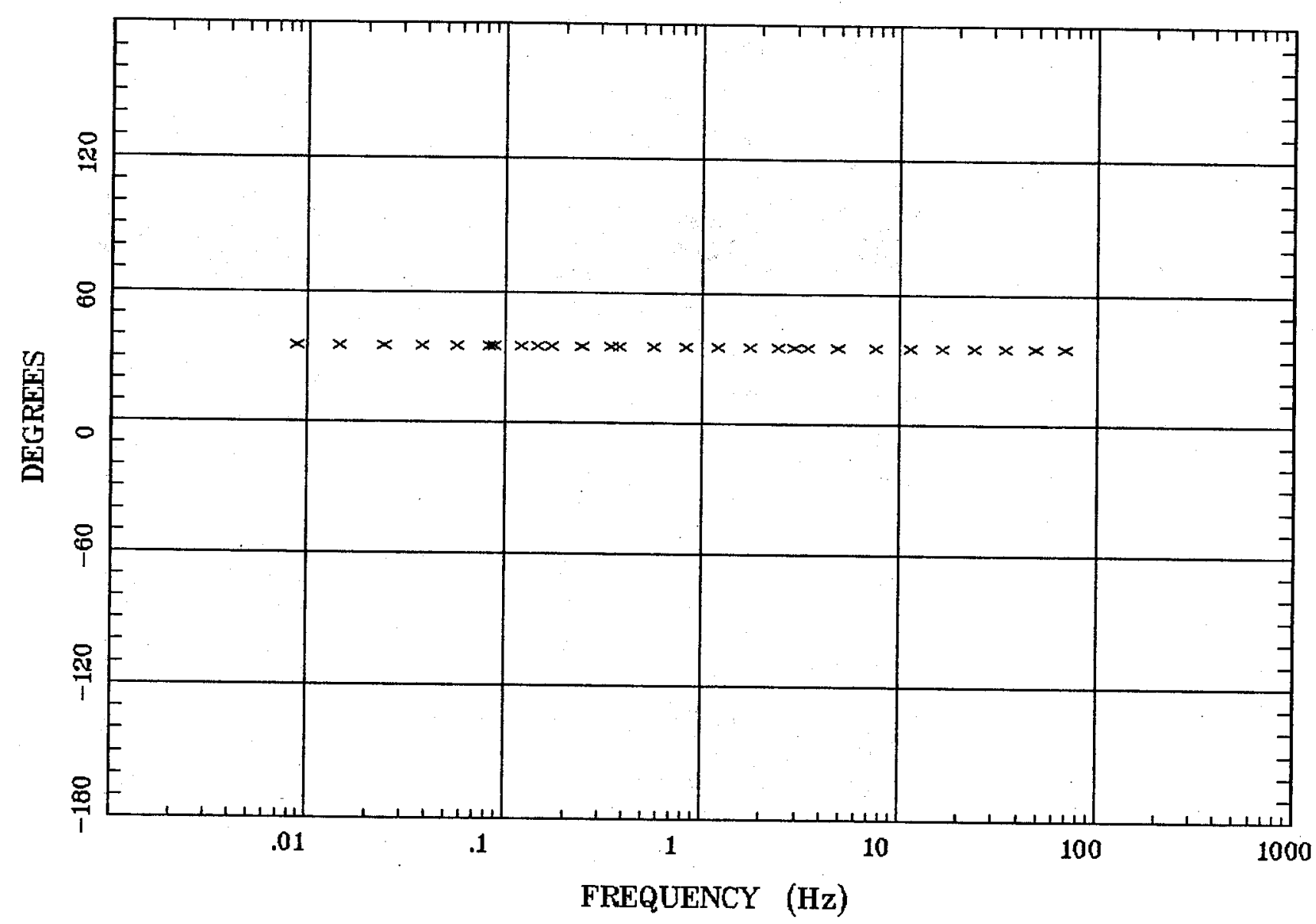

Client:

Remote: none

Acquired: 09:0 Jul 20, 2007

Survey Co:USGS
Rotation:

Filename: sl37m1.avg

Channels: Ch1 Ch2 Ch3 Ch4 Ch5 Ch3 Ch4 Plotted: 11:13 Nov 06, 2007

< EMI - ElectroMagnetic Instruments > 
Station 37

Alamosa, CO 100k

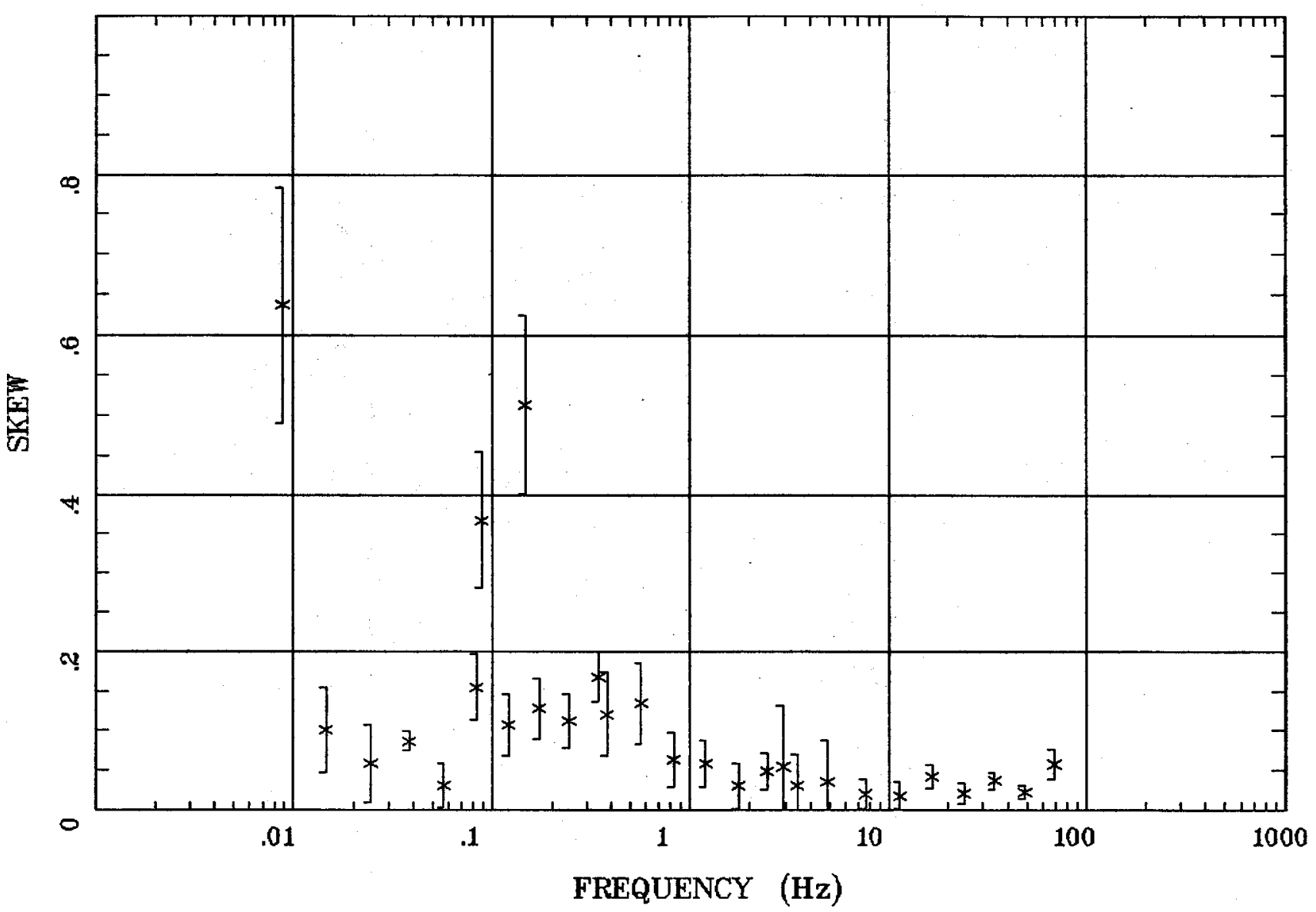

Client:

Remote: none

Acquired: 09:0 Jul 20, 2007

Survey Co:USGS

Rotation:

Filename: sl37m1.avg

Channels: Ch1 Ch2 Ch3 Ch4 Ch5 Ch3 Ch4

Plotted: 11:13 Nov 06, 2007

$<$ EMI - ElectroMagnetic Instruments 


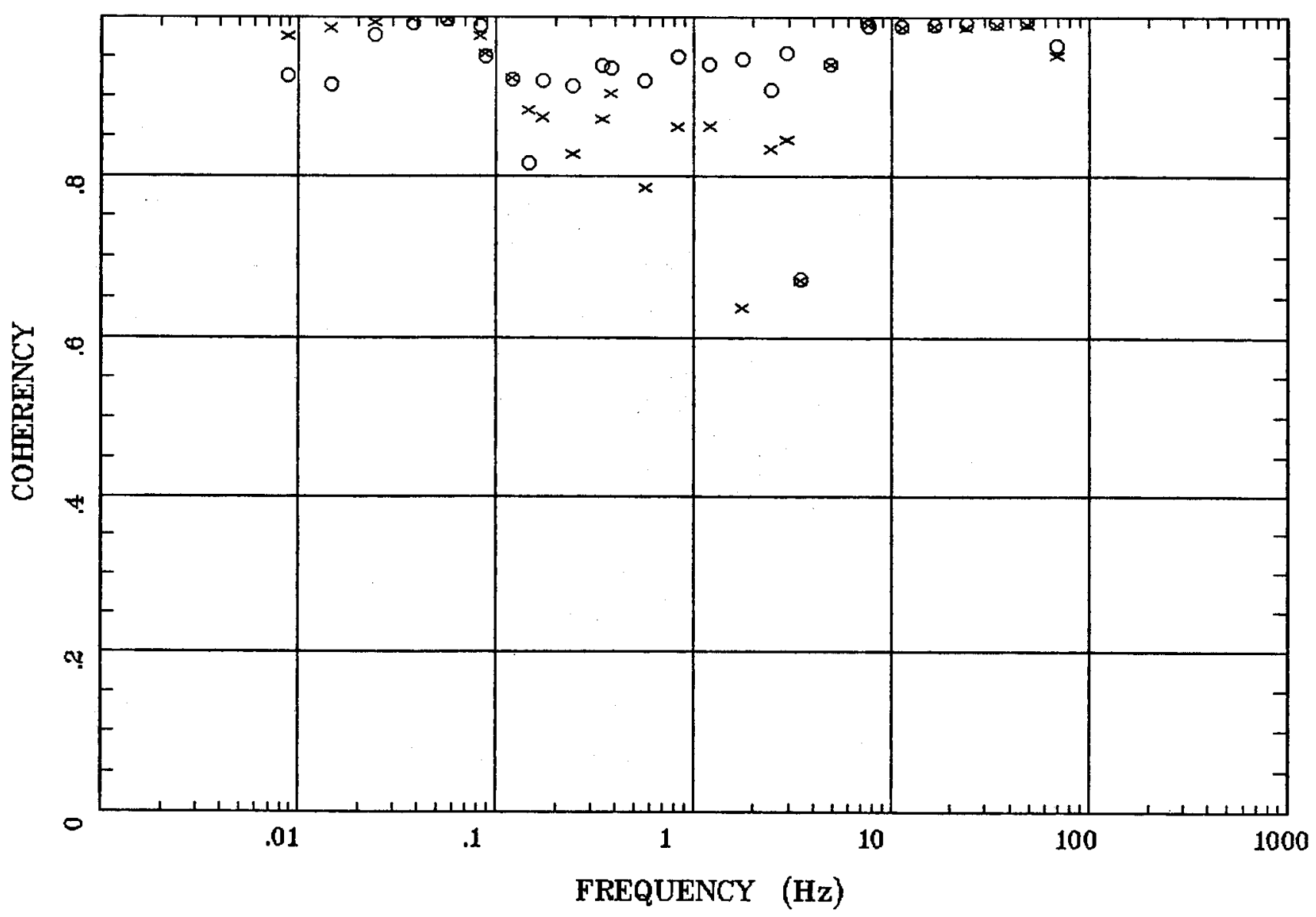

Client:

Remote: none

Acquired: 09:0 Jul 20, 2007

Survey Co:USGS
Rotation:

Filename: sl37m1.avg

Channels: Ch1 Ch2 Ch3 Ch4 Ch5 Ch3 Ch4 Plotted: 11:13 Nov 06, 2007

< EMI - ElectroMagnetic Instruments > 


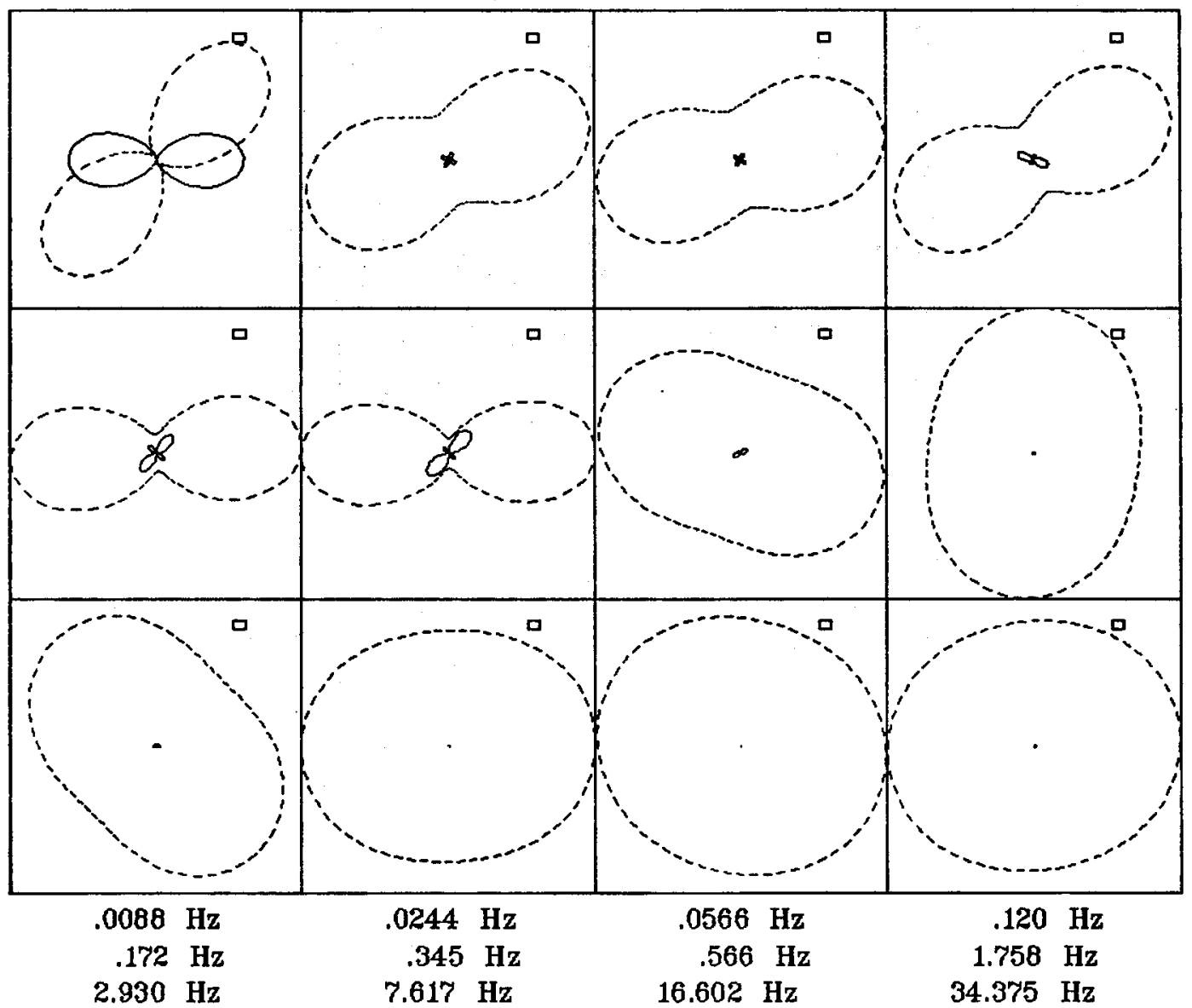

Client:

Remote: none

Acquired: 09:0 Jul 20, 2007 Survey Co:USGS
Rotation:

Filename: sl37m1.avg

Channels: Ch1 Ch2 Ch3 Ch4 Ch5 Ch3 Ch4 Plotted: 11:14 Nov 06, 2007

$<$ EMI - ElectroMagnetic Instruments > 
Alamosa, CO 100k

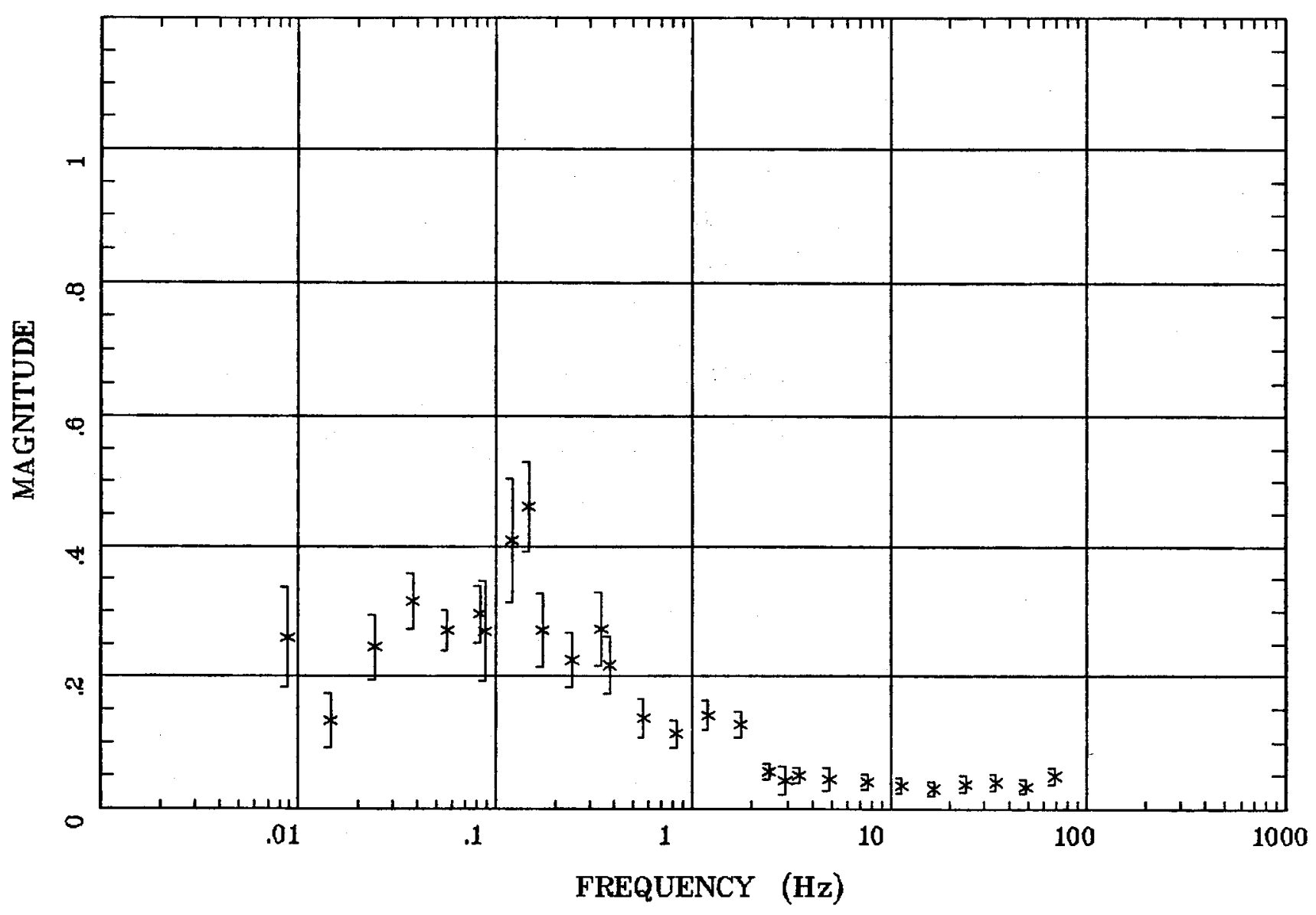

Client:

Remote: none

Acquired: 09:0 Jul 20, 2007 Survey Co:USGS
Rotation:

Filename: sl37m1.avg

Channels: Ch1 Ch2 Ch3 Ch4 Ch5 Ch3 Ch4

Plotted: 11:14 Nov 06, 2007

< EMI - ElectroMagnetic Instruments 
Alamosa, CO 100k

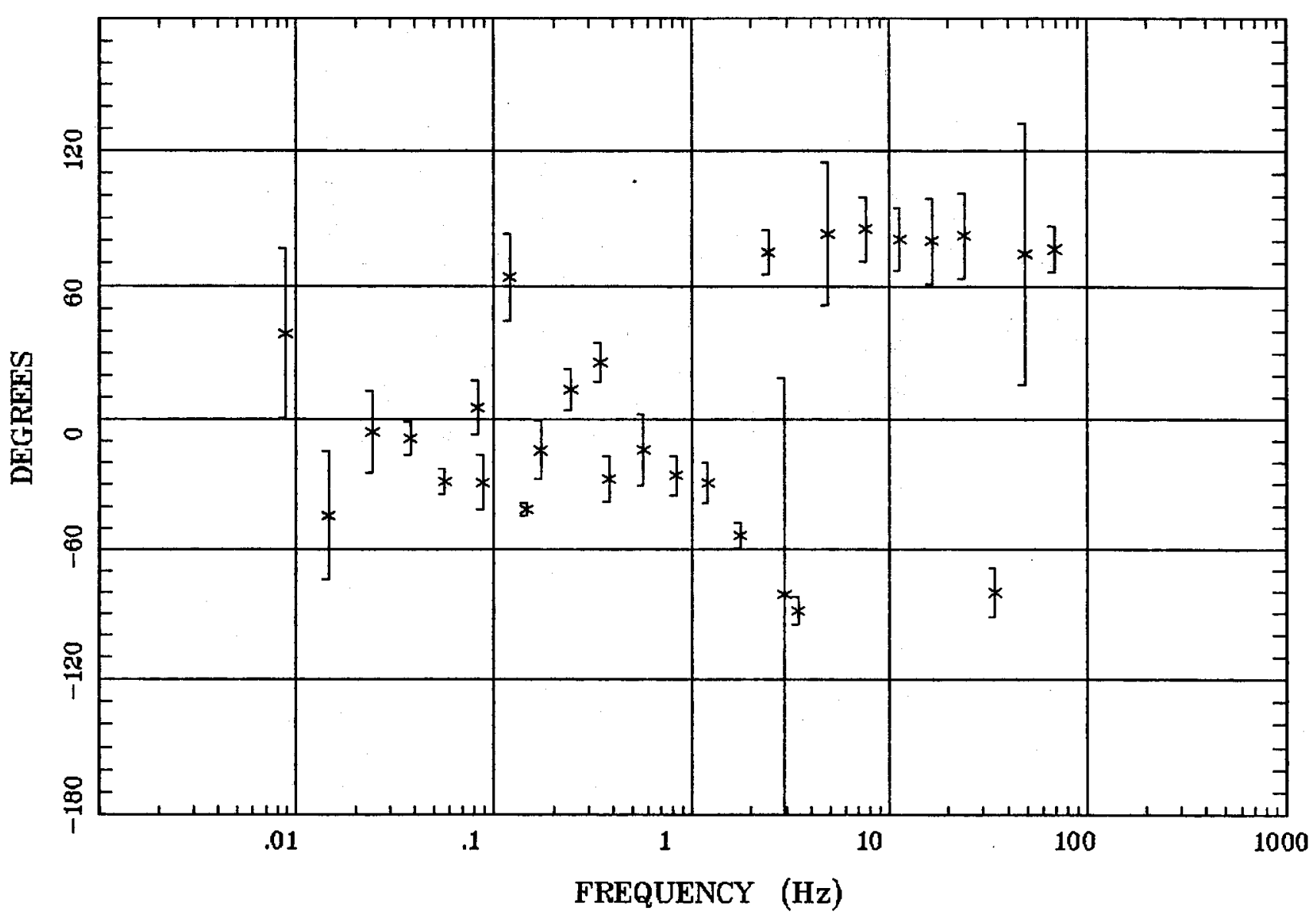

Client:

Remote: none

Acquired: 09:0 Jul 20, 2007 Survey Ca:USGS
Rotation:

Filename: sl37m1.avg

Channels: Ch1 Ch2 Ch3 Ch4 Ch5 Ch3 Ch4 Plotted: 11:14 Nov 06, 2007

$<$ EMI - ElectroMagnetic Instruments 


\section{HzHx.x Coh HzHy.o}

Alamosa, CO 100k

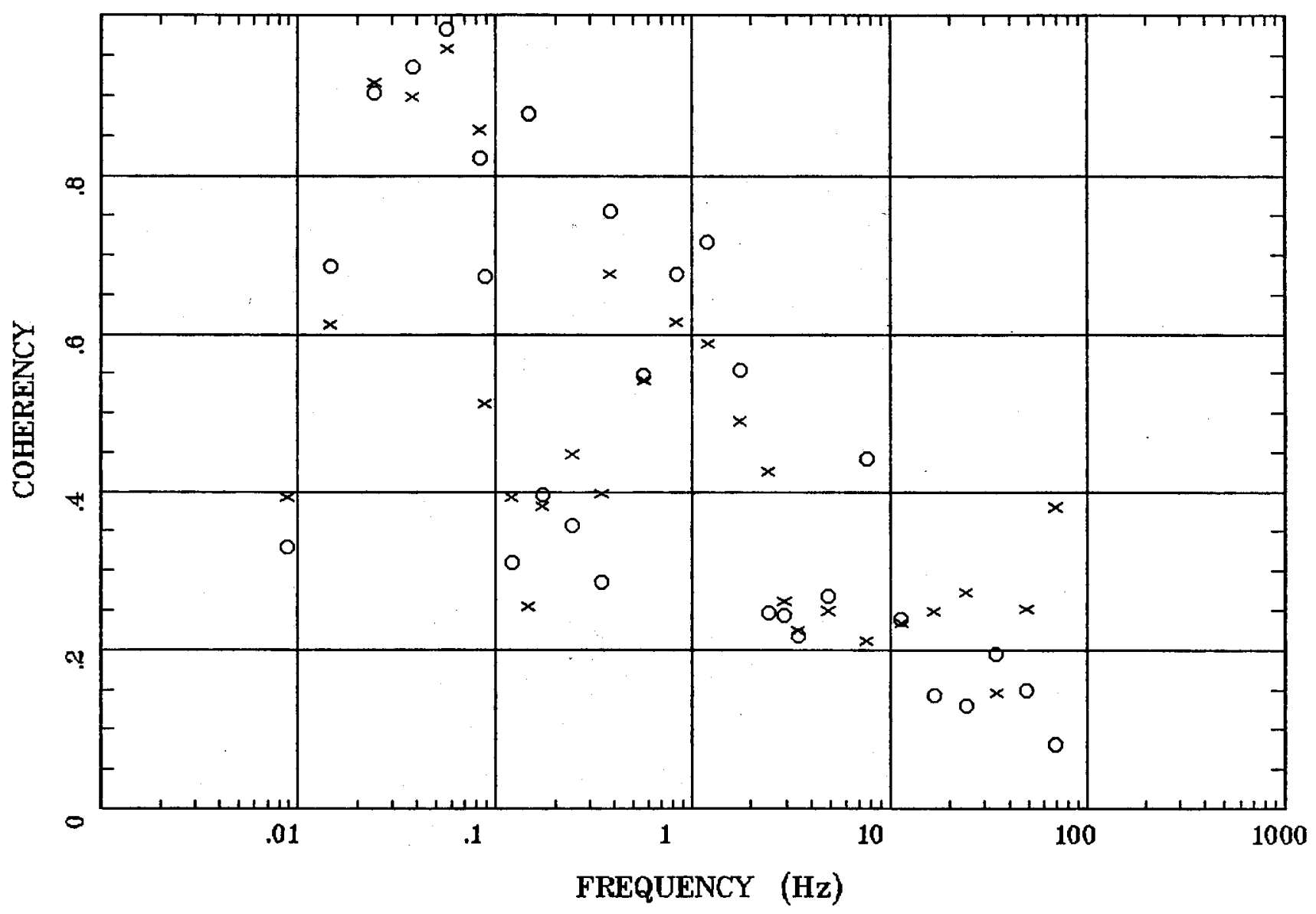

Client:

Remote: none

Acquired: 09:0 Jul 20, 2007 Survey Co:USGS
Rotation:

Filename: sl37m1.avg

Channels: Ch1 Ch2 Ch3 Ch4 Ch5 Ch3 Ch4

Plotted: 11:14 Nop 06, 2007

$<$ EMI - ElectroMagnetic Instruments 
APPARENT RESISTIVITY Alamosa, CO 100k

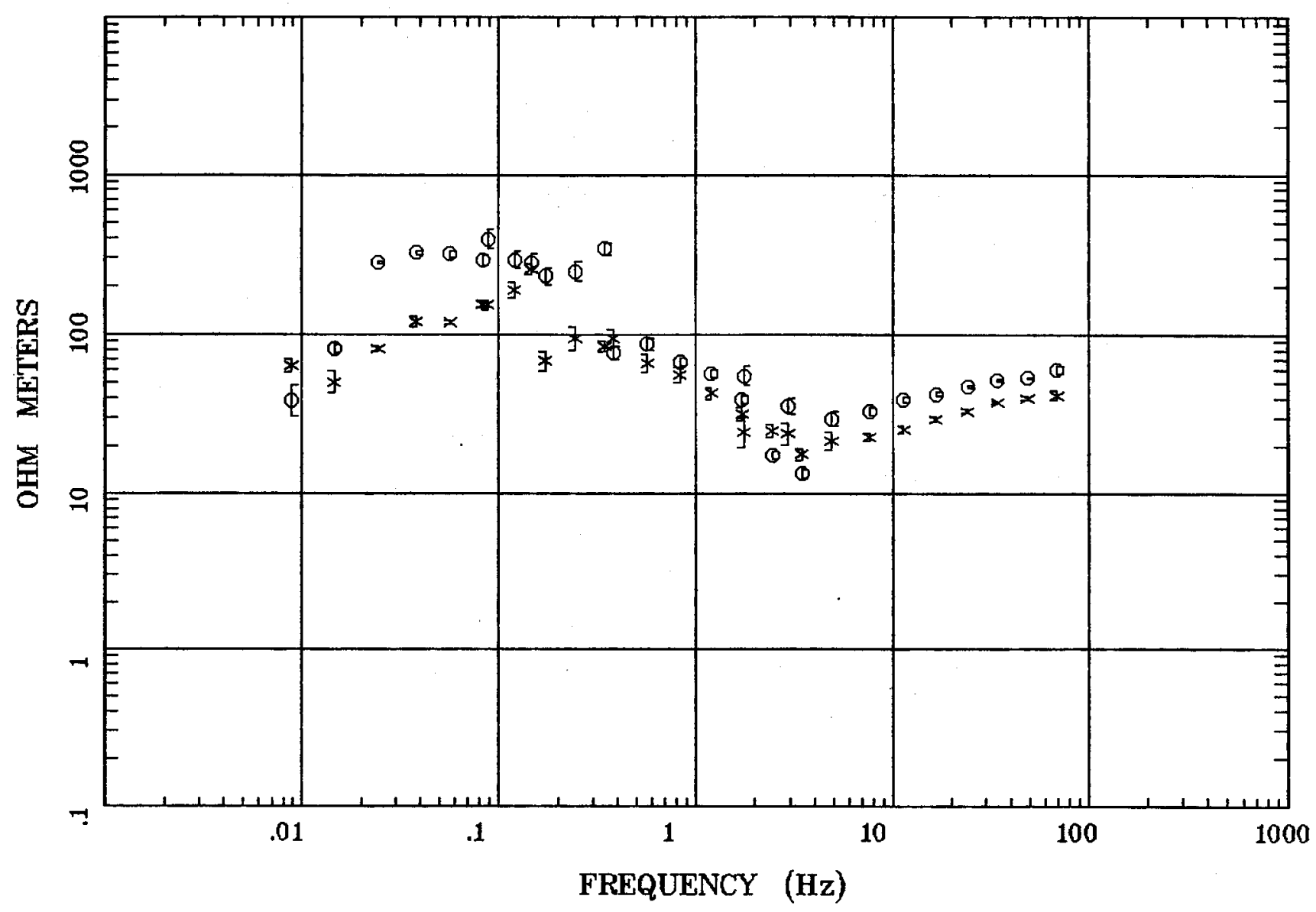

Client:

Remote: none

Acquired: $13: 5$ Jul 20, 2007 Survey Co:UsGS
Rotation:

Filename: sl38mall.avg

Channels: Ch1 Ch2 Ch3 Ch4 Ch5 Ch3 Ch4 Plotted: 11:14 Nov 06, 2007

< EMI - ElectroMagnetic Instruments > 
IMPEDANCE PHASE

Alamosa, CO 100k

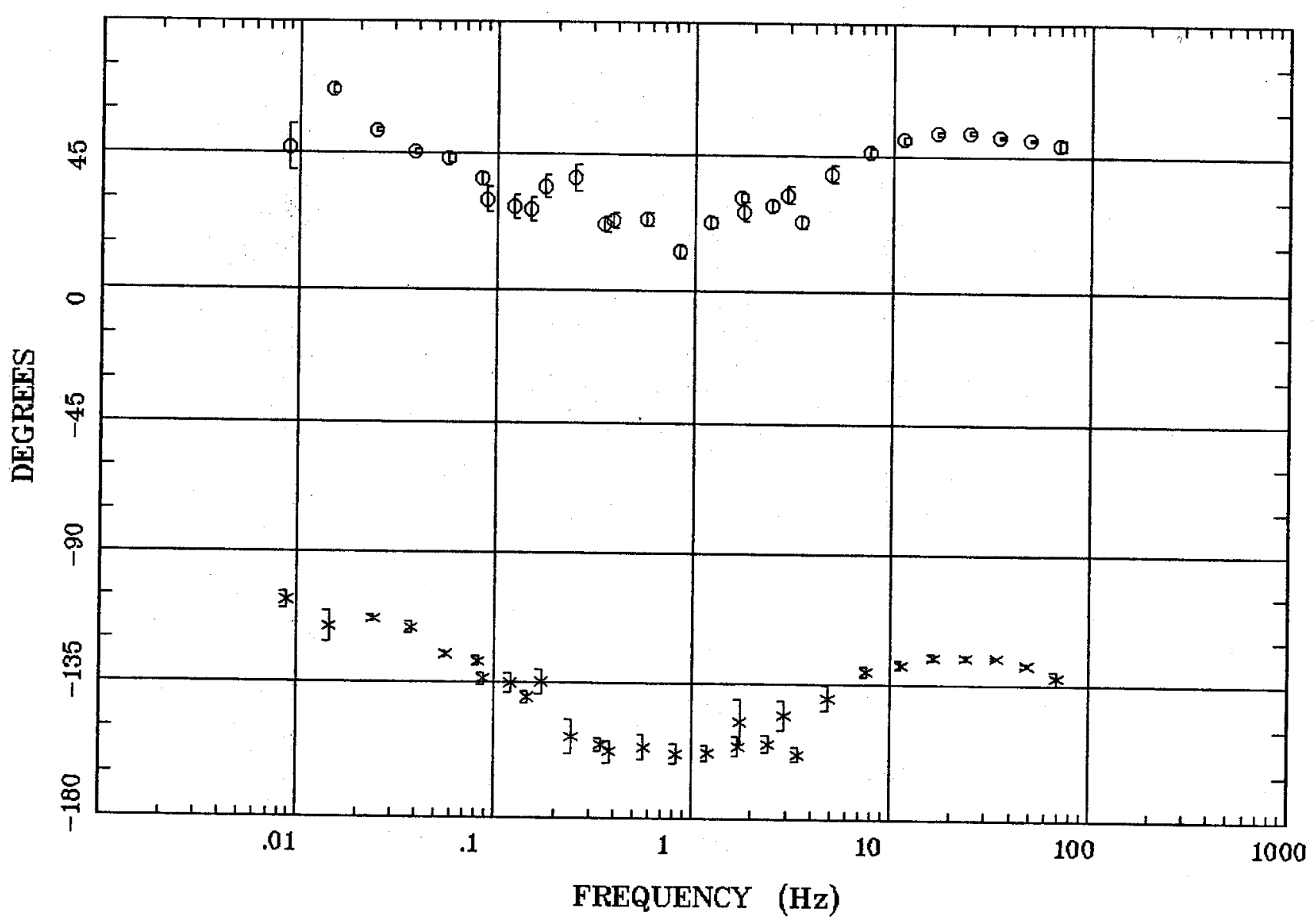

Client:

Remote: none

Acquired: 13:5 Jul 20, 2007

Survey Co:USGS
Rotation:

Filename: sl38mall.avg

Channels: Ch1 Ch2 Ch3 Ch4 Ch5 Ch3 Ch4

Plotted: 11:14 Nov 06, 2007

$<$ EMI - ElectroMagnetic Instruments > 
ROTATION ANGLE Alamosa, CO 100k

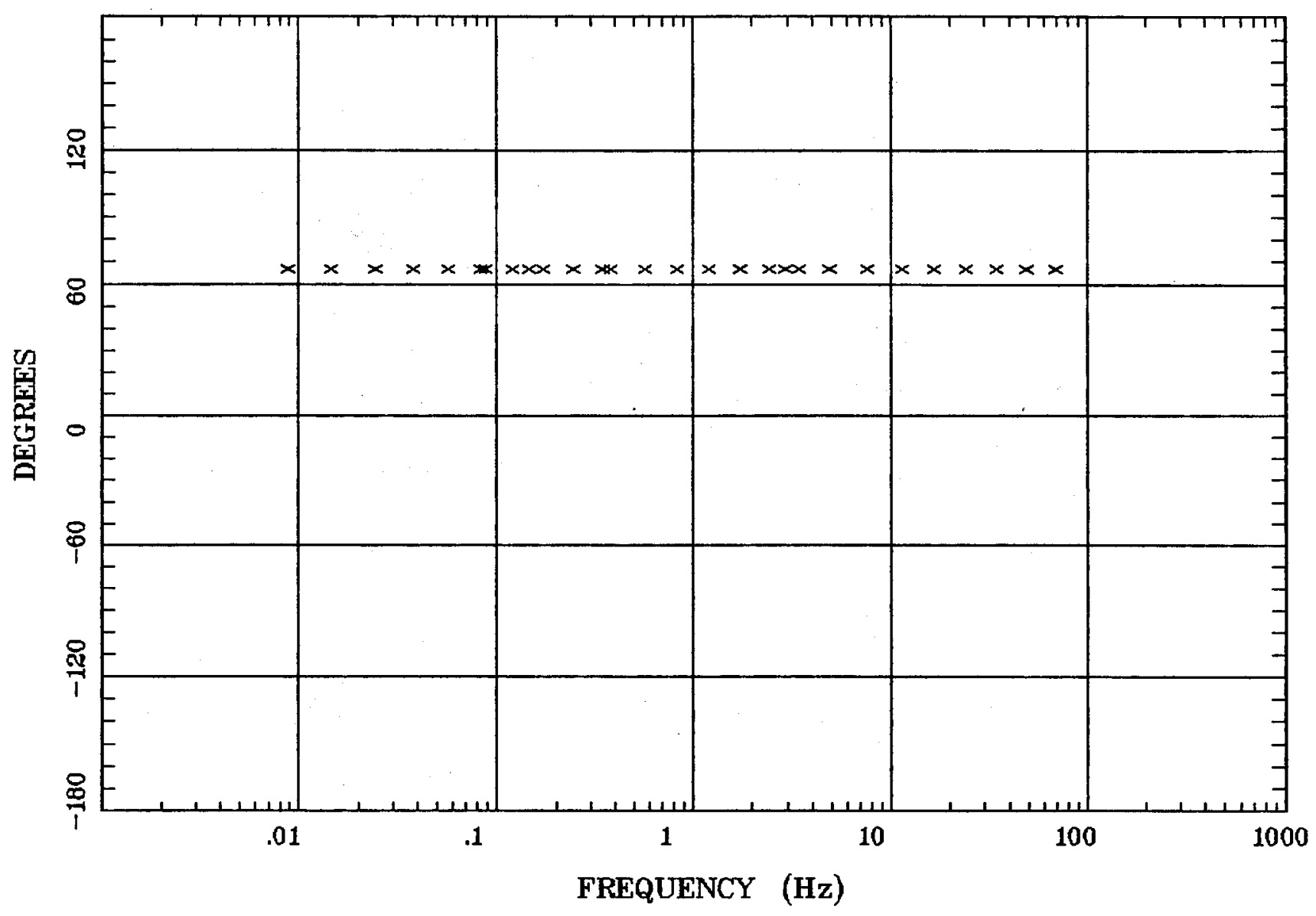

Client:

Remote: none

Acquired: $13: 5$ Jul 20, 2007

Survey Co:USGS
Rotation:

Filename: sl38mall.avg

Channels: Ch1 Ch2 Ch3 Ch4 Ch5 Ch3 Ch4

Plotted: 11:14 Nov 06, 2007

$<$ EMI - ElectroMagnetic Instruments 
IMPEDANCE SKEW

Alamosa, CO $100 \mathrm{k}$

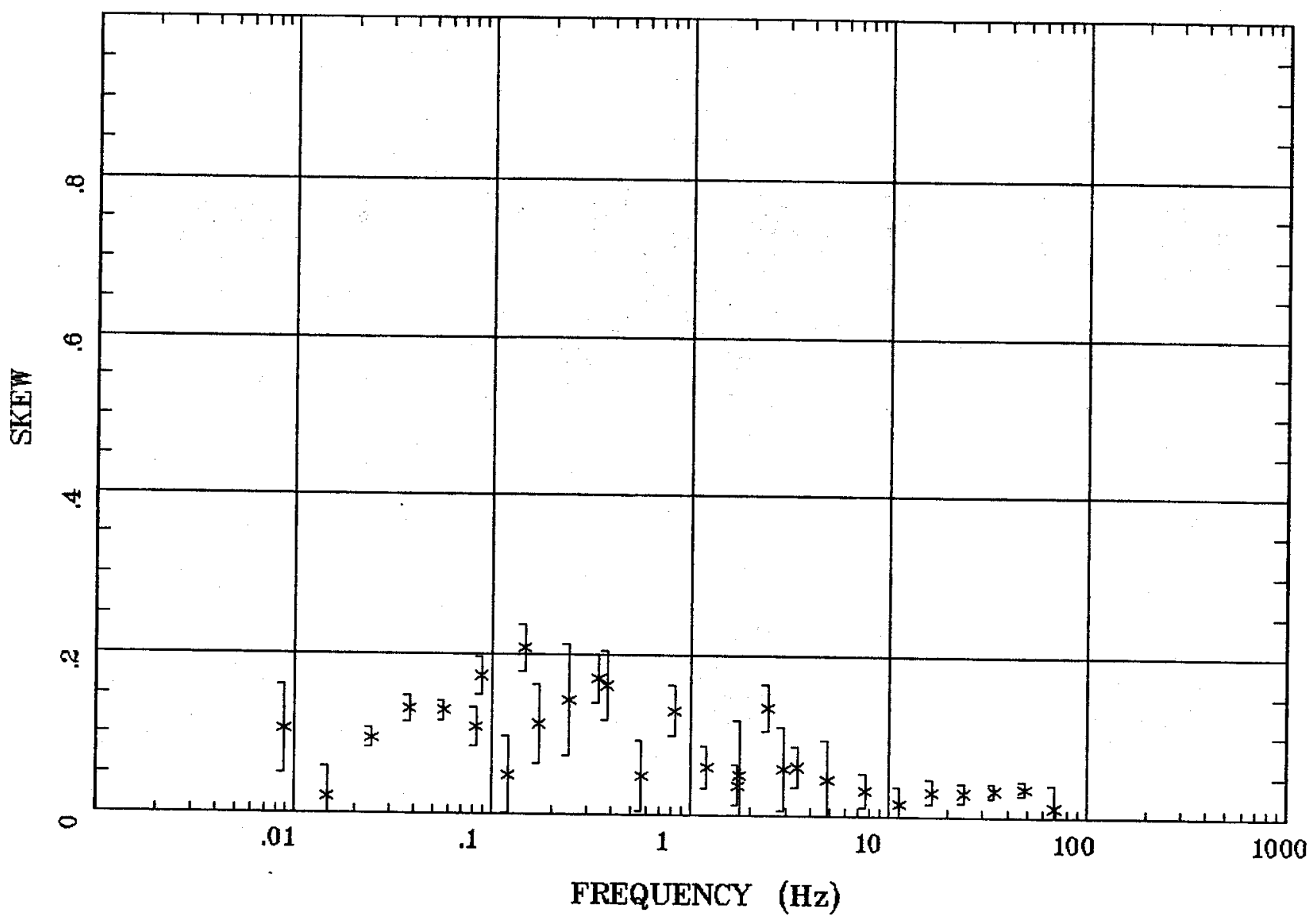

Client:

Remote: none

Acquired: 13:5 Jul 20, 2007

Survey Co:USGS
Rotation:

Filename: sl36mall.avg

Channels: Ch1 Ch2 Ch3 Ch4 Ch5 Ch3 Ch4 Plotted: 11:14 Nov 06, 2007

< EMI - ElectroMagnetic Instruments > 
E MULT Coh.

Alamosa, CO 100k

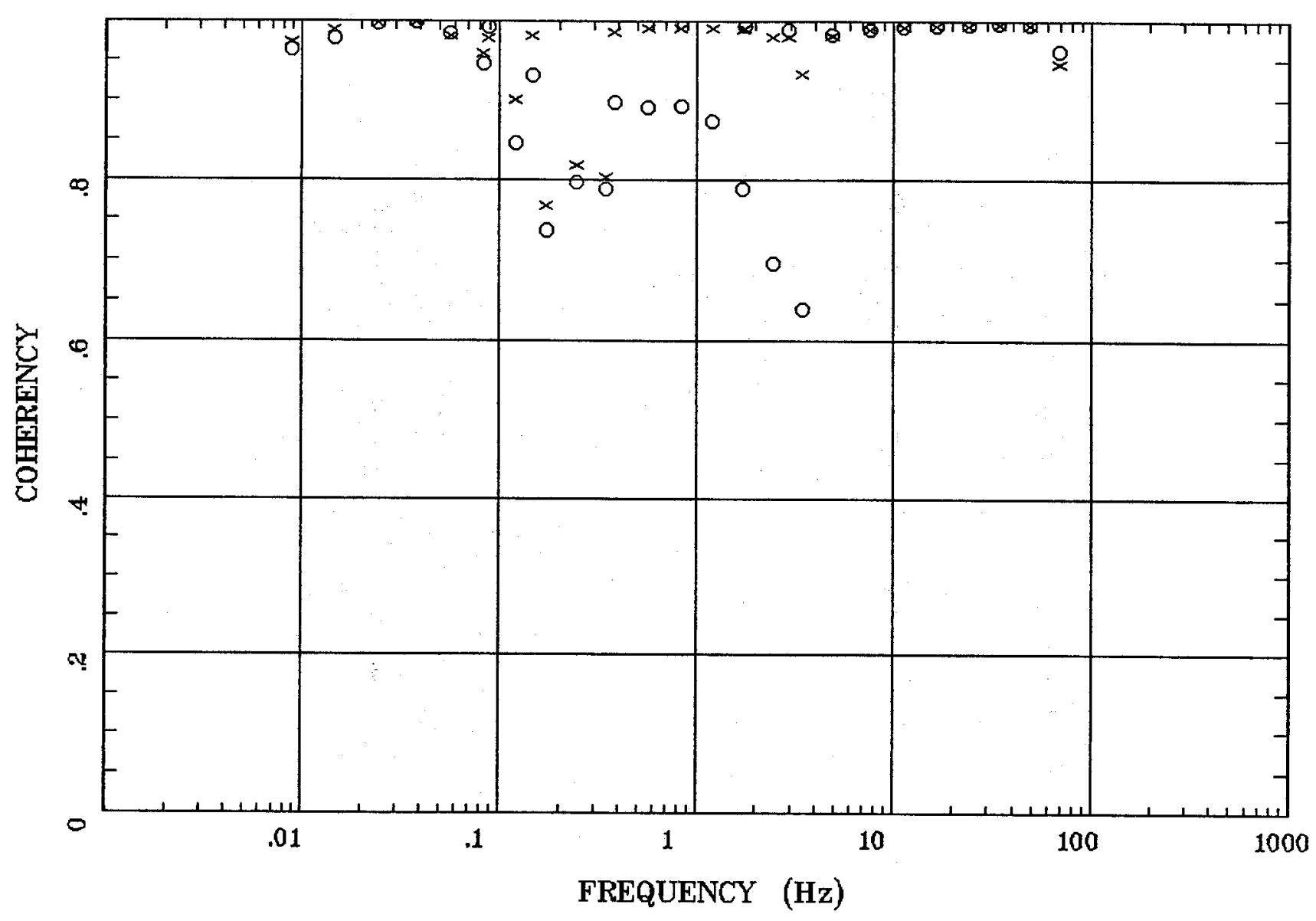

Client:

Remote: none

Acquired: 13:5 Jul 20, 2007

Survey Co:USGS
Rotation:

Filename: sl38mall.avg

Channels: Ch1 Ch2 Ch3 Ch4 Ch5 Ch3 Ch4 Plotted: 11:14 Nov 06, 2007

< EMI - ElectroMagnetic Instruments 
POLAR PLOTS

Alamosa, CO 100k

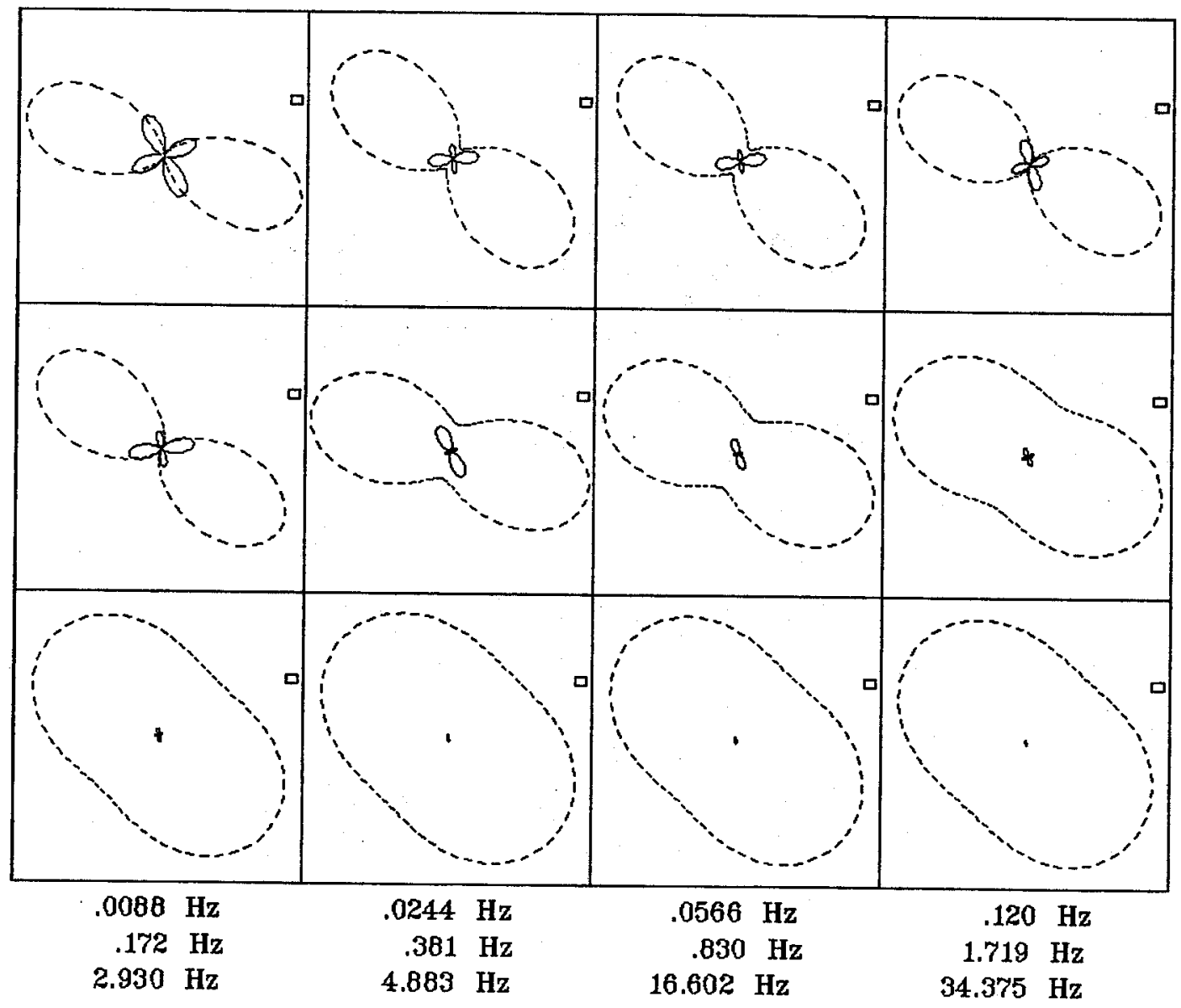

Client:

Remate: none

Acquired: 13:5 Jul 20, 2007

Survey Co:USGS
Rotation:

Filename: sl38mall.avg

Channels: Ch1 Ch2 Ch3 Ch4 Ch5 Ch3 Ch4

Plotted: 11:14 Nov 06, 2007

$<$ EMI - ElectroMagnetic Instruments 
Alamosa, CO $100 \mathrm{k}$

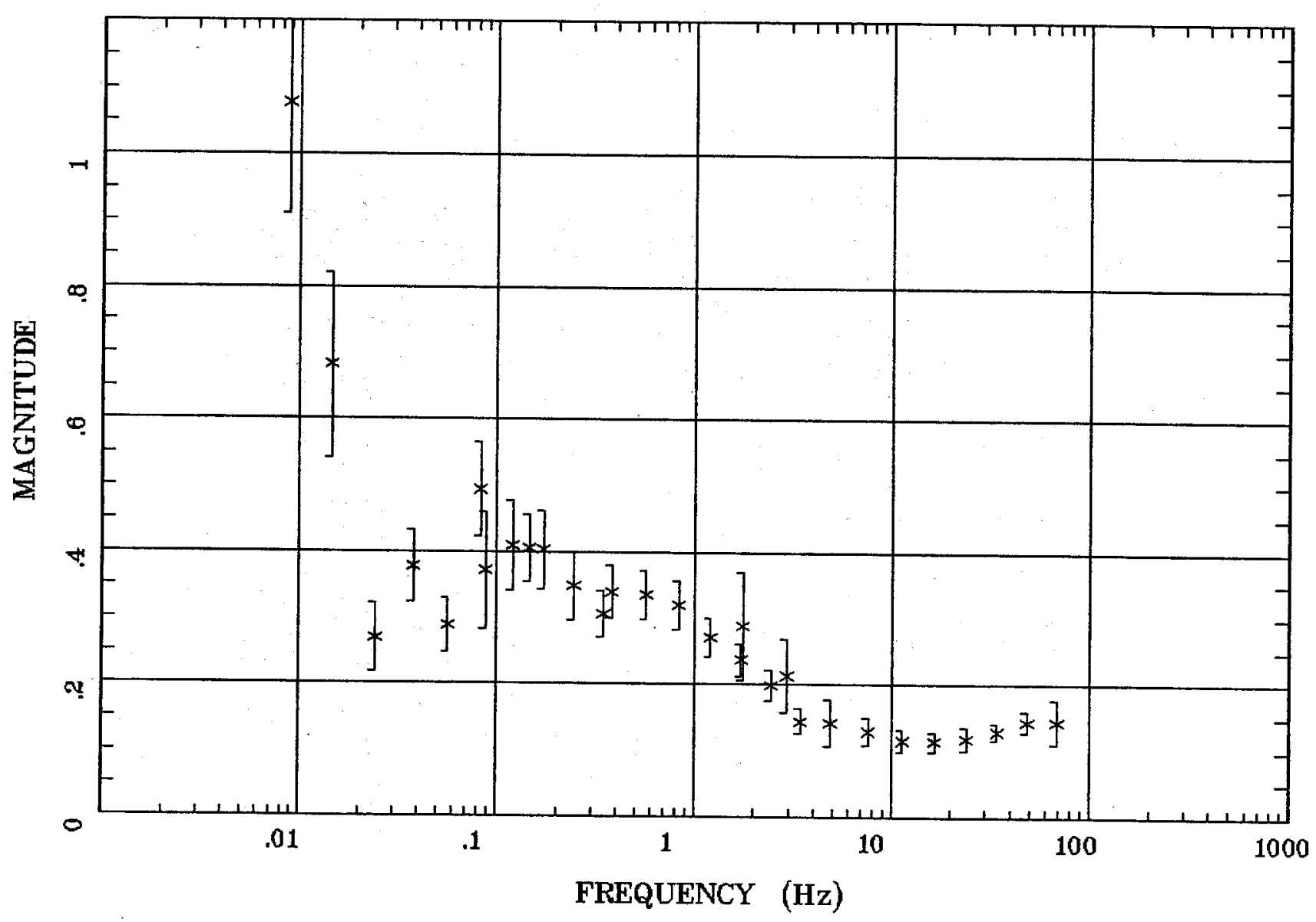

Client:

Remote: none

Acquired: 13:5 Jul 20, 2007

Survey Co:USGS
Rotation:

Filename: sl38mall.avg

Channels: Ch1 Ch2 Ch3 Ch4 Ch5 Ch3 Ch4

Plotted: 11:14 Nov 06, 2007

$<$ EMI - ElectroMagnetic Instruments 
Alamosa, CO 100k

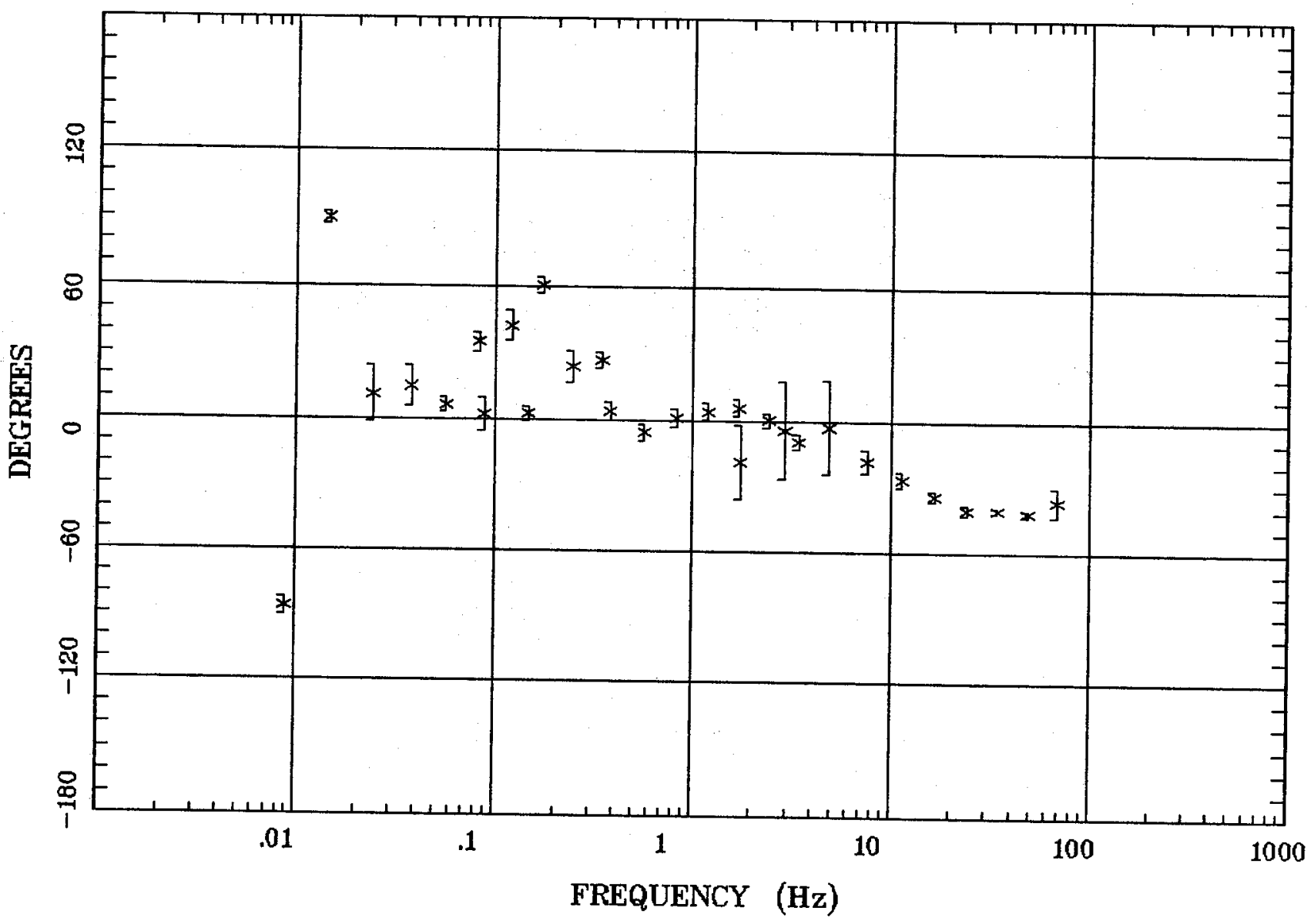

Client:

Remote: none

Acquired: 13:5 Jul 20, 2007 Survey Co:USGS
Rotation:

Filename: sl38mall.avg

Channels: Ch1 Ch2 Ch3 Ch4 Ch5 Ch3 Ch4 Plotted: 11:14 Nov 06, 2007

< EMI - ElectroMagnetic Instruments > 
Alamosa, CO 100k

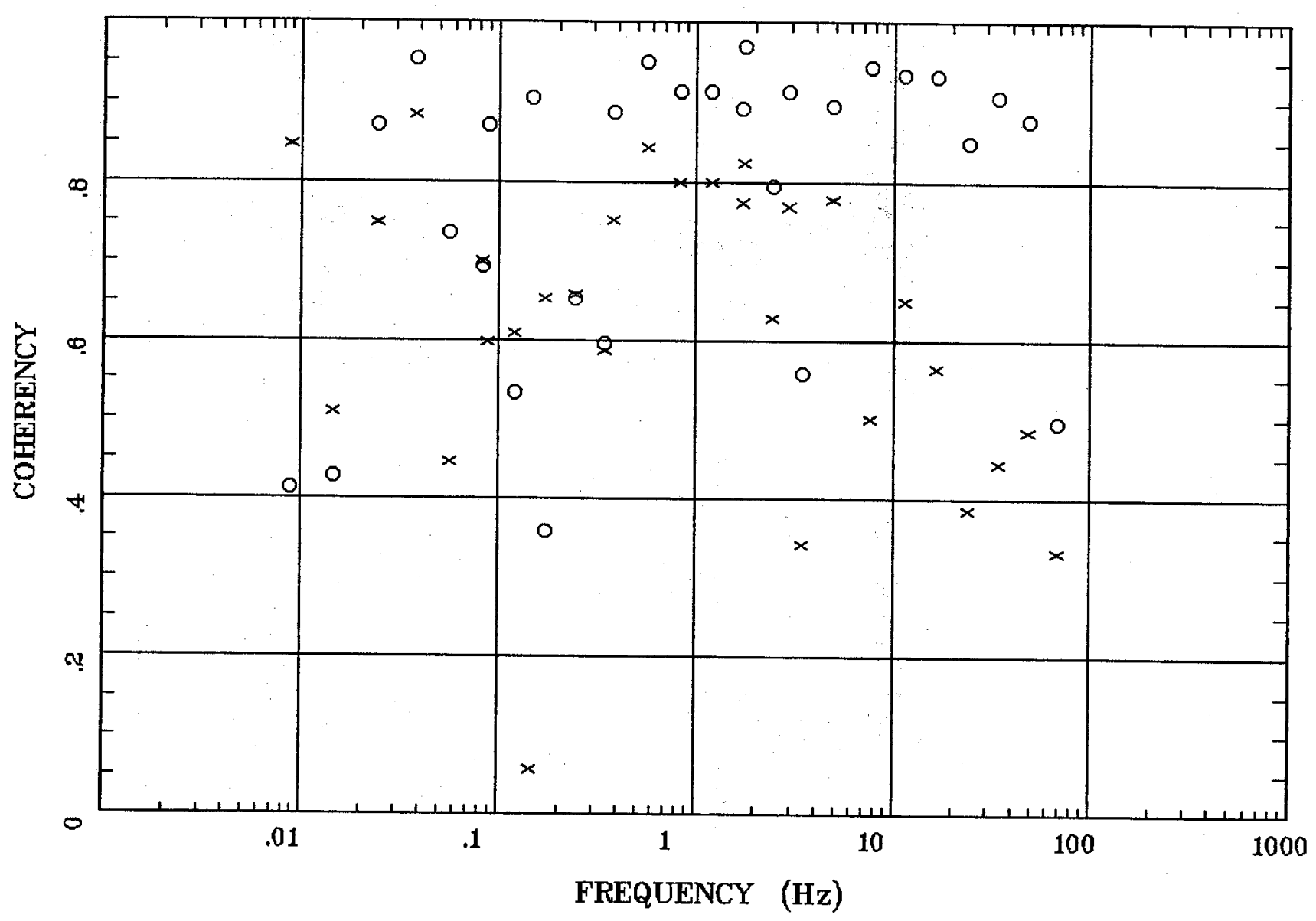

Client:

Remote: none

Acquired: 13:5 Jul 20, 2007

Survey Co:USGS
Rotation:

Filename: sl38mall.avg

Channels: Ch1 Ch2 Ch3 Ch4 Ch5 Ch3 Ch4

Plotted: 11:14 Nov 06, 2007

< EMI - ElectroMagnetic Instruments > 


\section{APPARENT RESISTIVITY \\ Alamosa, CO 100k}

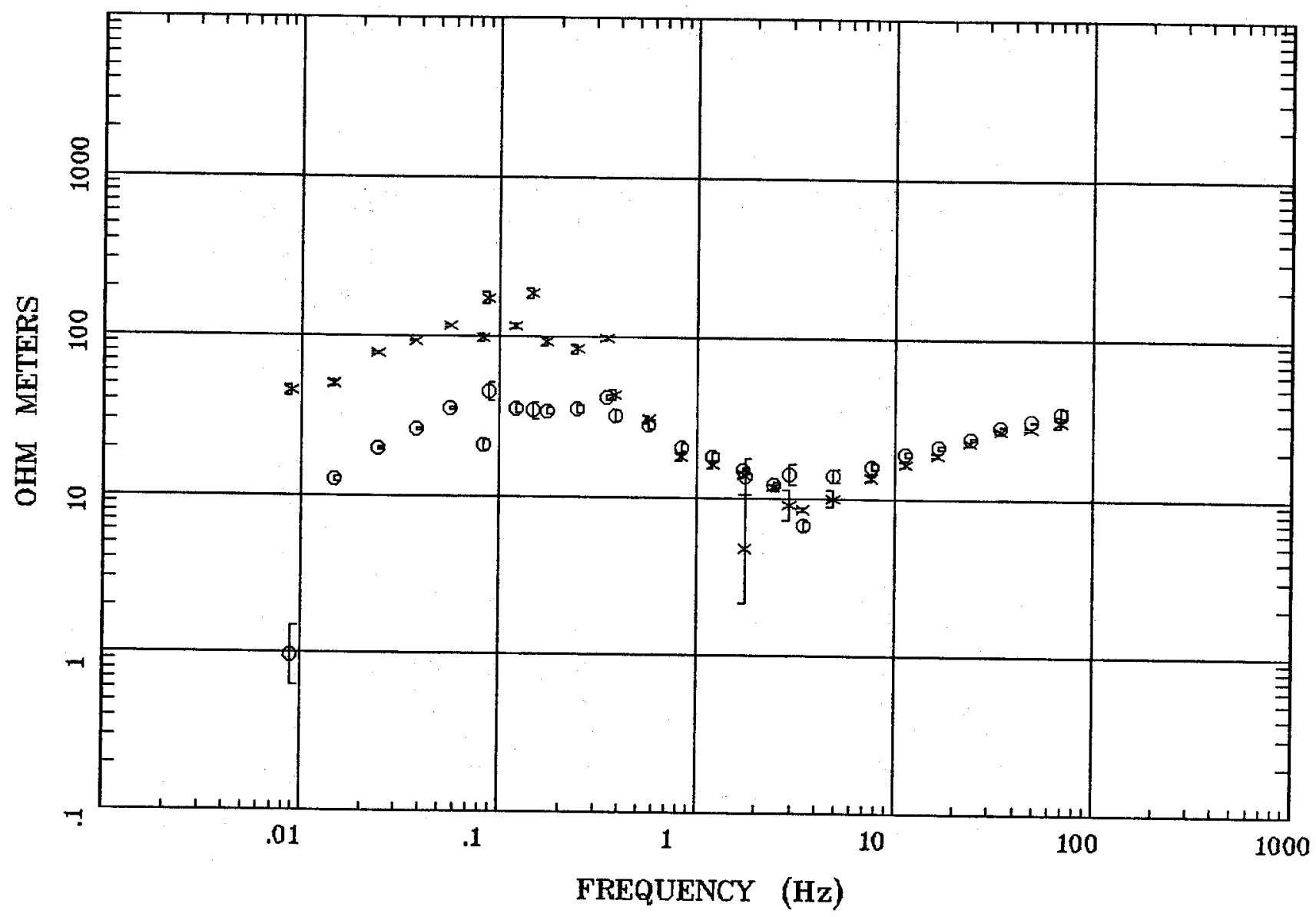

Client:

Remate: none

Acquired: 10:0 Jul 21, 2007

Survey Co:USGS
Rotation:

Filename: sl39mall.avg

Channels: Ch1 Ch2 Ch3 Ch4 Ch5 Ch3 Ch4

Plotted: 11:13 Nov 06, 2007

$<$ EMI - ElectroMagnetic Instruments > 
IMPEDANCE PHASE

Alamosa, CO 100k

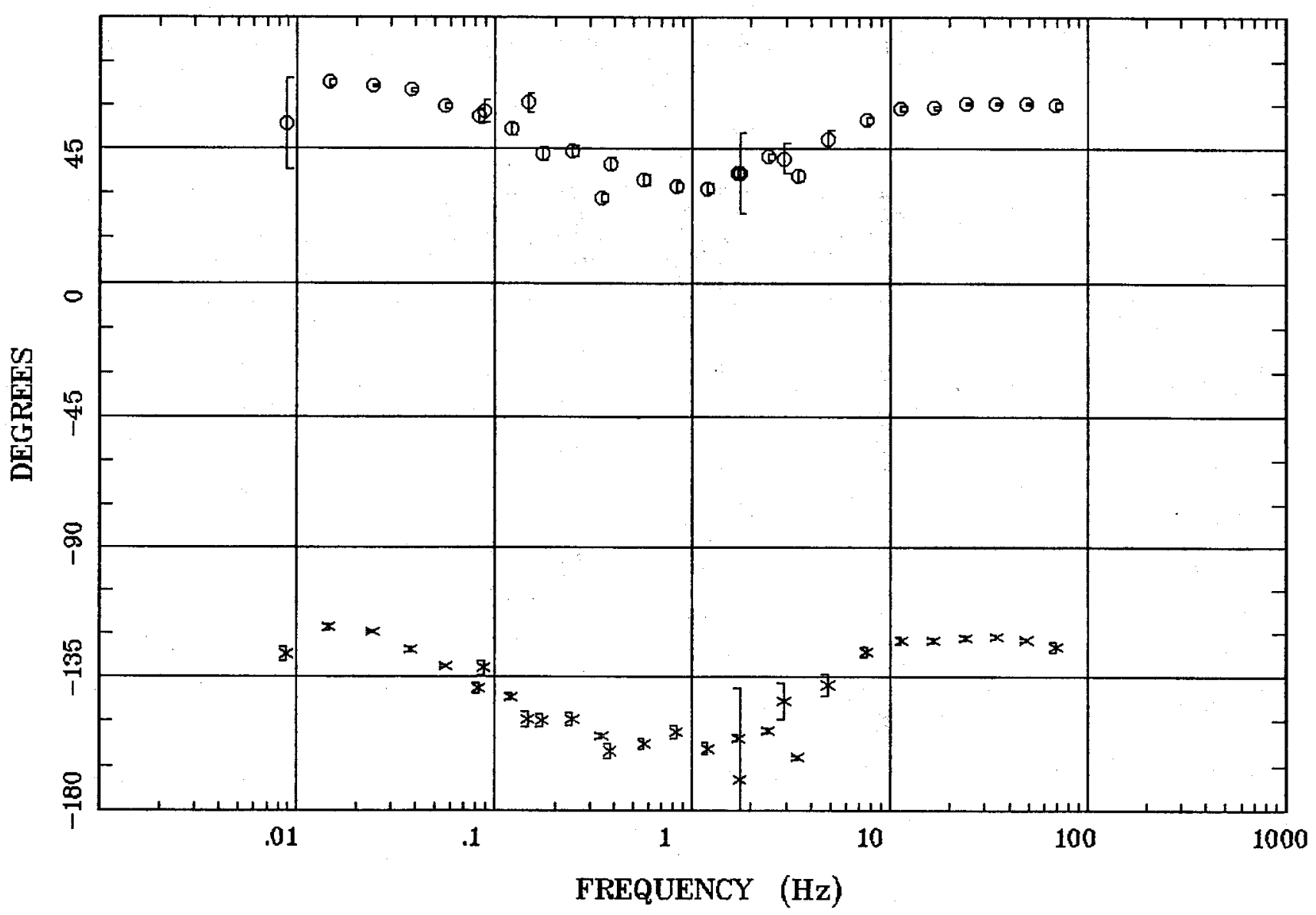

Client:

Remote: none

Acquired: 10:0 Jul 21, 2007

Survey Co:USGS
Rotation:

Filename: sl39mall.avg

Channels: Ch1 Ch2 Ch3 Ch4 Ch5 Ch3 Ch4 Plotted: 11:13 Nov 06, 2007

< EMI - ElectroMagnetic Instruments > 
ROTATION ANGLE

Alamosa, Co 100k

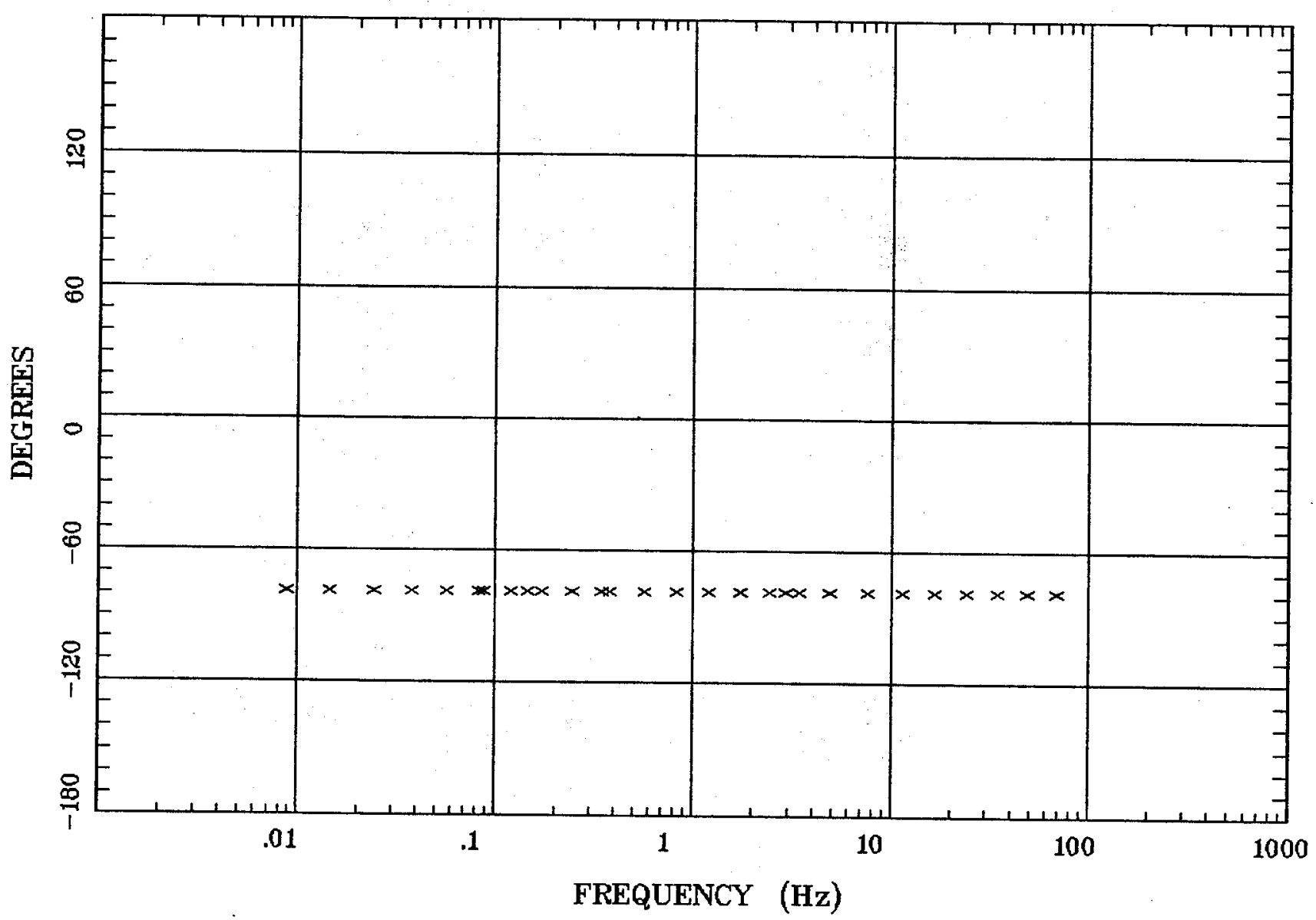

Client:

Remote: none

Acquired: 10:0 Jul 21, 2007 Survey Co:USGS
Rotation:

Filename: sl39mall.avg

Channels: Ch1 Ch2 Ch3 Ch4 Ch5 Ch3 Ch4

Plotted: 11:13 Nov 06, 2007

< EMI - ElectroMagnetic Instruments , 
Station 39

IMPEDANCE SKEW

Alamosa, Co 100k

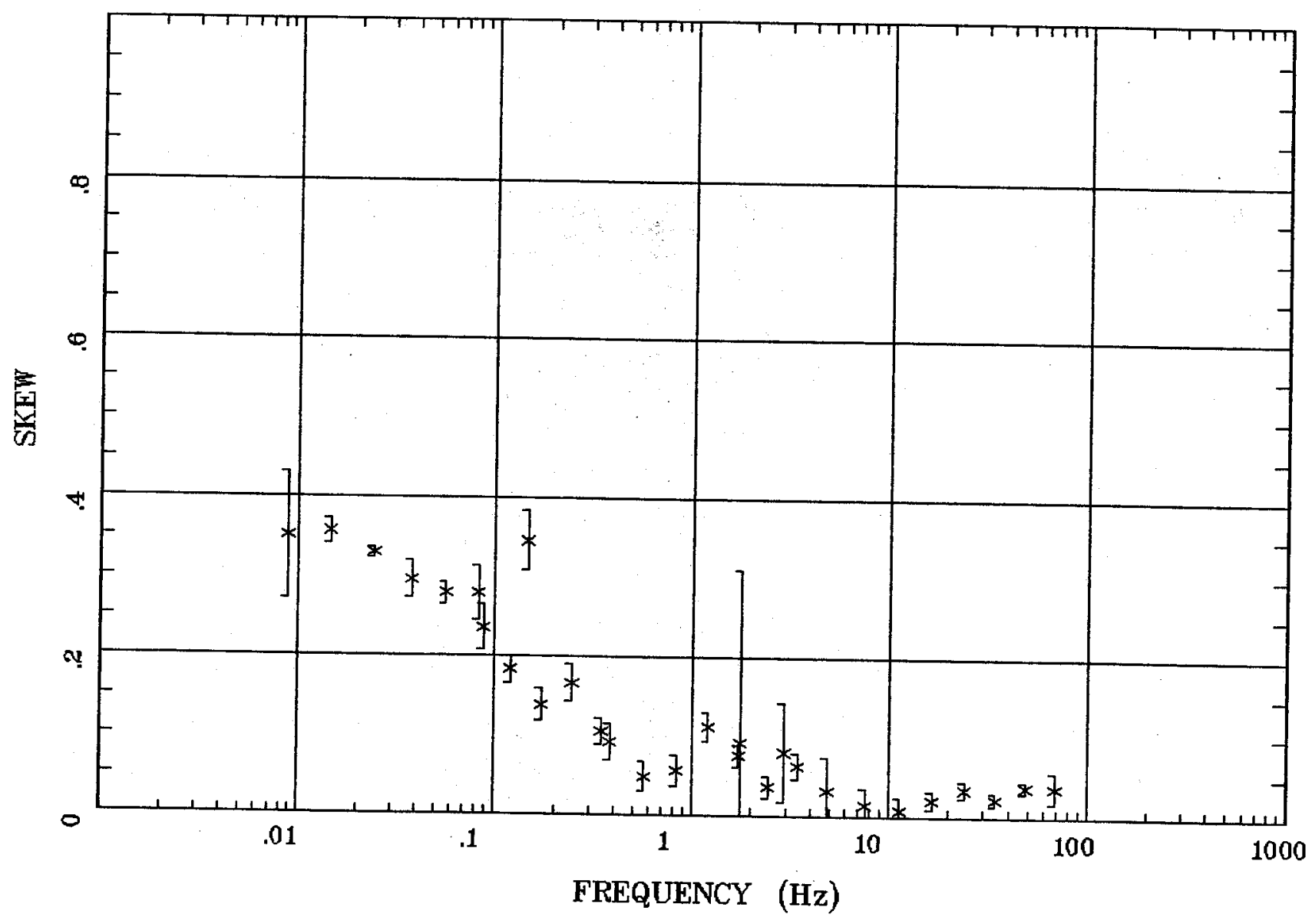

Client:

Remote: none

Acquired: 10:0 Jul 21, 2007 Survey Co:USGS
Rotation:

Filename: sl39mall.avg

Channels: Ch1 Ch2 Ch3 Ch4 Ch5 Ch3 Ch4 Plotted: 11:13 Nov 06, 2007

< EMI - ElectroMagnetic Instruments > 
E MULT Coh. Alamosa, co $100 \mathrm{k}$

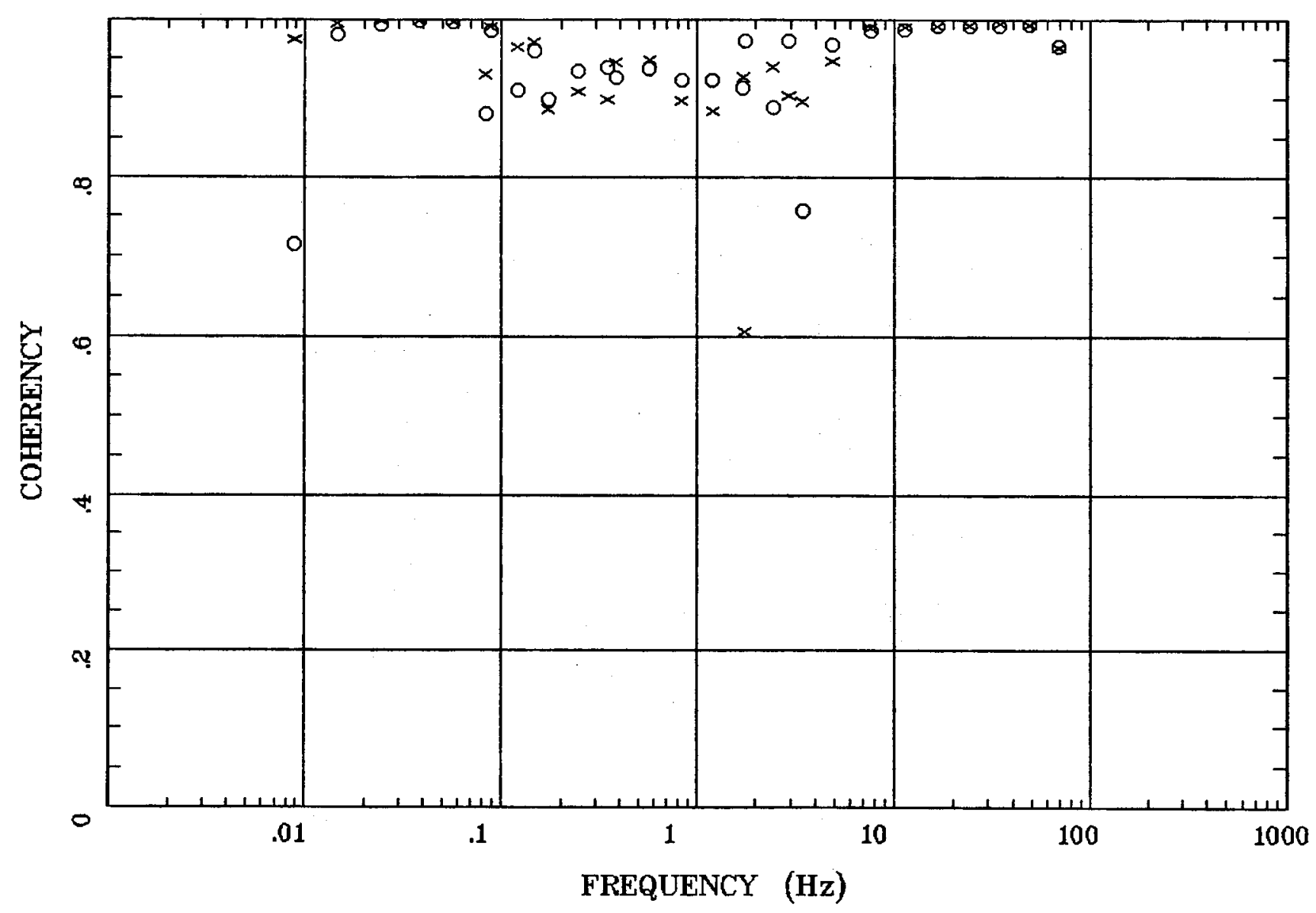

Client:

Remote: none

Acquired: 10:0 Jul 21, 2007

Survey Co:USGS
Rotation:

Filename: sl39mall.avg

Channels: Ch1 Ch2 Ch3 Ch4 Ch5 Ch3 Ch4

Plotted: 11:13 Nov 06, 2007

< EMI - ElectroMagnetic Instruments > 
Alamosa, CO 100k

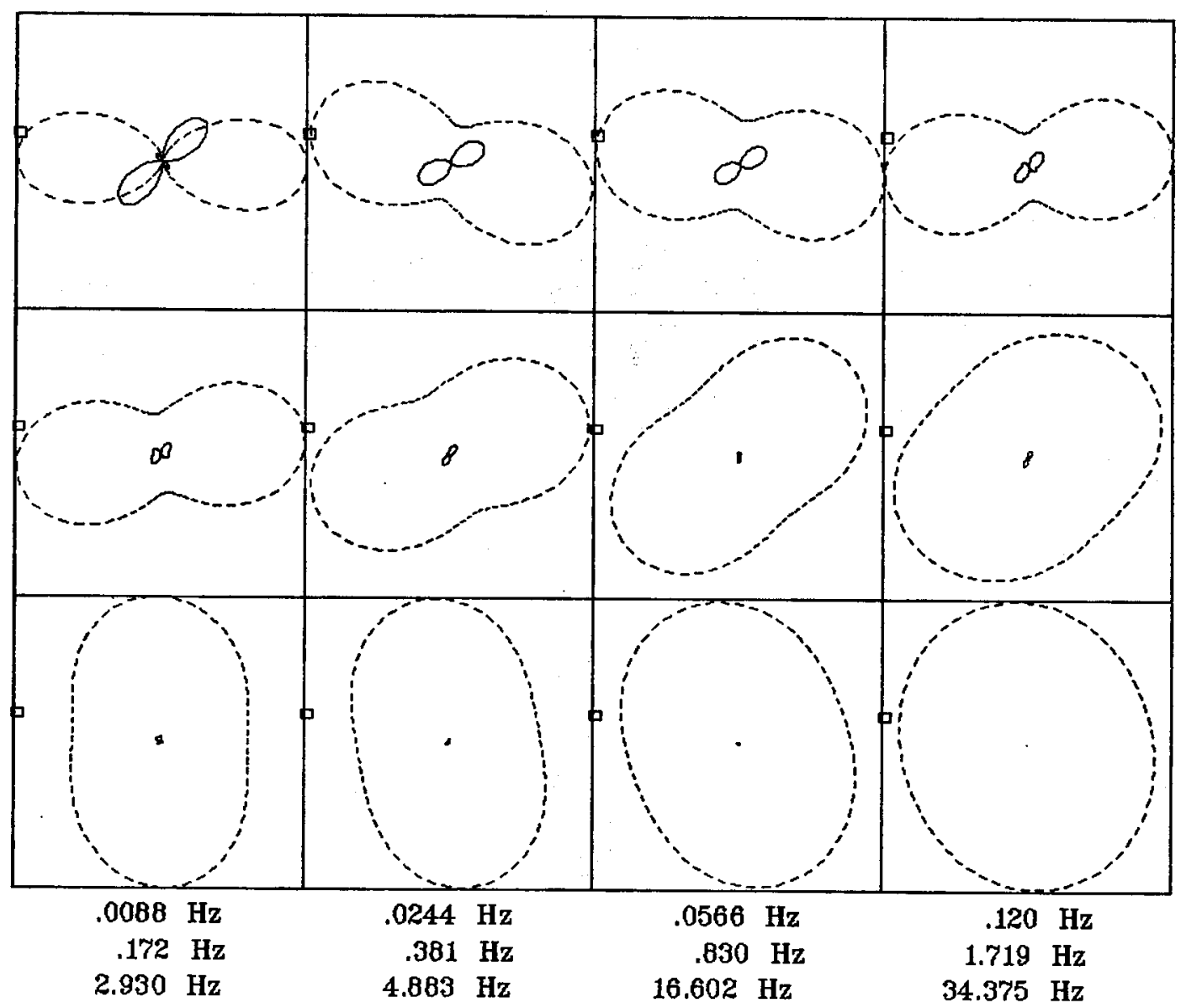

\section{Client:}

Remote: none

Acquired: 10:0 Jul 21, 2007 Survey Co:USGS
Rotation:

Filename: sl39mall.avg

Channels: Ch1 Ch2 Ch3 Ch4 Ch5 Ch3 Ch4 Plotted: 11:13 Nov 06, 2007

< EMI - ElectroMagnetic Instruments > 
TIPPER MAGNITUDE

Alamosa, Co 100k

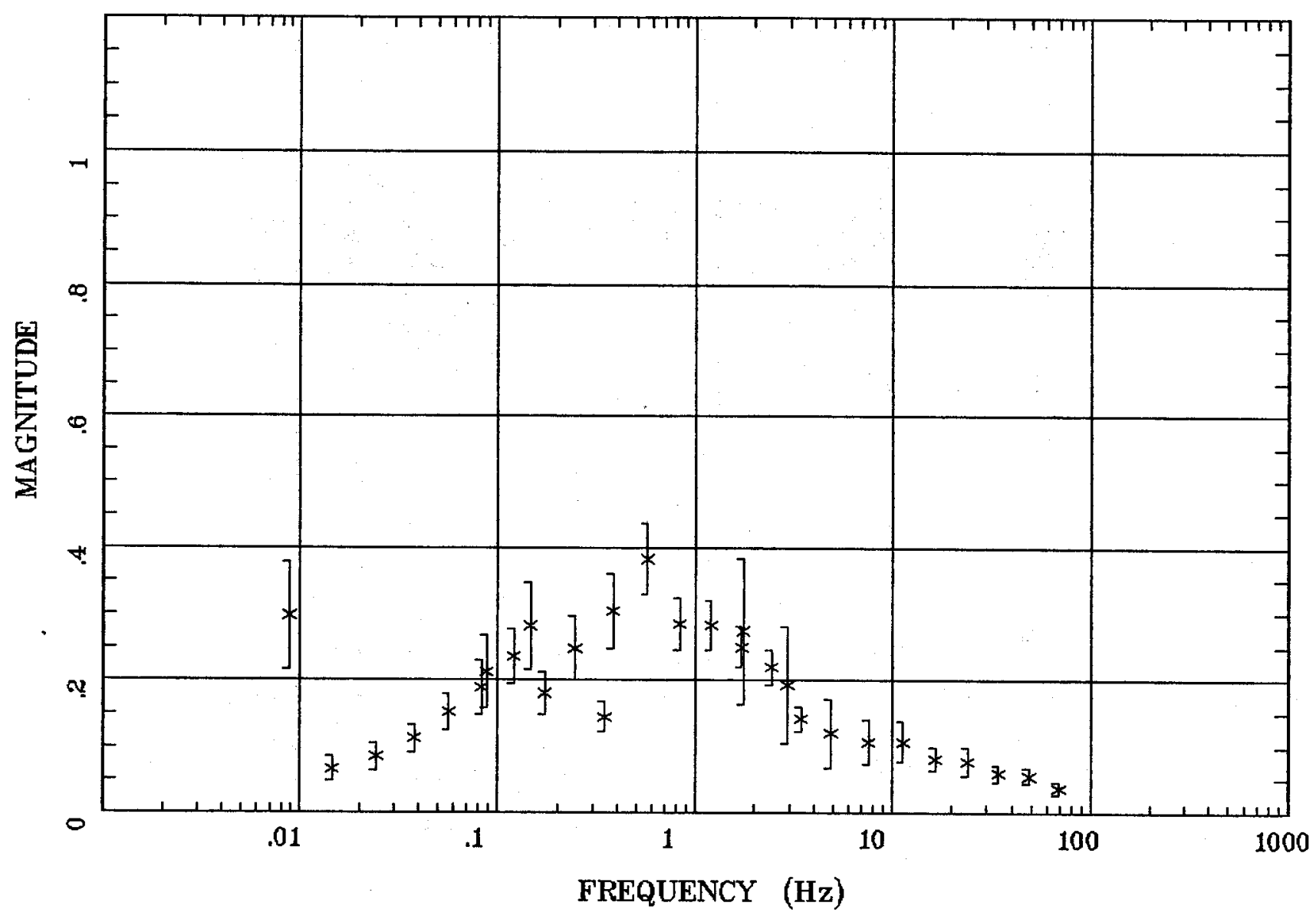

Client:

Remote: none

Acquired: 10:0 Jul 21, 2007

Survey Co:USGS
Rotation:

Filename: sl39mall.avg

Channels: Ch1 Ch2 Ch3 Ch4 Ch5 Ch3 Ch4

Plotted: 11:13 Nov 06, 2007

$<$ EMI - ElectroMagnetic Instruments 
Alamosa, CO 100k

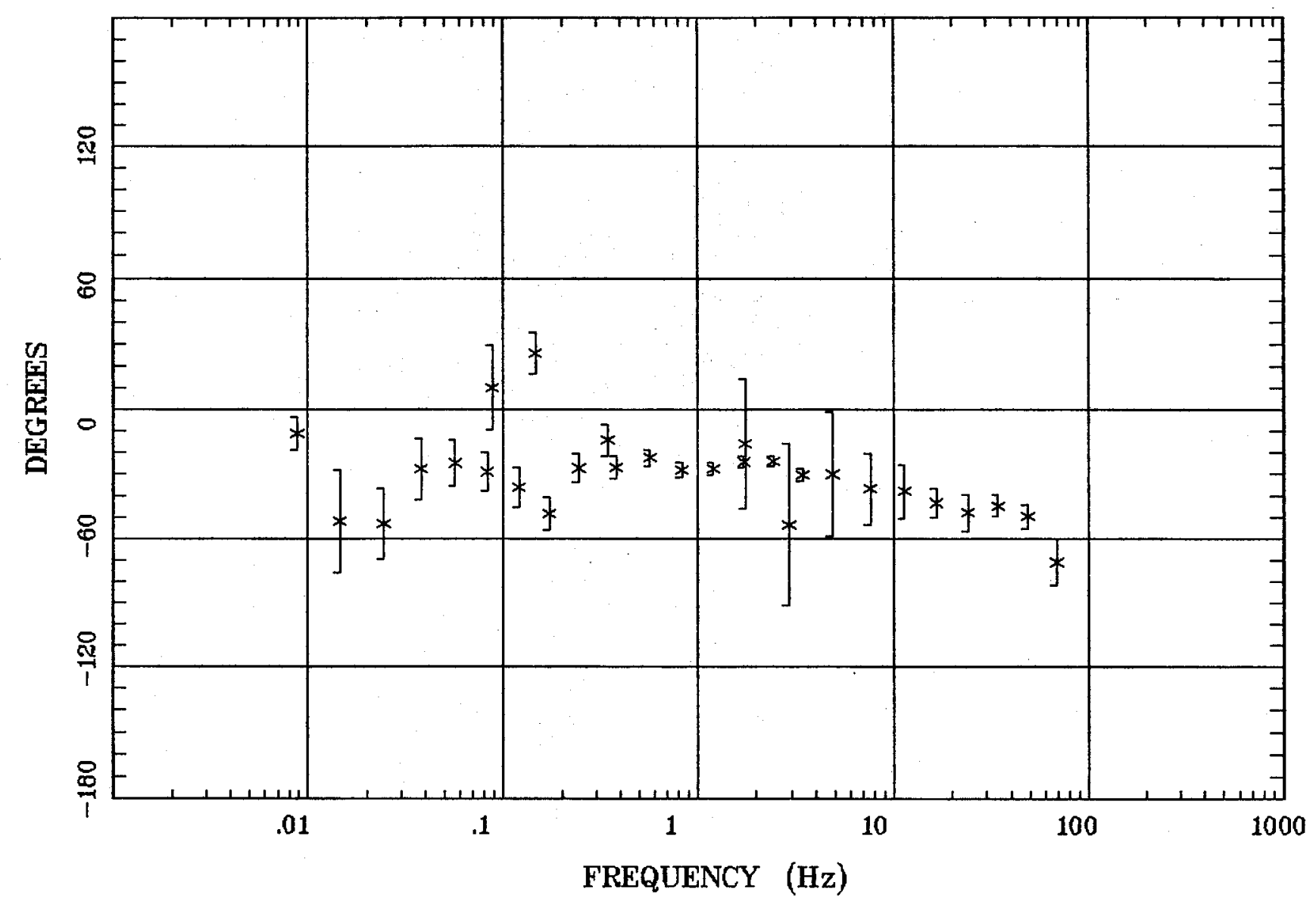

Client:

Remote: none

Acquired: 10:0 Jul 21, 2007 Survey Co:USGS
Rotation:

Filename: sl39mall.avg

Channels: Ch1 Ch2 Ch3 Ch4 Ch5 Ch3 Ch4 Plotted: 11:13 Nov 06, 2007

< EMI - ElectroMagnetic Instruments 
HzHx.x Coh HzHy.o Alamosa, CO 100k

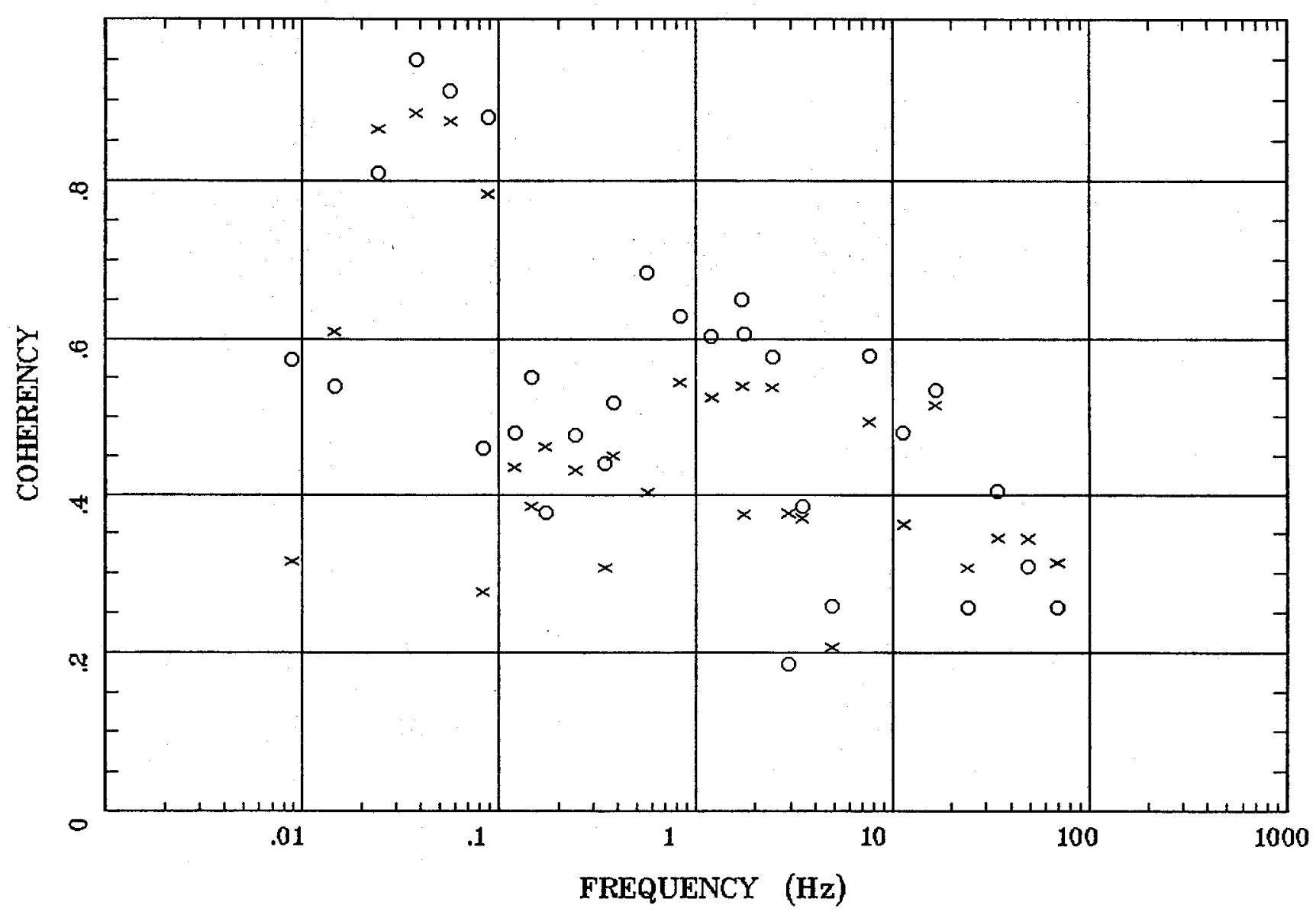

Client:

Remote: none

Acquired: 10:0 Jul 21, 2007 Survey Co:USGS
Rotation:

Filename: sl39mall.avg

Channels: Ch1 Ch2 Ch3 Ch4 Ch5 Ch3 Ch4 Plotted: 11:13 Nov 06, 2007

< EMI - ElectroMagnetic Instruments 
Alamosa, CO 100k

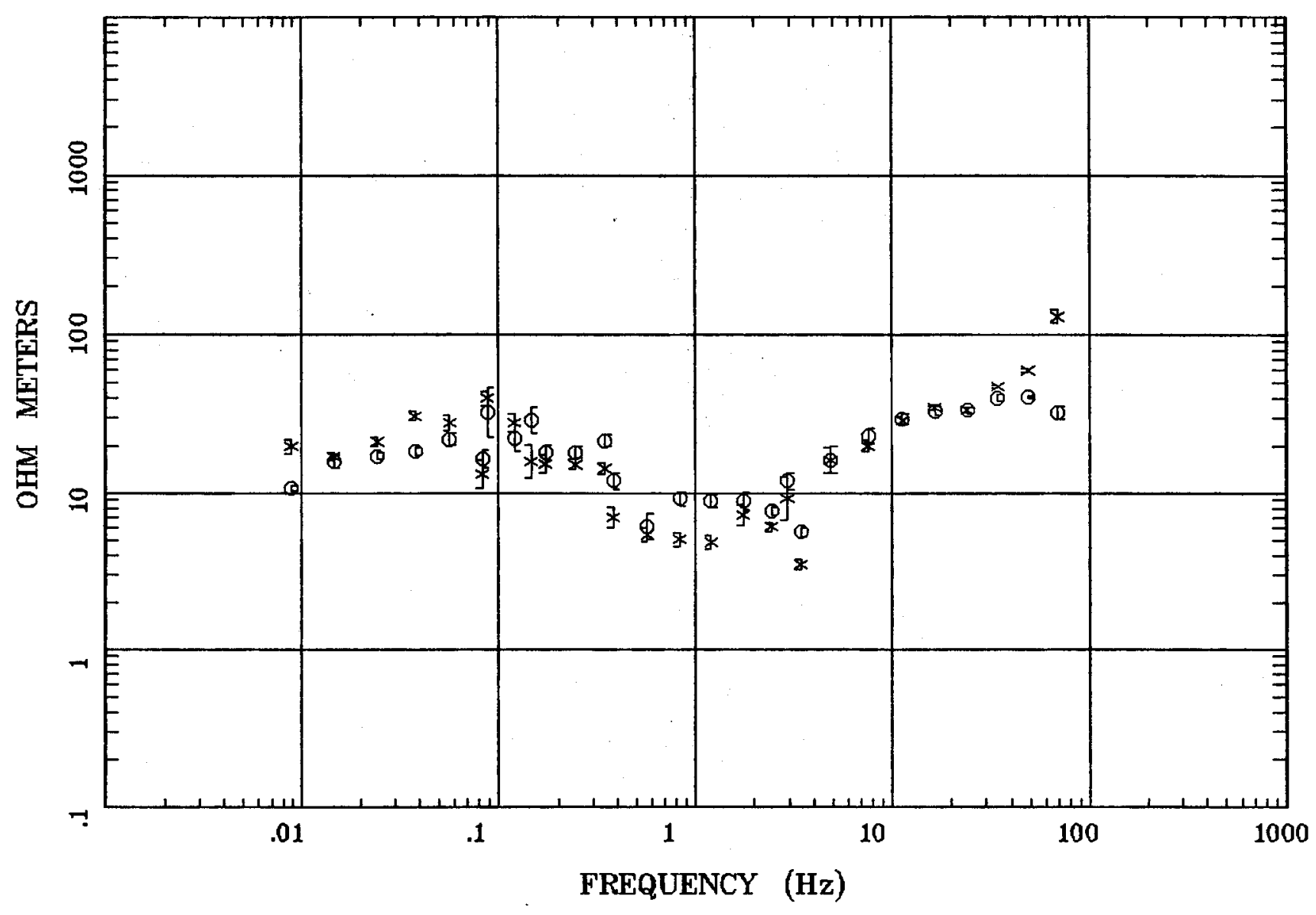

Client:

Remote: none

Acquired: 09:1 Jul 22, 2007

Survey Co:USGS
Rotation:

Filename: sl40m1.avg

Channels: Ch1 Ch2 Ch3 ch4 Ch5 Ch3 Ch4 Plotted: 11:12 Nov 06, 2007

< EMI - ElectroMagnetic Instruments 
IMPEDANCE PHASE

Alamosa, CO 100k

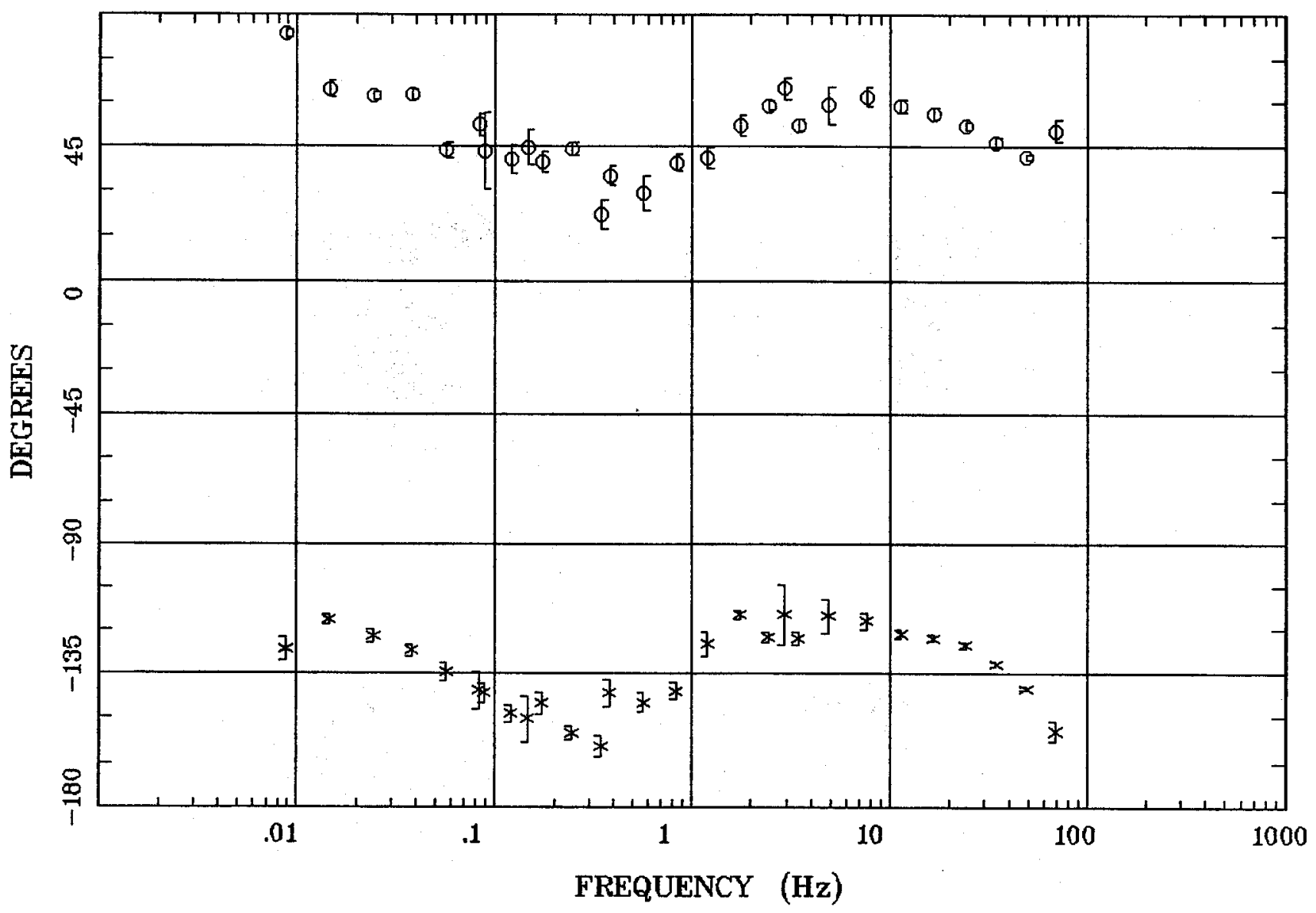

Client:

Remote: none

Acquired: 09:1 Jul 22, 2007

Survey Co:USGS
Rotation:

Filename: sl40m1.avg

Channels: Ch1 Ch2 Ch3 Ch4 Ch5 Ch3 Ch4

Plotted: 11:12 Nov 06, 2007

< EMI - ElectroMagnetic Instruments > 
Station $\mathbf{4 0}$

\section{ROTATION ANGLE}

Alamosa, CO 100k

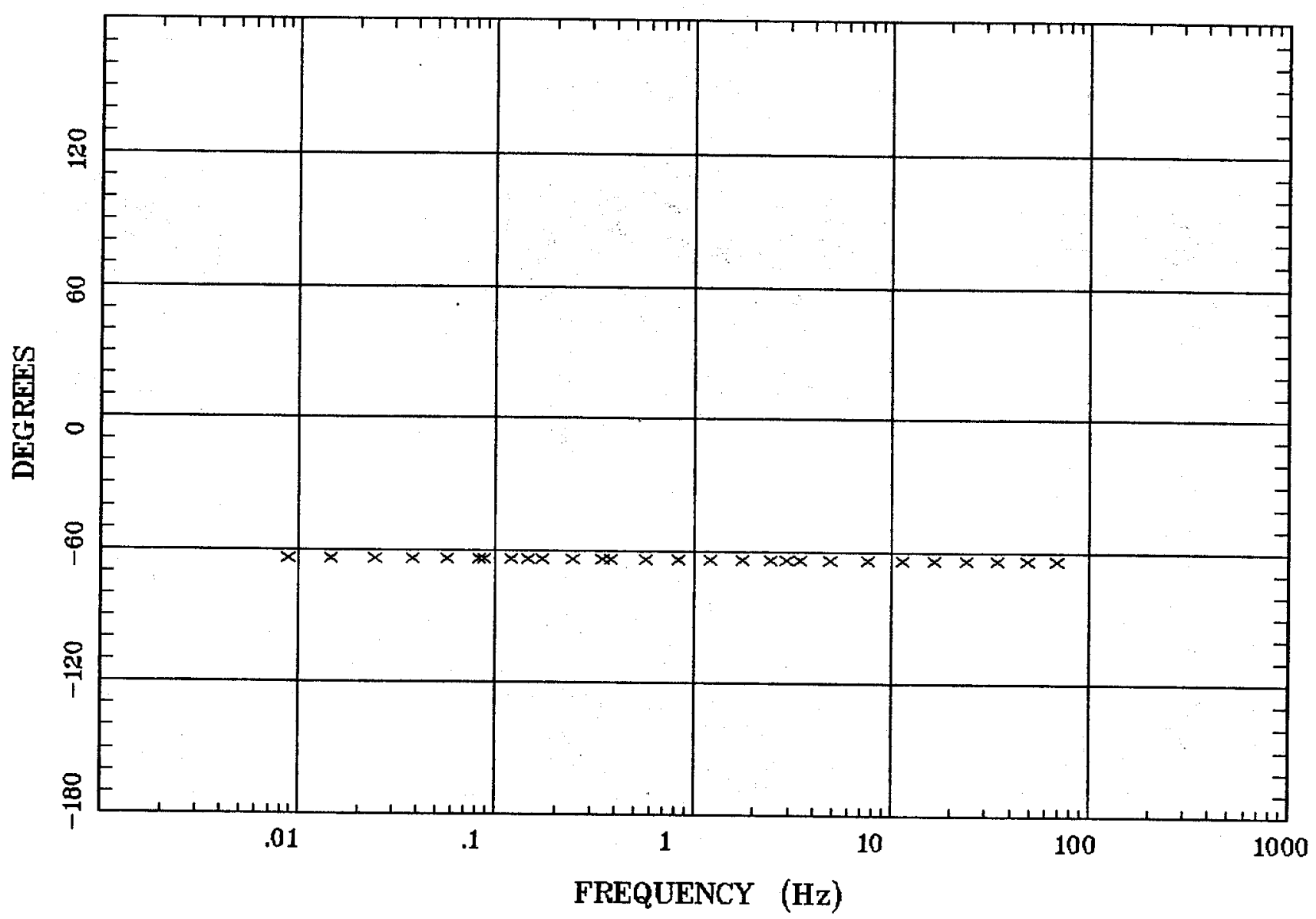

Client:

Remote: none

Acquired: 09:1 Jul 22, 2007

Survey Co:USGS
Rotation:

Filename: sl40m1.avg

Channels: Ch1 Ch2 Ch3 ch4 Ch5 Ch3 Ch4 Plotted: 11:12 Nov 06, 2007

$<$ EMI - ElectroMagnetic Instruments 
Alamosa, CO 100k

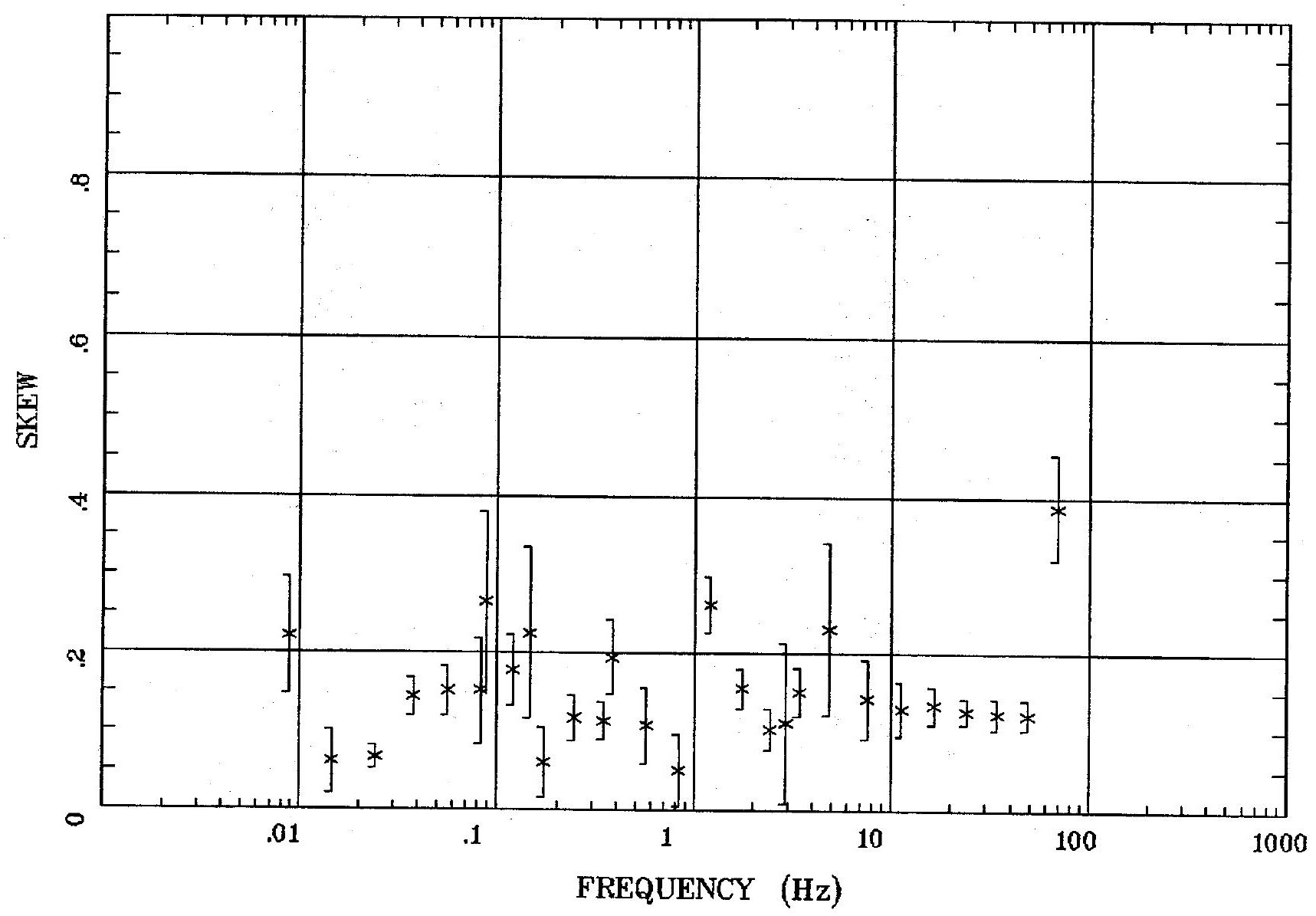

Client:

Remote: none

Acquired: 09:1 Jul 22, 2007 Survey Co:USGS
Rotation:

Filename: sl40m1.avg

Channels: Ch1 Ch2 Ch3 Ch4 Ch5 Ch3 Ch4 Plotted: 11:12 Nov 06, 2007

$<$ EMI - ElectroMagnetic Instruments 
E MULT Coh.

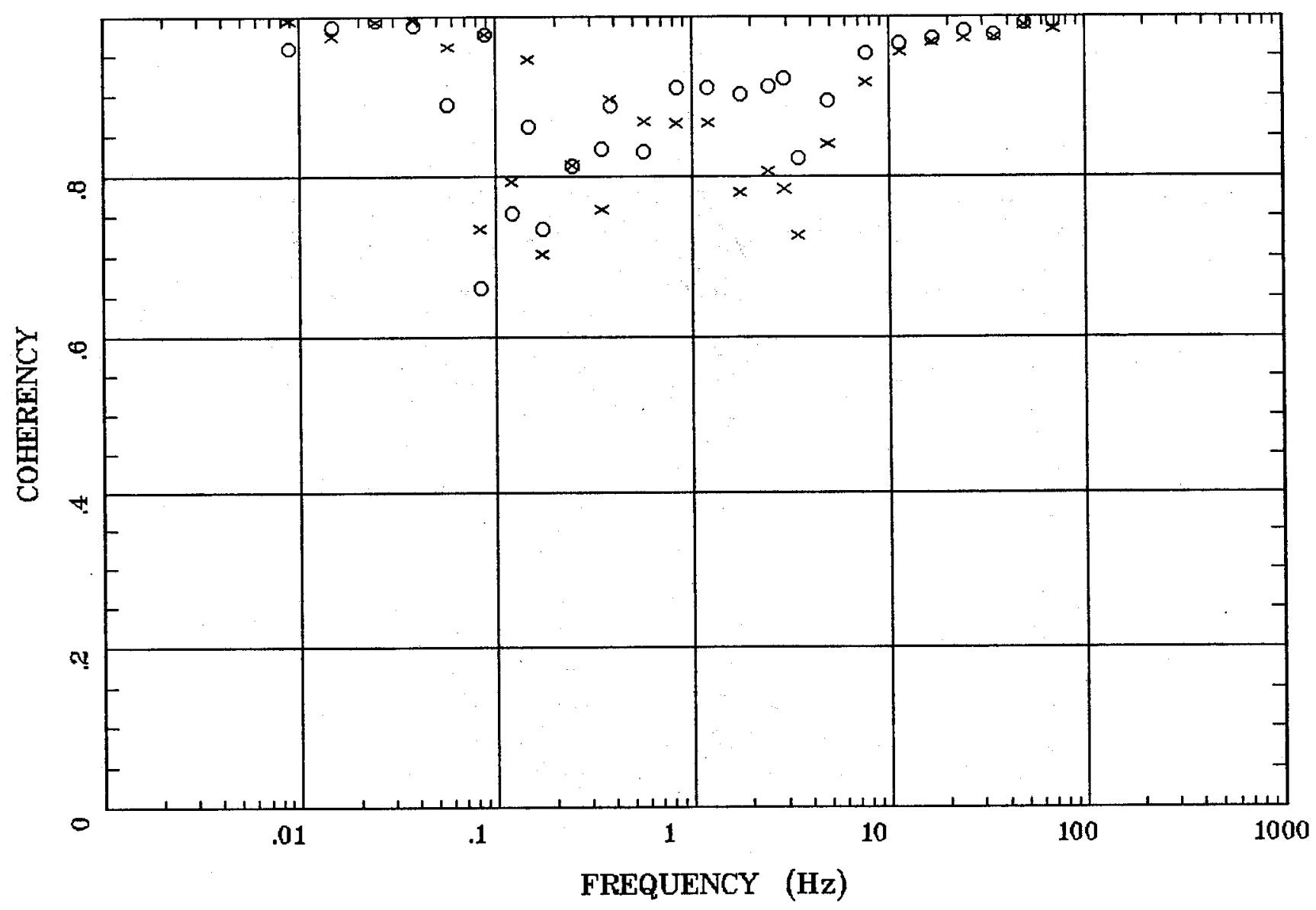

Client:

Remote: none

Acquired: 09:1 Jul 22, 2007 Survey Co:USGS

Alamosa, CO 100k 


\section{POLAR PLOTS}

Alamosa, CO 100k

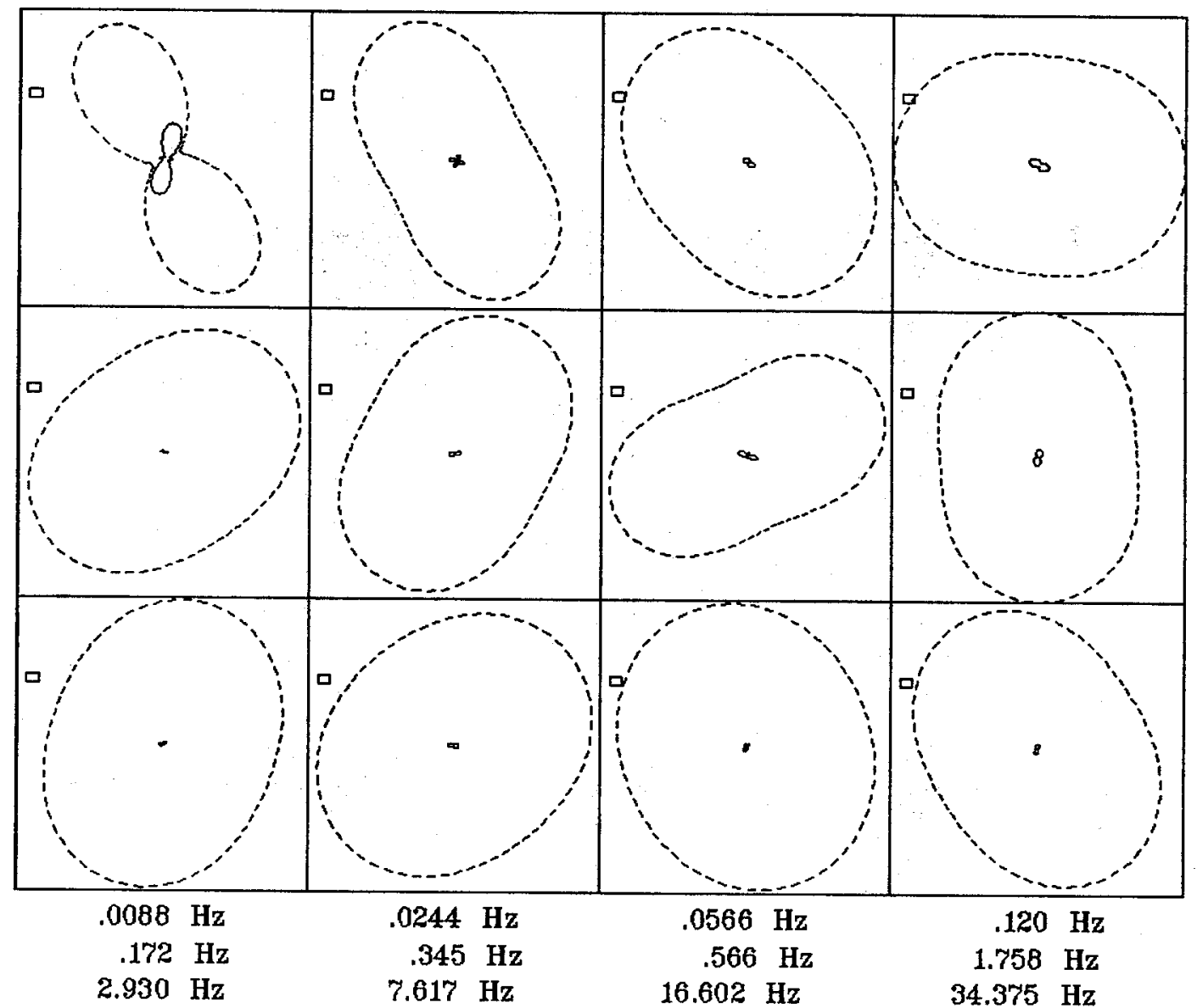

Client:

Rem ote: none

Acquired: 09:1 Jul 22, 2007

Survey Co:USGS
Rotation:

Filename: sl40m1.avg

Channels: Ch1 Ch2 Ch3 Ch4 Ch5 Ch3 Ch4 Plotted: 11:12 Nov 06, 2007

$<$ EMI - ElectroMagnetic Instruments 


\section{Station 40}

TIPPER MAGNITUDE

Alamosa, CO 100k

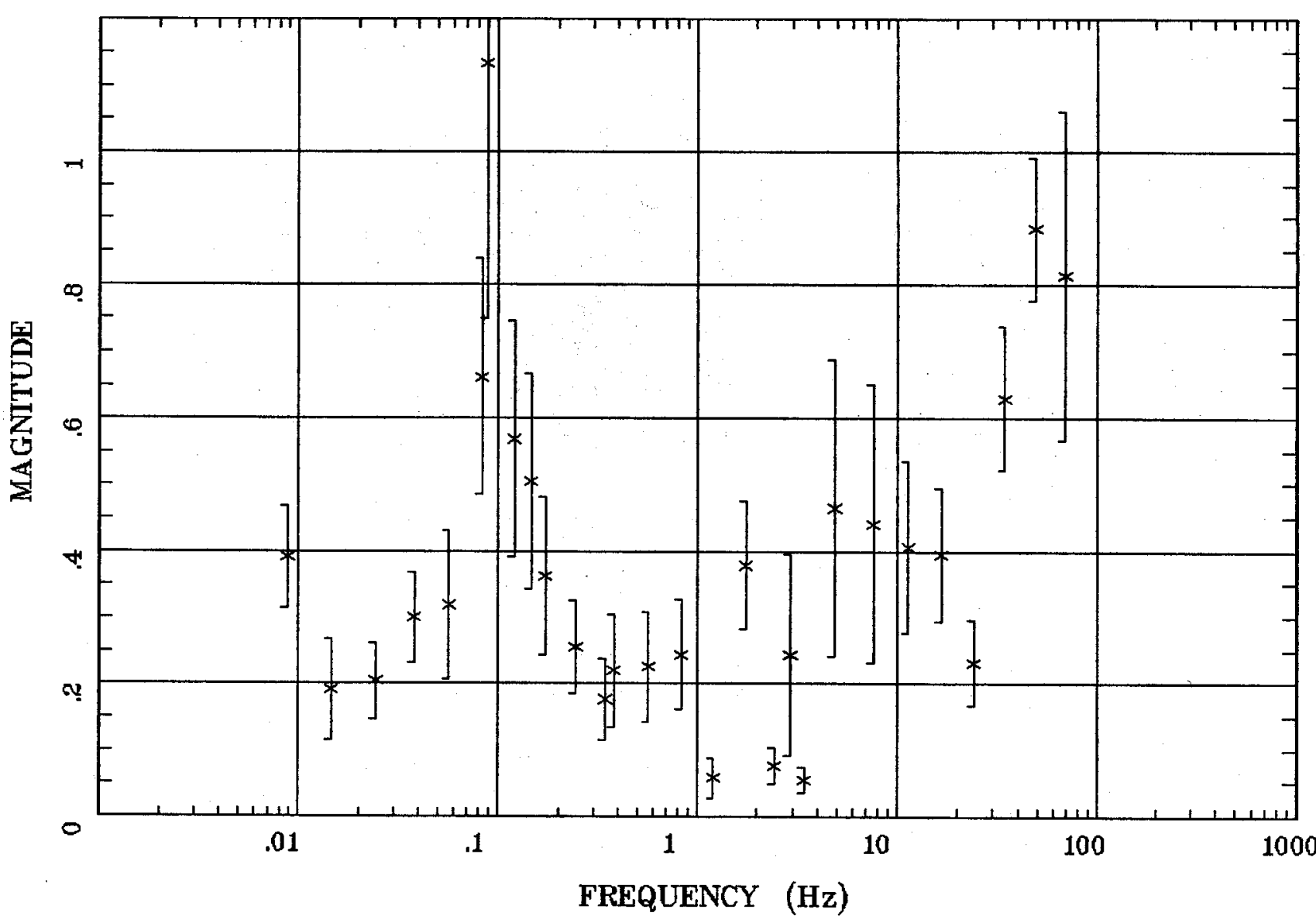

Client:

Remote: none

Acquired: 09:1 Jul 22, 2007

Survey Co:USGS
Rotation:

Filename: $s \mathrm{l} 40 \mathrm{~m} 1 . \mathrm{avg}$

Channels: Ch1 Ch2 Ch3 Ch4 Ch5 Ch3 Ch4

Plotted: 11:12 Nov 06, 2007

< EMI - ElectroMagnetic Instruments 
Alamosa, CO 100k

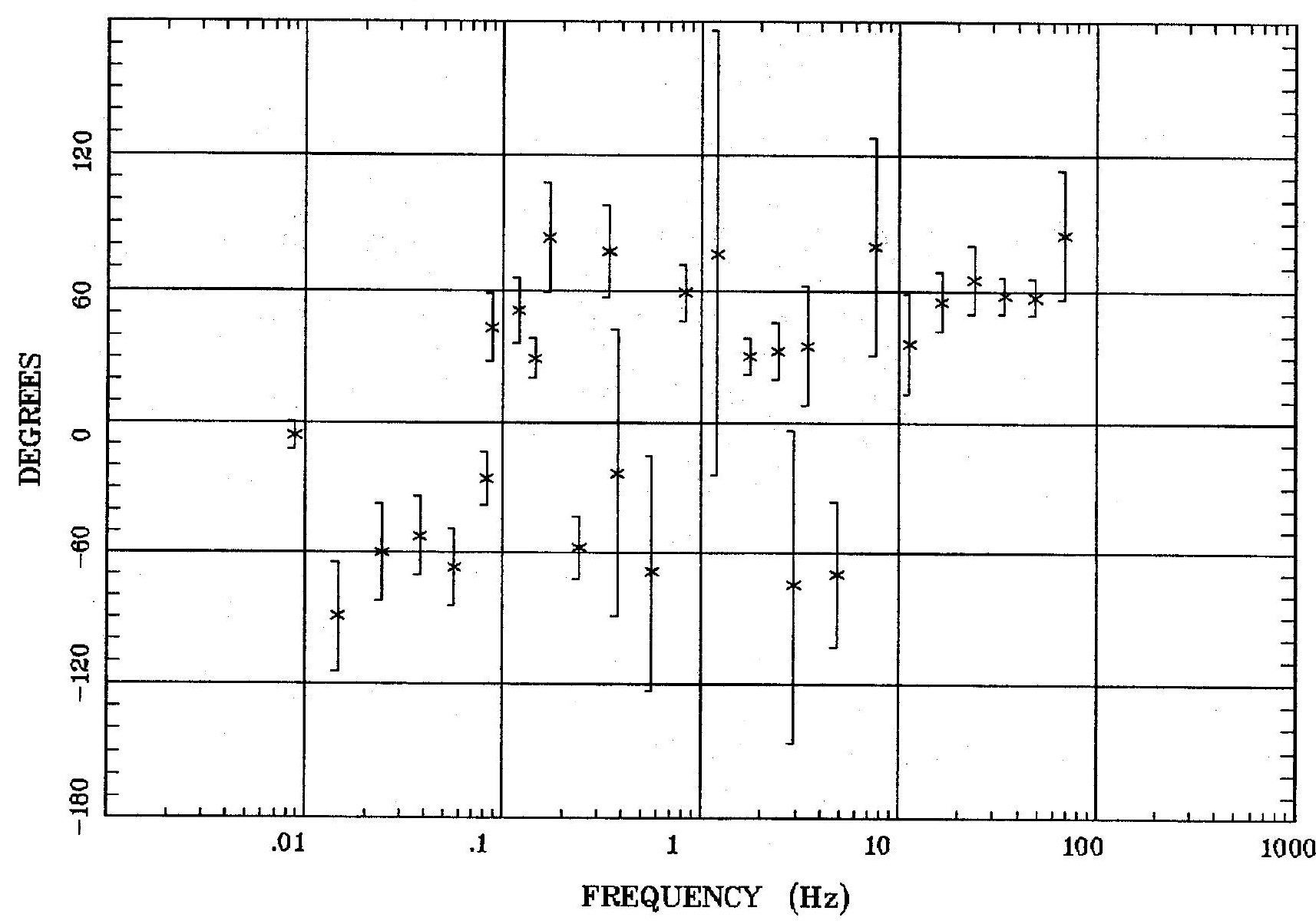

Client:

Remote: none

Acquired: 09:1 Jul 22, 2007 Survey Co:USGS
Rotation:

Filename: sl40m1.avg

Channels: Ch1 Ch2 Ch3 Ch4 Ch5 Ch3 Ch4 Plotted: 11:12 Nov 06, 2007

< EMI - ElectroMagnetic Instruments > 
HzHx.x Coh HzHy.o

Alamosa, CO 100k

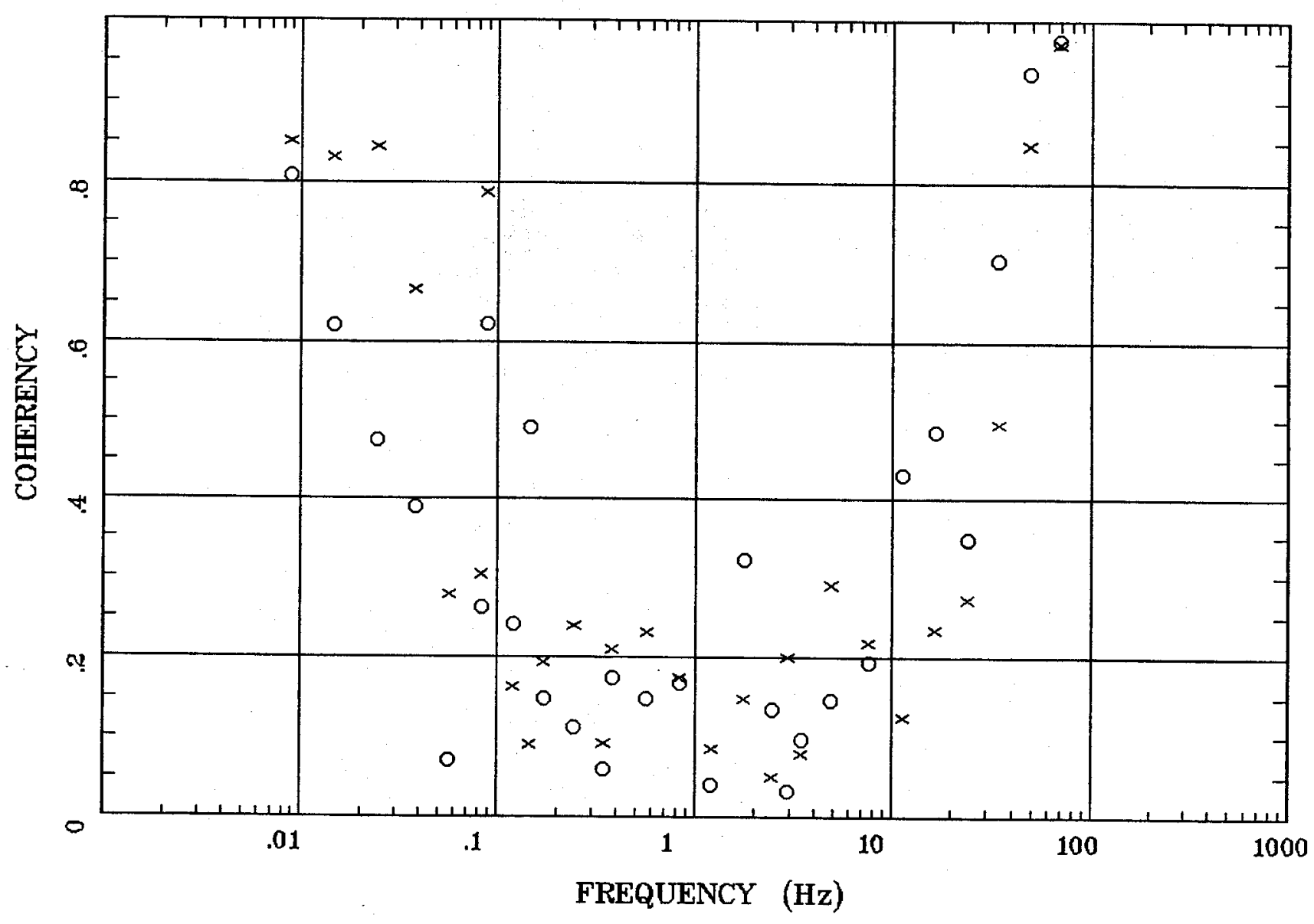

Client:

Remote: none

Acquired: 09:1 Jul 22, 2007 Survey Co:USGS
Rotation:

Filename: sl40m1.avg

Channels: Ch1 Ch2 Ch3 Ch4 Ch5 Ch3 Ch4 Plotted: 11:12 Nop 06, 2007

< EMI - ElectroMagnetic Instruments > 
Alamosa, Co $100 k$

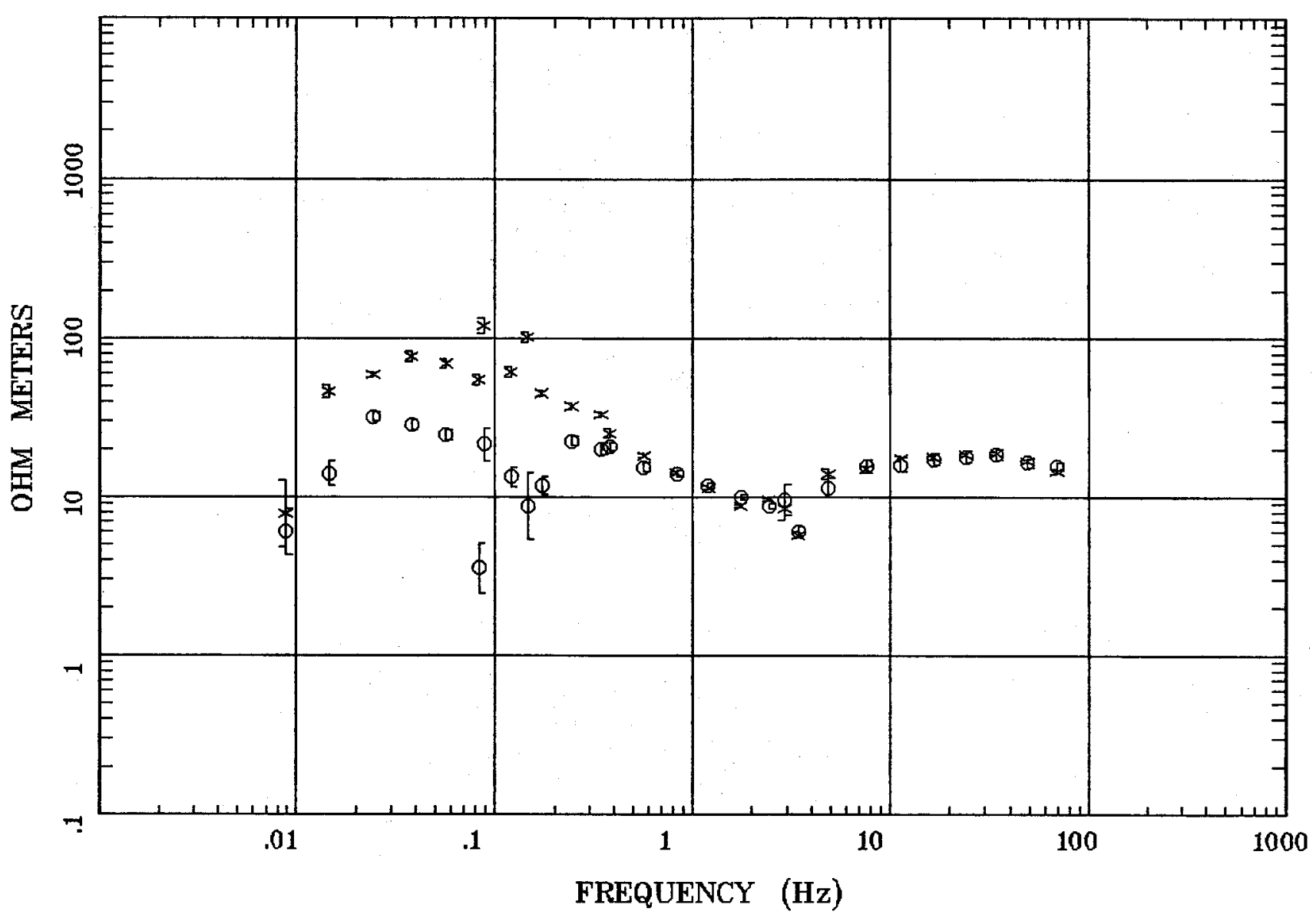

Client:

Remote: none

Acquired: 10:1 Jul 23, 2007 Survey Co:USGS
Rotation:

Filename: sl41m.avg

Channels: Ch1 Ch2 Ch3 Ch4 Ch5 Ch3 Ch4 Plotted: 11:19 Nov 06, 2007

< EMI - ElectroMagnetic Instruments > 


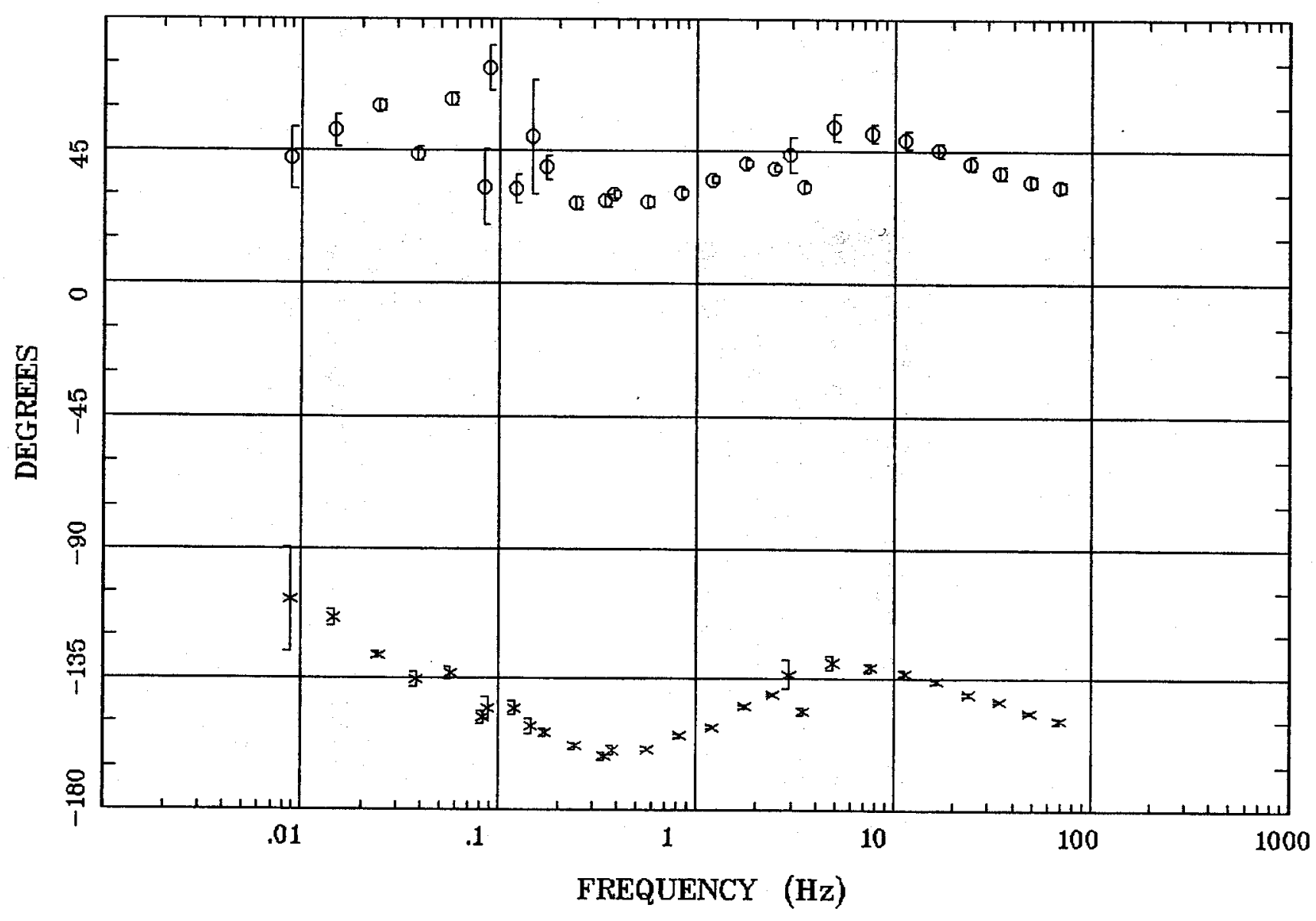

Client:

Remate: none

Acquired: 10:1 Jul 23, 2007

Survey Co:USGS
Rotation:

Filename: sl41m.avg

Channels: Ch1 Ch2 Ch3 Ch4 Ch5 Ch3 Ch4 Plotted: 11:19 Nov 06, 2007

$<$ EMI - ElectroMagnetic Instruments 


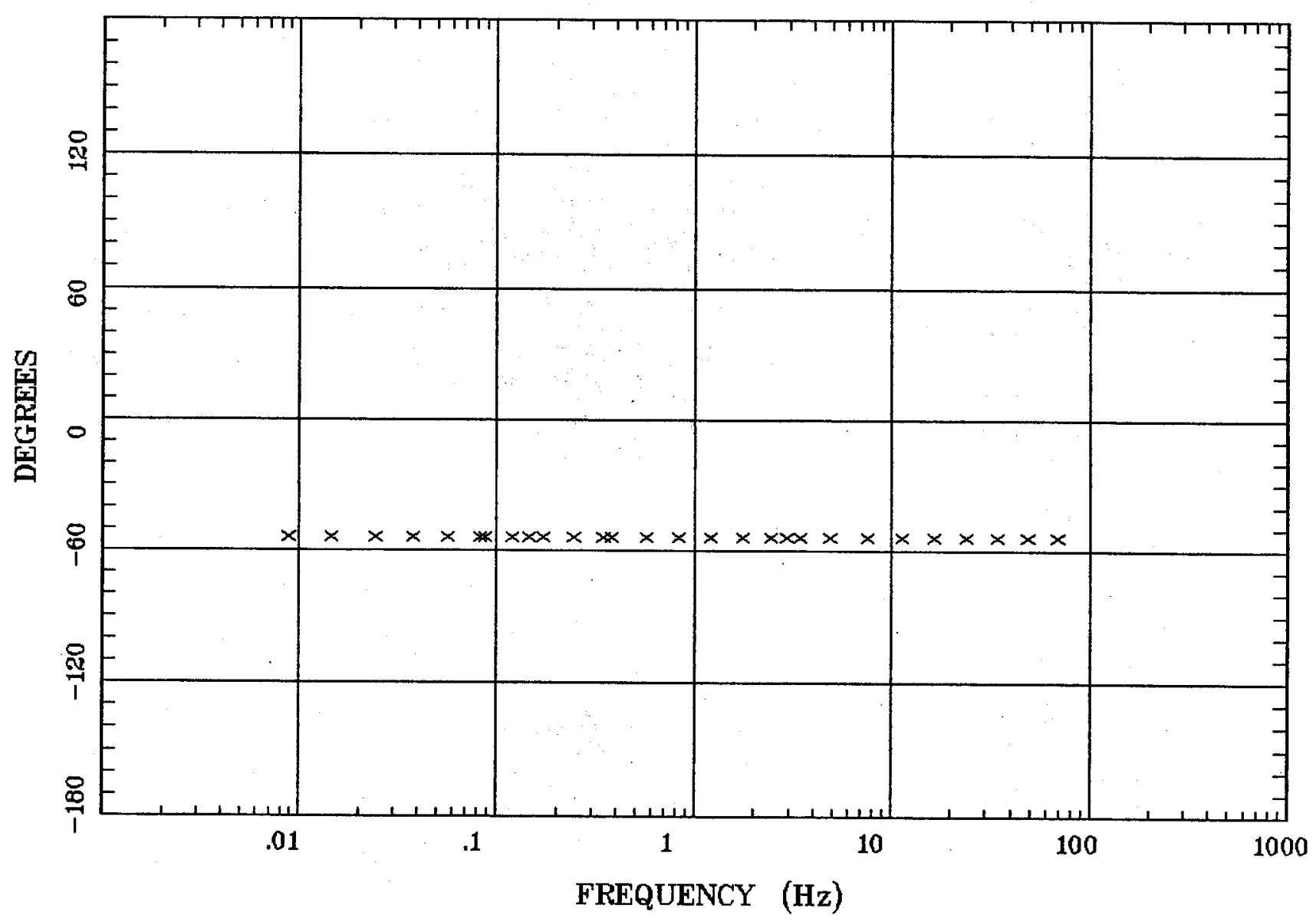

Client:

Remote: none

Acquired: 10:1 Jul 23, 2007

Survey Co:USGS
Rotation:

Filename: sl41m.avg

Channels: Ch1 Ch2 Ch3 Ch4 Ch5 Ch3 Ch4

Plotted: 11:19 Nov 06, 2007

$<$ EMI - ElectroMagnetic Instruments 


\section{Station 41}

Alamosa, CO 100k

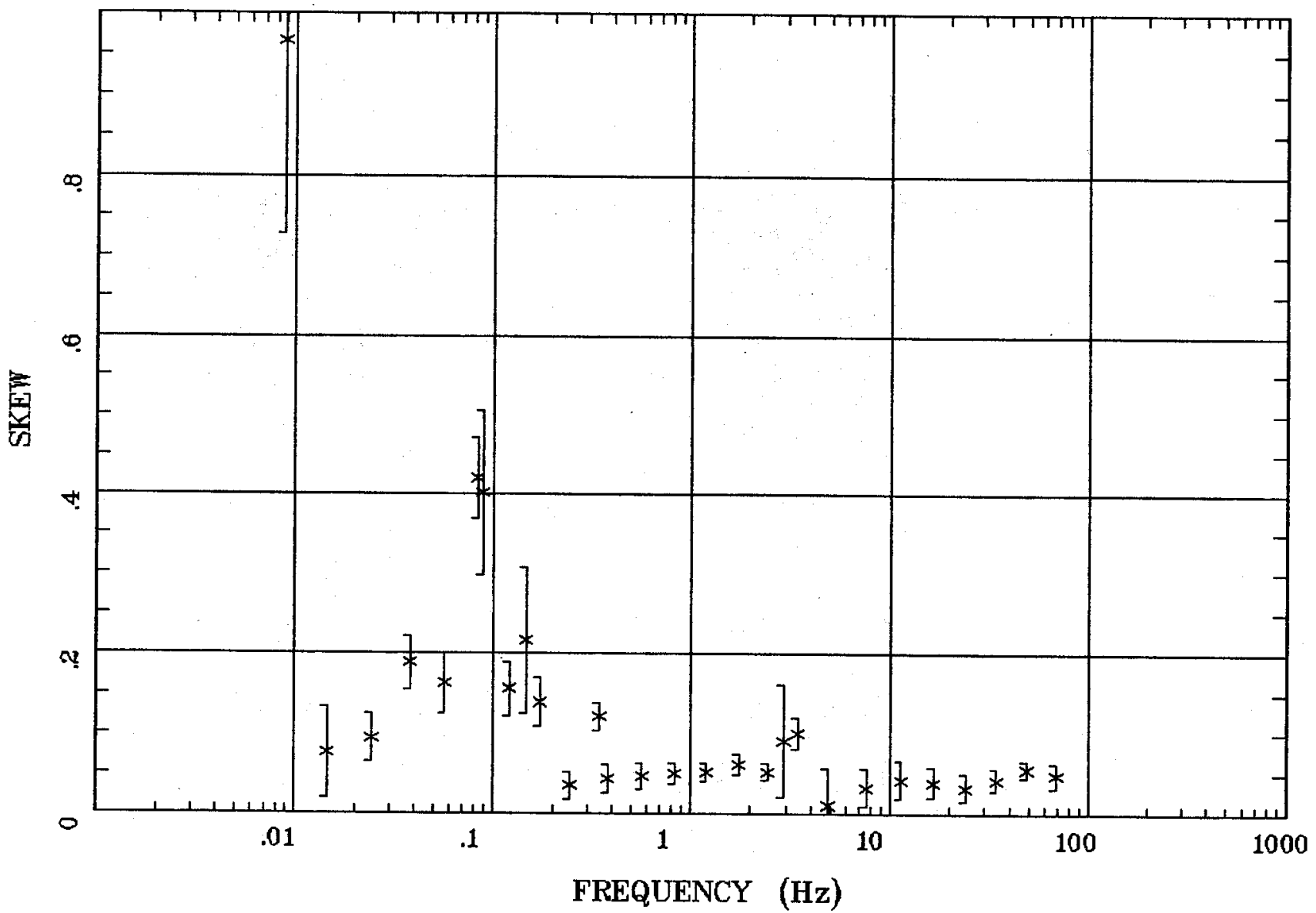

Client:

Remote: none

Acquired: 10:1 Jul 23, 2007 Survey Co:USGS
Rotation:

Filename: sl41m.avg

Channels: Ch1 Ch2 Ch3 Ch4 Ch5 Ch3 Ch4

Plotted: 11:19 Nov 06, 2007

< EMI - ElectroMagnetic Instruments > 
E MULT Coh.

Alamosa, CO 100k

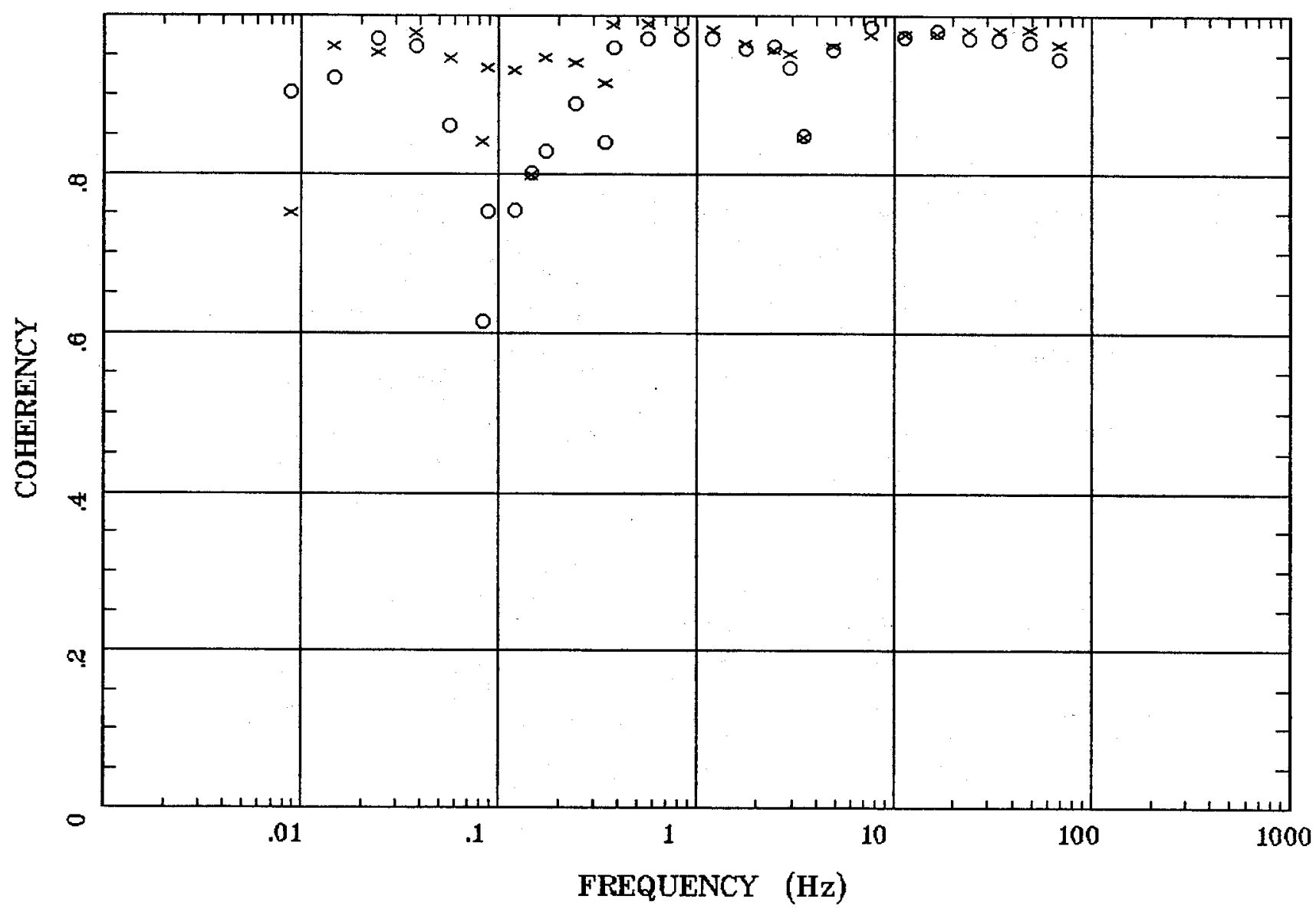

Client:

Remote: none

Acquired: 10:1 Jul 23, 2007 Survey Co:USGS
Rotation:

Filename: sl41m.avg

Channels: Ch1 Ch2 Ch3 Ch4 Ch5 Ch3 Ch4

Plotted: 11:19 Nop 06, 2007

< EMI - ElectroMagnetic Instruments 


\section{POLAR PLOTS}

Alamosa, Co $100 \mathrm{k}$

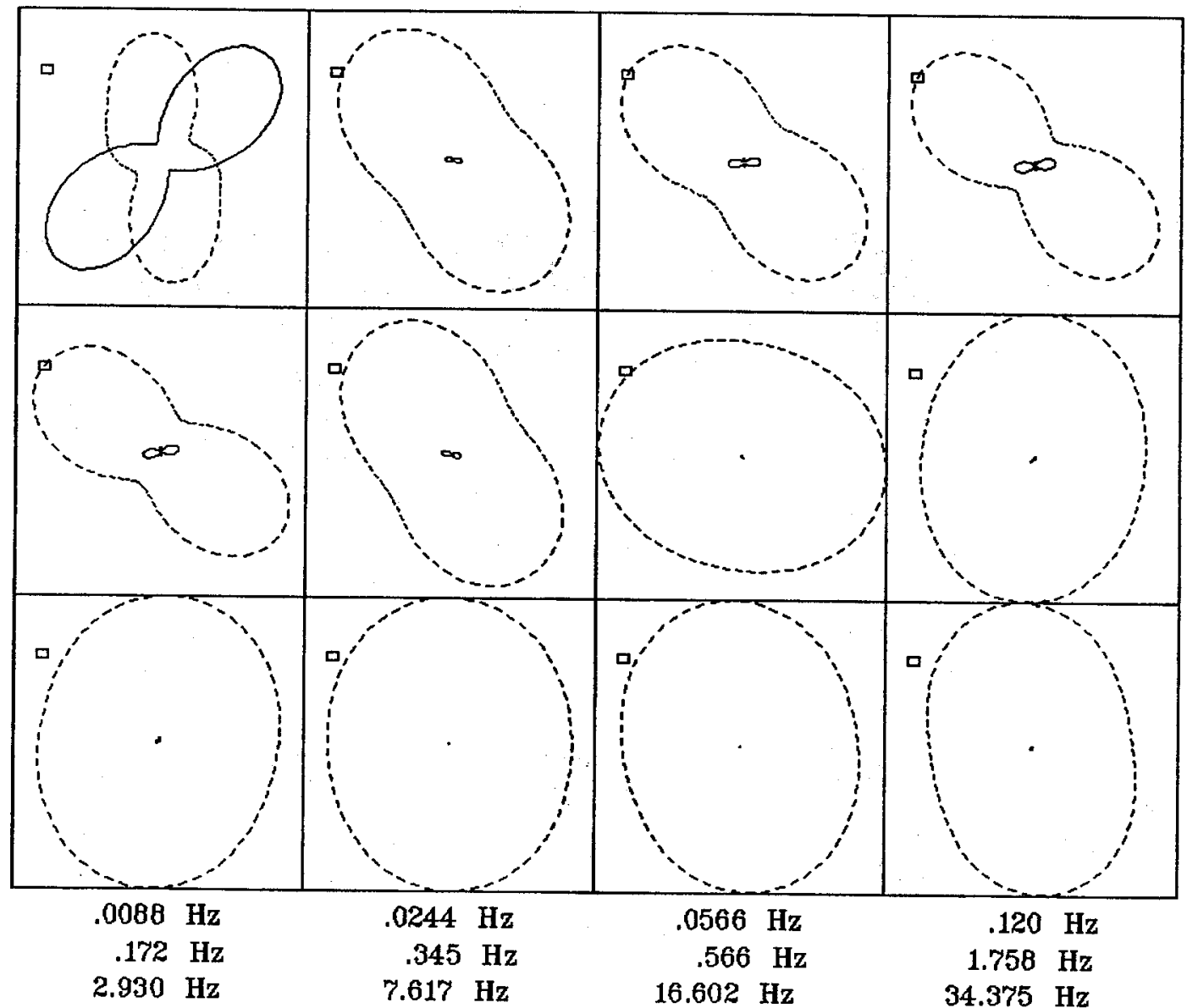

Client:

Remote: none

Acquired: 10:1 Jul 23, 2007 Survey Co:USGS
Rotation:

Filename: sl41m.avg

Channels: Ch1 Ch2 Ch3 Ch4 Ch5 Ch3 Ch4 Plotted: 11:19 Nov 06, 2007

< EMI - ElectroMagnetic Instruments 
Alamosa, CO 100k

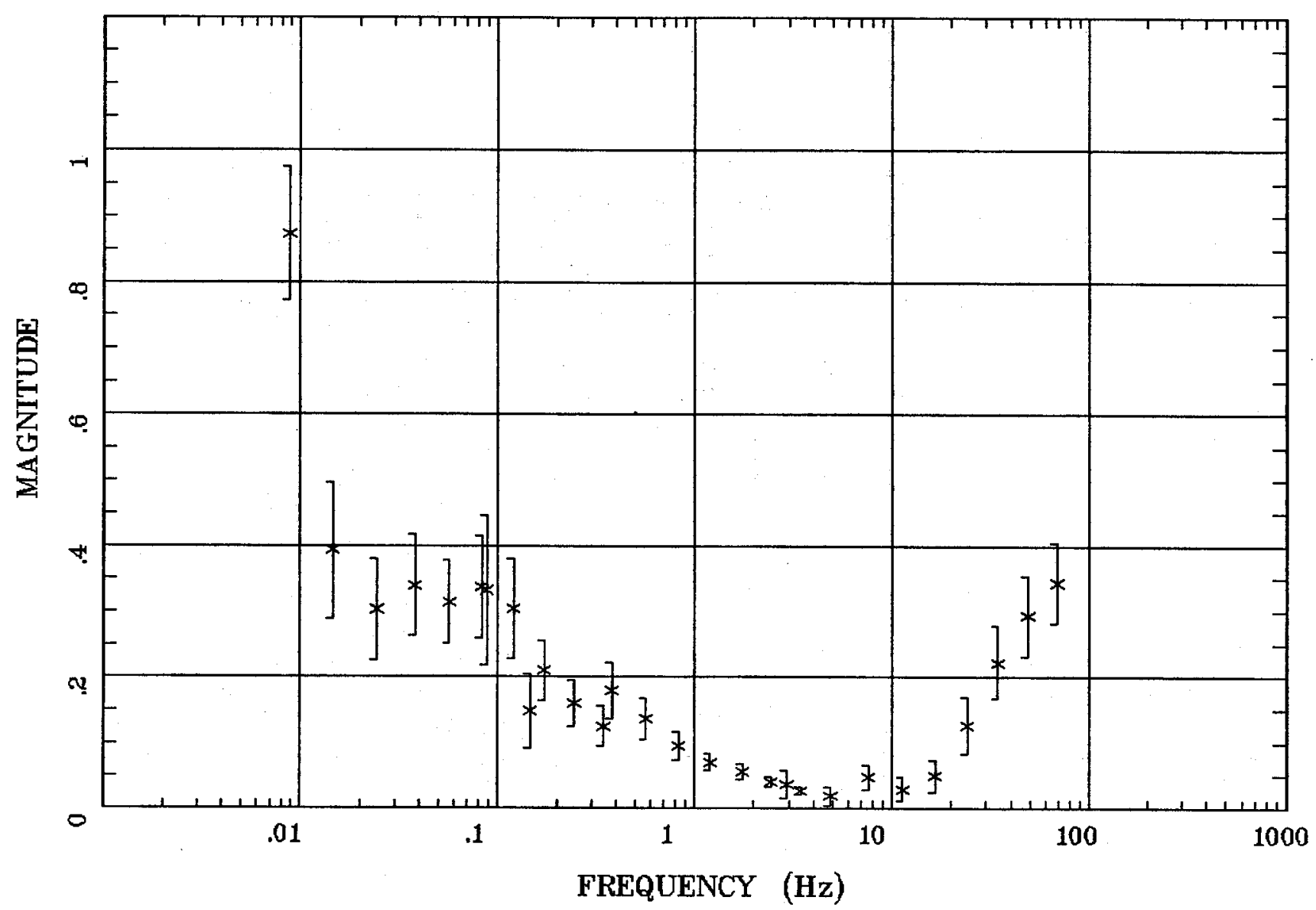

Client:

Remote: none

Acquired: 10:1 Jul 23, 2007

Survey Co:USGS
Rotation:

Filename: sl41m.avg

Channels: $\mathrm{Ch} 1 \mathrm{Ch} 2 \mathrm{Ch} 3 \mathrm{Ch} 4 \mathrm{Ch} 5 \mathrm{Ch} 3 \mathrm{Ch} 4$

Plotted: 11:19 Nov 06, 2007

< EMI - ElectroMagnetic Instruments 
Alamosa, CO 100k

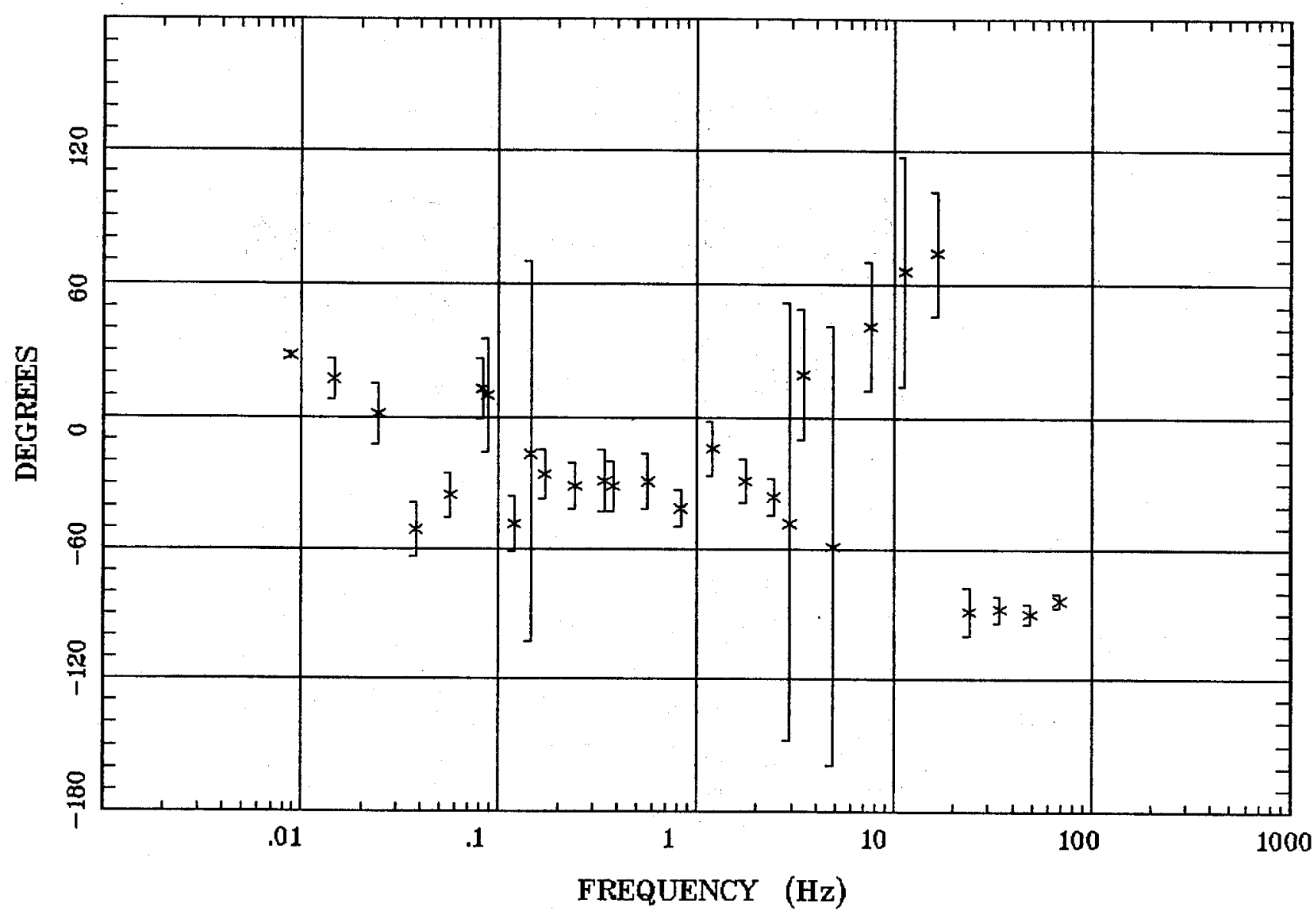

Client:

Remote: none

Acquired: 10:1 Jul 23, 2007 Survey Co:USGS
Rotation:

Filename: sl41m.arg

Channels: Ch1 Ch2 Ch3 Ch4 Ch5 Ch3 Ch4 Plotted: 11:19 Nov 06, 2007

$<$ EMI - ElectroMagnetic Instruments 
HzHx.x Coh HzHy.o

Alamosa, CO 100k

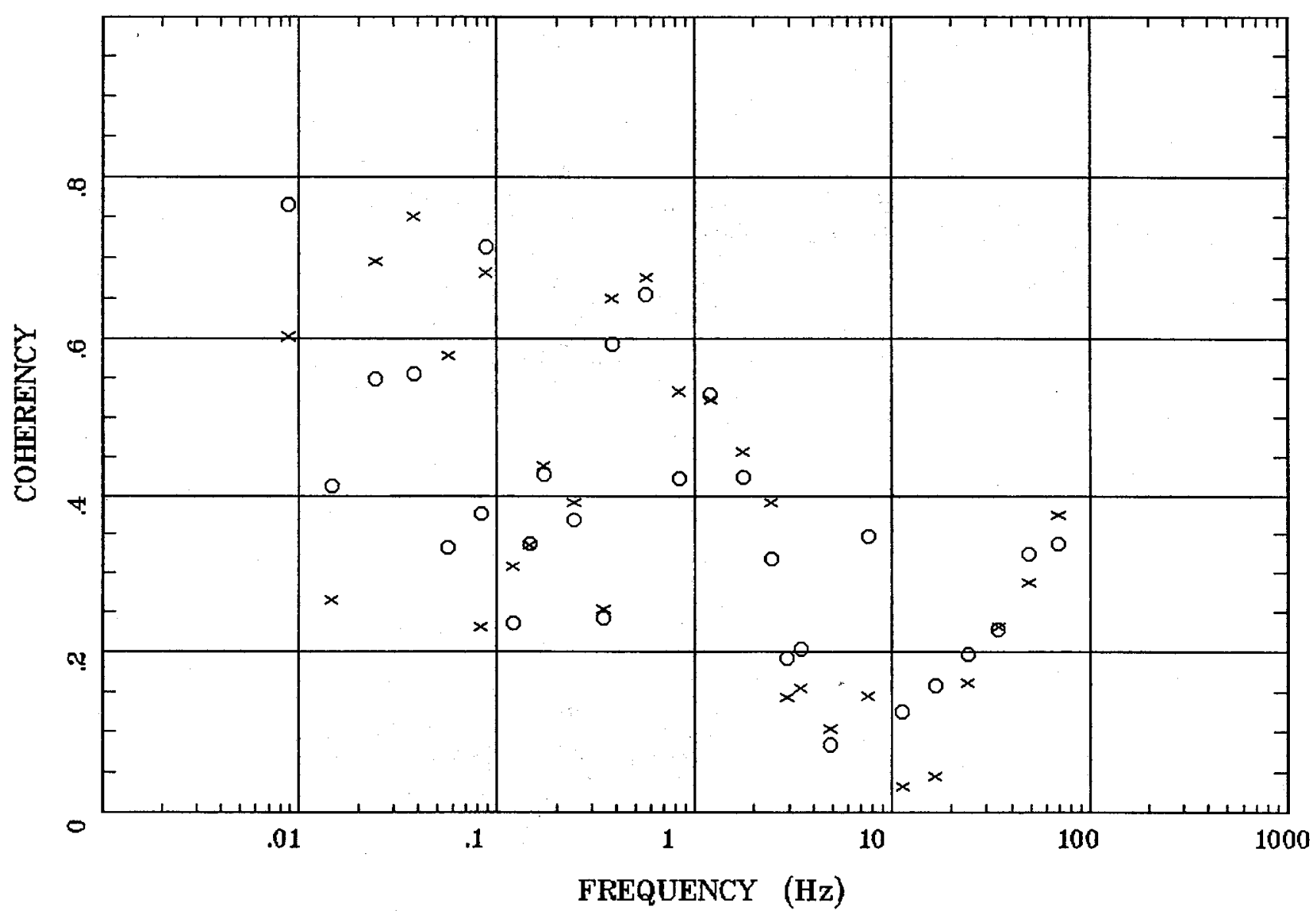

Client:

Remote: none

Acquired: 10:1 Jul 23, 2007

Survey Co:USGS
Rotation:

Filename: sl41m.avg

Channels: Ch1 Ch2 Ch3 Ch4 Ch5 Ch3 Ch4 Plotted: 11:19 Nov 06, 2007

$<$ EMI - ElectroMagnetic Instruments 


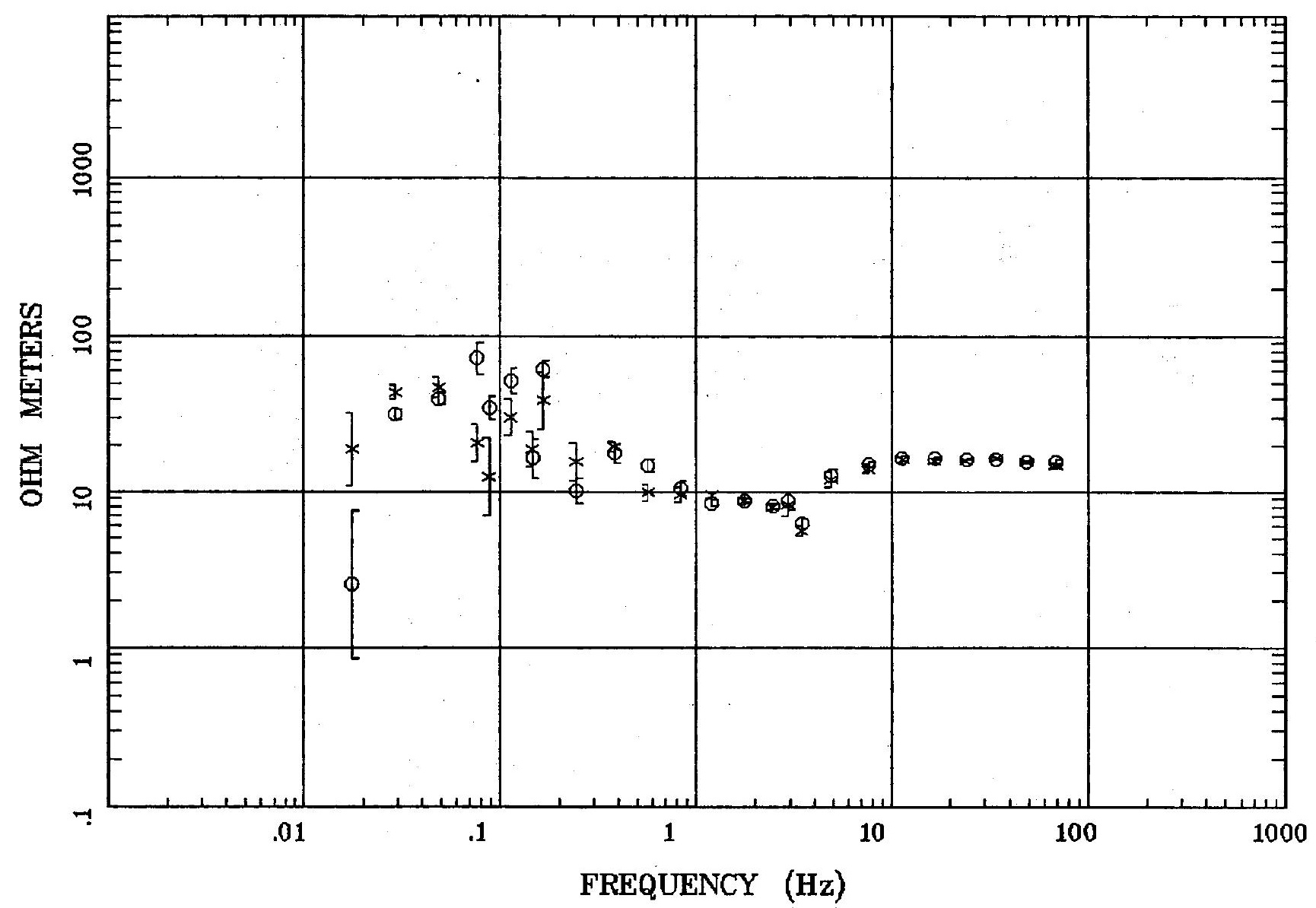




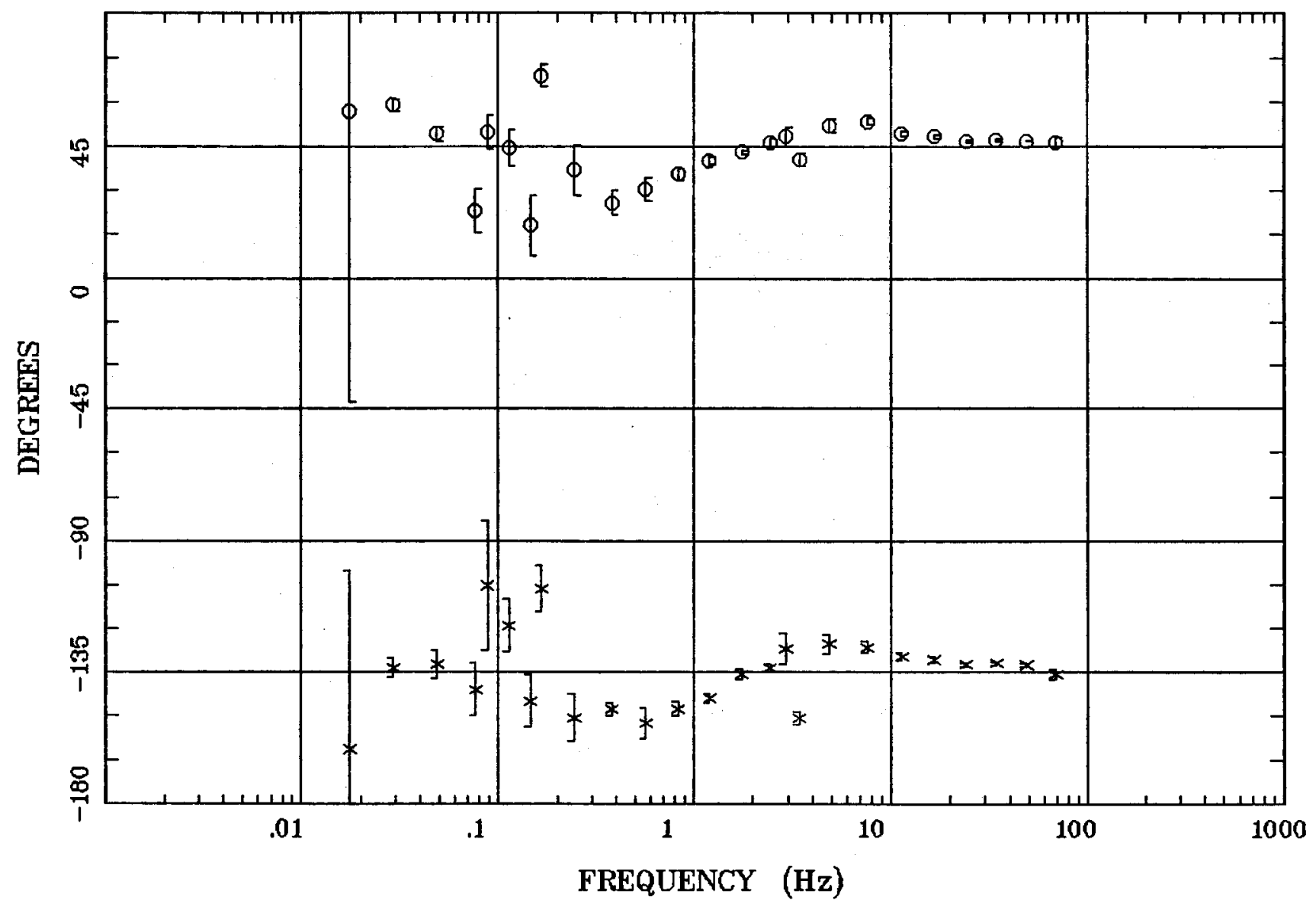

Client:

Remote: none

Acquired: 14:4 Jul 23, 2007 Survey Co:USGS
Rotation:

Filename: sl42m2.avg

Channels: Ch1 Ch2 Ch3 Ch4 Ch5 Ch3 Ch4 Plotted: 11:19 Nov 06, 2007

< EMI - ElectroMagnetic Instruments 


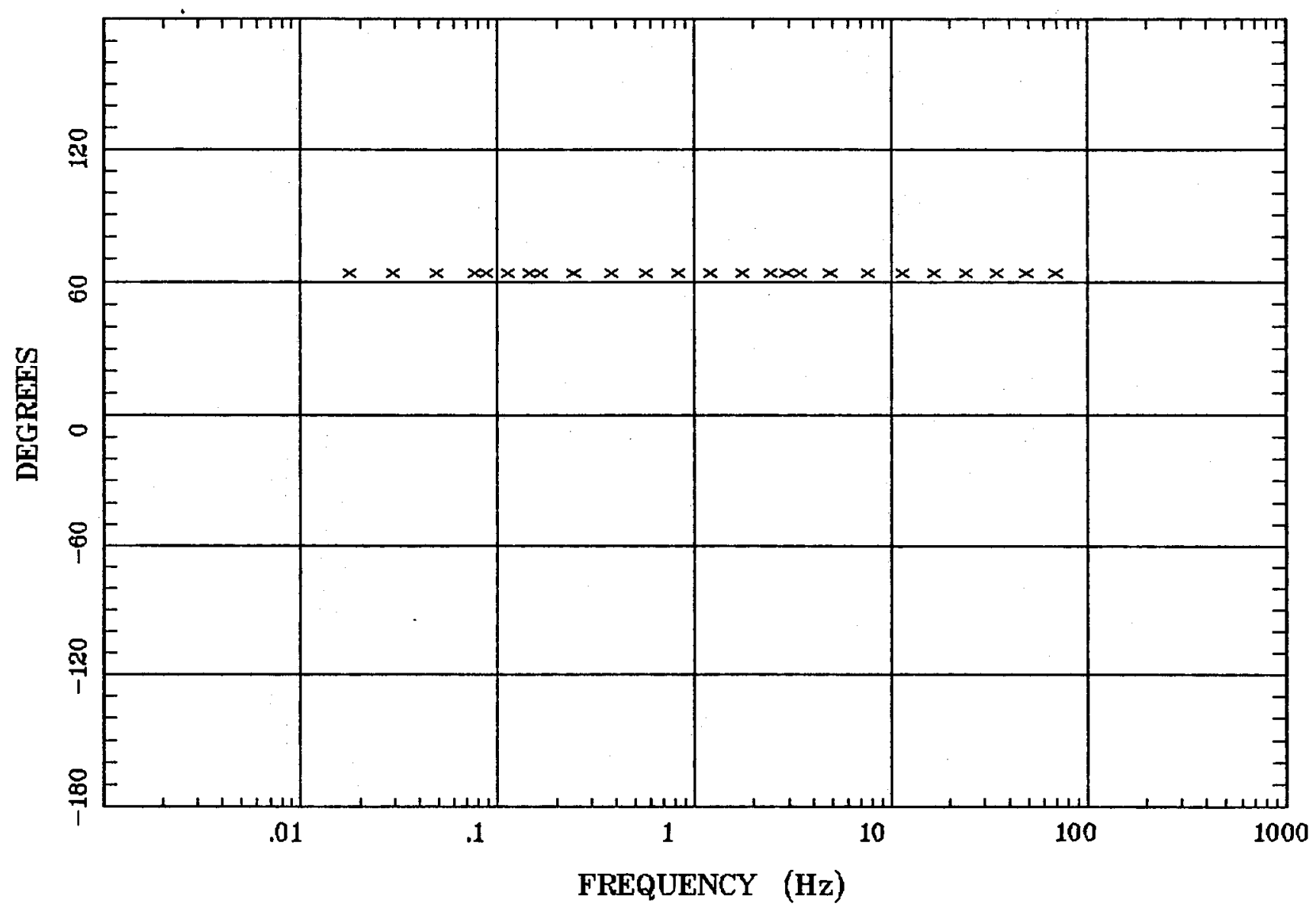

Client:

Remote: none

Acquired: 14:4 Jul 23, 2007 Survey Co:USGS
Rotation:

Filename: sl42m2.avg

Channels: Ch1 Ch2 Ch3 Ch4 Ch5 Ch3 Ch4 Plotted: 11:19 Nov 06, 2007

< EMI - ElectroMagnetic Instruments 


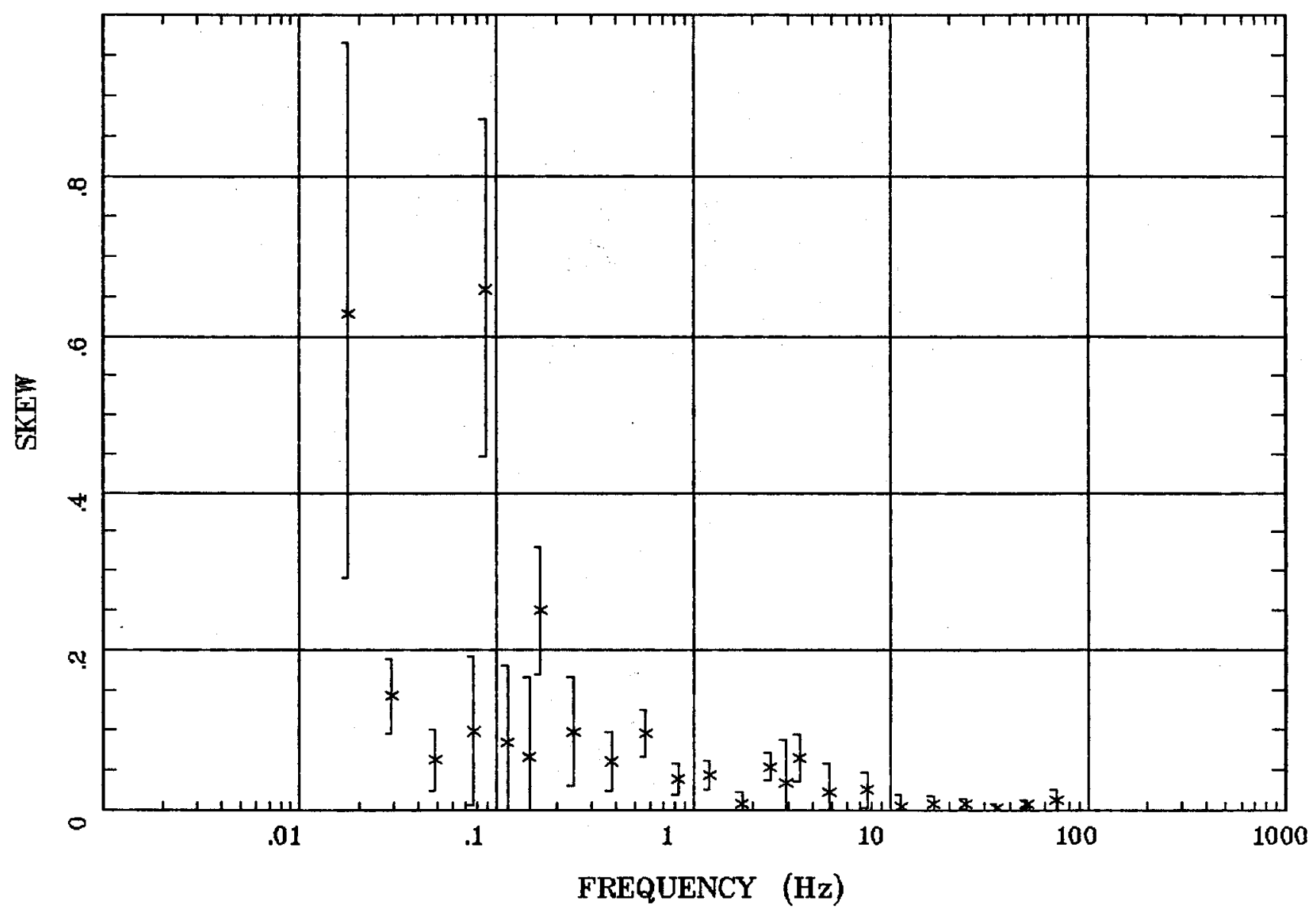

Client:

Remote: none

Acquired: 14:4 Jul 23, 2007 Survey Co:USGS
Rotation:

Filename: sl42m2.avg

Channels: Ch1 Ch2 Ch3 Ch4 Ch5 Ch3 Ch4

Plotted: 11:19 Nov 06, 2007

$<$ EMI - ElectroMagnetic Instruments 


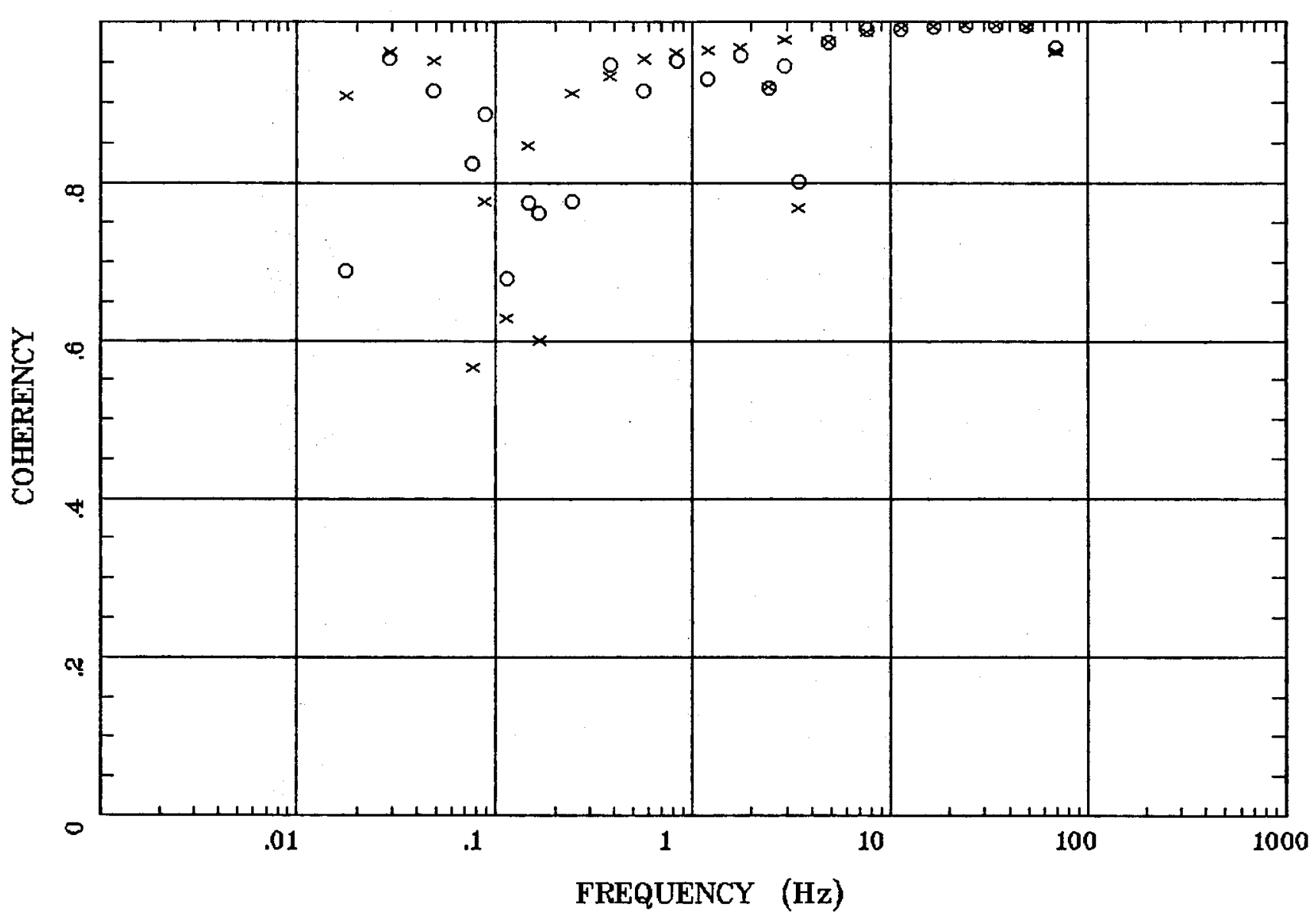

Client:

Remote: none

Acquired: 14:4 Jul 23, 2007 Survey Co:USGS
Rotation:

Filename: sl42na2.avg

Channels: Ch1 Ch2 Ch3 Ch4 Ch5 Ch3 Ch4 Plotted: 11:19 Nov 06, 2007

< EMI - ElectroMagnetic Instruments 
POLAR PLOTS

Alamosa, CO 100k

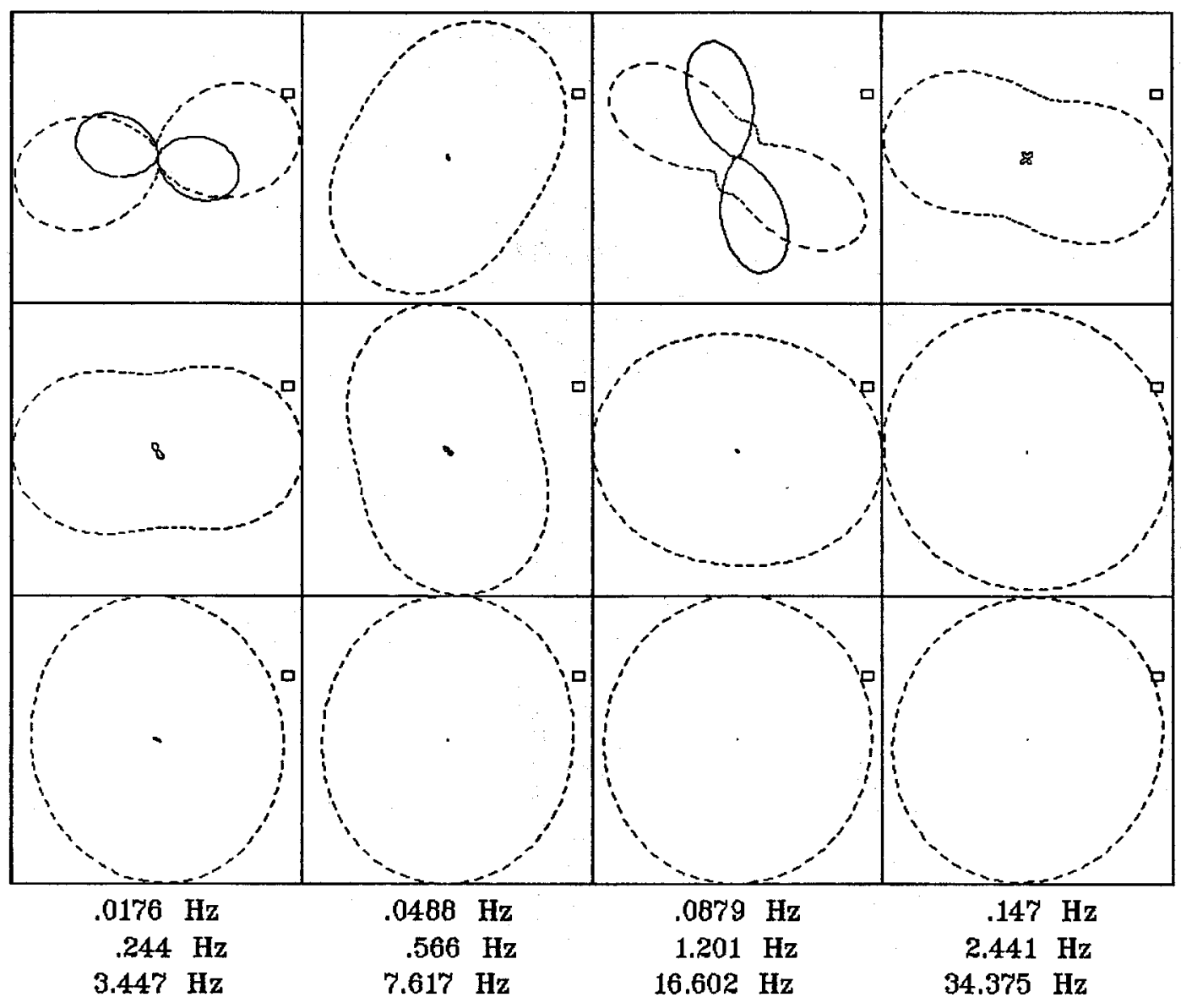

Client:

Remote: none

Acquired: 14:4 Jul 23, 2007

Survey Co:USGS
Rotation:

Filename: sl42m2.avg

Channels: Ch1 Ch2 Ch3 Ch4 Ch5 Ch3 Ch4

Plotted: 11:19 Nov 06, 2007

< EMI - ElectroMagnetic Instruments > 
Alamosa, CO 100k

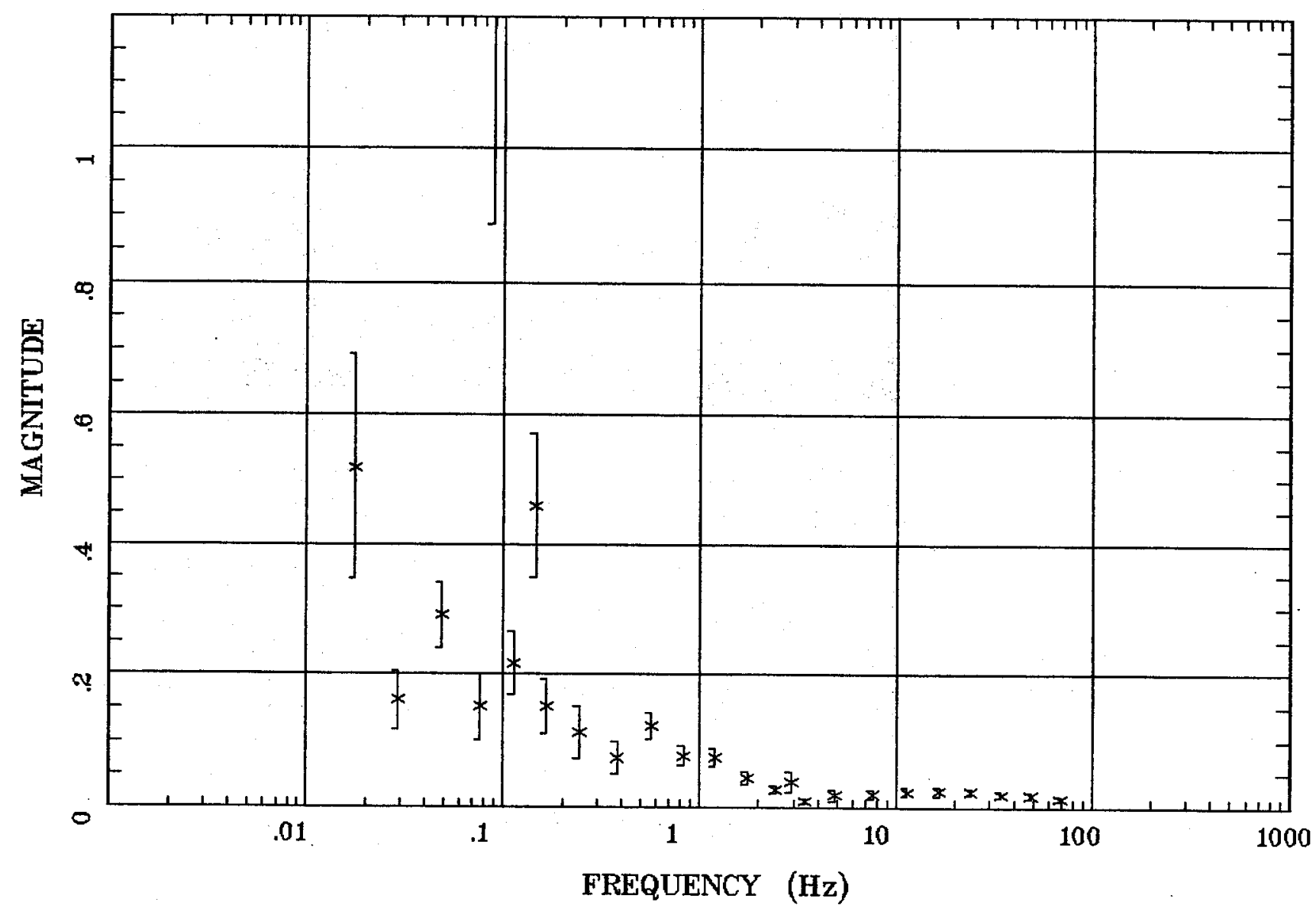

Client:

Remote: none

Acquired: 14:4 Jul 23, 2007 Survey Co:USGS
Rotation:

Filename: sl42m2.avg

Channels: Ch1 Ch2 Ch3 Ch4 Ch5 Ch3 Ch4 Plotted: 11:19 Nov 06, 2007

< EMI - ElectroMagnetic Instruments 
TIPPER STRIKE

Alamosa, CO 100k

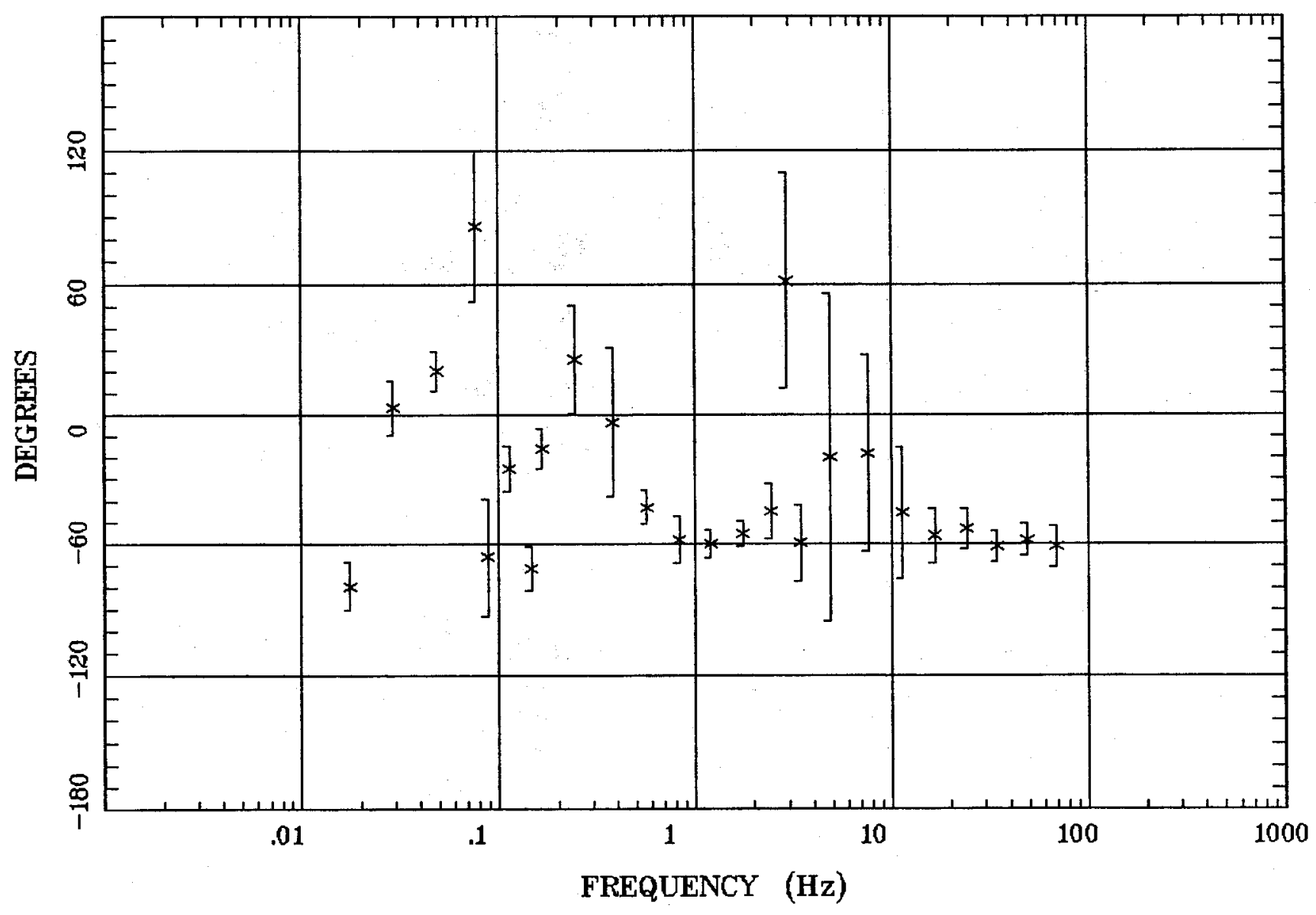

Client:

Remote: none

Acquired: 14:4 Jul 23, 2007

Survey Co:USGS
Rotation:

Filename: sl42m2.avg

Channels: Ch1 Ch2 Ch3 Ch4 Ch5 Ch3 Ch4

Plotted: 11:19 Nov 06, 2007

< EMI - ElectroMagnetic Instruments 
HzHx.x Coh HzHy.o Alamosa, Co 100k

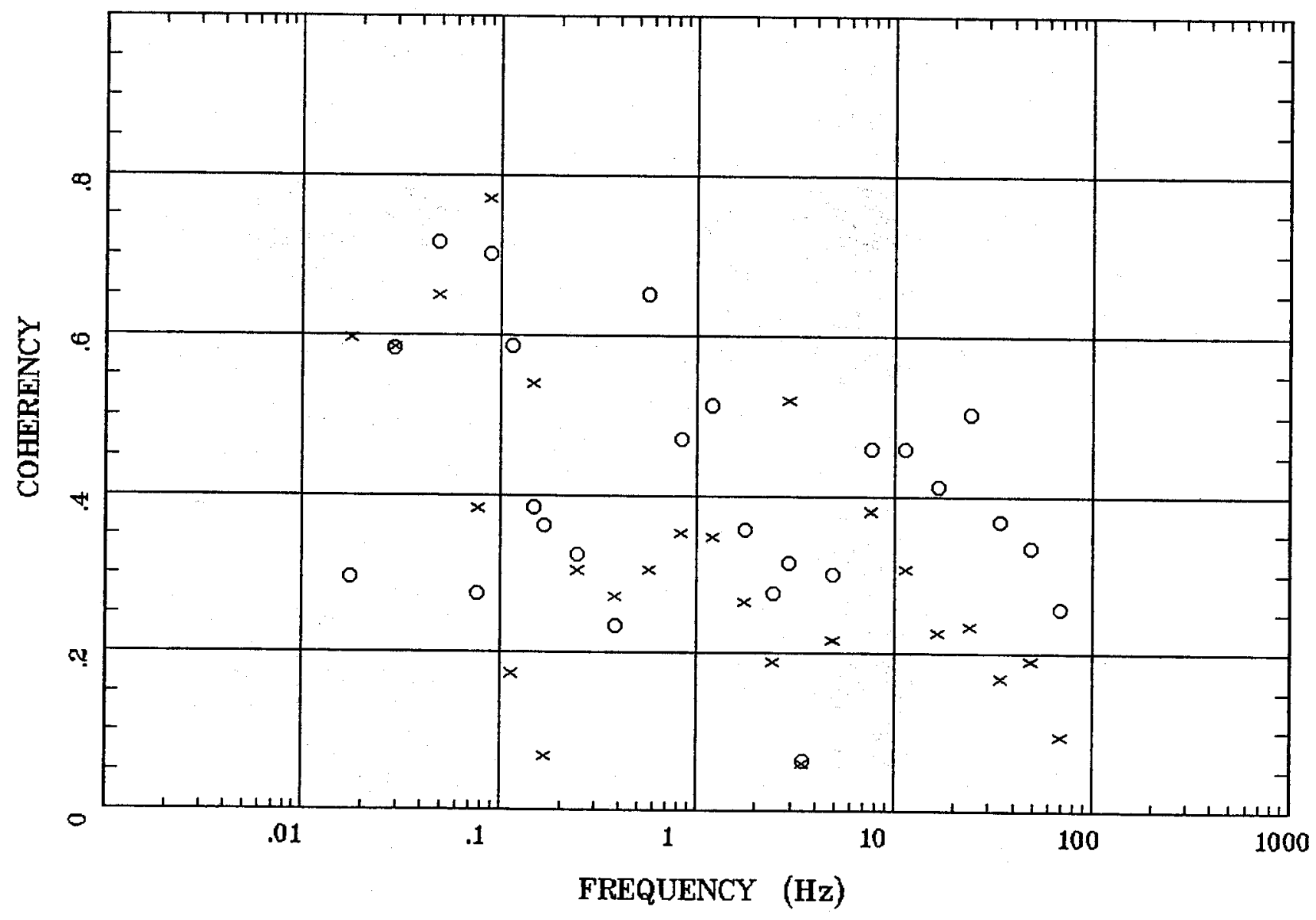

Client:

Remote: none

Acquired: 14:4 Jul 23, 2007

Survey Co:USGS
Rotation:

Filename: sl42m2.avg

Channels: Ch1 Ch2 Ch3 Ch4 Ch5 Ch3 Ch4

Plotted: 11:19 Nov 06, 2007

< EMI - ElectroMagnetic Instruments 
Station 43

APPARENT RESISTIVITY Alamosa, CO $100 \mathrm{k}$

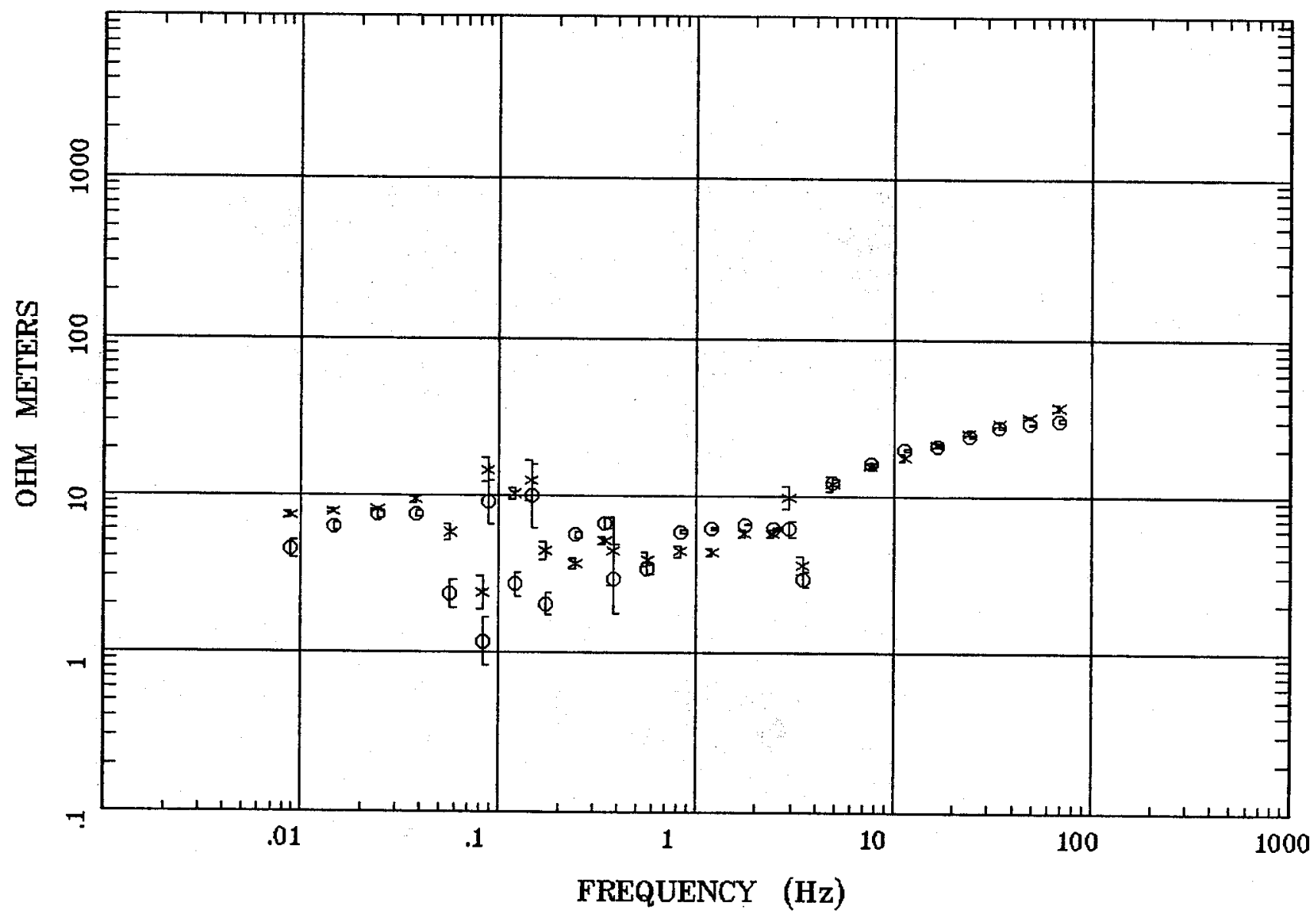

Client:

Remote: none

Acquired: 09:1 Jul 24, 2007

Survey Co:USGS
Rotation:

Filename: sl43m1.avg

Channels: Ch1 Ch2 Ch3 Ch4 Ch5 Ch3 Ch4

Plotted: 11:10 Nov 06, 2007

< EMI - ElectroMagnetic Instruments > 


\section{IMPEDANCE PHASE}

Alamosa, CO 100k

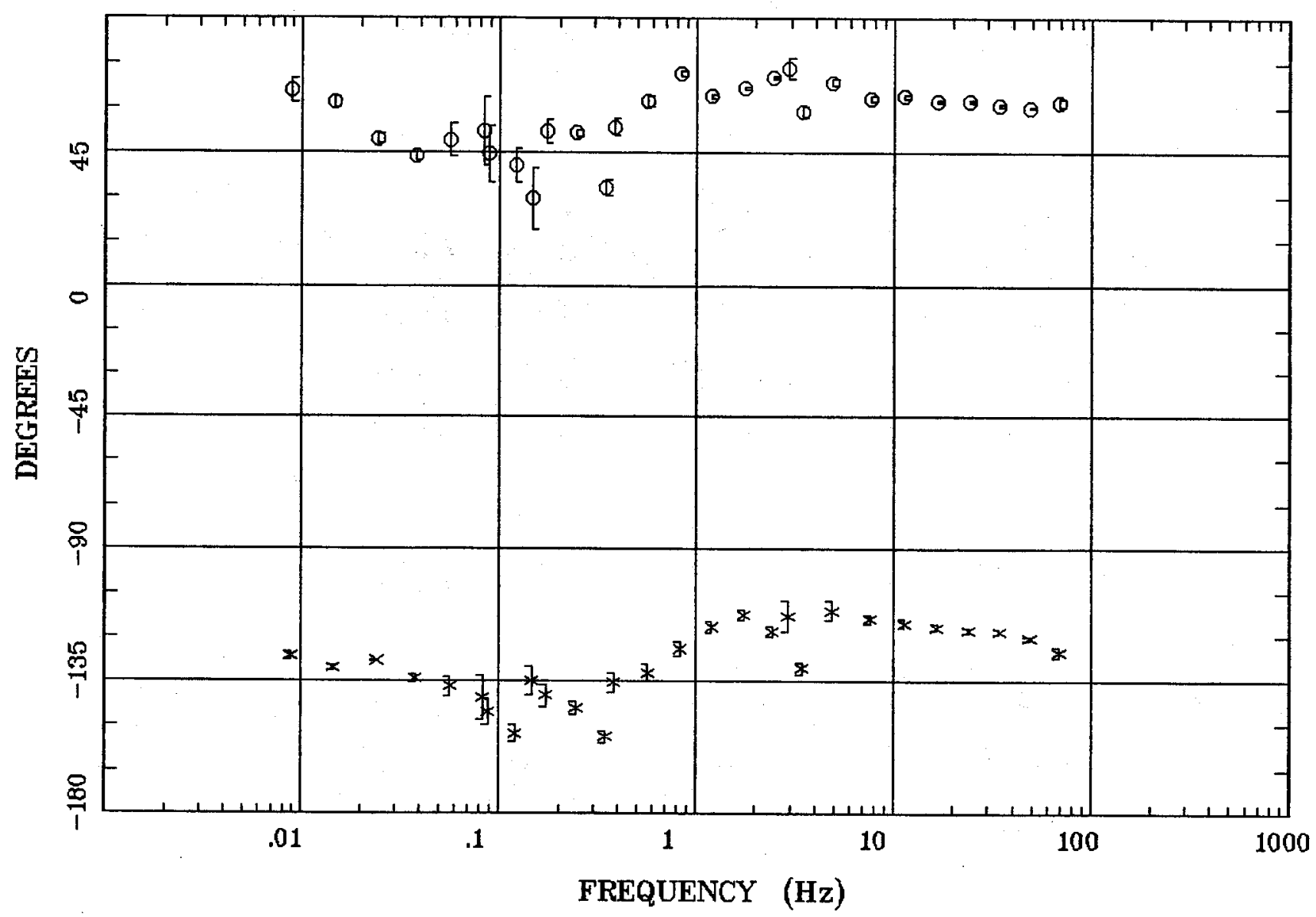

Client:

Remote: none

Acquired: 09:1 Jul 24, 2007 Survey Co:USGS
Rotation:

Filename: sl43m1.avg

Channels: Ch1 Ch2 Ch3 Ch4 Ch5 Ch3 Ch4 Plotted: 11:10 Nor 06, 2007

< EMI - ElectroMagnetic Instruments 


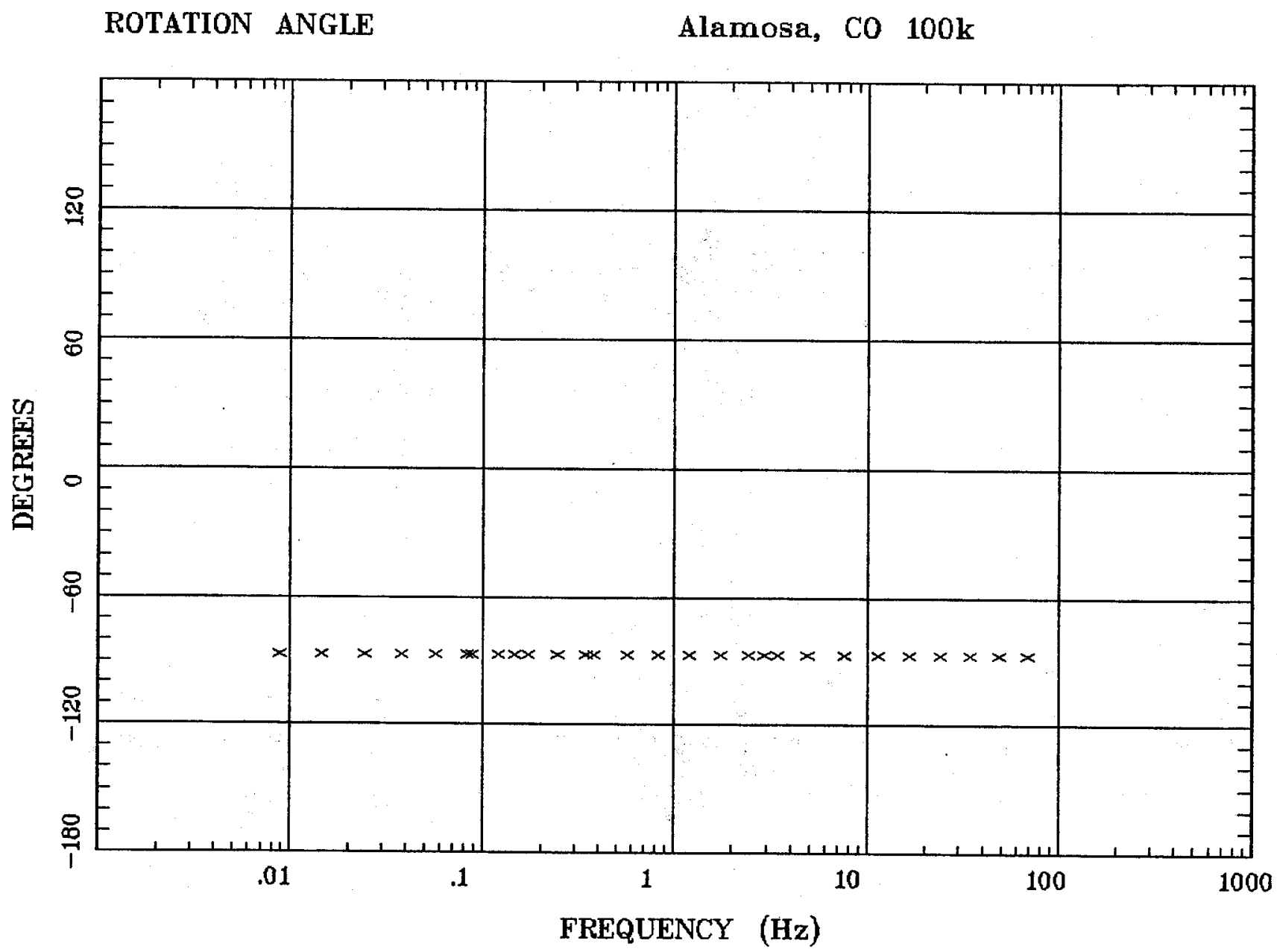

Client:

Remote: none

Acquired: 09:1 Jul 24, 2007

Survey Co:USGS
Rotation:

Filename: sl43m1.avg

Channels: Ch1 Ch2 Ch3 Ch4 Ch5 Ch3 Ch4

Plotted: 11:10 Nop 06, 2007

< EMI - ElectroMagnetic Instruments > 
Station $\mathbf{4 3}$

IMPEDANCE SKEW

Alamosa, CO 100k

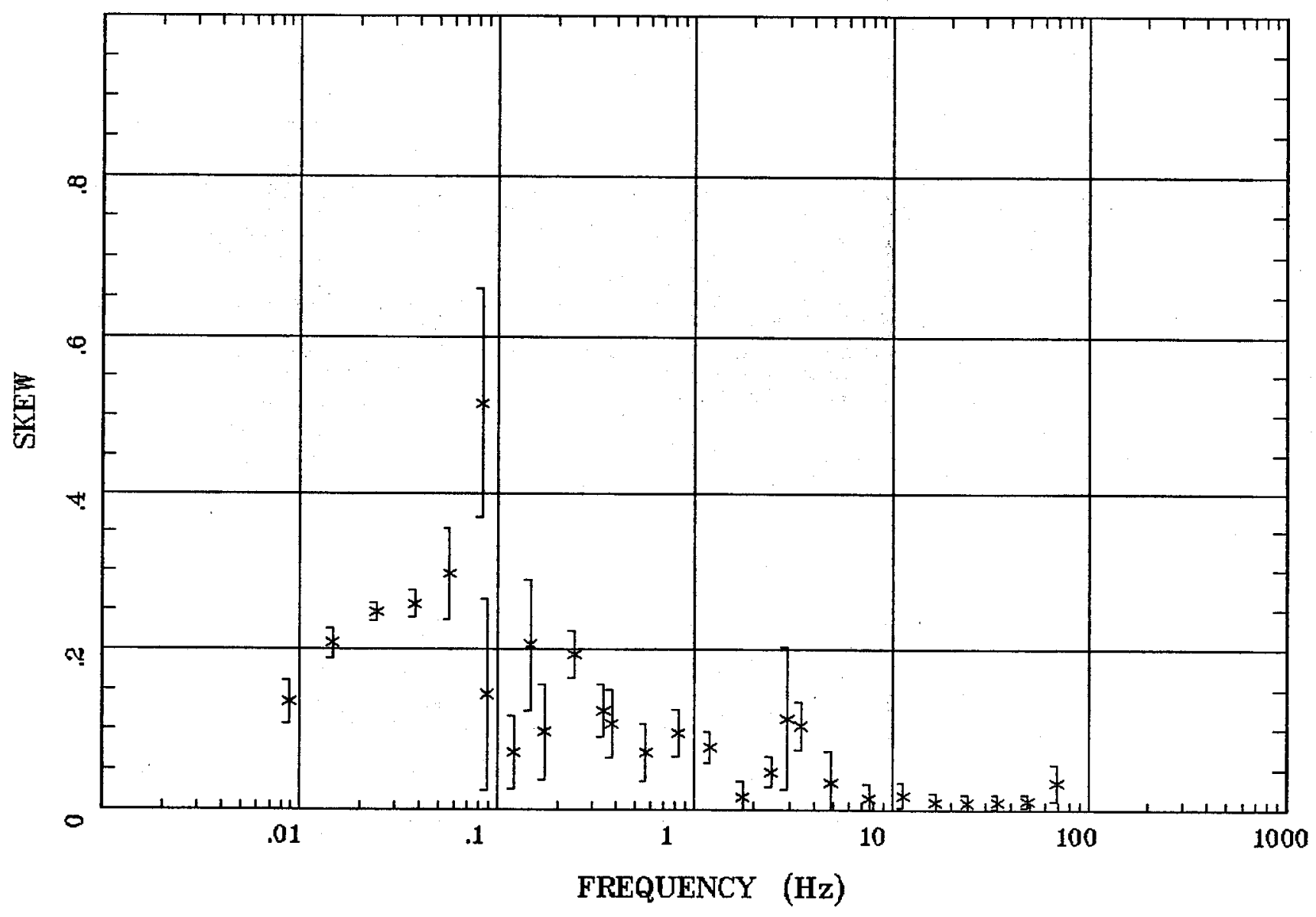

Client:

Rotation:

Remote: none

Acquired: 09:1 Jul 24, 2007

Survey Co:USGS

Filename: sl43m1.avg

Channels: Ch1 Ch2 Ch3 Ch4 Ch5 Ch3 Ch4

Plotted: 11:10 Nov 06, 2007

< EMI - ElectroMagnetic Instruments > 
E MULT Coh.

Alamosa, CO 100k

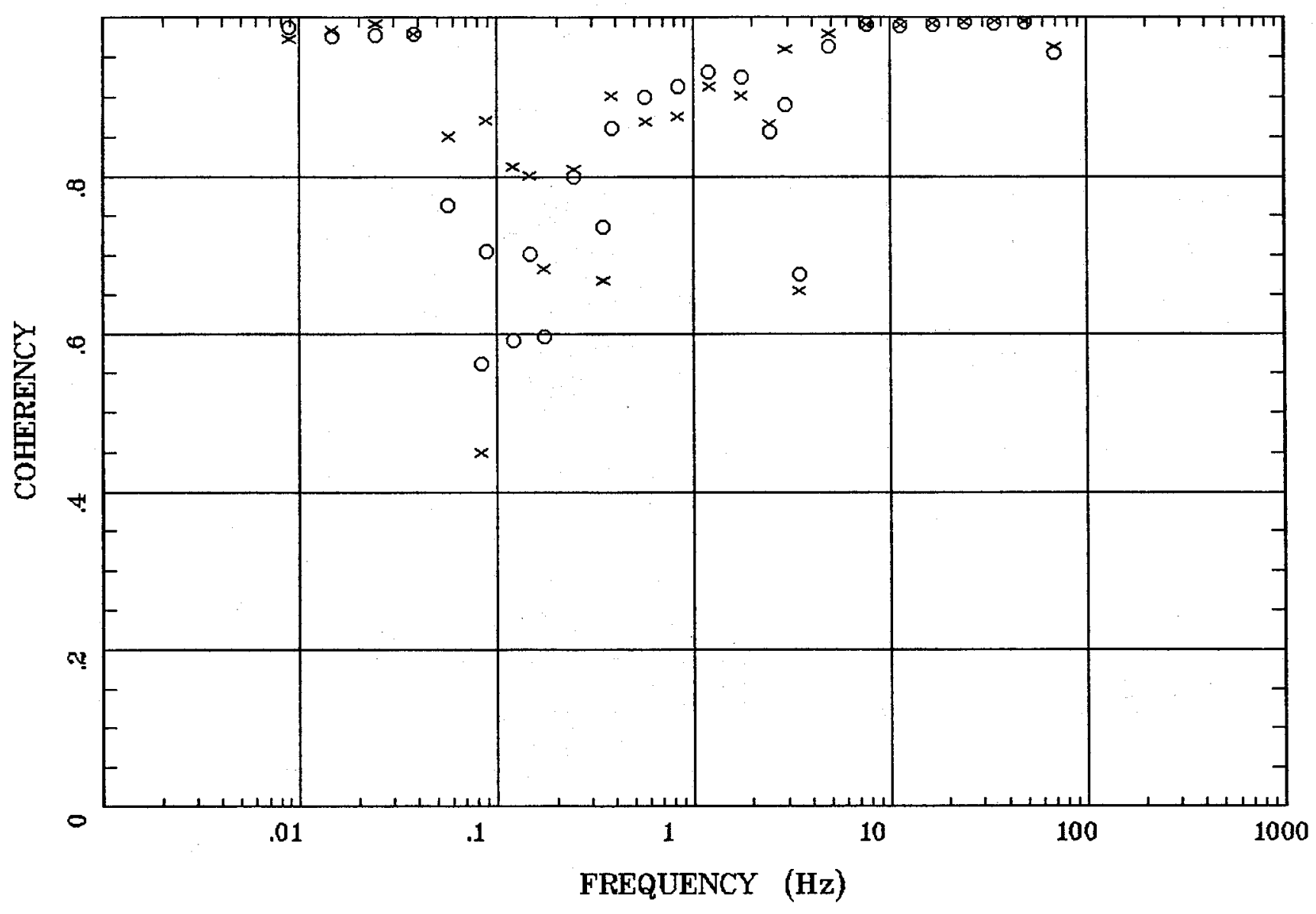

Client:

Remote: none

Acquired: 09:1 Jul 24, 2007

Survey Co:USGS
Rotation:

Filename: sl43m1.avg

Channels: Ch1 Ch2 Ch3 ch4 Ch5 Ch3 Ch4

Platted: 11:10 Nov 06, 2007

< EMI - ElectroMagnetic Instruments > 
POLAR PLOTS

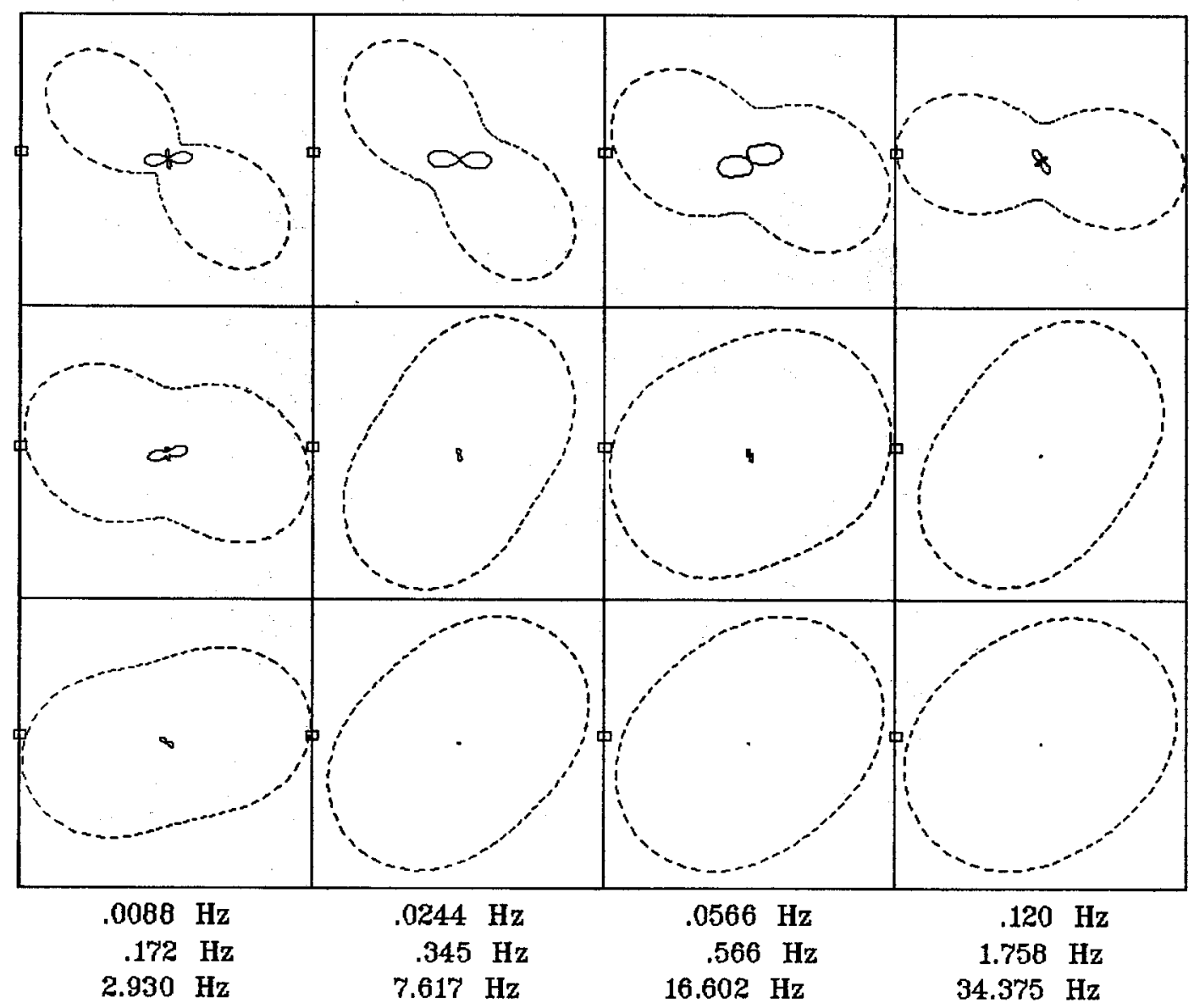

Client:

Remote: none

Acquired: 09:1 Jul 24, 2007 Survey Co:USGS
Alamosa, CO 100k

Rotation:

Filename: sl43m1.avg

Channels: Ch1 Ch2 Ch3 Ch4 Ch5 Ch3 Ch4 Plotted: 11:10 Nov 06, 2007

< EMI - ElectroMagnetic Instruments > 
TIPPER MAGNITUDE Alamosa, CO $100 \mathrm{k}$

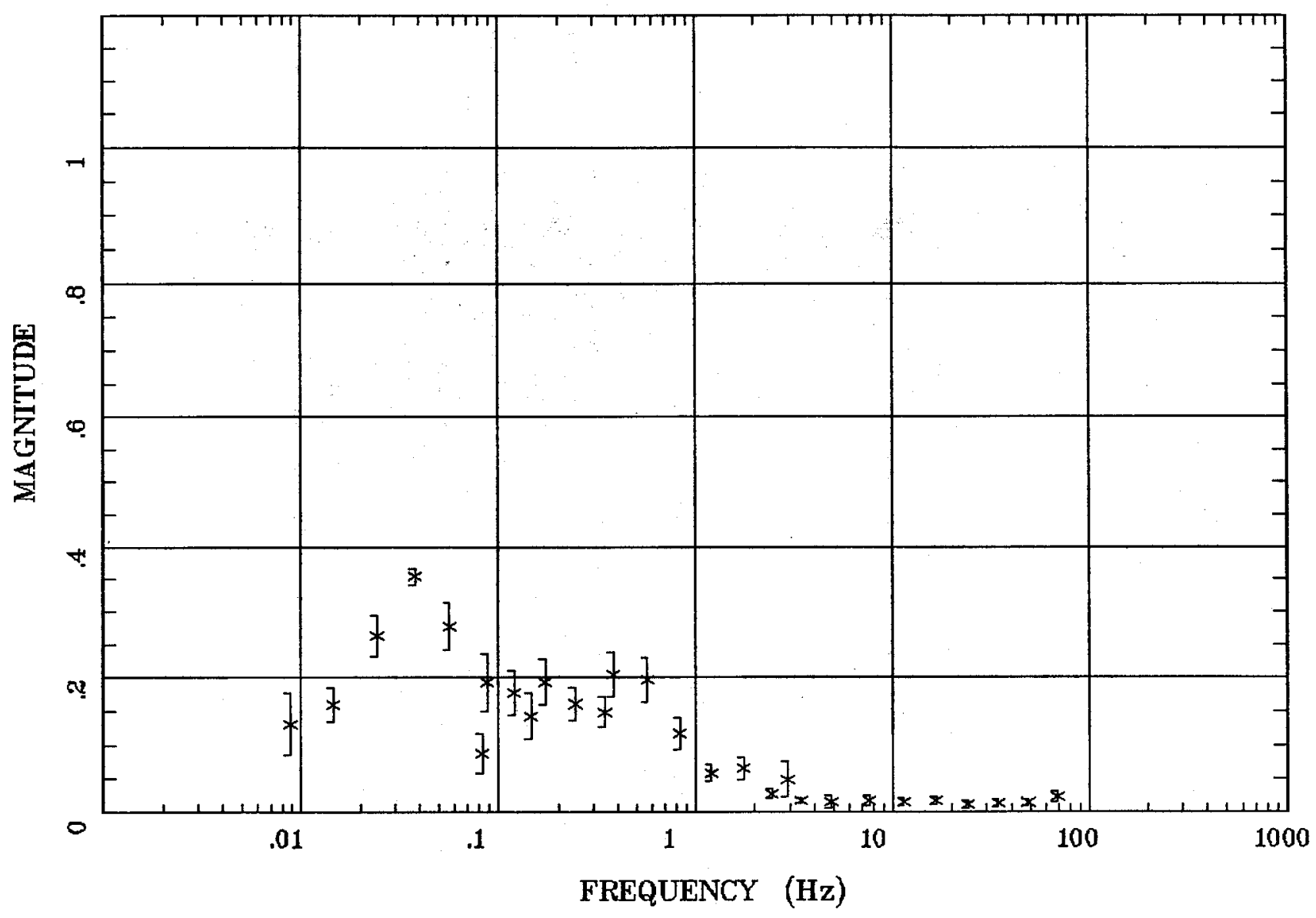

Client:

Remote: none

Acquired: 09:1 Jul 24, 2007

Survey Co:USGS
Rotation:

Filename: sl43m1.avg

Channels: Ch1 Ch2 Ch3 ch4 Ch5 Ch3 Ch4 Platted: 11:10 Nov 06, 2007

< EMI - ElectroMagnetic Instruments > 
TIPPER STRIKE

Alamosa, CO 100k

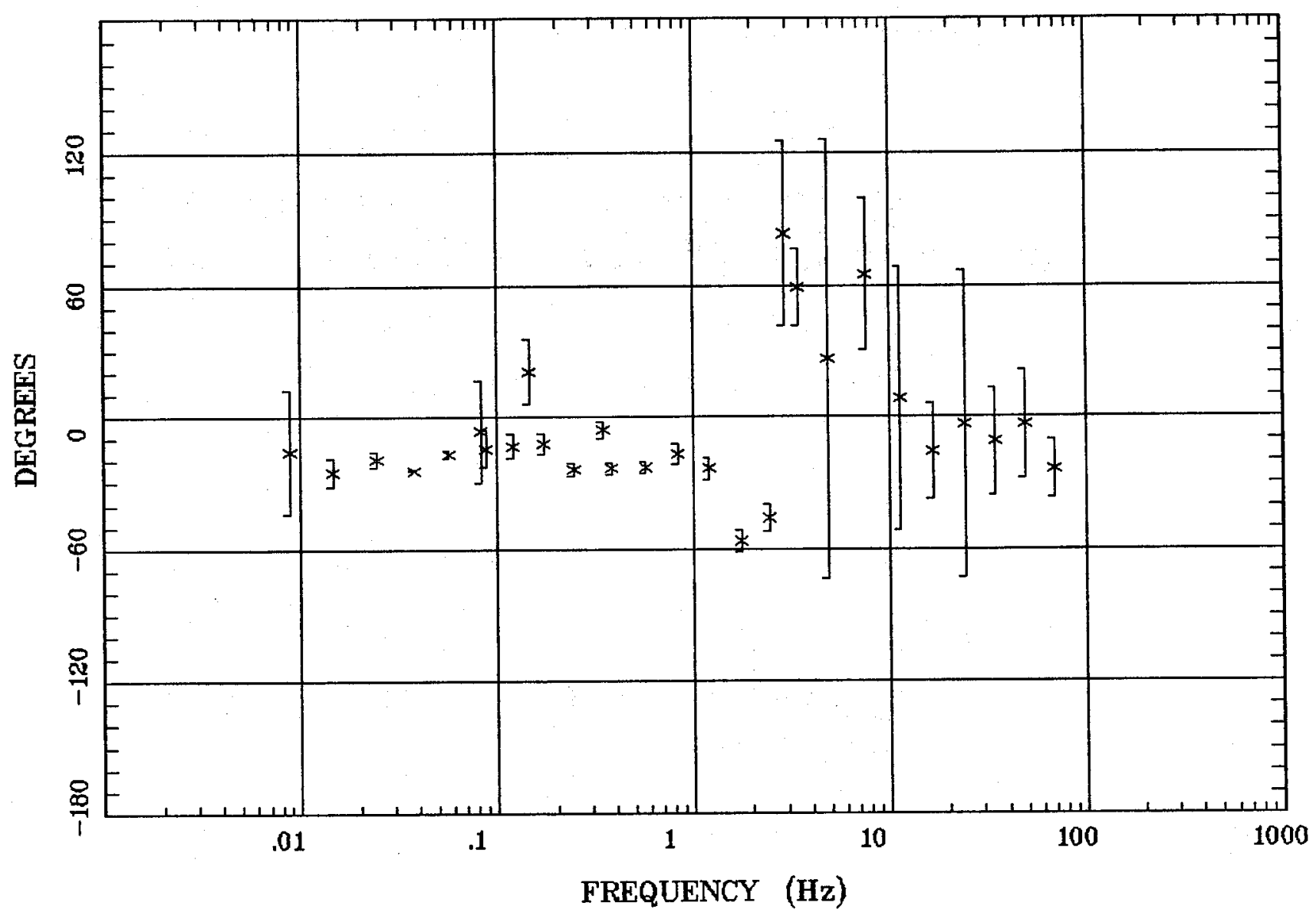

Client:

Remote: none

Acquired: 09:1 Jul 24, 2007 Survey Co:USGS
Rotation:

Filename: sl43m1.avg

Channels: Ch1 Ch2 Ch3 Ch4 Ch5 Ch3 Ch4 Plotted: 11:10 Nov 06, 2007

< EMI - ElectroMagnetic Instruments 
HzHx.x Coh HzHy.o

Alamosa, CO 100k

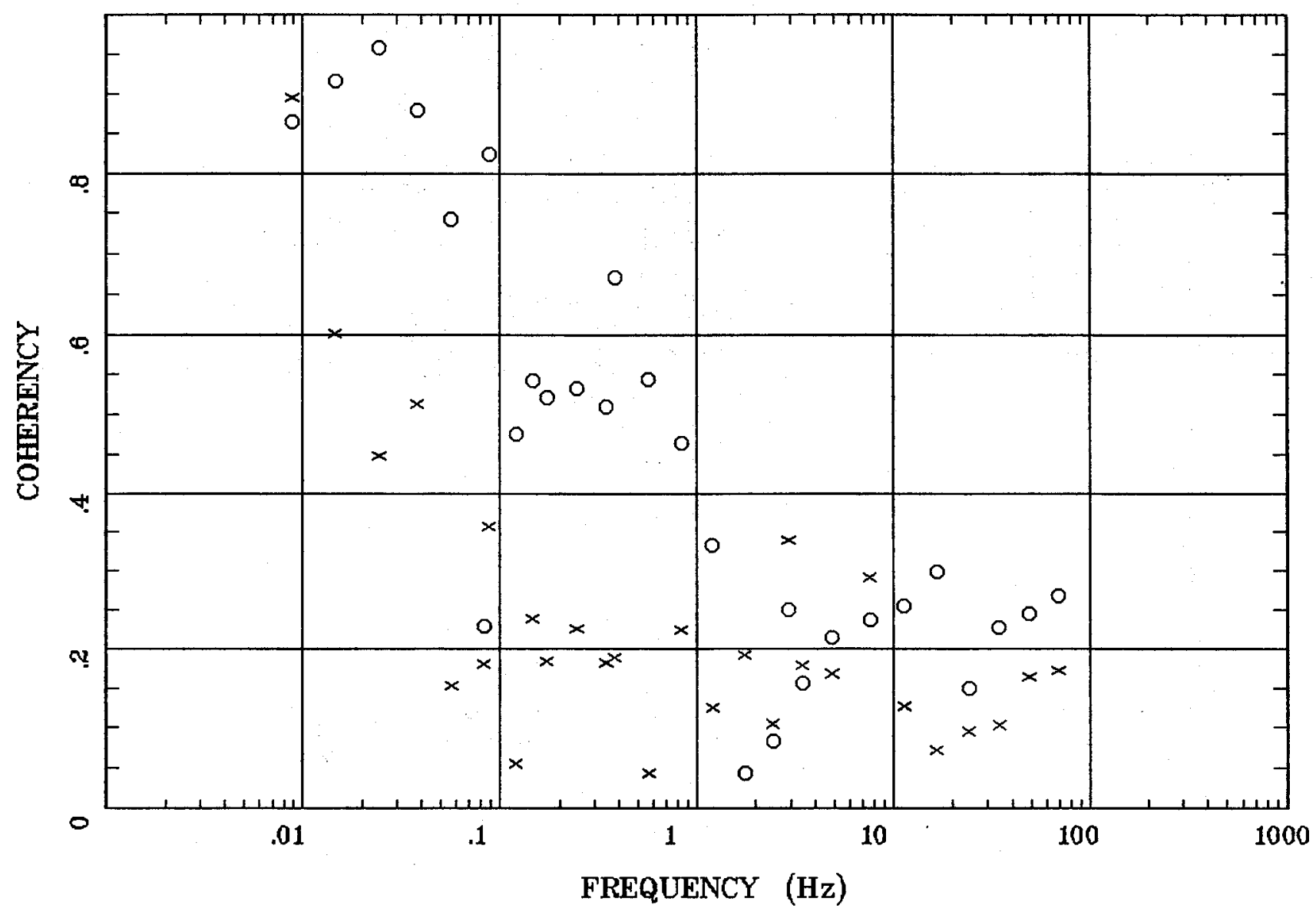

Client:

Remote: none

Acquired: 09:1 Jul 24, 2007 Survey Co:USGS

\section{Rotation:}

Filename: sl43m1.evg

Channels: Ch1 Ch2 Ch3 Ch4 Ch5 Ch3 Ch4 Plotted: 11:10 Nov 06, 2007

< EMI - ElectroMagnetic Instruments 


\section{Station 44}

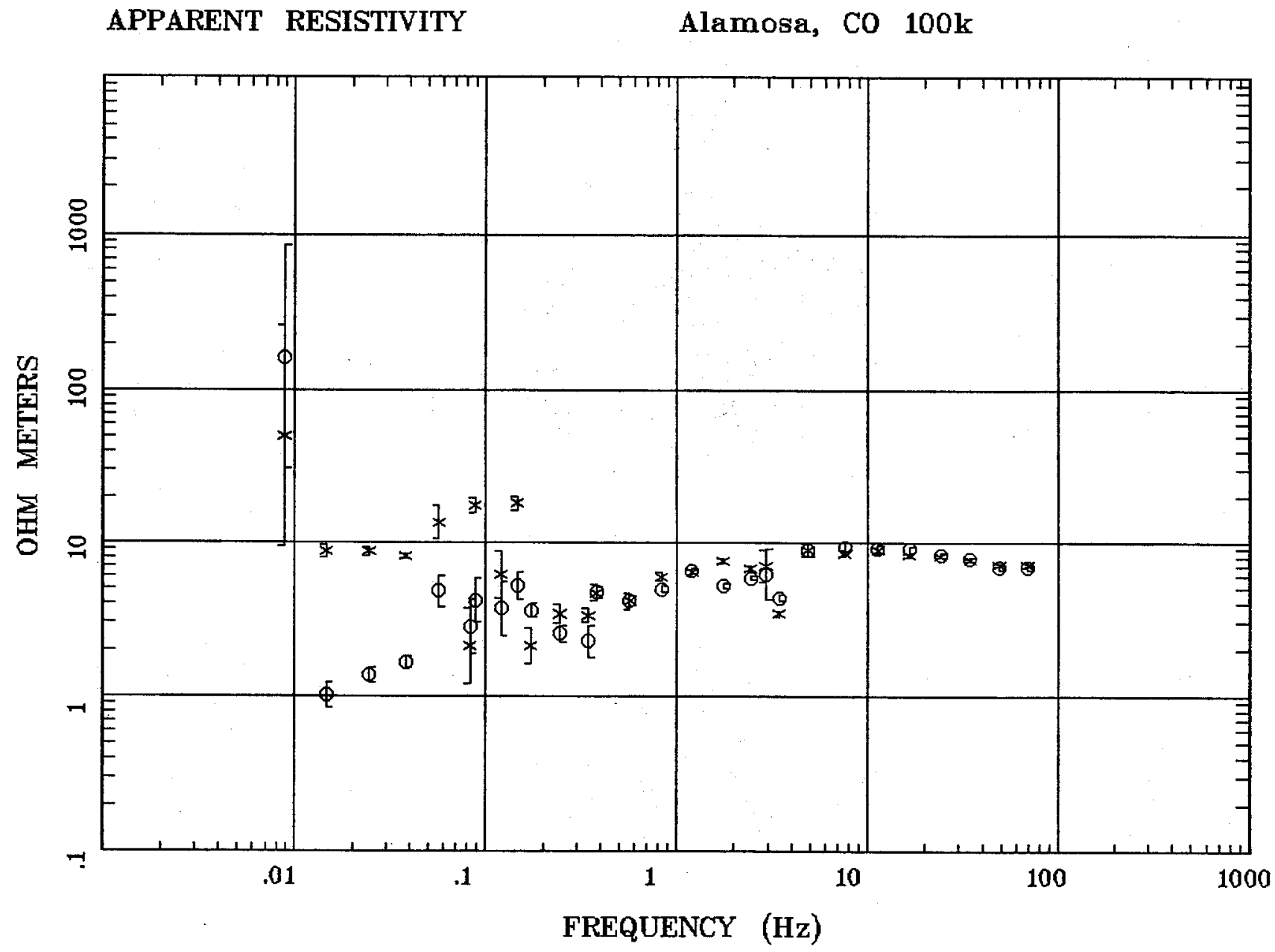

Client:

Remote: none

Acquired: 11:1 Jul 25, 2007

Survey Co:USGS
Rotation:

Filename: sl44m1.avg

Channels: Ch1 Ch2 Ch3 Ch4 Ch5 Ch3 Ch4 Plotted: 11:10 Nop 06, 2007

< EMI - ElectroMagnetic Instruments > 


\section{Station 44}

IMPEDANCE PHASE

Alamosa, CO 100k

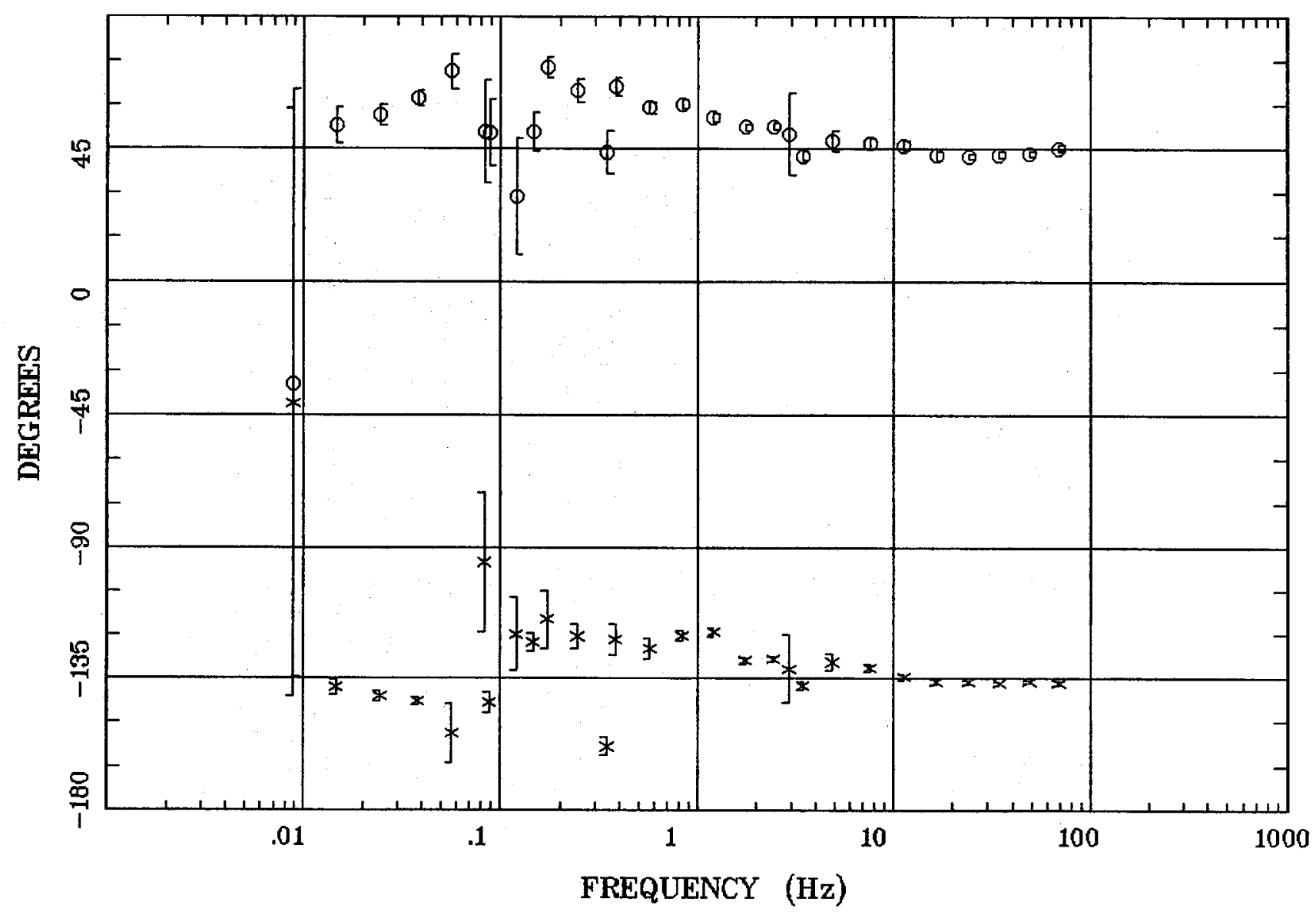

Client:

Remate: none

Acquired: 11:1 Jul 25, 2007 Survey Co:USGS
Rotation:

Filename: sl44m1.avg

Channels: Ch1 Ch2 Ch3 Ch4 Ch5 Ch3 Ch4 Plotted: 11:10 Nov 06, 2007

$<$ EMI - ElectroMagnetic Instruments 


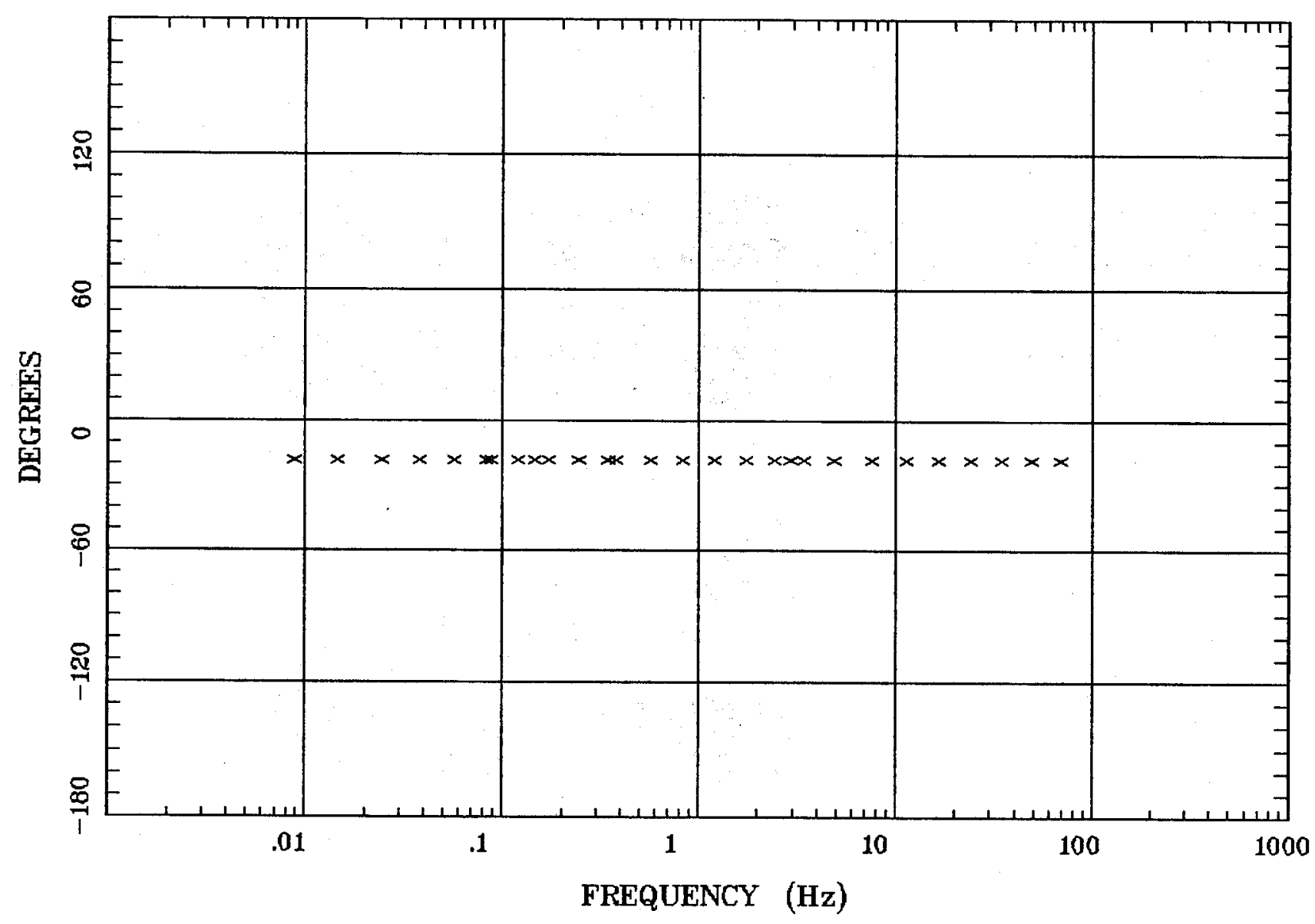

Client:

Remote: none

Acquired: 11:1 Jul 25, 2007 Survey Co:USGS

\section{Rotation:}

Filename: sl44m1.avg

Channels: Ch1 Ch2 Ch3 Ch4 Ch5 Ch3 Ch4

Plotted: 11:10 Nov 06, 2007

< EMI - ElectroMagnetic Instruments 
Alamosa, CO 100k

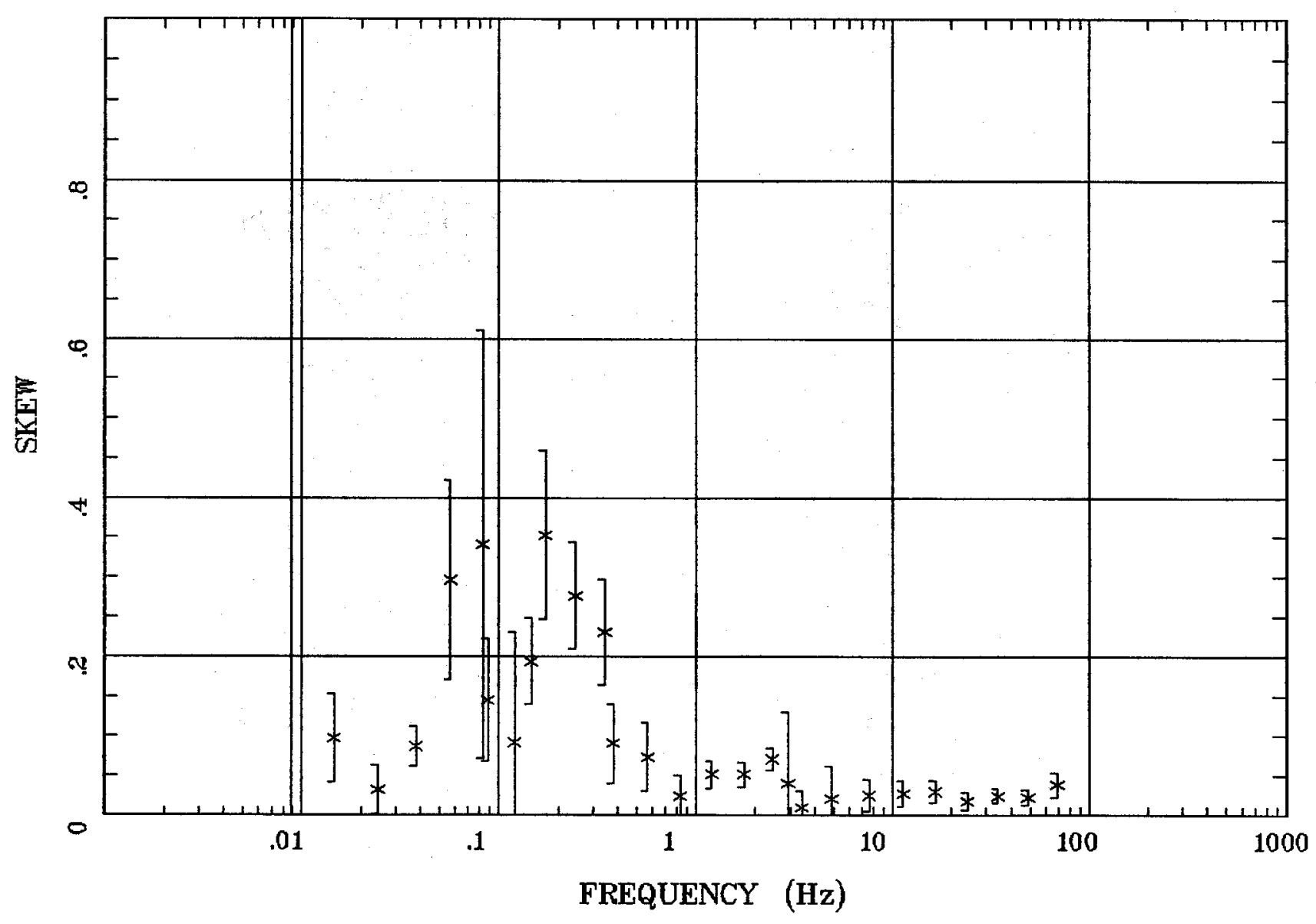

Client:

Remote: none

Acquired: 11:1 Jul 25, 2007 Survey Co:USGS
Rotation:

Filename: sl44m1.avg

Channels: Ch1 Ch2 Ch3 Ch4 Ch5 Ch3 Ch4 Plotted: 11:10 Nov 06, 2007

< EMI - ElectroMagnetic Instruments > 
Alamosa, CO 100k

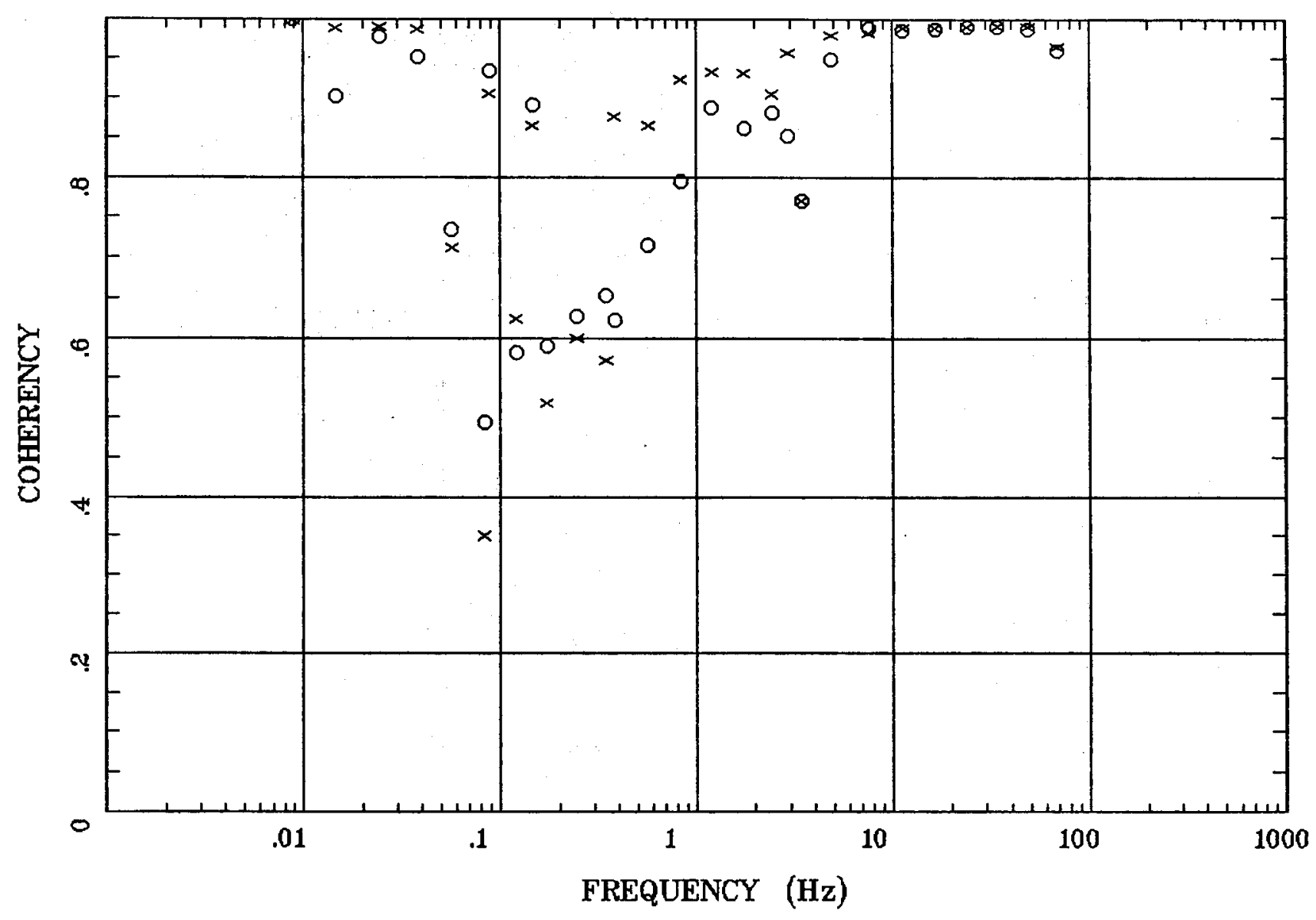

Client:

Remote: none

Acquired: 11:1 Jul 25, 2007

Survey Co:USGS
Rotation:

Filename: sl44m1.avg

Channels: Ch1 Ch2 Ch3 Ch4 Ch5 Ch3 Ch4 Plotted: 11:10 Nov 06, 2007

< EMI - ElectroMagnetic Instruments 


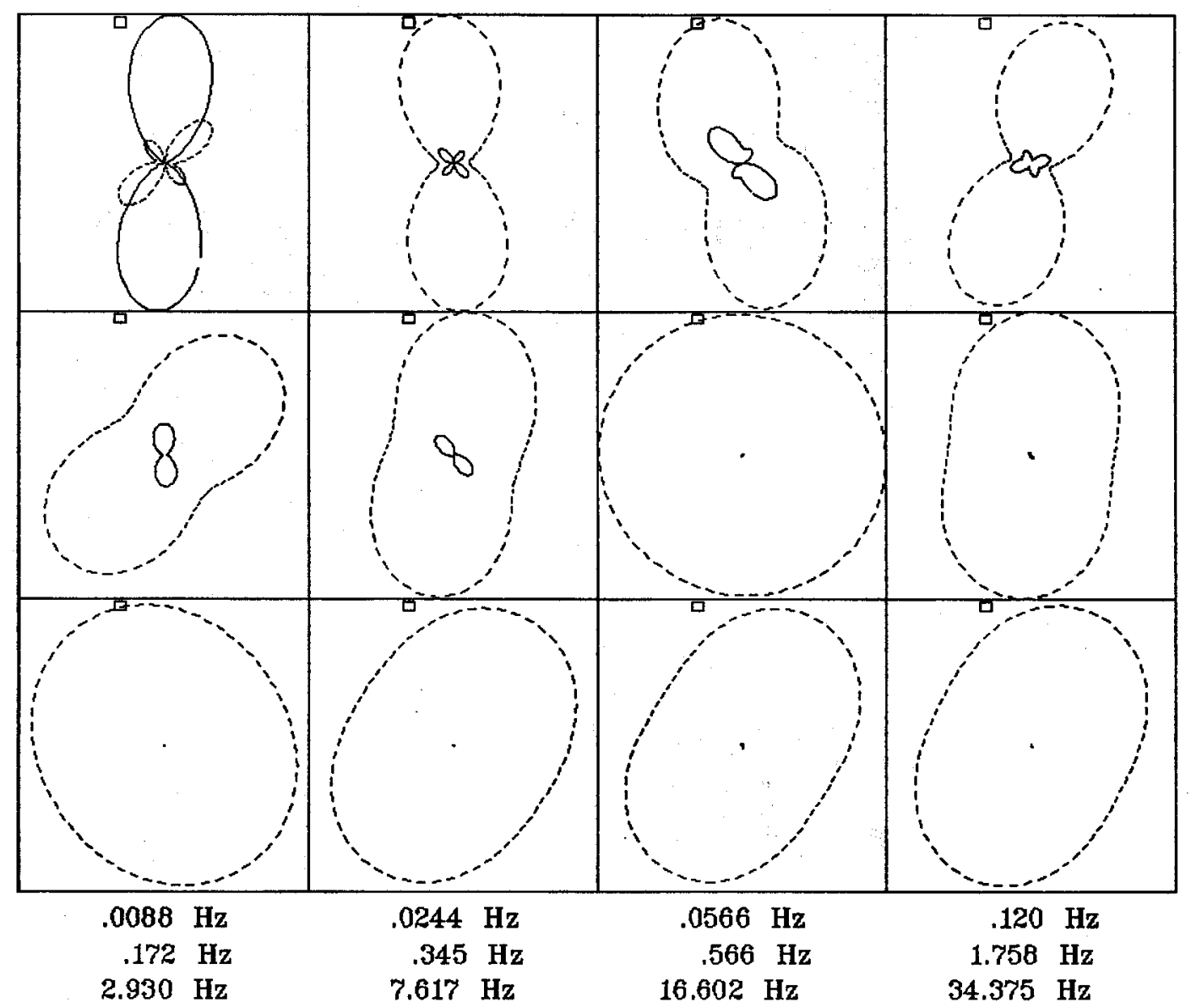

Client:

Remote: none

Acquired: 11:1 Jul 25, 2007 Survey Co:USGS
Rotation:

Filename: sl44m1.avg

Channels: Ch1 Ch2 Ch3 Ch4 Ch5 Ch3 Ch4 Plotted: 11:10 Nov 06, 2007

< EMI - ElectroMagnetic Instruments 


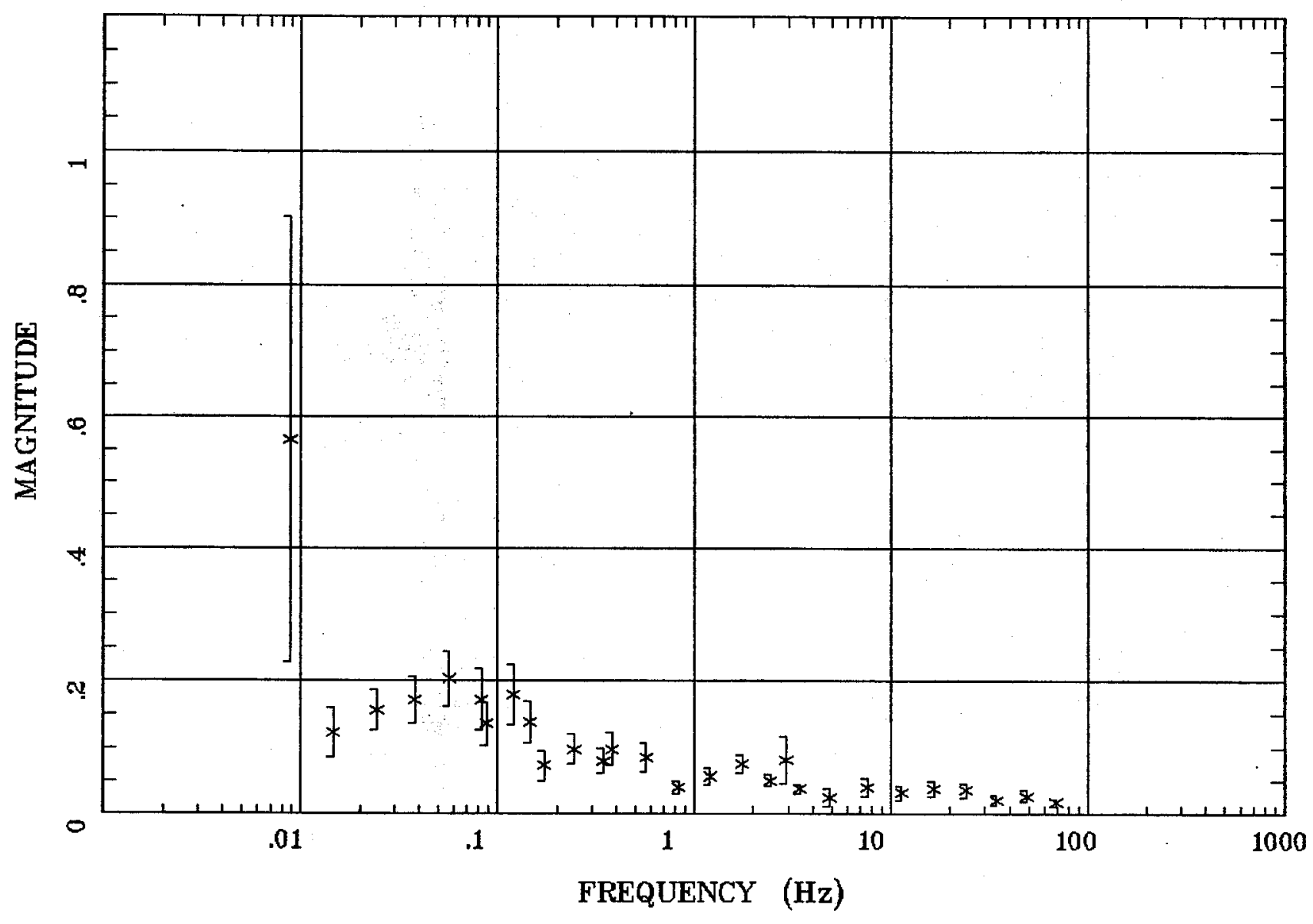

Client:

Remote: none

Acquired: 11:1 Jul 25, 2007 Survey Co:USGS

\section{Rotation:}

Filename: sl44m1.avg

Channels: Ch1 Ch2 Ch3 ch4 Ch5 Ch3 Ch4 Plotted: 11:10 Nov 06, 2007

< EMI - ElectroMagnetic Instruments 
TIPPER STRIKE

Alamosa, CO 100k

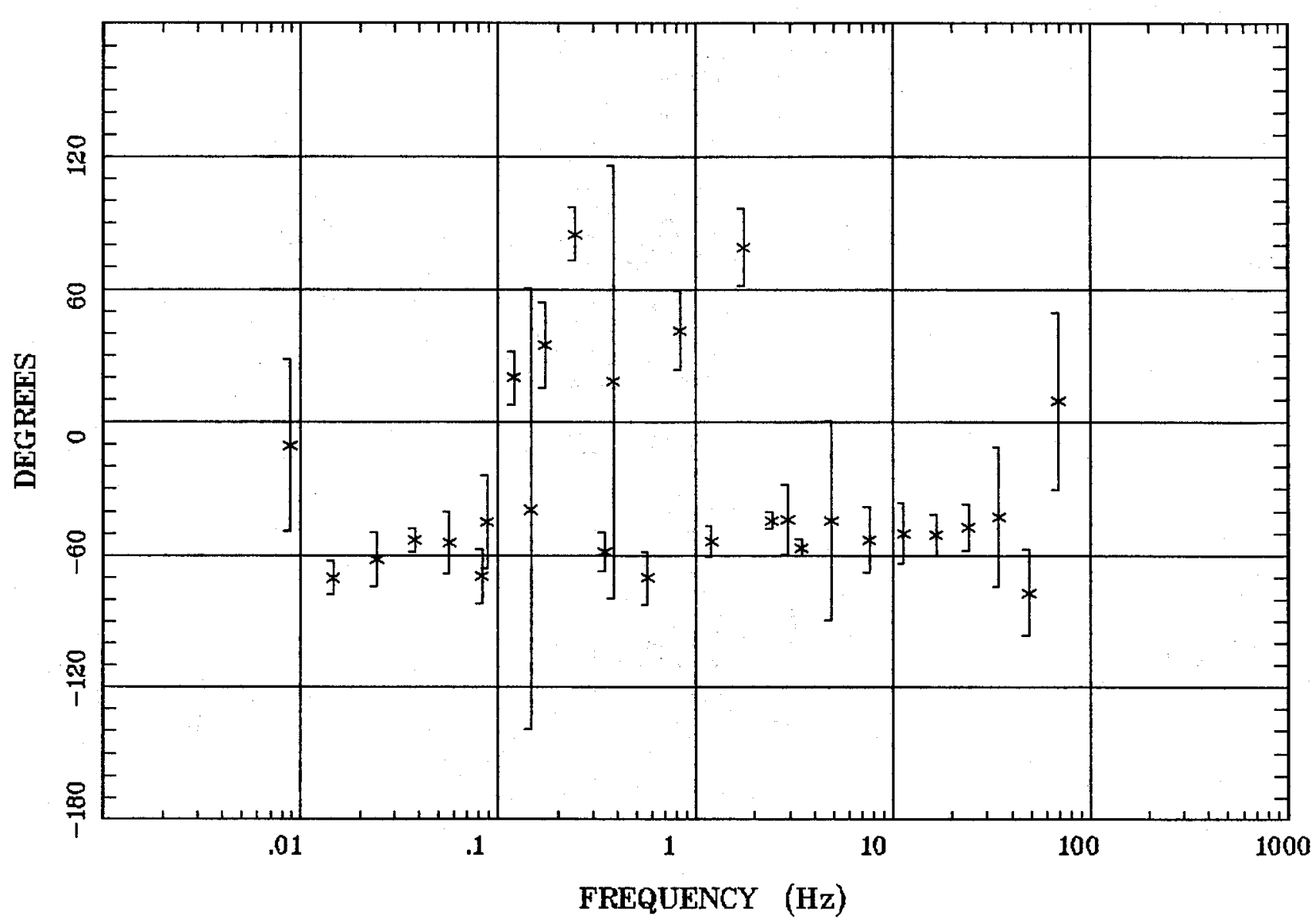

Client:

Remote: none

Acquired: 11:1 Jul 25, 2007 Survey Co:USGS
Rotation:

Filename: sl44m1.avg

Channels: Ch1 Ch2 Ch3 Ch4 Ch5 Ch3 Ch4 Plotted: 11:10 Nop 06, 2007

$<$ EMI - ElectroMagnetic Instruments > 
HzHx.x Coh HzHy.o

Alamosa, CO 100k

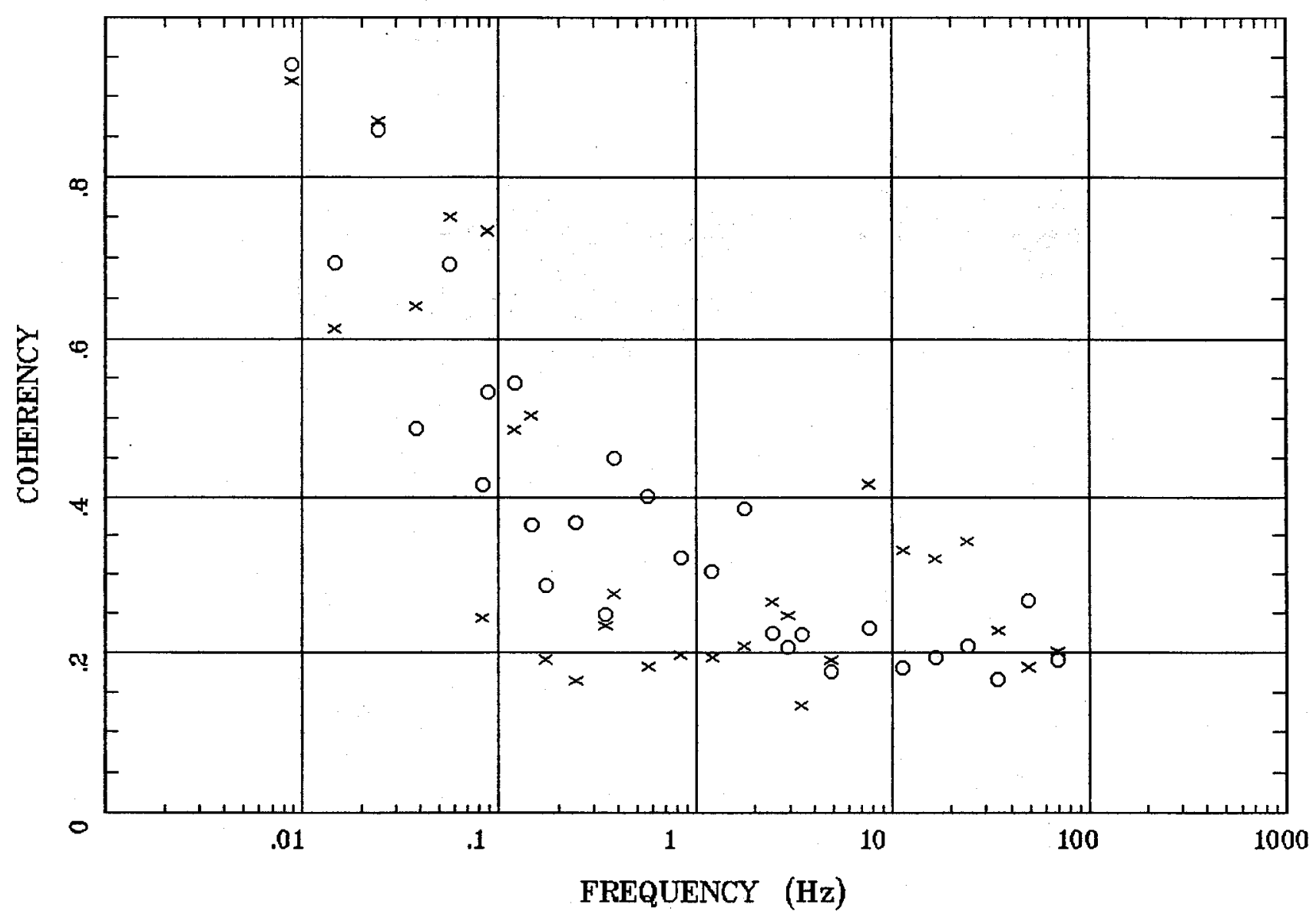

Client:

Remote: none

Acquired: 11:1 Jul 25, 2007

Survey Co:USGS
Rotation:

Filename: sl44m1.avg

Channels: Ch1 Ch2 Ch3 Ch4 Ch5 Ch3 Ch4 Plotted: 11:10 Nov 06, 2007

< EMI - ElectroMagnetic Instruments > 